\title{
Thermal-Fatigue and Thermo-Mechanical Equivalence for Transverse Cracking Evolution in Laminated Composites
}

\author{
Javier Cabrera Barbero \\ West Virginia University, jc0002@mix.wvu.edu
}

Follow this and additional works at: https://researchrepository.wvu.edu/etd

Part of the Computer-Aided Engineering and Design Commons, Engineering Mechanics Commons, Mechanics of Materials Commons, Polymer and Organic Materials Commons, Space Vehicles Commons, and the Structures and Materials Commons

\section{Recommended Citation}

Cabrera Barbero, Javier, "Thermal-Fatigue and Thermo-Mechanical Equivalence for Transverse Cracking Evolution in Laminated Composites" (2018). Graduate Theses, Dissertations, and Problem Reports. 3715. https://researchrepository.wvu.edu/etd/3715

This Dissertation is protected by copyright and/or related rights. It has been brought to you by the The Research Repository @ WVU with permission from the rights-holder(s). You are free to use this Dissertation in any way that is permitted by the copyright and related rights legislation that applies to your use. For other uses you must obtain permission from the rights-holder(s) directly, unless additional rights are indicated by a Creative Commons license in the record and/ or on the work itself. This Dissertation has been accepted for inclusion in WVU Graduate Theses, Dissertations, and Problem Reports collection by an authorized administrator of The Research Repository @ WVU.

For more information, please contact researchrepository@mail.wvu.edu. 


\title{
Thermal-FATigue AND Thermo-MeChaniCAL Equivalence for Transverse Cracking Evolution IN LAMinated Composites
}

\author{
Javier Cabrera Barbero \\ Dissertation submitted to the Benjamin M.Statler College of Engineering and Mineral \\ Resources at West Virginia University \\ in partial fulfillment of the requirements for the degree of \\ Doctor of Philosophy in Mechanical Engineering
}

\author{
Ever J. Barbero, Ph.D., Chair \\ Bruce S. Kang, Ph.D. \\ Eduardo M. Sosa, Ph.D. \\ Victor H. Mucino, Ph.D. \\ Adi Adumitroaie, Ph.D. \\ Department of Mechanical and Aerospace Engineering \\ Morgantown, West Virginia \\ December 6, 2018 \\ Keywords: Composite Materials, Damage, Matrix-Cracking, FEA, \\ Progressive Damage Models, Discrete Damage Mechanics model, \\ Thermal-fatigue, Thermal Cyclic Loads, Monotonic Cooling \\ Copyright 2018 Javier Cabrera Barbero
}




\begin{abstract}
Thermal-Fatigue and Thermo-Mechanical Equivalence for Transverse Cracking Evolution in Laminated Composites

Javier Cabrera Barbero

Carbon fiber reinforced plastics (CFRP) are potential materials for many aerospace and aeronautical applications due to their high specific strength/weight and a low coefficient of thermal expansion (CTE) resulting in a high long-term stability. Among candidate structures, the re-entry reusable launch vehicles (RLV), the fuel oxidant storage and transportation at cryogenic temperature, space satellites, and aircraft structure (frame, wings, etc...) can be highlighted. However, CFRP are prone to internal damage as a result of high residual stresses and thermal fatigue loading.
\end{abstract}

In this study, micro-cracking damage evolution in laminated composites subjected to monotonic cooling and thermal cyclic loads is developed through a theoretical model. Since matrix-damage predictions requires precise knowledge of the temperature-dependent properties, a detailed methodology to calculate the thermomechanical properties for both matrix and fibers of interest is included. Damage initiation and evolution is studied firstly under quasi-static cooling. The temperature dependence of the critical energy release rate (ERR) is also analyzed. Thermal fatigue of laminated composites is assessed based on low-cycle fatigue tests and the damage mechanisms involved are studied. A Master Paris's law is developed to predict matrix fatigue resistance as function of number of cycles regardless of layup and thermal ratio for both, low and high-cycle tests. Due to physical barriers that implies to perform a complete high-cycle thermal fatigue test, a methodology to simulate a thermal fatigue test using equivalent mechanical cyclic loads is developed to use the former as surrogate from later. 


\section{Acknowledgments}

I would like to express my sincere appreciation and gratitude to my advisor Dr. Barbero during all these learning years. It has been a privilege to work closely and learn from your lessons and advices for entire life. My gratitude to all my committe members for reading this Doctorate dissertation. My sincere thanks to Dr. Sosa for his time and support these years. My thanks to WVU and Dr.Mucino for the help received from faculty, particularly for the financial support as teaching assistant during last year.

I would like to express my sincere aprecciation for my friend Alex Mejia. It has been a privilage to meet someone with great human qualities and with whom I have shared fantastic moments.

Lastly, I would like to express my warmest sincere affection and love to my girlfriend, Diana Estaire for her unconditional support, goodness, patience, and love during this last year and half. 


\section{Dedication}

To my parents. Thank you for teaching me the most important life values. And thank you so much, for your encouragement, support and love all this time. 


\section{Contents}

1 Introduction $\quad 1$

1.1 Motivation . . . . . . . . . . . . . . . . . . 1

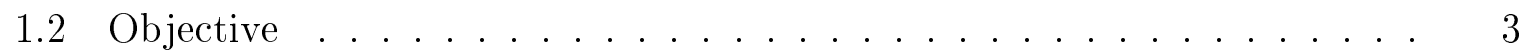

1.3 Literature Review . . . . . . . . . . . . . . . 5

1.3.1 Fatigue damage mechanisms ............ 5

1.3.2 Experimental characterization: S-N and Fatigue-Life Diagrams . . 8

1.3.3 Influence of constituents and laminate stacking sequence on S-N diagrams of composite materials . . . . . . . . . . 12

1.3.4 Fatigue of composites subjected to compression loading . . . . . 16

1.3.5 Modeling of Mechanical-Fatigue Damage . . . . . . . . . . 17

1.3.6 Modeling of Thermal-Fatigue Damage . . . . . . . . . 25

2 Discrete Damage Mechanics $\quad 31$

2.1 Theory formulation . . . . . . . . . . . . . . . . 32

2.2 Shear Lag Equations in Matrix Form . . . . . . . . . . . . . . 33

2.3 Solution of the Equilibrium Equation . . . . . . . . . . . . 34

2.4 Boundary Conditions for $\Delta T=0 \ldots \ldots \ldots \ldots \ldots \ldots$

2.4 .1 (a) Stress-free at the Cracks Surfaces . . . . . . . . . . . 36

2.4 .2 (b) External Loads . . . . . . . . . . . . . . 36

2.4 .3 (c) Homogeneous Displacements . . . . . . . . . . . . 36

2.5 Boundary Conditions for $\Delta T \neq 0 \ldots \ldots \ldots \ldots \ldots$

2.6 Degraded Laminate Stiffness and CTE . . . . . . . . . . . . 37

2.7 Degraded Lamina Stiffness . . . . . . . . . . . . . . . . . 38

2.8 Damage Activation Function . . . . . . . . . . . . . . . . 40

2.9 Solution Algorithm . . . . . . . . . . . . . . . . . . . 41

2.9 .1 Lamina Iterations . . . . . . . . . . . . . . . . . . . . . 42

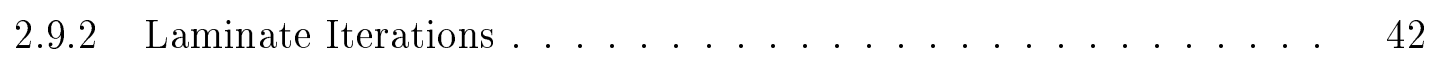


3 Temperature-dependent Properties 43

3.1 Constituent Properties . . . . . . . . . . . . . . . . . 45

3.1 .1 Epoxy $3501-6 \ldots \ldots \ldots \ldots \ldots$

$3.1 .2 \quad$ Epoxy $934 \ldots \ldots \ldots \ldots \ldots \ldots$

3.1.3 Epoxy ERL $1962 \ldots \ldots \ldots \ldots$. . . . . . . . . . . . . 49

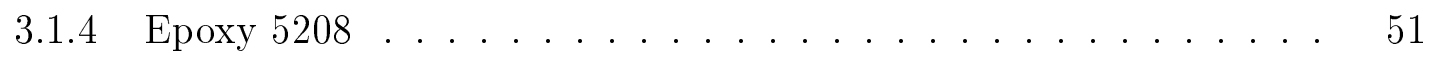

3.1 .5 AS4 Fiber ........................... 51

3.1 .6 T300 Fiber ........................ 53

3.1 .7 P75 Fiber ........................... 53

3.1 .8 Summary Constituent Properties . . . . . . . . . . 53

3.2 Coefficients of Thermal Expansion . . . . . . . . . . . . . . 54

3.2.1 Material System: T300/5208 . . . . . . . . . . . 56

3.2.2 Material System: P75/934 and T300/934 . . . . . . . 57

3.2.3 Material System: P75/1962 . . . . . . . . . . . 58

3.2.4 Material System: AS4/3501-6 . . . . . . . . . . 58

3.2.5 Summary CTE . . . . . . . . . . . . . . . 59

3.3 Finite Element Analysis . . . . . . . . . . . . . . . . 60

4 Monotonic cooling $\quad 66$

4.1 Critical Energy Release Rates . . . . . . . . . . . . . . 67

4.2 Results . . . . . . . . . . . . . . . . . . . . 72

4.2.1 P75/934 Carbon-Epoxy . . . . . . . . . . . . 72

$4.2 .2 \quad$ P75/ERL1962 Carbon-Epoxy _. . . . . . . . . . . 84

4.2 .3 AS4/3501-6 Carbon-Epoxy . . . . . . . . . . . . 87

4.2.4 T300/5208 Carbon-Epoxy . . . . . . . . . . . . . 90

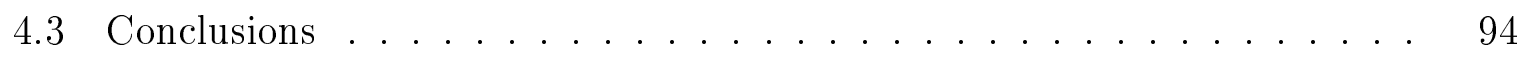

4.4 Conclusions . . . . . . . . . . . . . . . . . 96

5 Thermal Fatigue of Laminated Polymer-Matrix Composites 98

5.1 Materials and Methods . . . . . . . . . . . . . . . . 99

5.2 Methodology . . . . . . . . . . . . . . . . . 101

5.3 Separation of Variables . . . . . . . . . . . . . . . . . . 102

5.4 Fracture mechanisms of polymers at low temperature . . . . . . . . . 104

5.5 Mode II ERR effect . . . . . . . . . . . . . . . . . . . . . 107

5.6 Free-edge stress analysis . . . . . . . . . . . . . . . 110

5.7 Temperature range effect . . . . . . . . . . . . . . . 116

5.8 Paris Law . . . . . . . . . . . . . . . . . . . 117

5.9 Thermal Ratio . . . . . . . . . . . . . . . . . 122 
5.10 Fatigue Resistance . . . . . . . . . . . . . . . . . 124

5.11 Conclusions . . . . . . . . . . . . . . . . . 133

6 Thermo-Mechanical Equivalence $\quad 135$

6.1 Material System . . . . . . . . . . . . . . . . 136

6.2 Methods . . . . . . . . . . . . . . . . 136

6.2.1 Quasi-static cooling: $N=1 \ldots \ldots \ldots$. . . . . . . . . . . . . . . . . . . .

6.2.2 Thermal cycling loads: $N>1 \ldots \ldots \ldots . \ldots \ldots$

6.3 Biaxial Thermo-Mechanical Equivalence . . . . . . . . . . . . . . 141

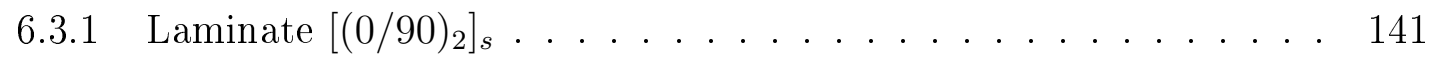

6.3 .2 Laminate $[0 / \pm 45 / 90]_{s} \ldots \ldots \ldots \ldots \ldots$. . . . . . . . . . 144

6.4 Unialxial Thermo-Mechanical Equivalence . . . . . . . . . . . . 146

6.4.1 Thermo-Mechanical Equivalence: Middle lamina . . . . . . . . . 147

6.4.2 Equivalent mechanical thickness . . . . . . . . . . . . . . . 149

6.5 Thermo-mechanical equivalence . . . . . . . . . . . . . . . . 154

6.5.1 Quasi-static Cooling Using $T_{r}=T_{\max } \ldots \ldots \ldots . \ldots . . \ldots 154$

6.5.2 Thermal Fatigue Using $T_{r}=T_{\max } \ldots \ldots \ldots \ldots$

6.6 Conclusions . . . . . . . . . . . . . . . . . . 162

7 Conclusions and Future work 163

7.0 .1 Future work . . . . . . . . . . . . . . 166

$\begin{array}{ll}\text { A Supplemental material } & 168\end{array}$

A.1 LaminaName . . . . . . . . . . . . . . . . . . 170

$\mathrm{A} .2 \mathrm{PBC} \ldots \ldots \ldots \ldots \ldots \ldots \ldots \ldots$

A.3 ParameterIntegrator . . . . . . . . . . . . . . . . 182

A.4 ExcelProperties . . . . . . . . . . . . . . . . . 184

A.5 Epsilonrecover . . . . . . . . . . . . . . . . 185

A.6 3DFreeEdge . . . . . . . . . . . . . . . 186

A.7 3DFreeEdge-2 . . . . . . . . . . . . . . . . . . 194

A.8 PBC-FreeEdge . . . . . . . . . . . . . . . . . 199

$\begin{array}{ll}\text { Bibliography } & 202\end{array}$ 


\section{List of Figures}

1.1 Examples of thermal cyclic loads in a space environment. . . . . . . . . 1

1.2 Average cycle of commercial aircrafts. . . . . . . . . . . . . . 2

1.3 A comparison between accelerated vs. real time thermal cycle for LEO [1]. 3

1.4 Transverse cracking in $\left[0 / \pm 70_{4} / 0_{0.5}\right]_{s}$ laminate of E-glass/epoxy subjected to $0.7 \%$ of strain $[2, \mathrm{Ch} .9] \ldots \ldots \ldots \ldots$

1.5 Damage mechanisms in unidirectional composites subjected to fatigue loads in fiber direction: (a) fiber breakage, (b) dispersed transverse cracks and (c) interfacial shear-normal failure. . . . . . . . . . . . 6

1.6 Primary and Secondary cracks on laminate composites subjected to fatigue loads. . . . . . . . . . . . . . . . . 7

1.7 Fatigue-life diagram for unidirectional composites under tensile loading parallel to fibers. . . . . . . . . . . . . . . . 9

1.8 Random fiber breakage in unidirectional composites under tensile loading. 9

1.9 Region I and II of the fatigue life diagram. . . . . . . . . . . . . . . 10

1.10 Two types of CFL diagrams based on Goodman and Bell-shape theories. 11

1.11 Fatigue-life diagram for glass and carbon epoxy laminates under tensile loading. . . . . . . . . . . . . . . . . . . 12

1.12 Fatigue-life diagram for off-axis and angle-plied laminates under tensile loading. . . . . . . . . . . . . . . . . . . 13

1.13 Fatigue-life diagram for cross-plied laminates of graphite-epoxy [3]. . . . 14

1.14 Fatigue-life diagram for a $[0 / \pm 45 / 90,-45]_{s}$ graphite-epoxy laminate [4]. 14

1.15 Baseline fatigue life-diagram modified according to a multiaxial state [5]. $\quad 15$

1.16 Fatigue behavior of fiber bundles subjected to cyclic loadings for both, glass-and carbon-fibers. . . . . . . . . . . . . . 18

1.17 Stiffness degradation stages on composites due to fatigue cyclic loadings [6]. 20

1.18 Strength distribution associated with specific residual strength relation [7]. 21

1.19 Interpretation of the residual strength equation subjected to tension-tension fatigue loads $[6] \ldots \ldots \ldots$. . . . . . . . . . . . . . . . . 24 
1.20 X-ray images for two material systems containing multiple transverse cracks at different orientations. . . . . . . . . . . . . . 26

1.21 Crack density vs. thermal cycle data for $\left[0_{2} / 90_{2}\right]_{s}$ and $\left[90_{2} / 0_{2}\right]_{s}$ T300/5208 laminate between -156 and $300^{\circ}[8]$. . . . . . . . . . 27

1.22 Strain energy release rate as function of crack size. . . . . . . . . . . 28

1.23 A Paris law for transverse crack density growth in G40-800/5620 under thermal and mechanical fatigue [9]. . . . . . . . . . . . . . 29

2.1 Representative volume element for DDM. . . . . . . . . . . . . . . 32

3.1 Estimated temperature-dependent modulus $E_{m}$ (top) and CTE (bottom) for Epoxy 3501-6 extrapolated to the whole temperature range of study $[-200,180 \mathrm{C}] \ldots \ldots \ldots \ldots \ldots \ldots \ldots$

3.2 Back calculation method to obtain the temperature-dependent matrix properties at any temperature $T_{i} \ldots \ldots \ldots \ldots$. . . . . . . 50

3.3 Estimated temperature-dependent modulus (top) and CTE (bottom) for Epoxy 934 and ERL 1962. . . . . . . . . . . . . . . . . .

3.4 Estimated temperature-dependent modulus (top) and CTE (bottom) for Epoxy 5208. . . . . . . . . . . . . . . . . . . 52

3.5 Back calculation method to obtain the fiber properties using set of experimental data at various temperatures $(\mathrm{N}) . \ldots \ldots 52$

3.6 Comparison between predicted and experimental data of transverse modulus $E_{2}$ for P75/934, AS4/3501-6, T300/934, and T300/5208 lamina. . .

3.7 Comparison between predicted and experimental data of transverse modulus $G_{12}$ for P75/934, AS4/3501-6, T300/934, and T300/5208 lamina. 55

3.8 Back calculation method to obtain the fiber and matrix CTE values. . . 57

3.9 Back calculation method to obtain the matrix CTE at any temperature $\left(T_{i}\right) .58$

3.10 Back calculation method to obtain the transverse CTE of the fiber from transverse lamina CTE as function of temperature.

3.11 Comparison of transverse lamina CTE $\alpha_{2}$ predicted with Levin's model (3.6) vs. experimental data for T300/934 with $V_{f}=0.57$, AS4/3501-6 with $V_{f}=0.67$, and P75/1962 with $V_{f}=0.52 \ldots \ldots \ldots$

3.12 Comparison between longitudinal lamina CTE $\alpha_{1}$ predicted with Levin's model (Eq. 3.6) and experimental data for T300/5208 with $V_{f}=0.68$ and P75/934 with $V_{f}=0.51 . \ldots \ldots \ldots \ldots$

3.13 Comparison micromechanics and FEA predictions of tangent and secant longitudinal CTE $\alpha_{1}$ for P75/934 $\left(V_{f}=0.51\right)$ and T300/5208 $\left(V_{f}=0.68\right)$. 
3.14 Comparison micromechanics and FEA predictions of tangent and secant transverse CTE $\alpha_{2}$ for P75/934 $\left(V_{f}=0.51\right)$ and T300/5208 $\left(V_{f}=0.68\right)$.

3.15 Comparison between micromechanics and FEA predictions of tangent and secant transverse CTE $\alpha_{2}$ for P75/1962 ( $\left.V_{f}=0.52\right)$, and AS4/3501-6 $\left(V_{f}=0.67\right)$.

$4.1 G_{I c}$ vs. temperature for P75/934 $(V f=0.65), \mathrm{P} 75 / 1962(V f=0.52)$, and AS4/3501-6 $\left(V_{f}=0.64\right)$. Two outliers data, at $-18 \mathrm{C}$ for AS4/3501-6 and at $-21 \mathrm{C}$ for P75/1962, not used. . . . . . . . . . . .

4.2 Crack density data vs. Temperature using middle $90_{2}^{\circ}$ lamina for laminate $\left[0_{2} / 90_{2}\right]_{s}$ P75/934 and interior lamina $90_{2}^{o}$ for laminate $\left[0_{2} / 45_{2} / 90_{2} /-45_{2}\right]_{s}$ $\mathrm{P} 75 / 1962$.

4.3 Crack density data vs. Temperature using interior $90_{4}^{\circ}$ lamina for laminate $\left[0_{4} / 45_{4} / 90_{4} /-45_{4}\right]_{s} \mathrm{AS} 4 / 3501-6$ and middle $90_{2}^{o}$ lamina for $\left[0_{2} / 90_{2}\right]_{s}$ T300/5208.

4.4 Crack density prediction vs. temperature for monotonic cooling of $[0 / \pm$ 45/90]s P75/934.

4.5 Crack density prediction vs. temperature for monotonic cooling of $[0 / 90 / \pm$ $45]_{s}$ P75/934.

4.6 Crack density prediction vs. temperature for monotonic cooling of $[0 / 45 / 90 /-$ $45]_{s}$ P75/934.

4.7 A comparison between crack density prediction vs. interior and edge $-45^{\circ}$ lamina data during cooling in $[0 / 45 / 90 /-45]_{s}$ P75/934 . . . . . . . . .

4.8 X-Ray photograph for laminate $\left[0_{2} / 90_{2}\right]_{s}$ P75/ERL1962 subjected to \pm 250 $\mathrm{F}$ and 3500 cycles [10]. Lines represent cracks for $0^{\circ}$ and $90^{\circ}$ laminas. . .

4.9 Crack density predictions vs. temperature for monotonic cooling of $[0 / 90 / \pm$ $45]_{s},[0 / \pm 45 / 90]_{s}$, and $[0 / 45 / 90 /-45]_{s}$ P75/934 . . . . . . . 77

4.10 Crack density predictions vs. temperature for monotonic cooling of $\left[\mathrm{O}_{2} / \pm\right.$

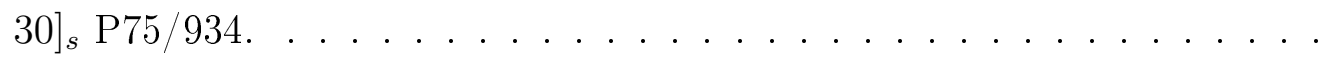

4.11 Evolution of damage activation function g and ERRs $G_{I}, G_{I I}$ during monotonic cooling for $\left[0_{2} / \pm 30\right]_{s}$ P75/934 laminate. . . . . . . . . . .

4.12 Tangent laminate CTE vs. temperature for monotonic cooling of $\left[0_{2} / 90_{2}\right]_{\mathrm{s}}$

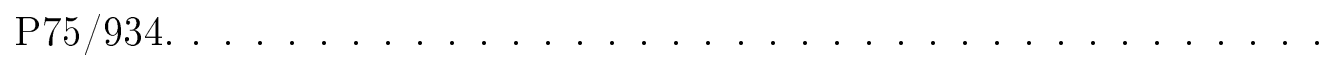

4.13 Tangent laminate CTE vs. temperature for monotonic cooling of $\left[0_{2} / \pm 30\right]_{s}$ P75/934. .

4.14 Tangent laminate CTE vs. temperature for monotonic cooling of $[0 / \pm$ $45 / 90]_{s}$ P75/934. 
4.15 Evolution of tangent laminate CTE as function of number of sub-laminas vs. temperature for $\left[0_{n} / 90_{n}\right]_{s}$ P75/934, with $n=1,2,4 \ldots \ldots . \ldots 3$

4.16 Crack density predictions vs. temperature for monotonic cooling of $\left[0_{2} / 45_{2} / 90_{2} /-\right.$ $\left.45_{2}\right]_{s}$ P75/ERLX1962. . . . . . . . . . . . . 85

4.17 A comparison between crack density prediction and experimental data for $90_{2}$ vs. temperature in $\left[0_{2} / 45_{2} / 90_{2} /-45_{2}\right]_{s}$ P75/ERLX1962 laminate. . 85

4.18 Tangent laminate CTE vs. temperature for monotonic cooling of $\left[0_{2} / 45_{2} / 90_{2} /-\right.$ $\left.45_{2}\right]_{s}$ P75/1962. . . . . . . . . . . . . 86

4.19 Crack density predictions vs. temperature for monotonic cooling of $\left[0_{4} / 45_{4} / 90_{4} /-\right.$ $\left.45_{4}\right]_{s}$ AS4/3501-6. Top: 904. Middle: 454. Bottom: $-45_{8} \ldots \ldots$. . . . 88

4.20 Tangent laminate CTE vs. temperature for monotonic cooling of $\left[0_{4} / 45_{4} / 90_{4} /-\right.$ $\left.45_{4}\right]_{s}$ AS4/3501-6. . . . . . . . . . . . . . . . 90

4.21 A comparison of laminate CTE $\alpha_{y}$ vs. temperature for monotonic cooling of $\left[0_{n} / 45_{n} / 90_{n} /-45_{n}\right]_{s}$ AS4/3501-6 with $n=1,2,4 \ldots \ldots \ldots$

4.22 Crack density prediction in $90^{\circ}$ and $0^{\circ}$ laminas vs. monotonic cooling with different LSS $\left[0_{3} / 90\right]_{s},\left[0_{2} / 90_{2}\right]_{s}$ and $\left[0 / 90_{3}\right]_{s}$ T300/5208. Top: predicted and experimental $\lambda_{90}$. Bottom: predicted $\lambda_{0} \ldots \ldots \ldots$

4.23 Tangent laminate CTE vs. temperature for monotonic cooling of $\left[0_{2} / 90_{2}\right]_{s}$ T300/5208. Note: $\alpha_{x y}=0 \ldots \ldots \ldots \ldots . \ldots . \ldots$

4.24 Transverse stresses $\sigma_{22}$ at $50 \mathrm{C}$ in different laminas with same lay-up given by FEA $[11] \ldots \ldots \ldots \ldots \ldots \ldots \ldots$. . . . . . . . . . . . . . . . . . . . . . .

5.1 Thermal strains for a cross-ply laminate during cooling from SFT to $T_{\min }$. Positive and negative arrows represent traction and compression, respectively. . . . . . . . . . . . . . . . . .

5.2 Temperature-dependent properties of epoxy 1962 in the range $[-156,121$ $\left.{ }^{\circ} C\right][12] . \ldots \ldots \ldots \ldots$

5.3 Fatigue resistance $f(N)$ as function of number of cycles for P75/1962 $\left[(0 / 90)_{2}\right]_{S}$ with $R_{T}=-156 / 121$. Experimental data is collected for middle $90_{2}^{o}$ lamina. . . . . . . . . . . . . . . . .

5.4 Fatigue resistance as function of number of cycles for P75/1962 [0/ \pm $45 / 90]_{S}$ with $R_{T}=-156 / 121$. Experimental data is collected for middle $90_{2}^{\circ}$ and interior $\pm 45^{\circ}$ laminas. . . . . . . . . . . .

5.5 ERR $G_{I}$ and $G_{I}$ vs. temperature during one thermal cycle for P75/1962 $[0 / \pm 45 / 90]_{S}$ in the range $\left[-156,121^{\circ} \mathrm{C}\right] . \mathrm{SFT}$ is $177^{\circ} \mathrm{C}[12] \ldots \ldots$

5.6 Representative 2D displacement crack field to illustrate the double thickness effect. 
5.7 Transverse stress $\sigma_{22}$ vs. temperature during one thermal cycle for P75/1962 $[0 / \pm 45 / 90]_{S}$ in the range $\left[-156,121^{\circ} \mathrm{C}\right] . \mathrm{SFT}$ is $177^{\circ} \mathrm{C}[12] \ldots \ldots$

5.8 Representative thermal fatigue test with time in the temperature range $\left[-156,121^{\circ} C\right]$. The thermal stresses are calculated from SFT at $177^{\circ} C$. 112

5.9 Front and side draw views from 3D finite element modelling. . . . . . . . 113

5.10 Longitudinal, transverse and shear free-edge stresses at $-156^{\circ} C$ for P75/1962 and LSS: $\left[(0 / 90)_{2}\right]_{S} . \ldots \ldots \ldots \ldots \ldots$

5.11 Transverse and shear free-edge stresses at $-156^{\circ} \mathrm{C}$ for P75/1962 and LSS: $[0 / \pm 45 / 90]_{S}$ and $[0 / 45 / 90 /-45]_{S}$ denoted by symbol $(*) \ldots . . .$.

5.12 Fatigue resistance $f(N)$ as function of number of cycles for P75/1962 $[0 / 45 / 90 /-45]_{S}$ with $R_{T}=-156 / 121, R_{T}=-101 / 66$, and $R_{T}=-46 / 10.118$

5.13 Crack density evolution vs. thermal cycling at different temperature ranges: $\left[-156,121^{\circ} \mathrm{C}\right],\left[-101,66^{\circ} \mathrm{C}\right]$, and $\left[-46,10^{\circ} \mathrm{C}\right]$.

$5.14 \mathrm{ERR} G_{I}$ as function of crack density $\lambda$ at two thermal ratios $R_{T}$ in the range $\left[-156,121^{\circ} \mathrm{C}\right]$ and $\left[-44,10^{\circ} \mathrm{C}\right]$ for $\left[(0 / 90)_{2}\right]_{S} \mathrm{P} 75 / 1962$.

5.15 Transverse microcrack density growth rate $(d \lambda / d N)$ as function of ERR range $\Delta G_{I}$ for P75/1962 during thermal fatigue with $R_{T}=-156 / 121$. The laminate layups are $\left[(0 / 90)_{2}\right]_{S},[0 / \pm 45 / 90]_{S}$ and $[0 / 45 / 90 /-45]_{S}$. Experimental crack density $\lambda$ belong to $90_{2}^{\circ}$ and $-45_{2}^{\circ}$ laminas respectively. 121

5.16 Transverse microcrack density growth rate $(d \lambda / d N)$ as function of ERR range $\Delta G_{I}$ for $\mathrm{P} 75 / 1962[0 / 45 / 90 /-45]_{S}$ during thermal fatigue with $R_{T}=-156 / 121, R_{T}=-101 / 66$, and $R_{T}=-46 / 10$. Experimental crack density $\lambda$ belong to thicker $-45_{2}^{o}$ laminas in both cases. . . . . . . . .

5.17 Comparison between master and regular Paris's law plot for P75/1962 $[0 / 45 / 90 /-45]_{S}$ with $R_{T}=-156 / 121, R_{T}=-101 / 66$, and $R_{T}=-46 / 10$. Experimental crack density $\lambda$ belong to $-45_{2}^{o}$ laminas in both cases. . . . 123

5.18 Thermal fatigue prediction using a modified Paris's law for a speciifc material system.

$5.19 G_{I}$ vs. crack density for $N=1$ and $T_{\min }=-156$ during cooling for $\left[(0 / 90)_{2}\right]_{s}$ P75/1962 with $R_{T}=-156 / 121 . \ldots \ldots \ldots$. . . . . .

5.20 Fatigue resistance $\mathrm{f}(\mathrm{N})$ for $\left[(0 / 90)_{2}\right]_{s}$ P75/1962 with $R_{T}=-156 / 121 \mathrm{ob}-$ tained through Paris's law and DDM model as illustrated in Figure 5.18.

5.21 Fatigue resistance $\mathrm{f}(\mathrm{N})$ for $\left[0_{2} / 90_{3}\right]_{s}$ P75/1962 with $R_{T}=-156 / 121$ obtained through Paris's law and DDM model as illustrated in Figure 5.18.

5.22 Fatigue resistance $\mathrm{f}(\mathrm{N})$ for $\left[0_{2} / \pm 45 / 90_{3}\right]_{s}$ P75/1962 with $R_{T}=-60 / 50$ obtained through Paris's law and DDM model as illustrated in Figure 5.18. 130 
5.23 Crack density evolution $\lambda$ vs. number of cycles $N$ for $\left[(0 / 90)_{2}\right]_{s}$ P75/1962 with $R_{T}=-156 / 121$ calculated with DDM and $f(N)$ reported in (5.16) and Table 5.3. Experimental data only available for middle $90_{2}^{\circ}$ lamina and low-cycle fatigue. . . . . . . . . . . . . . . .

5.24 Crack density evolution $\lambda$ vs. number of cycles $N$ for $\left[0_{2} / 90_{3}\right]_{s}$ P75/1962 with $R_{T}=-156 / 121$ calculated with DDM and $f(N)$ reported in (5.16) and Table 5.3. No experimental data is available to compare. . . . . . . .

5.25 Crack density evolution $\lambda$ vs. number of cycles $N$ for $\left[0_{2} / \pm 45 / 90_{3}\right]_{s}$ P75/1962 with $R_{T}=-60 / 50$ calculated with DDM and $f(N)$ reported in (5.16) and Table 5.3. No experimental data is available to compare. . . .

6.1 Proposed methodology to evaluate thermal fatigue through equivalent mechanical strains. Left side: Thermal fatigue. Right side: Mechanical fatigue.137

6.2 Comparison between crack density evolution $\lambda^{\text {th }}$ for $R_{T}=-156 / 121$ vs. crack density evolution $\lambda^{m e}$ subjected to equivalent mechanical strains $\epsilon_{T}^{m e}$ at RT for laminate $\left[(0 / 90)_{2}\right]_{s}$ P75/1962 in the range $\left[T_{\max }, T_{\min }\right] \ldots$. . .

6.3 Comparison between ERR $G_{I}^{t h}$ for $R_{T}=-156 / 121$ vs. $G_{I}^{m e}$ at RT subjected to equivalent mechanical strains $\epsilon_{T}^{m e}$ for laminate $\left[(0 / 90)_{2}\right]_{s}$ P75/1962 in the range $\left[T_{\min }, T_{\text {max }}\right] \ldots \ldots \ldots \ldots \ldots \ldots$

6.4 Comparison between longitudinal stress $\sigma_{1}^{\text {th }}$ for $R_{T}=-156 / 121$ vs. $\sigma_{1}^{m e}$ at RT subjected to equivalent mechanical strains $\epsilon_{T}^{m e}$ for laminate $\left[(0 / 90)_{2}\right]_{s}$ P75/1962 in the range $\left[T_{\min }, T_{\max }\right] \ldots \ldots \ldots \ldots$

6.5 Comparison between transverse stress $\sigma_{2}^{\text {th }}$ for $R_{T}=-156 / 121$ vs. $\sigma_{2}^{m e}$ at RT subjected to equivalent mechanical strains $\epsilon_{T}^{m e}$ for laminate $\left[(0 / 90)_{2}\right]_{s}$ P75/1962 in the range $\left[T_{\min }, T_{\max }\right] \ldots \ldots \ldots \ldots \ldots$

6.6 Evolution of equivalent mechanical strains $\epsilon_{T}^{m e}$ with $T$ for laminate $\left[(0 / 90)_{2}\right]_{s}$ $\mathrm{P} 75 / 1962$ in the range $\left[-156,121^{\circ} C\right]$. Reference temperature is set to $R T=23^{\circ} C \ldots \ldots \ldots \ldots \ldots \ldots \ldots \ldots \ldots \ldots \ldots \ldots \ldots \ldots \ldots$

6.7 A comparison between crack density evolution $\lambda^{\text {th }}$ with $R_{T}=-156 / 121$ vs. crack density evolution $\lambda^{m e}$ subjected to equivalent mechanical strains $\epsilon_{T}^{m e}$ at RT for laminate $[0 / \pm 45 / 90]_{s} \mathrm{P} 75 / 1962$ in the range $\left[T_{\min }, T_{\max }\right]$.

6.8 A comparison between transverse stress $\sigma_{2}^{\text {th }}$ with $R_{T}=-156 / 121$ vs. $\sigma_{2}^{m e}$ at RT subjected to equivalent mechanical strains $\epsilon_{i}^{m e}$ for laminate $[0 / \pm$ $45 / 90]_{s}$ P75/1962 in the range $\left[T_{\min }, T_{\max }\right] \ldots \ldots \ldots \ldots \ldots$. . . . . 147

6.9 Comparison between crack density evolution $\lambda_{90_{2}}^{t h}$ for $R_{T}=-156 / 121$ vs. crack density evolution $\lambda_{90_{2}}^{m e}$ subjected to uniaxial mechanical strains $\epsilon_{x}^{m e}$ at RT for laminate $\left[(0 / 90)_{2}\right]_{s} \mathrm{P} 75 / 1962$ in the range $\left[T_{\min }, T_{\max }\right] \ldots \ldots$. 
6.10 Comparison between $\operatorname{ERR} G_{I}^{t h}$ with $R_{T}=-156 / 121$ vs. $G_{I}^{m e}$ at RT for middle $90_{2}^{\circ}$ lamina subjected to equivalent mechanical strains $\epsilon_{x}^{m e}$ for laminate $\left[(0 / 90)_{2}\right]_{s}$ P75/1962 in the range $\left[T_{\min }, T_{\max }\right] \ldots \ldots \ldots \ldots$

6.11 Comparison between longitudinal stress $\sigma_{1}^{\text {th }}$ with $R_{T}=-156 / 121$ vs. $\sigma_{1}^{m e}$ at RT for middle $90_{2}^{\circ}$ lamina subjected to equivalent uniaxial mechanical strains $\epsilon_{x}^{m e}$ for laminate $\left[(0 / 90)_{2}\right]_{s} \mathrm{P} 75 / 1962$ in the range $\left[T_{\min }, T_{\max }\right]$. .

6.12 Comparison between transverse stress $\sigma_{2}^{\text {th }}$ with $R_{T}=-156 / 121$ vs. $\sigma_{2}^{m e}$ at RT for middle $90_{2}^{\circ}$ lamina subjected to equivalent uniaxial mechanical strains $\epsilon_{x}^{m e}$ for laminate $\left[(0 / 90)_{2}\right]_{s}$ P75/1962 in the range $\left[T_{\min }, T_{\max }\right]$. .

6.13 Comparison between crack density evolution $\lambda_{90_{2}}^{t h}$ for $R_{T}=-156 / 121$ vs. crack density evolution $\lambda_{90_{70} \%}^{m e}$ subjected to uniaxial equivalent mechanical strains $\epsilon_{x}^{m e}$ at RT for laminate $\left[(0 / 90)_{2}\right]_{s}$ P75/1962 in the range $\left[T_{\min }, T_{\text {max }}\right] .152$

6.14 Comparison between $\operatorname{ERR} G_{I}^{t h}$ for $R_{T}=-156 / 121$ vs. $G_{I}^{m e}$ at RT subjected to equivalent mechanical thickness $t_{m e}=0.70 t_{k}$ and strains $\epsilon_{x}^{m e}$ at RT for laminate $\left[(0 / 90)_{2}\right]_{s}$ P75/1962 in the range $\left[T_{\min }, T_{\max }\right] . \ldots$.

6.15 Comparison between longitudinal stress $\sigma_{1}^{\text {th }}$ for $R_{T}=-156 / 121$ vs. $\sigma_{1}^{m e}$ at RT subjected to uniaxial equivalent mechanical strains $\epsilon_{x}^{m e}$ at RT and $t_{m e}=0.70 t_{k}$ for laminate $\left[(0 / 90)_{2}\right]_{s} \mathrm{P} 75 / 1962$ in the range $\left[T_{\min }, T_{\max }\right]$.

6.16 Comparison between transverse stress $\sigma_{2}^{\text {th }}$ for $R_{T}=-156 / 121$ vs. $\sigma_{2}^{m e}$ subjected to uniaxial equivalent mechanical strains $\epsilon_{x}^{m e}$ at RT with $t_{m e}=$ $0.70 t_{k}$ for laminate $\left[(0 / 90)_{2}\right]_{s} \mathrm{P} 75 / 1962$ in the range $\left[T_{\min }, T_{\max }\right] \ldots$. . .

6.17 Comparison between ERR $G_{I}^{\text {th }}$ for $R_{T}=-40 / 30$ vs. $G_{I}^{m e}$ at $T_{r}=30^{\circ} \mathrm{C}$ subjected to equivalent mechanical strains $\epsilon_{x}^{m e}$ with $t_{m e}=0.87 t_{k}$ for laminate $\left[(0 / 90)_{2}\right]_{s}$ P75/1962 in the range $\left[T_{\min }, T_{\max }\right] \ldots \ldots \ldots$

6.18 Comparison between longitudinal stress $\sigma_{1}^{\text {th }}$ for $R_{T}=-40 / 30$ vs. $\sigma_{1}^{m e}$ at $T_{r}=30^{\circ} \mathrm{C}$ subjected to equivalent mechanical strains $\epsilon_{x}^{m e}$ with $t_{m e}=0.87 t_{k}$ for laminate $\left[(0 / 90)_{2}\right]_{s} \mathrm{P} 75 / 1962$ in the range $\left[T_{\min }, T_{\max }\right] \ldots \ldots \ldots$

6.19 Comparison between transverse stress $\sigma_{2}^{\text {th }}$ for $R_{T}=-40 / 30$ vs. $\sigma_{2}^{m e}$ at $T_{r}=30^{\circ} \mathrm{C}$ subjected to equivalent mechanical strains $\epsilon_{x}^{m e}$ at $R T=30^{\circ} \mathrm{C}$ and $t_{m e}=0.87 t_{k}$ for laminate $\left[(0 / 90)_{2}\right]_{s}$ P75/1962 in the range $\left[T_{\min }, T_{\max }\right] .157$

6.20 Fatigue degradation $f_{t h}(N)$ for $\left[(0 / 90)_{2}\right]_{s}$ P75/1962 with $R_{T}=-40 / 30$ calculated by Master Paris's law and DDM. . . . . . . . . . . . . 158

6.21 Crack density evolution $\lambda_{i}^{t h}(N)$ vs. number of cycles $N$ for $\left[(0 / 90)_{2}\right]_{s}$ P75/1962 with $R_{T}=-40 / 30$ calculated with DDM model 
6.22 Uniaxial equivalent mechanical strains $\epsilon_{x}^{m e}$ at discrete number of cycles $N$ vs. $T$ in the range $\left[-40,30^{\circ} C\right]$ for laminate $\left[0 / 90 / 0 / 90_{87 \%}\right]_{s} \mathrm{P} 75 / 1962$. Reference temperature is set to $T_{r}=30^{\circ} \mathrm{C}$. Results at $N=1285$ almost

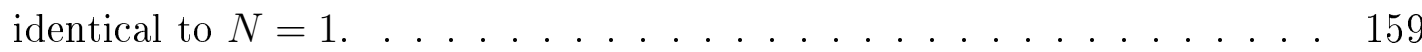

6.23 Comparison between ERR $G_{I}^{t h}$ for $R_{T}=-156 / 121$ vs. $G_{I}^{m e}$ at $T_{r}=$ $30^{\circ} \mathrm{C}$ with equivalent mechanical thickness $t_{m e}=0.87 t_{k}$ and strains $\epsilon_{x}^{m e}$ for laminate $\left[(0 / 90)_{2}\right]_{s}$ P75/1962 with $N=198746$ cycles. . . . . . . . . 160

6.24 Comparison between longitudinal stress $\sigma_{1}^{\text {th }}$ for $R_{T}=-40 / 30$ vs. $\sigma_{1}^{m e}$ at $T_{r}=30^{\circ} \mathrm{C}$ subjected to uniaxial equivalent mechanical strains $\epsilon_{x}^{m e}$ and $t_{m e}=0.87 t_{k}$ for laminate $\left[(0 / 90)_{2}\right]_{s}$ P75/1962 with $N=198746$ cycles. $\quad 161$

6.25 Comparison between transverse stress $\sigma_{2}^{\text {th }}$ for $R_{T}=-40 / 30$ vs. $\sigma_{2}^{m e}$ at $T_{r}=30^{\circ} \mathrm{C}$ subjected to equivalent mechanical strains $\epsilon_{x}^{m e}$ and $t_{m e}=0.867$ for laminate $\left[(0 / 90)_{2}\right]_{s}$ P75/1962 with $N=198746$ cycles. . . . . . . . . 161 


\section{List of tables}

1.1 Fatigue limit for different damage mechanims for epoxy. . . . . . . . . 16

3.1 Carbon fiber properties. . . . . . . . . . . . . 46

3.2 Quadratic temperature-dependent properties of Epoxy 3501-6 in the range $[-200,180]^{\circ}$ C . . . . . . . . . . . . . . 46

3.3 Quadratic temperature-dependent properties of Epoxy 934 in the range

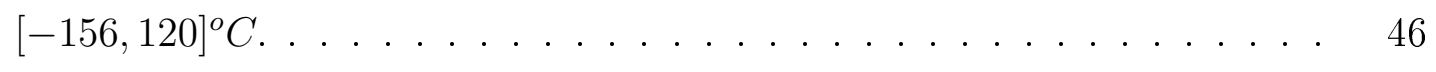

3.4 Quadratic temperature-dependent properties of Epoxy ERL 1962 in the range $[-156,120]^{\circ} \mathrm{C} \ldots \ldots \ldots \ldots \ldots \ldots$

3.5 Quadratic temperature-dependent properties of Epoxy 5208 in the range $[-156,120]^{\circ} \mathrm{\ldots} \ldots \ldots \ldots \ldots \ldots \ldots \ldots \ldots$

3.6 Comparison of experimental and FEA-calculated longitudinal lamina CTEs

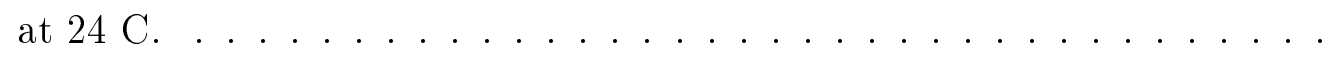

3.7 Comparison of experimental and FEA-calculated transverse lamina CTEs at 24 C . . . . . . . . . . . . . . . . . . . 64

4.1 Critical ERR $G_{I c}\left[\mathrm{~J} / \mathrm{m}^{2}\right]$, temperature $\left[{ }^{\circ} \mathrm{C}\right]$, see eq. $(3.2) \ldots \ldots \ldots . . . . \quad 72$

4.2 Quadratic temperature-dependent properties of P75/934 $\left(V_{f}=0.62[10\right.$, 13-15]) between $[-156,121]$ C. . . . . . . . . . . . 84

4.3 Quadratic temperature-dependent properties of P75/1962 $\left(V_{f}=0.52[10\right.$, $16,17])$ between $[-156,121]$ C . . . . . . . . . . . . .

4.4 Crack initiation temperature and maximum cracking density $\left(-190{ }^{\circ} \mathrm{C}\right)$ vs. number of sub-laimnas $(n)$ for AS4/3501-6 in $\left[0_{n} / 45_{n} / 90_{n} /-45_{n}\right]_{s}$ laminate. Subcript (e) means the exterior laminas $0_{n}$ and $-45_{n}$; Subcript (i) means interior laminas $90_{n}$ and $45_{n} \ldots \ldots \ldots \ldots$

4.5 Quadratic temperature-dependent properties of AS4/35016 ( $V_{f}=0.67$ [18, 19]) in the range $[-190, S F T]$ C . . . . . . . . . . . . . 91

4.6 Quadratic temperature-dependent properties of T300/5208 $\left(V_{f}=0.69[8\right.$, $20])$ in the range $[-156,121]$ C. . . . . . . . . . . . 
5.1 Cubic temperature-dependent properties of P75/1962 $\left(V_{f}=0.52\right.$ [10]) between $[-156,121]^{\circ} \mathrm{C}$. Temperature range for $G_{I c}$ is $[-156,-15]^{\circ} \mathrm{C} \ldots$. . 111

5.2 BCs for a 3D laminate simulation using solid elements C3D20R. . . . . 113

$5.3 f(N)$ parameters of P75/1962 ( $\left.V_{f}=0.52[10]\right)$. Subscript (e) and (i) represents exterior and interior laminas, respectively. Layup: A) $\left[(0 / 90)_{2}\right]_{s}$; B) $\left[0_{2} / 90_{3}\right]_{s}$; C) $\left[0_{2} / \pm 45 / 90_{3}\right]_{s} \ldots \ldots \ldots \ldots \ldots \ldots$

$6.1 f(N)$ parameters of P75/1962 $\left(V_{f}=0.52\right)$ under thermal fatigue. Subscript (e) and (i) represents exterior and interior laminas, respectively. Layup $(\mathrm{A}):\left[(0 / 90)_{2}\right]_{s} \ldots \ldots \ldots \ldots \ldots$ 


\section{Chapter 1}

\section{Introduction}

\subsection{Motivation}

Carbon fiber reinforced plastics (CFRP) are potential materials for many aerospace and aeronautical applications due to their high specific strength/weight and a low coefficient of thermal expansion (CTE) resulting in a high long-term stability. The anisotropy of composites allows a wide range of design where the CTE can be reduced significantly, and its lightweight lead to cost savings. Among candidate structures, the re-entry reusable launch vehicles (RLV), the fuel oxidant storage and transportation at cryogenic temperature $\left(-196^{\circ} \mathrm{C}\right.$ for nitrogen, or $-253^{\circ} \mathrm{C}$ for hydrogen), space satellites, and aircraft structure (frame, wings, etc...) can be highlighted. However, CFRP are prone to internal damage as a result of high residual stresses and thermal fatigue loading.

Composite materials in a space environment are subjected to solar radiation, vacuum, and cyclic temperature ranges depending on orbit of Earth, such as the low earth orbit (LEO), medium earth orbit (MEO), and geostationary earth orbit (GEO) as shown in

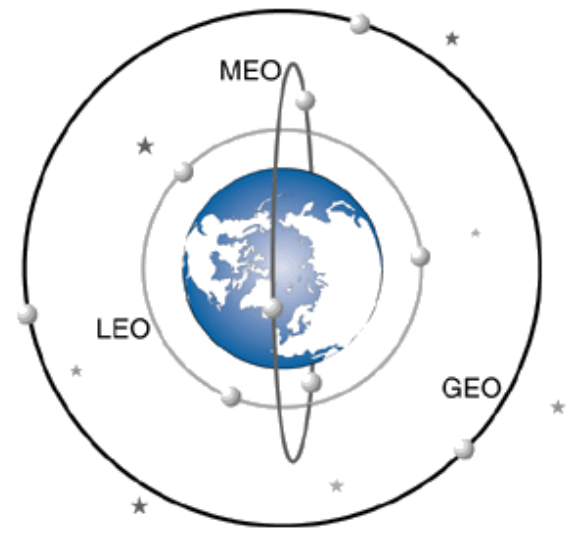

(a) Earth's orbit: GEO, LEO, and MEO.

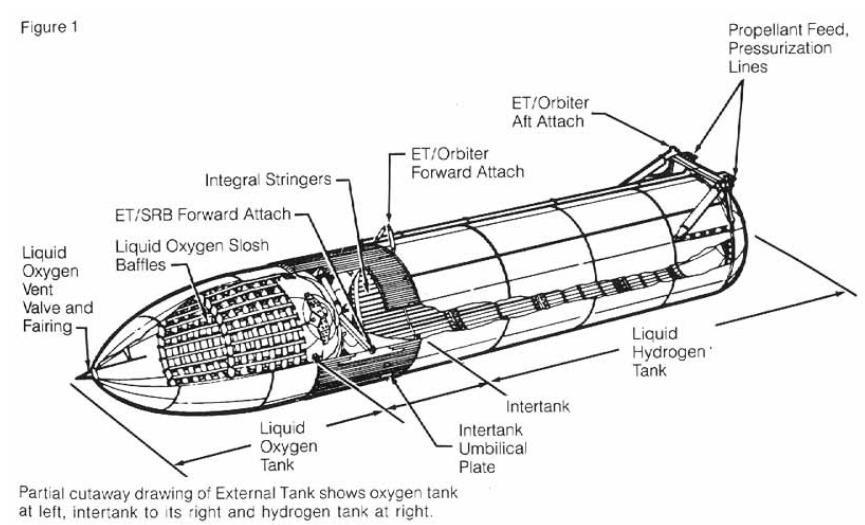

(b) External fuel tank for launch vehicles.

Figure 1.1: Examples of thermal cyclic loads in a space environment. 


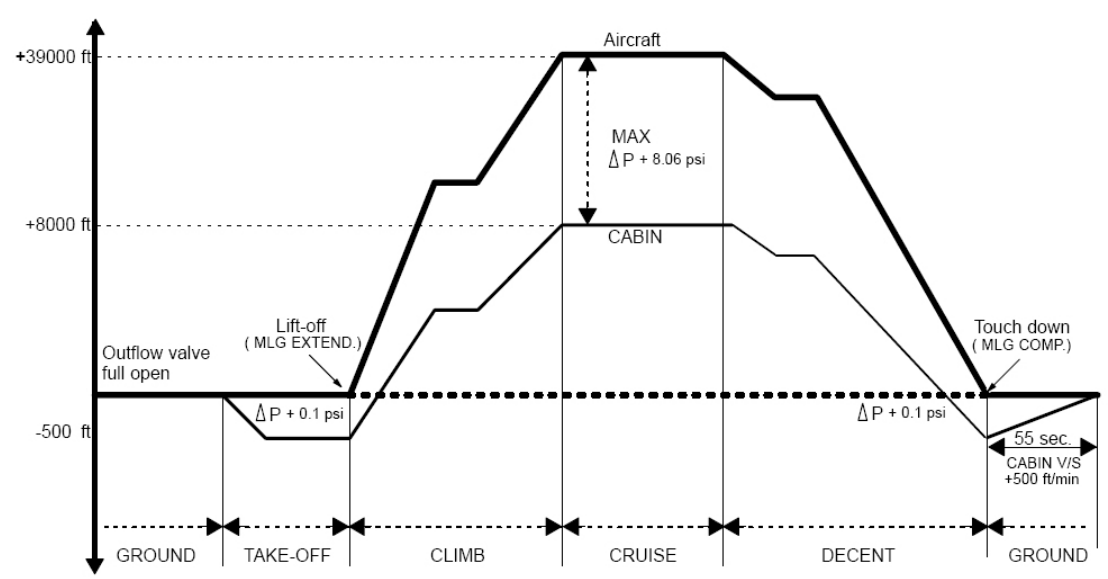

Figure 1.2: Average cycle of commercial aircrafts.

Figure 1.1.a. Although the cyclic temperature range, for a given cycle, can be tested using a heating/cooling system or coatings, unavoidable cyclic temperatures are developed. For instance, the temperature range varies from $\left[-156,121^{\circ} \mathrm{C}\right]$ to $\left[-101,65^{\circ} \mathrm{C}\right]$ degrees Celsius, and the number of cycles from 1 to 16 per day, for LEO and GEO, respectively.

A large number of investigations [1,8,10,21-27] have demonstrated internal damage in composite laminates mainly in form of microcracks at lamina level. Therefore, in order to predict the life of space structures, cyclic temperature ranges and residual stresses must be taken into account. Similar cases can be seen, for example, in the RLV entering and leaving of in the atmosphere of Earth, or fuel oxidant fluid tanks at cryogenic temperatures during transportation and storage, where a thermal cycle is generated each time they are filled and drained (see Figure 1.1.b). Furthermore, transverse cracking can produce gas leakage-paths or promote environmental attack conditions that might result in catastrophic failure.

Aircraft composite structures, such as the structural frame or wings, are also subjected to thermal cyclic loads and high residual stresses during its life. High temperature ranges result from take off, landing and cruising at $8000 \mathrm{ft}$ to $39000 \mathrm{ft}$ of altitude as shown in Figure 1.2. Hence, high cyclic thermal loads appear as a result of temperature range $\left[-70,40{ }^{\circ} C\right]$, as shown in Figure 1.2. Furthermore, these structures are subjected to oxidative and pressurized atmospheres increasing the aggressiveness of environment and enhancing a higher intralaminar cracking as well as other damage mechanisms [24,28].

While mechanical fatigue of composite materials has been extensively studied in the literature, thermal fatigue has been researched much less in comparison. Among multiple factors for this fact, we can highlight the lack of experimental fatigue data to correlate with analytical predictions or difficulties to find available temperature-dependent proper- 


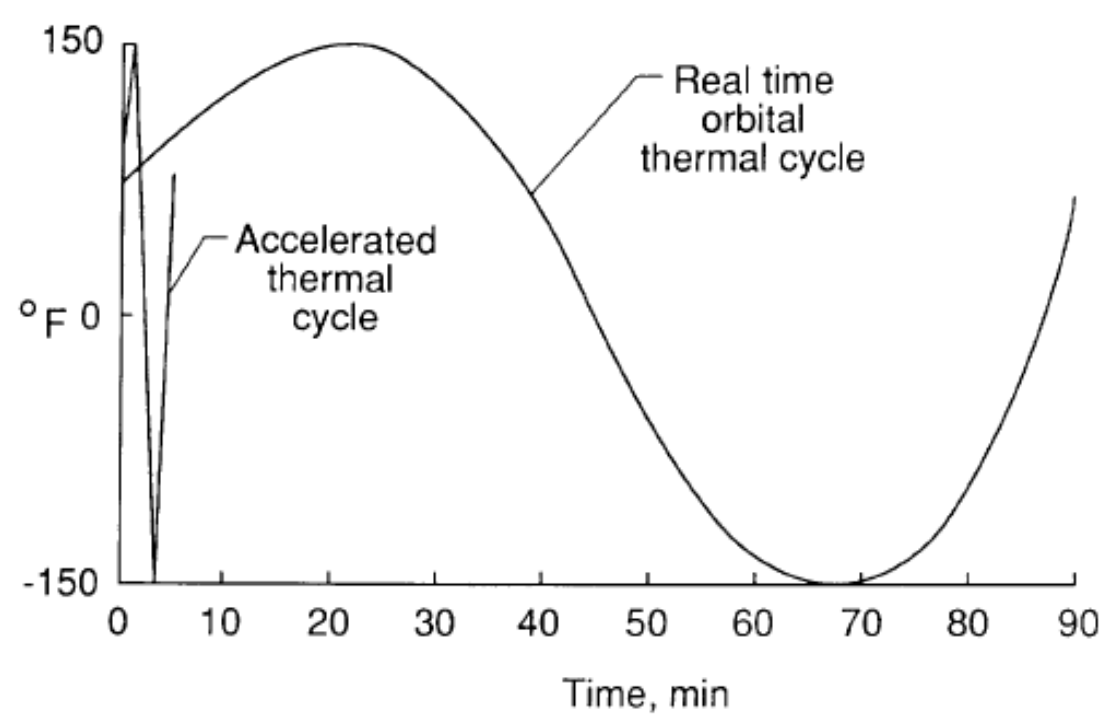

Figure 1.3: A comparison between accelerated vs. real time thermal cycle for LEO [1].

ties for the applied large temperature ranges in different applications. Unlike mechanical fatigue, the temperature range $\Delta T$, which is used as independent variable, varies depending on the application and must be tested in ovens controlling factors such as temperature gradient, powerful optical microscopic, etc... But not only the temperature range has influence, also the real time thermal cycle has a high impact. If we look again in the spacecraft, each cycle takes around 90-minute period with an amplitude of $\left[-101,65^{\circ} \mathrm{C}\right]$ to be completed for a LEO, as shown in Figure 1.3. Assuming a 30-year satellite life to be expected with a little less than 6000 cycles per year [1], the total cycles become 175,000 cycles. Although the number of cycles is not too large, each cycle can take around 15-minute period heating and cooling at a monotonic rate $[8,29,30]$. Therefore, a complete thermal fatigue test makes real time testing impossible from a practical point of view. Furthermore, it should be added extra time to measure either a macroscopic observable damage or a mechanical property. All the experimental data presented in the literature $[1,8,10,21-27]$ only covers between 500 and 5000 cycles. The same applies for aircraft composite structure, specially those aircrafts of second and third generation with a service life between 20 to 30 years. Although most popular airlines renew their fleet each 10 years, smaller companies invest on old airplanes having an already long life. It is thus of great importance that the service offered by these companies is safe after an average number of cycles between 30,000 and 60,000 cycles.

\subsection{Objective}

The objective of this dissertation is to implement a progressive damage model (PDM) for thermal static and fatigue loading. The proposed model is based on a discrete damage 
model (DDM) reported in [31-34], which has already been used to predict successfully transverse cracking under static mechanical loads. DDM was chosen for its simplicity to obtain the stiffness and CTE degradation from the critical energy release rate $G_{I c}$ and $G_{I I c}$ without any postulated damage function or empirical adjustment. In the pursuit of this goal, the thermal characterization of composite laminates under thermal loads will be implemented using temperature-dependent properties of each material system. The energy release rate (ERR) concept associated with crack opening displacements in mode I and II $\left(G_{I}\right.$ and $\left.G_{I I}\right)$ along with Griffith's failure criterion for an intralaminar crack will be used to predict crack initiation and evolution on laminate composites. In this way, both elastic and thermal properties of the damaged laminate will be calculated as function of the crack densities as well as the residual stresses for a given temperature range.

In order to predict transverse damage ${ }^{1}$ under thermal fatigue loading, the laminate composites will be studied with special attention during the first thermal cycle, in which a quasi-static load state can be assumed. The prediction of thermo-mechanical damage requires precise knowledge of temperature dependent-properties of the material, hence a methodology to back-calculate the constituent properties not available in the literature will be carried out through the use of micromechanics. Furthermore, predicted lamina properties will be compared with a finite element method (FEM) as benchmark solution. In this way, the ERR during the monotonic cooling will be calculated at lamina by lamina level and will compared with available experimental data to study the temperature-dependent fracture properties, $G_{I c}(T)$ and $G_{I I c}(T)$. Furthermore, the CTE of laminate composites will be calculated as function of temperature and crack density to obtain the residual stresses prior to thermal fatigue.

A thermal fatigue model will be implemented to predict crack evolution in laminate composites based on available experimental data. The cycle-dependent critical size concept will be used to evaluate the crack evolution and to adjust the critical ERR with number of cycles. In this way, the crack density saturation for both, thermal and mechanical fatigue will be compared. Furthermore, a Paris law similar to those in metal will be developed to calculate the crack density growth for a temperature range.

Finally, an effort to relate thermal and mechanical fatigue behavior will be carried out. The lack of experimental data for high-cycles in fatigue calls into question the analytical predictions based on only 500-2000 cycles of data. Therefore, new alternatives to highcycle fatigue thermal tests will be proposed using an analytical and numerical techniques. In the pursuit of this goal, the temperature-dependent properties must be implemented

\footnotetext{
${ }^{1}$ In the literature, transverse damage is frequently called matrix-cracking. In this dissertation, transverse damage is used to describe crack propagation along the fiber direction for any of the laminas which form the laminate.
} 
with accuracy. Only then, stress and strain fields can be obtained using a damage model to simulate thermal fatigue tests in terms of mechanical fatigue tests conditions at room temperature.

\subsection{Literature Review}

In this section, composite damage mechanisms under mechanical and thermal fatigue loads are reviewed and classified because similar transverse damage can be observed in mechanical fatigue and thermal fatigue. The fiber orientations will be unidirectional and angle-ply $( \pm \theta)$. Also, damage mechanisms in composite laminates for common stacking sequences such as quasi-isotropic, balanced and cross-ply laminates [35, Ch. 6] will be reviewed. Finally, the experimental characterization of composites under fatigue loading will be reviewed as well as the influence of its constituents and lamina orientation.

\subsubsection{Fatigue damage mechanisms}

According to [3,36-44], four main damage modes are observed during fatigue loading of composites subjected to controlled loads or strains, in which some of them might act in order, simultaneously, or in combination. The first damage mode observed is the intralaminar cracking as shown in Figure 1.4. This damage mechanism involves crack initiation and propagation in brittle polymers (e.g. epoxy) similar to those in metals. These cracks propagate quickly reaching the interface at the neighboring fibers or adjacent laminas. For unidirectional composites (i.e., a thick lamina such as $\left[0_{8}\right]$ ), transverse cracks perpendicular to fibers propagate until they reach the vicinity of fiber interface. Based on [36,37], different scenarios can occur as illustrated in Figure 1.5. At low strains, a sporadic break of a weak fiber or crack nucleation from internal flaws can give rise. In such cases, the crack growth is slow because a low stress concentration at the crack tip takes place as shown in Figure 1.5.a. When this happens, the crack progration may continue in the surrounding matrix but it is insufficient to break the neighboring fibers leading to shear-normal failure in the matrix-fiber interface due to stress concentration through a complex shear-lag mechanism [45] (see Figure 1.5.c). However, a clear increment in the number of cracks (see Figure 1.5.b) due to a fatigue phenomenon has been widely reported in the literature $[36,41,46]$. This discrete damage is described as a dispersed transverse failure mode [36,38]. For laminate composites (e.g angle-ply, cross-ply, etc...) transverse cracks along the fiber direction in off-axis laminas (predominant in $\theta^{\circ}$ laminas close to $90^{\circ}$ ) propagate up to reach the adjacent laminas. This dispersed transverse failure is described by the crack density $\lambda$ defined as the number of cracks per unit 


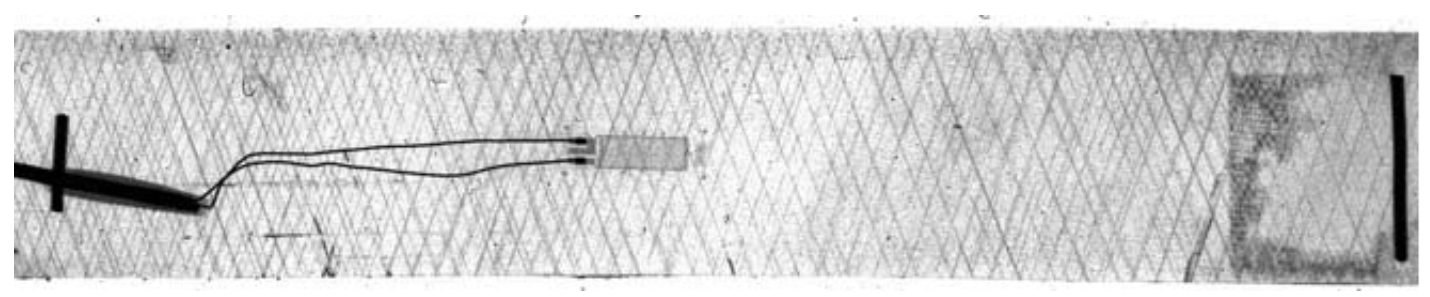

Figure 1.4: Transverse cracking in $\left[0 / \pm 70_{4} / 0_{0.5}\right]_{s}$ laminate of E-glass/epoxy subjected to $0.7 \%$ of strain [2, Ch.9]

length. Although the crack density is irregular and random at very low strains or even during its commissioning, such definition is well accepted in the literature for medium and high strains where $\lambda$ is almost equally spaced along the sample (see Figure 1.4). Based on [36], the crack density increase at a specific rate determined by the applied strain and the constraints provided by the neighboring laminas. Intralaminar cracking may continue in each lamina until an equilibrium state where a crack density saturation is reached. This equilibrium state has been also defined as a Characteristic Damage State (CDS) $[35,36,47]$, which depends on material system, the thickness and laminate stacking sequence (LSS). Transverse cracks in laminate composites subjected to static loads are often observed $[27,48,49]$, and matrix cracking is intensified by fatigue loading once a so-called strain fatigue limit $\epsilon_{f . l}$ is exceeded [36,37]. Due to the dependence of $\lambda$ with number of cycles, the composite strength, fatigue life, and further damage mechanisms may cause catastrophic or final failure.

(a)

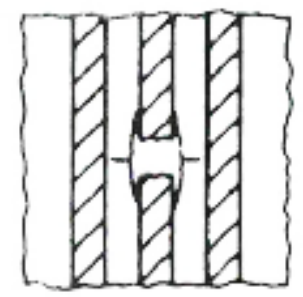

(b)

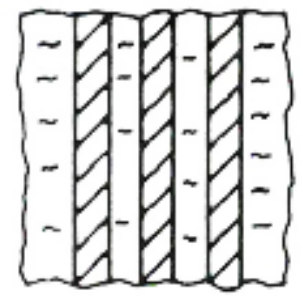

(c)

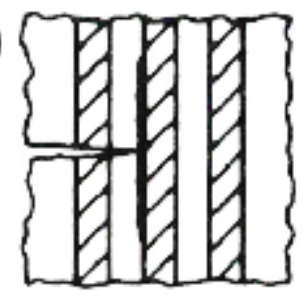

Figure 1.5: Damage mechanisms in unidirectional composites subjected to fatigue loads in fiber direction: (a) fiber breakage, (b) dispersed transverse cracks and (c) interfacial shearnormal failure.

The second damage mode in form of cracks perpendicular to the intralaminar cracking is observed in laminate composites as shown in Figure 1.6. In laminate composites, a high concentration of cracks along fibers (primary cracks ${ }^{2}$ ) may cause fiber-matrix debonding due to fiber splitting at both surfaces of the crack. Furthermore, secondary short cracks are generated along the interface between laminas where primary cracks occur [47] as shown in Figure 1.6.a and 1.6.b. Adding more cycles, these secondary cracks propagate perpendicular to the primary cracks causing crack coupling in which

\footnotetext{
${ }^{2}$ These primary cracks are the object of this study denoted by transverse damage in the title dissertation. Furthermore, transverse damage is also called matrix-cracking in the literature.
} 

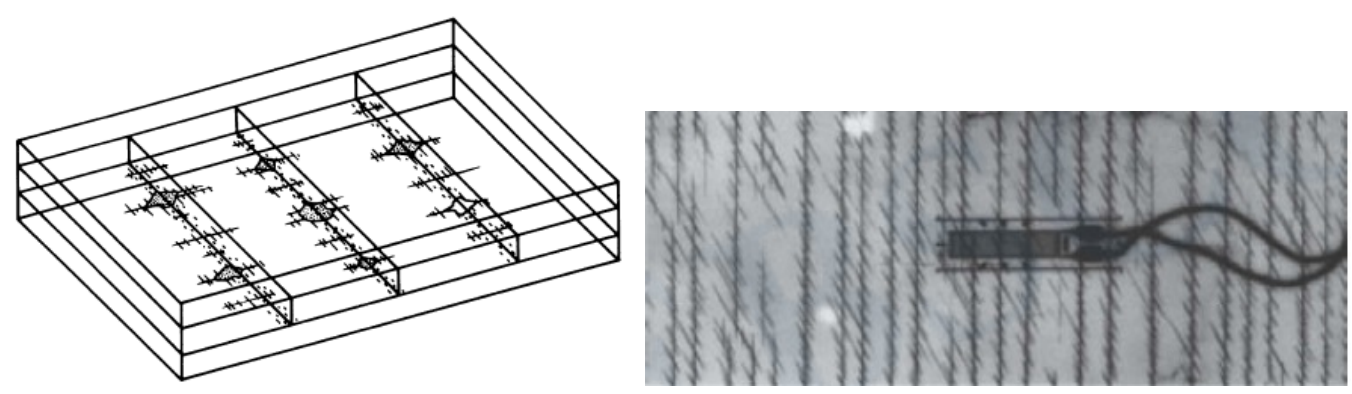

(a) Schematic diagram of a cross-ply lam- (b) Transverse cracking in a laminate composinate, showing primary and secondary ite showing undergoing short secondary cracks. transverse cracks with local delamination $[47]$.

Figure 1.6: Primary and Secondary cracks on laminate composites subjected to fatigue loads.

interfacial debonding near the edge of the laminate is produced due to shear and normal stresses out of plane, namely, free-edge stresses. As the loading history continues, the interfacial debonds increase due to high free-edge stresses (mixed growth in mode I and II) allowing the formation of delaminations denoted as the third damage mode $[50,51]$. Although the free-edge stresses in the primary cracks is the main reason to observe delamination in laminated composites, internal initial flaws at the lamina interface may develop anticipated delamination.

As the fatigue continues, the fourth damage mode in form of fiber breakage can be observed $[38,47]$. The failure of the fiber occurs when the strength of the weakest fiber is overcome and hence, its fatigue damage is associated with the number of fibers broken that may cause the laminate failure. However, though the final fracture of composite is associated with a large number of fibers broken, some fiber fractures occur during all stages of cyclic loading mainly in zones close to transverse cracks (high stress concentration). According to $[36,47]$, several scenarios can be presented during the fiber breakage. Fibers might break either at the weakest points along its length or in zones with high stress concentration located on the primary and secondary cracks, interfacial debonding and/or delamination. Where a single fiber breaks, shear stress concentrations close to the tip of the broken fiber may lead to local interfacial debonding in the surrounding matrix as shown in Figure 1.5.a. The length of the debonded area depends on the shear strength of matrix (fracture toughness in mode II). Furthermore, the high tensile stress in the surrounding matrix may also induce transverse disperse cracks in opening mode (fracture toughness in mode I) as shown in Figure 1.5.b. When the new crack is long enough to reach the next fiber interface (see Figure 1.5.c), the shear stress at its tip may allow new interface dobonding with the neighboring fibers as shown in Figure 1.5.c. In contrast to the equilibrium state of transverse cracks confined to the matrix alone, fiber debonding due to fatigue is characterized by a localized interfacial failure and usually 
causes sudden laminate failure.

Once the weakest fibers break, the final fracture of a composite follows a large fiber breakage in laminas where the principal tensile stresses are supported [6, 36, 47, 52]. Although the fiber breakage is usually preceded by other damage mechanics, more than one failure may occur simultaneously or the order of damage modes may change. For this reason, a non-progressive damage occurs and in many times, there is not more choice that to appeal to the statistics $[7,53,54]$. This stochastic nature can be seen for instance in laminate composites subjected to high strains history close to the average fracture strain of fibers $\epsilon_{c}$ where a non fatigue phenomenon occurs and the resulting damage is statistically controlled $[36,55]$. Substantial loss of stiffness during fatigue loading occurs and it may be considered to be a failure form anyway.

\subsubsection{Experimental characterization: S-N and Fatigue-Life Dia- grams}

Based on previous experience of fatigue life predictions, such as metals, the fatigue characterization of composites is obtained among other by $S$ - $N$ diagrams. Unlike metals, the applied strain $\epsilon$ is used as independent variable for testing so that both matrix and fiber are subjected to the same displacement. However, the applied stress $\sigma$ is sometimes chosen depending of applicability and functionality of composite structures. While metals are isotropic, the anisotropy of composites makes the stresses within the laminate to depend on volume fraction, elastic moduli, and internal damage. Furthermore, tests are restricted to a specific values of stress ratio $R$. This ratio can be defined as function of the maximum and minimum peak stress or strain as follows $[6,36]$

$$
F=\frac{\epsilon_{f . l .}}{\epsilon_{c}} \quad \text { or } \quad R=\frac{\sigma_{\min }}{\sigma_{\max }}
$$

where $\epsilon_{f . l}$. is the fatigue limit expressed as function of the damage mechanism and the composite fracture $\epsilon_{c}$. In Figure 1.7, a schematic fatigue-life diagram is illustrated for tensile fatigue of unidirectional composite. Three main regions similar as those S-N diagrams in metals are clearly differentiated with some particularities.

The first region (Region $I$ ) corresponds to the critical fracture strain of fibers, $\epsilon_{c}$, similar as $S_{u t}[56$, Ch.6] for metals. As shown in Figure 1.7, the horizontal band centered at $\epsilon_{c}$ corresponds to fiber breakage. This region represents a non-progressive fatigue damage due to random scatter of fiber breaks. Although not all fibers have the same strength, an average fracture strain $\epsilon_{c}$ is measured. Close to this strain, the weakest fiber will be the first to break. When this happens, high stress concentrations and/or other damage mechanisms may occur simultaneously which facilitate more fiber breakage and interfa- 


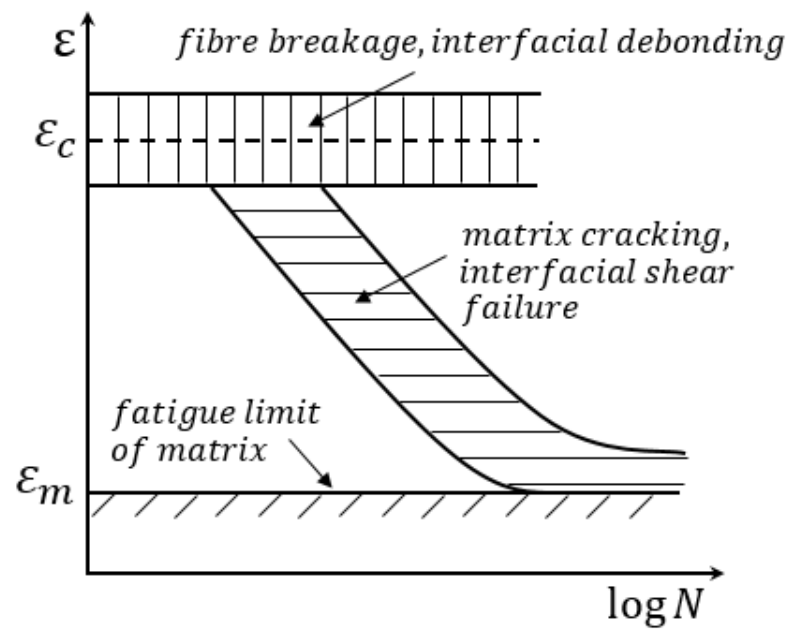

Figure 1.7: Fatigue-life diagram for unidirectional composites under tensile loading parallel to fibers.

cial debonding. Generally, the following fibers broken are located in the vicinity of fibers already broken due to high stress concentrations, or sometimes appears randomly within the laminate as illustrated in Figure 1.8. In any case, the lack of certainty to predict a logical trace way or cross section through the laminate to cause the entire laminate failure demands the use of statistic $[7,53,54]$. As result, the fiber breakage is fatigue-independent and the probability of obtaining an instantaneous critical fracture is represented by a horizontal band with mean value $\epsilon_{c}$, and lower and upper limit probability values equal to $5 \%$ and $95 \%$, respectively, as shown in 1.9.a.

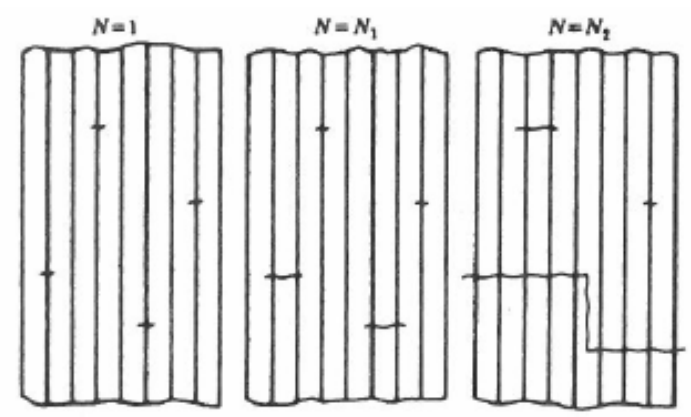

Figure 1.8: Random fiber breakage in unidirectional composites under tensile loading.

The second characteristic region (Region $I I$ ) corresponds to strains below the scatter band of the fracture strain $\epsilon_{c}$, as shown in Figure 1.7. In this region, as it happens in metals, progressive damage, clearly cycle-dependent, is developed. Damage mechanisms such as transverse cracking and interfacial debonding increase as number of cycles and sudden fiber breakage does not exist. The laminate degradation typically follows a power law where the crack initiation and evolution is essentially matrix-dependent but unlike metals, not just a critical crack but multiples damage modes interacting produce the collapse or laminate failure. However, though multiples damage mechanisms may occur, 


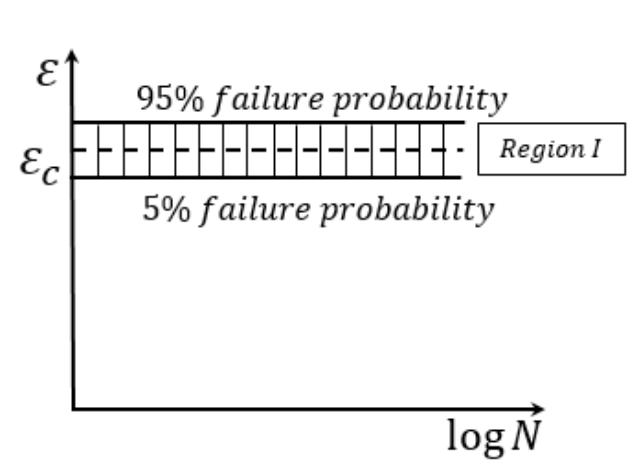

(a) Region I

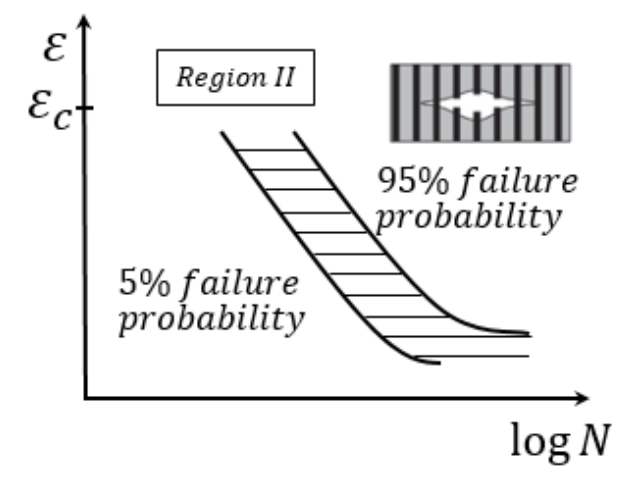

(b) Region II

Figure 1.9: Region I and II of the fatigue life diagram.

particularly at the end of fatigue life, crack density $\lambda$ is the main mechanism that results in premature or late fracture. Typically, this region is represented with a specific shape function such as linear-log or log-log function. Although the collected data for a specific Rratio may be quite complete, the practical need beyond those experimental measurements force to extrapolate using a power law or similar equations $[36,47,54,57]$. Moreover, the probability of a premature failure can be represented by a scatter band assuming a normal distribution according to ASTM [58], a simple two-parameter Wiebull distribution [59] or a Wiebull distribution [60] where a direct relationship between static and fatigue residual strength distribution exists. The fatigue life probability is represented by a scatter band with lower and upper limit values equal to $5 \%$ and $95 \%$, respectively, as shown in Figure 1.9.b.

The last characteristic region corresponds to strain values below which crack propagation may not occur and thus, the fatigue life is assumed to be infinite similar to those in metals $\left(S_{e}\right)\left[56\right.$, Ch.6]. This strain is defined as the fatigue limit $\epsilon_{f . l}$ and it is illustrated in Figure 1.7.

Another fatigue characterization of composites is obtained by Constant fatigue-life diagrams (CFL). These diagrams characterize the fatigue sensitivity based on the observable final fracture of composites subjected to alternating and mean stress, and thus they are closely related with $\mathrm{S}-\mathrm{N}$ diagrams. The main idea is to obtain the safe stress region at which for a given constant amplitude loading, the composite do not fail before a specified number of cycles $(N)$. While $\mathrm{S}-\mathrm{N}$ diagrams characterize the fatigue behavior for a single stress ratio $(\mathrm{R})$, CFL diagrams describe a high spectrum on the fatigue behavior for all R-values. Therefore, CFL diagrams represent a failure criteria in the ultimate strength of composite similar to metals, which have been extensively studied during the 19th century such as Goodman, Gerber, etc.. [56, Ch. 6].

Although the CFL diagrams can be a strong design criteria, their construction as well 


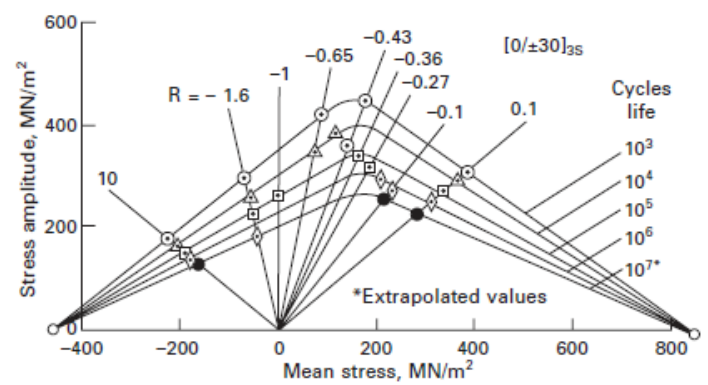

(a) Shifted CFL Goodman diagram for a carbon/epoxy laminate [61].

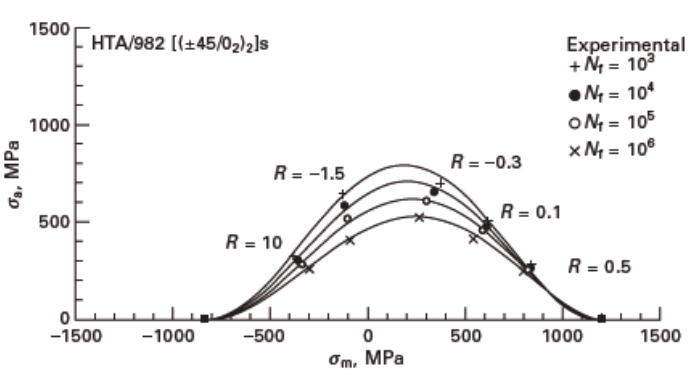

(b) General bell-shaped CFL diagram for a HTA/982 carbon/epoxy laminate [62]

Figure 1.10: Two types of CFL diagrams based on Goodman and Bell-shape theories.

as the effect of fatigue loading on the sensitivity of composites require a large amount of tests for many R-ratios. From a practical point of view, powerful fatigue-life theories are required to predict with reasonable accuracy the residual strength of composites saving time and cost. Many approaches in pursuit such goal are presented in the literature [6,61,63-67]. For all the theories, three main differences with respect those in metals such as Goodman or Gerber equations [56, Ch. 6] can be observed in Figure 1.10 including: a) tension and compression strength differences leading to asymmetry with the alternating stress axis , b) changes in shape (linear, quadratic,..) with increasing the number of cycles, and $c$ ) a highest alternating stress peak shifted to the right. Among all CFL diagrams stand out the linear theories corresponding to symmetric and asymmetric Goodman CFL diagrams [63] with good agreement for angle-plied $[ \pm 45]_{s}$ laminates, wood and polymer matrix composites. The shift and inclined Goodman diagrams [61,64] for balanced-plied laminates (see Figure 1.10.a) and the piece-wise CFL diagrams [65] for carbon/epoxy laminates. Similar theories for non-linear CFL diagrams use a Gerber's equation [6] or a characteristic bell-shaped CFL diagram [66], which has been shown to be valid for various types of multidirectional carbon/epoxy laminates (see Figure 1.10.b). However, the most recent approach was developed by [67], the so-called Anisomorphic CFL diagram where the experimental data required is only limited to the static strength values in tension and compression, and a reference $\mathrm{S}-\mathrm{N}$ curve for a particular stress ratio.

From a practical point of view, the fatigue characterization of composite based on S-N curves or CFL diagrams are of vital importance in the damage modeling of composites. Although a large number of tests are required, and thus time and cost consuming, the damage mechanisms involved during the fatigue loading are the basis of any model to predict either the strength and/or stiffness degradation. Regardless of the model, all of them require the use of experimental data to predict fatigue life using either a failure criteria, the continuous damage mechanics or through statistical functions. The thermal-fatigue of composites also involves multiaxial stress states characterized through its thermal ex- 
pansion coefficients and thus, it involves the understanding of damage mechanisms for isothermal mechanical fatigue. In fact, a macroscopic characterization such as the crack density or delamination is almost always required since the thermal-fatigue tests are excessively expensive and slow requiring analytical models to predict the final failure in many occasions.

\subsubsection{Influence of constituents and laminate stacking sequence on S-N diagrams of composite materials}

Unlike metals, the matrix and fiber stiffnesses of composites play an important role on fatigue-life diagrams. To understand the fatigue in angle-plied, cross-plied or other type of laminates, the fatigue of unidirectional composites must be understood first.

If we look at the unidirectional composite, the fracture strain of composite is limited by the fiber stiffness $\epsilon_{c}[35$, Ch. 4] and thus, the S-N diagram will depend on the type of fiber. For instance, in low stiffness fibers (i.e glass-fibers), $\epsilon_{m}$ typically fall below $\epsilon_{c}$, and the characteristic S-N diagram is shown in Figure 1.11.a, for different volume fractions $V_{f}$. In general, all data fall in region of transverse cracking, interfacial shear failure and delamination, so that a progressive damage occurs. Furthermore, as the $V_{f}$ decreases, the fatigue damage increases because higher number of damage mechanisms are involved, and $\epsilon_{c}$ lies away from the average fracture strain of composite leading a greater fatigue phenomenon. However, for high stiffness fibers (i.e carbon-fibers), $\epsilon_{m}$ may fall above $\epsilon_{c}$, and the characteristic S-N diagrams differ substantially as shown in Figure 1.11.b. In this case, all data may fall in the fiber breakage region and a non-progressive damage is developed, i.e. a stochastic nature. Therefore, once the carbon fibers breaks, matrix cannot support the fibers-load drop beyond (see Figure 1.11.b).
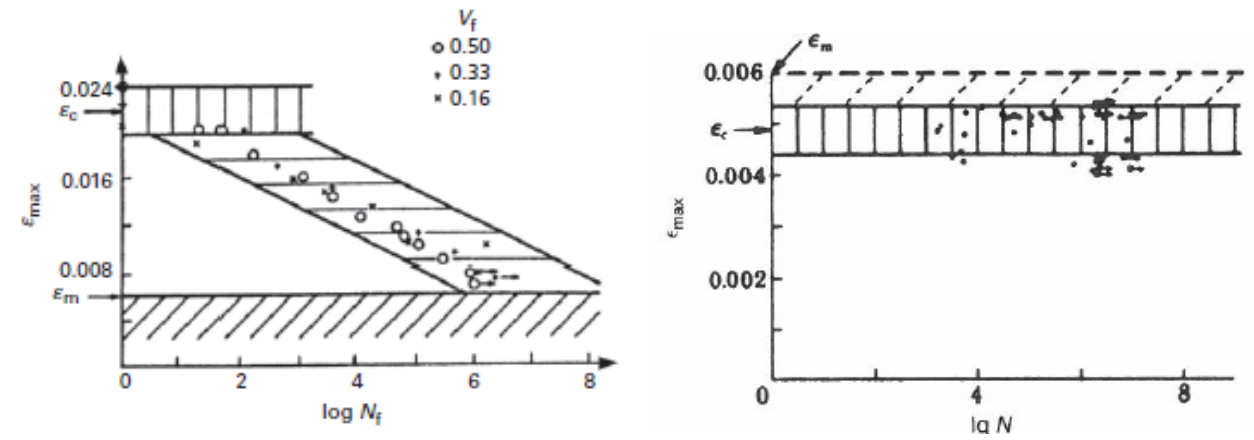

(a) Glass-fiber with low fiber stiffness [39] (b) Carbon-fiber with high fiber stiffness [68]

Figure 1.11: Fatigue-life diagram for glass and carbon epoxy laminates under tensile loading.

In off-axis fatigue for unidirectional composite, orientation vary between $0^{\circ}$ and $90^{\circ}$, 


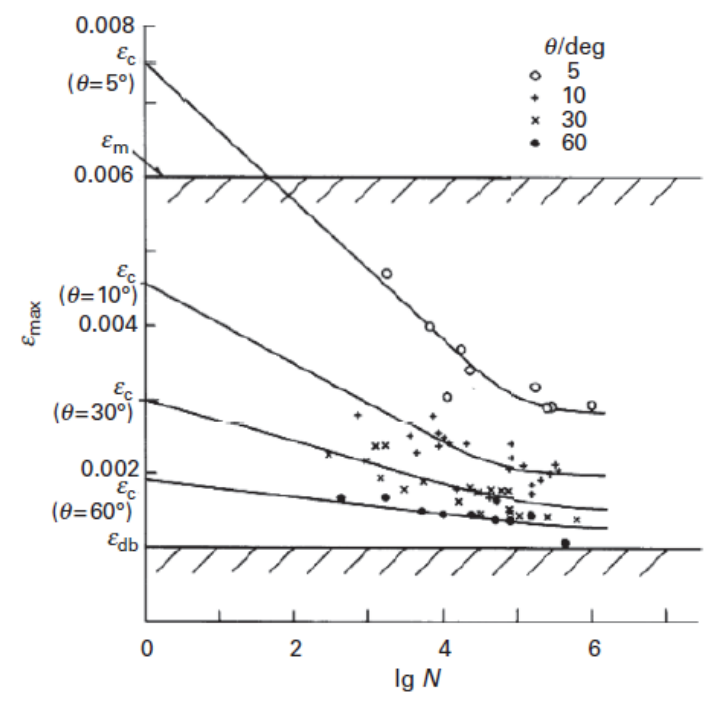

(a) Fatigue-life diagram for off-axis fatigue of (b) Comparison of fatigue limit between off-axis lamunidirectional composite glass-epoxy [40,70] ina (dotted line) and angle-ply laminates [69, 71]

Figure 1.12: Fatigue-life diagram for off-axis and angle-plied laminates under tensile loading.

and thus stress state vary from a pure tension to pure shear, or combination of both. Hence, a mixed crack growth is observed both in opening and sliding mode between matrix and fibers. An opening mode crack growth increases with increasing the off-axis angle, and it becomes critical at $90^{\circ}$ (tension normal to fibers) where pure transverse fiber debonding occurs [69]. At this point, the fatigue limit $\epsilon_{f . l}$ decreases so that all stresses focus on the interfacial debonding (not fiber breakage), and the fatigue limit is denoted as $\epsilon_{d . b}$, being in general lower than the fatigue limit of unreinforced (polymer) matrix $\epsilon_{m}$. For $0^{\circ}<\theta<90^{\circ}$ laminas, a transverse fiber debonding occurs first, followed by interfacial shear failure whose crack length will depend on off-axis angle. In Figure 1.12.a, it can be observed how the fracture strain $\epsilon_{c}$ almost disappear as off-axis angle laminas get close to $90^{\circ}$, and only transverse fiber debonding predominates. The fatigue limit $\left(\epsilon_{m} \approx 0.6 \%\right.$ for epoxy) decreases as off-axis angle increase up to reaches $\epsilon_{d . b .} \approx 0.1 \%$ at $90^{\circ}$ as shown in Figure 1.12.b with a dotted line for a glass-fiber composite.

The fatigue of angle-ply laminates follow the same pattern as off-axis fatigue of unidirectional lamina but with the added feature of delamination in the progressive damage region. In these type of laminates, the fatigue limit converges to same values at angles greater than $60^{\circ}$. It can be seen in Figure 1.12.b. that a significant improvement for angle-ply laminates with respect off-axis unidirectional laminas can be achieved for smaller angles $\left[0,60^{\circ}\right]$.

In cross-plied laminates, the first damage mechanisms to appear are transverse cracking and fiber debonding into $90^{\circ}$ laminas [72]. Typically, these laminates fail due to delamination that may occur when transverse cracking propagate to the adjacent lamina 


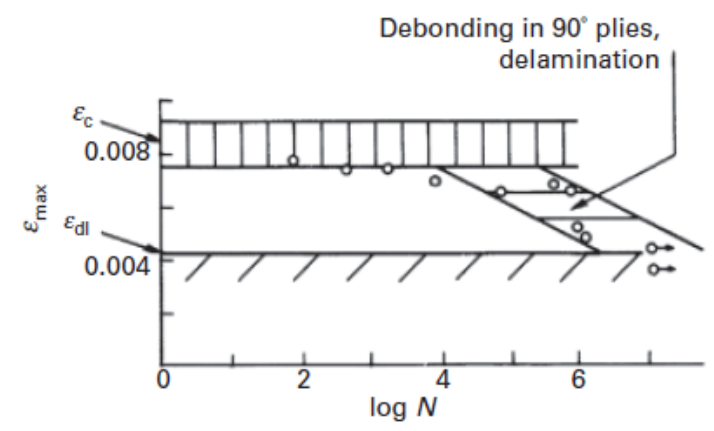

Figure 1.13: Fatigue-life diagram for cross-plied laminates of graphite-epoxy [3].

interfaces with high stress concentrations. At this point, the strength of the laminate is given by the statistic strength on $0^{\circ}$ laminas (fiber breakage). A fatigue-life diagram for cross-ply laminate is illustrated in Figure 1.13, where the fiber breakage scatter band (Weibull distribution) correspond to region I, and the progressive damage in $90^{\circ}$ laminas to region II. The fatigue limit correspond to strain at which transverse cracking and/or delamination occur, $\epsilon_{d . l}[3]$.

On laminates with combination of $0^{\circ}, 45^{\circ}$, and $90^{\circ}$ laminas, mechanically loaded in the fiber direction, the first damage mechanisms are found to be transverse cracking and transverse fiber debonding at $90^{\circ}$ laminas followed by delamination at $\pm 45^{\circ}$ laminas. When this happens, similarly to cross-ply laminates, an overstressing into $0^{\circ}$ laminas (Weibull distribution) is generated and thus, critical failure of composite may occur. A fatigue life diagram for these laminates is shown in Figure 1.14. The fatigue limit $\epsilon_{d . l}$. was found to be the minimum strain at which delamination due to interfacial debonding and transverse cracking occur.

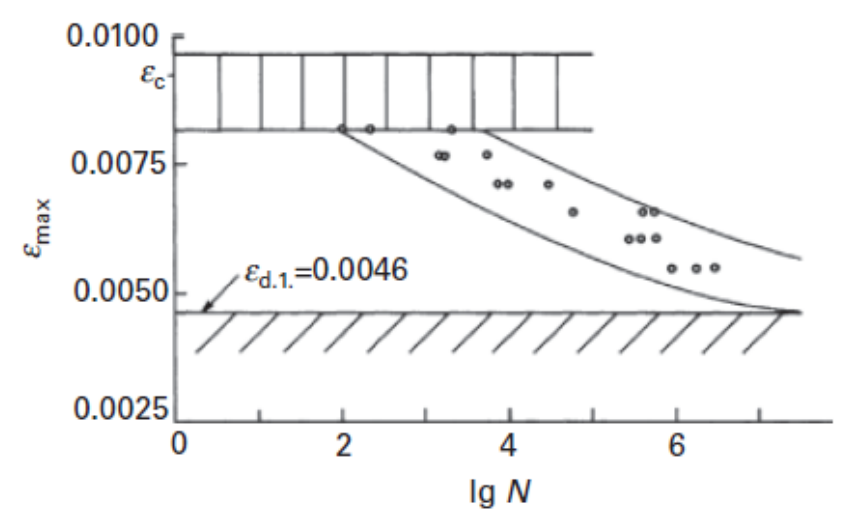

Figure 1.14: Fatigue-life diagram for a $[0 / \pm 45 / 90,-45]_{s}$ graphite-epoxy laminate [4].

Although unidirectional lamina and other type of laminate composites such as crossply, balanced or quasi-isotropic are commonly used, the unidirectional laminas are subjected to a continue change in the stress state. This is because either by specific design purpose (different LSS and off-axes laminas $\theta^{\circ}$ ) or a stress redistribution during to fa- 
tigue loading due to internal damage, the multiaxial stress state subjected for each lamina changes continuously. Therefore, depending on the magnitude of multiaxial state, the fatigue process can be analyzed in terms of main mechanisms induced by one stress over the other stresses.

In this way, a baseline fatigue life diagram can be established defining the so-called biaxial stress ratios for both cases, when stresses are dominant along fiber $\left(0^{\circ}\right)$ or matrix $\left(90^{\circ}\right)$. When $\sigma_{1}$ is dominant, the biaxial ratios are defined as $\gamma_{1}=\frac{\sigma_{2}}{\sigma_{1}}$ and $\gamma_{2}=\frac{\sigma_{12}}{\sigma_{1}}$, and they induce premature fatigue life as shown in Figure 1.15.a. When $\sigma_{2}$ is dominant, the biaxial ratios are defined as $\beta_{1}=\frac{\sigma_{1}}{\sigma_{2}}$ and $\beta_{2}=\frac{\sigma_{12}}{\sigma_{2}}$, and they will modify the off-axis fatigue life diagram $(0<\theta<90)$ as is shown in Figure 1.15.b, again reducing the fatigue life or even causing final fracture. In general, the laminas subjected to dominant stresses $\sigma_{1}$, are expected to cause the final fracture, also viewed as "critical elements". Then, the other off-axis laminas $(0<\theta<90)$ can be seen as "subcritical elements", and their failure enhance the overstressing in critical elements.

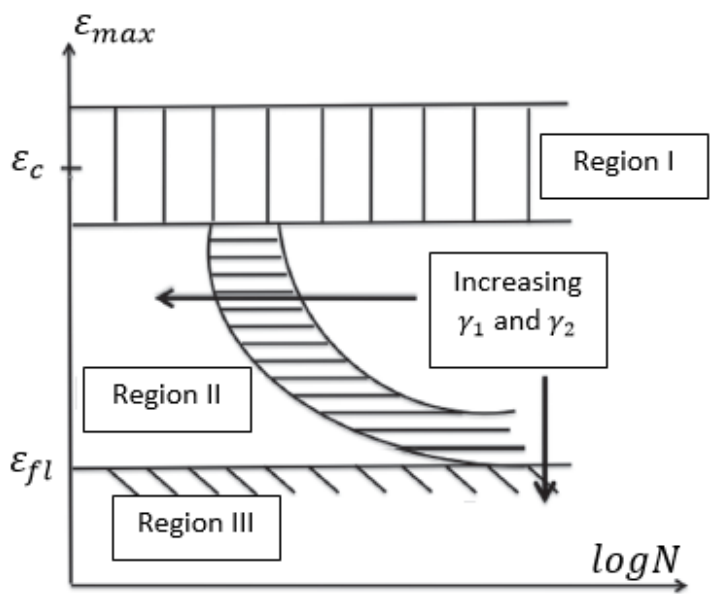

(a) On-axis fatigue life diagram modified by (b) Off-axis fatigue life diagram modified by factors $\lambda_{1}=\frac{\sigma_{2}}{\sigma_{1}}$ and $\lambda_{2}=\frac{\sigma_{6}}{\sigma_{1}}$.

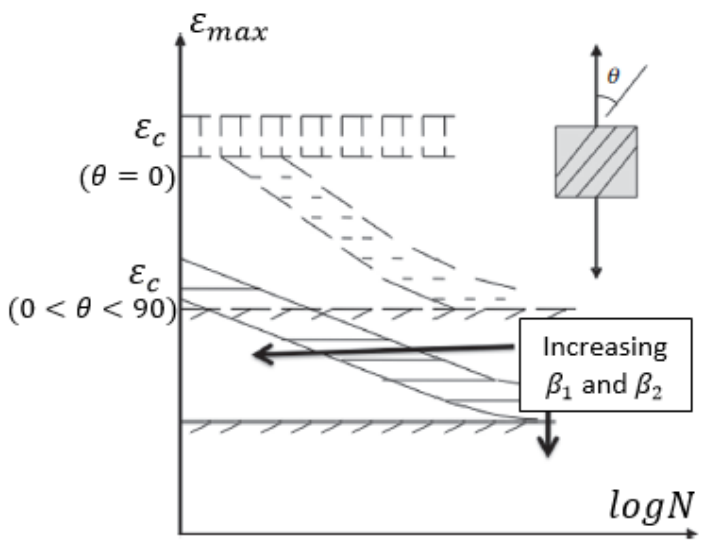

factors $\beta_{1}=\frac{\sigma_{1}}{\sigma_{2}}$ and $\beta_{2}=\frac{\sigma_{6}}{\sigma_{2}}$.

Figure 1.15: Baseline fatigue life-diagram modified according to a multiaxial state [5].

Based on the fatigue framework influence studied, a fatigue ratio is defined by (1.1) to give a general vision of the fatigue phenomenon involved. The S-N diagrams suggest therefore that fatigue is clearly matrix-dependent based on the operative damage mechanisms involved. Some fatigue limits given by [36] are shown in Table 1.1. 


\begin{tabular}{ll}
\hline Fatigue limit $\epsilon_{f . l .}$ & Damage Mechanism \\
\hline 0.006 & Transverse cracking \\
0.001 & Transverse Fiber Debond- \\
& ing \\
& Delamination caused by \\
0.0046 & debonding in the $90^{\circ}$ \\
& laminas \\
\hline
\end{tabular}

Tables 1.1: Fatigue limit for different damage mechanims for epoxy.

\subsubsection{Fatigue of composites subjected to compression loading}

For the case of compression along the fibers in UD composites fiber microbuckling [73] was observed as failure mechanism. Experimental observations [74-77] indicate that fibers tend to buckle under the influence of a local shear concentration in the matrix defects, misaligned or wavy fibers. As the loading history continue, the fiber buckling is intensified inducing microbuckling of the neighboring fibers and may lead a kink-band. When enough fibers break at the kink-bands, a critical state is reached causing the lamina failure. Despite its importance in real life, the present author focus in fatigue life of composite when they are subjected to tension loads. Since the thermal expansion coefficient of carbon fiber is negative, laminate composites subjected to thermal-fatigue undergo mainly positive strain, i.e. tension-tension (T-T) fatigue.

\subsubsection{Fatigue of glass- and carbon-fibers}

The fatigue behavior of fibers must be studied because it is a critical element which characterizes the final fracture and the fatigue strength of composites laminates [78]. Several researchers $[79,80]$ concluded that composites with a high modulus carbon fibers showed better fatigue behavior than those with a low modulus glass fibers.

Since the diameter of fibers are really small $(1-15 \approx \mu m)$, it is very difficult to apply true axial cyclic loadings to obtain accurate fatigue results based on a single fiber. On the one hand, the fiber undergoes slack after some cycles only avoidable using a cumulative extension load, but doing so is an incorrect representation of data using a progressive stress-strain curve $[5,81,82]$. Furthermore, all the fatigue measurements of a single fiber require complicated techniques which make use of advanced and expensive technology [83-85]. Despite of all these difficulties, the most common fatigue failure observed for a single fiber involves initiation of cracks at or near the surface [86].

On the other hand, a fiber bundle test is easier and presents less scatter than a single fiber test. Only the friction between them causes a faster cyclic degradation compared 
with a single fiber [87] that can be avoided using a cardboard with holes adequate spaced [88]. In this way, linear S-N curves was shown to represent well most brittle fibers [89] and thus, this methodology can be used to characterize the fatigue behavior. Also, it presents the advantage that only three to five samples are necessary to obtain reliable data in comparison to those tests using a single fiber (higher than 500) [5]. However, it was still observed that some scatter results were obtained due to internal flaws, surface defects, internal damage and the lack of standard methods [90]. For this reason, some authors [91] uses a statistic Weibull distribution to predict with reasonable reliability the fatigue strength of fibers.

In general, lamina composites with glass fibers have been shown to be much more sensitive to cyclic fatigue in the fiber direction than those with carbon fibers $[57,78]$. The fatigue behavior of glass fiber bundles is illustrated in Figure 1.16.a through a straincontrolled S-N curve. It can be seen that the stress level on the fiber bundles decreases first with a low rate while at higher number of cycles, fibers begin to fail quickly and the stress level decreases at much faster rate. However, unlike the glass fiber fatigue behavior, carbon fibers show little or no cyclic fatigue degradation as it is shown in Figure Figure 1.16.b. Several authors [90] reported that only around $2-4 \%$ of their initial strength was degraded during the cyclic loading or even, this fatigue degradation led to slight improvement of the Young's modulus of fibers [78,90,91]. This excellent behavior of carbon fibers is due to its perfect elastic nature and hence, fatigue residual deformation does not occur. Also, there are experimental evidence [88] that the tensile failure of fiber-glass composites is temperature dependent.

Although the fatigue characterization of fibers can be useful at a micro-scale using micromechanics, the majority of failures modes are missed (e.g. interfacial debonding, trasnverse cracks) and thus, the damage process involved in fatigue of unidirectional laminas is not well represented physically. Furthermore, it is impossible to characterize the fiber fatigue subjected to compression loads, i.e. buckling. For this reason, a experimental characterization of fatigue of unidirectinal laminas is preferred.

\subsubsection{Modeling of Mechanical-Fatigue Damage}

In general, fatigue of fiber-reinforced composites is quite complex where different types of damages (e.g., fiber fracture, transverse cracking, fiber-matrix debonding, delamination,...) may occur gradually or interacting each other generating different growth rates. Many models are established for a particular LSS, boundary conditions or cyclic loading, and their extrapolation to real structures is complex. Based on the diversity of models in the literature, beyond those based on pure macroscopic experimental observations (S-N and CFL curves), three main model categories are observed: fatigue life models based on 

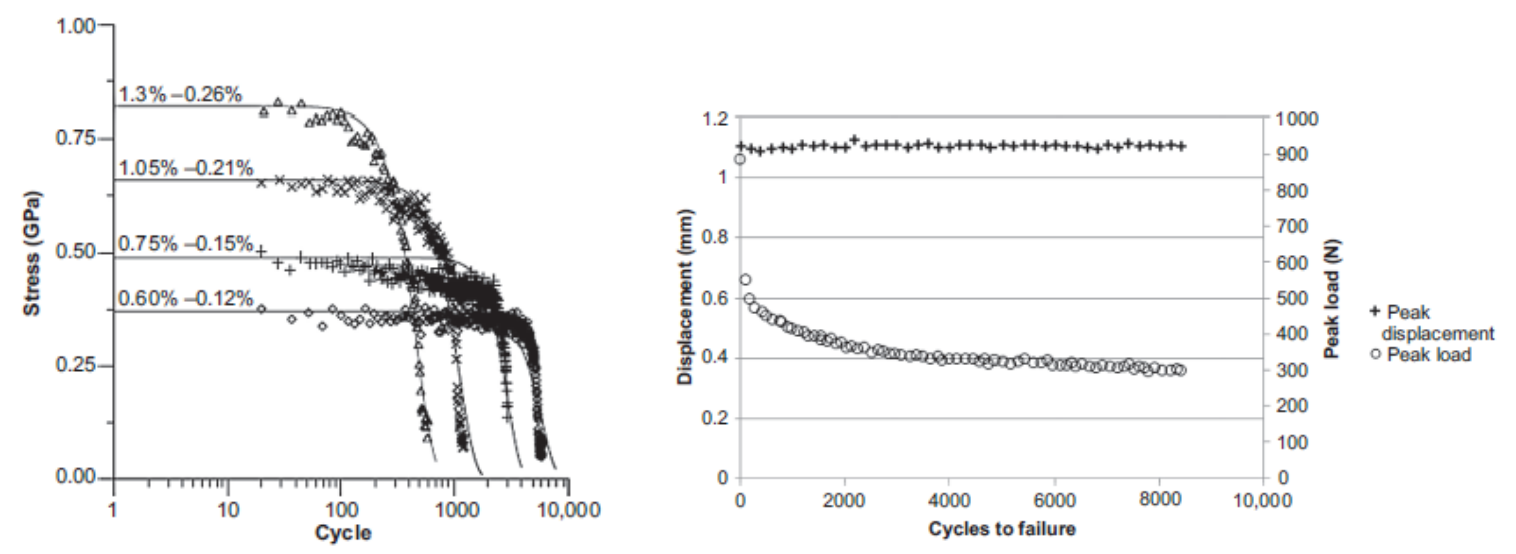

(a) $S$ - $N$ curve for E-glass fiber bundles in (b) $S$ - $N$ curve for T700 carbon fiber bundle in constant strain-controlled fatigue test. $R=0.2$ [78]. strain-controlled fatigue test ( $\epsilon=1.10 \%, R=0.2$ ) [91, 92].

Figure 1.16: Fatigue behavior of fiber bundles subjected to cyclic loadings for both, glass-and carbon-fibers.

a specific failure criteria, phenomenological stiffness/strength degradation models, and progressive damage models. An overview highlighting the main concepts and modeling to predict fatigue life is summarized.

\subsubsection{Fatigue life models}

Fatigue life models generally extract information from S-N or CFL curves to predict the fatigue behavior of unidirectional and laminate composites under multiaxial loads using a fatigue failure criteria. Therefore, they do not take into account damage accumulation. Although multiaxial loading tests are extensive in the literature [93,94], there are only few failure criteria able to predict with reasonable accuracy the fatigue life. This is due to the fact that the damage mechanisms that cause fatigue failure and thus the life prediction depends on the local stress field which in turn changes gradually with damage evolution inside the laminate. Therefore, a specific multiaxial state within the laminate will produce different local stresses as function of cycles [95, 96]. Moreover, the shear stress component has a high influence in the fatigue life prediction which couple different damage modes decreasing drastically the fatigue strength [94,97-99] but often is not well represented by FLM $[35,100,101]$.

According to literature [70,94,96,102-107], the majority of models use a polynomial failure criterion from those already existing at static loading such as Hashin and Rotem, Tsai-Hill, Tsai-Wu, Hoffman,... where the ultimate strength is a function of stress level, stress ratio and number of cycles $R_{s u}=f(R, \sigma, n)$. However, all these models are forced to make a parameters adjustment based on experimental S-N curves, which is time consuming and cost. Some researchers [108] proposed a semi-log S-N curve of the actual 
stress state from a reference S-N curve but it presents a high sensitivity depending on the chosen reference curve [94]. Others authors [109,110] propose a fatigue failure criteria based on a strain energy density function applied in two different ways. The methodology used by [111-113] calculate the total strain energy density of a generic multiaxial state in terms of uniaxial stress $\sigma_{x}$ using a transformation matrix [35] as follows

$$
\triangle W^{*}=\frac{1+R}{1-R}\left(\triangle \sigma_{x}\right)^{2}\left(\frac{\cos ^{4} \theta}{X^{2}}+\frac{\sin ^{4} \theta}{Y^{2}}+\frac{\cos ^{2} \theta \sin ^{2} \theta}{S^{2}}\right)
$$

where $\mathrm{R}$ is the stress ratio, and $\mathrm{X}, \mathrm{Y}$, and $\mathrm{S}$ are the strength limit for tension, compression and shear. Then, $\triangle W^{*}$ is related with fatigue life $\left(N_{f}\right)$ using the power law $\left(\triangle W^{*}=\right.$ $k N_{f}^{\alpha}$ ) given by [109]. On the other hand, a energy-based failure criteria as result of the main damage mechanisms observed during fatigue life (rectiliniar cracking and fiber failure, shear deformation and a mixed-mode crack growth) is presented in [110]. The final polynomial energy function is the energy contribution of each damage mode as follows

$$
\sigma_{1, a}^{2}\left(\frac{\frac{1}{E_{1}}-2 \frac{\nu_{12}}{E_{1}} \gamma_{1}+\frac{\gamma_{1}^{2}}{E_{2}}}{2 K_{S} E\left(N_{f}\right)}+\left[\frac{\gamma_{2}}{K_{6}\left(N_{f}\right)}\right]^{2}\right)=1
$$

where $\gamma_{1}, \gamma_{2}$ are the biaxial stress rates, and $K_{S E}, K_{6}$ parameters to be adjusted. More generic models based on normalized CFL diagrams are presented in [114]. Although these models only predict the fatigue life of laminate composite based on constant-amplitude loadings, their understanding and implementation in progressive models are of vital impotence to predict the final fracture, for either mechanical- or thermal-fatigue.

\subsubsection{Phenomenological models based on stiffness/strength degradation}

Experimental results in [41,115-117] demonstrate that observable changes in the composite stiffness have a clear impact on fatigue life and thus, it can be used as an indicator. They observed that polymeric laminates undergo clear changes in stiffness, differentiated in three stages as shown in Figure 1.17. At the first stage, a rapid decrease in the normalized stiffness $(2-5 \%)$ caused by primary cracks and some limited fiber breakage correspond to its $10-15 \%$ of life. As the cyclic loading continue, primary and secondary cracks with coupling lead to a continued, slow and linear normalized stiffness reduction (an additional 1-8\%) where the CDS and a $85-95 \%$ of fatigue life is reached. Finally, a sudden decrease on the normalized stiffness is observed at the end of its life characterized by delamination and final fracture. Therefore, this pattern in fatigue life can be used in the design of composite subjected to cyclic loads. With the goal to pursue the fatigue life prediction, phenomenological residual stiffness models (PRSM) have been developed [6, 47, 118-120]. 


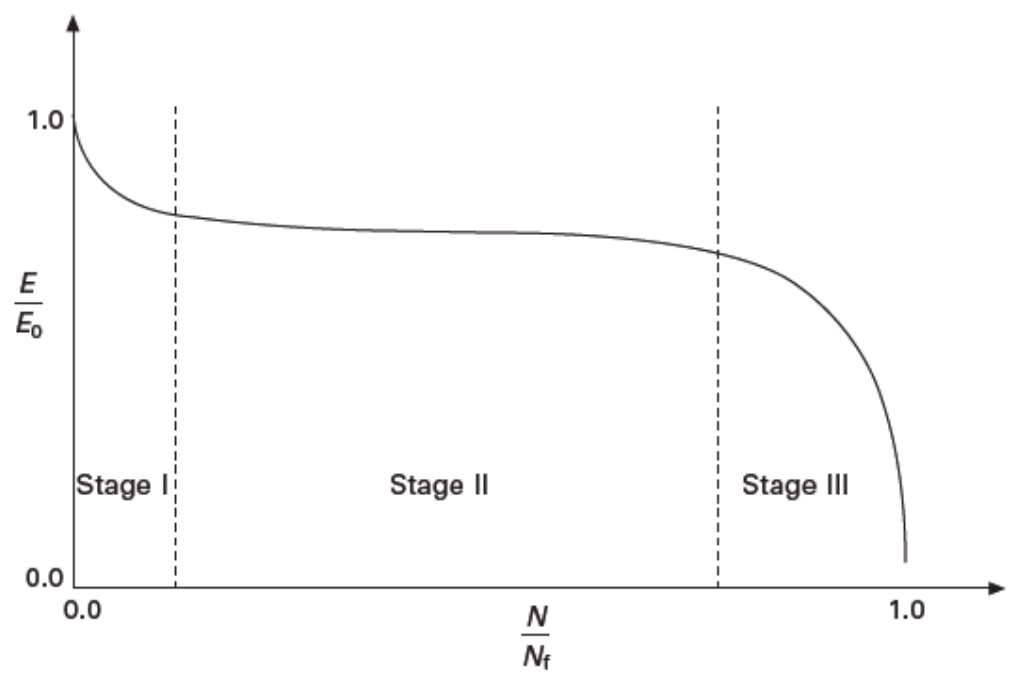

Figure 1.17: Stiffness degradation stages on composites due to fatigue cyclic loadings [6].

These models are based on numerical-experimental technique where the total damage of composite is obtained through of macroscopically observable degradation of elastic properties, typically in the longitudinal stiffness $E$. To account this degradation, a damage variable $D$ is proposed whose strain equivalence to a comparable undamaged volume under the effective stress $\tilde{\sigma}$ using the basics of continuum damage mechanics $(C D M)$ as follows

$$
D=1-\frac{E}{E_{o}} \quad \text { and thus } \quad \tilde{\sigma}=\frac{\sigma}{1-D}
$$

where $E_{o}$, and $E$ are the undamaged and damage modulus of elasticity, and $\tilde{\sigma}$, the effective stress. Under this consideration, several models [63,119,121-123] generally uses a continuum damage model to characterize the damage growth rate similar to the following power law

$$
\frac{d D}{d N}=A \frac{\tilde{\sigma}^{b}}{(1-D)^{c}}
$$

where $a, b$ and $c$ are material constants to be adjusted through the experimental results. A more complete residual stiffness approach was developed by $[120,124]$ where the damage growth rate is divided in the three characteristic stages I, II and III. Typically, the methodology first follows a parameter adjustment between experimental results and numerical modeling in stage I (transverse cracks) close to equation (1.5). Similar to stage I, a linear relation between stiffness degradation and number of cycles is established in stage II. Only a researcher [124] modeled the last stage until failure using the Tsai-Wu criterion where the effective stress $\tilde{\sigma}$ is introduced. In this way, an index failure rate $\Sigma$, defined as the inverse of the safety factor, simulate the associated residual strength as follows

$$
\left(\frac{\sigma}{\sum(1-D)}\right)^{2} \frac{1}{F_{1 t}\left|F_{1 c}\right|}+\frac{\sigma}{\sum(1-D)}\left(\frac{1}{F_{1 t}}-\frac{1}{\left|F_{1 c}\right|}\right)-1=0
$$


where $\sigma /(1-D)$ is the effective stress, $F_{1 t}$ and $F_{1 c}$ the corresponding tension and compression strength, and $\sum$ the index failure rate. An interesting phenomenological model to predict crack initiation and evolution was proposed by [23,125]. In this case, a twodimensional shear-lag model with a strain energy criteria based on the critical ERR $G_{I c}$ was proposed to establish a relationship between the elastic degradation properties and fatigue life. They observed that the crack density growth rate changes in three differentiate stages using a piece-wise function, and thus the crack density accumulation and saturation can be obtained integrating such function. The goal of this model is the use of $G_{I c}$ and how the crack density growth rate $\dot{\lambda}$ agrees with the theoretical concept of the critical size of internal defects with fatigue. In other words, internal flaws with lengths initially lower than the critical size increase up to reach such characteristic critical size under cyclic loading.

Other types of phenomenological models based on the residual strength have been developed distinguished in two categories: sudden death model or wearout model. The sudden death models $[41,126,127]$ determined the residual strength subjected to a high level of stress state, which drastically decreases as they approach to their fatigue life. The wearout models $[7,53,128,129]$ incorporate the "strength-life equal rank assumption" (SLERA), which assumes that the laminate's fatigue is proportional to its initial static strength and the final fracture is developed when residual strength reaches the maximum constant amplitude. Good wearout approaches developed by [7,53,130] assume that the residual strength and fatigue life can be well predicted using statistical Weibull distributions as shown in Figure 1.18.

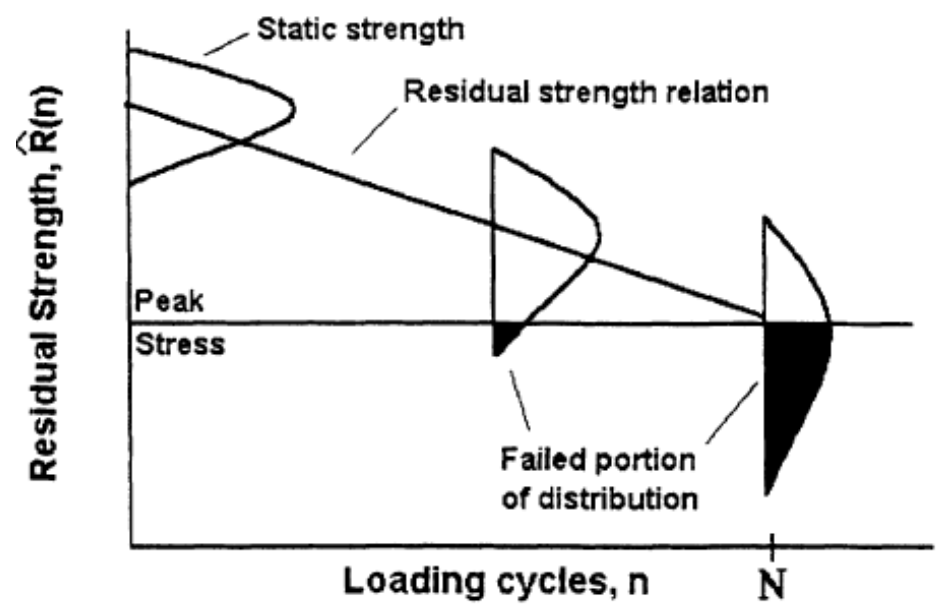

Figure 1.18: Strength distribution associated with specific residual strength relation [7].

Although phenomenological stiffness models lead to stress redistribution and can be used as non-destructive parameter to monitor de fatigue damage, they present drawbacks with respect a progressive damage models (PDM) such as $[6,31,35,35,101]$. For instance, 
they are frequently not valid for all stages, specially in stage III, and a universal failure criteria defining the critical stiffness degradation level to induce fatigue failure has not been defined yet. Some authors [41,131] define this critical modulus degradation when the secant modulus at the moment of failure in a static test is reached. Other instead [121] state that fatigue failure occurs when the fatigue resultant strain reaches the static ultimate strain. Furthermore, most models are one-dimensional and thus, only the longitudinal stiffness and not the remaining properties are taking into account which have a clear impact in fatigue failure. In addition, the residual stiffness models is based on laminate degradation rather than lamina stiffness degradation. While these phenomenological approaches are tied for a specifically LSS and experimental results, progressives damage models as in $[35,132]$ can calculate the stiffness degradation for each lamina and asses the fatigue effect on different LSS. However, the strength degradation models based on statistic functions (Weibull distribution) can be useful using the CDM so that damage variables $D$ can be calculated as function of a failure distribution, for instance in the critical elements ( $0^{\circ}$ laminas).

\subsubsection{Progressive damage models}

Fatigue life prediction of composite base on a progressive damage modeling (PDM) has also extensively reported in the literature such as in [6,133-140]. These models use a progressive algorithm which combines a stress analysis, failure analysis, and gradual material degradation as shown for instance in Figure 1.19. A local stress state generated by transverse cracking, coupling, and delamination is continuously updated in order to represent the physical process involved during the fatigue loading. As the number of cycles increase, the damage growth increases degrading the material properties lamina by lamina and a failure criteria is used to determine the fatigue life.

PDM based on the residual strength prediction These models use damage variables to predict the residual strength on laminate composites. Many authors [63,96, $133,141]$ use a failure criteria where the residual strength as function of cycles has been used instead of static strength. However, the residual strength of unidirectional laminas $\bar{R}_{i}=f(R, \sigma, n)$, changes with the actual local stress state and thus, a large number of experimental results to predict fiber failure is necessary [112,134]. Since the stress state in $0^{\circ}$ laminas changes gradually with damage variables (unsteady multiaxial state), the strength degradation present a high restriction. Some researchers [142,143] face this problem using a normalized function and adjusting some material parameters, which induce some suitable fatigue measurements $[62,134]$. Others $[6,111]$ face this problem predicting the fatigue life $N_{f}$ through a energy density function for any combination of stress ratio 
and stress state as explained in Section 1.3.5.1.

Since damage of composite laminate has a stochastic nature (independent damage modes or interacting with each other), some models are based on probabilistic functions such as Weibull distribution $[36,47]$. A statistic two-stage strength degradation model to predict fatigue life on laminate composites was presented by $[36,37]$. The first phase of damage is characterized by a non-interacting crack pattern (disperse) which eventually reach the crack density saturation (CDS). The second phase is initiated from localized damaged zones (coupling crack, interfacial debonds, delamination) increasing quickly the damage growth until final fracture. Once the probability of finding the number of cycles $N_{c}$ to reach the CDS (first stage) is obtained, the remaining residual strength is expressed in terms of a single crack, which would releases the same total stored elastic energy given by remaining damage modes. At this stage, the probability of finding the fatigue life which would cause to fail the laminate $N_{f}$ is expressed in terms of a power law distribution.

Another proposed methodology [144] suggests that the fatigue life in laminate composite can be determined by the residual strength degradation on critical elements $\left(0^{\circ}\right.$ laminas) taking into account the local stress state given by subcritical elements such as transverse cracking, interfacial debonds, and delaminations. The author assumes that the critical failure is initiated in regions with high localized stress at the fatigue life end rather than limited broken fibers at the early stage of primary cracks [145, 146]. Taking into account the residual strength cycle-dependent through unidirectional S-N diagrams and the stress state using a representative volume element (RVE) from subcritical elements, the residual strength of composites can be determined as shown the flowdiagram illustrated in Figure 1.19.

PDM based on the residual stiffness prediction Many stiffness degradation models under static loads have been extensively studied in the literature [6, 35, 144, 147-149]. Typically, intralaminar transverse cracking is the first mode of damage in polymer-matrix laminated composites [47]. Similar to the residual strength, the stiffness degradation is also cycle-dependent and precedes catastrophic modes of damage such as delamination, and fatigue life reduction [38]. Therefore, a stiffness reduction lamina by lamina in the progressive damage is necessary leading a continuous stress redistribution under multiaxial stress.

Under static loads, many attempts have been made to predict initiation and evolution of transverse cracks in laminated, polymer-matrix composites. Some models use a linear elastic fracture mechanics [147,150-153], a combination of fracture and strength $[154,155]$, a fracture toughness as damage criteria $[31,132,156]$, a variational approach $[157,158]$, or semi-empirical models based on adjustable parameters $[159,160]$. In any case, it has 


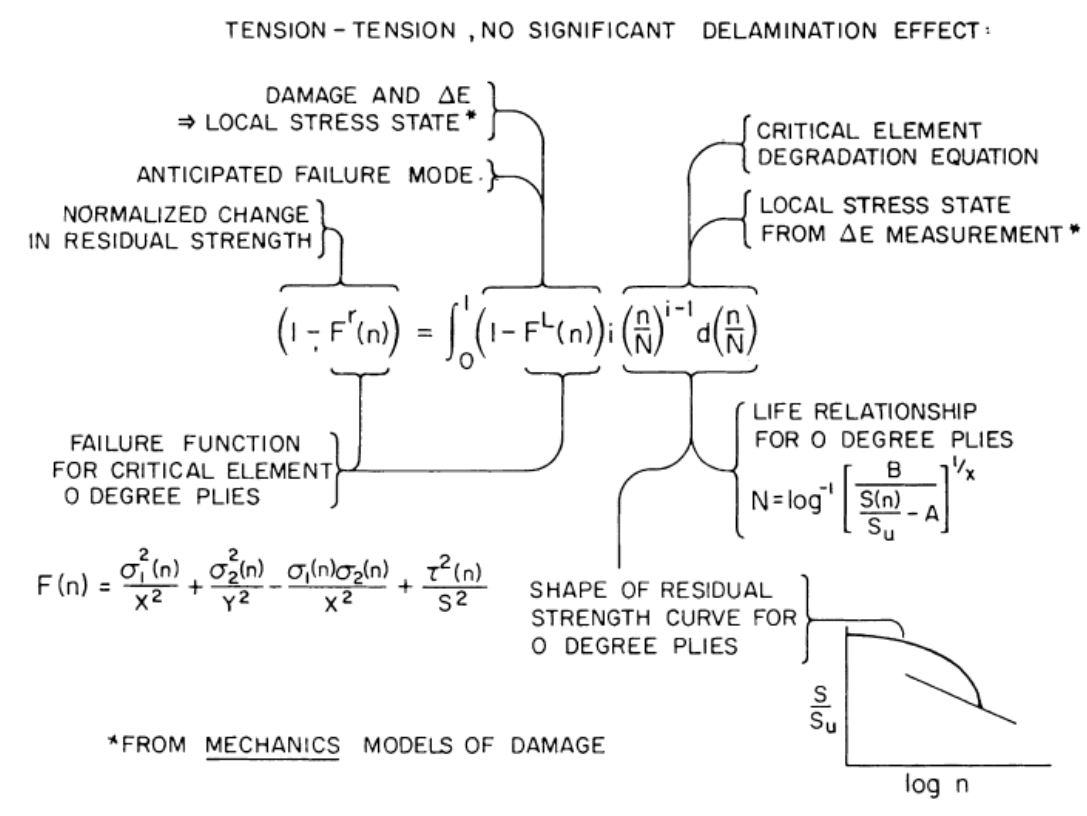

Figure 1.19: Interpretation of the residual strength equation subjected to tension-tension fatigue loads [6].

been shown experimentally that unidirectional loading of $\left[0_{m} / 90_{n}\right]_{s}$ cross-ply laminates produces transverse cracking in $90^{\circ}$ laminas when the load is applied in $0^{\circ}$ direction [161-166]. Similar behavior on cross-ply laminates under cyclic loadings has been also demonstrated $[6,38,47,134]$. However, under fatigue loading the CDS due to transverse cracking also precedes delamination or critical fracture of composite, and thus gradual stiffness degradation during the three stages (see Figure 1.17) must be computed.

Generally, the fatigue life until the CDS is reached and its corresponding laminate stiffness degradation are calculated in different ways. Some authors $[167,168]$ make use of the classical laminate theory (CLT) to obtain a stiffness degradation expression in the $90^{\circ}$ laminas as function of crack density, to then be compared with a master S-N curve and thereby, the fatigue life to CDS. Other researchers use a shear-lag model [38,169-171], a variational approach [22], a meso-scale damage analysis [28,31,139], continuum damage mechanics $[137,167]$ or a power law function to express the microscopic damage growth rate for both, stiffness and/or crack density [22,125,138,172-175]. The models based on damage growth rate are typically founded in the critical energy release rate $G_{I c}$, which is a material property and thus can be used as failure growth criteria for any LSS. Once the CDS is reached, the linear and sudden stiffness degradation due to delamination and final failure in stages II and III (see Figure 1.17) can be calculated for instance, through the use of $\mathrm{S}-\mathrm{N}$ curves on the critical elements ( $0^{\circ}$ laminas) [167,168,175], a semi-empirical model [134] adjusting parameters, a shear lag-analysis incorporating delamination [130], or using a damage growth rate for delamination or crack density [50, 135, 136, 176-179] 
expressed similar by to Paris's law as follows

$$
\frac{d \eta}{d N}=k\left(\frac{G_{\max }}{G_{I c}}\right)^{n}
$$

where $\eta$ can be the new delaminated area or crack density, and $G_{\max }, G_{I c}$ the maximum and critical energy release rate, respectively. The parameters $k$ and $n$ are adjusted through damage growth experimental data. Some recent models [180] calculate the stiffness degradation at micro-scale level (fiber and matrix) using CDM with damage variables $D$ adjusted through S-N diagrams of unidirectional laminas subjected to pure tensionfiber $\left(0^{\circ}\right)$, tension-matrix $\left(90^{\circ}\right)$, and shear $\left(45^{\circ}\right)$.

Theoretical formulations able to predict fatigue life under multiple block loading patters are necessary due to the spectrum load at which composites structures are subjected. The linear Palgrem-Miner rule is the most popular damage accumulation model which does not always lead to accurate results for composites. The summation of all partial damage coefficients are compared with a unity value which indicates whether the laminate fail or not [168]. Although some non-linear damage accumulation models were developed [181], they are fitted to experimental data of various block loadings. Therefore, they cannot be used for design purposes. Only a stress-independent non-linear cumulative damage model $[107,182]$ was developed for glass reinforced plastic (GRP) laminates. CFL diagrams can be used to predict accurately the fatigue life using linear or nonlinear damage rules as in [53,65].

\subsubsection{Modeling of Thermal-Fatigue Damage}

According to thermal fatigue investigations, most researchers $[1,8,10,21,29]$ agree on the effect of thermal cycling in composite materials. They conclude that laminate composite undergo mainly transverse cracks with some local but not significantly delamination on the specimens edge. These damage mechanisms, similar as those subjected to mechanical fatigue, are the result of thermal loads generated in each lamina due to mismatch in the coefficient thermal expansion (CTE) between laminas at different orientations. The transverse cracks starts from failures due to high residual thermal stresses at fibermatrix interface leading to ideal paths of crack propagation between two adjacent laminas. Therefore, cracks generated may no significantly alter the structural integrity but changes in the CTE $[1,183]$ may be large enough to make unacceptable the laminate composite for both, dimensional stability and critical applications such us fuel oxidant storage where gas-leakage may occur.

Unlike how it occurs in mechanical fatigue, experimental results for cross-ply [8$10,23,24,29,30,184]$ and quasi-isotropic laminates $[10,21,27,184]$ subjected to thermal 

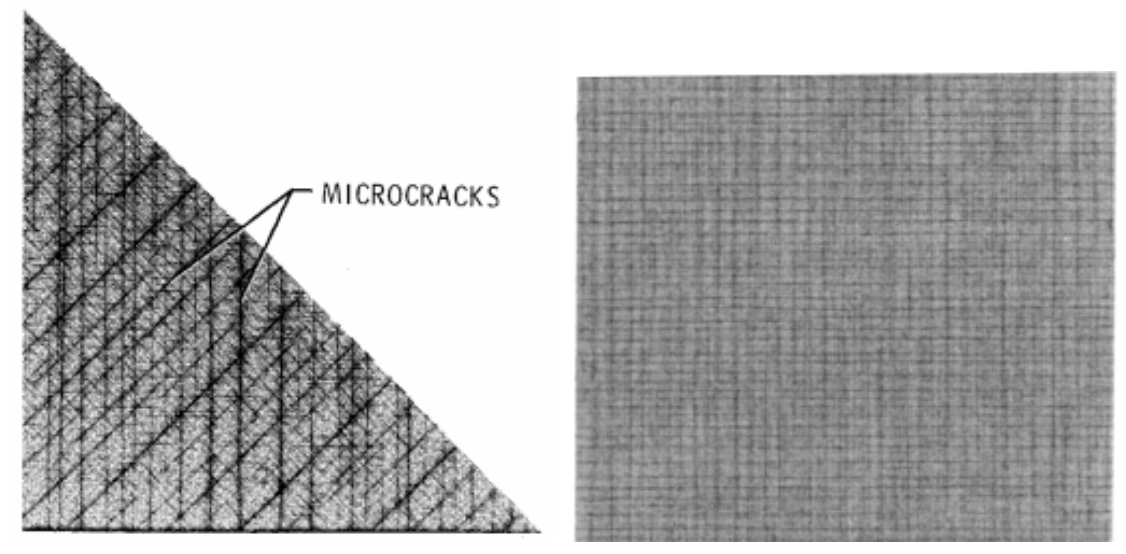

(a) C600/PMR-15 [0/45/90/ - 45] (b) P75/1962 [0/90 $]_{2 s}$ laminate sublaminate subjected to thermal cycling jected to thermal cycling after 3500 after 500 cycles between -156 and cycles between -156 and $120^{\circ}$ [10]. $300^{\circ}$ [21].

Figure 1.20: X-ray images for two material systems containing multiple transverse cracks at different orientations.

cycling show that predominant damage in form of transverse cracking (primary cracks) is generated not only in $90^{\circ}$ laminas but also in the adjacent laminas as shown in Figure 1.20. This microcracking effect is expected because in thermal fatigue, no reference coordinate system exists and all laminas undergo almost same strain in most cases. For instance this is clear on cross-ply laminates where microcracks usually span to the thickness and run parallel to the fibers.

Other characteristics such as residual strength in $0^{\circ}$ laminas and laminate bending stiffness was found to be almost undamaged because they are fiber controlled, and the thermal fatigue reported in the literature (i.e., around 500 cycles) is really small [1,21]. In addition, it was found in [1] that transverse cracking on laminates considerably increase when they are exposed to significant levels of radiation. The effect of thermal shock gradient on transverse cracking was studied by [30] resulting in minor differences with respect those specimens cycled at slow rate. However, experimental investigations [30] found lower crack densities, especially for inner laminas of cross-ply laminates, on cappededge specimens with respect those free-edge. Intense investigations reported in $[11,24,27]$ showed that the counting crack method from the free edge of the specimens by optical microscopy is highly influenced by the free-edge stress, and thus prediction using CLT may not match according to experimental crack density data, especially for inner laminas.

\subsubsection{Characteristic Damage State (CDS)}

The CDS on laminate composites was investigated for several researchers $[1,8,10]$ under thermal cyclic loads. Unlike the mechanical fatigue where the CDS is determined by 
the LSS and the material properties [23,125], the temperature also play an important role in the CDS under thermal fatigue. Experimental data for carbon/epoxy laminates in $[1,8,10,14,24,27,29,30]$ show an increment in the crack density $\lambda$ with the number of cycles which tends to reach an equilibrium state (CDS) at several thousand of cycles. Other authors went further comparing the induced CDS at static loads and under thermal 500 -cycles $\left(-156,120^{\circ}\right)$ through $\mathrm{FE}$ analysis in [8] as shown in Figure 1.21.a. In any case, the crack density $\lambda$ can be concluded to asymptotically approach the equilibrium state (CDS) at lower number of cycles in comparison with those subjected to mechanical fatigue. Only experimental results in $[30,184]$ show a slight growth on transverse cracking without reaching the CDS after 2600 cycles although a minimal growth rate at the end of cycling indicates fast approach to the equilibrium state. Therefore, the materialdependent properties and temperature range have a high influence in the CDS under thermal fatigue.

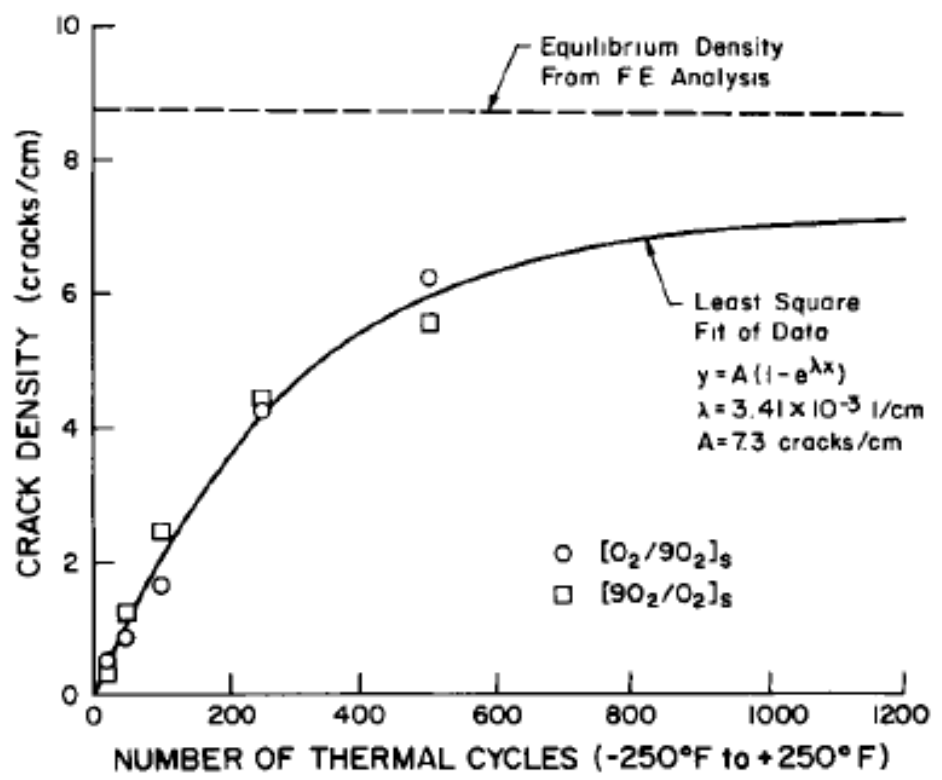

Figure 1.21: Crack density vs. thermal cycle data for $\left[0_{2} / 90_{2}\right]_{s}$ and $\left[90_{2} / 0_{2}\right]_{s}$ T300/5208 laminate between -156 and $300^{\circ}$ [8].

Since tests under thermal cycling that cause the failure of laminates require a large number of cycles (extremely time consuming), only thermal fatigue models based on transverse cracking until reaching the CDS are reported in the literature $[8,10,14,23,24$, 27-30]. Some authors [8] use CLT and temperature-dependent properties to calculate in situ transverse tensile strength $F_{2 t}^{i s}$ as function of temperature (see Figure 1.21), and in this way predict the initial temperature at which the first crack is generated. This methodology is insufficient in the thermal fatigue framework for several reasons. First, the in situ strength is only able to predict the first crack and thus, it is not able to calculate the CDS. Second, the in situ strength is a function of lamina thickness and 
it depends on the LSS. And third, it does not take into account free-edge stress where the thermal stress $\sigma_{2}$, in the $90^{\circ}$ laminas have been demonstrated to be greater at the edge (experimental) than interior [11,27]. Therefore, in situ strength at the edge must be calculated instead of the interior stress using CLT.

However, the majority of models $[29,185,186]$ use strain energy release rate $G_{I}$ to predict the crack onset and evolution during thermal cycling. This fracture mechanic concept is based on the assumption that internal flaws with a critical size $l_{c}$ within the laminate will grow when the internal stored energy $G_{I}$ reaches a critical value $G_{I c}$, as shown in Figure 1.22. This critical ERR is a material property and thus, it does not depend on thickness or LSS. Typically, only a opening growth mode is considered especially on cross-ply laminates $[9,24,27,29,185,187]$. Some authors [18, 188] studied the monotonic cooling of laminates to adjust both, shear-lag parameters and $G_{I c}$. Then, experimental data with thermal cycling $[10,22,29,30,184,186,189]$ is collected to adjust $G_{I c}$ as function of number of cycles.

Among the different proposals presented in the literature, some authors use a onedimensional [10,185] or two-dimensional [29] shear-lag model or a mesomodel [25] to calculate the stored energy in the laminate to be compared with $G_{I c}$, where a direct relationship was observed. They found that a micro-damage accumulation during the first fatigue cycles was necessary to generate transverse cracks in those laminates where the energy release rate was lower than $G_{I c}$. Only after a given number of cycles, the first fatigue crack was formed. In other words, a degradation on critical ERR with number of thermal cycles occurs. From a theoretical point of view, this means that internal flaws, which initially have small sizes, grow gradually up to reach the critical size $l_{c}$ (see Figure 1.22). Some authors [24] point to shrinkage of the matrix and oxidation phenomenon as potential points of nucleation and growth of internal defects as the thermal cycles increase.

Therefore, some researchers [125] calculated the crack density growth rate with the number of cycles obtaining a phenomenological law to predict the crack initiation and evolution. Others $[10,185,186]$ proposed a quadratic expression with the number of cycles to account the thermal fatigue effect on $G_{I c}$, i.e. decreasing with the number of cycles. However, more accurate models [190] assumes not only a degradation of $G_{I c}$ due to cyclic thermal loads but also a progressive oxidation in oxygen-rich environments. Experimental results in $[24,190]$ demonstrate the accelerating effect of damage built-up on oxidative environments inducing early transverse cracking. As result, a coupling between oxidation and thermal fatigue induces higher crack densities or a large degradation of $G_{I c}$, which can be written as a cumulative reduction by factors such as thermo-mechanical cycling loads, oxidation, etc... Although the strain energy criteria based on $G_{I c}$ is a 


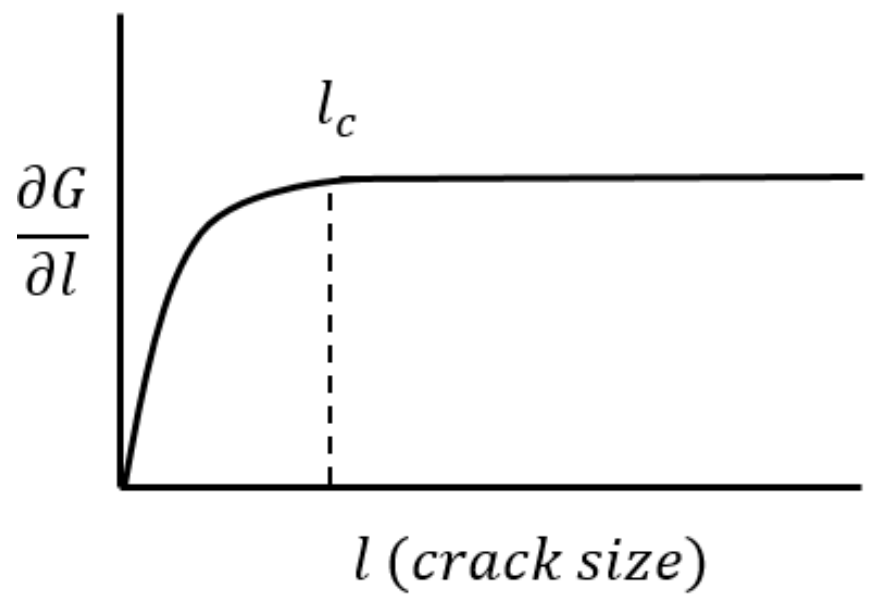

Figure 1.22: Strain energy release rate as function of crack size.

success in the author's opinion, several assumptions make it uncertain. For instance, the critical $G_{I c}$ values used in the literature were obtained in term of the in situ transverse strength $[10,185,186]$ or a specific LSS [125]. Sometimes, the $G_{I c}$ values were scaled with available transverse strength data $\left(F_{2 t}\right)$ and $G_{I c}$ values from other material systems [186]. Furthermore, none of models used the temperature dependent properties to characterize the matrix cracking effect under thermal cycling.

Other authors $[9,22]$ use a variational approach to obtain a relationship between transverse cracking and $G_{I}$. In this way, a modified Paris law was used that all data fall in a single master curve. Since the critical $G_{I c}$ is a material property, it is assumed that a master curve can be used to predict crack initiation an evolution for any material system. However, some results in [9] show different master curves for each temperature range in a certain material system. Also, a dependence with the temperature range is observed in both, the CDS and crack density growth rate. They showed that the crack saturation and the crack growth increases as $\Delta T$ increase. However, it cannot be concluded that the temperature range has an influence in the CDS because only one thousand cycles were compiled. Finally, they compared two Paris law due to mechanical and thermal fatigue showing clear differences on the crack growth rate, and thus a clear dependence of $G_{I c}$ with temperature can be concluded as shown in Figure 1.23.

The most recent works presented by $[187,191]$ use a complicated global-local model for stiffness prediction of symmetric damaged laminates [187] taken into account crack initiation and propagation. On one hand, the crack initiation is characterized by a stochastic nature starting from internal flaws $[187,191]$ and can be estimated using a the linear elastic fracture mechanics (LEFM) as function of the average transverse stress (not interacting between cracks). Since the transverse tensile strength $F_{2 t}$ follows a probabilistic function [7,53], the crack density as function of the average transverse stress $\sigma_{T}$ can be 


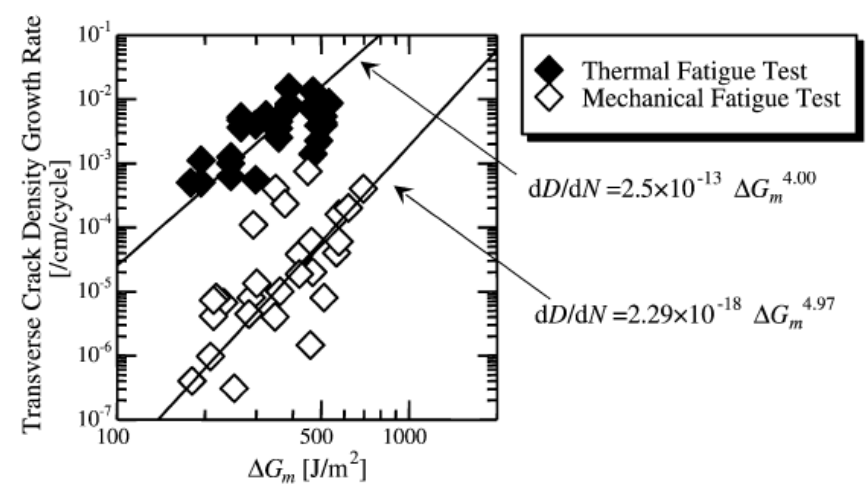

Figure 1.23: A Paris law for transverse crack density growth in G40-800/5620 under thermal and mechanical fatigue [9].

estimated by a Weibull distribution (1.8). On the other hand, the $G_{I}$ can be calculated using the crack open displacement (COD) method using average displacements [187]. In this way, a Paris law for individual crack growth in thermal cycling can be used to establish the crack propagation [22]. Although crack initiation can be described by the stochastic nature of $F_{2 t}$, the crack resistance strength is also well know to be thicknessdependent due to interlaminar interfaces and thus, a ERR criterion must be chosen. In this line, the Weibull distribution to obtain a representative statistical failure function of $G_{I c}$ is contradictory by definition with the theoretical meaning of $G_{I c}$, namely, a material property. However, the stochastic nature of nucleation and growth of cracks up to reach a critical size $l_{c}$, is inherent in this probability function, and thus a good prediction method.

In spite of good predictions reported in [187,191], small deviations at first and last thermal cycles is obtained mainly for two reasons. First, the temperature dependent properties are not taken into account and thus, the Weibull distribution, which is adjusted with $F_{2 t}$ at room temperature, is overestimated. And second, the Weibull distribution is adjusted assuming that the crack density saturation is equal to that in mechanical loading $\left(\lambda_{C D S}\right)$. Based on experimental results reported in the literature $[1,8,10,14,24,29,30]$, the CDS is observed to be lower at thermal cycling than mechanical fatigue.

$$
\frac{\lambda\left(\sigma_{T}, N\right)}{\lambda_{C D S}}=1-\exp \left[-k N^{\alpha}\left(\frac{\sigma_{T}}{Y_{t}}\right)\right]
$$




\section{Chapter 2}

\section{Discrete Damage Mechanics}

Among the multitude of damage models available, discrete damage mechanics (DDM), [31]) is attractive for this study because, in addition to the usual elastic properties, it requires only two values of critical energy release rate (critical ERR), $G_{\text {Ic }}$ and $G_{I I c}$, to predict both damage initiation and evolution due to transverse and in-plane shear stresses for general laminates subjected to general loads [192]. Furthermore, DDM has already been demonstrated to be mesh independent $[32,100]$ unlike other progressive damage model already implemented in commercial FEM softwares such as ANSYS [101] or Abaqus [35]. Therefore, DDM is an objective constitutive model without need to use a guessing characteristic length $L_{c}$ in order to reduce mesh dependency [193].

DDM is based on an analytical solution [132] of the displacement field inside a representative volume element (RVE, Figure 2.1) encompassing the laminate thickness t with $\mathrm{N}$ laminas, a unit length along the fiber direction of the cracking lamina $k=c$, and a distance $2 l=1 / \lambda$. The lamina coordinate system is denoted by $x_{1}, x_{2}$ and $x_{3}$ and the laminate coordinate system by $x, y$ and $z$ as shown in Figure 2.1. Each lamina in the laminate is susceptible to transverse cracking which is controlled by a damage activation function $g(\lambda, \epsilon, \triangle T)$, discussed in detail in Section 2.8. Such function determine whether the total elastic strain energy stored in the laminate is enough to generate a new crack. Such energy stored is a function of the crack density $\lambda$, and the applied strain $\epsilon$ and/or $\Delta T$, which is the difference between the reference and operation temperature.

The applied thermal-mechanical strain is applied in a successive increments until reaching a certain strain value or temperature range $\Delta T$. In each load step, the damage activation function is calculated for each lamina. If $g(\lambda, \epsilon, \triangle T)<0$, then no damage occurs but whether $g(\lambda, \epsilon, \triangle T)>0$, a new crack/s are generated parallel to the fiber orientation.

Once that a new crack appears, a return mapping algorithm is then used to determine the current crack density of the lamina. This procedure is repeated until all laminas within 
the laminate have been analyzed and finally converged. At this point, the laminate does not suffer more transverse cracking until more strain or temperature is applied. As the result of damage, the crack density increases and the stress is redistributed into the remaining laminas causing a loss of laminate stiffness and a variation in the laminate thermal expansion (CTE). This laminate response is then used to homogenize the damage lamina by lamina. Therefore, the homogenization of cracked lamina and an iterative procedure allows all laminas $k=1 \ldots N$ in a laminate to be cracking simultaneously with different crack density $\lambda_{k}$ values at a given time.

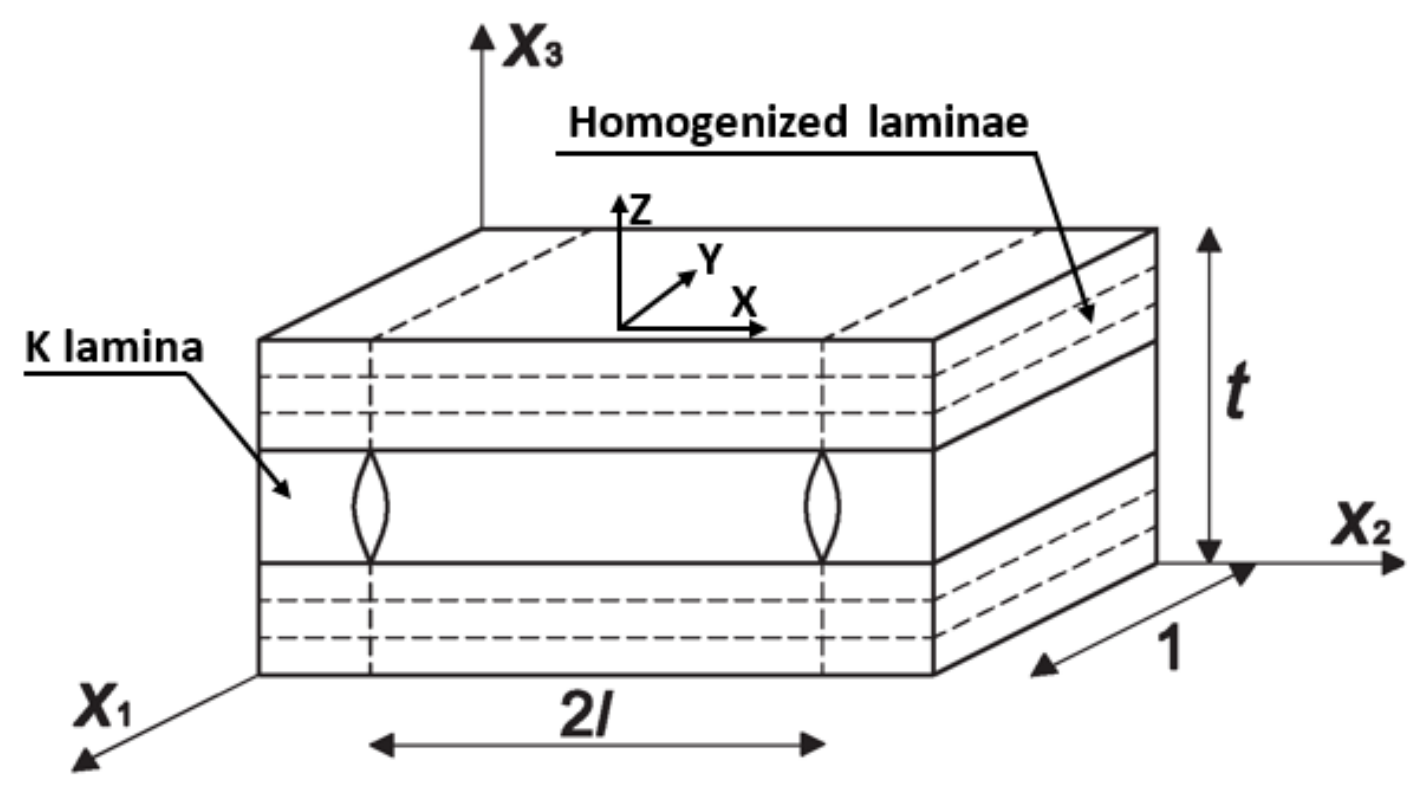

Figure 2.1: Representative volume element for DDM.

\subsection{Theory formulation}

The most practical laminates are symmetric so that they are the most efficient to design structures loaded by membrane loads [35, Chapter 12]. Therefore, no bending moments are applied to the laminate

$$
\frac{\partial w^{i}}{\partial x}=\frac{\partial w^{i}}{\partial y}=0
$$

where the superscript (i) refers to the ith lamina.

The bottom and top surfaces of the laminate are stress-free and the laminate is thin enough to be considered as a plane stress state. The thickness average of any mechanical variable is defined as

$$
\widehat{\phi}=\frac{1}{h_{i}} \int_{h_{i}} \phi d x_{3}
$$

where $\phi$ can be any parameter such as $\sigma, \epsilon, Q, \ldots$ This definition is useful for obtaining 
the overall reduced stiffness properties based on the averaged displacements.

For a cracking lamina $k$, the constitutive equation is

$$
\widehat{\sigma}_{i}^{k}=Q_{i j}^{k}\left(\widehat{\epsilon}_{j}-\widetilde{\alpha}_{j}^{k} \Delta T\right)
$$

where $\widetilde{\alpha}_{j}^{k}$ is the undamaged CTE of lamina $(\mathrm{k})$,

$$
\widehat{\sigma}^{k}=\left\{\begin{array}{c}
\widehat{\sigma}_{1}^{k} \\
\widehat{\sigma}_{2}^{k} \\
\widehat{\sigma}_{12}^{k}
\end{array}\right\}
$$

the average stresses of lamina $(\mathrm{k})$,

$$
\widehat{\epsilon}^{k}=\left\{\begin{array}{c}
\widehat{u}_{, 1}^{k} \\
\widehat{v}_{, 2}^{k} \\
\widehat{u}_{, 2}^{k}+\widehat{v}_{, 1}^{k}
\end{array}\right\}
$$

the average strain field of lamina $(\mathrm{k})$, and, $1 ;, 2$ represents partial derivatives as usual.

The constitutive equations for the remaining laminas $(m \neq k)$ can be calculated by using equation (2.3) with the reduced stiffness matrix, $Q_{i j}^{m}$, written in terms of their previously calculated damage values $D_{2}^{m}, D_{6}^{m}$, defined later in Section 2.7, and rotated to the $\mathrm{k}$ coordinate system using the usual transformation [35]

$$
Q^{m}=[T(-\theta)]\left[\begin{array}{ccc}
\widetilde{Q}_{11}^{m} & \left(1-D_{2}^{m}\right) \widetilde{Q}_{22}^{m} & 0 \\
\left(1-D_{2}^{m}\right) \widetilde{Q}_{12}^{m} & \left(1-D_{2}^{m}\right) \widetilde{Q}_{22}^{m} & 0 \\
0 & 0 & \left(1-D_{6}^{m}\right) \widetilde{Q}_{66}^{m}
\end{array}\right][T(\theta)]^{T}
$$

where the damage values belong to a diagonal second order damage tensor defined in [194].

\subsection{Shear Lag Equations in Matrix Form}

The intralaminar shear stresses are assumed to be linear as follows

$$
\tau_{13}^{i}\left(x_{3}\right)=\tau_{13}^{i-1, i}+\left[\tau_{13}^{i, i+1}(x)-\tau_{13}^{i-1, i}(x)\right] \frac{x_{3}-x_{3}^{i-1, i}}{h^{i}}
$$

and

$$
\tau_{23}^{i}\left(x_{3}\right)=\tau_{23}^{i-1, i}+\left[\tau_{23}^{i, i+1}(x)-\tau_{23}^{i-1, i}(x)\right] \frac{x_{3}-x_{3}^{i-1, i}}{h^{i}}
$$

where $x_{3}$ is in a lamina coordinate system (see Figure 2.1). $\tau_{13}^{i-1, i}$ is the shear stress at the interface between the $\mathrm{i}-1$ th and the ith lamina, and $x_{3}^{i-1, i}$ is the position of the interface 
between i-1th and ith lamina. This assumption is common to several other analytical models and it is called the Shear Lag assumption [195].

The shear lag equations are obtained from the constitutive equations for out-of-plane shear strains and stresses by means of weighted averages as follows

$$
\begin{aligned}
\left\{\begin{array}{c}
\widehat{u}^{(i)}-\widehat{u}^{(i-1)} \\
\widehat{v}^{(i)}-\widehat{v}^{(i-1)}
\end{array}\right\} & =\frac{h_{(i-1)}}{6}\left[\begin{array}{cc}
S_{45} & S_{55} \\
S_{44} & S_{45}
\end{array}\right]^{(i-1)}\left\{\begin{array}{c}
\tau_{23}^{i-2, i-1} \\
\tau_{13}^{i-2, i-1}
\end{array}\right\} \\
& +\left[\frac{h_{(i-1)}}{3}\left[\begin{array}{cc}
S_{45} & S_{55} \\
S_{44} & S_{45}
\end{array}\right]^{(i-1)}+\frac{h_{(i)}}{3}\left[\begin{array}{cc}
S_{45} & S_{55} \\
S_{44} & S_{45}
\end{array}\right]^{(i)}\right]\left\{\begin{array}{c}
\tau_{23}^{i-1, i} \\
\tau_{13}^{i-1, i}
\end{array}\right\} \\
& +\frac{h_{(i)}}{6}\left[\begin{array}{cc}
S_{45} & S_{55} \\
S_{44} & S_{45}
\end{array}\right]^{(i)}\left\{\begin{array}{c}
\tau_{23}^{i, i+1} \\
\tau_{13}^{i, i+1}
\end{array}\right\}
\end{aligned}
$$

from which the intralaminar shear stresses are expressed as

$$
\begin{array}{r}
\tau_{23}^{i, i+1}-\tau_{23}^{i-1, i}=\sum_{j=1}^{n-1}\left[[H]^{-1}{ }_{2 i-1,2 j-1}-[H]^{-1}{ }_{2 i-3,2 j-1}\right]\left\{\widehat{u}^{(j+1)}-\widehat{u}^{(j)}\right\} \\
+\left[[H]^{-1}{ }_{2 i-1,2 j}-[H]^{-1}{ }_{2 i-3,2 j}\right]\left\{\widehat{v}^{(j+1)}-\widehat{v}^{(j)}\right\} \\
\tau_{13}^{i, i+1}-\tau_{13}^{i-1, i}=\sum_{j=1}^{n-1}\left[[H]^{-1}{ }_{2 i, 2 j-1}-[H]^{-1}{ }_{2 i-2,2 j-1}\right]\left\{\widehat{u}^{(j+1)}-\widehat{u}^{(j)}\right\} \\
+\left[[H]^{-1}{ }_{2 i, 2 j}-[H]^{-1}{ }_{2 i-2,2 j}\right]\left\{\widehat{v}^{(j+1)}-\widehat{v}^{(j)}\right\}
\end{array}
$$

in terms of the $2(N-1)$ by $2(N-1)$ coefficient matrix $H$, which is the assemblage of equation (2.9). These relationships are then used in the equilibrium equations (2.11) and (2.12) to substitute the average displacements $\widehat{u}$ and $\widehat{v}$, for $\tau_{13}$, and $\tau_{23}$.

\subsection{Solution of the Equilibrium Equation}

The equilibrium equations for each lamina can be stated as follows

$$
\begin{aligned}
& \widehat{\sigma}_{1,1}^{(i)}+\widehat{\tau}_{12,2}^{(i)}+\left(\tau_{13}^{i, i+1}-\tau_{13}^{i-1, i}\right) h_{i}{ }^{-1}=0 \\
& \widehat{\tau}_{12,1}^{(i)}+\widehat{\sigma}_{2,2}^{(i)}+\left(\tau_{23}^{i, i+1}-\tau_{23}^{i-1, i}\right) h_{i}{ }^{-1}=0
\end{aligned}
$$

Then, substituting equations (2.9) and (2.10) into 3D equilibrium equations (2.11) and (2.12), the average displacements $\widehat{u}$ and $\widehat{u}$ can be obtained solving a partial differential equations (PDE) in the following form 


$$
\begin{aligned}
& \widehat{u}^{(i)}=a_{i} \sinh \lambda_{e} x_{2}+a x_{1}+b x_{2} \\
& \widehat{v}^{(i)}=b_{i} \sinh \lambda_{e} x_{2}+b x_{1}+a^{*} x_{2}
\end{aligned}
$$

where $e$ is the eigenvalue numbers. Thus, the general solution can be written as

$$
\left\{\begin{array}{c}
\hat{u}^{(1)} \\
\hat{u}^{(2)} \\
\cdot \\
\cdot \\
\cdot \\
\hat{u}^{(n)} \\
\hat{v}^{(1)} \\
\hat{v}^{(2)} \\
\cdot \\
\cdot \\
\cdot \\
\hat{v}^{(n)}
\end{array}\right\}=\sum_{e=1}^{2 N} A_{e}\left\{\begin{array}{c}
a_{1} \\
a_{2} \\
\cdot \\
a_{n} \\
b_{1} \\
b_{2} \\
\cdot \\
\cdot \\
\cdot \\
b_{n}
\end{array}\right\} \sinh \left(\eta_{e} x_{2}\right)+\left\{\begin{array}{c}
a \\
a \\
\cdot \\
\cdot \\
a \\
b \\
b \\
\cdot \\
\cdot \\
\cdot \\
b
\end{array}\right\} x_{1}+\left\{\begin{array}{c}
b \\
b \\
\cdot \\
b \\
a^{*} \\
a^{*} \\
\cdot \\
\cdot \\
\cdot \\
a^{*}
\end{array}\right\} x_{2}
$$

where $A_{e}, a, b$ and $a *$ in the general solution (2.14) are the coefficients that need to be found to generate the particular solution for each set of boundary conditions [196].

The next step is to evaluate each term in (2.11) and (2.12) using (2.14). This leads to the next eigenvalue system problem

$$
\left[\begin{array}{ll}
\alpha_{1} & \beta_{1} \\
\alpha_{2} & \beta_{2}
\end{array}\right]\left\{\begin{array}{l}
a_{j} \\
b_{j}
\end{array}\right\}+\eta^{2}\left[\begin{array}{ll}
\zeta_{26} & \zeta_{22} \\
\zeta_{66} & \zeta_{26}
\end{array}\right]\left\{\begin{array}{l}
a_{j} \\
b_{j}
\end{array}\right\}=\left\{\begin{array}{l}
0 \\
0
\end{array}\right\}
$$

with eigenvalues $\lambda_{e}$ and the eigenvectors $\left\{\begin{array}{l}a \\ b\end{array}\right\}$. This equation system yields $2 N$ eigenvalues and $2 N$ eigenvectors. The $2 N-2$ non trivial eigenvalues correspond to the hyperbolic sine solutions, while the two trivial eigenvalues correspond to the linear solutions.

\subsection{Boundary Conditions for $\Delta T=0$}

First consider the case of mechanical loads and no thermal loads. To find the values of $A_{e}, a, a *, b$, the following boundary conditions are enforced: (a) stress-free at the crack surfaces, (b) external loads, and (c) homogeneous displacements. The boundary condi- 
tions are then assembled into an algebraic system as follows

$$
[B]\left\{A_{e}, a, a^{*}, b\right\}^{T}=\{F\}
$$

where $[B]$ is the coefficient matrix of dimensions $2 N+1$ by $2 N+1 ;\left\{A_{e}, a, a^{*}, b\right\}^{T}$ represents the $2 N+1$ unknown coefficients, and $\{F\}$ is the RHS or force vector, also of dimension $2 N+1$.

\subsection{1 (a) Stress-free at the Cracks Surfaces}

The surfaces of the cracks are stress-free

$$
\begin{aligned}
& \int_{-1 / 2}^{1 / 2} \widehat{\sigma}_{2}^{(k)}\left(x_{1}, l\right) \mathrm{d} x_{1}=0 \\
& \int_{-1 / 2}^{1 / 2} \widehat{\tau}_{12}^{(k)}\left(x_{1}, l\right) \mathrm{d} x_{1}=0
\end{aligned}
$$

\section{4 .2 (b) External Loads}

In the direction parallel to the surface of the cracks (fiber direction $x_{1}$ ) the load is supported by all the laminas

$$
\frac{1}{2 l} \sum_{i=1}^{N} h_{i} \int_{-l}^{l} \widehat{\sigma}_{1}^{(i)}\left(1 / 2, x_{2}\right) d x_{2}=h \widehat{\sigma}_{1}
$$

In the direction normal to the crack surface ( $x_{2}$ direction) only the uncracking (homogenized) laminas carry load

$$
\begin{gathered}
\sum_{m \neq k} h_{m} \int_{1 / 2}^{1 / 2} \widehat{\sigma}_{2}^{(m)}\left(x_{1}, l\right) d x_{1}=h \widehat{\sigma}_{2} \\
\sum_{m \neq k} h_{m} \int_{1 / 2}^{1 / 2} \widehat{\tau}_{12}^{(m)}\left(x_{1}, l\right) d x_{1}=h \widehat{\tau}_{12}
\end{gathered}
$$

\subsection{3 (c) Homogeneous Displacements}

For a homogenized symmetric laminate, membrane loads produce a uniform displacement field through the thickness, i.e. all the uncracking laminas are subjected to the same 
displacement

$$
\begin{array}{ll}
\widehat{u}^{(m)}\left(x_{1}, l\right)=\widehat{u}^{(r)}\left(x_{1}, l\right) & ; \quad \forall m \neq k \\
\widehat{v}^{(m)}\left(x_{1}, l\right)=\widehat{v}^{(r)}\left(x_{1}, l\right) \quad ; \quad \forall m \neq k
\end{array}
$$

where $r$ is an uncracked lamina taken as reference. In the computer implementation, lamina 1 is taken as reference unless lamina 1 is cracking, in which case lamina 2 is taken as reference.

\subsection{Boundary Conditions for $\Delta T \neq 0$}

Next, consider the case of thermal loads, which add a constant term to the boundary conditions. Constant terms do not affect the matrix $[B]$, but rather subtract from the forcing vector $\{F\}$, as follows

$$
\{F\}_{\Delta T \neq 0}=\left\{\begin{array}{c}
\Delta T \sum_{j=1,2,6} Q_{1 j}^{(k)} \widetilde{\alpha}_{j}^{(k)} \\
\Delta T \sum_{j=1,2,6} Q_{1 j}^{(k)} \widetilde{\alpha}_{j}^{(k)} \\
\Delta T \sum_{i \neq(k)} \sum_{j=1,2,6} Q_{1 j}^{(i)} \widetilde{\alpha}_{j}^{(i)} \\
\Delta T \sum_{i \neq k} \sum_{j=1,2,6} Q_{2 j}^{(i)} \widetilde{\alpha}_{j}^{(i)} \\
\Delta T \sum_{i \neq k} \sum_{j=1,2,6} Q_{6 j}^{(i)} \widetilde{\alpha}_{j}^{(i)} \\
0 \\
0 \\
\ldots \\
\ldots \\
0
\end{array}\right\}
$$

In this way, the strain calculated for a unit thermal load $(\Delta T=1)$ is the degraded CTE of the laminate for the current crack density set $\lambda$.

\subsection{Degraded Laminate Stiffness and CTE}

First, we calculate the degraded stiffness of the laminate $Q=\mathbf{A} / h$ for a given crack density $\lambda_{k}$ in a cracked lamina $k$, where $\mathbf{A}$ is the in-plane laminate stiffness matrix, and $h$ is the thickness of the laminate. The thickness-averaged strain field in all laminas can be obtained by using the equation (2.14). At this point, the homogenization problem 
replaces the cracks from the $R V E$ by a reduction of stiffness of the homogenized material. Taking the volume average of the $R V E$ as follows,

$$
\bar{\phi}=\int_{V} \phi d v
$$

the constitutive equations are expressed in terms of stress and strain averaged. Since the CDM principle states that the applied strain is equal to the average strain at one point far enough from the cavity of cracks, the elastic constitutive equation is simplified as

$$
\widehat{\epsilon}=S \sigma_{j}^{o}
$$

where $\sigma_{j}^{o}$ is the stress applied to the laminate.

Then, the compliance of the laminate $S$ in the coordinate system of lamina $k$ can be calculated one column at a time by solving for the strains (2.14) for three load cases, $a$, $b$, and $c$, all with $\Delta T=0$, as follows

$$
\sigma_{a}^{o}=\left\{\begin{array}{c}
1 \\
0 \\
0
\end{array}\right\} ; \quad \sigma_{b}^{o}=\left\{\begin{array}{c}
0 \\
1 \\
0
\end{array}\right\} ; \quad \sigma_{c}^{o}=\left\{\begin{array}{c}
0 \\
0 \\
1
\end{array}\right\} ; \quad \Delta T=0
$$

Then, the compliance matrix of the laminate in the lamina $\mathrm{k}$ coordinate system as a function of the crack density is assembled as

$$
S\left(\lambda_{\text {matrix }}\right)=\left[\begin{array}{ccc}
{ }^{a} \epsilon_{1} & { }^{b} \epsilon_{1} & { }^{c} \epsilon_{1} \\
{ }^{a} \epsilon_{2} & { }^{b} \epsilon_{2} & { }^{c} \epsilon_{2} \\
{ }^{a} \gamma_{12} & { }^{b} \gamma_{12} & { }^{c} \gamma_{12}
\end{array}\right]
$$

To get the degraded CTE of the laminate, one sets $\widehat{\sigma}^{o}=\{0,0,0\}^{T}$ and $\Delta T=1$. The resulting strain is equal to the CTE of the laminate, i.e., $\left\{\alpha_{x}, \alpha_{y}, \alpha_{x y}\right\}^{T}=\left\{\epsilon_{1}, \epsilon_{2}, \gamma_{12}\right\}^{T}$.

\subsection{Degraded Lamina Stiffness}

The stiffness of lamina $m$, with $m \neq k$, in the coordinate system of lamina $k$ (see Figure 2.1), is given by (2.6) in terms of the previous calculated values $D_{2}^{(m)}, D_{6}^{(m)}$, given by (2.31). The stiffness of the cracking lamina $Q^{(k)}$ is yet unknown. Note that all quantities are expressed in the coordinate system of lamina $k$. 
The laminate stiffness is defined by the contribution of the cracking lamina $k$ plus the contribution of the remaining $N-1$ laminas, as follows

$$
Q=Q^{(k)} \frac{h_{k}}{h}+\sum_{m=1}^{n}\left(1-\delta_{m k}\right) Q^{(m)} \frac{h_{m}}{h}
$$

where the delta of Dirac is defined as $\delta_{m k}=1$ if $m=k$, otherwise 0 . The left-hand side (LHS) of (2.29) is known from (2.28) and all values of $Q^{(m)}$ can be easily calculated so that the $m$ laminas are not cracking at the moment. Therefore, one can calculate the degraded stiffness $Q^{(k)}$ of lamina $k$ as follows

$$
Q^{(k)}=\frac{h}{h_{k}}\left[Q-\sum_{m=1}^{n}\left(1-\delta_{m k}\right) Q^{(m)} \frac{h_{m}}{h}\right]
$$

where $Q$ without a superscript is the stiffness of the laminate. To facilitate later calculations, the stiffness $Q^{(k)}$ can be written in terms of the stiffness of the undamaged lamina and damage variables $D_{2}^{(k)}, D_{6}^{(k)}$, using equation (2.6). The damage factor can be written as follows

$$
D_{j}^{(k)}\left(\lambda_{k}, \epsilon^{0}\right)=1-Q_{j j}^{(k)} / \widetilde{Q}_{j j}^{(k)} \quad ; \quad j=2,6 ; \text { no sum on } j
$$

where $\widetilde{Q}^{(k)}$ is the original value of the undamaged property, and $Q^{(k)}$ is the degraded (homogenized) value computed in (2.30), both expressed in the coordinate system of lamina $k$.

The coefficient of thermal expansion of the cracking lamina $k$ can be calculated in a similar way as follows

$$
\alpha^{(k)}=\frac{1}{h_{k}} S^{(k)}\left(h Q \alpha-\sum_{m \neq k} h_{m} Q^{(m)} \alpha^{(m)}\right)
$$

with $S=\left[Q^{(k)}\right]^{-1}$. The corresponding thermal damage variable is written as

$$
D_{j}^{\alpha(k)}=1-\alpha_{j}{ }^{(k)} / \widetilde{\alpha}_{j}^{(k)} \quad ; \quad j=2,6
$$

Once the damages for lamina $\mathrm{k}$ are known, they are used in the next laminate iteration as the new homogenized properties of the lamina. 


\subsection{Damage Activation Function}

The DDM model [31] is able to predict the crack density of a lamina through the damage activation function $\mathrm{g}(\lambda, \epsilon, \triangle T)$, which is essentially the Griffin fracture criteria,

$$
G(\lambda, \epsilon, \triangle T) \geq G_{c}
$$

where $G$ is the energy release rate (ERR) for the given laminate state $(\lambda, \epsilon, \triangle T)$ and $G_{c}$ is the critical ERR specific of each material system.

Since intralaminar cracks may propagate in mode I (opening) and mode II (shearing), the ERR needs to be decomposed into $G_{I}$ and $G_{I I}$. Therefore, $G_{I}$ is calculated with $\epsilon=\left\{0, \epsilon_{2}, 0\right\}$ and $G_{I I}$ is calculated with $\epsilon=\left\{0,0, \gamma_{12}, 0\right\}$ [197]. The proposed mode separation is consistent with the method of mechanical work during crack closure in classical fracture mechanics [198].

The damage activation function may consider with interaction between mode I and mode II in a proposed functional form [199] as follows

$$
g(\lambda, \epsilon, \triangle T)=(1-r) \sqrt{\frac{G_{I}(\lambda, \epsilon, \Delta T)}{G_{I c}}}+r \frac{G_{I}(\lambda, \epsilon, \Delta T)}{G_{I c}}+\frac{G_{I I}(\lambda, \epsilon, \Delta T)}{G_{I I c}}-1 \leq 0
$$

where

$$
r=\frac{G_{I c}}{G_{I I c}}
$$

Note that critical ERR $G_{I c}, G_{I I c}$ are the only material properties to predict both initiation and evolution of crack density. No hardening exponents or any other damage evolution material properties are needed to describe the kinetic evolution of damage.

The energy release rates associated with a new crack in the middle of the RVE can be calculated by computing the laminate stiffness and CTE for the current state, and for a trial crack density that is the double current crack density. To obtain the energy associated to those states we use the Griffith's energy principle applied on its discrete (finite) form in order to describe the behavior of crack growth, as follows

$$
\begin{aligned}
G_{I} & =-\frac{\Delta U_{I}}{\Delta A} \\
G_{I I} & =-\frac{\Delta U_{I I}}{\Delta A}
\end{aligned}
$$

where $\Delta U_{I}, \Delta U_{I I}$ are the change in laminate strain energy during mode I and mode II finite crack growth, respectively; and $\Delta A$ is the newly created (finite) crack area, which 
is one half of the new crack surface. Counting crack area as one-half of crack surface is consistent with the classical fracture mechanics convention for which fracture toughness $G_{c}$ is twice of Griffith's surface energy $\gamma_{c}$. Thus, the ERR values $G_{I}, G_{I I}$ in modes $i=I, I I$ are calculated as

$$
G_{i}=\frac{U_{i, a}-U_{i, b}}{\Delta A_{c}}
$$

where $U_{I, a}, U_{I, b}, U_{I I, a}, U_{I I, b}$ are the elastic strain energies in modes I and II, for crack densities $\lambda_{a}$ and $\lambda_{b}=2 \lambda_{a}$, and $\Delta A$ is the increment of crack area when a new crack propagates (Sect. 8.4.7 in [200]). Mode decomposition is achieved by splitting the strain energy $U$ into mode I (opening) and II (shear) as follows

$$
\begin{gathered}
U_{I}=\frac{V_{R V E}}{2 H} \sum_{k=1}^{n} t_{k}\left(\epsilon_{2}-\widetilde{\alpha}_{2}^{(k)} \delta T\right) Q_{2 j}^{(k)}\left(\epsilon_{j}-\widetilde{\alpha}_{j}^{(k)} \delta T\right) \\
U_{I I}=\frac{V_{R V E}}{2 H} \sum_{k=1}^{n} t_{k}\left(\epsilon_{6}-\widetilde{\alpha}_{6}^{(k)} \delta T\right) Q_{6 j}^{(k)}\left(\epsilon_{j}-\widetilde{\alpha}_{j}^{(k)} \delta T\right)
\end{gathered}
$$

where $H=\sum_{k=1}^{n} t_{k}, \epsilon_{6}=\gamma_{12}$, and $\widetilde{\alpha}^{(k)}$ are the undamaged CTE of lamina $k$. Equation (2.39) is cast in the coordinate system of the cracking lamina $k$ so that $\epsilon_{2}$ is mode I (crack opening) and $\epsilon_{6}$ is mode II (crack shear). Laminate ultimate failure is predicted by a fiber damage and failure criterion [201].

DDM assumes local uniformity of crack spacing and linear distribution of intralaminar stresses. Despite these restrictions, predicted results correlate extremely well with available data for a broad variety of material systems (Carbon and Glass reinforced composites) [34, 202], laminate stacking sequences (LSS) [32,33, 100, 156, 192, 203-205] and loading conditions including open hole tension data up to failure [201,206-208].

Since the size of the RVE $\left(1 \times t \times 1 / \lambda_{k}\right)$ is dictated by the crack density $\lambda_{k}$, not by the element size, and the solution is in terms of displacements, not stress or strain, the predictions of the DDM constitutive model are mesh-density and element-type independent. The only effect of mesh density is on the quality of the stress/strain field. This is a remarkable advantage with respect to cohesive zone constitutive models, which produce results that are mesh-density and element-type dependent, as shown in [35, 202, 209].

\subsection{Solution Algorithm}

The solution algorithm consists of (a) strain steps, (b) laminate-iterations, and (c) laminaiterations. The state variables for the laminate are the array of crack densities for all 
laminas $i$ and the membrane strain $\epsilon$. At each load (strain) step, the strain on the laminate is increased and the laminas are checked for damage.

\subsubsection{Lamina Iterations}

When transverse cracking is detected in lamina $k$, a return mapping algorithm (RMA) is invoked to iterate and adjust the crack density $\lambda_{k}$ in lamina $k$ in such a way that, $g_{k}$ returns to zero while maintaining equilibrium between the external forces and the internal forces in the laminas. The iterative procedure works as follows. At a given strain level $\epsilon$ for the laminate and given $\lambda_{k}$ for lamina $k$, calculate the value of the damage activation function $g_{k}$ and the damage variables, which are both functions of $\lambda_{k}$. The RMA calculates the increment (decrement) of crack density as

$$
\Delta \lambda_{k}=-g_{k} / \frac{\partial g_{k}}{\partial \lambda}
$$

until $g_{k}=0$ is satisfied within a given tolerance, for all $k=1 \ldots N$, where $N$ is the number of laminas in the laminate. The analysis starts with a negligible value of crack density present in all laminas $(\lambda=0.02 \mathrm{cracks} / \mathrm{mm}$ were used in the examples $)$ due to defects inherent into materials.

\subsubsection{Laminate Iterations}

To calculate the stiffness reduction of a cracked lamina ( $k$-lamina), all of the other laminas ( $m$-laminas) in the laminate are considered not damaging during the course of laminaiterations in lamina $k$, but with damaged properties calculated according to the current values of their damage variables $D_{i}^{(m)}$. Given a trial value of $\lambda_{k}$, the analytical solution provides $g_{k}, D_{i}^{(k)}$ for lamina $k$ assuming all other laminas do not damage while performing lamina iterations in lamina $k$. Since the solution for lamina $k$ depends on the stiffness of the remaining laminas, a converged iteration for lamina $k$ does not guarantee convergence for the same lamina once the damage in the remaining laminas is updated. In other words, within a given strain step, the stiffness and damage of all the laminas are interrelated and they must all converge. This can be accomplished by laminate-iterations; that is, looping over all laminas repeatedly until all laminas converge to $g=0$ for all $k$. 


\section{Chapter 3}

\section{Temperature-dependent Properties}

Due to difference in coefficient of thermal expansion (CTE) among laminas with different orientations, thermal stress and strain are induced, which often cause transverse cracking of the laminas. Prediction of thermo-mechanical damage requires precise knowledge of the temperature-dependent properties of the material, including elastic and fracture properties. Temperature ranges of interest include Low Earth Orbit (LEO [-100 to 66 C]) [210], Geostationary Earth Orbit (GEO [-156 to 121]) [211-217], and cryogenic tanks (Liquid Nitrogen, Oxygen, Helium, $\mathrm{CO}_{2}$, Hydrogen, etc.). Waste Heat Recovery devices [24 to $200 \mathrm{C}$ ] are also susceptible to transverse cracking when cooling down from their operating temperature $[218,219]$.

The material properties of polymer matrix composites change with temperature, mainly due to the temperature-dependent properties of the polymer. Measurement of strength and thermal expansion of laminated composites as a function of temperature is reported for example in [183,220-224]. Temperature dependence of lamina properties are reported for example in [13,17, 225-228].

Since temperature-dependent data for most material systems is scarce, micromechanics is often used to predict lamina data from fiber and matrix data. Temperaturedependent properties for fiber and matrix are also difficult to find, but once lamina data for a few material system are found, the fiber and matrix properties can be back calculated, then used to predict lamina properties for other combinations of fiber/matrix at similar, yet different, values of fiber volume fraction. Although many micromechanics models exist, most have been derived for isotropic fibers, although carbon fibers are transversely isotropic.

The lamina properties that are most sensitive to temperature are the matrix dominated properties, namely $E_{2}(T), G_{12}(T)$, and $\alpha_{2}(T)$. Prediction of these properties are affected by the transverse properties of the fiber $E_{T}, G_{T}, \alpha_{T}$ as well as those of the polymer. Therefore, it is important to use a micromechanics model that accounts for transverse 
isotropy of the fiber. Furthermore, it is advantageous to use a micromechanics model that can predict all five properties of the transversely isotropic lamina using a unified formulation and that yields accurate predictions without requiring adjustable parameters such as stress partitioning [200], etc. The periodic microstructure model (PMM) [229] satisfies all of the aforementioned requirements and it has been extensively validated for elastic and creep/relaxation behavior of unidirectional composites [229-231]. PMM is described in App. 2 of [200] and implemented in [232].

For isotropic fibers, the transverse CTE of the lamina can be estimated by the rule of mixtures [200,233] as follows

$$
\alpha_{2}=\alpha_{3}=\left(1+\nu_{m}\right) \alpha_{m}\left(1-V_{f}\right)+\left(1+\nu_{f}\right) \alpha_{f} V_{f}-\alpha_{1} \nu_{12}
$$

where $\nu_{f}, \nu_{m}, \nu_{12}$ are the Poisson's ratio of fiber, matrix, and composite, respectively; $\alpha_{f}, \alpha_{m}, \alpha_{2}$ are the CTE of the fiber, matrix, and transverse direction of the lamina, respectively; and $V_{f}$ is the fiber volume fraction. Strife and Prewo [234] proposed a modification to account for transversely isotropic properties of the fiber. Their modification is not derived from mechanics or physical principles but just a substitution of $\nu_{A}, \alpha_{T}$ for $\nu_{f}, \alpha_{f}$ in the rule of mixtures formula [233]. Predictions using either [233] (3.1) or [234] are somewhat accurate for some materials such as Kevlar/Epoxy but no so for other materials [234]. The model proposed by Levin [235] (see eq. (3.6) is intrinsically exact and able to calculate the three CTE values for an orthotropic lamina, but relies on the estimated elasticity tensor for the lamina. Therefore, the accuracy of Levin's method is only limited by the accuracy of the estimate for the elasticity tensor. Levin's work was extended in [236] for three-phase composites and used in an extensive study of thermal properties of composite materials in [212,237].

The matrix is always assumed to be isotropic with elastic properties $E_{m}(T), \nu_{m}(T)$, and $\alpha_{m}(T)$, were $T$ is the temperature. On the other hand, the fibers are assumed to be transversely isotropic with properties $E_{A}, E_{T}, G_{A}, \nu_{A}, \nu_{T}, \alpha_{A}, \alpha_{T}$. Carbon fibers are reported to be almost temperature independent [211-217] in the temperature ranges for LEO [-100-66] C and GEO [-156-121] C.

Poisson's ratio $\nu_{m}$ of polymers is influenced by the free volume available in the material. At high temperatures, the polymer chains become rubbery and the Van der Waals forces, which control the inter-chain bonds, are weaker leading to higher Poisson's ratio. Conversely, at low temperatures, the polymer becomes brittle with lower Poisson's ratio. Some authors report Poisson's ratio almost constant with temperature for composite laminas with epoxy resins [238,239]. Others bracket the Poisson ratio's of polymer at low temperatures between 0.29 and 0.39 [240]. 
Below the glass transition temperature $T_{g}$, the molecular structure of polymers transitions from a rubbery to glassy state, and becomes more and more rigid and brittle at lower temperatures. The elastic modulus $E_{m}$ increases markedly and the strain to failure $\epsilon_{m u}$ decreases [241-244] at colder temperatures, culminating at cryogenic liquid nitrogen $\left(\mathrm{LN}_{2}\right)$ temperature $(-196 \mathrm{C})$ [245], which is the lowest limit considered in this work.

Carbon-fiber composite data has been used to demonstrate the applicability of the proposed methodology. The material systems used in this study are: Cytec P75/934, Amoco P75/1962, Narmco T300/5208, and Hexcel AS4/3501-6. Some material property data that are necessary for thermo-mechanical analysis are unavailable in the literature and the type of data missing is not the same for every material system. For example, the transverse CTE of AS4 and T300 fibers are not available. The temperature range of lamina and matrix data varies drastically among different sources. Sometimes there is a single data point available at low temperature using liquid Helium (-231 C) [243, 246] or the range of temperature is too narrow [241,242,247]. Some experimental data show no higher rigidity at cryogenic temperature [248], or even softening [249]. No definite conclusion is available in the literature about rigidity below $-200 \mathrm{C}$.

Since availability of material properties are different for each fiber, matrix, and material system (lamina), the proposed methodology must be flexible to adapt to the availability of data. Consequently, a generic outline of the proposed methodology is presented first in Sections 3.1 and 3.2 followed by detailed, self-contained descriptions of the complete process for each fiber, matrix, and composite material system (lamina). In an effort to have complete descriptions of the parameter estimation process for each material, some repetition of calculation steps must be tolerated.

\subsection{Constituent Properties}

The methodology used to calculate the properties of the constituents (fiber and matrix) is presented in this section. If not available in the literature, the elastic properties of the constituents (fiber and matrix) are, in this work, back calculated using periodic microstructure micromechanics (PMM, App. 2 in [200])

Properties of carbon fibers used in this work are shown in Table 3.1. The longitudinal modulus $E_{A}$ and CTE $\alpha_{A}$ are collected from manufacturer data sheets [250-252]. The rest of fiber properties $\left(E_{T}, G_{A}, \nu_{A}, \nu_{T}\right)$ and transverse fiber CTE $\alpha_{T}$ are back calculated as explained in this section and Section "Coefficients of Thermal Expansion", respectively.

Temperature-dependent properties of Epoxy are shown in Tables 3.2, 3.3, 3.4, and 3.5, back calculated from unidirectional lamina data, as explained in this section and Section "Coefficients of Thermal Expansion". In all cases, the matrix properties $\left(E_{m}, \nu_{m}, \alpha_{m}\right)$ are 
Tables 3.1: Carbon fiber properties.

\begin{tabular}{cccc}
\hline Property & AS4 & T300 & P75 \\
\hline$E_{A}[\mathrm{GPa}]$ & 231.000 & 231.000 & 517.000 \\
$E_{T}[\mathrm{GPa}]$ & 23.453 & 26.864 & 11.158 \\
$G_{A}[\mathrm{GPa}]$ & 15.764 & 81.662 & 10.636 \\
$\nu_{A}$ & 0.253 & 0.156 & 0.269 \\
$\nu_{T}$ & 0.371 & 0.287 & 0.306 \\
$\alpha_{A}\left[10^{-6} / \mathrm{C}\right]$ & -0.630 & -0.600 & -1.46 \\
$\alpha_{T}\left[10^{-6} / \mathrm{C}\right]$ & 5.997 & 11.086 & 12.500 \\
\hline
\end{tabular}

represented by a quadratic polynomial

$$
P(T)=P_{a}+P_{b} T+P_{c} T^{2}
$$

where $P$ is the property, $T$ is the temperature, and $P_{a}, P_{b}, P_{c}$ are the coefficients. In order to get reliable values, experimental data at low, room, and high temperatures are necessary. The back calculation method provides the property $\mathrm{P}(\mathrm{T})$ at various temperatures so that the predicted lamina properties fit available experimental data as explained in this section and Section "Coefficients of Thermal Expansion". Then, property values ar various temperatures are subsequently fitted with (3.2). Quadratic polynomial were used also by $[13,15,20,212,222,237,253]$.

Tables 3.2: Quadratic temperature-dependent properties of Epoxy 3501-6 in the range $[-200,180]^{\circ} C$.

\begin{tabular}{c|ccc}
\hline Property & $P_{a}$ & $P_{b}$ & $P_{c}$ \\
\hline$E_{m}[\mathrm{MPa}]$ & 4580.4836 & -10.6103 & 0 \\
$\nu_{m}$ & 0.3812 & $3.856410^{-5}$ & 0 \\
$\alpha_{m}\left[10^{-6} / C\right]$ & 38.3445 & 0.1224 & 0 \\
\hline
\end{tabular}

Tables 3.3: Quadratic temperature-dependent properties of Epoxy 934 in the range $[-156,120]^{\circ} \mathrm{C}$.

\begin{tabular}{c|ccc}
\hline Property & $P_{a}$ & $P_{b}$ & $P_{c}$ \\
\hline$E_{m}[\mathrm{MPa}]$ & 5032.7732 & -16.7561 & 0.0251 \\
$\nu_{m}$ & 0.3659 & $-1.110810^{-4}$ & $-8.608010^{-7}$ \\
$\alpha_{m}\left[10^{-6} / C\right]$ & 38.7655 & 0.1524 & $-1.3255310^{-4}$ \\
\hline
\end{tabular}


Tables 3.4: Quadratic temperature-dependent properties of Epoxy ERL 1962 in the range $[-156,120]^{\circ} \mathrm{C}$

\begin{tabular}{c|ccc}
\hline Property & $P_{a}$ & $P_{b}$ & $P_{c}$ \\
\hline$E_{m}[\mathrm{MPa}]$ & 5032.7732 & -16.7561 & 0.0251 \\
$\nu_{m}$ & 0.3659 & $-1.110810^{-4}$ & $-8.608010^{-7}$ \\
$\alpha_{m}\left[10^{-6} / C\right]$ & 49.3143 & 0.1594 & $-4.50910^{-4}$ \\
\hline
\end{tabular}

Tables 3.5: Quadratic temperature-dependent properties of Epoxy 5208 in the range $[-156,120]^{\circ} \mathrm{C}$.

\begin{tabular}{c|ccc}
\hline Property & $P_{a}$ & $P_{b}$ & $P_{c}$ \\
\hline$E_{m}[\mathrm{MPa}]$ & 4828.7124 & -5.4846 & $-5.216410^{-3}$ \\
$\nu_{m}$ & 0.4072 & $-3.333210^{-4}$ & $7.911910^{-7}$ \\
$\alpha_{m}\left[10^{-6} / C\right]$ & 36.65977 & 0.1887 & $-9.544110^{-5}$ \\
\hline
\end{tabular}

Fiber properties $\left(E_{T}, G_{A}, \nu_{A}, \nu_{T}\right)$ and matrix properties $\left(E_{m}, \nu_{m}\right)(3.2)$ are adjusted so that the lamina properties $\left(E_{1}, E_{2}, G_{12}, \nu_{12}, \nu_{23}\right)$ predicted using PMM fit available experimental lamina data $\left(E_{1}^{d}, E_{2}^{d}, G_{12}^{d}, \nu_{12}^{d}\right)$ available in the literature. Superscript " $d$ " means "data". The properties are adjusted by minimizing the error $D$ calculated as follows

$$
D=\frac{1}{N} \sqrt{\sum^{N}\left[\left(\frac{E_{1}-E_{1}^{d}}{E_{1}^{d}}\right)^{2}+\left(\frac{E_{2}-E_{2}^{d}}{E_{2}^{d}}\right)^{2}+\left(\frac{G_{12}-G_{12}^{d}}{G_{12}^{d}}\right)^{2}+\left(\frac{\nu_{12}-\nu_{12}^{d}}{\nu_{12}^{d}}\right)^{2}\right]}
$$

where $N$ is the number of lamina data points at a given temperature, and superscript $d$ means data. In order to give the same weight to all properties, each term is normalized as shown. Elastic properties from literature or manufacturer data sheet, if available, are used as initial guess for the minimization algorithm.

Denoting by $x$ the value of any of the material properties of interest, and by $D$ the error (3.3), the value of property $x$ is found when the error $D$ is less than the function tolerance (i.e., error tolerance) [254] tol $^{\text {fun }}=10^{-8}$ and the change in property $\Delta x$ is less than the step size tolerance $t o l^{x}=10^{-8}$.

Not all material systems can be characterized exactly with the procedure described above. Variations in the procedure are necessary to make use of the available data, which varies from material to material. In the following, four matrices and four fibers are characterized, illustrating how to adapt the proposed procedure to make best use use of the available data. 


\subsubsection{Epoxy 3501-6}

A large amount of experimental elastic data $\left(E_{1}^{d}, E_{2}^{d}, G_{12}^{d}, \nu_{12}^{d}\right)$ exits at room temperature (RT) and high temperature (HT) for AS4/3501-6 unidirectional lamina [18, 183,213,223, $225,228,255,256]$ but at low temperature, only longitudinal modulus data $E_{1}^{d}$ at $-54 \mathrm{C}$ is available [19]. No matrix-dominated $\left(E_{2}^{d}, G_{12}^{d}, \nu_{12}^{d}\right)$ could be found at low temperature. Back calculation of $E_{m}(-54 C)$ and $\nu_{m}(-54 C)$ from $E_{1}^{d}(-54 C)$ is not possible because $E_{1}$ is a fiber dominated property but $E_{m}, \nu_{m}$ are matrix-dominated properties. Therefore, the temperature-dependent properties $E_{m}, \nu_{m}$ for Epoxy 3501-6 were adjusted based on available neat resin data $[257,258]$. In this way, matrix coefficients $(3.2)$ for $E_{m}, G_{m}$ of Epoxy 3501-6 are obtained by interpolation in the range [24,150 C] of the data available in $[257,258]$. Linear interpolation is sufficiently accurate in this case. The Poisson's ratio $\nu_{m}$ is calculated in terms of $E_{m}, G_{m}$ using the isotropic relationship $\nu_{m}=E_{m} /\left(2 G_{m}-1\right)$. In this paper, temperature ranges are given from hot to cold because that is the way cooling takes place.

Calculated values of Poisson's ratio $\nu_{m}$ turn out to be virtually constant with temperature. Since the temperature-dependent properties are linearly fitted, based on neat resin data, and they vary smoothly with temperature, they are extrapolated to the whole temperature range of study [-200,180 C] as shown in Figure 3.1. For predictions, the temperature range in this paper starts at $180 \mathrm{C}$ because that is the most common glass transition temperature of the materials studied. The coldest temperature is $-200 \mathrm{C}$ for illustrative purposes only.

\subsubsection{Epoxy 934}

Elastic properties $E_{m}, \nu_{m}$ of Epoxy 934 at high $(121 \mathrm{C})$ and room temperature (RT) are taken from the experimental neat resin data in [259]. Then, the elastic properties $E_{m}, \nu_{m}$ at low temperature $(-156 \mathrm{C})$ of Epoxy 934 are obtained by minimizing the error (3.3) between T300/934 lamina data $\left(E_{1}^{d}, E_{2}^{d}, G_{12}^{d}, \nu_{12}^{d}\right)$ available in [227] and predicted lamina properties $\left(E_{1}, E_{2}, G_{12}, \nu_{12}, \nu_{23}\right)$ calculated using PMM micromechanics (App. 2 in [200]). The methodology used is illustrated in Figure 3.2 by a flowchart. The tolerance [254] used is tol $^{x}=$ tol $^{\text {fun }}=10^{-8}$.

T300 fiber properties used as input data in PPM are taken from Table 3.1. Once the elastic properties at room, high, and low temperature have been obtained, the coefficients (3.2) are calculated by a quadratic interpolation in the range $[-156,121 \mathrm{C}]$ and reported in Table 3.3. The values found for these coefficients are very close to the values reported in [212]. The resulting plot is shown in Figure 3.3. Unlike Figure 3.1, the curves in Figure 3.3 are not linear and thus extrapolation outside of the range of the experimental 

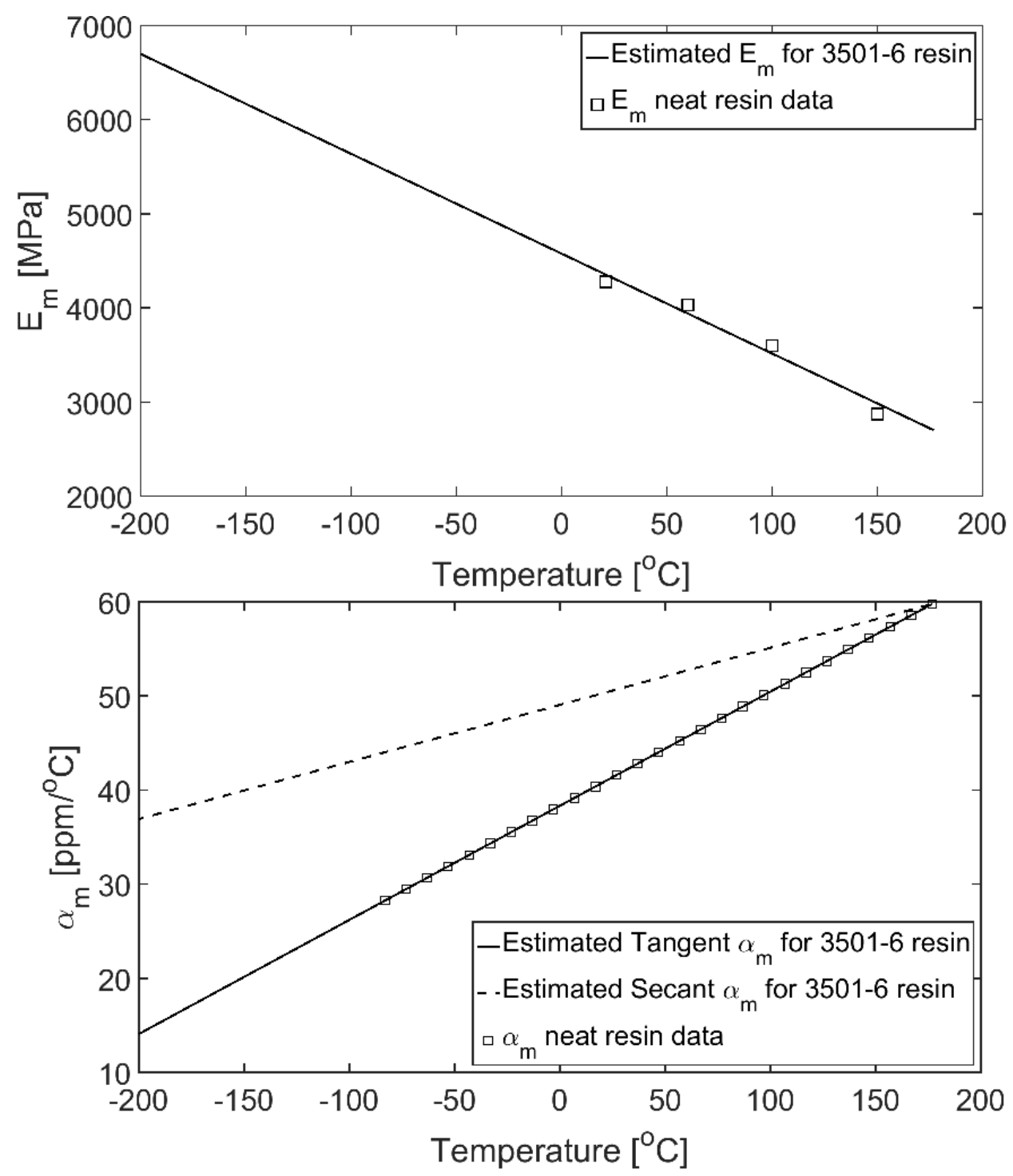

Figure 3.1: Estimated temperature-dependent modulus $E_{m}$ (top) and CTE (bottom) for Epoxy 3501-6 extrapolated to the whole temperature range of study [-200,180 C].

data may yield exaggerated values. Therefore, for calculation of crack density outside the temperature range of the experimental data from which the temperature dependence is found, the matrix properties are assumed to be constant and equal to the end values of the experimental data, as shown in Figure 3.3.

\subsubsection{Epoxy ERL 1962}

Epoxy ERL 1962 is similar to Epoxy 934 with added rubbery particles to increase fracture toughness. Lamina data from the literature $[10,17,186,188]$ for composites using these two resins (934 and ERL 1962) and the same type of fiber have almost identical properties. Only a slightly lower modulus for ERL 1962 than Epoxy 934 was reported in [10]. Lacking experimental data revealing temperature-dependent properties for neat 


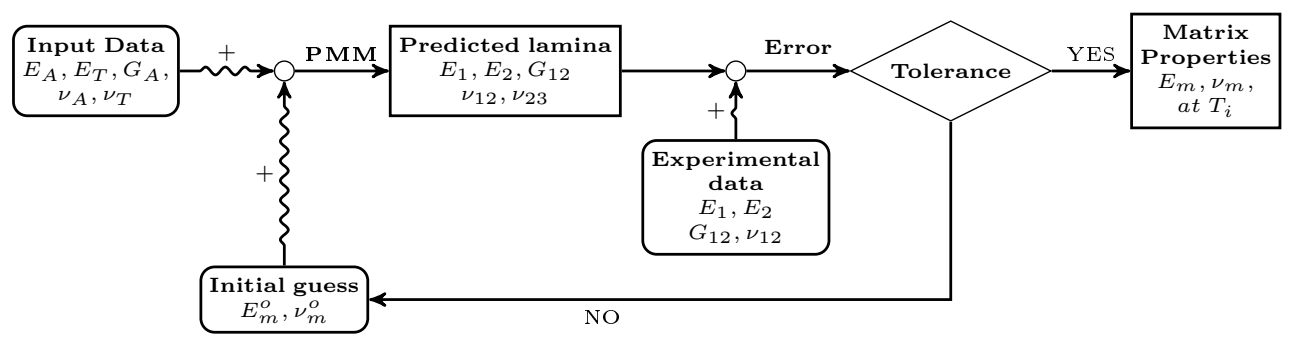

Figure 3.2: Back calculation method to obtain the temperature-dependent matrix properties at any temperature $T_{i}$.
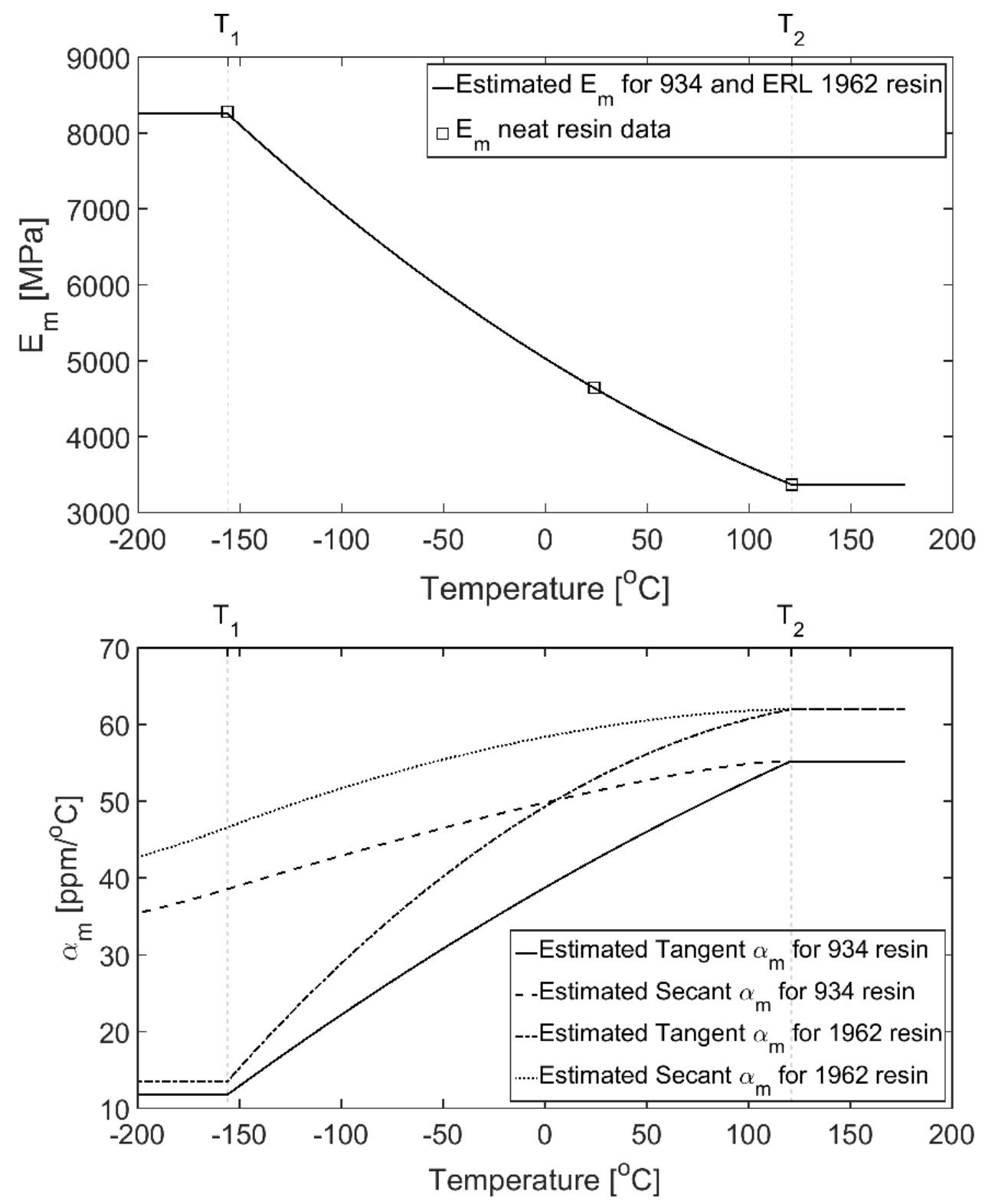

Figure 3.3: Estimated temperature-dependent modulus (top) and CTE (bottom) for Epoxy 934 and ERL 1962. 
resin or unidirectional laminas using ERL 1962 matrix, the temperature-dependent elastic properties of Epoxy ERL 1962 are assumed in this study to be equal to those of Epoxy 934, but temperature-dependent CTEs are still adjusted to experimental data as shown in Section "Material system: P75/1962".

\subsubsection{Epoxy 5208}

Elastic properties $E_{m}, \nu_{m}$, of Epoxy 5208 are back calculated from lamina elastic data in [220,221] at cryogenic, room, and high temperatures $(-156,24$, and $121 \mathrm{C})$. The Poisson's ratio reported in [221] is so high that leads to $\nu_{m}>0.5$ for temperatures below -100 C. Such values are incoherent for isotropic polymers at low temperature [239, 240]. For this reason, the lamina Poisson's ratio $\nu_{12}^{d}=0.24$ at RT was taken from [222] and assumed equal to 0.3 at cryogenic temperature $(-156 \mathrm{C})$, which are typical values for brittle epoxy polymers at very low temperatures [240].

Once the experimental data are collected, the elastic properties $E_{m}, \nu_{m}$ of Epoxy 5208 at each temperature $(-156,24$, and $121 \mathrm{C})$ are back calculated by minimizing the error (3.3) between experimental lamina data $\left(E_{1}^{d}, E_{2}^{d}, G_{12}^{d}, \nu_{12}^{d}\right)$ available in [20,221,222] and predicted lamina properties $\left(E_{1}, E_{2}, G_{12}, \nu_{12}, \nu_{23}\right)$ using PMM micromechanics (App. 2 in [200]). The procedure is illustrated in Figure 3.2 by a flowchart, with tolerance [254] tol ${ }^{x}$ and $t o l^{\text {fun }}=10^{-8}$. The T300 fiber properties used as input data in PMM are taken from Table 3.1. Finally, the matrix coefficients (3.2) of Epoxy 5208 are obtained by a quadratic interpolation of the values obtained at $-156,24$, and $121 \mathrm{C}$, then reported in Table 3.5 and depicted in Figure 3.4. Similarly to Figure 3.3, the curves in Figure 3.4 are nonlinear. Therefore, outside the range of the experimental data from which the temperature dependence is found, the matrix properties are assumed to be constant and equal to the end values of the experimental data, as shown in Figure 3.4.

\subsubsection{AS4 Fiber}

The longitudinal modulus $E_{A}$ of AS4 fiber is obtained from manufacturer data sheet $[252,260]$. The remaining elastic properties of AS4 fiber are back calculated from material system AS4/3501-6 using a set of experimental data at room (RT) and high $\left(121^{\circ} \mathrm{C}\right)$ temperature. The matrix properties $E_{m}, \nu_{m}$ of Epoxy 3501-6 at room and high temperature are obtained from $[257,258]$. The rest of elastic fiber properties $\left(E_{T}, G_{A}, \nu_{A}, \nu_{T}\right)$ are back calculated using set of experimental data at both temperatures by minimizing the error (3.3) between unidirectional lamina data $\left(E_{1}^{d}, E_{2}^{d}, G_{12}^{d}, \nu_{12}^{d}\right)$ of AS4/3501-6 in [256] and predicted lamina properties $\left(E_{1}, E_{2}, G_{12}, \nu_{12}, \nu_{23}\right)$ using PMM micromechanics (App. 2 in [200]). The methodology used is shown in Figure 3.5 by a flowchart with 

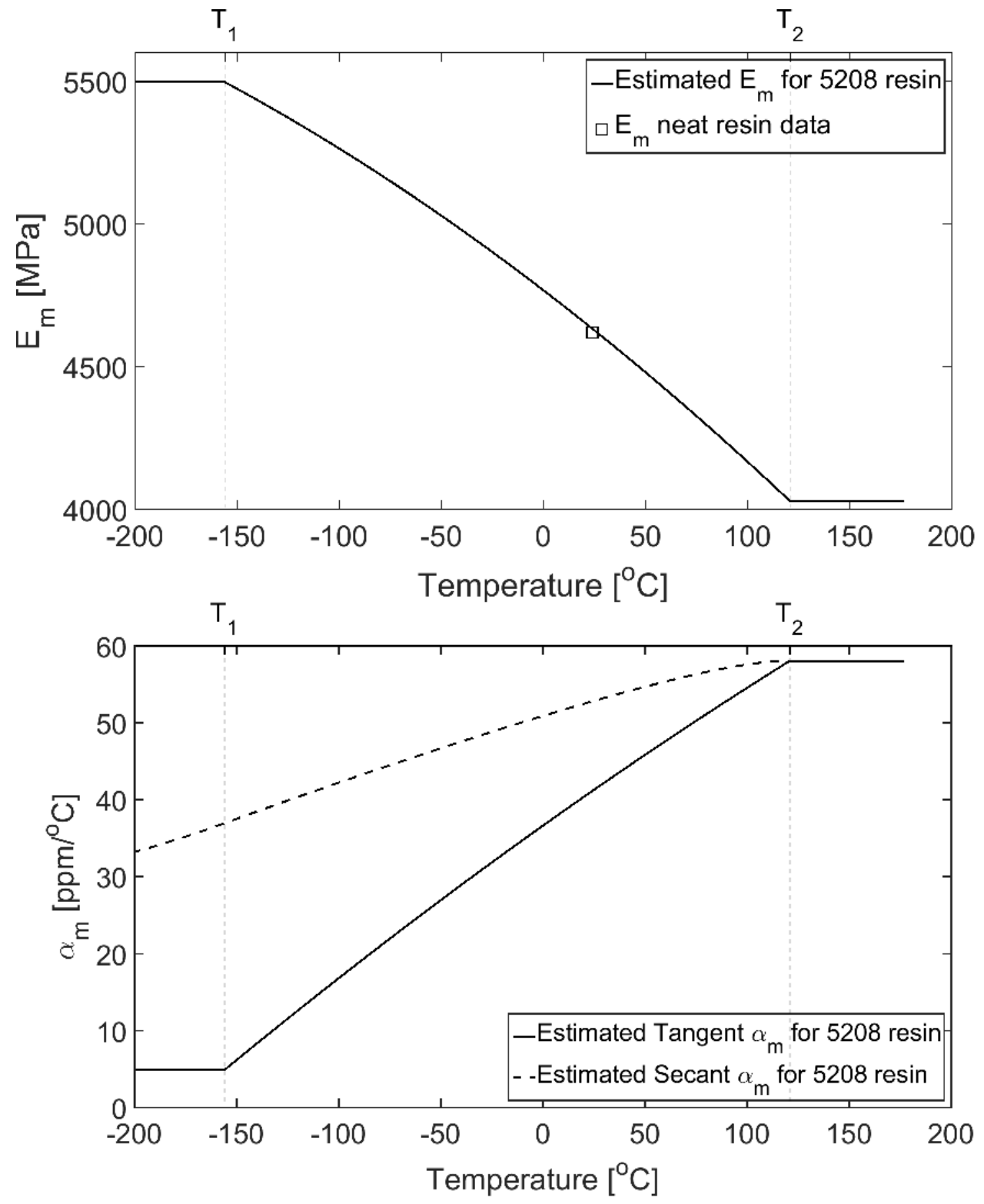

Figure 3.4: Estimated temperature-dependent modulus (top) and CTE (bottom) for Epoxy 5208. 


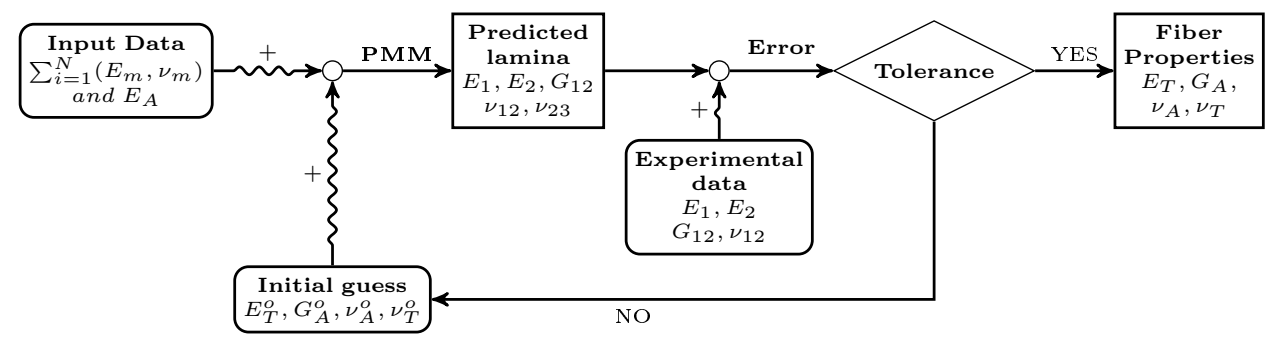

Figure 3.5: Back calculation method to obtain the fiber properties using set of experimental data at various temperatures $(N)$.

tolerance [254] ol $^{x}$ and $t o l^{\text {fun }}=10^{-8}$. The final AS4 fiber properties are reported in Table 3.1.

\subsubsection{T300 Fiber}

The longitudinal modulus $E_{A}$ of T300 fiber is obtained from manufacturer data sheet [251]. The remaining elastic properties of T300 fiber are back calculated from material system T300/5208 at room temperature. The matrix properties $E_{m}, \nu_{m}$, of Epoxy 5208 at room temperature are obtained from [261]. The rest of elastic fiber properties $\left(E_{T}, G_{A}, \nu_{A}, \nu_{T}\right)$ are back calculated at room temperature by minimizing the error (3.3) between unidirectional lamina data $\left(E_{1}^{d}, E_{2}^{d}, G_{12}^{d}, \nu_{12}^{d}\right)$ of T300/5208 in [20,221,222] and lamina properties $\left(E_{1}, E_{2}, G_{12}, \nu_{12}, \nu_{23}\right)$ predicted with PMM (App. 2 in [200]). The procedure is illustrated in Figure 3.5 by a flowchart. The resulting properties for T300 fiber are reported in Table 3.1.

\subsubsection{P75 Fiber}

The average fiber modulus reported in the literature for (unsized) P75 [10, 14, 17, 260, 262-266] and (sized) P75S [267] is $E_{A}=517 \mathrm{GPa}$. Using the longitudinal modulus $E_{A}=517 \mathrm{GPa}$ and the properties of Epoxy 934 (Table 3.3), the rest of elastic properties $\left(E_{T}, G_{A}, \nu_{A}, \nu_{T}\right)$ for P75 fiber are back calculated by minimizing (3.3) between unidirectional lamina data $\left(E_{1}^{d}, E_{2}^{d}, G_{12}^{d}, \nu_{12}^{d}\right)$ of both $\mathrm{P} 75 / 934$ and P75/1962 available in $[10,13,17,186,268]$ and lamina properties $\left(E_{1}, E_{2}, G_{12}, \nu_{12}, \nu_{23}\right)$ predicted using PMM micromechanics (App. 2 in [200]). All the properties of P75 are back calculated using data from literature at room temperature. The procedure is illustrated in Figure 3.5 by a flowchart, with tolerance [254] $\mathrm{tol}^{x}$ and tol $^{\text {fun }}=10^{-8}$. The resulting values are reported in Table 3.1. 


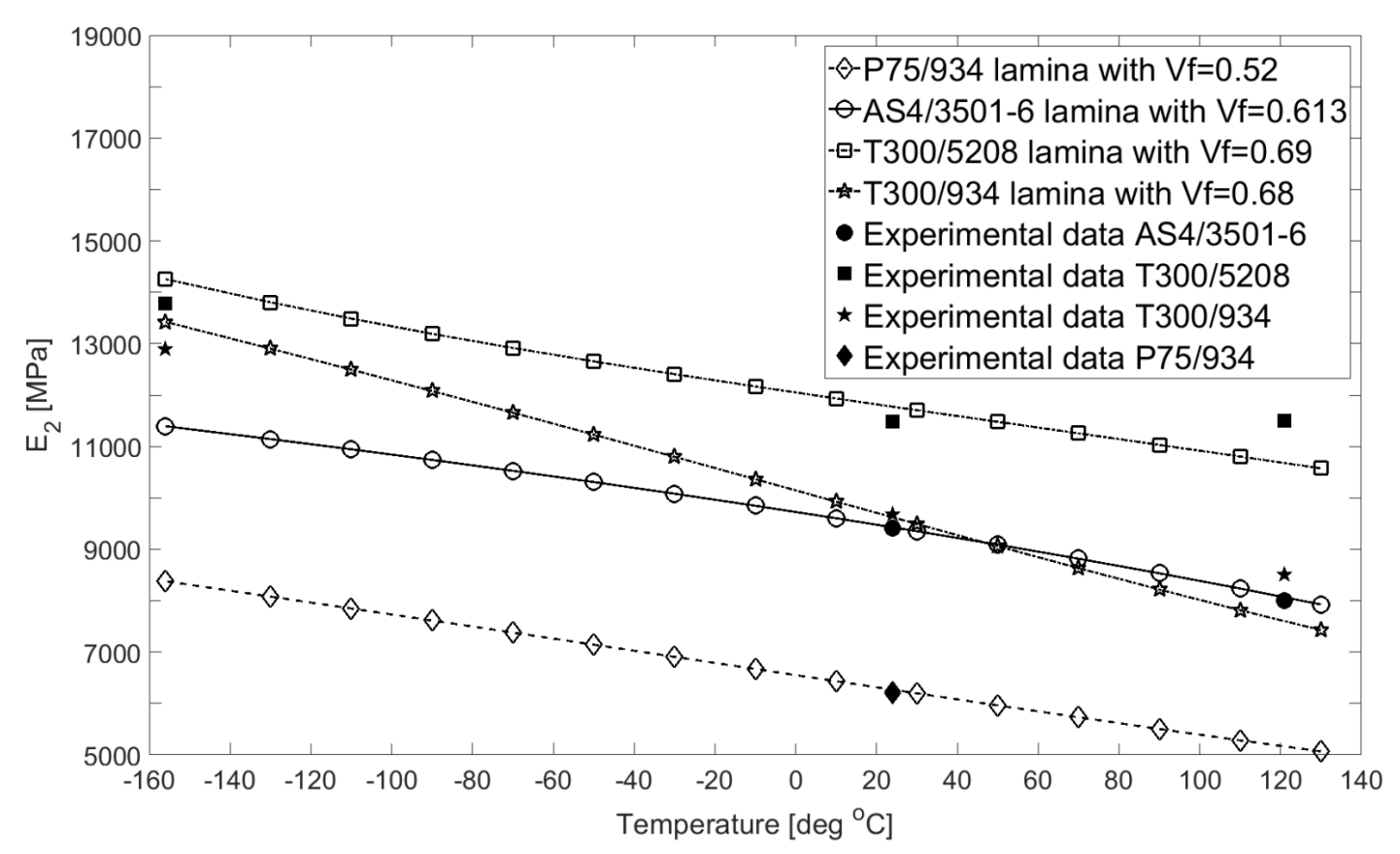

Figure 3.6: Comparison between predicted and experimental data of transverse modulus $E_{2}$ for P75/934, AS4/3501-6, T300/934, and T300/5208 lamina.

\subsubsection{Summary Constituent Properties}

Once the fiber and matrix properties are adjusted, one can predict elastic lamina properties using PMM micromechanics (App. 2 in [200]) and compare with available experimental data. Comparison between model predictions and experimental data for transverse modulus $E_{2}$ as a function of temperature are shown in Figure 3.6. Comparison between model predictions and experimental data for in-plane shear modulus $G_{12}$ as a function of temperature are shown in Figure 3.7.

Since fiber properties are assumed to be temperature-independent, the adjusted properties $\left(E_{T}, G_{T}, \nu_{A}, \nu_{T}\right)$ are constant values that minimize the error between prediction and experimental data at several temperatures. In other words, the constant fiber properties are found in such a way that the deviation from predicted lamina data is as small as possible over the entire data set that may include data for several temperatures. The opposite occurs for matrix properties $\left(E_{m}(T), \nu_{m}(T)\right)$, which are temperature-dependent. For matrix properties, different values of $\left(E_{m}(T), \nu_{m}(T)\right)$ are found at each temperature, and then fitted with the quadratic polynomial (3.2), as a function of temperature.

The proposed methodology can be used to back calculate the constituent properties for any combination of fibers and polymers. However, the available material properties for each fiber and matrix are different and thus, the methodology must be flexible adapted. 


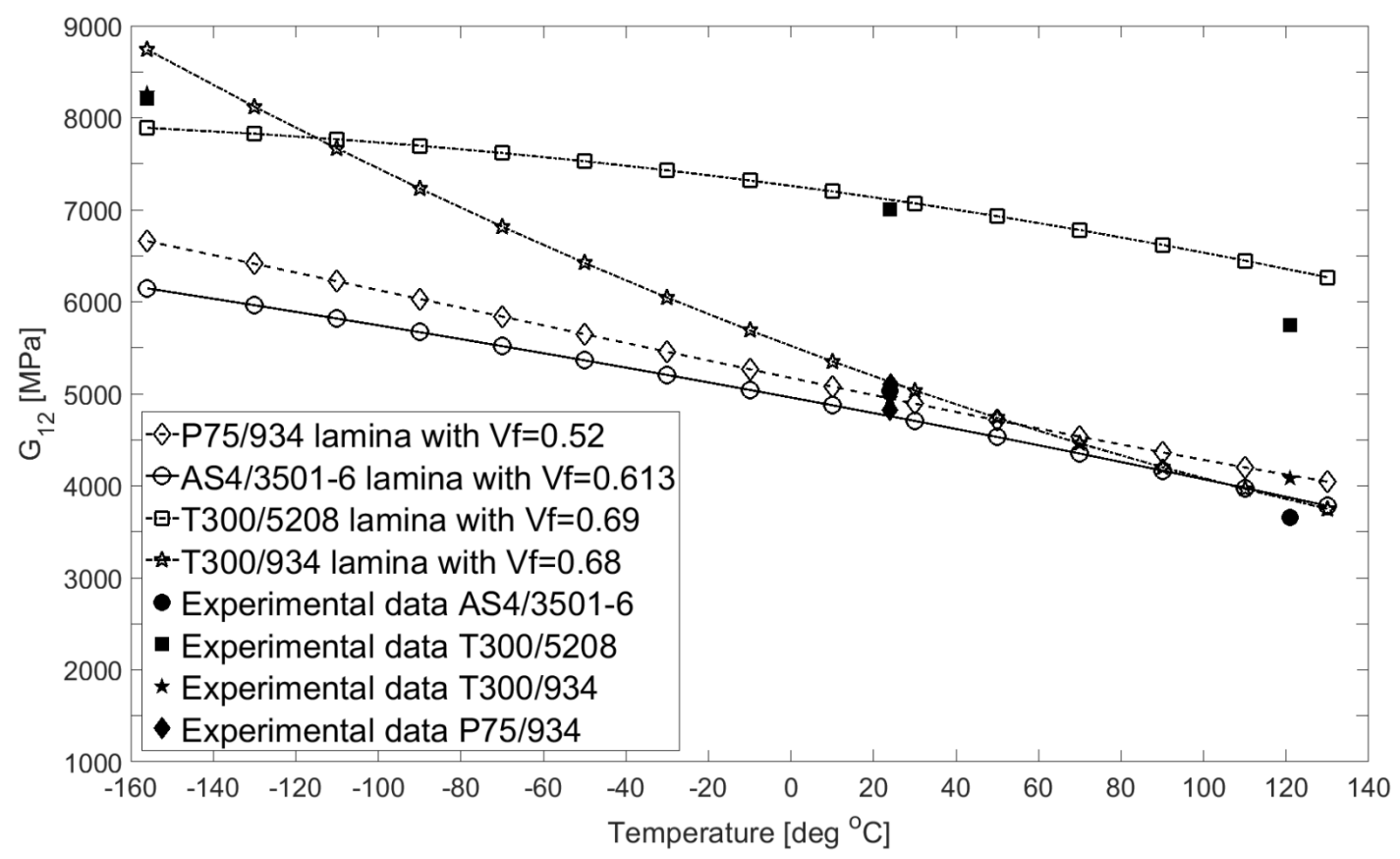

Figure 3.7: Comparison between predicted and experimental data of transverse modulus $G_{12}$ for P75/934, AS4/3501-6, T300/934, and T300/5208 lamina.

\subsection{Coefficients of Thermal Expansion}

The coefficients of thermal expansion (CTE) in the longitudinal and transverse directions of a lamina are defined as

$$
\alpha_{i}=\frac{\partial \epsilon_{i}}{\partial T} \quad \text { with } \quad i=1,2
$$

where $\epsilon_{i}$ are the components of strain and $T$ is the temperature. In this work $\alpha_{i}$ denote tangent CTEs (also called instantaneous CTE). The secant CTE is defined as follows

$$
\bar{\alpha}_{i}=\frac{1}{T-S F T} \int_{S F T}^{T} \alpha_{i} d T
$$

where $S F T$ is the stress free temperature. Equation (3.4) is useful because it directly relates the experimental thermal strain data of a unidirectional lamina with its CTE at any temperature without the need for specifying a reference temperature.

Levin [269] derived an exact solution for effective CTEs of a composite with twophases: fiber (transversely isotropic (TI)) and matrix (isotropic). Levin's Model (LM) relates volume average $\langle\cdot\rangle$ stresses and strains in a representative volume element (RVE) to obtain the effective CTEs as follows

$$
\alpha_{i}=\alpha_{i j}=\left\langle\alpha_{i j}\right\rangle+\left(\alpha_{i j}^{f}-\alpha_{i j}^{m}\right)\left(S_{i j k l}^{f}-S_{i j k l}^{m}\right)^{-1}\left(S_{i j k l}-\left\langle S_{i j k l}^{f}\right\rangle\right) \text { with } i=j
$$


where $S_{i j k l}$ are the elastic compliances, $\alpha_{i j}$ are the CTEs, and the subscripts $f$ and $m$ denote fiber and matrix, respectively. Equation (3.6) requires the effective elastic compliance $S_{i j k l}$ as a function of temperature, which in this work is obtained through PMM (App. 2 in [200]). Hence, the elastic properties of the constituents as a function of temperature must be obtained before calculating the thermal properties. For isotropic matrix and TI fibers, $\alpha_{i j}=0$ for $i \neq j$, and a single subscript suffices for all components of CTE.

Since the type of experimental data available varies from material to material, there are cases for which the CTE values for fiber $\alpha_{A}, \alpha_{T}$, and/or matrix $\alpha_{m}(T)$ are available from experimental data for fiber and/or matrix. However, in most cases they are not directly available, and thus they have to be adjusted by minimizing the error function

$$
D_{t}=\frac{1}{N} \sqrt{\sum^{N}\left[\left(\frac{\alpha_{i}-\alpha_{i}^{d}}{\alpha_{i}^{d}}\right)^{2}\right]} \quad \text { with } i=1,2
$$

between experimental lamina $\mathrm{CTE} \alpha_{i}^{d}$ data (available in the literature) and lamina CTE $\alpha_{i}$ predicted using (3.6). The subscripts $i=1,2$ denote longitudinal and transverse CTE, respectively, and $N$ is the number of data values available. In order to give the same weight to all properties, the error function is normalized for each term.

Since longitudinal CTE $\alpha_{1}$ is a fiber dominated property, the volume fraction is chosen to match the predicted longitudinal CTE with experimental data $\alpha_{1}^{d}$ at room temperature, which is available in the literature for all material systems considered in this study.

CTE from literature or manufacturer data sheet, if available, are used as initial guess for minimization. The CTE of matrix $\alpha_{m}(T)$ are always back calculated using the transverse lamina CTE $\alpha_{2}^{d}$ because the later is matrix dominated. Once the CTE $\alpha_{m}(T)$ are obtained at various temperatures using (3.7), a quadratic interpolation is carried out to obtain the polynomial's coefficients in (3.2). Manufacturer values of $\alpha_{m}(R T)$, if available, are used as initial guess for the error minimization algorithm.

Denoting by $x$ the value of any CTE of interest, and by $D_{t}$ the error (3.7), the value of property $x$ is found when the error $D_{t}$ is less than the function tolerance (i.e., error tolerance) [254] tol $^{\text {fun }}=10^{-8}$ and the change in property $\Delta x$ is less than the step size tolerance tol $^{x}=10^{-8}$.

Since availability of data varies among material systems, not all material systems can be characterized exactly with the procedure described above. In fact, variations in the procedure are necessary to make use of the available data, which varies from material to material. In the following, five material systems (T300/5208, P75/934, T300/934, P75/1962, and AS4/3501-6) are characterized, illustrating how to adapt the proposed 
procedure to make best use of the available data.

\subsubsection{Material System: T300/5208}

The axial CTE $\alpha_{A}$ of T300 fiber is obtained from literature [212,270] and manufacturer data sheet [251]. Data for transverse CTE $\alpha_{T}$ of T300 fiber is not available. Therefore, for this material system only, the transverse CTE $\alpha_{T}$ of T300 fibers and temperaturedependent CTE $\alpha_{m}(T)$ of Epoxy 5208 are back calculated in three steps.

First, the transverse CTE $\alpha_{T}$ of T300 fiber and the RT CTE of the matrix $\alpha_{m}^{0}(R T)$ are back calculated by minimizing the error (3.7) using both the longitudinal and transverse lamina CTEs at RT. In this way, the transverse CTE $\alpha_{T}$ of T300 fiber and the RT CTE of the matrix $\alpha_{m}^{0}(R T)$ can be adjusted so that the lamina CTEs $\alpha_{1}, \alpha_{2}$, predicted using (3.6) match experimental CTEs $\alpha_{1}^{d}, \alpha_{2}^{d}$, for T300/5208 lamina from [20,212,220]. The matrix CTE at RT from [261] is used as initial guess for $\alpha_{m}^{0}(R T)$. The methodology used is illustrated in Figure 3.8. Tolerances used [254] are $t^{2} l^{x}=t^{\prime} l^{\text {fun }}=10^{-4}$. At the end of this first step, the CTEs of T300 fiber are reported in Table 3.1.

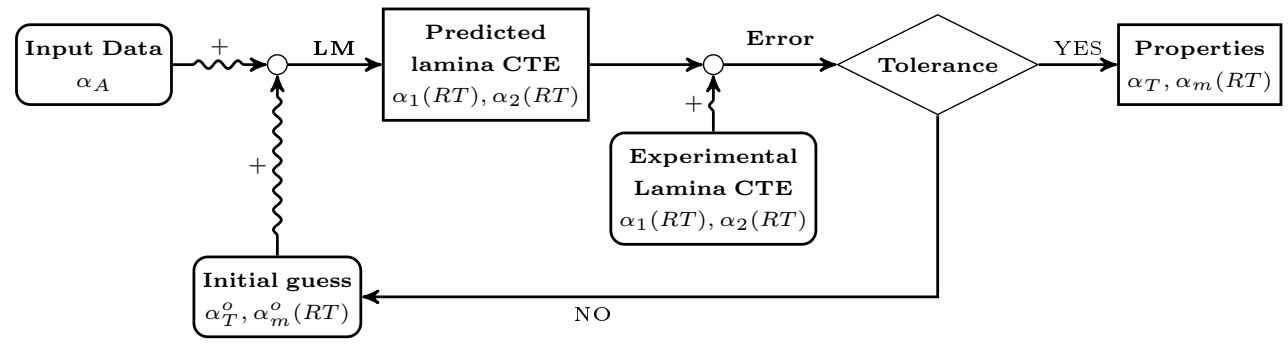

Figure 3.8: Back calculation method to obtain the fiber and matrix CTE values.

Second, the temperature-dependent CTE $\alpha_{m}(T)$ of Epoxy 5208 is back calculated at various temperatures (in the temperature range $[-130,120 \mathrm{C}]$ ) by minimizing the error (3.7) between experimental lamina CTE in the transverse direction $\alpha_{2}^{d}$ for T300/5208 lamina in [212] and predicted lamina CTE $\alpha_{2}$ using micromechanics (3.6). The procedure is illustrated by a flowchart in Figure 3.9. The matrix CTE previously calculated at room temperature $\alpha_{m}^{0}(R T)$ is used as initial guess. A schematic of the procedure is shown in Figure 3.9. Tolerance [254] used are tol $^{x}=$ tol ${ }^{\text {fun }}=10^{-8}$.

Third, once the temperature-dependent CTE $\alpha_{m}(T)$ of Epoxy 5208 is calculated for a large number of temperature data points, the matrix coefficients (3.2) are obtained by a quadratic interpolation of those results. Then, the CTE of Epoxy 5208 as function of temperature is reported in Table 3.5. 


\subsubsection{Material System: P75/934 and T300/934}

The CTE values $\alpha_{A}, \alpha_{T}$, of P75 fiber are obtained from literature and manufacturer data sheet $[14,212,250,271]$. Identical values were found in various literary resources and thus they are assumed to be valid for this study. Temperature-dependent CTE of Epoxy 934 could not be calculated using the data for P75/934 in [13] due to lack of experimental data points at cryogenic temperatures. Instead, data for material system T300/934 in [212] with temperature range $[-156,121 \mathrm{C}]$ is used to calculate the temperature-dependent CTE of Epoxy 934. Therefore, the temperature-dependent CTE $\alpha_{m}(T)$ of Epoxy 934 are back calculated at various temperatures by minimizing the error (3.7) between experimental lamina CTE in the transverse direction $\alpha_{2}^{d}$ for T300/934 lamina in [212] and predicted lamina CTE $\alpha_{2}$ using micromechanics (3.6). The methodology used is illustrated in Figure 3.9 using tolerance [254] tol $^{x}=$ tol $^{\text {fun }}=10^{-8}$.

Once the matrix properties $\alpha_{m}(T)$ of Epoxy 934 are calculated for a large number of temperature data points, the matrix coefficients (3.2) are obtained by a quadratic interpolation of those results. The CTE of Epoxy 934 as function of temperature is reported in Table 3.3. The predicted values $\alpha_{1}, \alpha_{2}$, as a function of temperature for P75/934 lamina are plotted in Section "Finite Element Analysis".

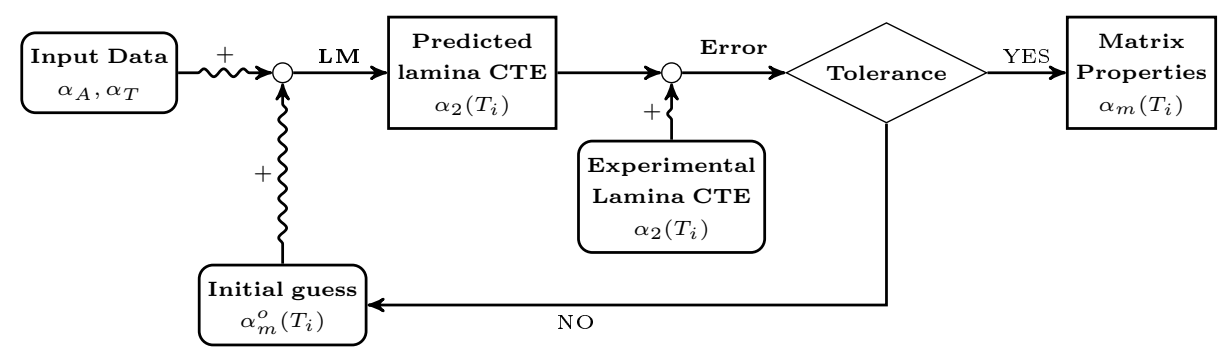

Figure 3.9: Back calculation method to obtain the matrix CTE at any temperature $\left(T_{i}\right)$.

\subsubsection{Material System: P75/1962}

The temperature-dependent properties $\alpha_{m}(T)$ of Epoxy ERL 1962 are back calculated at various temperatures by minimizing the error (3.7) between experimental lamina CTE in the transverse direction $\alpha_{2}^{d}$ for P75/1962 lamina in [17,19], and lamina CTE $\alpha_{2}$ predicted by micromechanics (3.6). The procedure is illustrated by a flowchart in Figure 3.9 using tolerance [254] tol $^{x}=$ tol $^{\text {fun }}=10^{-8}$.

The CTEs values of P75 fiber used in (3.6) are already reported in Table 3.1. Due to the availability of thermal strain data $\epsilon_{i}$ for this particular material system $(\mathrm{P} 75 / 1962$ lamina), [17,19] the CTE $\alpha_{2}^{d}$ data is calculated from thermal strain data $\epsilon_{i}$ using (3.4). Since $\epsilon_{i}$ data is quadratic in the temperature range [-150,120 C], the resulting CTE is also quadratic in the same temperature range. Once the temperature-dependent CTE $\alpha_{m}(T)$ 
of Epoxy ERL 1962 has been back calculated for a large number of temperature data points, the matrix coefficients (3.2) are obtained by a quadratic interpolation of those results. Then, the CTE of Epoxy ERL 1962 as function of temperature is reported in Table 3.4

\subsubsection{Material System: AS4/3501-6}

The axial CTE $\alpha_{A}$ of AS4 fiber is obtained from manufacturer data sheet [252]. The temperature-dependent properties $\alpha_{m}(T)$ of Epoxy 3501-6 are taken from [215, 225, 272] in the temperature range $[-90,150 \mathrm{C}]$, which can be represented well by a linear function of temperature. Since the transverse CTE $\alpha_{T}$ of AS4 fiber is not available, it is back calculated by minimizing the error (3.7) between the predicted lamina CTE $\alpha_{2}$ using micromechanics (3.6), and experimental lamina CTE $\alpha_{2}^{d}$ for AS4/3501-6 lamina available in [225]. The procedure used is shown in Figure 3.10 using tolerance [254] tol $^{x}=$ tol $^{\text {fun }}=$ $10^{-8}$. The transverse CTE of AS4 fiber is reported in Table 3.1.

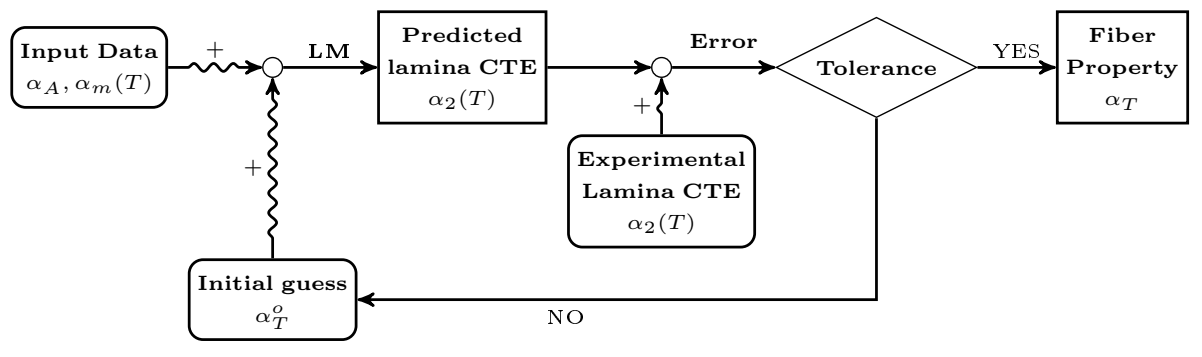

Figure 3.10: Back calculation method to obtain the transverse CTE of the fiber from transverse lamina CTE as function of temperature.

\subsubsection{Summary CTE}

Once the matrix CTE are adjusted, one can predict lamina CTE using (3.6) and compare with available experimental data (from sources cited above for each material system). Comparison between predicted lamina CTE using (3.6) and experimental data $\alpha_{1}^{d}, \alpha_{2}^{d}$ is shown in Figures 3.11-3.12. The comparison in Figure 3.11 is excellent with $\alpha_{2}$ in the range [5-45] 10-6/C. In Figure 3.12, predicted and experimental values of $\alpha_{1}$ do not match so well, except at room temperature. The deviation may be attributed to possible temperature-dependence of the transverse CTE of the fibers $\alpha_{T}(T)$, but such temperature dependency in impossible to ascertain without additional experimental data, which is not available.

The proposed methodology can be used to back calculate the thermal properties for any combination of fibers and polymers. However, the available material properties for each fiber and matrix are different and thus, the methodology must be flexible adapted. 


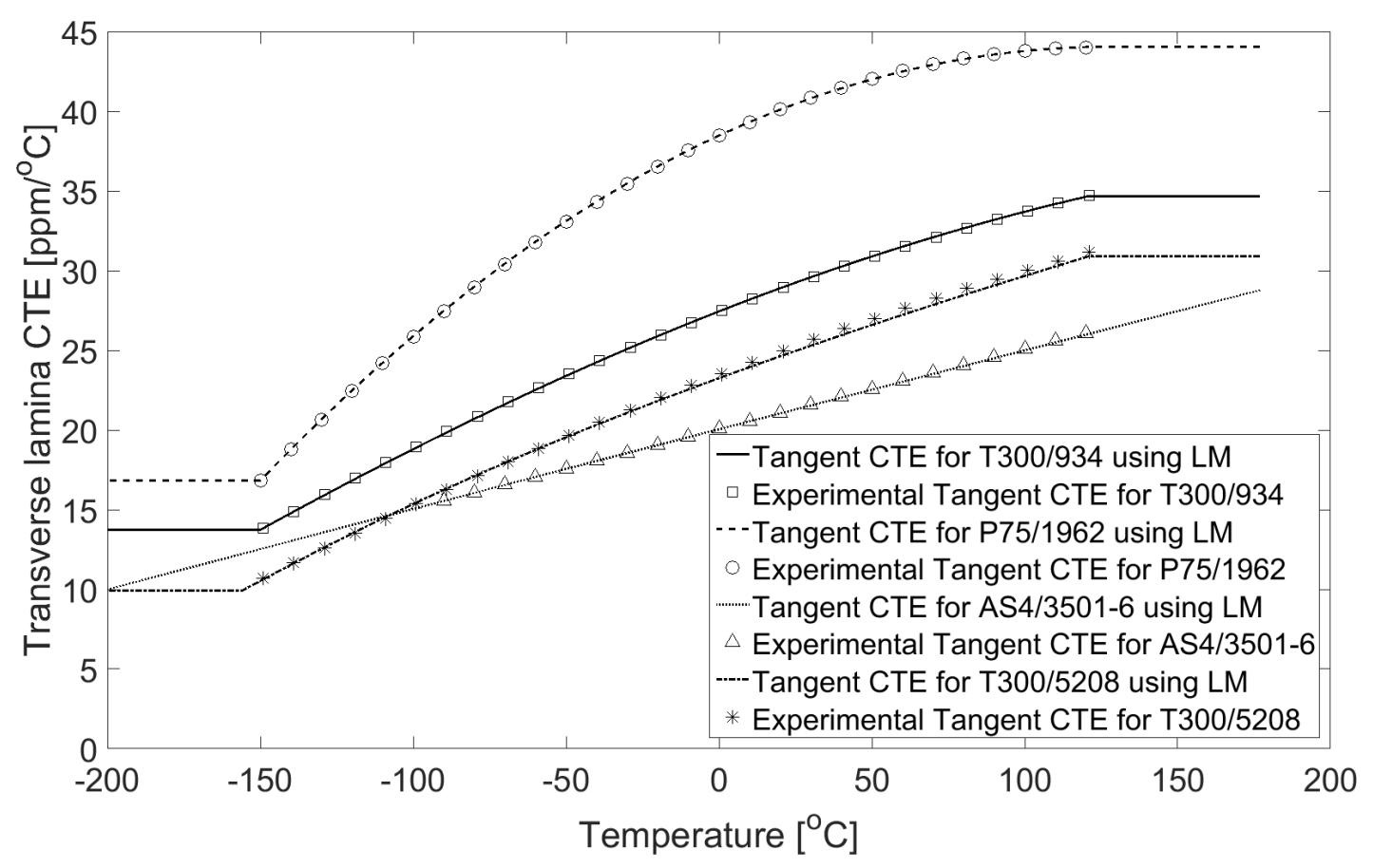

Figure 3.11: Comparison of transverse lamina CTE $\alpha_{2}$ predicted with Levin's model (3.6) vs. experimental data for T300/934 with $V_{f}=0.57$, AS4/3501-6 with $V_{f}=0.67$, and P75/1962 with $V_{f}=0.52$.

\subsection{Finite Element Analysis}

In this section, the effective CTEs as function of temperature for a composite lamina are calculated using finite element analysis (FEA). The results are used to asses the accuracy of the micromechanics model (3.6) for CTE. A summary of the methodology is included, and comparison between micromechanics and FEA predictions is presented.

To obtain the effective CTEs for the whole temperature range, monotonic cooling is simulated from the glass transition temperature $T_{g}$ of the polymer down to cryogenic temperatures (-200 C).

To represent a transversely isotropic lamina with 3D solid elements, the microstructure is assumed to have the fibers arranged in an hexagonal array, and from that microstructure a representative volume element (RVE) limited by a cuboid is represented, as it can be seen in Figures 6.3-6.5 in [193]. The dimensions of the RVE are calculated to achieve the desired volume fraction $V_{f}$, as explained in Example 6.2 in [193].

Since longitudinal CTE $\alpha_{1}$ is a fiber dominated property, the volume fraction is chosen to match the predicted longitudinal CTE with experimental data $\alpha_{1}^{d}$ at room temperature, which is available in the literature for all material systems considered in this study.

Periodic boundary conditions (PBC) are imposed to the RVE in order to enforce continuity of displacements. To avoid over constraining at edges and vertices, master nodes $(\mathrm{MN})$, one for each face of the RVE in $x_{1}, x_{2}$, and $x_{3}$ directions, are used to couple 


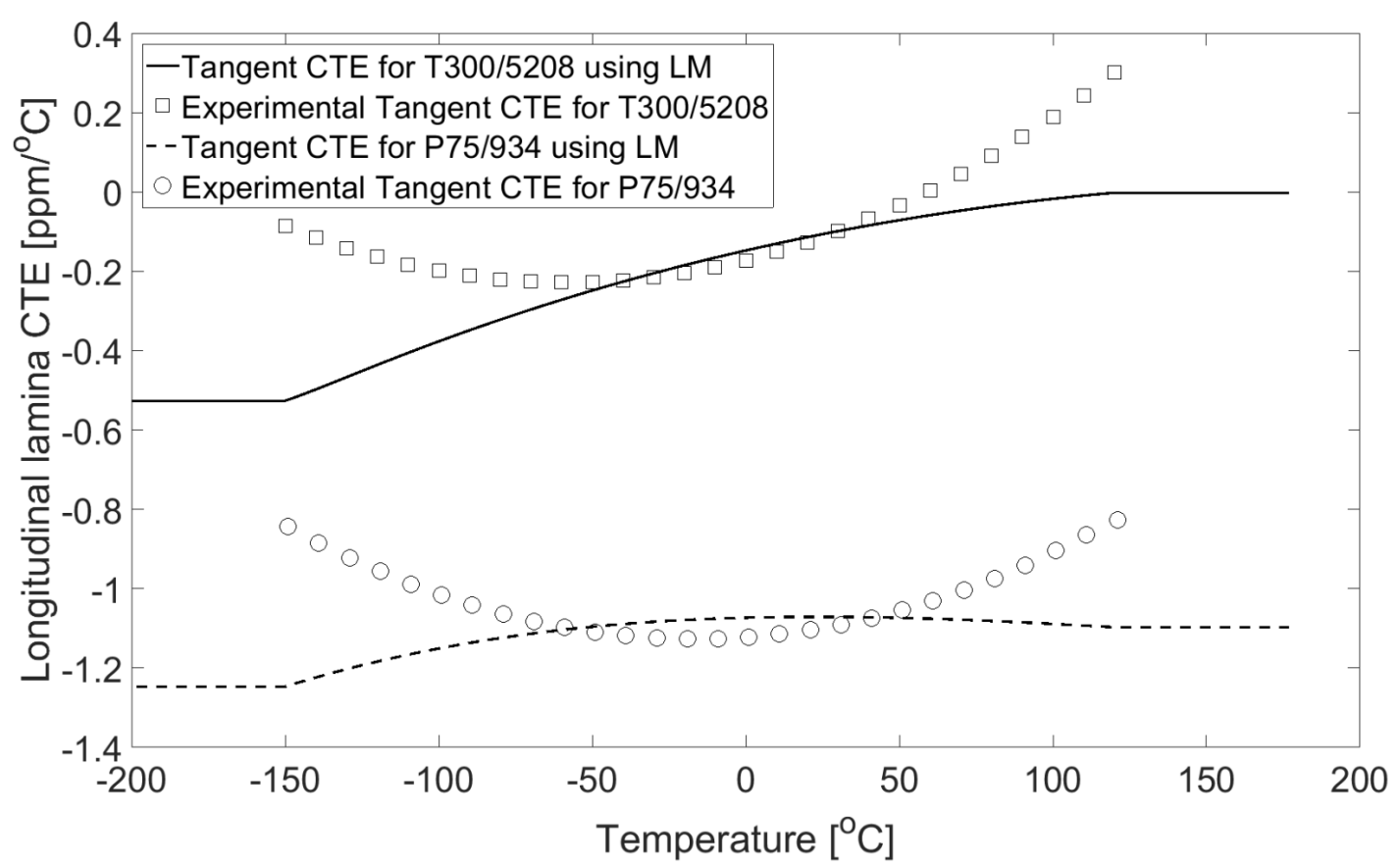

Figure 3.12: Comparison between longitudinal lamina CTE $\alpha_{1}$ predicted with Levin's model (Eq. 3.6) and experimental data for T300/5208 with $V_{f}=0.68$ and P75/934 with $V_{f}=0.51$.

the DOF through constraints equations. The BCs thus become

$$
\begin{array}{cc}
\text { symmetry } & \text { uniform displacements } \\
u_{1}\left(0, x_{2}, x_{3}\right)=0 ; & u_{1}\left(a_{1}, x_{2}, x_{3}\right)=u_{1}^{M N_{X_{1}}} \\
u_{2}\left(x_{1}, 0, x_{3}\right)=0 ; & u_{2}\left(x_{1}, a_{2}, x_{3}\right)=u_{2}^{M N_{X_{2}}} \\
u_{3}\left(x_{1}, x_{2}, 0\right)=0 ; & u_{3}\left(x_{1}, x_{2}, a_{3}\right)=u_{3}^{M N_{X_{3}}}
\end{array}
$$

where $M N_{X_{1}}, M N_{X_{2}}$ and $M N_{X_{3}}$ are the master nodes (reference points) in $x_{1}, x_{2}$ and $x_{3}$ directions, respectively. The RVE occupies the volume with dimensions: $0 \leq x_{1} \leq a_{1}$, $0 \leq x_{2} \leq a_{2}$, and $0 \leq x_{3} \leq a_{3}$. The MNs are tied to surfaces defined by $x=a_{1}, y=$ $a_{2}, z=a_{3}$. No displacements or loads are specified at the MN, so that the RVE is free to expand/contract with thermal expansion but subject to compatibility conditions with the surrounding continuum.

The temperature-dependent properties of the matrix $E_{m}, \nu_{m}, \alpha_{m}$, are defined as a set of $N$ temperature-property data pairs as $\left(T_{1}, P_{1}\right),\left(T_{2}, P_{2}\right), \ldots,\left(T_{N}, P_{N}\right)$. The values are obtained using (3.2) and Tables 3.2-3.5. These values are discretized with $\Delta T=1 \mathrm{C}$ to simplify the computations and interpretation of results. Outside the range $[-156,120]$ for which experimental data is available, the properties of the matrix are assumed to be constant and equal to the first (or last) experimental data pair (Figures 3.3,3.4).

Two python scripts ('ParameterIntegrator.py' and 'Excelproperties.py') are used to create the input property tables for the matrix material. All Python scripts 
are available as supplemental materials on Appendix A. Since the matrix properties are defined by piece-wise functions (Figures 3.3,3.4), the resulting lamina properties are also piece-wise functions (Figures 3.13-3.15). The transversely isotropic properties of the fibers are assumed to be constant over the entire temperature range. Curing-induced shrinkage of the epoxy resin is not taken into account.

FEA analysis was performed with Abaqus 6.14, using small displacement, linear elastic material, and 3D elements C3D8R. A Python script ('LaminaName.py') is used to generate the FEA model. A mapped mesh was constructed providing identical mesh on opposite surfaces. The PBC are implemented as constraints equations between master nodes and surfaces with normals along the $x, y, z$ directions, respectively. A Python script ('PBC.py') is used to automate such process. Symmetric BC were applied to surfaces defined by $x=0, y=0, z=0$.

Finally, a Python script ('Epsilonrecover.py') is used to calculate the accumulated thermal strains at temperature $T$ via volume averages from mesh elements $j$ as

$$
\epsilon(T)=\frac{1}{V_{R V E}} \int_{V_{R V E}} \hat{\epsilon}(x, y, z) d V=\frac{1}{V_{R V E}} \sum_{j=1}^{\text {elements }} \hat{\epsilon}^{j} V_{i}^{j}
$$

Computational micromechanics is used in this section as described in Ch. 6 in [193]. In this way, constituent properties can be assigned separately to the constituents (fiber and matrix) and the FEA model can be subjected to a variation of temperature. Then, FEA calculates the strain $\hat{\epsilon}(x, y, x)$ at all Gauss integration points inside the representative volume element (RVE) and the average strain over the RVE is easily computed as in $(3.9)$.

The tangent CTE $\alpha(T)$ is a function of temperature in (3.4) and the secant CTE $\bar{\alpha}(T)$ is also a function of temperature (3.5), using the stress-free temperature (SFT) as reference temperature. For each increment of temperature $T$, Abaqus calculates the accumulated strain in terms of the secant CTE (as stated in [155]) i.e.,

$$
\epsilon^{a c c}(T)=\bar{\alpha}(T) \times(T-S F T)
$$

and the user has to calculate tangent CTE by differentiation in (3.4).

Both $\alpha_{m}$ and $\bar{\alpha}_{m}$ are smooth continuous functions in the interval $\left[T_{1}, T_{2}\right]$ for which experimental data exists (see labels $T_{1}, T_{2}$ in Figs. 3.3 and 3.4), but they are constant outside that range, i.e. in the ranges $\left[-200, T_{1}\right]$ and $\left[T_{2}, S F T\right]$. Recall that the properties are assumed constant outside the range for which experimental data exist, as shown in Figures 3.3-3.4. Since a piece-wise function is not differentiable at the transition points $T_{1}$ and $T_{2},(3.4)$ cannot be used and the tangent CTE at those temperatures is undefined. 
To solve the indetermination, we propose to provide Abaqus with the tangent rather than the secant, i.e., substitute $\alpha(T)$ for $\bar{\alpha}(T)$ in (3.10). In this situation, Abaqus calculates a fictitious strain $\epsilon^{a c c *}(T)$, as per the following equation

$$
\epsilon^{a c c *}(T)=\alpha(T) \times(T-S F T)
$$

which is not the actual accumulated strain but a fictitious value. However, dividing this fictitious value by the temperature interval $(T-S F T)$, i.e., rewriting (3.11) as

$$
\alpha(T)=\frac{\epsilon^{a c c *}(T)}{(T-S F T)}
$$

the desired result is obtained, namely the tangent CTE, while avoiding the differentiation (3.4), and thus a potential error is eliminated.

Using the aforementioned procedure, effective CTEs $\alpha_{1}, \alpha_{2}$ are calculated using FEA and then compared with experimental data and with predicted lamina CTE using (3.6) for all the material systems considered in this study. Comparison between FEA-calculated and experimental values $\alpha_{1}$ and $\alpha_{2}$ at room temperature from [13,17, 20,212, 220,272] are reported in Table 3.6 and 3.7. The predictions compare very well with experimental data for all the material system studied. The only anomaly observed is for longitudinal lamina CTE for T300/934 shown in Table 3.6, which may be due to a slight deviation in the fiber volume fraction. Longitudinal lamina CTE is very sensitive to fiber volume fraction. For example, just increasing fiber volume fraction by $2 \%$, the predicted value drops $0.06910^{-6} / \mathrm{C}$, thus reducing the difference.

Tables 3.6: Comparison of experimental and FEA-calculated longitudinal lamina CTEs at

\begin{tabular}{|c|c|c|c|c|c|}
\hline \multirow[b]{2}{*}{$\begin{array}{l}\text { Material } \\
\text { System }\end{array}$} & \multirow[b]{2}{*}{$\begin{array}{c}V_{f} \\
{[\%]}\end{array}$} & Experimental & FEA & \multirow[b]{2}{*}{$\%$ Error } & \multirow[b]{2}{*}{ Reference } \\
\hline & & $\begin{array}{c}\alpha_{1} \\
{\left[10^{-6} / \mathrm{C}\right]}\end{array}$ & $\begin{array}{c}\alpha_{1} \\
{\left[10^{-6} / \mathrm{C}\right]}\end{array}$ & & \\
\hline T300/5208 & 68 & -0.113 & -0.113 & 0.1 & [212] \& Fig. 13 \\
\hline T300/5208 & 70 & -0.166 & -0.163 & 1.8 & {$[20,220]$} \\
\hline T300/934 & 57 & -0.001 & 0.151 & 111.9 & [212] \\
\hline P75/934 & 51 & -1.051 & -1.071 & 1.8 & {$[13]$} \\
\hline P75/1962 & 52 & -0.987 & -0.984 & 0.3 & [17] \\
\hline AS4/3501-6 & 67 & - & -0.194 & - & [272] \& Fig. 11 \\
\hline
\end{tabular}
$24 C$.

Comparison between predicted lamina CTE using (3.6) and FEA is shown in Figures 3.13-3.15. The CTE predictions by both methods are very close. Longitudinal CTE is compared in Figure 3.13 and transverse CTE is compared Figures 3.14-3.15. It can be seen that Levin's model is as accurate as FEA. Since Levin's model is analytical, it is 
Tables 3.7: Comparison of experimental and FEA-calculated transverse lamina CTEs at 24 C.

\begin{tabular}{|c|c|c|c|c|c|}
\hline \multirow[b]{2}{*}{$\begin{array}{l}\text { Material } \\
\text { System }\end{array}$} & \multirow[b]{2}{*}{$\begin{array}{c}V_{f} \\
{[\%]}\end{array}$} & Experimental & FEA & \multirow[b]{2}{*}{$\%$ Error } & \multirow[b]{2}{*}{ Reference } \\
\hline & & $\begin{array}{c}\alpha_{2} \\
{\left[10^{-6} / \mathrm{C}\right]}\end{array}$ & $\begin{array}{c}\alpha_{2} \\
{\left[10^{-6} / \mathrm{C}\right]}\end{array}$ & & \\
\hline T300/5208 & 68 & 25.236 & 24.960 & 1.1 & [212] \& Fig. 13 \\
\hline T300/5208 & 70 & 23.327 & 23.752 & 1.7 & {$[20,220]$} \\
\hline T300/934 & 57 & 29.340 & 29.170 & 0.6 & [212] \\
\hline P75/934 & 51 & 34.531 & 34.061 & 1.4 & [13] \\
\hline P75/1962 & 52 & 40.405 & 40.493 & 0.21 & [17] \\
\hline AS4/3501-6 & 67 & 21.212 & 21.335 & 0.6 & [272] \& Fig. 11 \\
\hline
\end{tabular}

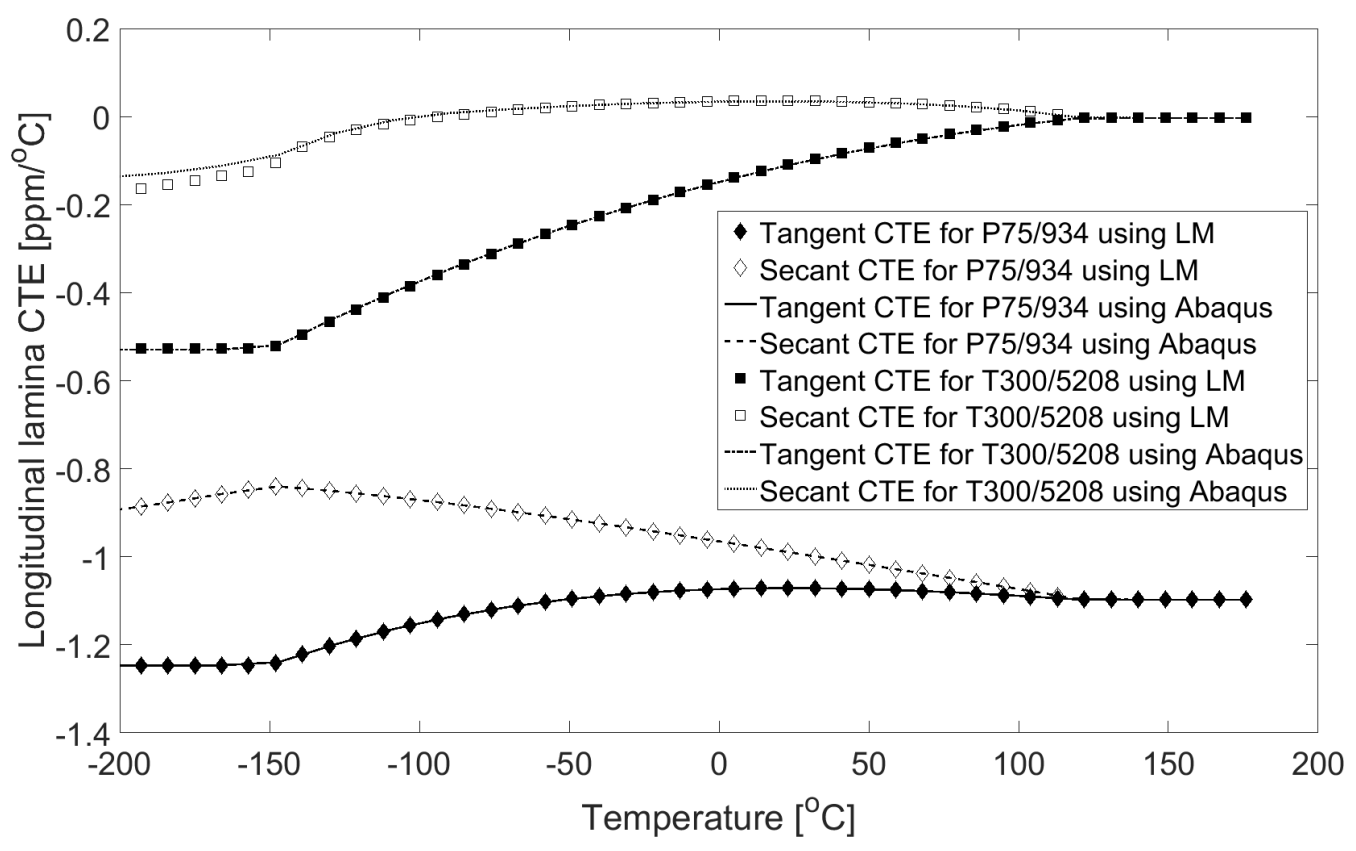

Figure 3.13: Comparison micromechanics and FEA predictions of tangent and secant longitudinal CTE $\alpha_{1}$ for P75/934 $\left(V_{f}=0.51\right)$ and T300/5208 $\left(V_{f}=0.68\right)$.

then used for all remaining calculations in this work. 


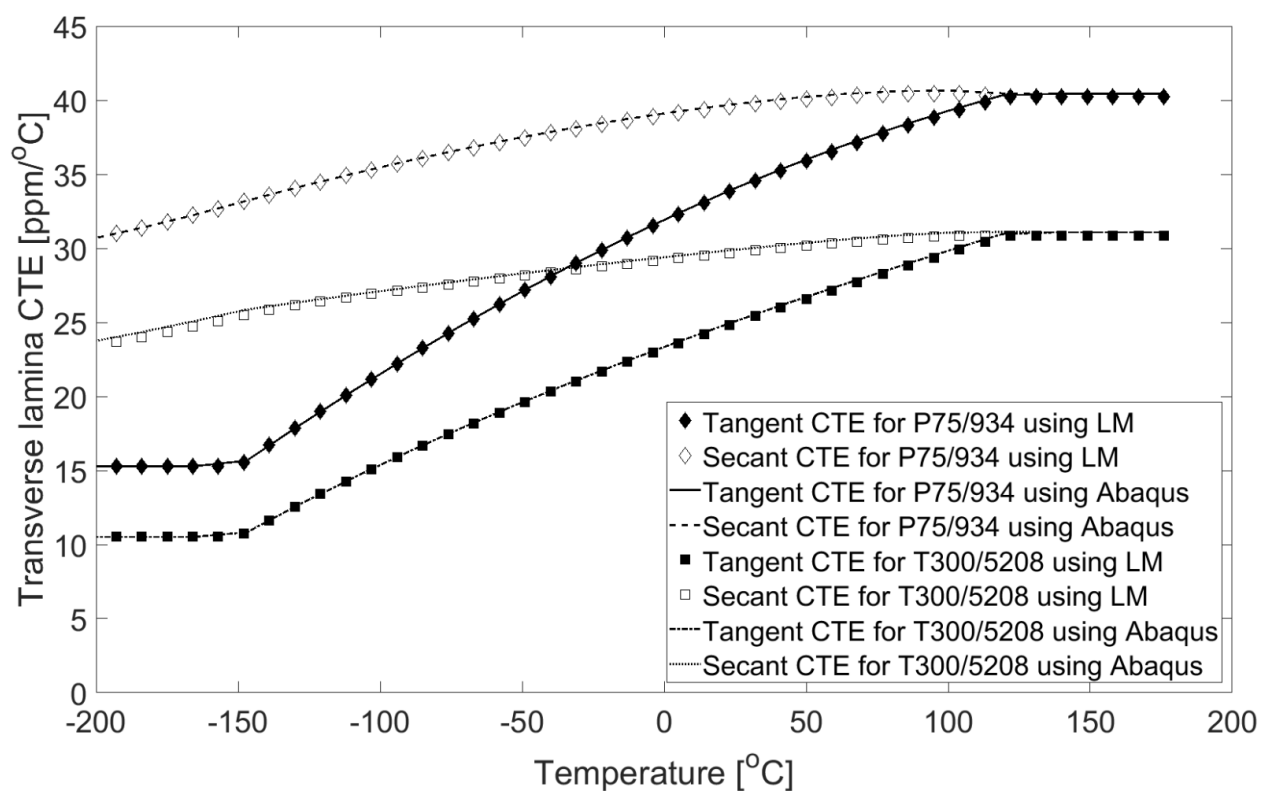

Figure 3.14: Comparison micromechanics and FEA predictions of tangent and secant transverse CTE $\alpha_{2}$ for P75/934 $\left(V_{f}=0.51\right)$ and T300/5208 $\left(V_{f}=0.68\right)$.

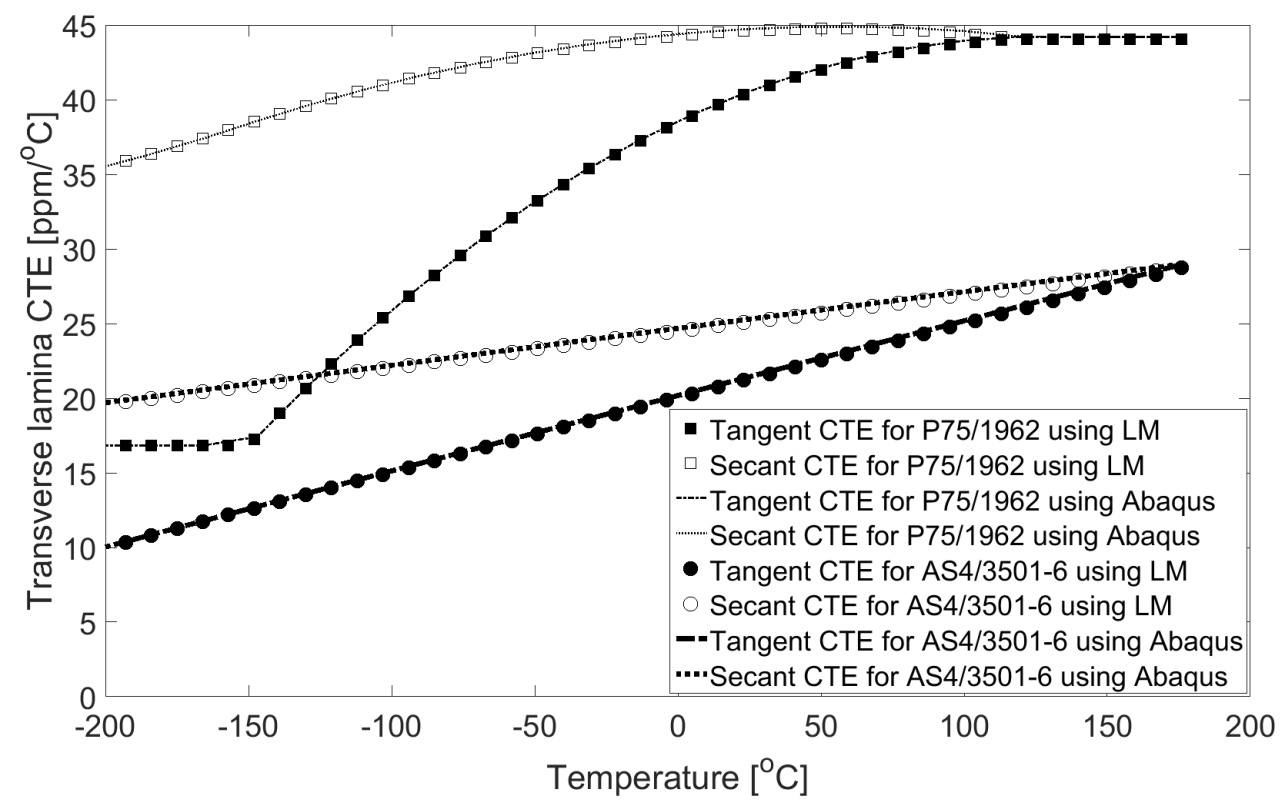

Figure 3.15: Comparison between micromechanics and FEA predictions of tangent and secant transverse CTE $\alpha_{2}$ for Pr5/1962 $\left(V_{f}=0.52\right)$, and AS4/3501-6 $\left(V_{f}=0.67\right)$. 


\section{Chapter 4}

\section{Monotonic cooling}

In order to evaluate thermal fatigue of composite materials, transverse damage initiation and evolution in laminated composites during the first thermal cycle must be studied first. Although some experimental data is obtained using liquid nitrogen [1], mos of them are subjected to constant cooling/heating rate to ensure uniform temperature gradient in the material. Furthermore, despite of periodic nature for thermal cyclic loads, the first thermal cycle can be simulated by a quasi-static cooling for two reasons. First, the highest transverse damage occurs at the lowest temperature where the residual stresses are maximum as it will be shown later. Second, there are no a fatigue effects during the first cycle because it takes a number of cycles to see the effects of fatigue. Therefore, it will be assumed that first thermal cycle can be simulated monotonic cooling from the SFT to the lowest temperature of the thermal fatigue situation under study.

In order to simulate a monotonic cooling, a powerful analytical model able to predict with accuracy the laminate behavior is required incorporating the temperature-dependent properties of material system presented in Chapter 3. For this study, discrete damage mechanics (DDM) is chosen due to its simplicity and accuracy on laminate composites subjected to thermo-mechanical loads $[31,32,34]$. Furthermore, only two values of critical energy release rate (critical ERR), $G_{I c}$ and $G_{I I c}$ are needed to successfully predict both damage initiation and evolution as explained in more detail in Chapter 2.

Standard methods to measure the interlaminar $G_{I c}$ and $G_{I I c}$ can be found in the literature for delamination (e.g. ASTM D5528). However, these properties are not the same as those for intralaminar critical ERR used to predict transverse damage initiation and evolution. Since no standard methods exist to measure the intralaminar critical ERR, the objective of this chapter is to propose a methodology to determine the intralaminar $G_{I c}$ and $G_{I I c}$ required by DDM. In this way, both mechanical and thermal response of laminated composite as function of crack density and temperature, as well as the residual thermal stresses prior to thermal fatigue can be obtained. 


\subsection{Critical Energy Release Rates}

To a first approximation, intralaminar cracking of unidirectional laminated composites can be described by the modified Griffith's criterion [273,274] for brittle materials undergoing small plastic deformations and blunting of the crack tip. Refinements to this approximation increase the complexity of the model to achieve more accuracy [275]. However, the modified Griffith's criterion has been extensively validated for predicting initiation and accumulation of damage in the form of intralaminar cracks for a variety of material systems $[100,156,164,166,186,276]$. As it was commented in previous chapter, polymers become brittle at low temperature, and thus the onset and development of new cracks can be described by Linear Elastic Fracture Mechanics, whose crack initiation is controlled by fracture toughness $K_{I c}$. Once the crack starts, it suddenly propagates up to adjacent laminas. Assuming the width much larger than the thickness (plane-strain), the critical ERR $G_{I c}$ can be related to the fracture toughness as follows

$$
\begin{array}{r}
G_{I_{c}}=\frac{K_{I c}^{2}}{E}\left(1-\nu^{2}\right) \\
K_{I c}=\sigma_{t} \alpha \sqrt{\pi a}
\end{array}
$$

where $E$ is the Young's modulus, $\nu$ the Poisson's ratio, $\sigma_{t}$ the tensile strength, $\alpha$ a parameter to account for the geometry of the specimen, and $a$ the crack length.

Looking at (5.1), it would appear that $G_{I c}$ should be temperature dependent because $E$ and $\nu$ are temperature dependent. However, it remains to ascertain the temperature dependence of $K_{I c}$. If both $E$ and $K_{I c}^{2}$ were to increase/decrease at the same rate, then $G_{I c}$ would be virtually constant.

According to the literature, $K_{I c}$ generally increases at cryogenic temperatures for a large variety of polymers [243,277-279] and specifically for epoxy [246,248,280,281]. The physical phenomenon that can explain this increment of the critical ERR is reported in $[240,281,282]$. On one hand, the specific heat conduction of plastics is very small at low temperature [242,244,280,283,284], behaving as insulating material. Thus, heat conduction is impaired and the crack tip is subject to approximately adiabatic conditions. On the other hand crack propagation is unstable, reaching high speeds, up to $1 / 3$ of the transverse sound velocity in brittle materials such as epoxy at low and cryogenic temperatures. Due to crack propagation speed, friction, chain scissions, and high-rate deformation, heat is generated that causes temperature to rise at the crack tip under adiabatic conditions. High temperature induces a plastic zone at the crack tip that absorbs energy and arrests the crack until additional external load and deformation increases the ERR sufficiently to start the crack again. This is corroborated by arrest lines [244] 
that can be observed, which are left behind the path followed by the crack propagating through the material in this fashion.

The rate of growth of $K_{I c}$ with cooling could be ascertained from (5.2) in terms of the tensile strength $\sigma_{t}$, which increases at low temperatures [280], while the tensile strain $\epsilon_{t}$ decreases [241-244]. However, lacking experimental data for $K_{I c}$ and $\sigma_{t}$ at low and cryogenic temperatures for the polymers of interest (Epoxies 3501-6, 5208, 934, and 1962), an alternative method is needed to estimate the critical ERR $G_{I c}$. Therefore, in this work, the critical ERR values are adjusted so that the DDM damage model predicts the same crack density as available experimental crack density data $\lambda^{d}$ by minimizing the following error $D$ function

$$
D=\frac{1}{N} \sqrt{\sum_{j=1}^{N}\left(\lambda_{j}-\lambda_{j}^{d}\right)}
$$

where $N$ is number of data points at a given temperature, and $\lambda_{j}$ is the crack density data for specimen $j$.

In order to study the temperature dependence of $G_{I c}$, two different approximations are used in this section. In the first approximation, the critical ERR $G_{I c}$ is assumed to be temperature dependent and thus adjusted by minimizing the error $D(4.3)$ at each temperature for which experimental data is available. Then, a polynomial is adjusted though the values of $G_{I c}$ obtained at those temperatures. To adjust a polynomial over the temperature range of interest $[-200,180 \mathrm{C}]$, only data for material systems that have been tested at several temperatures over that range can be used. For example, data that only exists for a single temperature cannot be used to characterize temperaturedependence.

In the second approximation, the critical ERR $G_{I c}$ is assumed to be temperature independent (constant). Therefore, all data $\lambda^{d}$ can be used regardless of whether data from a given source is available for just one or for multiple temperatures. Furthermore, if it can be shown that a constant (temperature independent) value of critical ERR $G_{I c}$ is sufficiently accurate to predict crack density vs. temperature, then the amount of testing needed to characterize a material system can be reduced with respect to $G_{I c}$ being a function of temperature. The specific details of both procedures are described next:

Assuming temperature-dependent $G_{I c}$, the critical ERR $G_{I c}$ is adjusted by minimizing the error $D$ (4.3) between the predicted crack density $\lambda$ and experimental crack density data $\lambda^{d}$ for each temperature for which experimental data is available $[18,20$, 186,188]. Prediction of crack density is performed using the Discrete Damage Mechanics (DDM) formulation (Ch. 8 in [200]). Implementations of this formulation for commercial FEA software exist in the form of plugins for Abaqus [193] and ANSYS [194]. Abaqus is 


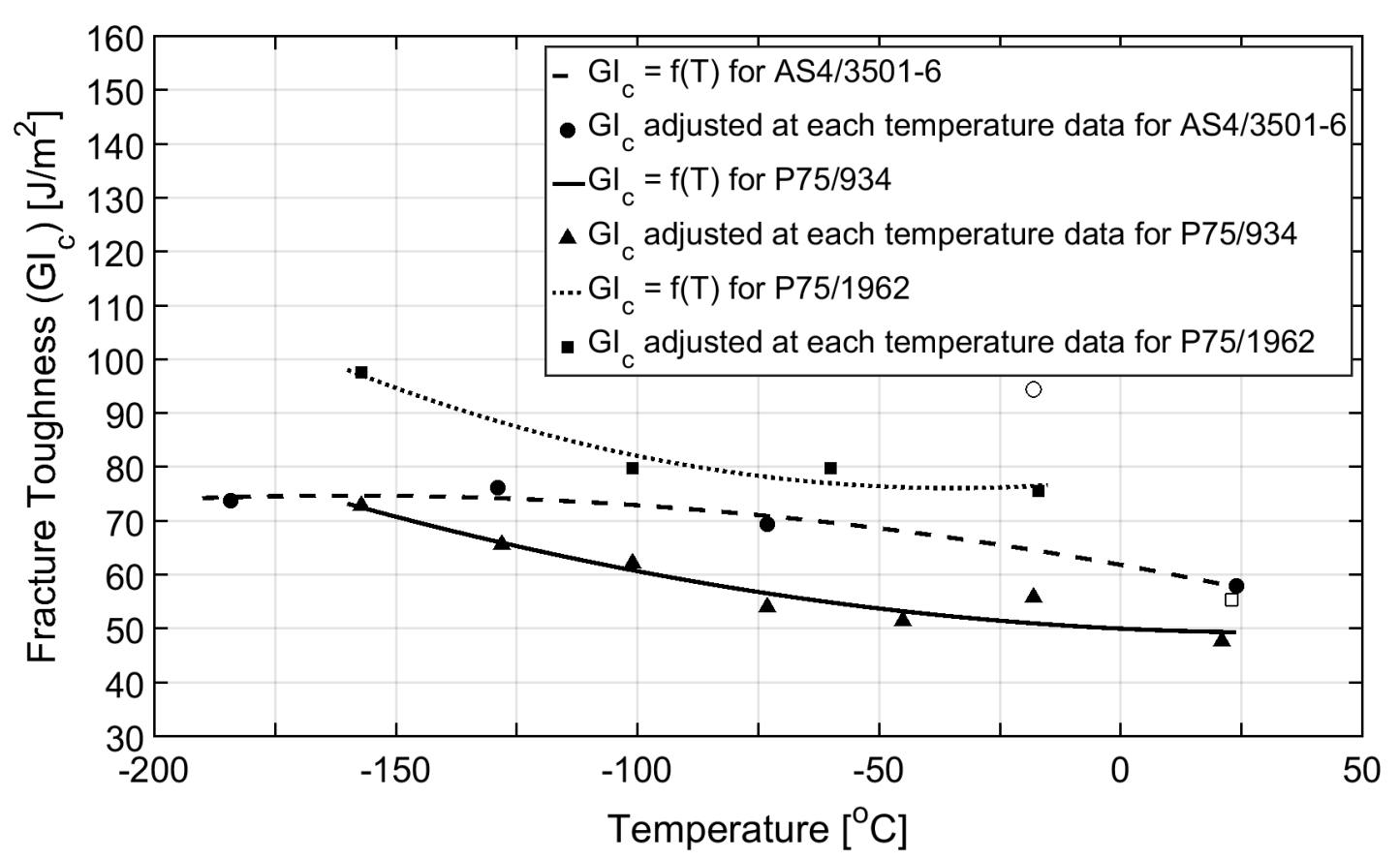

Figure 4.1: $G_{I c}$ vs. temperature for P75/934 $(V f=0.65)$, P75/1962 ( $\left.V f=0.52\right)$, and AS4/3501-6 $\left(V_{f}=0.64\right)$. Two outliers data, at $-18 C$ for $A S 4 / 3501-6$ and at $-21 C$ for P75/1962, not used.

used in this study.

$G_{I c}$ values for material systems P75/934 $\left[0_{2} / 90_{2}\right]_{S}, \mathrm{P} 75 / 1962\left[0_{2} / 45_{2} / 90_{2} /-45_{2}\right]_{S}$, and AS4/3501-6 $\left[0_{4} / 45_{4} / 90_{4} /-45_{4}\right]_{S}$, obtained at discrete temperatures are then fitted with a quadratic polynomial as shown in Figure 4.1. Material system T300/5208 $\left[0_{2} / 90_{2}\right]_{S}$ undergoes negligible cracking until -156 C [20], so it is not included in the figure.

Some outlier data points are reported for AS4/3501-6 and P75/1962 around -18 C and $23 \mathrm{C}$, respectively. These outliers correspond to data with a large scatter so they were not used in this study. For all cases, a quadratic interpolation was found to accurately represent $G_{I c}(T)$ as a function of temperature. According to Figure 4.1, $G_{I c}$ at low temperature increases between $26.91 \%$ and $39.46 \%$ with respect RT.

Assuming temperature-independent $G_{I c}$, the critical ERR $G_{I c}$ is adjusted using all sets of experimental crack density $\lambda^{d}$ available. A comparison between the predicted crack density and experimental data subjected to monotonic cooling is shown in Figures 4.24.3 using both constant $G_{I c}$ and temperature-dependent $G_{I c}(T)$. Only constant $G_{I c}$ was used for T300/5208 due to lack of experimental data at low temperatures for this material system. However, temperature dependence of the constituents is taken into account for all cases. Prediction of crack density vs. temperature are quite good with either constant $G_{I c}$ or temperature-dependent $G_{I c}$ for all materials systems except P75/934 and P75/1962, for which accuracy at cryogenic temperature improves when temperature-dependent $G_{I c}$ 


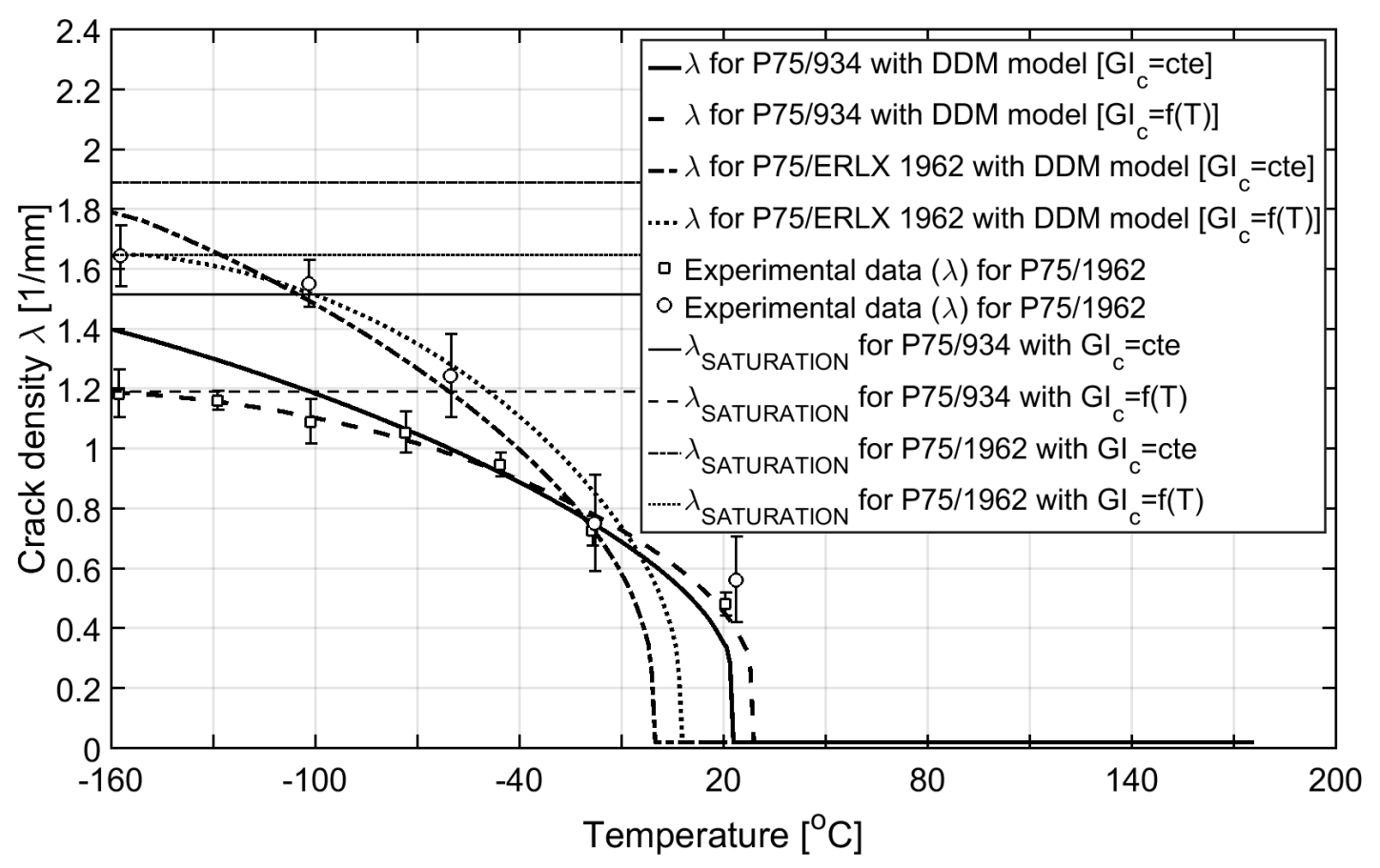

Figure 4.2: Crack density data vs. Temperature using middle $90_{2}^{\circ}$ lamina for laminate $\left[0_{2} / 90_{2}\right]_{s}$ Pr5/934 and interior lamina $90_{2}^{o}$ for laminate $\left[0_{2} / 45_{2} / 90_{2} /-45_{2}\right]_{s}$ Pr5/1962.

is used.

For P75/934, P75/1962, and T300/934, the experimental data was measured at the edge of the specimens $[18,20,186,188]$. For AS4/3501-6, experimental data was measured at both the edge and the interior of specimens [18]. $G_{I c}$ is calculated from interior data for AS4/3501-6 but edge data is also shown in Figure 4.3 for comparison. Interior data was used, if available, because the agreement between predicted and experimental crack density is better, and X-ray data (used to detect interior cracks) is usually more reliable that optical edge inspection.

Saturation crack density is here defined as the asymptotic value of crack density as temperature approaches extremely low temperature. Saturation crack density is shown in Figures 4.2-4.3 to illustrate the expected behavior at lower temperatures than those for which experimental data is available. It can be seen in Figures 4.2-4.3 that the rate of damage with cooling, defined as

$$
\dot{\lambda}=-\frac{\partial \lambda}{\partial T}
$$

decreases over the whole temperature range. That is, less and less damage is induced by the same decrement of temperature $\Delta T$ as the temperature decreases. This is due to four factors. First, damage accumulation reduces the transverse stiffness $E_{2}$, thus larger strains can occur at the same stress level in the cracking lamina. Second, $E_{2}$ increases with cooling (Figure 3.6), which works opposite to the previous effect. Third, 


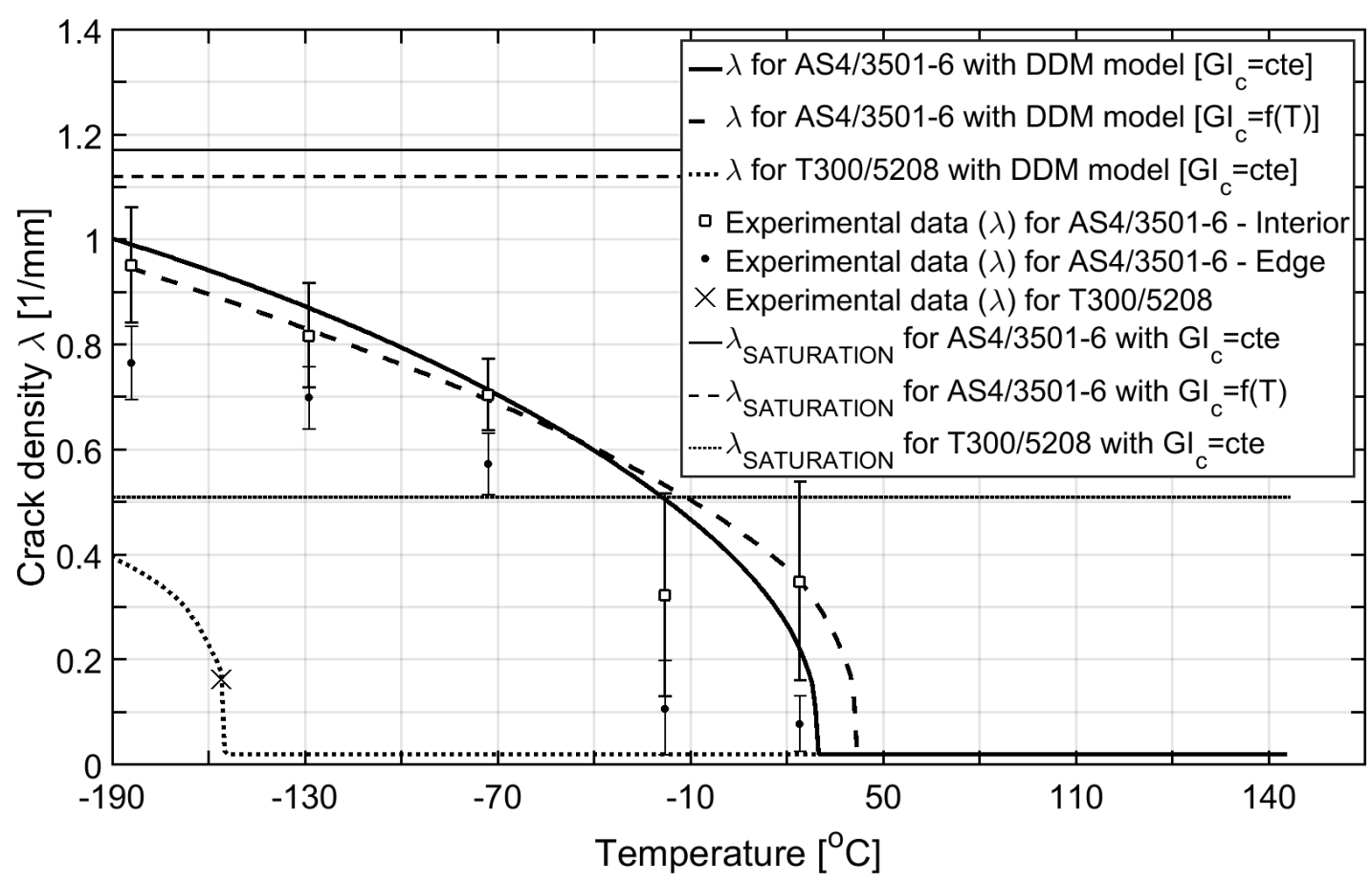

Figure 4.3: Crack density data vs. Temperature using interior $90_{4}^{\circ}$ lamina for laminate $\left[0_{4} / 45_{4} / 90_{4} /-45_{4}\right]_{s}$ AS4/3501-6 and middle $90_{2}^{\circ}$ lamina for $\left[0_{2} / 90_{2}\right]_{s}$ T300/5208.

the transverse CTE $\alpha_{2}$ decreases with cooling (see Figures 3.14-3.15), so larger reductions of temperature can be tolerated with the same increment of damage. When these three effects are combined, it seems that constant $G_{I c}$ is the answer, with the reduction of damage rate at lower temperature being captured quite well by the model, although some differences can be observed at cryogenic temperature for P75/934 and P75/1962. The fourth factor is the increase of critical ERR with cooling depicted in Figure 4.1, where it can be seen that the temperature dependence of $G_{I c}$ is more pronounced for P75/934 and P75/1962. For the other materials systems, the temperature dependence is less pronounced and thus predictions of crack density with constant $G_{I c}$ are better. Note that an increase of $G_{I c}$ with cooling (Figure 4.2) further reduces the rate of damage at lower temperatures. Both temperature-dependent and independent properties of critical ERR for the material system studied are shown in Table 4.1.

The proposed methodology can be used to calculate the temperature dependence of the critical ERR for both thermoset and thermoplastic polymer composites only if they behave as brittle materials at cryogenic temperatures as explained in Chapter 3. Furthermore, the temperature dependent properties must be calculated with precise knowledge prior to study the temperature dependence of the critical ERR $G_{I c}$. 
Tables 4.1: Critical ERR $G_{I c}\left[J / m^{2}\right]$, temperature [ $\left.{ }^{\circ} \mathrm{C}\right]$, see eq. (3.2).

\begin{tabular}{cccccc}
\hline & \multicolumn{4}{c}{ Temp. dependent } & Temp. \\
\cline { 2 - 5 } Material & $P_{a}$ & $P_{b}$ & $P_{c}$ & Range $\left[{ }^{\circ} C\right]$ & independent \\
\hline P75/934 & 50.0561 & $-4.300610^{-2}$ & $6.374910^{-4}$ & {$[-160,20]$} & 53.4050 \\
P75/1962 & 77.8054 & $9.621110^{-2}$ & $1.394810^{-3}$ & {$[-160,-15]$} & 84.4808 \\
AS4/3501-6 & 61.9052 & $-1.609710^{-1}$ & $-5.041210^{-4}$ & {$[-190,20]$} & 68.0664 \\
T300/5208 & - & - & - & - & 226.0000 \\
\hline
\end{tabular}

\subsection{Results}

Predictions using thermo-mechanical DDM [31,35] are compared with experimental crack density data to validate the proposed methodology assuming temperature-independent $G_{I c}$ and including the temperature-dependent properties of the constituents. Further, laminate CTEs are predicted as function of crack density and temperature during the monotonic cooling. The analyzed material systems are: P75/934 [185,186,285], P75/1962 [10,188], AS4/3501-6 [18], and T300/5208 [8,20].

\subsubsection{P75/934 Carbon-Epoxy}

\subsubsection{Crack Density}

Predictions of three P75/934 laminates with same angle-ply laminas but different laminate stacking sequence $(\mathrm{LSS})[0 / \pm 45 / 90]_{s},[0 / 90 / \pm 45]_{s}$, and $[0 / 45 / 90 /-45]_{s}$ are presented in Figure 4.4-4.6. Experimental crack data for all three laminates is collected from [185,188]. It can be seen that predicted values compare reasonable well with experimental data. Such data points are generally obtained at three or four temperatures from several specimens. Crack density $\lambda$ was determined by edge inspection, or interior data X-ray inspection. Sometimes experimental data shows large variability. The thickness of the $90^{\circ}$ lamina has a clear impact on damage onset but not on saturation crack density. When the $90^{\circ}$ lamina is located at the middle plane, namely a thicker $\left(90_{2}\right)$ lamina, it cracks earlier during cooling $\left(-23 \mathrm{C}\right.$ for $[0 / \pm 45 / \mathbf{9 0}]_{s}$ in Figure 4.4) and crack density grows with slow rate. When $90^{\circ}$ lamina is located inside the laminate, namely a thinner (90) lamina, it cracks later $\left(-107 \mathrm{C}\right.$ and $-96 \mathrm{C}$, for $[0 / \mathbf{9 0} / \pm 45]_{s}$ and $[0 / 45 / \mathbf{9 0} /-45]_{s}$, in Figures 4.5 and 4.6, respectively) and crack density grows faster. This is because for DDM, laminas located at the middle plane or surface crack have higher thickness. That is, the middle ply has double thickness while the local 2D displacements field $\left(u_{i}\right)$ of top/bottom surface ply behaves as a lamina with double thickness because it is on a free surface (Figure 5.6.b).

The same pattern is repeated with respect $0^{\circ}, 45^{\circ}$, and $-45^{\circ}$ laminas as shown in Figures 4.4-4.6. The evolution and onset of cracks in $0^{\circ}$ lamina is predicted to be almost 
the same $(-43,-21$, and $-24 \mathrm{C})$ for all LSS as shown in Figures 4.4-4.6, because $0^{\circ}$ laminas are always on the surface. Note that no experimental data collected for $0^{\circ}$ lamina was found in the literature despite of large damage cracking to which they are subjected. Single surface laminas $\left(0^{\circ}\right)$ behave as center $[90]_{s}$ pairs.

In the same way, damage initiation and cracks evolution in $+45^{\circ}$ lamina is predicted to be the same $(-102,-107$, and $-95 \mathrm{C})$ for all LSS as shown in Figures 4.4-4.6, because the $+45^{\circ}$ laminas are interior (not surface and not center pairs). Experimental data compare well with predicted crack density when interior data is collected $\left([0 / \mathbf{4 5} / 90 /-45]_{s}\right.$ laminate) as shown in Figure 4.7. A better agreement between experimental data and predicted cracks can significantly be appreciated when cracks are measured using an interior X-ray inspection for inner $\pm 45^{\circ}$ laminas. Based on [18], this fact is attributed to differences in the transverse stresses at the edge between $90^{\circ}$ and $\pm 45^{\circ}$ laminas, according to a free-edge analysis focused on membrane loads using a 3D model [286]. Looking the free-edge of $90^{\circ}$ lamina group and using a laminate global coordinate system (c.s), the transverse stresses $\left(\sigma_{x}\right)$ for $\pm 45^{\circ}$ laminas group at the edge corresponds to shear stresses, which are virtually zero due to the free thermal expansion of the laminate. The transverse stress to the fiber for $\pm 45^{\circ}$ laminas group rise to the expected values (CLPT) only when they are far enough from the edge $(\approx 2 \mathrm{~mm})$. Therefore, fewer cracks are generated at the edge and larger error between edge data and predicted cracks can be seen as shown in Figures 4.4-4.6. This effect is attenuated for thinner laminas.

The $-45^{\circ}$ lamina repeats the same pattern as $90^{\circ}$ lamina as is shown in Figures 4.4-4.6. When lamina at $-45^{\circ}$ is located at the middle plane, namely a thicker $\left(-45_{2}\right)$ lamina, it cracks earlier during the cooling $\left(-41 \mathrm{C}\right.$ and $-30 \mathrm{C}$ for $[0 / 90 / \pm \mathbf{4 5}]_{s}$ and $[0 / 45 / 90 / \mathbf{- 4 5}]_{s}$ in Figures 4.5 and 4.6, respectively) and the crack density grows with slow rate. When $-45^{\circ}$ lamina is located inside the laminate, namely a thinner $(-45)$ lamina, it cracks later $\left(-102 \mathrm{C}\right.$ for $[0 / \pm \mathbf{4 5} / 90]_{s}$ in Figure 4.4$)$ but crack density grows with greater rate than the $-45^{\circ}$ lamina located at the middle. However, the crack density for both case converges to similar values between 1.7 to 1.9 [cracks $/ \mathrm{mm}]$ with independence of the LSS.

The crack density evolution within the laminate for $[0 / 90 / \pm 45]_{s},[0 / \pm 45 / 90]_{s}$, and $[0 / 45 / 90 /-45]_{s}$ P75/934 is shown in Figure 4.9. Unlike a laminate mechanically loaded, transverse damage is present in all laminas of the composite, while in a quasi-isotropic and symmetric laminate loaded mechanically in x-direction, only the $90^{\circ}$ lamina group crack. Due to the free thermal expansion of the laminate, a reference c.s does not exist and transverse cracking is also generated in $0^{\circ}, 45^{\circ}$, and $-45^{\circ}$. Since there are not constraints, all laminas are subjected to the same thermal expansion (cooling). Although no experimental data for $0^{\circ}$ laminas are reported in $[18,188]$, an X-ray image from [10] with transverse cracking in $0^{\circ}$ and $90^{\circ}$ laminas is shown in Figure 4.8. Due to free thermal 


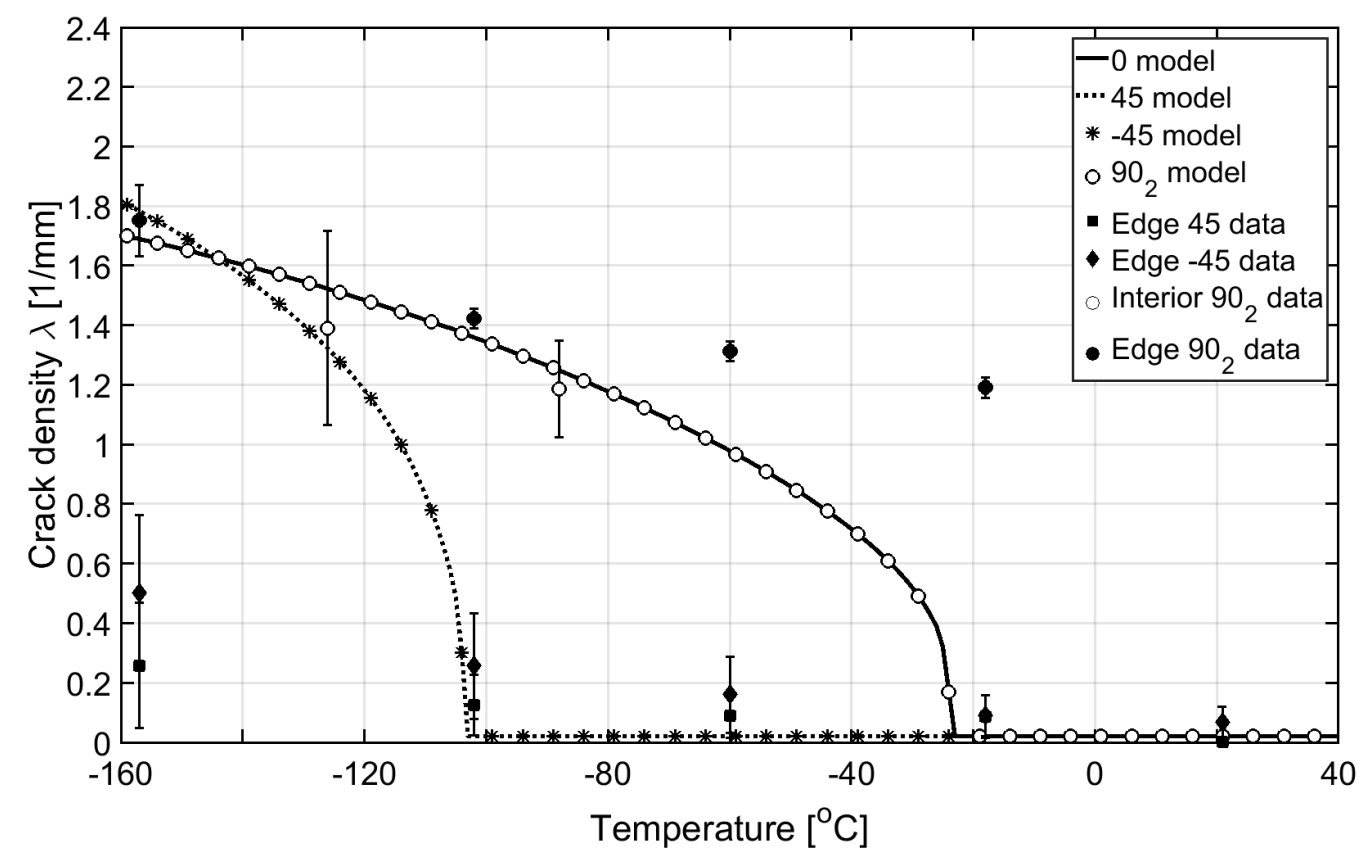

Figure 4.4: Crack density prediction vs. temperature for monotonic cooling of $[0 / \pm 45 / 90]_{s}$ P75/934.

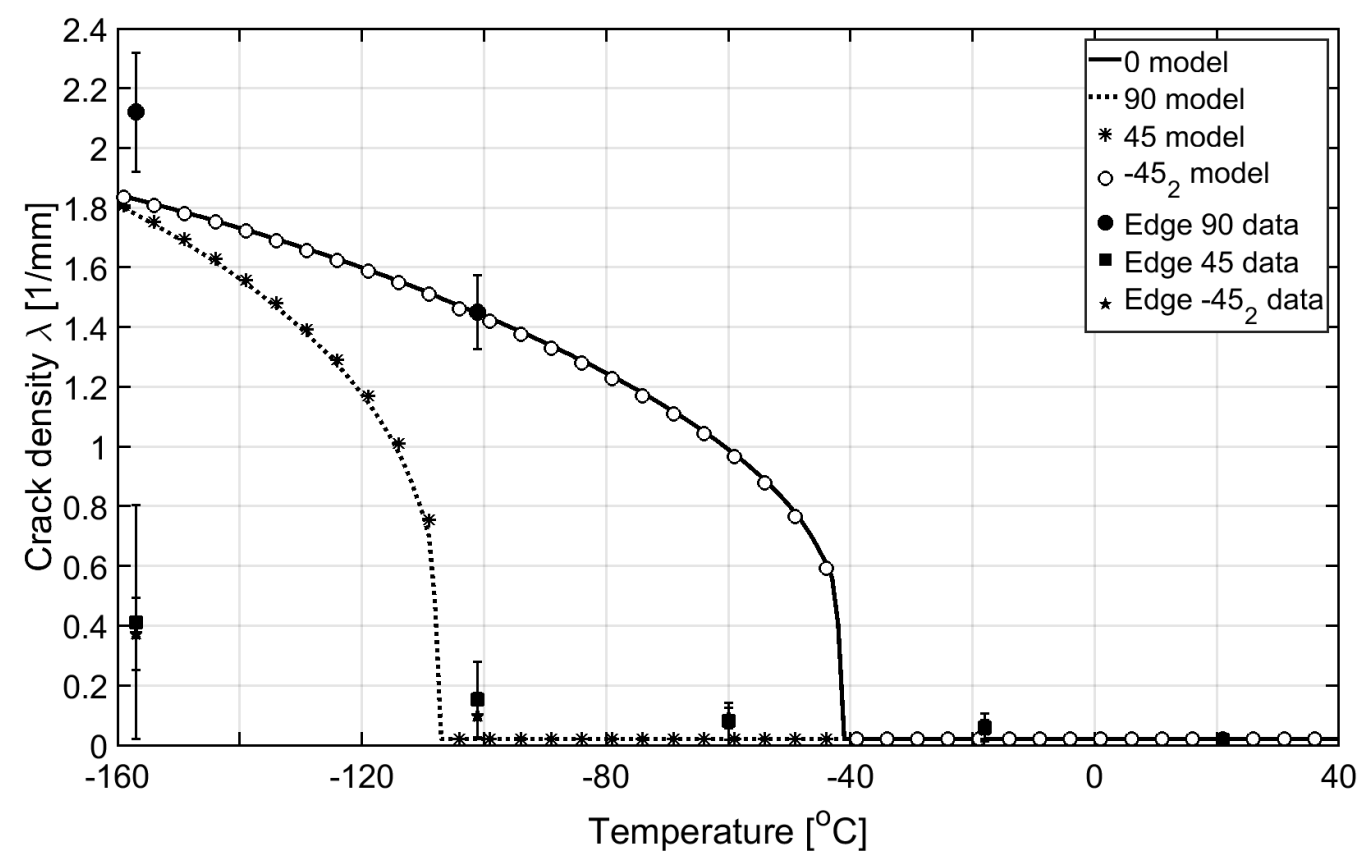

Figure 4.5: Crack density prediction vs. temperature for monotonic cooling of $[0 / 90 / \pm 45]_{s}$ P75/934. 


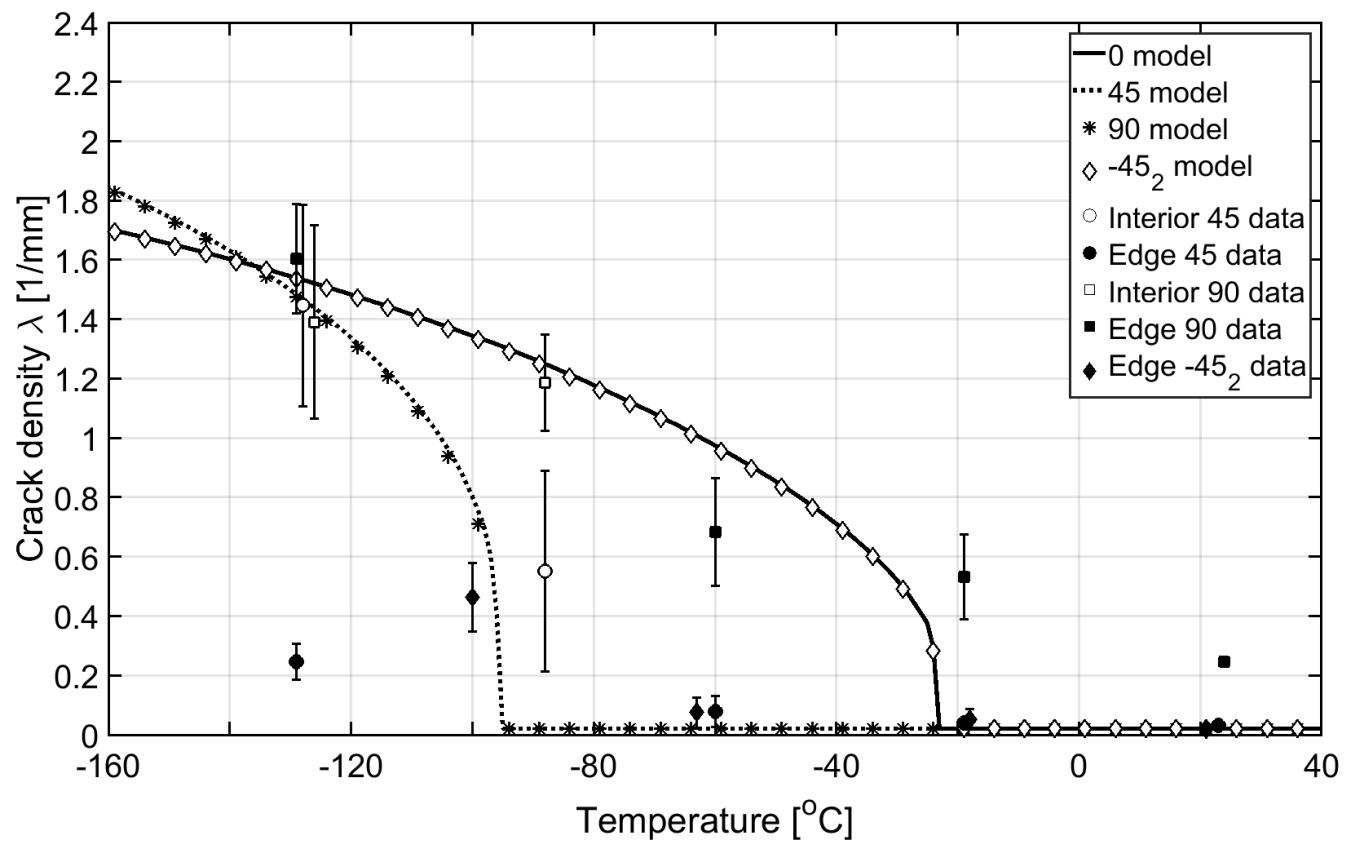

Figure 4.6: Crack density prediction vs. temperature for monotonic cooling of $[0 / 45 / 90 /-$ $45]_{s}$ P75/934.

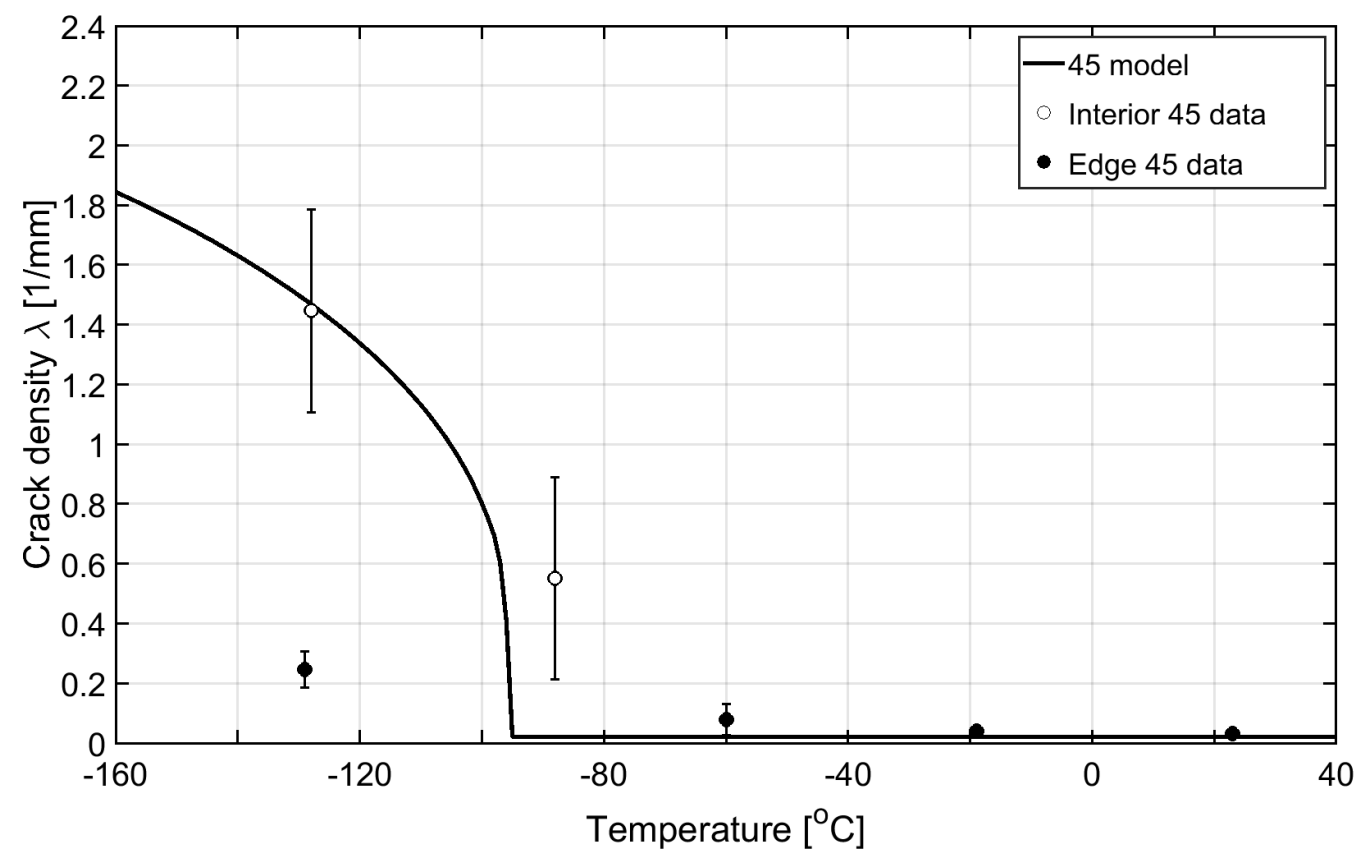

Figure 4.7: A comparison between crack density prediction vs. interior and edge $-45^{\circ}$ lamina data during cooling in $[0 / 45 / 90 /-45]_{s}$ P75/934. 


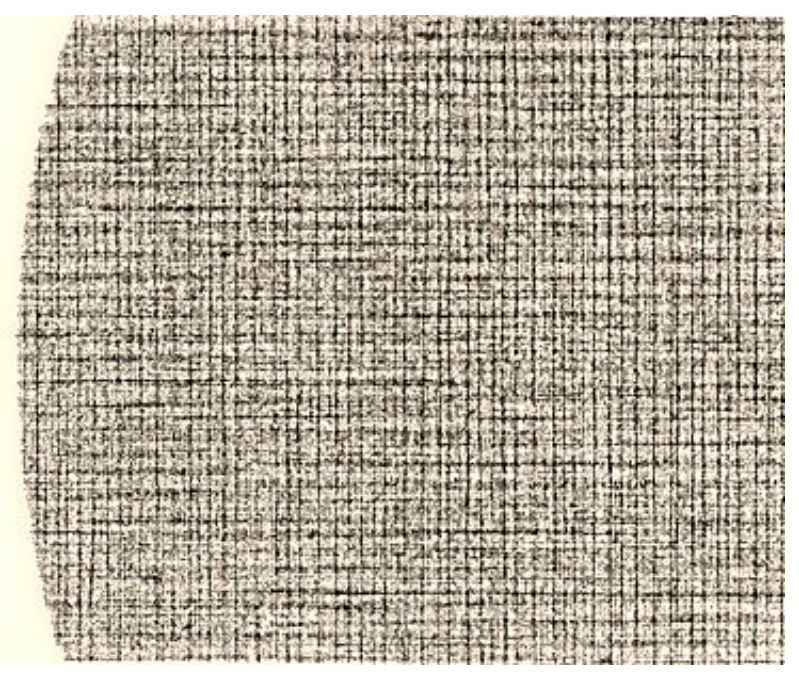

Figure 4.8: X-Ray photograph for laminate $\left[0_{2} / 90_{2}\right]_{s}$ P75/ERL1962 subjected to $\pm 250 \mathrm{~F}$ and 3500 cycles [10]. Lines represent cracks for $0^{\circ}$ and $90^{\circ}$ laminas.

expansion of laminate and symmetry, both $0^{\circ} \& 90^{\circ}$ undergo transverse cracking.

In Figure 4.9 , the $0^{\circ}$ and $-45^{\circ}$ laminas $\left([\mathbf{0} / 90 / \pm \mathbf{4 5}]_{s}\right)$ start cracking earlier $(-41$ C). Since they have double thickness, higher strain energy is released upon cracking. On the other hand, the $90^{\circ}$ and $45^{\circ}$ laminas start cracking later $(-106 \mathrm{C})$ once the stress have been redistributed into the laminate and the ERR reaches the critical $G_{I c}$. Since $90^{\circ}$ and $45^{\circ}$ laminas have the same thickness (one ply), they start cracking at the same temperature with higher cracking rate. The crack density for all laminas at $-160 \mathrm{C}$ converge to similar values around $1.8[\mathrm{cracks} / \mathrm{mm}]$.

The same pattern can be seen in Figure 4.9 for $[0 / \pm 45 / 90]_{s}$ and $[0 / 45 / 90 /-45]_{s}$ P75/934. In laminate $[0 / \pm 45 / 90]_{s}$, the $0^{\circ}$ and $90^{\circ}$ laminas start cracking earlier with lower cracking rate while $\pm 45^{\circ}$ laminas start cracking later with a higher cracking rate. The crack density for all laminas at $-160 \mathrm{C}$ converge to similar values around 1.8 [cracks $/ \mathrm{mm}]$. In laminate $[0 / 45 / 90 /-45]_{s}$, the crack density evolution is similar to $[0 / 90 / \pm 45]_{s}$ laminate because both have the same orientations at the surface and at the mid-plane with $0^{\circ}$ and $-45^{\circ}$, respectively. From a design point of view, $[0 / 90 / \pm 45]_{s}$ laminate is the best stacking sequence because it is crack free until $-41 \mathrm{C}$. The other two configurations start cracking earlier at $-23 \mathrm{C}$.

Crack density evolution for $\left[0_{2} / \pm 30\right]_{s}$ P75/934 is shown in Figure 4.10. Experimental data [188] compare well with predicted crack density except for $0^{\circ}$ lamina, which no data was found. It can be observed that transverse cracking only occurs for $0^{\circ}$ and $-30_{2}^{\circ}$ laminas group at $T_{2}=-73 \mathrm{C}$ and $T_{1}=-114 \mathrm{C}$ for $0^{\circ}$ and $-30_{2}^{\circ}$ lamina, respectively. No matrix cracking is predicted for $30^{\circ}$ lamina shown with solid dot in Figure 4.10. In order to explain the non-cracking in $30^{\circ}$ ply, the damage activation function $(\mathrm{g})$ values for each lamina is shown in Figure 4.11 (left). When a lamina is non-cracking, this means that 

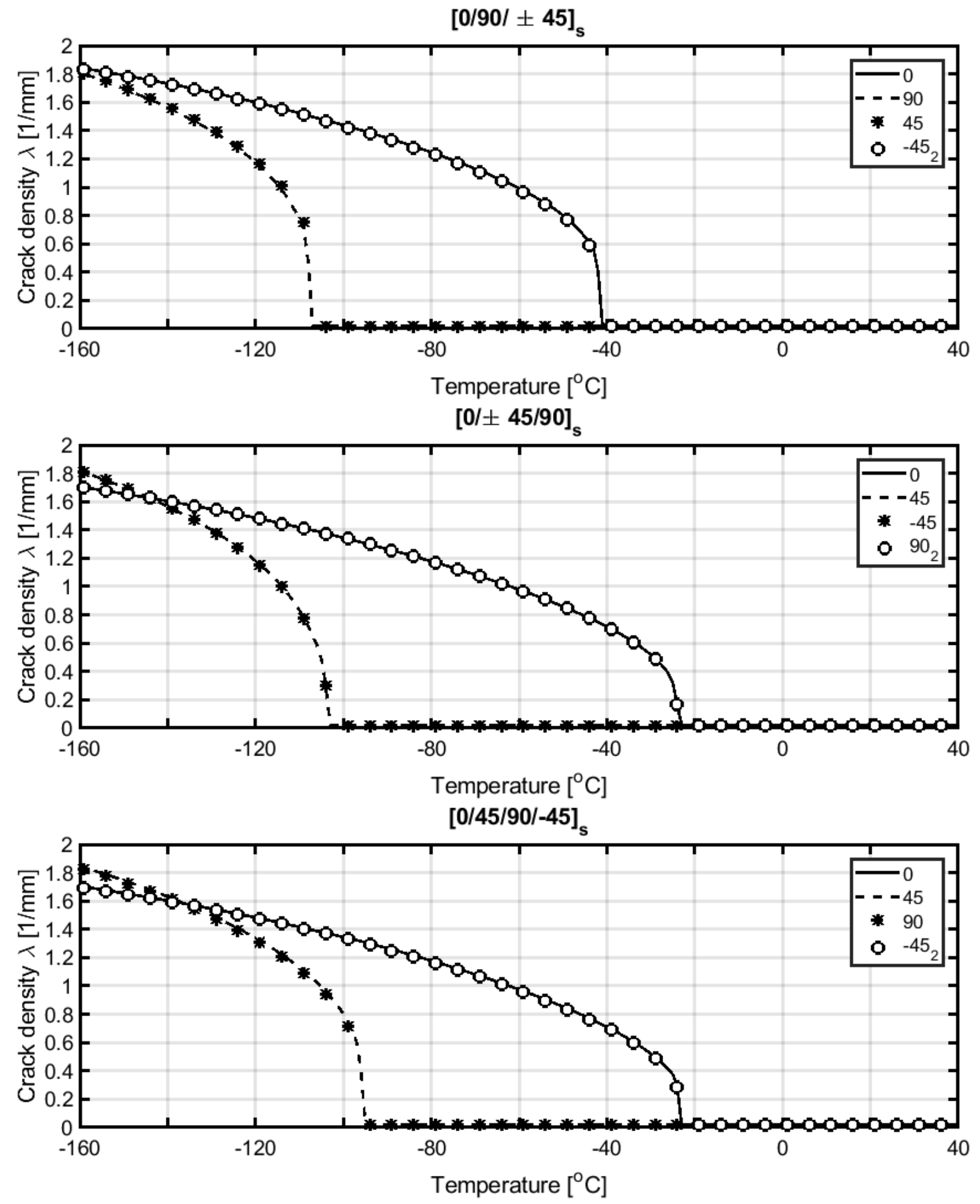

Figure 4.9: Crack density predictions vs. temperature for monotonic cooling of $[0 / 90 / \pm 45]_{s}$, $[0 / \pm 45 / 90]_{s}$, and $[0 / 45 / 90 /-45]_{s}$ P75/934. 


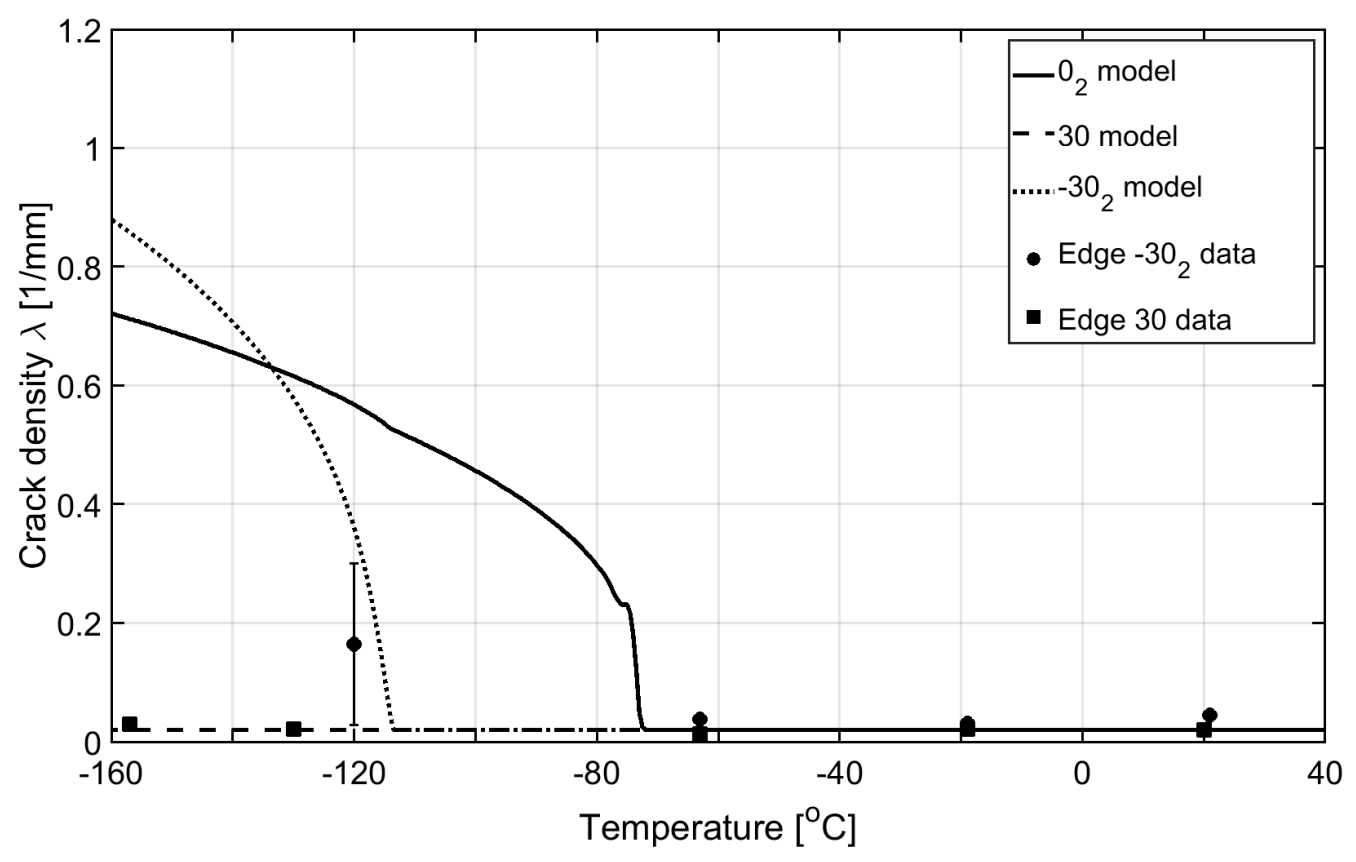

Figure 4.10: Crack density predictions vs. temperature for monotonic cooling of $\left[0_{2} / \pm 30\right]_{s}$ Pr5/934.

the ERR is not large enough to reach the onset of cracking as shown for $30^{\circ}$ lamina (see Figure 4.11). The g function value need to reach a value equal to 1.0 so that the ERR is big enough to generate a new crack into the lamina. The $g$ function increases smoothly during the cooling until $0^{\circ}$ lamina begins cracking. When this happens, a higher ERR rate for balanced $\pm 30^{\circ}$ laminas occurs but not enough for $30^{\circ}$ lamina whose $\mathrm{g}$ value is 0.936, and no cracks are generated.

Since $\left[0_{2} / \pm 30\right]_{s}$ laminate is balanced, laminate shear strains $\epsilon_{x y}$ are zero until transverse cracking begins due to free thermal expansion. Only when $-30_{2}$ lamina cracks, small shear strains in comparison with longitudinal and transverse laminate strains $\left(\epsilon_{x}\right.$ and $\epsilon_{y}$ ) appear on the laminate as shown in Figure 4.13 later. Note that cracks in $0_{2}$ lamina do not affect to laminate shear strains $\epsilon_{x y}$. However, differences between $\epsilon_{x}$ and $\epsilon_{y}$ induce shear strains at lamina level for \pm 30 laminas group unlike quasi-isotropic laminates. Therefore, $G_{I I}$ is released during monotonic cooling as shown in Figure 4.11 (right). Although a mixed-mode I and II can occurs, no cracks density data was found for +30 lamina and good agreement between predictions and crack data is obtained using only $G_{I c}$. Therefore, a large $G_{I I c}$ was assumed in order to avoid an overestimate in laminate crack density, which it already match well using only $G_{I c}$. Furthermore, $G_{I}$ is around 5-6 times the obtained $G_{I I}$ during all cooling and thus, crack opening in mode I is assumed to be dominant in laminate composites subjected to cooling. 


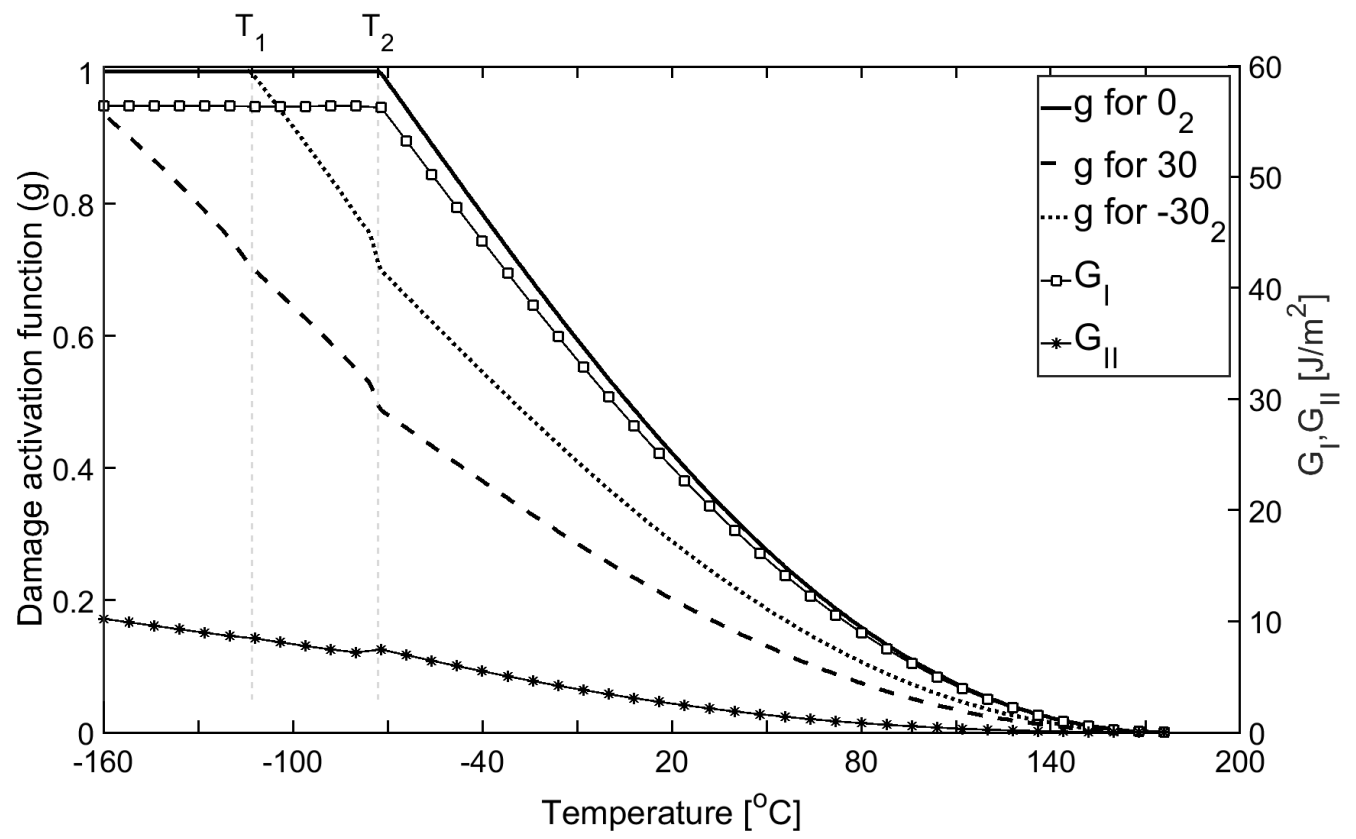

Figure 4.11: Evolution of damage activation function $g$ and $E R R s G_{I}, G_{I I}$ during monotonic cooling for $\left[0_{2} / \pm 30\right]_{s}$ Pr5/934 laminate.

\subsubsection{CTE}

The tangent laminate thermal expansions $(C T E)$ in global coordinates of the laminate $\left(\alpha_{x}, \alpha_{y}\right.$, and $\left.\alpha_{x y}\right)$ are shown in Figure 4.12 for $\left[0_{2} / 90_{2}\right]_{s}$ P75/934 in the range [-160,SFT] C. This cross-ply laminate behave as isotropic in $x$ and $y$ direction with no coupling between shear and extension. When laminate is free to expand, both $0^{\circ}$ and $90^{\circ}$ laminas undergo the same displacement and thus, they crack at the same time. Analyzing Figure 4.12 , it can be seen that laminate $C T E$ in $x$ and $y$ direction remain equal during the monotonic cooling. The laminate CTEs $\left(\alpha_{x}\right.$ and $\left.\alpha_{y}\right)$ remain equal in the range [121,Tg] $\mathrm{C}$ (right side) where the temperature-dependent data are assumed constant equal to the last data point available (see Figure 4.12). Then, the laminate CTEs $\left(\alpha_{x}\right.$ and $\left.\alpha_{y}\right)$ vary according to the laminate temperature-dependent data shown in Table 4.2. Once the cross-ply laminate start cracking at $23 \mathrm{C}$, both laminate CTEs drop fast as function of both, crack density and temperature-dependent properties. When laminate start cracking, the crack density in both laminas grow fast and the slope of the laminate CTEs vary strongly until the cracking rate decreases. Then, they remain virtually constant up to $-160 \mathrm{C}$. Note that laminate CTE remain negative upon cracking, which it is highly influenced by the volume fraction $\left(V_{f}=0.62\right)$. Since both laminas keep the same transverse cracking rate, there are not shear deformations in the laminate and thus, the laminate CTE $\alpha_{x y}$ remain equal to zero during the whole monotonic cooling.

The tangent laminate thermal expansion $(C T E)$ in global coordinates of the laminate $\left(\alpha_{x}, \alpha_{y}\right.$, and $\left.\alpha_{x y}\right)$ are shown in Figure 4.13 for $\left[0_{2} / \pm 30\right]_{s}$ P75/934 between $[-160, \mathrm{SFT}]$ 
C. As it was shown in Figure 4.11, only $0_{2}^{\circ}$ and $-30_{2}^{\circ}$ laminas undergo enough ERR to generate cracks and thus, both laminate CTEs $\left(\alpha_{x}\right.$ and $\left.\alpha_{y}\right)$ are not equal. As explained previously, the laminate CTES $\left(\alpha_{x}\right.$ and $\left.\alpha_{y}\right)$ remain equal in the range [121,SFT] $\mathrm{C}$ (right side) where the temperature-dependent data are assumed constant equal to the last data point available (see Figure 4.13). Then, the laminate CTEs $\left(\alpha_{x}\right.$ and $\left.\alpha_{y}\right)$ vary according to the laminate temperature-dependent data until the onset of cracking in $0^{\circ}$ lamina at $-73 \mathrm{C}$. Once the $-30_{2}^{o}$ lamina starts cracking, the laminate CTEs $\left(\alpha_{x}\right.$ and $\left.\alpha_{y}\right)$ vary as function of both, crack density and temperature-dependent properties. In this case, only the cracks of $-30_{2}^{\circ}$ laminas starting at $-114 \mathrm{C}$ has a small impact into longitudinal laminate CTE $\left(\alpha_{x}\right)$ because cracks in $0_{2}^{o}$ ply does not affect. However, the transversal laminate CTE $\left(\alpha_{y}\right)$ is affected for both, $0_{2}^{o}$ and $-30_{2}^{o}$ plies cracks and thus, the $\alpha_{y}$ slope varies earlier $(-73 \mathrm{C})$ and faster than $\alpha_{x}$. Since laminate is balanced, laminate CTE $\left(\alpha_{x y}\right)$ remain zero until $-30_{2}^{\circ}$ ply starts cracking. However a minimal damage impact can be appreciated in Figure 4.13, and $\alpha_{x y}$ is really small.

Similar to Figure 4.13, the tangent laminate $C T E \mathrm{~s}$ in global coordinates $\left(\alpha_{x}, \alpha_{y}\right.$, and $\left.\alpha_{x y}\right)$ are shown in Figure 4.14 for $[0 / \pm 45 / 90]_{s}$ P75/934 between $[-160, \mathrm{SFT}] \mathrm{C}$. In this case, three different phases can be appreciated. First, longitudinal and transverse laminate $C T E \mathrm{~s}\left(\alpha_{x}\right.$ and $\left.\alpha_{y}\right)$ remain equal in the range [121,SFT] $\mathrm{C}$ (right side on Figure 4.14) where the temperature-dependent data do not exist. Once the laminate temperature dependence starts at $121 \mathrm{C}$, laminate CTEs $\left(\alpha_{x}\right.$ and $\left.\alpha_{y}\right)$ vary according to Table 4.2. Second, both $\alpha_{x}$ and $\alpha_{y}$ vary as function of temperature and the crack density prediction. Once surface and middle laminas $\left(0^{\circ}\right.$ and $\left.90_{2}^{\circ}\right)$ start cracking, both slope of $\alpha_{x}$ and $\alpha_{y}$ decrease fast due to the onset of damage. In this case, only $\alpha_{x}$ is affected by $90_{2}^{\circ}$ lamina cracks while $\alpha_{y}$ is affected by $0^{\circ}$ lamina cracks. As it can be seen in Figure 4.9, the crack density for both laminas is predicted to be the same so crack differences do not exist and both laminate $C T E$ s drop with same rate too. Third, the inside laminas $\left( \pm 45^{\circ}\right)$ start cracking at same temperature with equal cracking rate (see Figure 4.9), so both laminate $C T E \mathrm{~s}\left(\alpha_{x}\right.$ and $\left.\alpha_{y}\right)$ drop parallel up to $-160 \mathrm{C}$. Since all laminas crack in pairs keeping the same cracking rate, $0^{\circ}$ and $90^{\circ}$ laminas first followed by angle-plies \pm 45 (see Figure 4.9), no laminate shear strain $\epsilon_{x y}$ appear and thus, the laminate CTE $\alpha_{x y}$ remain equal to zero during the whole monotonic cooling.

The tangent laminate CTEs in global coordinates $\left(\alpha_{x}, \alpha_{y}\right.$, and $\left.\alpha_{x y}\right)$ are shown in Figure 4.15 for $\left[0_{n} / 90_{n}\right]_{s}$ P75/934 between $[-160$, SFT] C, where $n$ is the number of plies for each $\theta$ lamina. Ply thickness is kept constant with $0.127 \mathrm{~mm}$, and laminate thickness is $t=0.127 n \mathrm{~mm}$. Experimental data including tangent thermal expansions are not available for laminate $C T E$, so comparisons are not possible. The influence of ply number $n$ for each $\theta$-lamina can be appreciated in Figure 4.15, where both laminate 


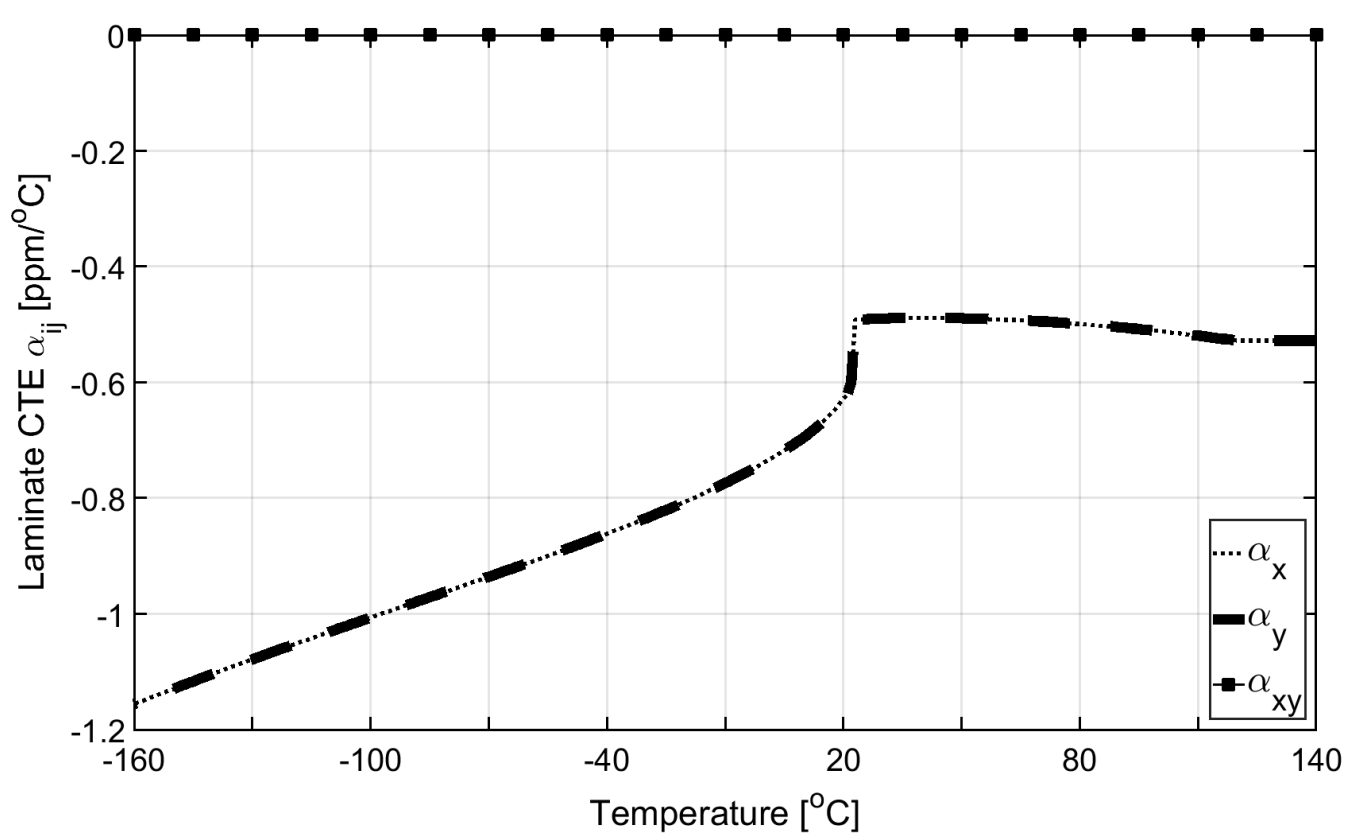

Figure 4.12: Tangent laminate CTE vs. temperature for monotonic cooling of $\left[0_{2} / 90_{2}\right]_{s}$ P75/934.

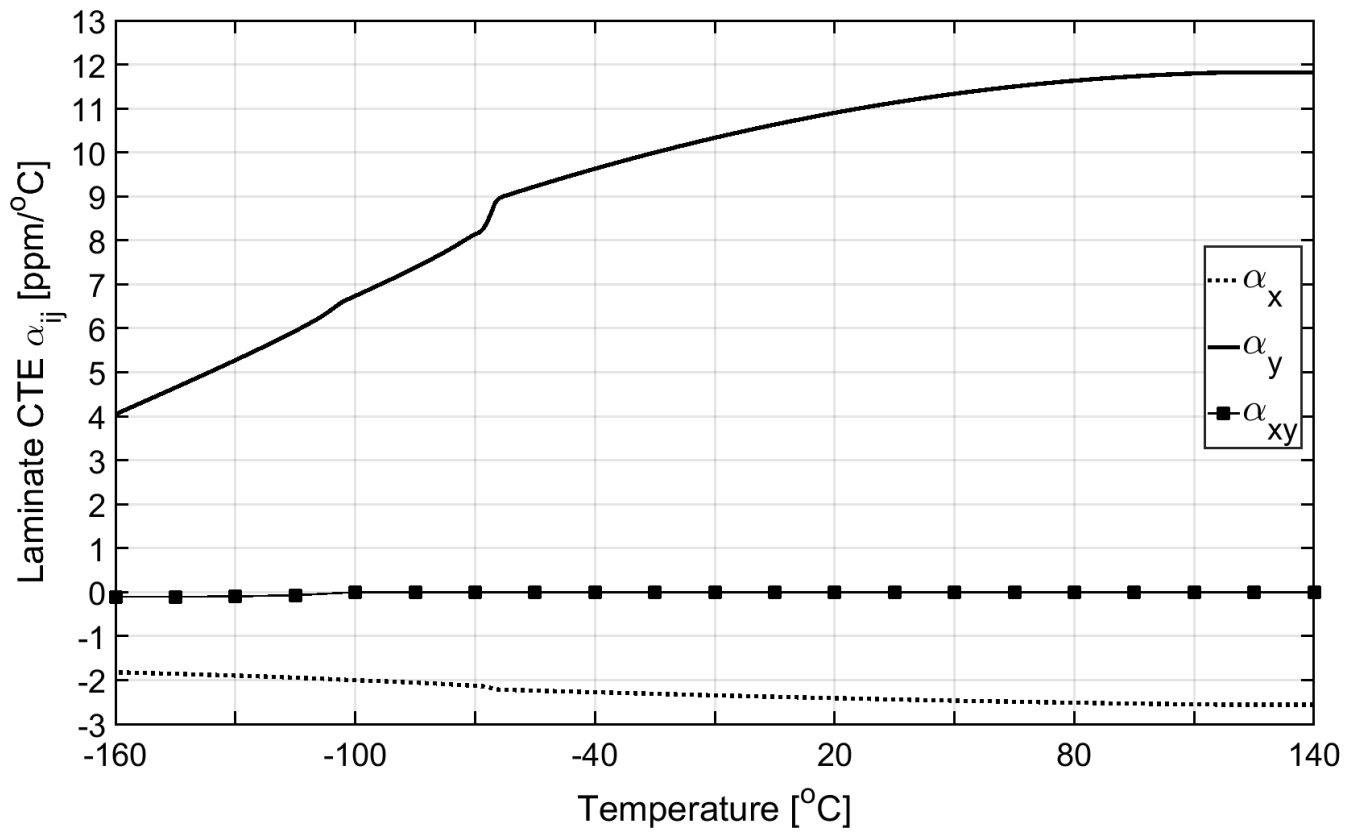

Figure 4.13: Tangent laminate CTE vs. temperature for monotonic cooling of $\left[0_{2} / \pm 30\right]_{s}$ P75/934. 


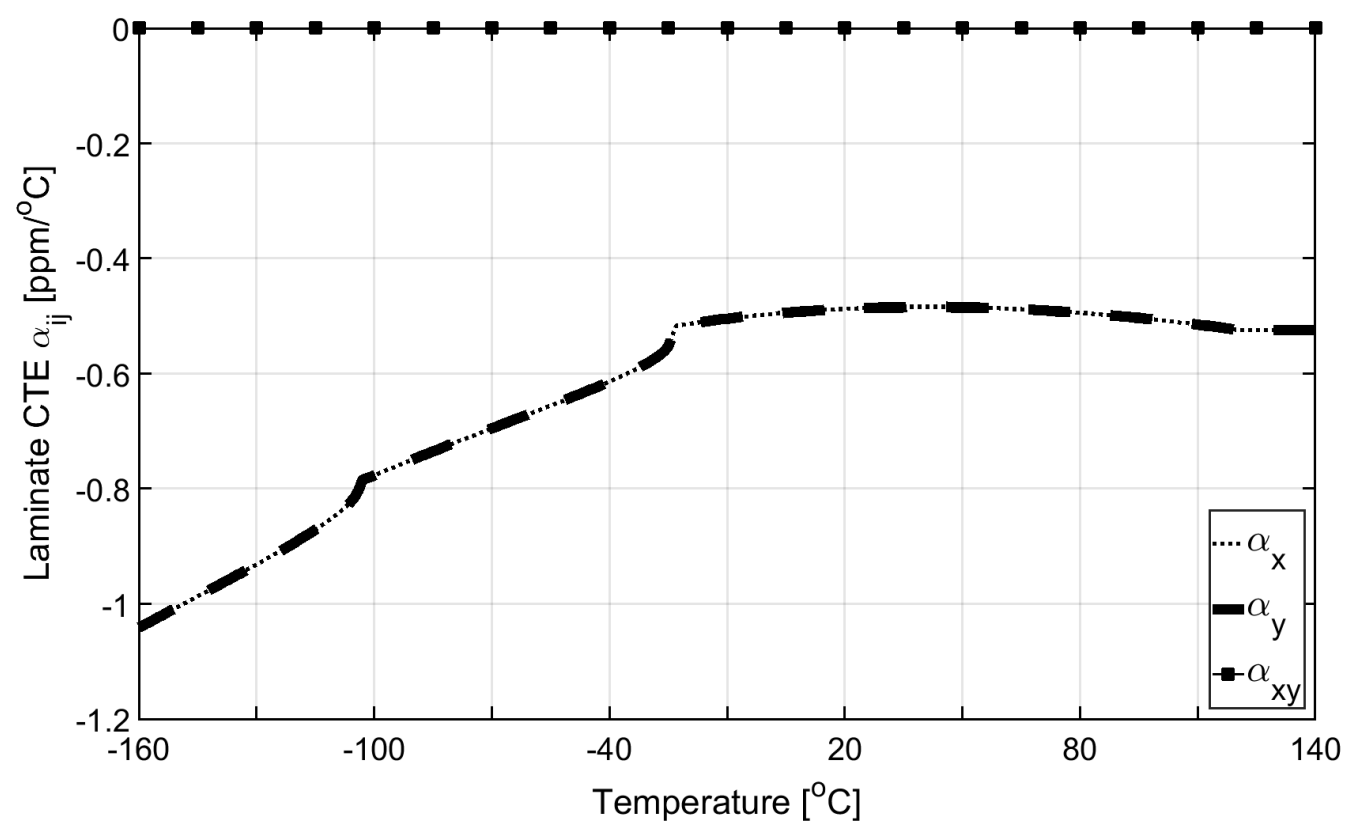

Figure 4.14: Tangent laminate CTE vs. temperature for monotonic cooling of $[0 / \pm 45 / 90]_{s}$ P75/934.

CTEs $\left(\alpha_{x}\right.$ and $\left.\alpha_{y}\right)$ drop once the onset of cracking begins at $-40,23$ and $67 \mathrm{C}$ according to $n=1,2,4$ respectively. As a general rule, whether lamina thickness decreases, the lamina is more resistant to cracking and the fall of $\alpha_{x}$ and $\alpha_{y}$ are delayed to a lower temperature. Furthermore, with fewer number of plies $n$, crack growth rate trends to be higher and thus, laminate $C T E$ s drop faster as well.

The temperature-dependent properties of P75/934 are shown in Table 4.2. Such properties were calculated using periodic microstructure model [35, PMM, App. 2] and Levin's work [269] as mentioned in Section 3.1 and 3.2. The temperature-dependent properties for P75 carbon fiber are taken from Table 3.1, while 934 epoxy properties are taken from Table 3.3. All the elastic $\left(E_{1}, E_{2}, G_{12}, \nu_{12}, \nu_{23}\right)$ and thermal $\left(\alpha_{1}, \alpha_{2}\right)$ laminate properties are adjusted by a quadratic polynomial (3.2). 

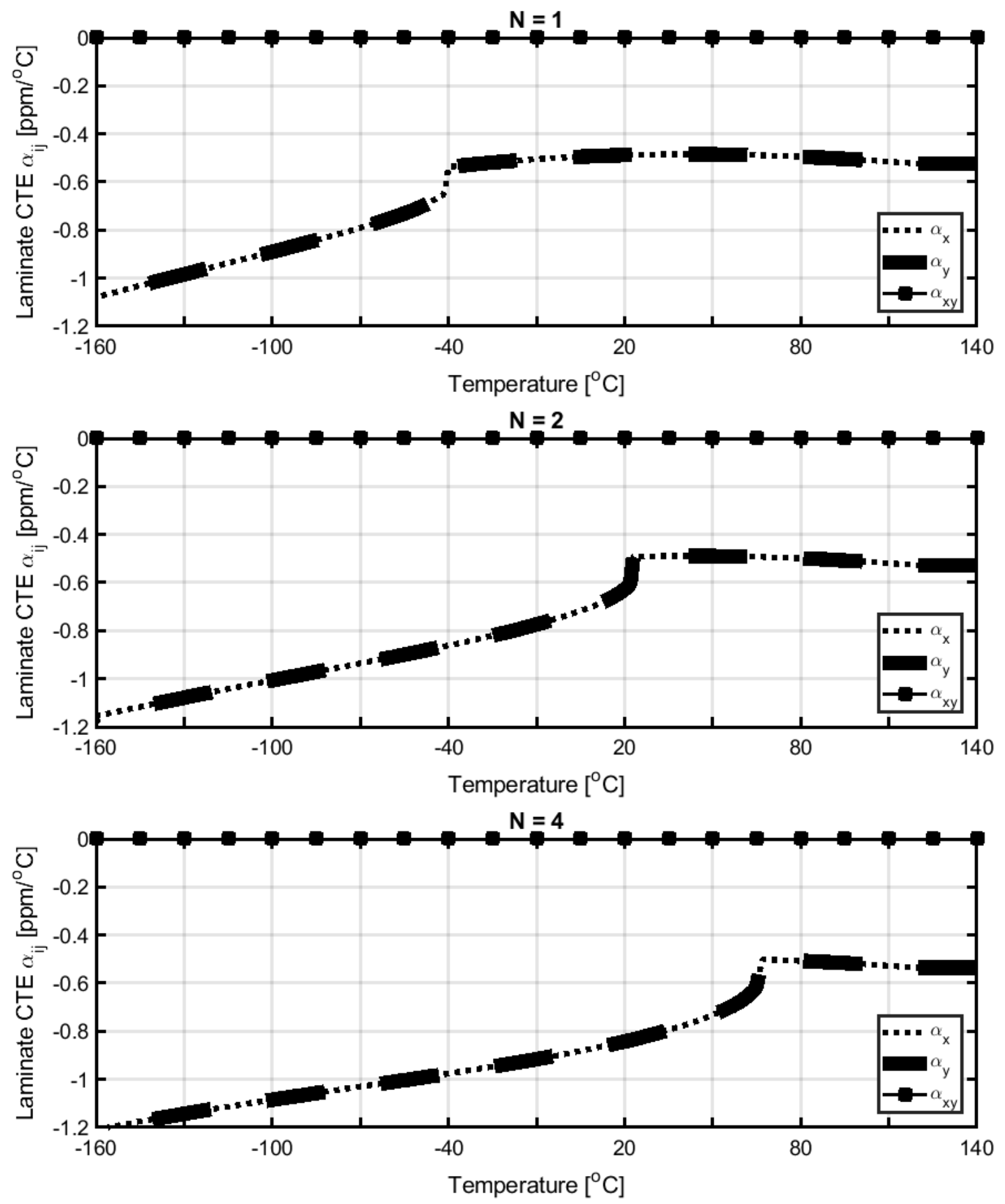

Figure 4.15: Evolution of tangent laminate CTE as function of number of sub-laminas vs. temperature for $\left[0_{n} / 90_{n}\right]_{s}$ P75/934, with $n=1,2,4$. 
Tables 4.2: Quadratic temperature-dependent properties of P75/934 $\left(V_{f}=0.62[10,13-15]\right)$ between $[-156,121] C$.

\begin{tabular}{ccccc}
\hline \multicolumn{5}{c}{ Temperature dependent properties } \\
\hline Property & $P_{a}$ & $P_{b}$ & $P_{c}$ & Reference \\
\hline$E_{1}[\mathrm{MPa}]$ & 337824.6634 & -5.9298 & $8.7283 \mathrm{E}-03$ & Sec. 3.1 \\
$E_{2}[\mathrm{MPa}]$ & 7163.2212 & -9.7344 & $-2.5418 \mathrm{E}-03$ & Sec. 3.1 \\
$G_{12}[\mathrm{MPa}]$ & 4983.8657 & -9.2594 & $4.3316 \mathrm{E}-03$ & Sec. 3.1 \\
$\nu_{12}$ & 0.3021 & $-5.4423 \mathrm{E}-05$ & $-2.7713 \mathrm{E}-07$ & Sec. 3.1 \\
$\nu_{23}$ & 0.5258 & $-6.5145 \mathrm{E}-05$ & $-9.2675 \mathrm{E}-07$ & Sec. 3.1 \\
$\alpha_{1}\left[10^{-6} / \mathrm{C}\right]$ & -1.2322 & $1.8294 \mathrm{E}-04$ & $-3.1555 \mathrm{E}-06$ & Sec. 3.2 and 3.3 \\
$\alpha_{2}\left[10^{-6} / \mathrm{C}\right]$ & 26.4532 & $6.1956 \mathrm{E}-02$ & $-1.1729 \mathrm{E}-04$ & Sec. 3.2 and 3.3 \\
$G_{I c}\left[\mathrm{~J} / \mathrm{m}^{2}\right]$ & 53.4050 & - & - & Sec. 4.1 \\
\hline & Temperature independent properties & {$[186]$} \\
\hline$F_{1 t}[\mathrm{MPa}]$ & \multicolumn{5}{c}{$[130]$} \\
$F_{1 c}[\mathrm{MPa}]$ & 620 & {$[10]$} \\
$F_{2 c}[\mathrm{MPa}]$ & \multicolumn{5}{c}{140} & {$[10]$} \\
SFT $[\mathrm{C}]$ & \multicolumn{5}{c}{0.127} \\
Ply thickness $[\mathrm{mm}]$
\end{tabular}

\subsubsection{P75/ERL1962 Carbon-Epoxy}

\subsubsection{Crack Density}

A comparison between experimental data and crack density predictions for $\left[0_{2} / 45_{2} / 90_{2} /-\right.$ $\left.45_{2}\right]_{s}$ P75/1962 is shown in Figure 4.16-4.17. Experimental crack data [16,188] compare well with predicted crack density for $90_{2}^{\circ}$ lamina (see Figure 4.17), while large error can be appreciated for $\pm 45^{\circ}$ laminas (see Figure 4.16). This fact is highly influenced by the cracks measurement through the edge inspection. As commented in Section 4.2.1, fewer cracks are counted at the edge due to the free-edge stress generated in $\pm 45^{\circ}$ laminas and thus, higher discrepancies can be observed when no interior data is available. All laminas are subjected to crack initiation and evolution as it was shown in Figure 4.9 using the same LSS but different material system (P75/934). The surface and middle laminas $\left(0_{2}^{o}\right.$ and $\left.-45_{4}^{o}\right)$ start cracking at the same temperature $(40 \mathrm{C})$ keeping equal cracking rate. The same pattern can be seen for inside laminas $\left(45_{2}^{\circ}\right.$ and $\left.90_{2}^{\circ}\right)$ at $0 \mathrm{C}$. Since balanced angle-plies \pm 45 start cracking at different temperature, laminate shear strain $\epsilon_{x y}$ is generated during the monotonic cooling, which will affect to the $\alpha_{x y}$ of the laminate.

\subsubsection{CTE}

The tangent laminate CTEs in global coordinates $\left(\alpha_{x}, \alpha_{y}\right.$, and $\left.\alpha_{x y}\right)$ are shown in Figure 4.18 for $\left[0_{2} / 45_{2} / 90_{2} /-45_{2}\right]_{s}$ P75/1962 between $[-160, S F T]$ C. First, longitudinal and 


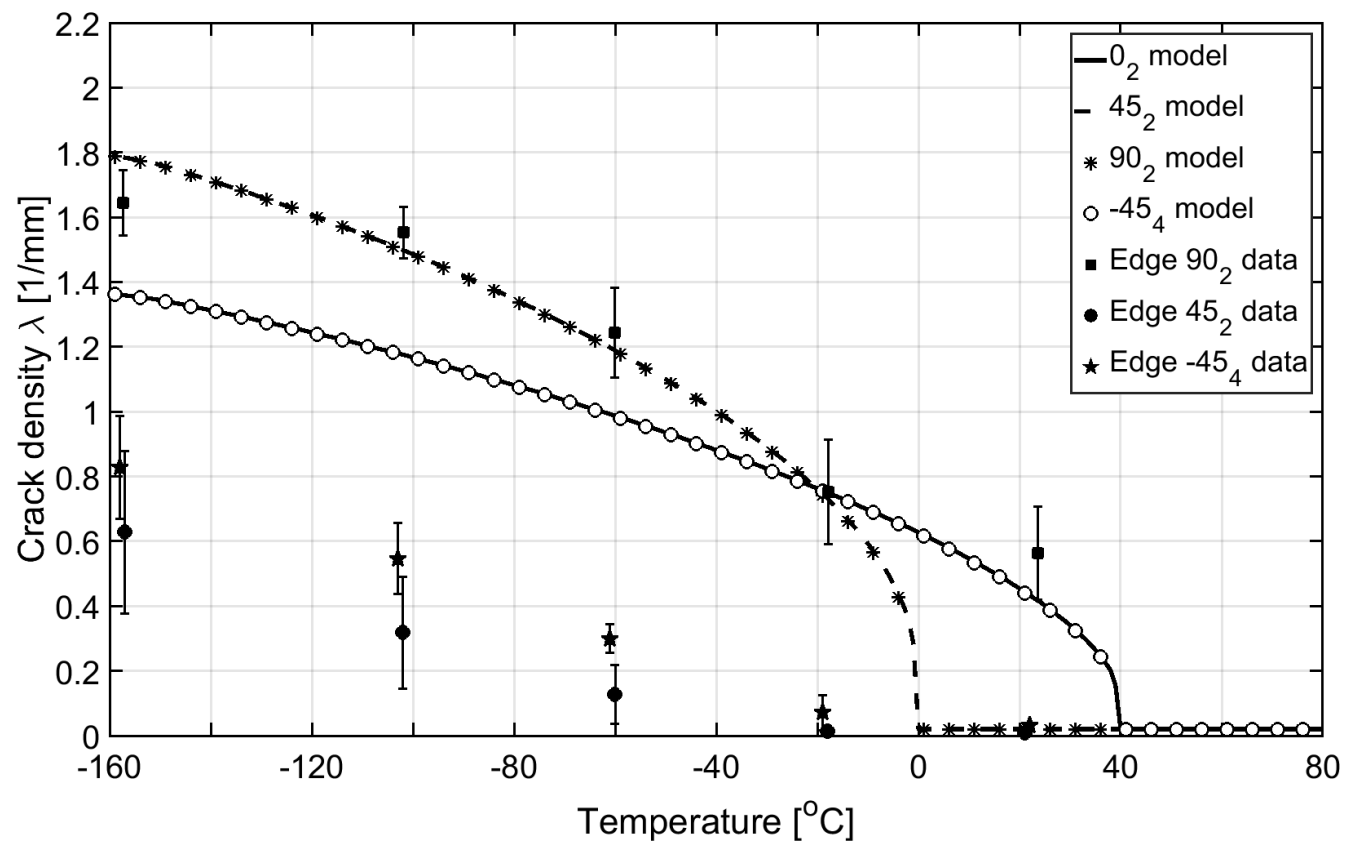

Figure 4.16: Crack density predictions vs. temperature for monotonic cooling of $\left[0_{2} / 45_{2} / 90_{2} /-45_{2}\right]_{s}$ P75/ERLX1962.

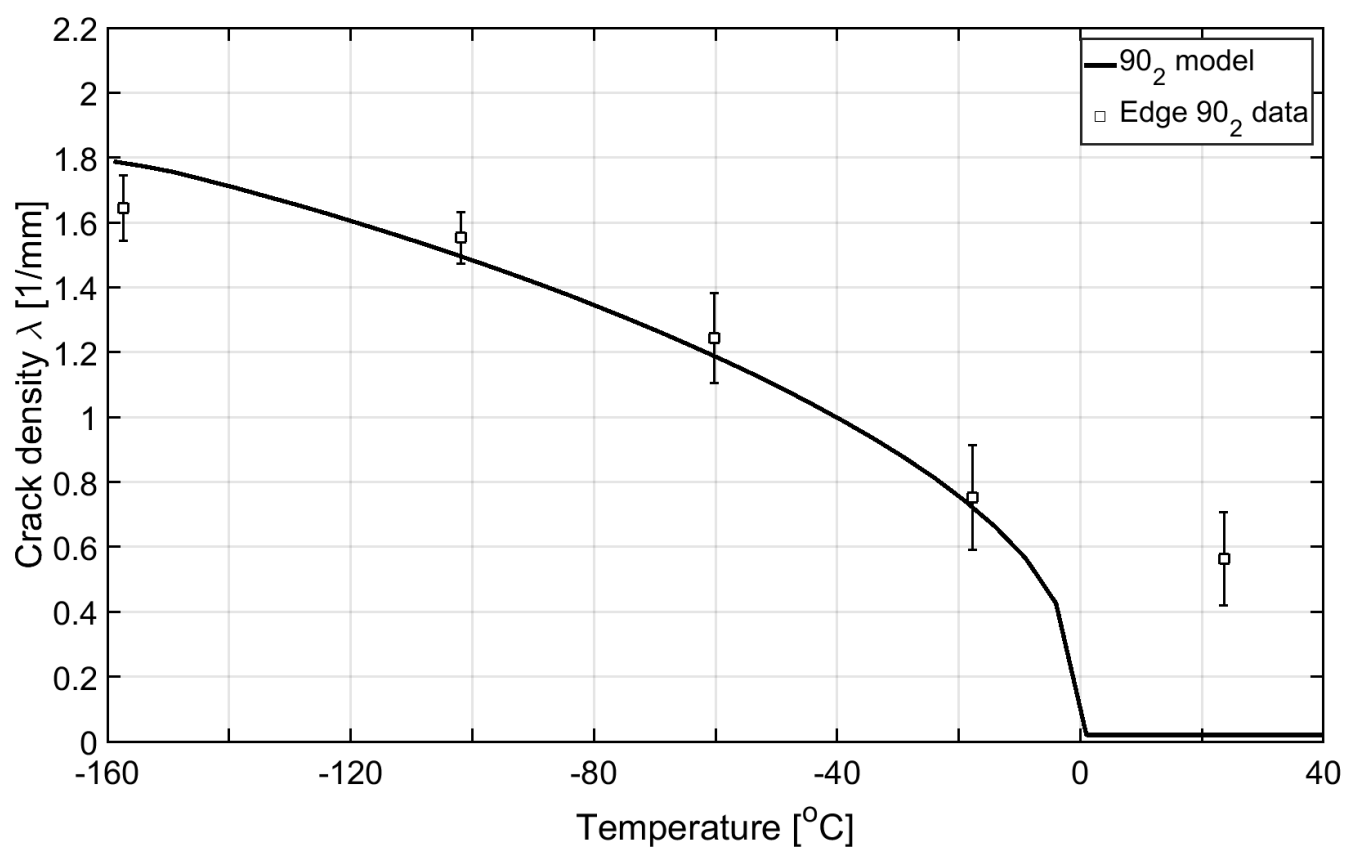

Figure 4.17: A comparison between crack density prediction and experimental data for $90_{2}$ vs. temperature in $\left[0_{2} / 45_{2} / 90_{2} /-45_{2}\right]_{s}$ P75/ERLX1962 laminate. 


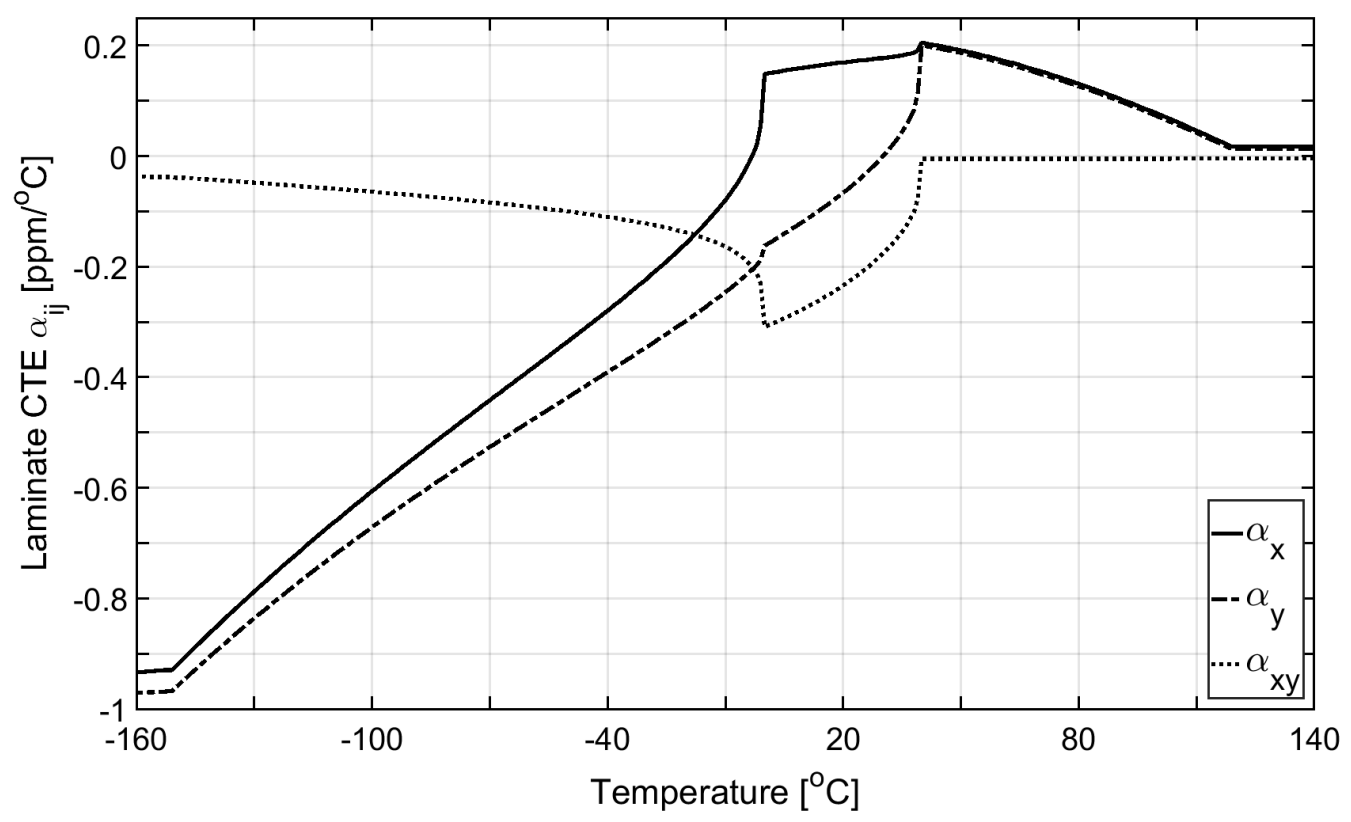

Figure 4.18: Tangent laminate CTE vs. temperature for monotonic cooling of $\left[0_{2} / 45_{2} / 90_{2} /-\right.$ $\left.45_{2}\right]_{s}$ P75/1962.

transverse laminate $C T E \mathrm{~s}\left(\alpha_{x}\right.$ and $\left.\alpha_{y}\right)$ remain equal in the range [121, Tg] $\mathrm{C}$ (right side on Figure 4.18) where the temperature-dependent data do not exist. In the temperature range $[40,121] \mathrm{C}$, both $\alpha_{x}$ and $\alpha_{y}$ vary according to the laminate temperature-dependent data shown in Table 4.3. Once laminas start cracking, the laminate CTEs change as function of temperature and crack density. In this laminate, the balanced \pm 45 laminas crack at different temperature because they are located at middle/inside respectively and thus, there exist shear strains and slope rate differences between $\alpha_{x}$ and $\alpha_{y}$ as it can be observed in Figure 4.18. The longitudinal CTE $\alpha_{x}$ hardly varies with the $0_{2}^{o}$ and $-45_{4}$ lamina cracks. However, $\alpha_{x}$ is mostly influenced by the inside $45_{2}$ and $90_{2}$ laminas cracks once they start cracking. Conversely, the transverse CTE $\alpha_{y}$ is highly influenced by cracks of both laminas and thus, $\alpha_{y}$ drops with a higher cracking rate during cooling. When inside laminas also start to crack, both laminate CTEs keep dropping with higher rates up to $-160 \mathrm{C}$. Close to $-160 \mathrm{C}$, both laminate $C T E$ s become constant because the predicted crack density almost reaches crack saturation. Since shear strains are produced by different crack density between \pm 45 laminas, the laminate CTE $\alpha_{x y}$ value becomes nonzero from $40 \mathrm{C}$, where only $-45_{4}^{\circ}$ lamina start cracking. As the crack density differences between balanced \pm 45 laminas become less, $\alpha_{x y}$ approaches zero at $-160 \mathrm{C}$.

The temperature-dependent properties of P75/1962 are shown in Table 4.3 calculated using periodic microstructure model [35, PMM, App. 2] and Levin's work [269] as mentioned in Section 3.1 and 3.2. The temperature-dependent properties for P75 carbon fiber are taken from Table 3.1, while 1962 epoxy properties are taken from Table 3.4. The elastic $\left(E_{1}, E_{2}, G_{12}, \nu_{12}, \nu_{23}\right)$ and thermal $\left(\alpha_{1}, \alpha_{2}\right)$ laminate properties are adjusted by 
a quadratic polynomial (3.2).

Tables 4.3: Quadratic temperature-dependent properties of P75/1962 $\left(V_{f}=0.52[10,16,17]\right)$ between $[-156,121] C$.

\begin{tabular}{|c|c|c|c|c|}
\hline \multicolumn{5}{|c|}{ Temperature dependent properties } \\
\hline Property & $P_{a}$ & $P_{b}$ & $P_{c}$ & Reference \\
\hline$E_{1}[\mathrm{MPa}]$ & 271270.586 & -8.1099 & $1.1894 \mathrm{E}-02$ & Sec. 3.1 \\
\hline$E_{2}[\mathrm{MPa}]$ & 6554.2638 & -11.6689 & 4.9329E-04 & Sec. 3.1 \\
\hline$G_{12}[\mathrm{MPa}]$ & 3998.0213 & -8.8436 & $6.1187 \mathrm{E}-03$ & Sec. 3.1 \\
\hline$\nu_{12}$ & 0.3147 & $-6.9707 \mathrm{E}-05$ & $-4.0521 \mathrm{E}-07$ & Sec. 3.1 \\
\hline$\nu_{23}$ & 0.5557 & $-1.009 \mathrm{E}-04$ & $-1.1402 \mathrm{E}-06$ & Sec. 3.1 \\
\hline$\alpha_{1}\left[10^{-6} / \mathrm{C}\right]$ & -0.9721 & 1.5237E-04 & $-8.9154 \mathrm{E}-06$ & Sec. 3.2 and 3.3 \\
\hline$\alpha_{2}\left[10^{-6} / \mathrm{C}\right]$ & 38.4688 & 8.9483E-02 & $-3.6463 \mathrm{E}-04$ & Sec. 3.2 and 3.3 \\
\hline$G_{I c}\left[\mathrm{~J} / m^{2}\right]$ & 84.4810 & - & - & Sec. 4.1 \\
\hline \multicolumn{5}{|c|}{ Temperature independent properties } \\
\hline$F_{1 t}[\mathrm{MPa}]$ & & 848 & & [17] \\
\hline$F_{1 c}[\mathrm{MPa}]$ & & 434 & & [17] \\
\hline$F_{2 c}[\mathrm{MPa}]$ & & 27 & & {$[10,186]$} \\
\hline $\mathrm{SFT}[\mathrm{C}]$ & & 177 & & [17] \\
\hline Ply thickness [mm] & & 0.127 & & {$[10,16]$} \\
\hline
\end{tabular}

\subsubsection{AS4/3501-6 Carbon-Epoxy}

\subsubsection{Crack Density}

A comparison between experimental data and crack density predictions for $\left[0_{4} / 45_{4} / 90_{4} /-\right.$ $\left.45_{4}\right]_{s}$ AS4/3501-6 is shown in Figure 4.19. Experimental crack initiation and evolution data in [18] compare well with predicted crack density for all laminas of the composite. Generally, a better agreement can be seen when interior data (X-ray inspection) is compared due to the free-edge stress generated in angle-plies $\left(\theta^{\circ}\right)$. No experimental data for $0^{\circ}$ lamina was reported. The surface and middle laminas $\left(0_{4}^{\circ}\right.$ and $\left.-45_{8}^{\circ}\right)$ start cracking at the same time $(66 \mathrm{C})$ keeping equal cracking rate during the whole monotonic cooling. Same pattern can be seen for inside laminas $\left(45_{4}^{\circ}\right.$ and $\left.90_{4}^{\circ}\right)$ at $30 \mathrm{C}$. The maximum crack density corresponds for inside laminas $\left(45_{4}^{\circ}\right.$ and $\left.90_{4}^{\circ}\right)$ with a value of 1 [cracks $\left./ \mathrm{mm}\right]$ while the others two laminas reach a crack density value equal to 0.75 [cracks $/ \mathrm{mm}]$. Since the surface and middle plies $\left(0_{4}^{o}\right.$ and $-45_{8}^{\circ}$ ) have double thickness, higher ERR is produced and the crack initiation occurs first. Then, the inside laminas get a higher cracking rate where a load redistribution in the laminate is generated. Since the angle-plies 45 and -45 start cracking at different temperature, laminate shear strain $\epsilon_{x y}$ is generated during the monotonic cooling, which will affect the $\alpha_{x y}$ of the laminate.

The influence of ply-number $n$ in $\left[04_{4} / 45_{4} / 90_{4} /-45_{4}\right]_{s}$ AS4/3501- 6 for each $\theta$-lamina is illustrated in Table 4.4. The temperature at which crack initiation starts and the maxi- 

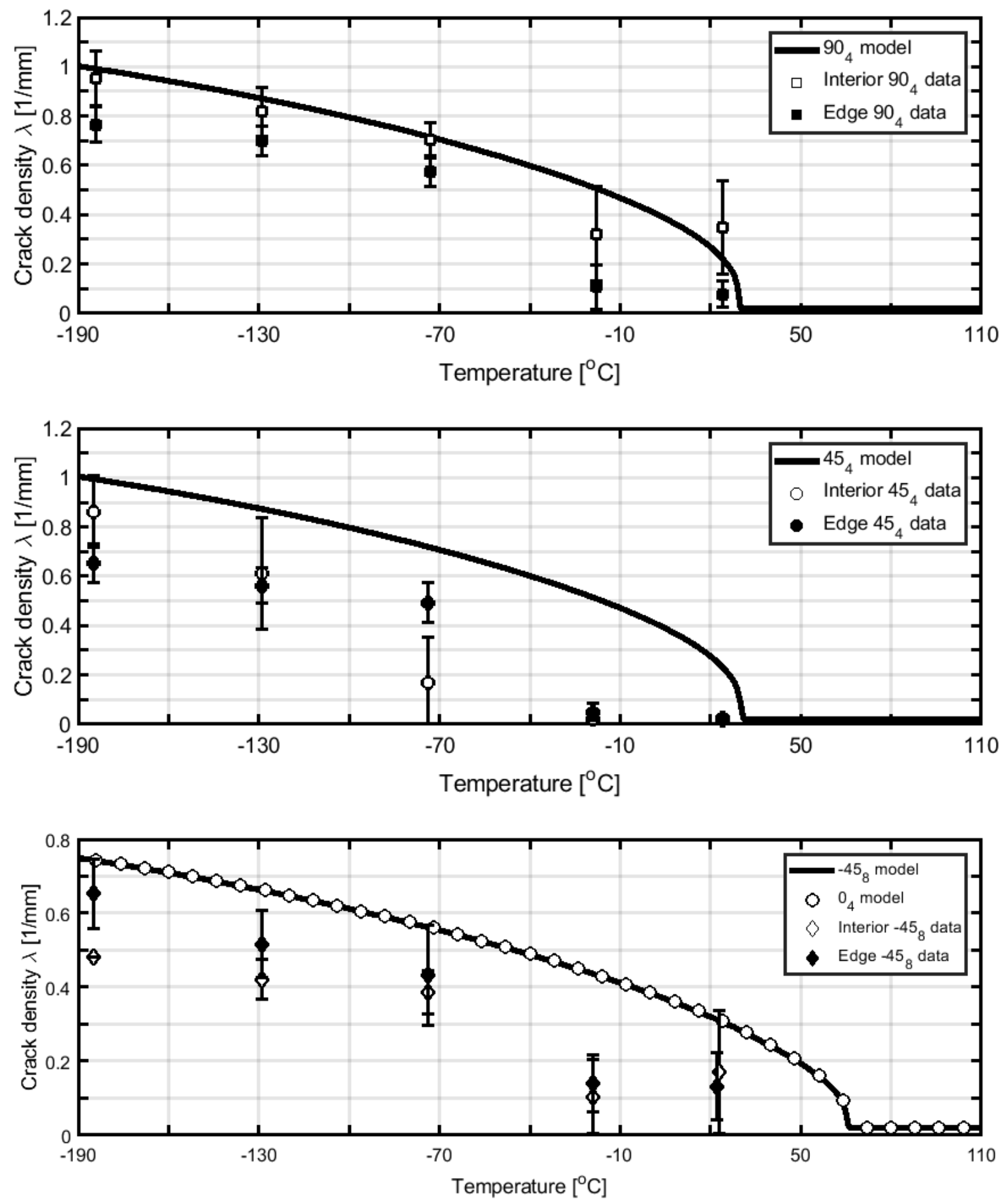

Figure 4.19: Crack density predictions vs. temperature for monotonic cooling of $\left[00_{4} / 45_{4} / 90_{4} /-45_{4}\right]_{s}$ AS4/3501-6. Top: $90_{4}$. Middle: $45_{4}$. Bottom: $-45_{8}$. 
mum crack density at $-190 \mathrm{C}$, are compared for both, exterior $\left(0_{4}^{\circ}\right.$ and $\left.-45_{8}^{\circ}\right)$ and interior $\left(45_{4}^{\circ}\right.$ and $90_{4}^{\circ}$ ) laminas. Ply thickness is constant with a value equal to $0.134 \mathrm{~mm}$. Lamina thickness is $t=0.134 n \mathrm{~mm}$. As general rule, thicker laminas $(n=4)$ move forward the crack initiation at early temperatures leading the cracking rate decreases as well as the maximum crack density within the laminate. On the other hand, thinner laminas $(n=1)$ become more resistant and delay the crack initiation to lower temperatures but they proceed with higher cracking rates and crack densities obtained are raised.

Tables 4.4: Crack initiation temperature and maximum cracking density $\left(-190{ }^{\circ} C\right)$ vs. number of sub-laimnas (n) for AS4/3501-6 in $\left[0_{n} / 45_{n} / 90_{n} /-45_{n}\right]_{s}$ laminate. Subcript (e) means the exterior laminas $0_{n}$ and $-45_{n}$; Subcript (i) means interior laminas $90_{n}$ and $45_{n}$.

\begin{tabular}{ccccc}
\hline \multicolumn{5}{c}{ Crack initiation vs. Ply thickness } \\
\hline$n$ & $T_{e} \mathrm{C}$ & $\lambda_{e}[$ cracks $/ \mathrm{mm}]$ & $T_{i} \mathrm{C}$ & $\lambda_{i}[\mathrm{cracks} / \mathrm{mm}]$ \\
\hline 1 & -61 & 1.327 & -154 & 1.170 \\
2 & +15 & 1.064 & -38 & 1.339 \\
4 & +65 & 0.751 & +30 & 0.998 \\
\hline
\end{tabular}

\subsubsection{CTE}

The tangent laminate $C T E \mathrm{~s}$ in global coordinates $\left(\alpha_{x}, \alpha_{y}\right.$, and $\left.\alpha_{x y}\right)$ are shown in Figure 4.20 for $\left[00_{4} / 45_{4} / 90_{4} /-45_{4}\right]_{s}$ AS4/3501-6 between [-160,SFT] C. Laminate CTEs as function of temperature and crack density are very similar as those shown in Figure 4.18 for $\left[0_{2} / 45_{2} / 90_{2} /-45_{2}\right]_{s}$ P75/1962 but with thiner laminas $(n=2)$. The longitudinal and transverse laminate CTEs $\left(\alpha_{x}\right.$ and $\left.\alpha_{y}\right)$ remain equal in the range [66,SFT] $\mathrm{C}$ (right side in Figure 4.20) because the laminate is quasi-isotropic, symmetric and no transverse cracking exists. In the temperature range $[30,66] \mathrm{C}$, both laminate $C T E \mathrm{~s}\left(\alpha_{x}\right.$ and $\left.\alpha_{y}\right)$ vary according to the laminate temperature-dependent data shown in Table 4.5. The balanced \pm 45 laminas crack at different temperature because they are located at middle/inside respectively and thus, shear strains and slope differences between $\alpha_{x}$ and $\alpha_{y}$ can be observed in Figure 4.20. The longitudinal CTE $\alpha_{x}$ is only influenced by the $-45_{8}^{o}$ lamina cracks with a low damage impact until the inside laminas start cracking. On the other hand, the transversal CTE $\alpha_{y}$ is highly influenced by both, the $0_{4}^{\circ}$ and $-45_{8}^{\circ}$ laminas and thus, $\alpha_{y}$ slope drops with higher rate. When inside laminas also crack, both laminate $C T E$ s keep dropping with higher rates up to $-160 \mathrm{C}$. At this temperature, the laminate $C T E$ s values approach to zero and they are practically identical because the crack density distribution get close to symmetry. Since shear strains are produced by the gap of cracking between balanced \pm 45 laminas, the laminate CTE $\alpha_{x y}$ value becomes non-zero from 60 $\mathrm{C}$, where only the $-45_{8}^{o}$ lamina start cracking. As the crack density differences between balanced \pm 45 laminas become smoother, $\alpha_{x y}$ approaches to zero. 


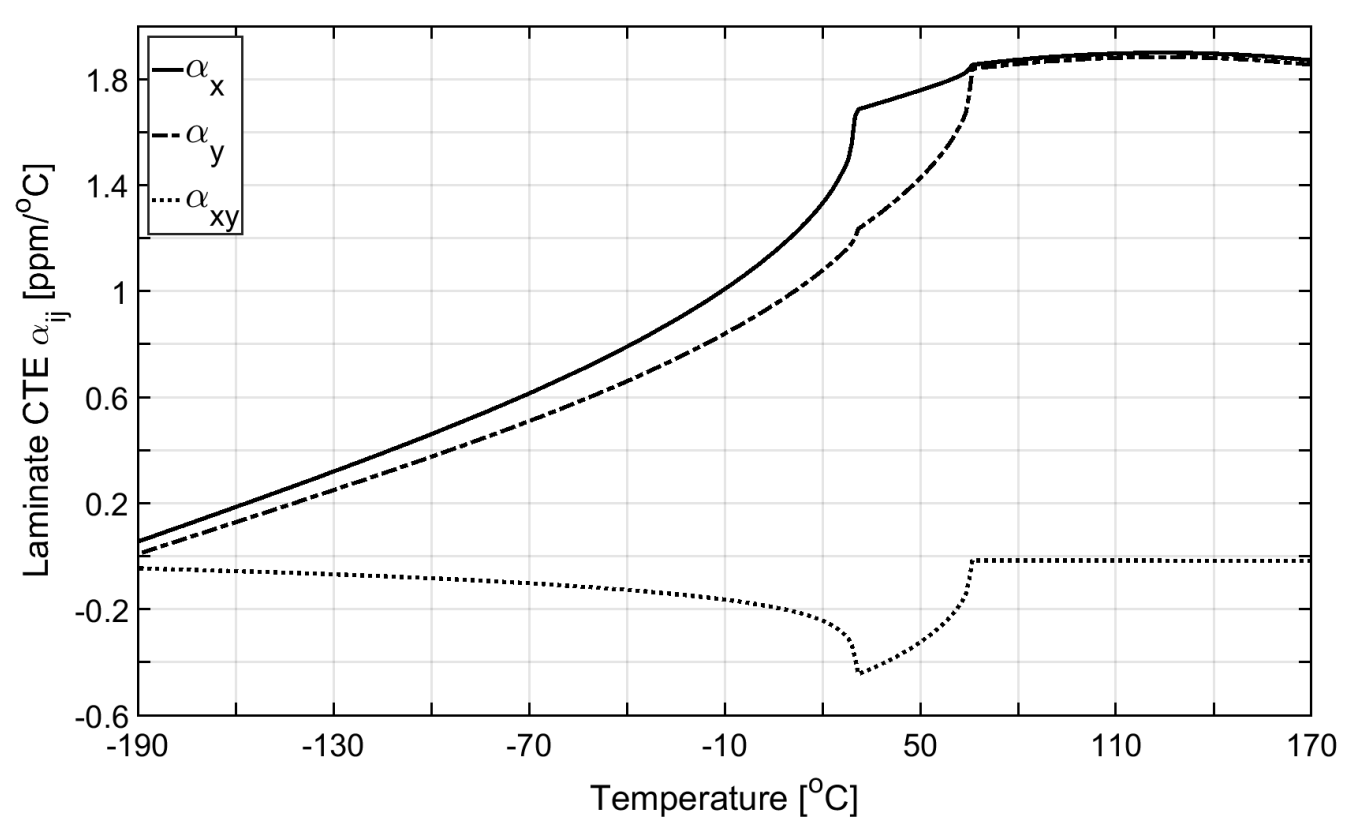

Figure 4.20: Tangent laminate CTE vs. temperature for monotonic cooling of $\left[0_{4} / 45_{4} / 90_{4} /-\right.$ $\left.45_{4}\right]_{s}$ AS4/3501-6.

The influence of ply-number $n$ in the transversal tangent CTE $\left(\alpha_{y}\right)$ for $\left[04_{4} / 45_{4} / 90_{4} /-\right.$ $\left.45_{4}\right]_{s}$ AS4/3501-6 is shown in Figure 4.21. Similar to Table 4.4, the laminate CTE variation is conditioned by the temperature at which crack initiation starts. As the lamina thickness become smaller $(n=1)$, the variation of transversal laminate $C T E$ is delayed to lower temperatures $(-61 \mathrm{C})$ while for thicker laminas $(n=4)$ such variation starts earlier $(65 \mathrm{C})$. However, similar slope rates are generated for any case.

The temperature-dependent properties of AS4/3501-6 are shown in Table 4.5 calculated using periodic microstructure model [35, PMM, App. 2] and Levin's work [269] as mentioned in Section 3.1 and 3.2. The temperature-dependent properties for AS4 carbon fiber are taken from Table 3.1, while 3501-6 epoxy properties are taken from Table 3.2. The elastic $\left(E_{1}, E_{2}, G_{12}, \nu_{12}, \nu_{23}\right)$ and thermal $\left(\alpha_{1}, \alpha_{2}\right)$ laminate properties are adjusted by a quadratic polynomial (3.2).

\subsubsection{T300/5208 Carbon-Epoxy}

\subsubsection{Crack Density}

A comparison of three T300/5208 laminates with same angle-ply laminae but different laminate stacking sequence (LSS) $\left[0_{3} / 90\right]_{s},\left[0_{2} / 90_{2}\right]_{s}$, and $\left[0 / 90_{3}\right]_{s}$ are presented in Figure 4.22. Only experimental data for $90^{\circ}$ laminas are available in the literature [20]. Crack density distribution $\lambda$ were determined by edge inspection after 20 temperature cycles [20], assuming that thermal fatigue for 20 cycles is negligible. It can be seen that predicted values compare well with experimental data. The laminates are cross-ply with symmetry, 


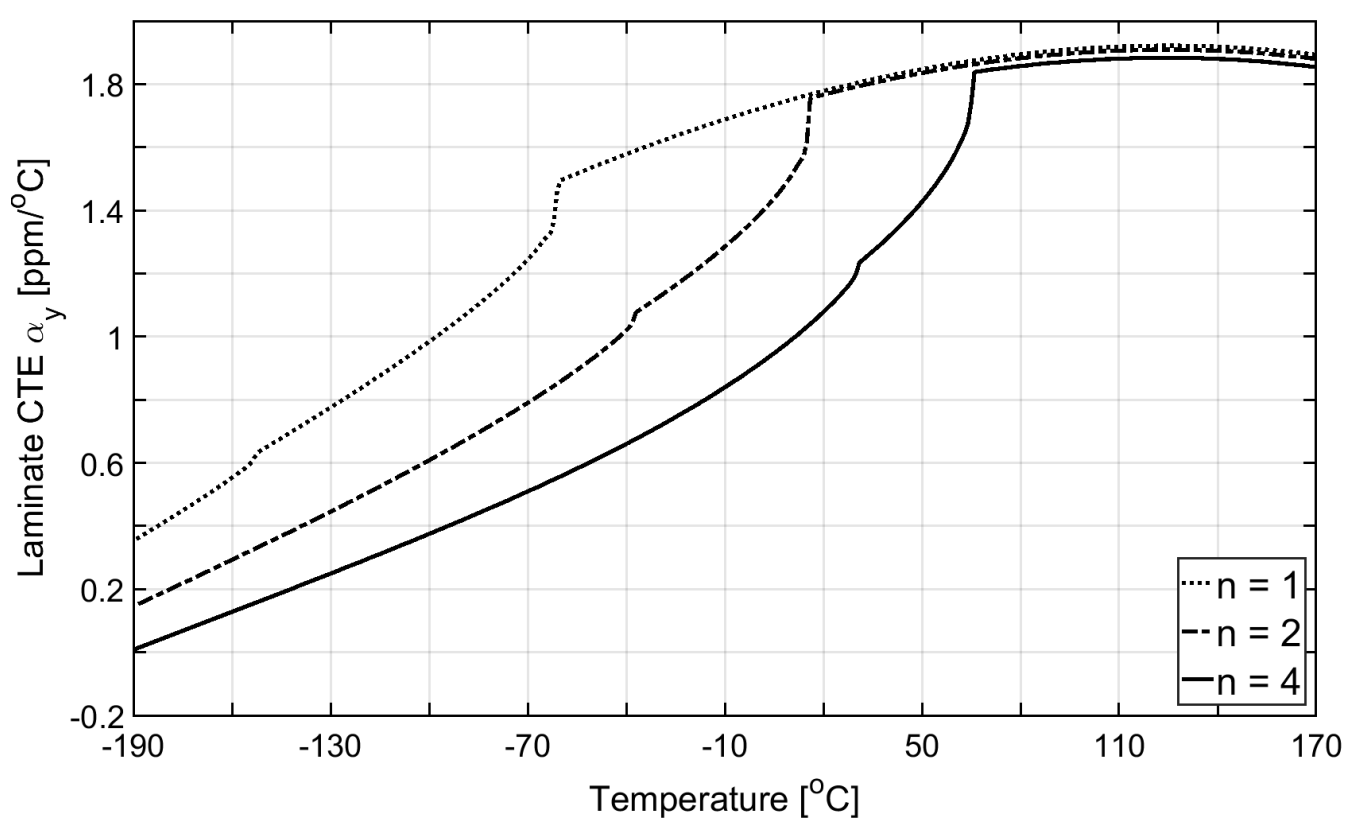

Figure 4.21: A comparison of laminate CTE $\alpha_{y}$ vs. temperature for monotonic cooling of $\left[0_{n} / 45_{n} / 90_{n} /-45_{n}\right]_{s}$ AS4/3501-6 with $n=1,2,4$.

Tables 4.5: Quadratic temperature-dependent properties of AS4/35016 $\left(V_{f}=0.67[18,19]\right)$ in the range [-190, SFT] C.

\begin{tabular}{|c|c|c|c|c|}
\hline \multicolumn{5}{|c|}{ Temperature dependent properties } \\
\hline Property & $P_{a}$ & $P_{b}$ & $P_{c}$ & Reference \\
\hline$E_{1}[\mathrm{MPa}]$ & 142719 & -4.1751 & - & Sec. 3.1 \\
\hline$E_{2}[\mathrm{MPa}]$ & 9683.123 & -12.4703 & $-1.1139 \mathrm{E}-02$ & Sec. 3.1 \\
\hline$G_{12}[\mathrm{MPa}]$ & 4926.6731 & -8.3811 & $-5.0312 \mathrm{E}-03$ & Sec. 3.1 \\
\hline$\nu_{12}$ & 0.2989 & $9.7916 \mathrm{E}-06$ & - & Sec. 3.1 \\
\hline$\nu_{23}$ & 0.576 & 4.2195E-05 & - & Sec. 3.1 \\
\hline$\alpha_{1}\left[10^{-6} / \mathrm{C}\right]$ & -0.0819 & $5.1375 \mathrm{E}-04$ & $-3.9878 \mathrm{E}-06$ & Sec. 3.2 and 3.3 \\
\hline$\alpha_{2}\left[10^{-6} / \mathrm{C}\right]$ & 22.6855 & $5.9408 \mathrm{E}-2$ & - & Sec. 3.2 and 3.3 \\
\hline$G_{I c}\left[\mathrm{~J} / m^{2}\right]$ & 68.066 & - & - & Sec. 4.1 \\
\hline \multicolumn{5}{|c|}{ Temperature independent properties } \\
\hline$F_{1 t}[\mathrm{MPa}]$ & & 1806 & & [19] \\
\hline$F_{1 c}[\mathrm{MPa}]$ & & 154 & & [19] \\
\hline$F_{2 c}[\mathrm{MPa}]$ & & 221 & & [18] \\
\hline SFT $[\mathrm{C}]$ & & 177 & & [18] \\
\hline Ply thickness [mm] & & 0.134 & & [18] \\
\hline
\end{tabular}



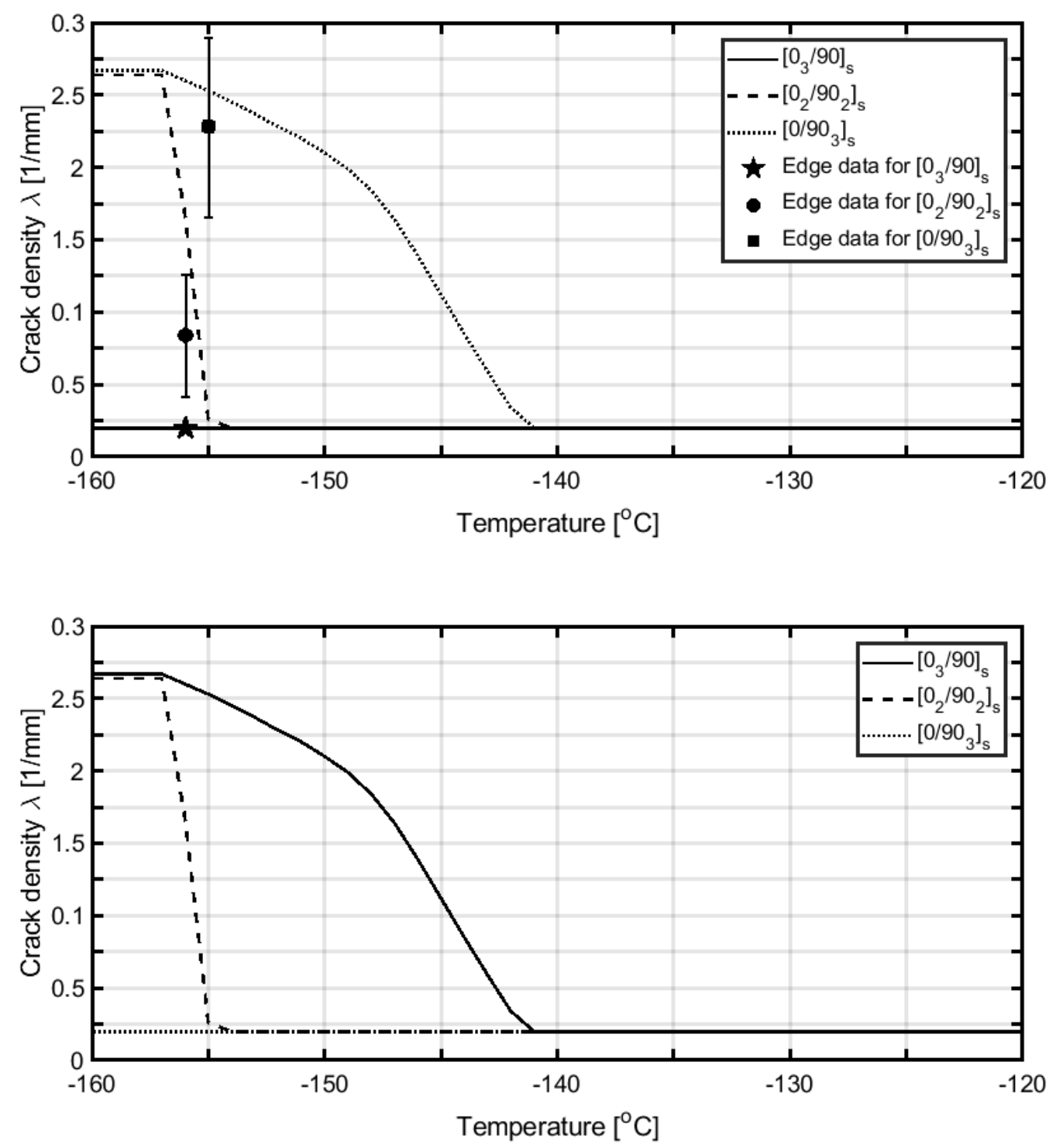

Figure 4.22: Crack density prediction in $90^{\circ}$ and $0^{\circ}$ laminas vs. monotonic cooling with different LSS $\left[0_{3} / 90\right]_{s},\left[0_{2} / 90_{2}\right]_{s}$ and $\left[0 / 90_{3}\right]_{s}$ T300/5208. Top: predicted and experimental $\lambda_{90}$. Bottom: predicted $\lambda_{0}$

so no coupling between shear and extension exists. As expected, the thickest lamina cracks first independently of the lamina $\left(0^{\circ}\right.$ or $\left.90^{\circ}\right)$. For $\left[0_{3} / 90\right]_{s}$ laminate, the thickest $0_{3}^{o}$ lamina generate a higher constraint effect over the $90^{\circ}$ lamina delaying crack initiation in $90_{2}$ lamina and even avoiding cracking during the whole monotonic cooling. The opposite effect is seen for $\left[0 / 90_{3}\right]_{s}$ laminate where the $90_{3}^{\circ}$ lamina cracks first $(-142 \mathrm{C})$ and quickly reaches its crack density saturation. For $\left[0_{2} / 90_{2}\right]_{s}$, all laminas are subjected to the same strain due to free thermal expansion of the laminate and thus, both $0^{\circ}$ and $90^{\circ}$ laminas crack at the same temperature $(-156 \mathrm{C})$ with equal cracking rates. In the same way as $\left[0 / 90_{3}\right]_{s}$ laminate, both laminas reach their crack density saturation quickly. 


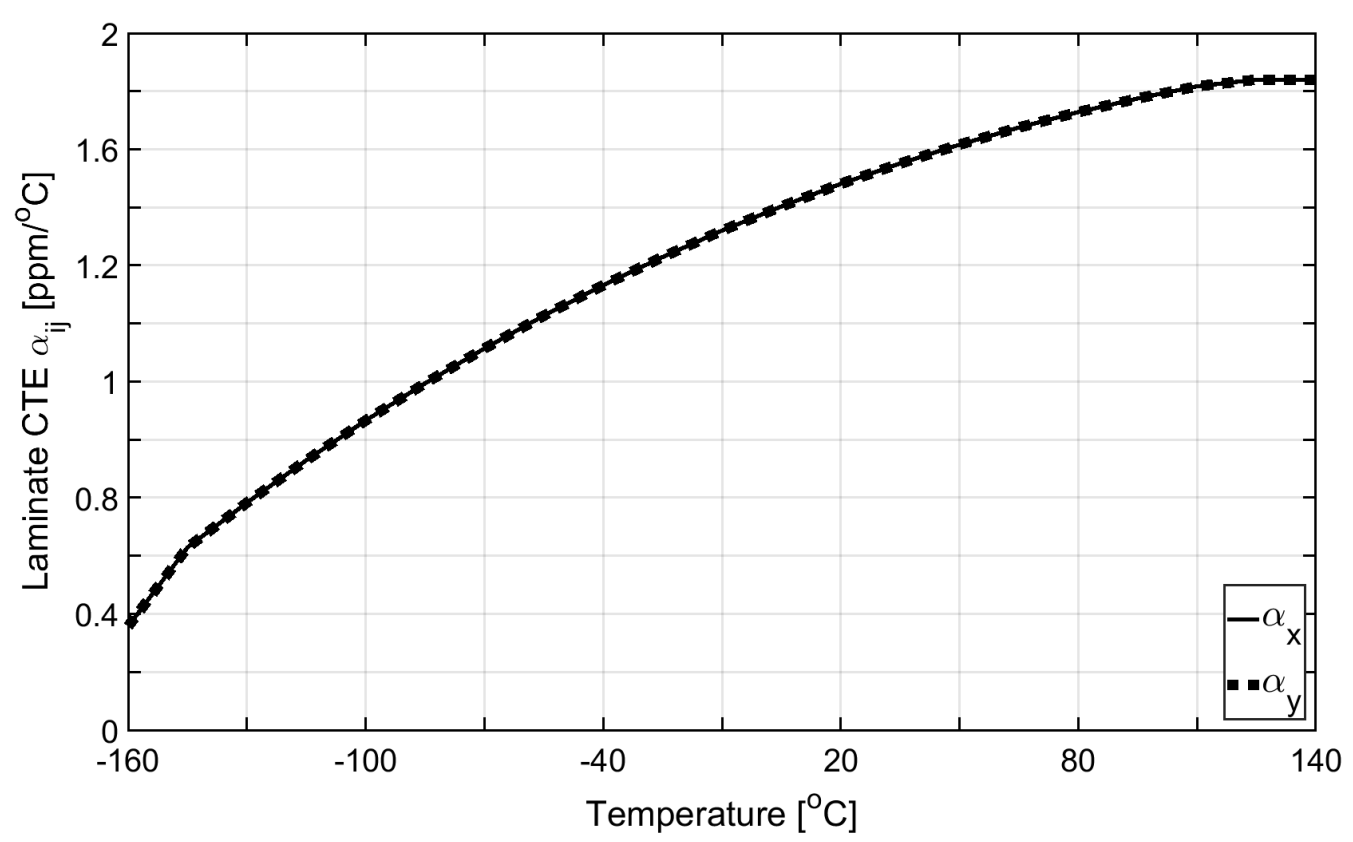

Figure 4.23: Tangent laminate CTE vs. temperature for monotonic cooling of $\left[0_{2} / 90_{2}\right]_{s}$ T300/5208. Note: $\alpha_{x y}=0$.

\subsubsection{CTE}

Tangent laminate CTEs in global coordinates of the laminate $\left(\alpha_{x}, \alpha_{y}\right.$, and $\left.\alpha_{x y}=0\right)$ are shown in Figure 4.23 for $\left[0_{2} / 90_{2}\right]_{s}$ T300/5208 between $[-160$, SFT] C. Since laminate is cross-ply and symmetric, the thermal strains $\epsilon_{x}=\epsilon_{y}$ are the same and thus, no coupling between shear and extension exists. As it can be seen in Figure 4.23, both laminate $C T E$ s remain equal during the whole monotonic cooling because no transverse cracking is generated until they cracks at $-156 \mathrm{C}$. In this range, the laminate $C T E$ s vary according to the laminate temperature-dependent data shown in Table 4.6. Once the cross-ply laminate start cracking at $-156 \mathrm{C}$, a suddenly drop for both laminate CTEs is observed until $-160 \mathrm{C}$. Note that $\alpha_{x}$ and $\alpha_{y}$ still match when both laminas are cracked because no reference system exists and the keep the same cracking rate. Since both laminas crack equally, not shear deformations appear and thus, $\alpha_{x y}$ remains equal to zero during the whole monotonic cooling.

The temperature-dependent properties of T300/5208 are shown in Table 4.6 calculated using periodic microstructure model [35, PMM, App. 2] and Levin's work [269] as mentioned in Section 3.1 and 3.2. The temperature-dependent properties for T300 carbon fiber are taken from Table 3.1, while 3501-6 epoxy properties are taken from Table 3.5. The elastic $\left(E_{1}, E_{2}, G_{12}, \nu_{12}, \nu_{23}\right)$ and thermal $\left(\alpha_{1}, \alpha_{2}\right)$ laminate properties are adjusted by a quadratic polynomial (3.2). 
Tables 4.6: Quadratic temperature-dependent properties of T300/5208 $\left(V_{f}=0.69\right.$ [8,20]) in the range $[-156,121] C$.

\begin{tabular}{|c|c|c|c|c|}
\hline \multicolumn{5}{|c|}{ Temperature dependent properties } \\
\hline Property & $P_{a}$ & $P_{b}$ & $P_{c}$ & Reference \\
\hline$E_{1}[\mathrm{MPa}]$ & 160298.5983 & -1.9908 & $-1.8104 \mathrm{E}-03$ & Sec. 3.1 \\
\hline$E_{2}[\mathrm{MPa}]$ & 12338.3635 & -12.0962 & $9.9186 \mathrm{E}-03$ & Sec. 3.1 \\
\hline$G_{12}[\mathrm{MPa}]$ & 8492.0421 & -6.8925 & $-1.46 \mathrm{E}-02$ & Sec. 3.1 \\
\hline$\nu_{12}$ & 0.2269 & $-1.3521 \mathrm{E}-04$ & $3.2461 \mathrm{E}-07$ & Sec. 3.1 \\
\hline$\nu_{23}$ & 0.5653 & $-4.4407 \mathrm{E}-04$ & $1.5286 \mathrm{E}-06$ & Sec. 3.1 \\
\hline$\alpha_{1}\left[10^{-6} / \mathrm{C}\right]$ & -0.1673 & $1.7203 \mathrm{E}-03$ & $-4.7315 \mathrm{E}-06$ & Sec. 3.2 and 3.3 \\
\hline$\alpha_{2}\left[10^{-6} / \mathrm{C}\right]$ & 22.8995 & 6.9351E-02 & $-8.1085 \mathrm{E}-05$ & Sec. 3.2 and 3.3 \\
\hline$G_{I c}\left[\mathrm{~J} / m^{2}\right]$ & 269 & - & - & Sec. 4.1 \\
\hline \multicolumn{5}{|c|}{ Temperature independent properties } \\
\hline$F_{1 t}[\mathrm{MPa}]$ & & 1440 & & {$[8]$} \\
\hline$F_{1 c}[\mathrm{MPa}]$ & & 1500 & & [287] \\
\hline$F_{2 c}[\mathrm{MPa}]$ & & 246 & & [287] \\
\hline SFT $[\mathrm{C}]$ & & 177 & & {$[20]$} \\
\hline Ply thickness [mm] & & 0.127 & & [20] \\
\hline
\end{tabular}

\subsection{Conclusions}

Intralaminar crack opening in mode II, $G_{I I c}$, is common on laminated composites subjected to static loads [31,288], however transverse cracks for most of LSS composites subjected to thermal loads can be predicted using only $G_{I c}$. This is due to the fact that no shear strain appears when laminates are free to expand, at least until first cracks occur for both symmetric cross-ply and quasi-isotropic laminates. Even when $\pm 45^{\circ}$ laminas are present, for instance in quasi-isotropic laminates, no reference coordinate system exist, and all laminas are subjected to same strain. In addition, most of $G_{I I c}$ values obtained in the literature for both intralaminar and interlaminar are higher than $G_{I c}$, with larger crack densities when a mixed-mode I and II occur [32,34,35, 100].

Based on the results shown in Section 4.2, the LSS has influence on the shear strains that may appear. For example, in the case of $[0 / \pm 45 / 90]_{s}$ P75/934 (see Figure 4.9), both \pm 45 laminas crack at the same temperature with identical cracking growth rate avoiding shear strains during monotonic cooling. Also in Figure 4.14 where $\alpha_{x y}$ remains equal to zero. By contrast, in the case of $\left[0_{2} / 45_{2} / 90_{2} /-45_{2}\right]_{s}$ P75/1962 (see Figure 4.16), the \pm 45 laminas crack at different temperature inducing small shear strains for a finite temperature interval as shown in Figure 4.18. For such case, the shear strains are generated due to the lag of crack initiation between both \pm 45 laminas (internal and middle position). However, $\alpha_{x y}$ approaches to zero as soon the crack density in both laminas get close each other. Therefore, the LSS has a some influence with respect the transverse cracking mixed-mode I and II but the magnitude of shear strain $\epsilon_{x y}$ is small. 
For all cases in Sec. 4.2, the predicted crack density in \pm 45 laminas group presents good agreement with experimental data when cracks are measured using a interior X-ray inspection (see Figure 4.7 and 4.19). However, in some cases where cracks were obtained using an optical microscopy inspection at the edge of the specimens, the crack density is over estimated (see Figure 4.4 and 4.5, 4.16, 4.19). Based on these results, two conclusions can be drawn.

First, the transverse damage can be predicted well using only $G_{I c}$ because transverse cracking in mixed-mode I and II would generate higher ERRs, and thus equal or greater crack densities. However, the external experimental data (edge of the specimen) are already over estimated using only GIc. Therefore, cracks are not produced in mixedmode or the ERR generated in mode II is relatively small compared to ERR in mode I as illustrated in Figures 4.11. For instance in Figure 4.18, $\alpha_{x y}$ is close to zero during almost all monotonic cooling and thus, small shear strains appear.

Second, there must be a connection between the traverse tensile stresses $\sigma_{22}$ given by CLT in each lamina, and the free-edge stresses. According to several researchers $[11,24,185]$, the transverse cracking damage on carbon laminate composites subjected to thermal loads is highly influenced by the free-edge stresses. Several specimens with same lay-up but different LSS (i.e, different cutting edge plane inspection) were tested $[11,27]$, and the same external crack density was found regardless of LSS but, large discrepancies when cracks were measured at the edge from internal laminas leading to large scatter data on interior laminas \pm 45 or 90 . While $90^{\circ}$ laminas were found to have some cracks starting from edge, short or even not cracks were measured in \pm 45 from edge. A comparison between $\sigma_{22}$ given by CLT and a 3D FEA at the edge was performed to explain this phenomenon as shown in Figure 4.24. They found that $\sigma_{22}$ stress at the edge on interior 90 layers is approximately a $24 \%$ higher than the $\sigma_{22}$ stress given by CLT and thus, cracks begins from edge and propagate along specimen length. However, the $\sigma_{22}$ stress at the edge on interior \pm 45 laminas decreases a $57 \%$ and thus, short cracks begin from the interior of specimen without getting propagate to the edge into a single crack. Therefore, the inspection through polished edges of samples is not an adequate method to count cracks on laminates subjected to pure thermal fatigue when the \pm 45 laminas are embedded inside.

Furthermore, only two potential lay-ups $\left[0_{2} / \pm 30\right]_{s}$ for P75/934 and $\left[0_{2} / \pm 60_{2}\right]_{s}$ for P75/1962, which are candidate to produce a mixed-mode I and II, were found in the literature, but the data was measured from edge and thus not so reliable. Since there is not evidence of transverse cracking in mode II, it is assumed as valid that crack initiation and evolution can be predicted well using only $G_{I c}$ in the temperature range studied. 


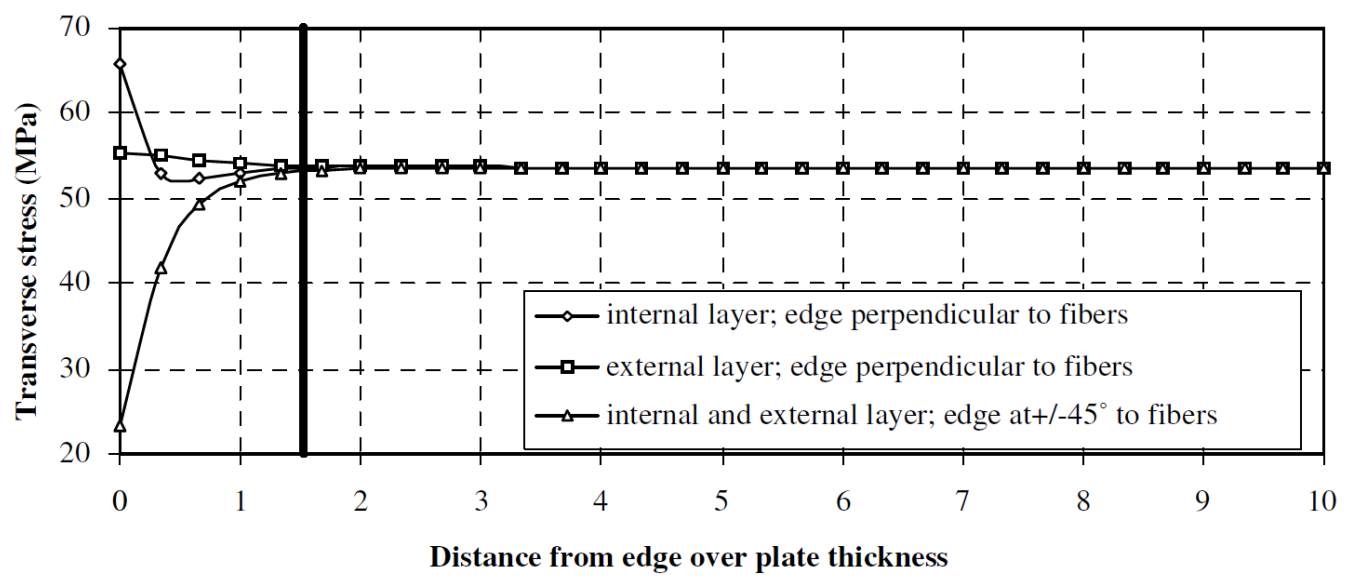

Figure 4.24: Transverse stresses $\sigma_{22}$ at $50 \mathrm{C}$ in different laminas with same lay-up given by FEA [11].

\subsection{Conclusions}

Since elastic and CTE properties of polymers are temperature-dependent, they induce temperature-dependency on all the effective properties of laminas and laminates. However, the temperature dependency of fiber-dominated properties is small because the fiber-properties are virtually independent of temperature or their variation with temperature is very small.

The temperature dependence of matrix-dominated properties can be accurately represented by a quadratic function and in some cases the variation is so slight that a linear function suffices.

Although the experimental data is scarce non-existent in some cases and displays great scatter in other cases, a systematic procedure is developed and applied to extract in-situ properties for both fibers and polymers encompassing four composite material systems while taking into account their temperature dependence.

Finite element analysis confirms the accuracy of the analytical micromechanics model selected for this study. Once the fiber and polymer properties are found, micromechanics allows computation of all lamina effective properties for the temperature range of interest. However, care should be taken not to extrapolate outside the temperature range of the experimental data used for material characterization, particularly when nonlinear equations are used to model the data. Predictions outside this range are thus made assuming constant values for all properties outside the temperature range of the experimental data.

When laminates are mechanically loaded, damage initiation and accumulation up to crack saturation are characterized by two values of critical ERR in modes I (opening) and II (shear). However, cooling of quasi-isotropic laminates produces only mode I cracking because the thermal contraction is the same in every direction, and cross-ply laminates crack in mode I only because there is no shear induced. Therefore, only $G_{I c}$ was used in 
for this study.

The critical ERR $G_{I c}$ is easily obtained by minimizing the error between crack density prediction and available data. A constant value of critical ERR produces satisfactory predictions of crack density vs. temperature. To eliminate the small discrepancy on saturation crack density at cryogenic temperature requires adjusting the critical ERR with a quadratic equation. From a practical point of view, being able to produce satisfactory estimates of damage with a constant value of critical ERR is advantageous because it reduces the amount of experimentation needed to adjust the critical ERR.

Some of the experimental crack-density data is inconclusive about crack saturation for some material systems, namely AS4/3501-6 and T300/5208. In other words, for those material systems the temperature at which data is available is not low enough to show crack density leveling off. However, model predictions clearly show that crack saturation is likely in all cases. This is because the critical ERR does not change much with cooling, but transverse CTE drops significantly with cooling (Figures 3.14-3.15), thus depriving the system from the main driver for thermo-mechanical transverse cracking. 


\section{Chapter 5}

\section{Thermal Fatigue of Laminated Polymer-Matrix Composites}

A broad variety of composite structures, such as aircraft, satellites, cryogenic storage and so on, are subjected to damage accumulation when they are thermally loaded. Due to difference in the coefficient of thermal expansion (CTE) among laminas with different orientations, thermal stresses and strains are induced, often resulting in transverse cracking that can precipitate other types of damage such as fiber-matrix debonding and delamination between laminas. Furthermore, these cracks cause degradation of material properties as well as changes in the CTE of the laminate, which may affect its structural integrity, efficiency, or may result in eventual failure.

Numerous experimental results $[1,8,10,20,23,27,29,185,188,189,289]$ show evidence of transverse cracking when composite laminates are loaded thermally either through cooling at a constant rate $[18,185,188]$ (monotonic cooling) or through cyclic thermal loads $[10,23,27,29]$. Furthermore, transverse cracking is well known to appear in the first stage of damage during cyclic loading $[38,47]$ until an equilibrium state called characteristic damage state $(C D S)$ is reached. Typically, CDS is a laminate property independent of loading history where crack saturation is reached after a large number of cycles [47]. However, based on low-cycle fatigue predictions and experimental data in the literature, laminates under thermal cyclic loads seems to asymptotically reach a lower crack density saturation value (CDS) for low-cycle fatigue compared with the CDS reached in laminates subjected to static loads or mechanical fatigue $[8,10,18]$.

For the sake of clarity and without lack of generality, let's consider a quasi-isotropic (QI) laminate. Under uniaxial mechanical loading (fatigue or static), the stress and strain fields in each lamina results from imposed strains that leads to damage patterns starting in the form of transverse cracking in $90^{\circ}$ laminas. However, composite laminates under free thermal expansion are subjected to biaxial stress states in each lamaina of the 


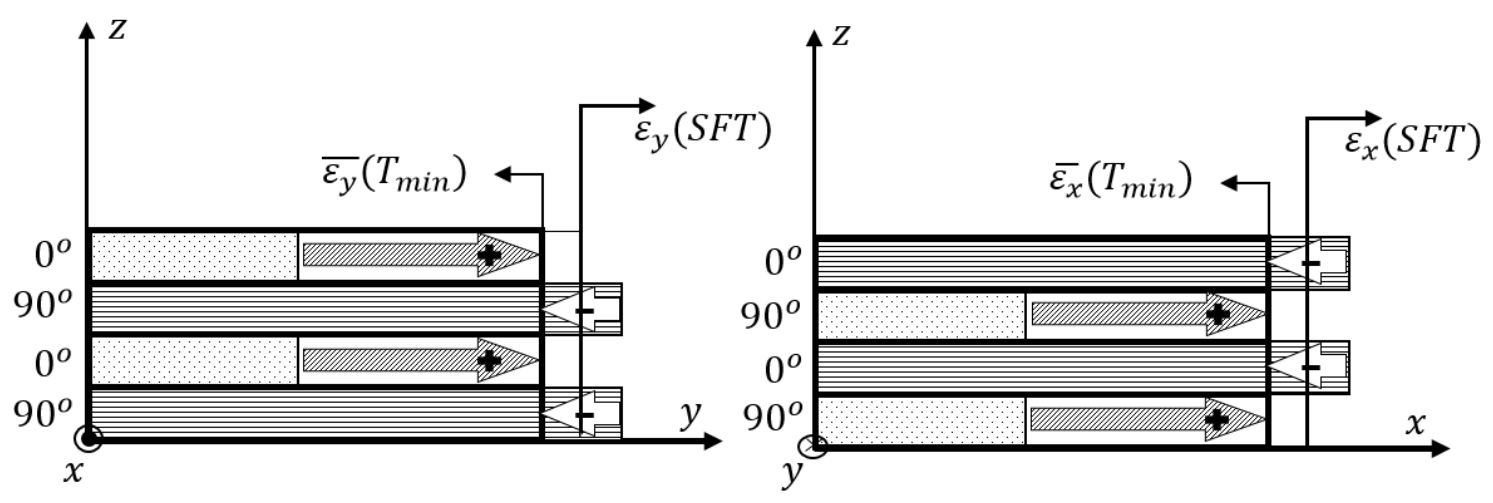

Figure 5.1: Thermal strains for a cross-ply laminate during cooling from SFT to $T_{m i n}$. Positive and negative arrows represent traction and compression, respectively.

laminate that are independent of orientation, which leads to lamina by lamina damage, i.e. transverse cracking in $0^{\circ}, 90^{\circ}$, and $\pm \theta$ laminas.

CTEs along the fiber $\alpha_{1}$ and perpendicular to fiber $\alpha_{2}$ are negative and positive, respectively. Hence, during cooling, thermal strains become positive along fiber whereas they are negative perpendicular to fiber, as shown in Figure 5.1. Since $\alpha_{1}$ is much smaller than $\alpha_{2}$, the laminate contracts with average strain $\bar{\epsilon}_{i}$ at $T_{\text {min }}$. However, such final $\bar{\epsilon}_{i}$ results from internal equilibrium between laminas leading to transverse cracking regardless of orientation. Note that $S F T$ is the stress free temperature at which no thermal stresses appear so that all laminas are initially aligned together.

The elastic properties of the lamina are temperature-dependent mostly due to the polymer matrix, which is the constituent most sensitive to temperature. The matrix goes from a rubbery state at high temperature to brittle state at low/cryogenic temperature. The lamina properties that are most affected by temperature are $E_{2}(T), G_{12}(T)$, and $\alpha_{2}(T)$, which are matrix-dominated and affected by the transverse properties of the fiber. As a result, the stiffness $E_{2}$ perpendicular to the fiber increases significantly at low temperatures while the transverse CTE $\alpha_{2}$ of the lamina decreases significantly at cryogenic temperatures [12] as shown in Figure 5.2. Furthermore, due to the temperaturedependent properties, some differences such as crack saturation (CDS) and crack growth rate are observed on thermally loaded laminates [10,186] vs. those subjected to mechanical loads. However, similar damage mechanisms are observed in both thermal and mechanical fatigue.

\subsection{Materials and Methods}

The material system used in this study is Amoco P75/1962, and the material properties of the fiber and matrix are collected from [12] in the temperature range $\left[-156,12{ }^{\circ} \mathrm{C}\right]$. 

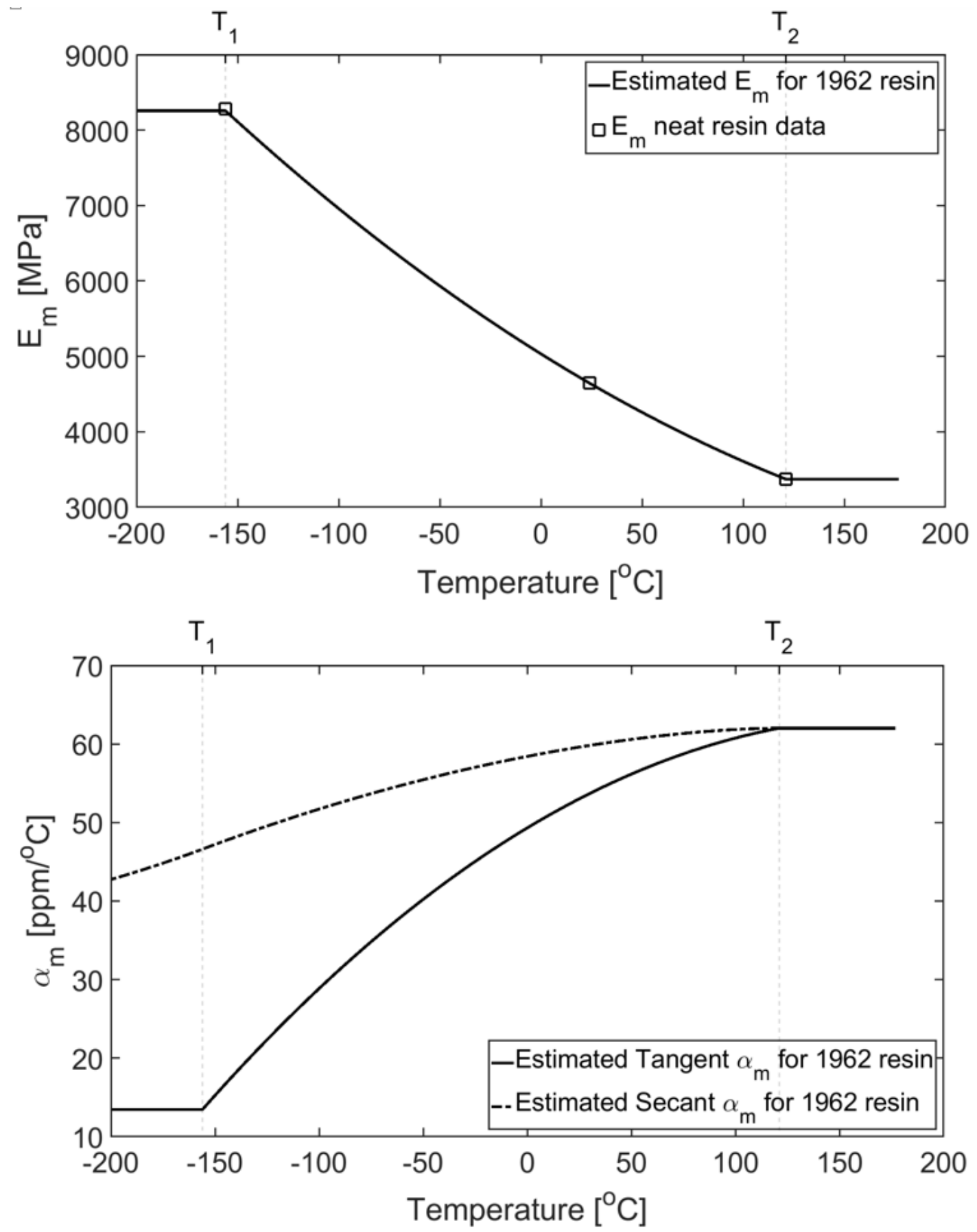

Figure 5.2: Temperature-dependent properties of epoxy 1962 in the range [-156, $\left.121^{\circ} \mathrm{C}\right]$ [12]. 
Lamina mechanical properties are calculated using periodic microstructure model (PMM) [290, A.2] while lamina CTE's are obtained using Levin's Model (LM) [269] [290, Sect.4.4] with volume fraction $V_{f}=0.52[10,188,189]$. Crack density $\lambda[$ cracks $/ \mathrm{mm}]$ in each lamina is predicted with Discrete Damage Mechanics (DDM) [31] [290, Sect.8.4].

\subsection{Methodology}

The objective in this work is to develop a theoretical model to predict transverse cracking in laminate composites subjected to thermal cyclic loads, namely thermal fatigue. Experimental results reported in $[10,188,189]$ are used to compare predictions with available crack densities $\lambda^{d}$ measured at the free-edge at a discrete number of thermal cycles $\mathrm{N}$.

A modified Griffith's criterion [274] is used to predict transverse cracking. Since polymers become brittle at low temperatures, the onset and propagation of new cracks can be predicted by Linear Elastic Fracture Mechanics (LEFM) where crack initiation is controlled by the fracture toughness $K_{I c}$. Assuming the width of the specimen to be much larger than the thickness (plane-strain), the critical energy release rate (ERR) $G_{I c}$ is related to the fracture toughness $[273,274]$ as follows

$$
\begin{array}{r}
G_{I_{c}}=\frac{K_{I c}^{2}}{E}\left(1-\nu^{2}\right) \\
K_{I c}=\sigma \alpha \sqrt{\pi a}
\end{array}
$$

where $E$ is the Young's modulus, $\nu$ the Poisson's ratio, $\sigma$ the stress at which cracks propagate for a crack of length $a$, and $\alpha$ is a parameter to account for the geometry of the specimen. Among the multitude of damage models available, discrete damage mechanics (DDM) [31] is attractive for this study because in addition to the temperaturedependent properties, it only requires the critical ERR $G_{I c}$ and $G_{I I c}$ to predict both damage initiation and evolution due to transverse and in-plane shear loading.

According to experimental results $[10,188,189]$, higher crack densities are reported as the number of cycles increase causing stiffness degradation and consequent reduction of ERR below the level required to propagate new cracks. Thus, the material's resistance to cracking must be degraded to allow for an increase of crack density with number of cycles N. Since there is no available experimental data for fracture toughness $K_{I c}$ at cryogenic temperatures as function of the number of cycles for the polymers of interest (Epoxy 1962), an alternative method is required to adjust the critical ERR $G_{I c}$, which is necessary for the damage model. In this work, $G_{I c}$ is adjusted by minimizing the error function D between DDM predictions and available experimental crack density data $\lambda^{d}$ 
as follows

$$
D=\frac{1}{M} \sqrt{\sum_{j=1}^{M}\left(\lambda_{j}-\lambda_{j}^{d}\right)}
$$

where $\mathrm{M}$ is the number of data points and $\lambda_{j}$ is the crack density.

Before adjusting $G_{I c}$, it is important to highlight that the critical ERR is temperaturedependent because both the matrix properties $(E, \nu)$ and fracture toughness $K_{I c}$ increase at low temperature [12]. On the other hand, transverse damage during the first cycle $(N=1)$ is fatigue insensitive and crack density for $N=1$ can be predicted by the quasistatic cooling model presented in Ch. 4 [12]. For this reason, the temperature dependence of $G_{I c}$ and its effect on crack density during cooling are assessed under monotonic cooling prior to thermal fatigue prediction as explained in Chapter 3 and 4 .

The highest crack density generated during one thermal cycle corresponds to the lowest temperature, i.e. $T_{\text {min }}$, at which both the ERR $G_{I}$ and thermal stresses are maximum. Therefore, the critical ERR $G_{I c}$, which controls crack propagation, corresponds to the lowest temperature, i.e. $E\left(T_{\min }\right), \nu\left(T_{\min }\right)$, and $K_{I c}\left(T_{\min }\right)$. Note that this is expected because the highest thermal strains occur at $\Delta T=T_{\min }-S F T$.

The material's resistance to cracking during thermal fatigue must degrade after a given number of cycles. Experimental crack densities are collected after a number of cycles have been completed. Since the highest crack density is reached at $T_{\text {min }}$, experimental crack density collected at discrete number of cycles $\mathrm{N}$ can be predicted using $G_{I c}\left(T_{m i n}\right)$.

For monotonic cooling at $N=1$, predictions of crack density assuming $G_{I c}^{\prime}$ to be independent of temperature produce good results, as reported in [12], where superscript $"$ " denotes $N=1$. While transverse stiffness $E_{2}$ increases at low temperature, $E_{2}$ decreases due to the development of new cracks and the two effects balance each other. Further, the transverse CTE $\alpha_{2}$ decreases significantly with cooling, which allows the material to be more resistant to damage as the temperature decreases. In the meantime, the critical ERR $G_{I c}^{\prime}$ increases slightly with cooling as reported in [12].

Therefore, transverse cracking due to monotonic thermal loads can be predicted using $G_{I c}^{\prime}\left(T_{\min }\right)$ for $N=1$. For thermal fatigue, either $G_{I c}$ decreases with $\mathrm{N}$ or other factors come into play.

\subsection{Separation of Variables}

In thermal fatigue, transverse cracking $\lambda$ is governed by temperature-dependent properties, number of cycles, and thermal amplitude load. Therefore, it will be assumed that 
$G_{I c}$ can be adjusted through a function using separation of variables according to the following expression

$$
G_{I c}=g(T) f\left(N, R_{T}\right)
$$

where $\mathrm{T}$ is temperature, $\mathrm{N}$ is the number of cycles, and $R_{T}=T_{\min } / T_{\max }$ is the thermal ratio. Since the crack density $\lambda$ increases with the number of cycles, the critical ERR values $G_{I c}$ in (5.4) will be adjusted at a discrete number of cycles N to evaluate the material's resistance to cracking due to thermal fatigue.

The temperature function $g(T)$ in (5.4) is assumed to be $g(T)=G_{I c}^{\prime}\left(T_{\min }\right)$, which is independent of the number of cycles. Although $R_{T}$ depends on the temperature amplitude, the thermal stresses are always calculated from the stress free temperature, namely $\Delta T_{i}=T_{i}-S F T$ for $T_{i}=T_{\min }$ when $R_{T}$ is fixed. Furthermore, the highest crack density occurs at the lower temperature, so that $G_{I c}^{\prime}\left(T_{m i n}\right)$ can be adjusted by minimizing the error D between DDM prediction and crack density data at the first cycle, i.e. $\lambda_{N=1}^{d}$ where superscript " $\mathrm{d}$ " denotes "data" to differentiate from predicted crack density $\lambda$. For $\mathrm{P} 75 / 1962$ at $T_{\min }=-156^{\circ} \mathrm{C}, G_{I c}^{\prime}\left(T_{\min }\right)=181.87\left[\mathrm{~J} / \mathrm{m}^{2}\right]$ by minimizing the error $\mathrm{D}(5.3)$ using data from [10,186,189].

Since no fatigue phenomenon is noticeable during the first cycle, the fatigue resistance function is $f\left(N, R_{T}\right)=1$. With $G_{I c}^{\prime}\left(T_{\min }\right)$ obtained from monotonic cooling at $N=1$, the evolution of $G_{I c}$ as function of number of cycles (5.4) can be expressed as follows

$$
G_{I c}\left(N, R_{T}, T_{\text {min }}\right)=G_{I c}^{\prime}\left(T_{\text {min }}\right) f\left(N, R_{T}\right)
$$

Next, $G_{I c}$ values in (5.5) are calculated by minimizing the error D at discrete number of cycles $\mathrm{N}$, for which experiment data $\lambda^{d}$ is available [10,186,189].

In order to study how the fatigue resistance $f(N)$ evolves for fixed $R_{T}$, a thermal fatigue test in the range $\left[-156,12{ }^{\circ} \mathrm{C}\right]$ and laminate stacking sequence (LSS) $\left[(0 / 90)_{2}\right]_{S}$ is used because more data points are available. Then, the dependent variable $G_{I c}$ in (5.5) is normalized as $G_{I c} / G_{I c}^{\prime}\left(T_{\min }\right)$ in order to calculate $f(N)$. The fatigue resistance $f(N)$ seems to fit well a linear function in semi-logarithm scale, as shown in Figure 5.3, but the discrepancy at both ends of the number of cycles demands further consideration. The coefficients of a Simple Linear Regression (SLR) are estimated transforming to a logarithmic scale as follows

$$
y=\beta_{1} \log _{10}(N)+1
$$

where residuals are assumed to follow a normal distribution $\sim\left(0, \sigma^{2}\right)$ of mean equal to 


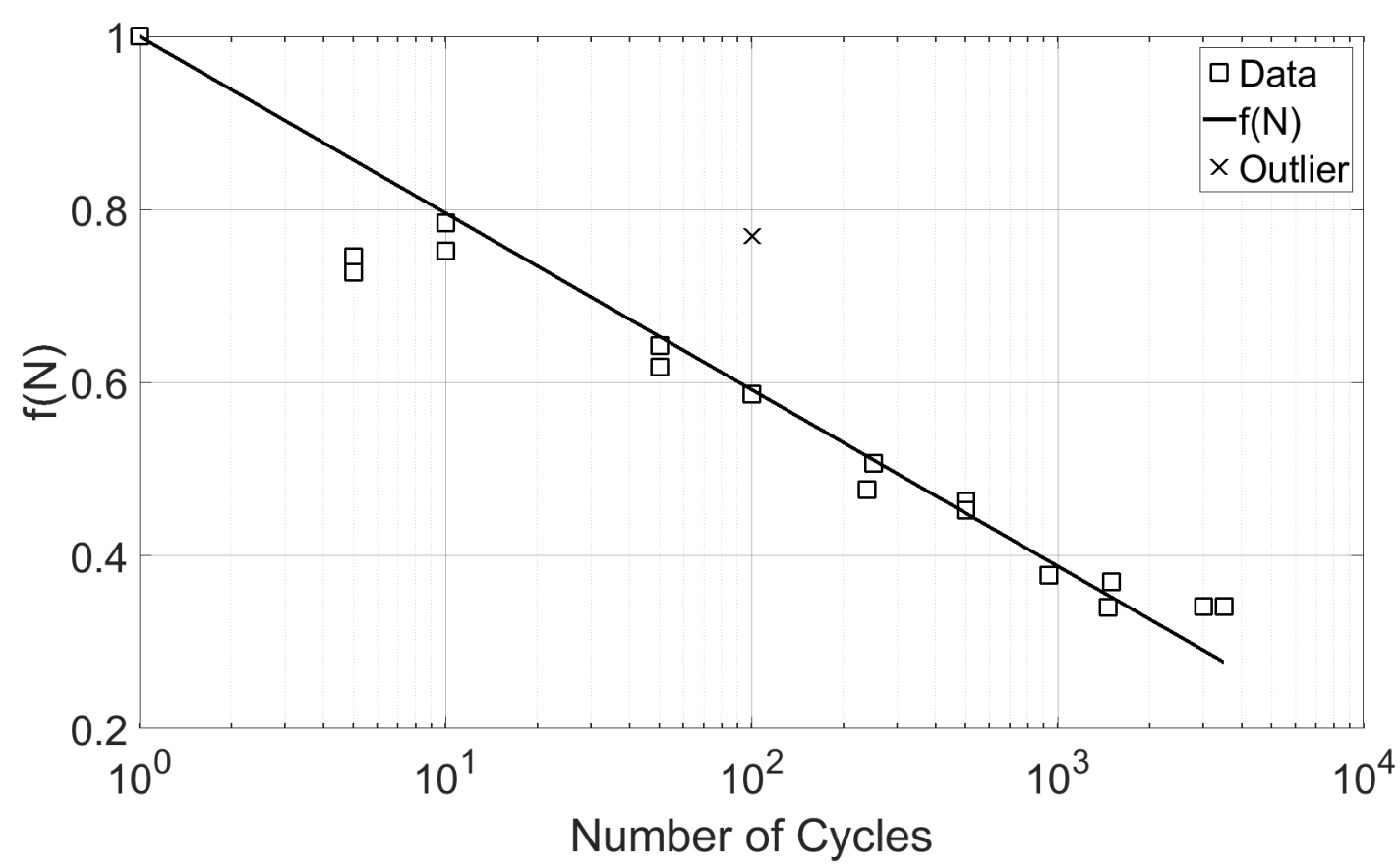

Figure 5.3: Fatigue resistance $f(N)$ as function of number of cycles for P75/1962 $\left[(0 / 90)_{2}\right]_{S}$ with $R_{T}=-156 / 121$. Experimental data is collected for middle $90_{2}^{o}$ lamina.

zero and variance $\sigma^{2}$ [291]. The constant coefficient is expected to be equal to one because no fatigue phenomenon occurs during the first cycle $(f(N) \approx 1)$, so that $G_{I c}=G_{I c}^{\prime}\left(T_{\min }\right)$ at $N=1$, and $\beta_{1}=-0.204$. An outlier data point is reported in Figure 5.3 at $N=100$ cycles. This point was ignored based on two statistical methods Cook's distance and DFFITS [291,292] providing evidence that in fact it is an outlier. Only data for middle $90_{2}^{o}$ lamina is available.

\subsection{Fracture mechanisms of polymers at low tempera- ture}

In this section we discuss the fracture mechanisms of brittle materials such as metals and polymers at low temperatures, as well as the fatigue resistance $f(N)$ from a theoretical point of view. Generally speaking, fracture mechanics focus its attention on flaws, imperfections, and voids which already exist in the material. Under this consideration, several failure mechanisms for crack propagation can be identified.

Typically, crack nucleation in crystalline materials is generated through dislocations in the material lattice due to shear stresses. As the number of dislocations and their interaction increases, internal void size grows leading to crack nucleation, which becomes a path for crack propagation. This mechanism is referred in the literature as shearing. In LEFM, illustrious researchers such as Taylor, Orowan, and Griffith postulated the 
intrinsic existence of initial voids in the material. These imperfections allow internal dislocation at stress levels much lower than yield stress, that result into fatigue cracking below yield stress.

Unlike metals, which have a crystalline-granular structure, polymers are formed by molecular chains connected by covalent bonds. The continuous oscillation of the molecular chains, which arrange themselves in equilibrium, determine the properties of polymers. However, such oscillation becomes restricted as the temperature decreases. Below a specific temperature, called the glass transition temperature ( $\mathrm{Tg}$ ), molecular motions are highly restricted so that free volume is reduced and polymer chains are compacted into a glassy state. As temperature decreases, polymers chains are efficiently packed leading to greater density into a set of organized crystalline laminas. Therefore, polymers can be assumed to behave as brittle materials at low temperatures.

The fracture of brittle polymers is caused by cavitation in the form of microvoids due to stress or deformation. When a brittle/crystalline polymer is loaded, initial crystalline laminas break into smaller blocks forming microfibrils along the principal stress axis, which span the faces of voids. The breakage of these fibrils lead to development of cracks in form of crazes [293]. Such crazes develop into cracks similarly to those in brittle metals along the direction of main stress/strain axes. Some researchers [294,295] point out that the onset of crazes depends on the stress state, crystallinity level, and environment conditions. Therefore, under multiaxial stress conditions, crack propagation is very sensitive to the hydrostatic stress component, and crazes become an ideal path for crack propagation.

Since polymer matrices, such as epoxy can be assumed to be isotropic and brittle at low temperatures, crack propagation can be assessed by LEFM [293]. While it is true, even for brittle materials, that a small energy is consumed by the blunting process at the crack tip, the correlation given by Irwin and Orowan [273,274] is still valid when the inelastic deformations are small compared with the crack size. Under these conditions, the cracktip stress distribution can be calculated by using the Irwin formulation [274] associated to each mode of propagation (mode I,II, and III). Consequently, crack propagation can be controlled by fracture toughness $K_{I c}$, which is a material property that can be related to the critical ERR $G_{I c}$ using (5.1).

Looking into equation (5.2), LEFM works under the premise that initial flaws or voids already exist in the material. Operating with equations (5.2) and (5.1), the crack size can be expressed as follows

$$
a=\frac{1}{\pi} \frac{G_{I c} E_{m}}{\left(1-\nu_{m}^{2}\right)(\beta \sigma)^{2}}
$$


where $E_{m}$ and $\nu_{m}$ are matrix properties, $G_{I c}$ the critical ERR, $\beta$ a geometric factor, and $\sigma$ the stress at crack propagation. Based on equation (5.7), the minimum size $a$ to propagate an existent crack can be estimated as follows. First the critical value $G_{I c}$, which is a material property, can be adjusted using equation (5.3) to fit experimental data $\lambda^{d}(T)$ with the DDM model. Second, the temperature-dependent properties $E_{m}$ and $\nu_{m}$ can be easily obtained using PMM [290]. And third, the stress $\sigma$ at which the first crack begins to propagate can be calculated by DDM. Therefore, crack propagation depends on the probability that initial flaws already existing in the material reach a critical size that can be estimated as follows

$$
a \geq a_{c}=\frac{1}{\pi} \frac{G_{I c} E_{m}}{\left(1-\nu_{m}^{2}\right)\left(\sigma_{i n i}\right)^{2}}
$$

where $\sigma_{i n i}$ is the stress for crack density $\lambda_{i n i}$, which is the crack density at $N=1$, and $\beta=1$ because DDM accounts for geometric effects. Therefore, crack densities measured at the end of the first cycle are assumed to come from initial flaws or voids whose size is greater than the critical size $a_{c}$.

Under thermal fatigue, the fatigue resistance $f\left(N, R_{T}\right)$ may be interpreted as the capacity of material to nucleate new flaws or increase the size of defects $a$ already in the material under certain loading conditions and thermal ratio $R_{T}$. As it can be seen in Figure 5.3, $f(N)$ decreases with number of cycles so that the critical $G_{I c}$ in (5.4) decreases, allowing the model to predict higher crack densities for higher number of cycles $[10,188,189]$. In other words, $f(N)$ can be interpreted as an analytical parametrization to reduce the $G_{I c}$ needed to propagate new cracks. Note that the critical $G_{I c}^{\prime}\left(T_{\min }\right)$ is a material property and thus, it cannot change with number of cycles, but $f(N)$ can.

From a theoretical point of view, the nucleation and growth of existing crazes developed in crystalline polymers (Epoxy at low temperatures) depends on the local stress state given by distortion and hydrostatic pressure. Furthermore, the stress distribution at the crack tip trend to infinity as shown in [274]. Therefore, as long as the number of cycles increase, even under low thermal cyclic loads, new cracks are expected to propagate when their initial length reaches the critical value $a_{c}$ given in (5.8).

In order to illustrate the growth of initial flaw size $a$ as function of number of cycles $N$, the following scenario is presented. Given a LSS subjected to low $R_{T}$, transverse damage initiation occurs at first cycle if $G_{I} \geq G_{I c}$. If such condition is not satisfied, large number of cycles must be performed until cracks start to propagate as reported in $[23,296]$. Therefore, since $G_{I c}^{\prime}\left(T_{\min }\right)$ is a material property and the stress state remains constant for fixed $R_{T}$, new cracks will propagate only when their initial flaw length reach a critical value $a_{c}$ given by (5.8). This means that void nucleation during the first few thermal 
cycles may be required.

As the number of cycles increases and new cracks propagate in the laminate, the material undergoes stiffness degradation and consequent reduction of the transverse stress $\sigma_{22}$ between two neighbor cracks, represented by $\sigma$ in equation (5.7). Operating with equation (5.8) and assuming $G_{I c}$ and the temperature-dependent moduli at $T_{\min }$ to be invariants for a fixed $R_{T}$, a lower $\sigma_{22}$ would require a larger flaw size $a_{c}$ to propagate a new crack as expressed below

$$
\sigma_{22}=\sqrt{\frac{G_{I c}^{\prime}\left(T_{\min }\right) K}{a_{c}}} ; \quad K=\frac{E_{m}\left(T_{\min }\right)}{\pi\left(1-\nu_{m}\left(T_{\min }\right)\right)}
$$

where $\mathrm{K}$ depends on the material properties and temperature.

Since $G_{I c}^{\prime}\left(T_{\min }\right)$ is a material property, $K$ is a constant, and $a_{c}$ is unknown, equation (5.8) is rewritten as

$$
\sigma_{22}=\sqrt{G_{I c}^{\prime}\left(T_{\min }\right) K f(N)}
$$

and $f(N)$ is adjusted so that predicted crack density $\lambda(N)$ fits experimental data $\lambda_{i}(N)$.

Comparing equation (5.9) and (5.10), the fatigue resistance $f(N)$ is inversely proportional to critical size $\propto 1 / a_{c}$. While is true that $a_{c}(N)$ is unknown, $\lambda$ increases with number of cycles $\mathrm{N}$, causing stiffness degradation and thus, leading to lower $\sigma_{22}$ between two neighbor cracks (stress relation). According to equation (5.9), existing flaws under lower $\sigma_{22}$ propagate only if a larger flaw size $a_{c}$ is reached. Therefore, $a_{c}(N)$ increases leading to lower fatigue resistance $f(N)$ in the range $1<f(N)<0$ as shown in Figure 5.3.

\subsection{Mode II ERR effect}

Fatigue resistance $f(N)$ for laminate $[0 / \pm 45 / 90]_{S}$ P75/1962 is reported in Figure 5.4 separately for $45^{\circ},-45^{\circ}$, and $90_{2}^{\circ}$ laminas. The $f(N)$ functions obtained from each lamina fit well by SLR in (5.6). However, $f(N)$ seems to degrade more for the $-45^{\circ}$ lamina with steepest slope $\beta_{1}$. A data point at $N=500$ cycles is found to be an outlier based on both Cook's distance and DFFITS [291,292]. The critical $G_{I c}$ values in (5.5) needed for SLR are obtained by minimizing the error D (5.3) at discrete number of cycles $\mathrm{N}$ for which experimental data $\lambda^{d}$ is available in [10]. No data for exterior $0^{\circ}$ lamina is available.

For a given LSS, all laminas are subjected to same thermal ratio $R_{T}$ and number of cycles. Hence, the differences on $f(N)$ could be affected by two factors: the cracking mode and the local stress state.

Regarding the cracking mode, the propagation of cracks can be represented by mode I (crack opening), mode II (crack shear), or interacting mix-mode I and II. Since quasi- 


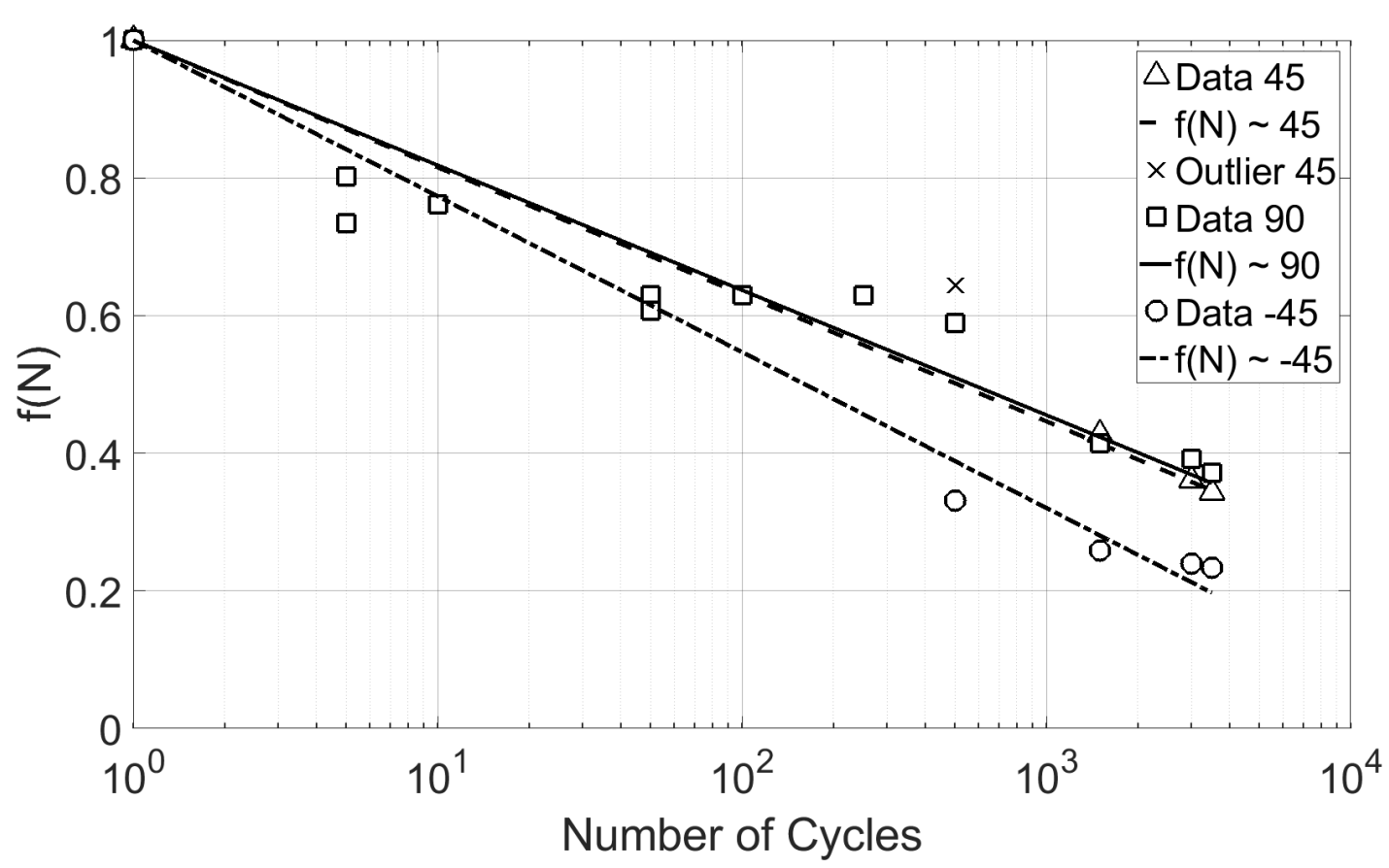

Figure 5.4: Fatigue resistance as function of number of cycles for P75/1962 $[0 / \pm 45 / 90]_{S}$ with $R_{T}=-156 / 121$. Experimental data is collected for middle $90_{2}^{\circ}$ and interior $\pm 45^{\circ}$ laminas.

isotropic laminates contain laminas with $\pm \theta$ orientation, they are candidate to generate transverse cracking in a mix-mode I and II unlike cross-ply laminates. In order to illustrate this, a comparison between $G_{I}$ and $G_{I I}$ obtained during one thermal cycle for P75/1962 $[0 / \pm 45 / 90]_{S}$ in the range $\left[-156,121^{\circ} \mathrm{C}\right]$ is shown in Figure 5.5.

As it can be seen in Figure 5.5, the $G_{I I}$ is zero. Therefore, transverse cracking in quasi-isotropic laminates subjected to thermal fatigue (free expansion) depends only on mode I regardless of lamina orientation. In addition, laminas located at the middle plane $\left(90_{2}^{\circ}\right)$ and surface $\left(0^{\circ}\right)$ crack earlier than other laminas due to their thickness. That is, $90_{2}^{\circ}$ ply has double thickness (Figure 5.6.a) while the local 2D displacements field $\left(u_{i}\right)$ of $0^{\circ}$ surface ply behaves as a lamina with double thickness because it is on a free surface (Figure 5.6.b). While this may be not the real solution, it is a close solution.

On the other hand, the onset and growth of crazes developed in crystalline polymers (low temperatures) depends on the local stress state being highly affected by the principal stresses such as $\sigma_{22}$, i.e. a crack opening mode. Hence, $f(N)$ could be affected by local stress state during thermal fatigue. If this is truth, each lamina may require an different $f(N)$.

Since quasi-isotropic laminates contain laminas in $\pm \theta$ orientation, they are candidates to be subjected to complex stress states. To illustrate this, a comparison between $\sigma_{22}$ of each lamina obtained during one thermal cycle for P75/1962 $[0 / \pm 45 / 90]_{S}$ in the range $\left[-156,121^{\circ} \mathrm{C}\right]$ is shown in Figure 5.7. 


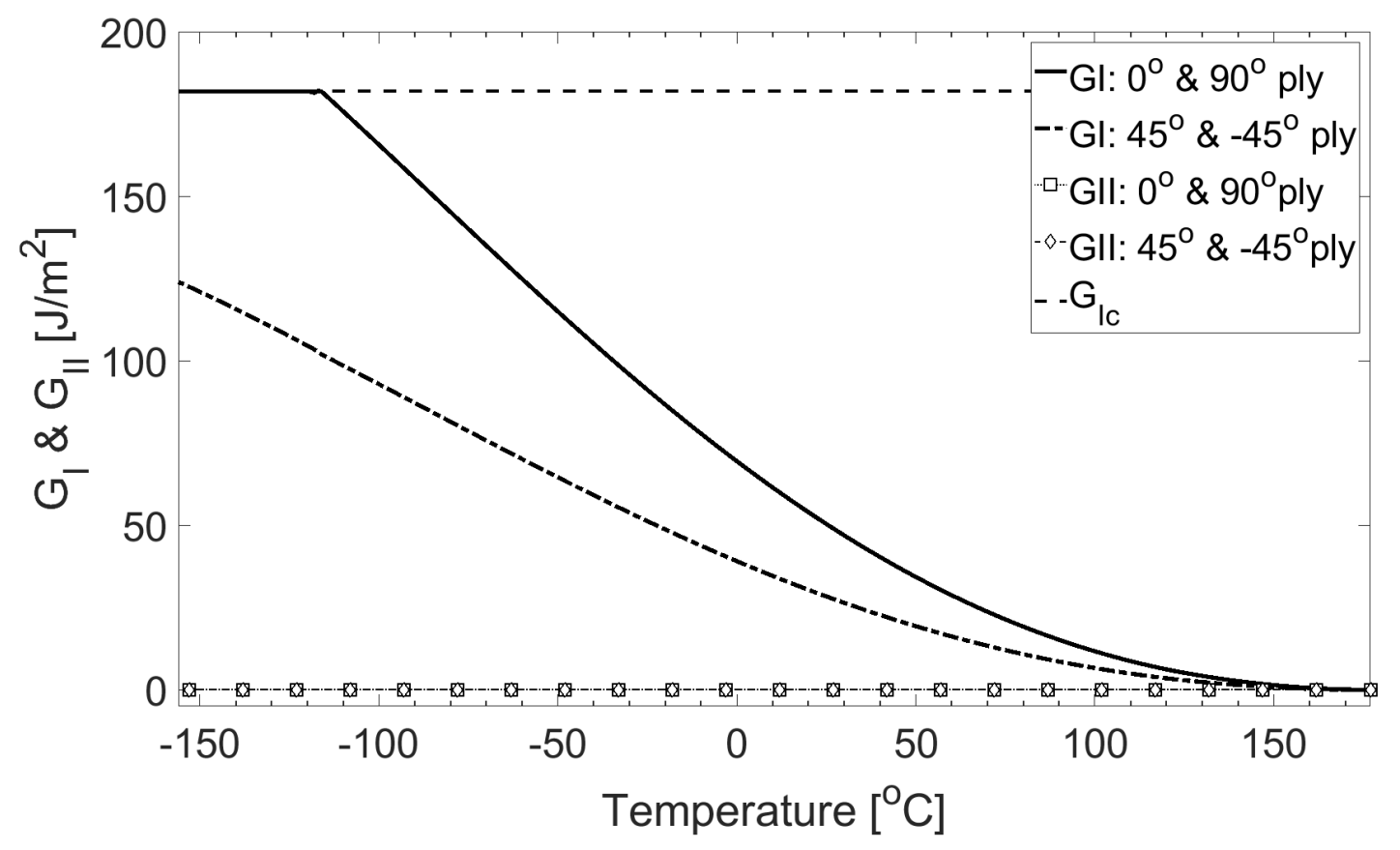

Figure 5.5: $E R R G_{I}$ and $G_{I}$ vs. temperature during one thermal cycle for P75/1962 [0/ \pm $45 / 90]_{S}$ in the range $\left[-156,121^{\circ} \mathrm{C}\right]$. SFT is $177^{\circ} \mathrm{C}$ [12].

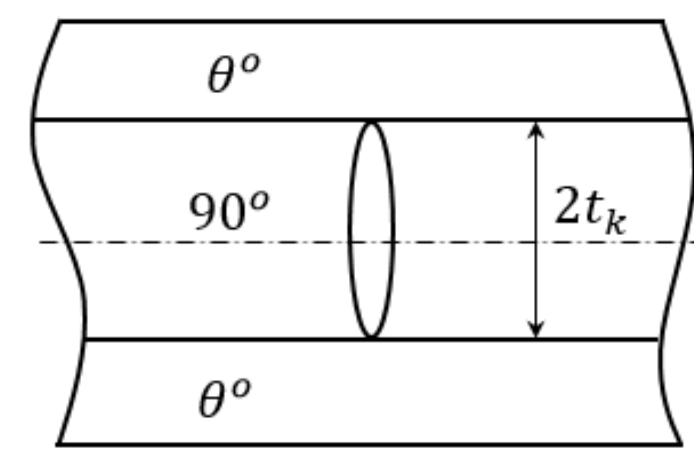

(a)

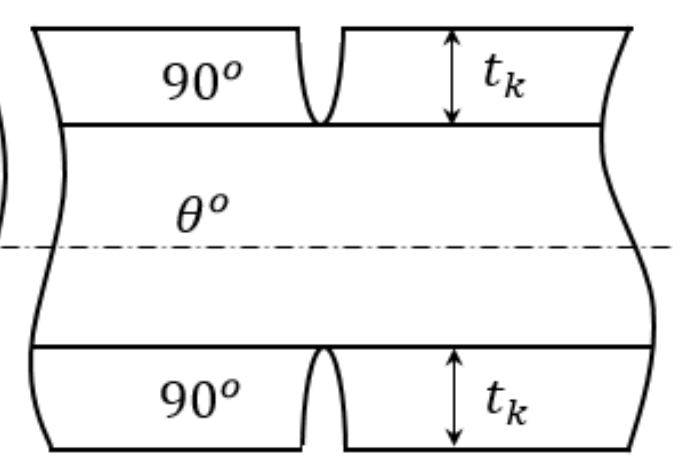

(b)

Figure 5.6: Representative 2D displacement crack field to illustrate the double thickness effect. 


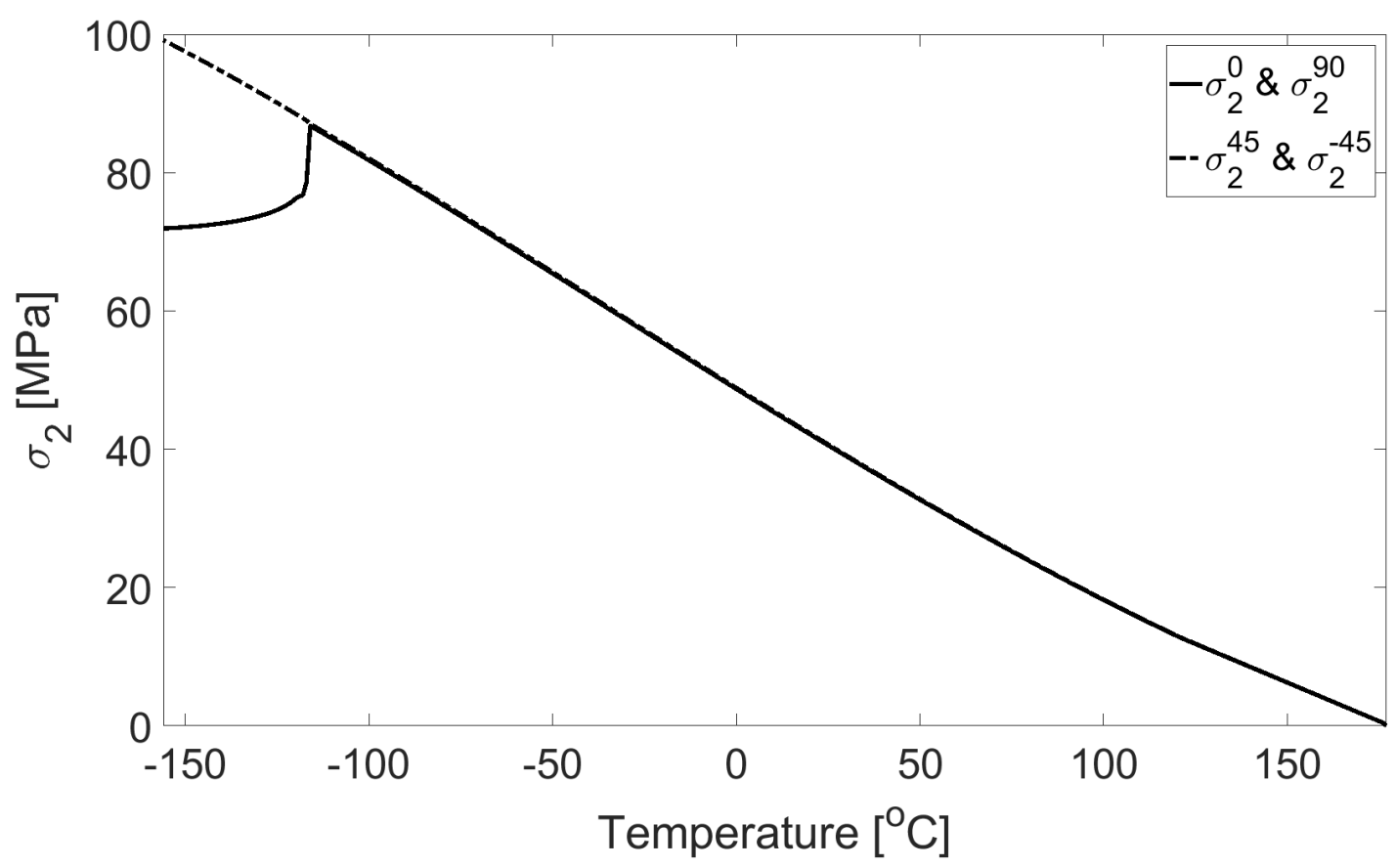

Figure 5.7: Transverse stress $\sigma_{22}$ vs. temperature during one thermal cycle for P75/1962 $[0 / \pm 45 / 90]_{S}$ in the range $\left[-156,121^{\circ} \mathrm{C}\right] . S F T$ is $177^{\circ} \mathrm{C}[12]$.

As it can be seen in Figure 5.7, all laminas are subjected to the same $\sigma_{22}$ regardless of lamina orientation. That is, there is not a reference coordinate system on laminates subjected to free thermal expansion. However, the $G_{I}$ in $0^{\circ}$ and $90_{2}^{\circ}$ laminas reach the critical ERR $G_{I c}^{\prime}$ before the thermal cycle has been completed and thus, they crack relaxing $\sigma_{22}$, which decreases as shown in Figure 5.7.

Although the stress state is virtually the same regardless of lamina orientation, $0^{\circ}$ and $90_{2}^{\circ}$ laminas crack earlier than other laminas leading to different $\sigma_{22}$ between laminas over the entire thermal load. Therefore, this stress gap may induce different $f(N)$ for each lamina as shown in Figure 5.4 for $-45^{\circ}$ lamina. However, $f(N)$ in $\pm \theta$ laminas should go together because $G_{I}$ for both laminas is identical during cooling and they crack simultaneous. In addition, stress free-edge may affect $f(N)$ in $\pm \theta$ laminas and thus, it must be considered.

\subsection{Free-edge stress analysis}

Since crack density is measured from edge, edge effects may distort the data for some laminas. In this section, the free-edge stresses of composite laminates are obtained through finite element analysis (FEA). The results are used to explain the disparity of $f(N)$ between $-45^{\circ}$ and the rest of the laminas in Figure 5.4.

The intralaminar stresses at free-edge are computed using a 3D FEA to evaluate its effect on transverse cracking. Furthermore, the laminate stacking sequence (LSS) will 
Tables 5.1: Cubic temperature-dependent properties of P75/1962 $\left(V_{f}=0.52\right.$ [10]) between $[-156,121]^{\circ} \mathrm{C}$. Temperature range for $G_{I c}$ is $[-156,-15]^{\circ} C$.

\begin{tabular}{cccc}
\hline \multicolumn{4}{c}{ Temperature dependent properties } \\
\hline Property & $P^{a}$ & $P^{b}$ & $P^{c}$ \\
\hline$E_{1}[\mathrm{MPa}]$ & 271270.586 & -8.1099 & $1.1894 \mathrm{E}-02$ \\
$E_{2}[\mathrm{MPa}]$ & 6554.2638 & -11.6689 & $4.9329 \mathrm{E}-04$ \\
$G_{12}[\mathrm{MPa}]$ & 3998.0213 & -8.8436 & $6.1187 \mathrm{E}-03$ \\
$\nu_{12}$ & 0.3147 & $-6.9707 \mathrm{E}-05$ & $-4.0521 \mathrm{E}-07$ \\
$\nu_{23}$ & 0.5557 & $-1.009 \mathrm{E}-04$ & $-1.1402 \mathrm{E}-06$ \\
$\alpha_{1}^{\text {tangent }}\left[10^{-6} / \mathrm{C}\right]$ & -0.9767 & $1.5237 \mathrm{E}-04$ & $-8.9154 \mathrm{E}-06$ \\
$\alpha_{1}^{\text {tangent }}\left[10^{-6} / \mathrm{C}\right]$ & 38.4688 & $8.9483 \mathrm{E}-02$ & $-3.6463 \mathrm{E}-04$ \\
$G_{I c}\left[\mathrm{~J} / \mathrm{m}^{2}\right]$ & 77.8054 & $9.6211 \mathrm{E}-02$ & $1.3948 \mathrm{E}-03$ \\
\hline
\end{tabular}

be studied under three configurations: $\left[(0 / 90)_{2}\right]_{S},[0 / \pm 45 / 90]_{S}$, and $[0 / 45 / 90 /-45]_{S}$. The temperature range $\left[-156,121^{\circ} \mathrm{C}\right]$ is selected because it induces the largest thermal stresses. The stress-free temperature (SFT) is chosen to be the polymerization one at $177^{\circ} \mathrm{C}$, as shown in Figure 5.8. A constant distribution of temperature through the specimen is assumed because experimental data tests are performed heating/cooling at low constant rate $(\approx 20$ minutes/cycle) $[10,188,189]$. The temperature-dependent properties of Amoco P75/1962 are shown in Table 5.1 represented by a quadratic polynomial [12]. Ply thickness is taken to be constant with a value equal to $0.127 \mathrm{~mm}$.

A square laminate with dimensions large enough was chosen to avoid any interactions at the edge. Far away from the edge, classic laminate theory is valid and thus, the intralaminar stresses at each lamina can be computed using DDM model. Since DDM predicts transverse cracking in some laminas during a thermal cycling as shown in Figures 5.5 and 5.7, a 2D FEA simulation is performed to obtain the transverse thermal stresses $\sigma_{22}$ of an undamaged specimen. The transverse thermal stresses induced in the laminate using the 2D simulation are found to be equal in each lamina regardless the LSS (free thermal expansion). Such thermal stresses are maximum at $-156^{\circ} \mathrm{C}$ (98.65 $\left.\mathrm{MPa}\right)$ and minimum at $121^{\circ} \mathrm{C}(12.85 \mathrm{MPa})$, as shown in Figure 5.8. The 2D FEA results match perfectly with DDM results for \pm 45 laminas, for which no cracks are generated as shown in Figure 5.7. No shear stresses are obtained and thus, only crack propagation in mode $\mathrm{I}$ is generated, as noted in Section 5.5.

In order to obtain edge effects on the intralaminar in-plane stresses, a 3D FEA of an undamaged laminate composite subjected to free thermal expansion is performed. A square laminate was selected to be represented by 3D solid elements with dimensions: $0 \leq x \leq 2 a, 0 \leq x_{2} \leq 2 b$, and $0 \leq x_{3} \leq 2 H$. The thickness $H$ is the sum of each lamina thickness corresponding to laminate's half, $H=\sum_{1}^{N} t k_{i}$. The size ratio of the composite is rather large $(a / H \geq 10)$ to avoid any interactions at the edge. Only one-eighth of 


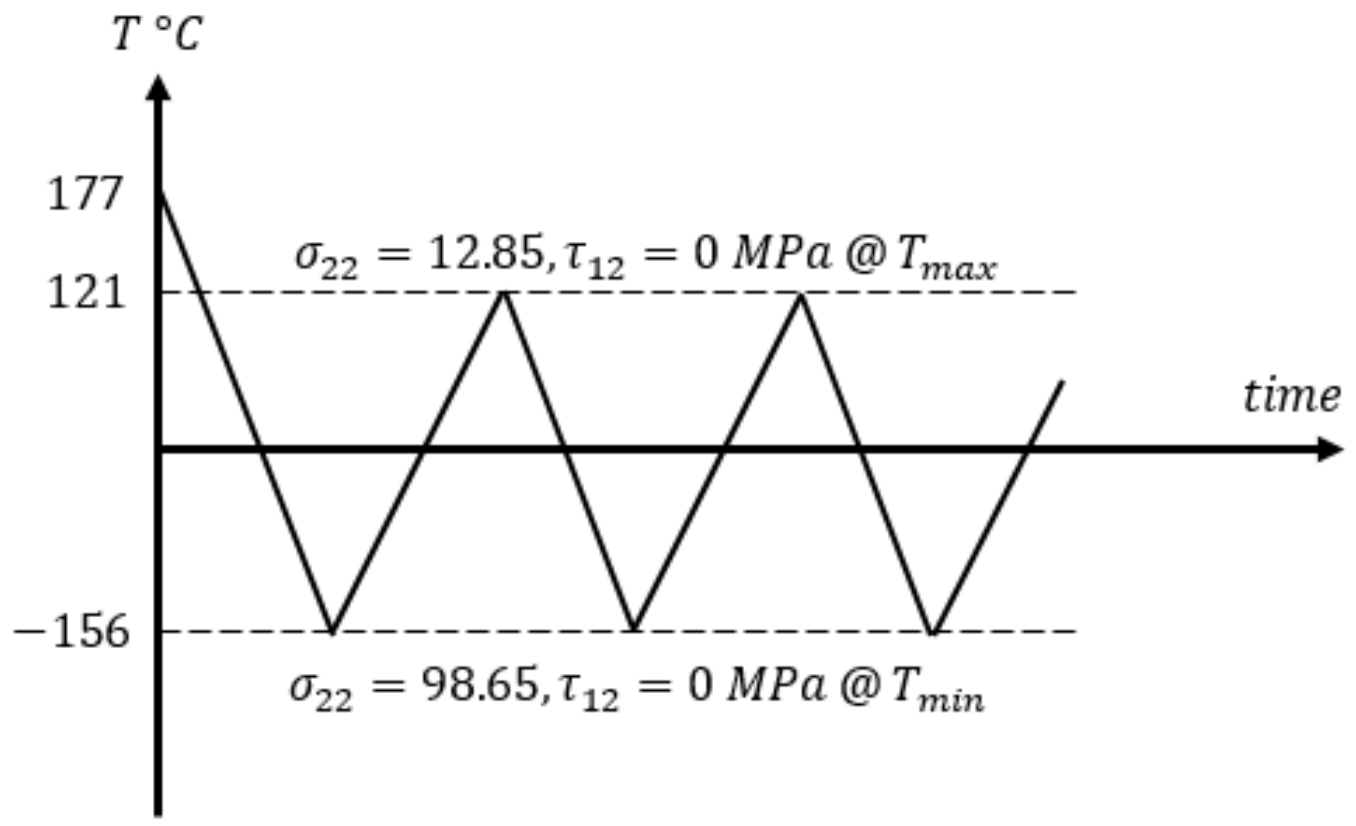

Figure 5.8: Representative thermal fatigue test with time in the temperature range $\left[-156,121^{\circ} \mathrm{C}\right]$. The thermal stresses are calculated from SFT at $177^{\circ} \mathrm{C}$.

the laminate is modeled due to symmetry with respect the planes of symmetry: $X$ sym, Y sym, and Zsym, as shown in Figure 5.9.

To satisfy the isostrain assumption in the laminate, a set of reference nodes $\left(R N_{i}\right)$ are selected at each lamina, i.e. at $h_{i}=[N t k,(N-1) t k, \ldots, t k]$. These $R N_{i}$, which are located at edge $\left(a, b, h_{i}\right)$ to avoid over constraining (Figure 5.9), are used to couple the DOF in $x_{1}$ and $x_{2}$ directions with respect both faces of the laminate described by the planes $I s o^{x}$ and $I s o^{y}$ as shown in Figure 5.9. Note that nodes located at the freeedge between laminas must be free to move without restrictions, otherwise interlaminar stresses would be imposed. Furthermore, symmetric laminates under thermal stress do not undergo curvature and thus, all nodes that belong to surfaces defined by $x_{3}=h_{i}$ remain tied at the same plane denoted by $K_{u r_{h_{i}}^{z}}^{z}$ in Figure 5.9. Since isostrain conditions couple the $x_{1}$ and $x_{2}$ directions simultaneously, a master node $(M N)$ located at point $(a, b, H)$ as shown in Figure 5.9, is selected to couple the DOF of $R N_{i}$ in $x_{1}$ and $x_{2}$ directions. In this way, $M N$ is free to move enforcing continuity of displacements over the entire laminate. The BCs are shown in Table 5.2.

The temperature-dependent properties of Amoco P75/1962, are defined as a set of $\mathrm{N}$ temperature-property data pairs as (T1; P1), (T2; P2),..., (TN; PN). The values are obtained from Table 5.1. These values are discretized with $\Delta T=1^{\circ} C$ to simplify the computations. Outside the range [-156,121], for which experimental data is not available, the properties of the laminate are assumed to be constant and equal to the last data pair. 

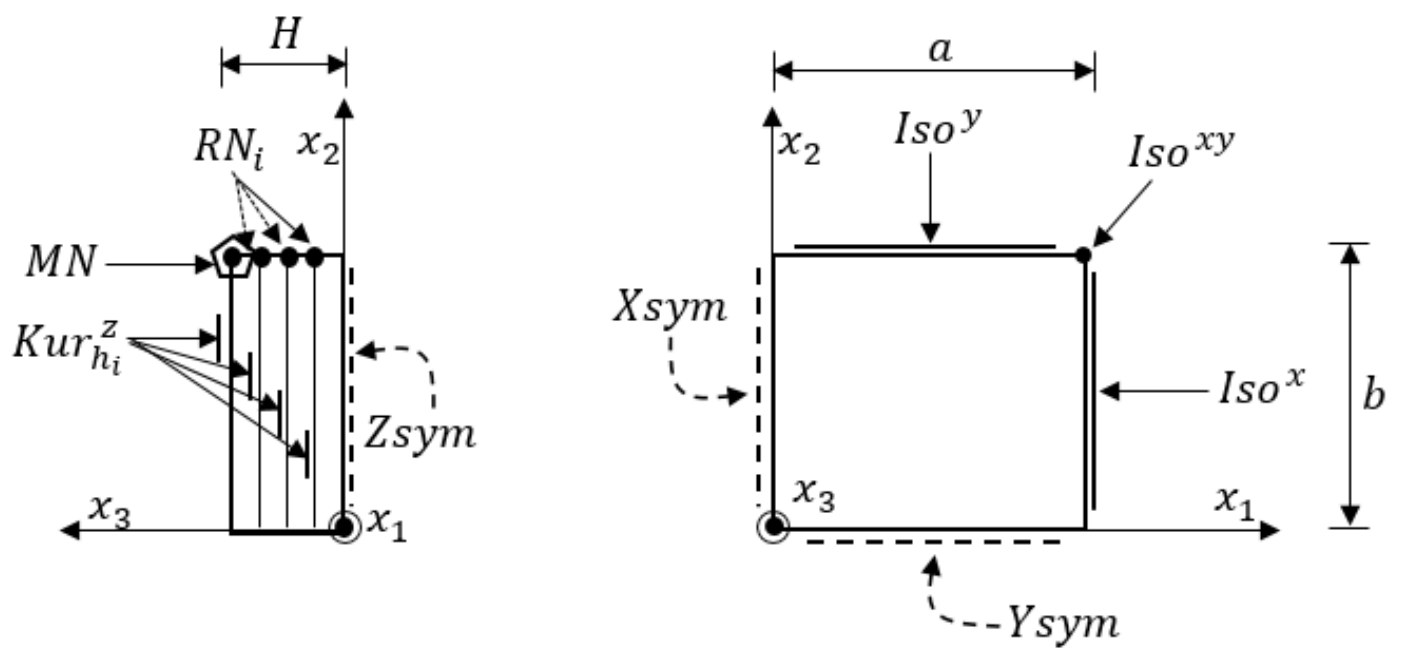

Figure 5.9: Front and side draw views from $3 D$ finite element modelling.

Tables 5.2: BCs for a 3D laminate simulation using solid elements C3D20R.

Symmetry BCs:

Plane $X \operatorname{sym}\left(x_{1}=0\right)$ :

Plane $Y \operatorname{sym}\left(x_{2}=0\right)$ :

Plane $Z \operatorname{sym}\left(x_{3}=0\right)$ :

Isostrain \& Curvature BCs:

Plane $\operatorname{Iso}^{x}\left(x_{1}=a\right)$ :

Plane $\operatorname{Iso}^{y}\left(x_{2}=b\right)$ :

Planes $\operatorname{Kur}_{h_{i}}^{z}\left(x_{3}=h_{i}\right)$ :
Constraint equations

$$
\begin{aligned}
& u_{1}\left(0, x_{2}, x_{3}\right)=0 \\
& u_{2}\left(x_{1}, 0, x_{3}\right)=0 \\
& u_{3}\left(x_{1}, x_{2}, 0\right)=0
\end{aligned}
$$

Constraint equations

$$
u_{1}\left(a, x_{2}, h_{i}\right)=u_{1}^{R N}\left(a, b, h_{i}\right)
$$$$
u_{2}\left(x_{1}, b, h_{i}\right)=u_{2}^{R N}\left(a, b, h_{i}\right)
$$$$
u_{3}\left(x_{1}, x_{2}, h_{i}\right)=u_{3}^{R N}\left(a, b, h_{i}\right)
$$

$u_{i}^{R N}\left(a, b, h_{i}\right)=u_{i}^{M N}(a, b, H) ; i=1,2$.

FEA analysis was performed with Abaqus 6.14, using small displacements, linear elastic material, and quadratic 3D elements C3D20R reduced integration. The mesh is refined in areas close to the edge where free-edge effects may appear. Each lamina thickness is modeled with four C3D20R elements to capture with accuracy the interlaminar stresses as well as the Poisson's effect. The laminate is subjected to a thermal load from SFT $\left(177^{\circ} \mathrm{C}\right)$ up to $-156^{\circ} \mathrm{C}$. The intralaminar stresses are obtained from the core (planes of symmetry) to the free-edge.

Transverse $\left(\sigma_{22}\right)$, longitudinal $\left(\sigma_{11}\right)$, and shear $\left(\tau_{12}\right)$ thermal stresses from the freeedge $(x=0)$ are shown in Figure 5.10 for a cross-ply P75/1962 laminate and stacking sequence $\left[(0 / 90)_{2}\right]_{S}$. Since the laminate is only subjected to a thermal load from SFT to $-156^{\circ} \mathrm{C}$, the intralminar stresses match both, the CLT values given by DDM in Figure 5.7 (undamaged \pm 45 laminas) and the $2 \mathrm{D}$ FEA simulation whose results are shown in Figure 5.8. Taking into account the complex BCs described in Table 5.2, the 3D simulation is considered to be validated.

Looking into Figure 5.10, some inferences can be made. First, the transverse stress $\sigma_{22}$ in cross-ply laminates is affected by the free-edge, and it is independent of orientation. 
The $\sigma_{22}$ increases about $14 \%$ at the free-edge but it vanishes to a distance approximately $0.8 \mathrm{~mm}$ from edge. Similar results are given in [24] for $90^{\circ}$ laminas. However, no freeedge effects in [24] are shown in $0^{\circ}$ laminas even though the laminate is subjected to free thermal expansion and all laminas should be expand the same. As it can be seen in Figure 5.10, all laminas are subjected to the same strain and thus, $\sigma_{22}$ is the same with regard the orientation, $0^{\circ}$ and $90^{\circ}$.

Second, the longitudinal stress $\sigma_{11}$ must satisfy the free-edge stress condition $\left(\sigma_{11}=0\right.$ at $x=0$ ) as shown in Figure 5.10. And third, the free-edge effect over the shear stresses $\tau_{12}$ is negligible during the whole thermal cycle as shown in Figure 5.10.

The $\tau_{12}$, which tends to zero near the free edge, are the same and of opposite sign with respect $0^{\circ}$ and $90^{\circ}$ laminas, respectively. Therefore, cracks propagate from edge to center of the plate and fatigue resistance $f(N)$ can be obtained by counting crack densities at the edge in case of cross-ply laminates regardless of lamina orientation.

\section{Longitudinal and Transverse stress}
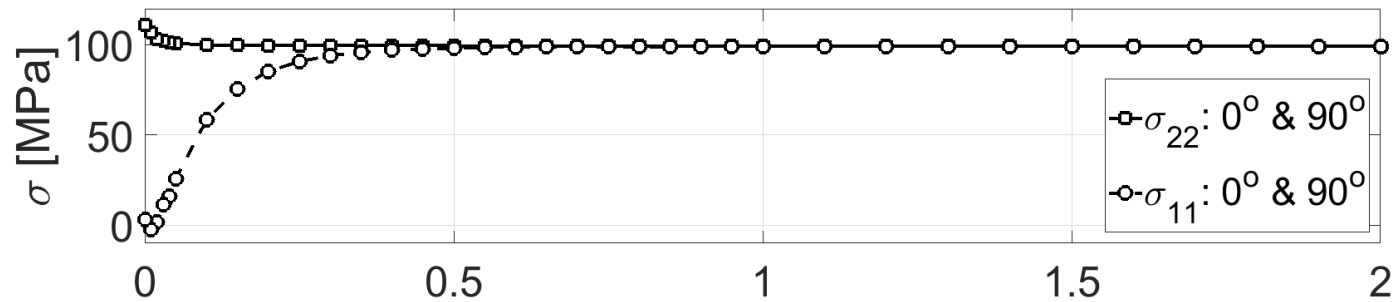

Distance from edge [mm]

Shear stress

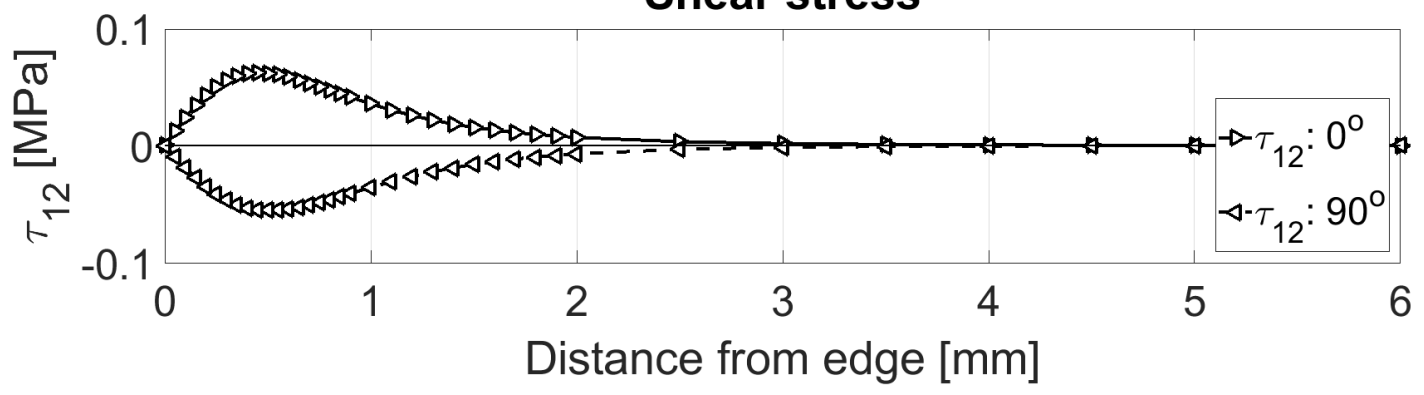

Figure 5.10: Longitudinal, transverse and shear free-edge stresses at $-156^{\circ} \mathrm{C}$ for P75/1962 and LSS: $\left[(0 / 90)_{2}\right]_{S}$.

Intralaminar thermal stresses $\sigma_{22}$ and $\tau_{12}$ vs. edge's distance are plotted in Figure 5.11 for angle-ply P75/1962 laminates $[0 / \pm 45 / 90]_{S}$ and $[0 / 45 / 90 /-45]_{S}$ similar as those given in [27]. As shown in Figure 5.11, the transverse stress $\sigma_{22}$ depends on the lamina orientation $\left(90^{\circ}\right.$ or $\left.\pm 45^{\circ}\right)$ and thus, the stress distribution is affected by the free-edge. Same results are obtained for both LSS. In $90^{\circ}$ laminas, $\sigma_{22}$ increases almost a $20 \%$ at the free-edge whereas in $\pm 45^{\circ}$ laminas, $\sigma_{22}$ falls sharply off on the edge about $70 \%$. This means that cracks, which initially start at the edge in $90^{\circ}$ laminas, propagate towards the plate's core. Hence, crack densities measured form edge can be used to predict well 

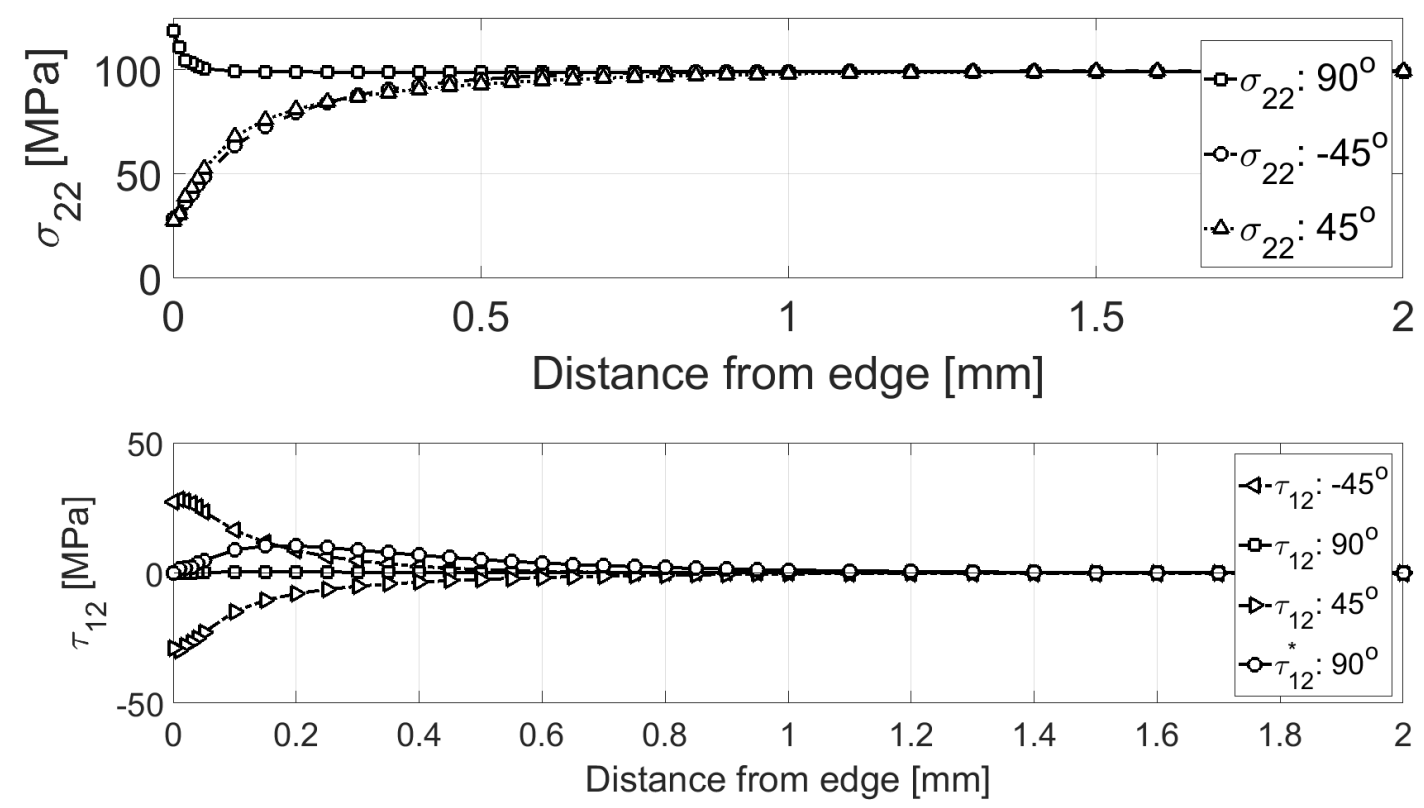

Figure 5.11: Transverse and shear free-edge stresses at $-156^{\circ} \mathrm{C}$ for P75/1962 and LSS: $[0 / \pm 45 / 90]_{S}$ and $[0 / 45 / 90 /-45]_{S}$ denoted by symbol $(*)$.

fatigue resistance $f(N)$.

However, cracks counted from edge in $\pm 45^{\circ}$ laminas is a wrong praxis because cracks, which initially start inside the lamina may not propagate to the edge. On the other hand, the shear stresses $\tau_{12}$ are lower than $\sigma_{22}$.

For $90^{\circ}$ laminas, $\tau_{12}$ represents about $11 \%$ of $\sigma_{22}$ and thus, an interacting mode I and II may enhance crack propagation enhancing crack density. For $[0 / 45 / 90 /-45]_{S}$, no shear stresses $\left(\tau_{12}^{*}\right)$ are obtained and only a crack-opening mode I $G_{I}$ is generated. However for $\pm 45^{\circ}$ laminas, $\tau_{12}$ is about same order of magnitude with respect $\sigma_{22}$ (both $\approx 30 \mathrm{MPa}$ ) and a pure interacting mode I and II is obtained. This interaction mode in $\pm 45^{\circ}$ laminas may enhance the crack propagation from the free-edge similar as those in $90^{\circ}$ laminas. This fact would explain the reason why $f(N)$ in $\pm 45^{\circ}$ laminas is sometimes predicted well obtaining similar results as those back calculated in $90^{\circ}$ laminas as shown in Figures 5.4 and 5.12.

Based on results shown in Figure 5.10 and 5.11, crack density data in $90^{\circ}$ laminas is considered to be the best lamina orientation to adjust $f(N)$. For $\pm 45^{\circ}$ laminas, an interacting mode I and II is obtained at the free-edge which vanishes to distance $\approx 1.4$ $\mathrm{mm}$. Therefore, experimental crack density data in $\pm 45^{\circ}$ laminas is not recommended as good praxis to obtain $f(N)$ unless an alternative method such as X-ray or acoustic emissions is used. Only in those cases where $45^{\circ}$ lamina is embedded in the middle, namely double thickness, crack propagation may approach those in $90^{\circ}$ laminas. 


\subsection{Temperature range effect}

The influence of thermal ratio on fatigue resistance $f(N)$ is studied in this section under several temperature ranges of interest such as Geostationary Earth Orbit (GEO[-156,121 $\left.{ }^{\circ} \mathrm{C}\right]$ ), Low Earth Orbit (LEO[-101,66 $\left.{ }^{\circ} \mathrm{C}\right]$ ), and Thermally Controlled Orbit (TCO[-46,10 $\left.\left.{ }^{o} \mathrm{C}\right]\right)$.

In order to study the thermal ratio effect $R_{T}$, the function $f(N)$ for Amoco P75/1962 $[0 / 45 / 90 /-45]_{S}$ laminate at three different temperature ranges $\left[-156,121{ }^{\circ} \mathrm{C}\right],[-101,66$ $\left.{ }^{\circ} \mathrm{C}\right]$, and $\left[-46,10^{\circ} \mathrm{C}\right]$ is shown in Figure 5.12. The fatigue resistance function $f(N)$ is expected to degrade faster for higher temperature ranges because the residual thermal stresses at each temperature $T_{i}$ are calculated from $S F T$ as $\alpha_{i} \Delta T_{i}=\alpha_{i}\left(T_{i}-S F T\right)$. Therefore, larger thermal stresses are obtained at lower temperatures, which coincidentally have higher crack density data as reported in $[10,188,189]$. This effect can be seen in Figure 5.12 where the slope become steeper as temperature range increases, specially for $90^{\circ}$ laminas. However, a disagreement can be seen in $f(N)$ looking at $\pm 45^{\circ}$. Ideally, $f(N)$ adjusted from $\pm \theta$ laminas should not present differences with respect $90^{\circ}$ laminas because thermal (cooling) loading has no preferential orientation and thus, all laminas in cross-ply (CP) and quasi-isotropic (QI) laminates are subjected to similar thermal stress. However, differences in $\sigma_{22}$ with respect to $90^{\circ}$ laminas and edge effects in $\pm \theta$ laminas may induce discrepancies as shown in Figure 5.12. Such differences are more pronounced in thinner $45^{\circ}$ laminas compared with thicker $-45_{2}^{\circ}$ laminas as it was commented in Section 5.6. Actually, $f(N)$ values looks to be virtually the same between $-45_{2}^{o}$ and 90 laminas at higher and medium temperature range.

In addition, the temperature range effect with $R_{T}=-46 / 10$ in $f(N)$ presented in Figure 5.12 is not clear. On one hand, the adjusted $f(N)$ by using $45^{\circ}$ lamina seems to be weaker than those at higher temperature range, e.g. $\left[-101,66{ }^{\circ} C\right]$. Contrary to the expectations, fatigue resistance $f(N)$ seems to be more severe in the range $[-46,10$ ${ }^{\circ} C$ ] where lower thermal stresses are generated. And on the other hand, $f(N)$ seems to apparently follows another relation at low temperatures rather than equation (5.6). Instead, it fits well with classical linear function as follows

$$
y=\beta_{1} N+1
$$

Therefore, to study the temperature range effect an alternative method must be developed. Only data for thicker laminas seem to be consistent because higher crack nucleations are developed for higher temperature ranges whereas nonsense results are obtained for thinner laminas. Therefore, the influence of LSS should be studied in greater depth, for example using Paris law. In addition, $f(N)$ shape function disagree at low temper- 
atures (e.g. $\left.R_{T}=-46 / 10\right)$ and no conclusion can be inferred from the experimental data.

\subsection{Paris Law}

In this section both laminate stacking sequence (LSS) and temperature range effects on fatigue resistance $f(N)$ are studied using Paris's law. Crack density vs. number of cycles $\lambda(N)$ for three values of thermal ratio $R_{T}$ are shown in Figure 5.13. Crack density saturation (CDS) reached for low number of cycles and its value is significant lower than for mechanical fatigue or static tests both of which reach $C D S \approx 1 / t_{k}$.

Similar to fatigue damage in metals, a Paris's law to predict transverse cracking evolution under thermal or mechanical cycling loads for an arbitrary layup was initially proposed in [297]. The ERR range $\Delta G$ can be used instead of the stress intensity factor range $\Delta K$ and crack density $\lambda$ instead of crack length $a$.

Unlike metals, laminated composites do not fail by growth of a single matrix crack. Instead, the crack growth rate $a$ is expressed in terms of $\lambda$ that release the same total stored elastic energy as a single large crack. Based on [298], quantum fracture mechanics is used in this work to substitute $G_{I}(\lambda, T)$ by the mean value

$$
G_{I}(\lambda, \Delta \lambda, T)=\sqrt{\left\langle G^{2}(\lambda, T)\right\rangle_{\lambda}^{\lambda+\Delta \lambda}}
$$

where $\langle\cdot\rangle=(1 / \Delta \lambda) \int_{\lambda}^{\lambda+\Delta \lambda} \cdot d \lambda$. Therefore, Paris's law is expressed using (5.12) as follows

$$
\frac{d \lambda}{d N}=A \Delta G_{I}(\lambda, T)^{\alpha}
$$

for a given temperature range $\left[T_{\min }, T_{\max }\right]$, and where $A$ and $\alpha$ are two power fitting material parameters for a specific material system.

Several researchers studied the relationship between transverse microcracks growth rate and ERR to evaluate fatigue damage under thermal [179, 297,299] and mechanical [166,300] loads. Most of studies obtain reasonable results using an energetic fracture mechanic method such as variational approach [179,299,301] or a 2D shear-lag analysis [23]. However, the calculation of ERR in both methodologies involves the adjustment of additional material parameters and/or experimental tests to calculate average transverse stress to be compared with the ultimate lamina strength, which in turn depends on the thickness and LSS (in-situ strength).

In contrast, in this study the ERR $G_{I}$ is calculated using DDM model which does not need the adjustment of any material parameter beyond $G_{I c}$. Furthermore, the lamina 

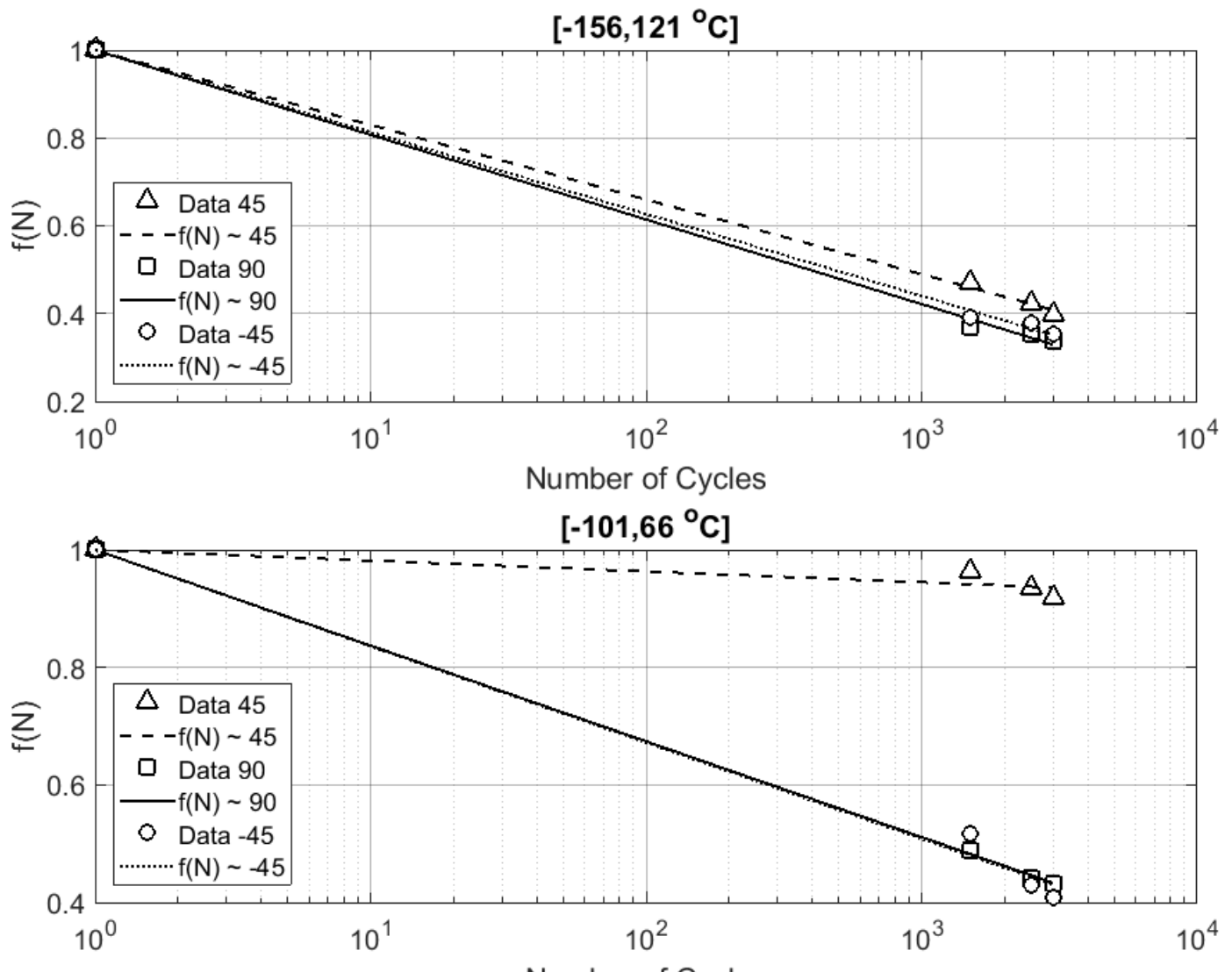

Number of Cycles

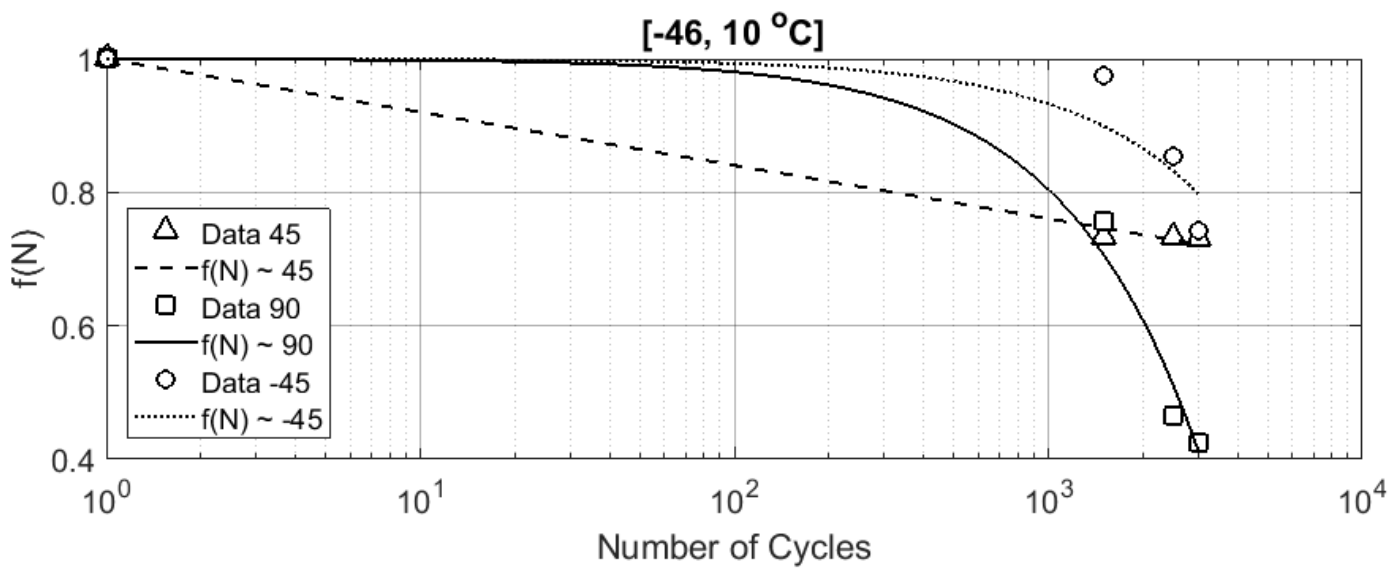

Figure 5.12: Fatigue resistance $f(N)$ as function of number of cycles for P75/1962 $[0 / 45 / 90 /-45]_{S}$ with $R_{T}=-156 / 121, R_{T}=-101 / 66$, and $R_{T}=-46 / 10$. 


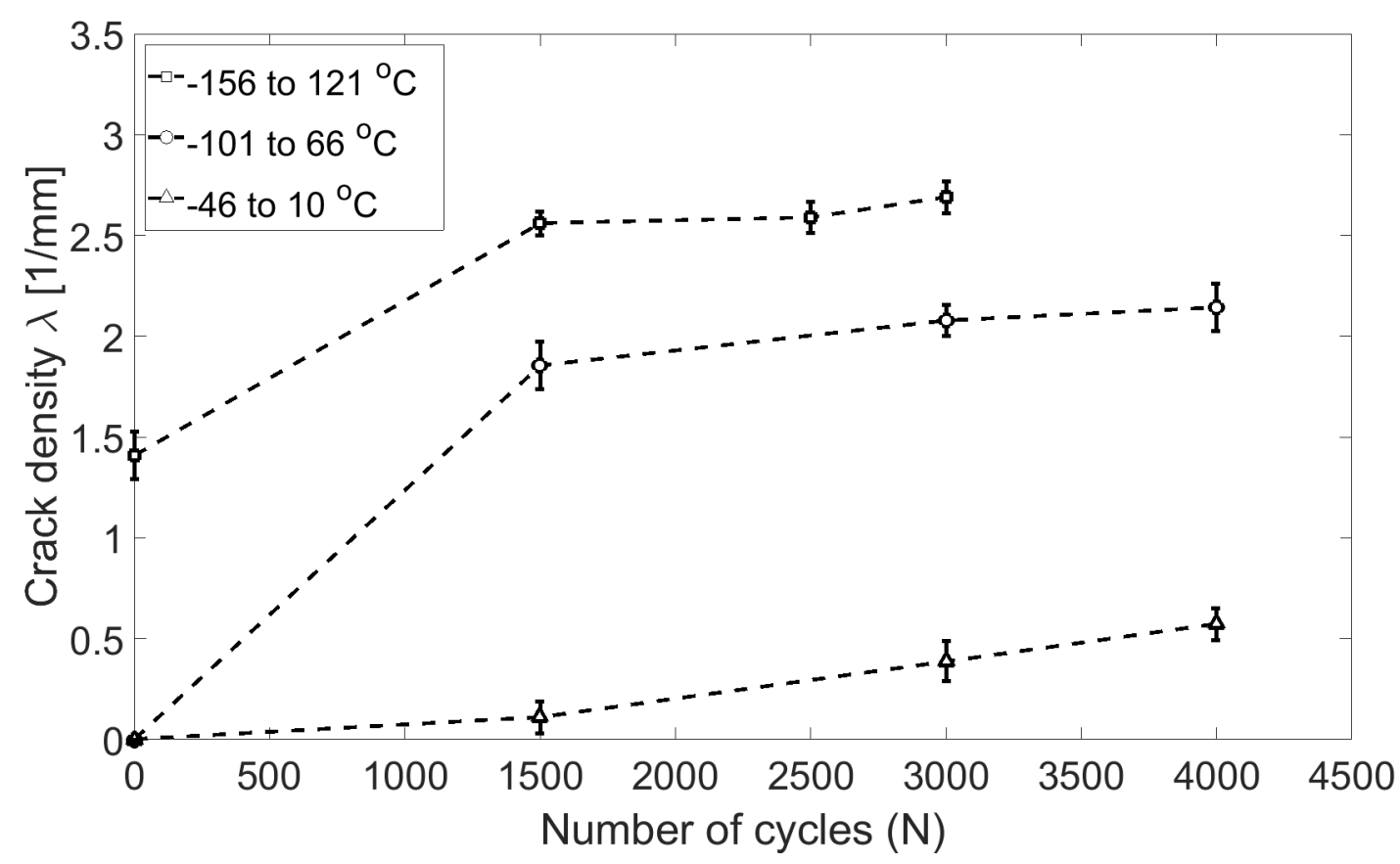

Figure 5.13: Crack density evolution vs. thermal cycling at different temperature ranges: $\left[-156,121^{\circ} \mathrm{C}\right],\left[-101,66^{\circ} \mathrm{C}\right]$, and $\left[-46,10^{\circ} \mathrm{C}\right]$.

thickness effect is internally taken into account by solving the displacement field through the equilibrium equations. In addition, the temperature-dependent properties from Table 5.1 are included and $\lambda$ is the only state variable needed. Therefore, the ERR $G_{I}$ can be easily calculated for any laminate configuration as shown in Figure 5.14.

The ERR $G_{I}$ calculated as function of crack density $\lambda$ for laminate $\left[(0 / 90)_{2}\right]_{S}$ P75/1962 at various temperatures are reported in Figure 5.14 for $90_{2}^{\circ}$ laminas. To illustrate the application of Paris's law, two thermal ratios $R_{T}$ are selected over the ranges $\left[-156,121^{\circ} \mathrm{C}\right]$ and $\left[-44,10^{\circ} C\right]$, which correspond to $\left(\Delta G_{I}\right)_{1}$ and $\left(\Delta G_{I}\right)_{2}$, respectively at $\lambda=1.0 \mathrm{~mm}^{-1}$.

Crack-growth ratio calculated with Paris's Law proposed in (5.13) is shown in Figure 5.15 with $\Delta G_{I}$ calculated with DDM as illustrated in Figure 5.14, and $d \lambda / d N$ from experimental data. Experimental crack density data come from $[1,10,186]$ with LSS: $\left[(0 / 90)_{2}\right]_{S},[0 / \pm 45 / 90]_{S}$, and $[0 / 45 / 90 /-45]_{S}$.

Based on the results shown in Figure 5.14, a higher ERR range $\Delta G_{I}$ in the range $\left[-156,121^{\circ} \mathrm{C}\right]$ with respect the range $\left[-44,10^{\circ} \mathrm{C}\right]$ i.e., $\left(\Delta G_{I}\right)_{1}>>\left(\Delta G_{I}\right)_{2}$, will produce a faster crack-growth rate $d \lambda / d N$ as shown in Figure 5.15. Both $T_{\min }$ and $T_{\max }$ influence the ERR range. If $\Delta G_{I}$ is the only controlling parameter, the use of a $T_{\max }$ close to $T_{\min }$ for a given $R_{T}$, will yield low crack growth rate as shown in Figure 5.15, even if laminates are exposed to cryogenic temperatures.

Furthermore, it can be seen in Figure 5.14 that $G_{I}$ remains virtually constant in the range $0<\lambda<0.35 \mathrm{~mm}^{-1}$ and thus, a constant crack growth rate during the first few thermal cycles is expected because $\Delta G_{I}$ hardly changes. For small $\lambda$, the effective shear- 


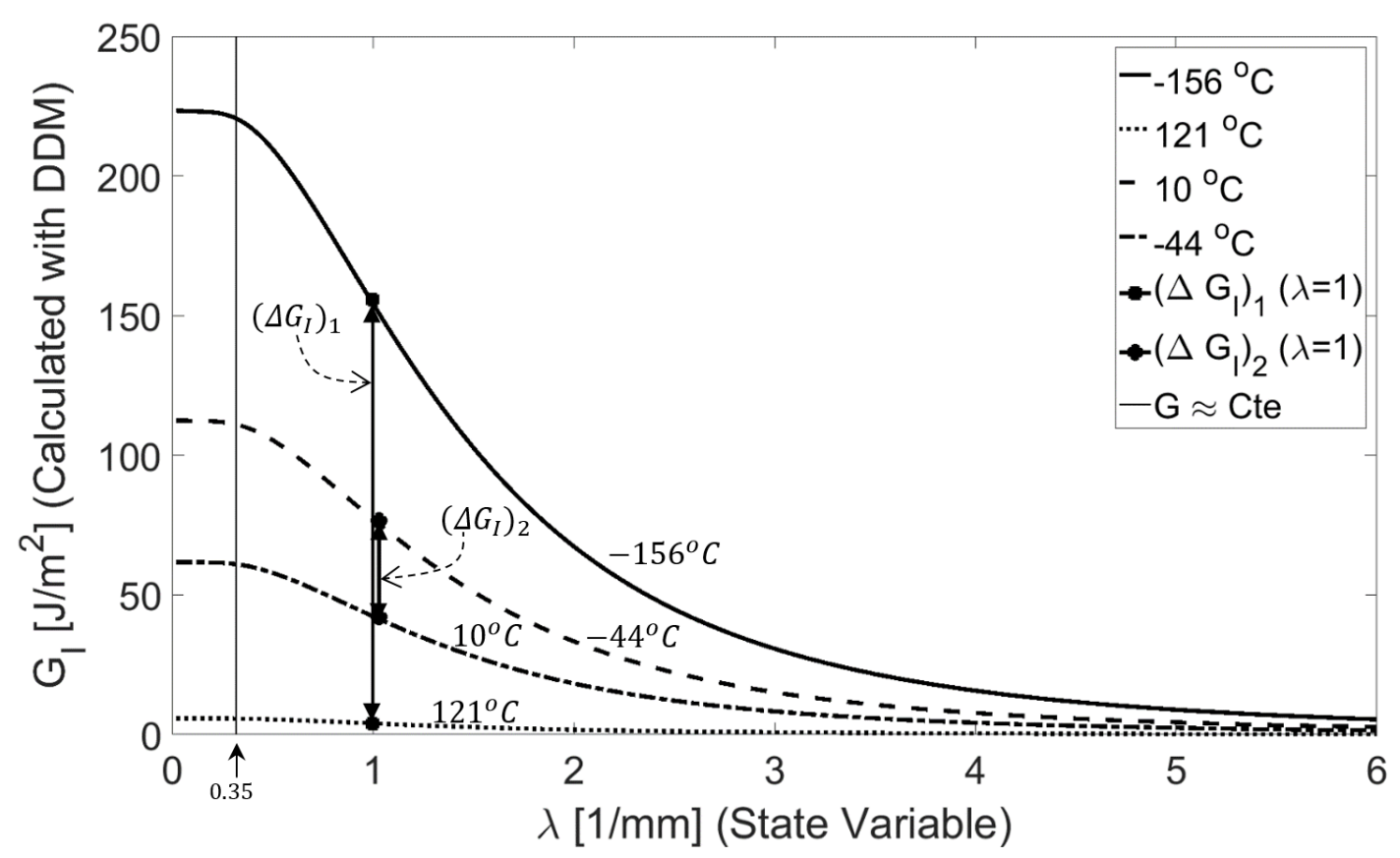

Figure 5.14: $E R R G_{I}$ as function of crack density $\lambda$ at two thermal ratios $R_{T}$ in the range $\left[-156,121^{\circ} \mathrm{C}\right]$ and $\left[-44,10^{\circ} \mathrm{C}\right]$ for $\left[(0 / 90)_{2}\right]_{S}$ Pr5/1962.

lag is small compared with the distance between two neighbor cracks [302] and thus, the crack interaction is negligible leading to minor difference on $\Delta G_{I}$.

Excellent correlation between Paris's law and experimental data shown in Figure 5.15 suggests that $\Delta G_{I}$ is the only driving forece that controls the fatigue resistance $f(N)$ under thermal cycling loads, yielding a linear relation on a log-log scale, with $\lambda$ being the only state variable. The collected experimental crack density data come from $[1,10,186]$ using data from the thicker laminas (laminas at middle) and LSS: $\left[(0 / 90)_{2}\right]_{S},[0 / \pm 45 / 90]_{S}$, and $[0 / 45 / 90 /-45]_{S}$.

Based on the correlation observed in Figure 5.15 for different LSS, it is postulated that Paris's law can be used to predict fatigue resistance $f(N)$ regardless of laminate configuration in symmetric cross-ply and quasi-isotropic laminates. This means that a Paris's law plot can be used to correlate a material system in a master curve where $f(N)$ can be obtained by calculating $\Delta G_{I}$ lamina by lamina once the material parameters $A$ and $\alpha$ are estimated using SLR as shown in Figure 5.15. Outlier data points reported in Figure 5.15 are ignored based on both Cook's distance and DFFITS statistical methods. The scatter band with dash lines in Figure 5.15 represents a $90 \%$ confidence interval on micro-cracking fatigue damage prediction.

The outlier data points shown in Figure 5.15 may indicate the existence of three regions. That is, a fast crack-growth rate region (damage initiation), a constant slope region, and a slow crack-growth rate region (CDS). In the first stage for low $N$, a large $\Delta G_{I}$ leads to fast growth rate represented by the outliers data on the top right of Fig- 


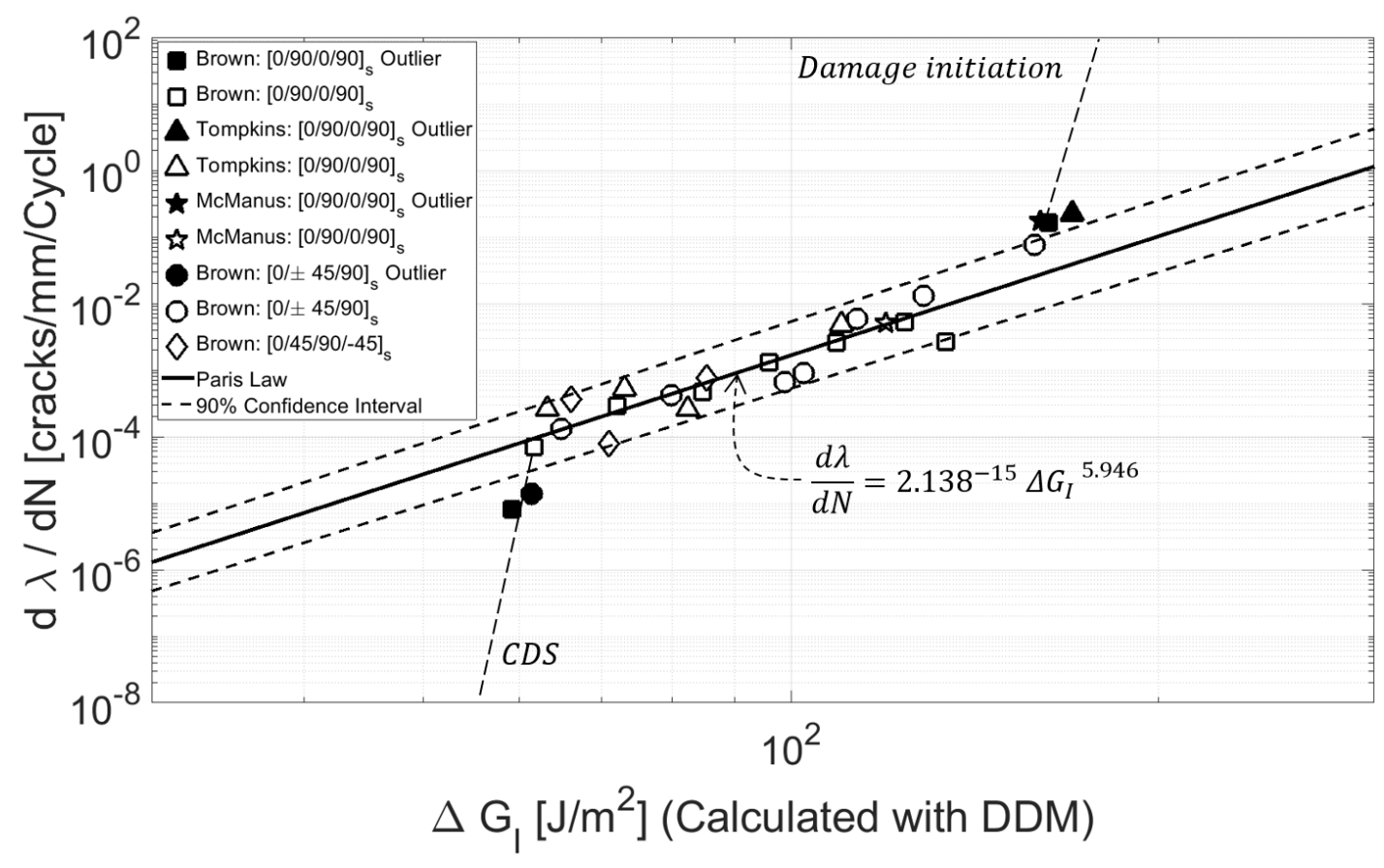

Figure 5.15: Transverse microcrack density growth rate $(d \lambda / d N)$ as function of ERR range $\Delta G_{I}$ for P75/1962 during thermal fatigue with $R_{T}=-156 / 121$. The laminate layups are $\left[(0 / 90)_{2}\right]_{S},[0 / \pm 45 / 90]_{S}$ and $[0 / 45 / 90 /-45]_{S}$. Experimental crack density $\lambda$ belong to $90_{2}^{\circ}$ and $-45_{2}^{\circ}$ laminas respectively.

ure 5.15. Initially, no interaction between neighbor cracks is present and the ERR $G_{I}$ remains virtually constant as shown in Figure 5.14 for $\lambda<0.35 \mathrm{~mm}^{-1}$. Thus, few cycles are needed to nucleate new cracks and $\lambda$ increases quickly during the first cycles until it falls into the linear relation. Furthermore, it can be seen that $\Delta G_{I} \approx G_{I c}^{\prime}\left(T_{\min }\right)$ in the first cycles, as illustrated by the damage initiation line on the top right of Figure 5.15 because the fatigue phenomenon is negligible for low $\mathrm{N}$ and the behavior approaches quasi-static cooling. The thermal stress at first stage is high and early cracks can be attributed to initial flaws whose crack size $a>a_{c}$.

In the second stage, after $\lambda$ reaches $\approx 0.35 \mathrm{~mm}^{-1}, \Delta G_{I}$ decreases (Figure 5.14) and the crack growth rate follows Paris's law (5.13).

Finally, a high crack density $\lambda$ leads to lower $\Delta G_{I}$ (Figure 5.14, far right) and the crack growth rate reduces sharply. The third stage (CDS) is represented by the left outliers data in Figure 5.15 when the CDS of the laminate is reached. In this region, thermal stress decreases drastically due to stiffness degradation, thus reducing the nucleation and propagation of flaws.

Unlike classical Paris's Law in metals, these three regions in Paris's Law for thermal fatigue start from the right side in Figure 5.15. In metals, a large single crack size $a$ leads to high $\Delta K$ resulting in a faster growth rate. Instead in composites, the same stored energy released by a single large crack is substituted by a high $\lambda$. However, as 
$\lambda$ increases, laminate stiffness decreases and neighbor cracks begins to interact so that the available $\Delta G_{I}$ to form new cracks decreases (Figure 5.14). This leads to slower crack-growth rate as shown in Figure 5.15.

\subsection{Thermal Ratio}

To study the effect of thermal ratio effect on transverse micro-cracking during thermal fatigue, Paris's law (5.13) is plotted as function of $\Delta G_{I}$ at several temperature ranges in Figure 5.16. The scatter band obtained in Figure 5.15 is used again to verify that all data fit in a single master curve regardless temperature range. Data subjected to highest and medium temperature range fall into the scatter band while lowest range disagree.

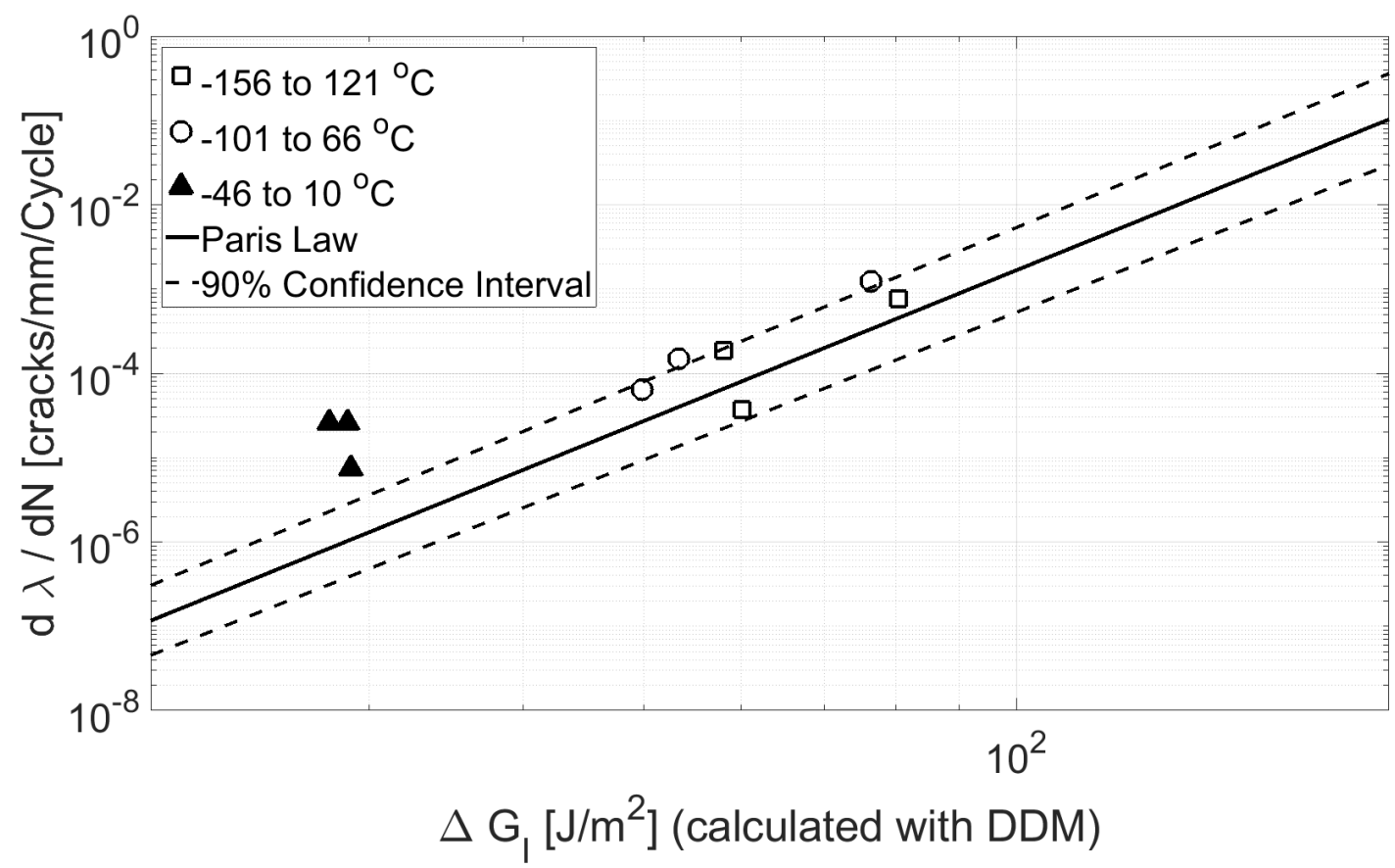

Figure 5.16: Transverse microcrack density growth rate $(d \lambda / d N)$ as function of ERR range $\Delta G_{I}$ for P75/1962 $[0 / 45 / 90 /-45]_{S}$ during thermal fatigue with $R_{T}=-156 / 121, R_{T}=$ $-101 / 66$, and $R_{T}=-46 / 10$. Experimental crack density $\lambda$ belong to thicker $-45_{2}^{\circ}$ laminas in both cases.

A likely explanation is that the Paris's plot in Figure 5.16 does not take into account the temperature dependence of $G_{I c}^{\prime}\left(T_{\min }\right) . \Delta G_{I}$ is calculated with DDM as function of state variable $\lambda$, and $d \lambda / d N$ comes directly from experimental data. Therefore, $\Delta G_{I}$ loses relevant information when $G_{I c}$ varies with temperature.

According to [12], $G_{I c}$ can increase about $30 \%$ between room temperature and $-156^{\circ} \mathrm{C}$. As a result, if $\Delta G_{I}$ is kept constant, the value of $G_{I c}^{\prime}$ at $-46^{\circ} C$ decreases, leading to higher crack propagation compared with those at highest and medium temperature range where $G_{I c}^{\prime}\left(T_{\min }\right)$ is greater. Therefore, the crack growth rate at lower temperature ranges seem 
to increase. This fact can be seen in Figure 5.16 for data in the range $\left[-46,10^{\circ} \mathrm{C}\right]$ which falls outside the $90 \%$ confidence interval. It is therefore proposed that data for a specific material system (regardless layup or temperature range) should be predicted using a Master Paris's law by normalizing the ERR range as follows

$$
\frac{d \lambda}{d N}=A\left(\frac{\Delta G_{I}(\lambda, T)}{G_{I c}^{\prime}\left(T_{\min }\right)}\right)^{\alpha}
$$

In order to confirm this hypothesis, the Paris's law plot in Figure 5.15 is normalized using (5.14) and shown in Figure 5.17. The scatter band is recalculated being $A$ the only parameter affected. The critical ERR $G_{I c}^{\prime}\left(T_{\min }\right)$ as function of temperature for P75/1962 is reported in Ch. 4 and [12].

As it can be seen in Figure 5.17, all data points fall into the scatter band and thus, a Master Paris Law defined by (5.14) can be used to predict microcracking fatigue damage regardless temperature range. Experimental data points from Figure 5.16 are plotted with solid symbols in their original position (Figure 5.16) and shifted (open symbols) for temperature ranges $\left[-101,66^{\circ} \mathrm{C}\right]$ and $\left[-46,10^{\circ} \mathrm{C}\right]$ as shown in Figure 5.17 to illustrate the differences. Since data in Figure 5.16 is already subjected to the temperature range $\left[-156,121^{\circ} \mathrm{C}\right]$, only open symbols are shown for that range.

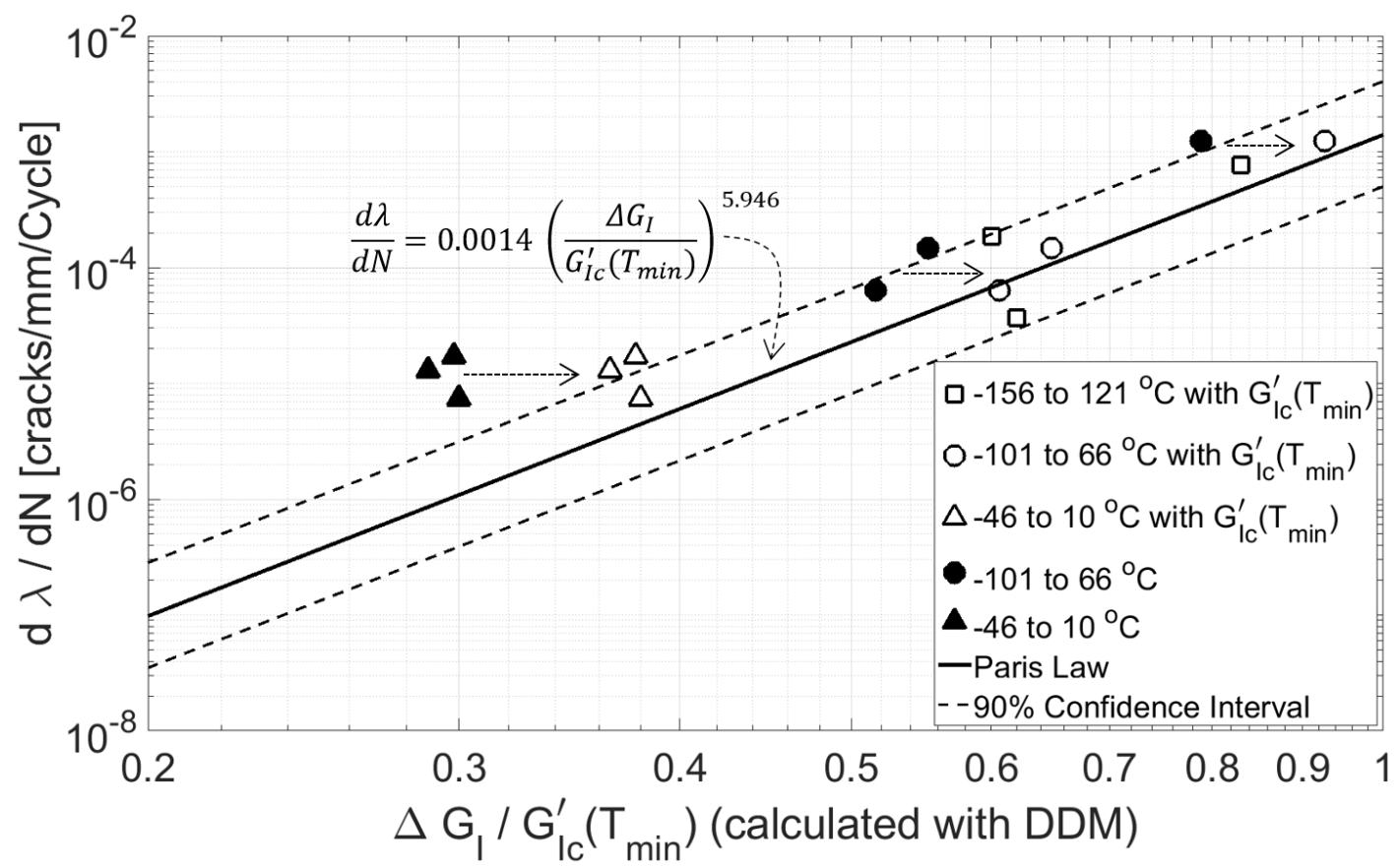

Figure 5.17: Comparison between master and regular Paris's law plot for P75/1962 $[0 / 45 / 90 /-45]_{S}$ with $R_{T}=-156 / 121, R_{T}=-101 / 66$, and $R_{T}=-46 / 10$. Experimental crack density $\lambda$ belong to $-45_{2}^{o}$ laminas in both cases.

Based on Figure 5.17, the Master Paris's law must be normalized using equation (5.14) to account for temperature dependency of $G_{I c}^{\prime}\left(T_{\min }\right)$. Looking at Figure 5.14, one could 
envision a situation where the same $\Delta G_{I}$ could be obtained with different $R_{T}$ playing with the temperature range set $\left[T_{\min }, T_{\max }\right]$. Therefore, an identical $\Delta G_{I c}$ with different $T_{\min }$ could lead to the same crack growth rate unless the temperature dependence of $G_{I c}^{\prime}\left(T_{\min }\right)$ is taken into account as shown in Figure 5.17.

For each cycle, the highest $\lambda$ is determined by $T_{\min }$ at which both maximum ERR and stresses occurs. On the other hand, $R_{T}=T_{\min } / T_{\max }$ determines the range $\Delta G_{I}$ which in turn controls the crack growth rate $d \lambda / d N$ as shown in Figure 5.16 and 5.17.

As the material damages $(\lambda \rightarrow \infty)$ the ERR range $\Delta G_{I}$ reduces (Figure 5.14) due to both loss of stiffness and crack interaction. Then, the crack-growth rate $d \lambda / d N$ reduces (Figure 5.15) reaching almost saturation crack density (CDS) at 2000-4000 cycles (Figure 5.13).

\subsection{Fatigue Resistance}

As proposed in (5.5) the fatigue and temperature dependent $G_{I c}$ is assumed to be the product of the critical ERR $G_{I c}^{\prime}\left(T_{\min }\right)$ for $N=1$ and the fatigue resistance function $f(N)$. A master Paris's law can be used not only to account for layup and temperature range effect but also to calculate the fatigue resistance function $f(N)$ for a given material system and LSS using the algorithm illustrated in Figure 5.18. In order to calculate $f(N)$, some steps must be considered.

First, a quasi-static cooling analysis must be performed to calculate the crack density $\lambda_{1}^{k}$ in each lamina during the first cycle. This is because for $N=1$, the fatigue phenomenon is negligible so that $G_{I c}^{\prime}=G_{I c}\left(T_{\min }\right)$. Therefore, the evolution of damage $\lambda$ prior to thermal fatigue can be calculated with DDM simulating monotonic cooling from $S F T$ to $T_{\min }$. According to Griffith's criterion [274], a lamina will crack if

$$
\zeta=\frac{G_{I}^{k}}{G_{I c}^{\prime}} \geq 1 \text { at } T_{\min }
$$

where $k$ represent each lamina of the laminate.

In some cases, the ERR $G_{I}^{k}$ of lamina $k$ may be insufficient $(\zeta<1)$ to generate the first crack (called first ply failure in the literature). For such cases, propagation of the first crack under thermal cycling loads requires void nucleation during a few cycles until first cracks propagates. In other words, the size $a$ of initial flaws needs to grow until they reach the critical size $a_{c}$ as explained by equation (5.9).

The damage initiation criterion $\zeta$ can be observed in Figure 5.19 for $\left[(0 / 90)_{2}\right]_{s}$ P75/1962 with $R_{T}=-156 / 121$. Both exterior $0^{\circ}$ and middle $90_{2}^{\circ}$ laminas satisfy the condition $\zeta>1$ and thus, damage initiation occurs at first cycle, i.e. quasi-static cooling. In fact, $\lambda_{i}^{k}$ can 


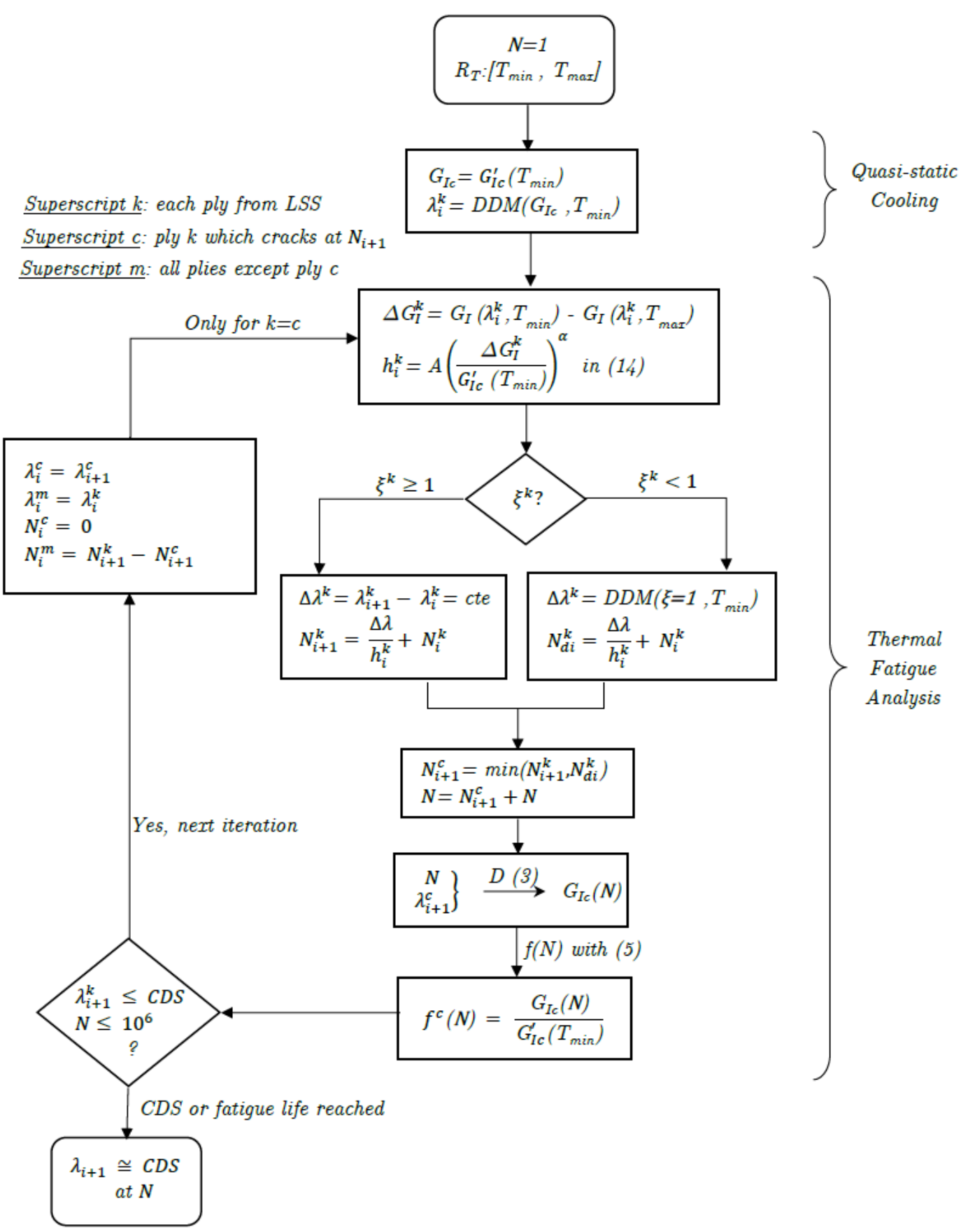

Figure 5.18: Thermal fatigue prediction using a modified Paris's law for a speciifc material system. 
be easily estimated at the intersection point between $G_{I}$ and $G_{I c}$ lines in Figure 5.19. The crack density $\lambda_{i}^{k}$ increases suddenly in few cycles because $G_{I}$ remain virtually constant in the range $0<\lambda<0.6$ as shown in Figure 5.19. Hereinafter, fatigue resistance $f(N)$ can be calculated by a master Paris's law (5.14) following the procedure illustrated in Figure 5.18.

However, interior $0^{\circ}$ and $90^{\circ}$ laminas with $\zeta<1$ need some void nucleation before the first crack occurs. When this occurs, cracks propagate and $\zeta \approx 1$ with $f(N)$ for interior laminas lower than unit value. In fact, it is later calculated that damage in interior laminas starts at $N_{d i}=262$ cycles with $f(N)=0.6$ (Figure 5.20).

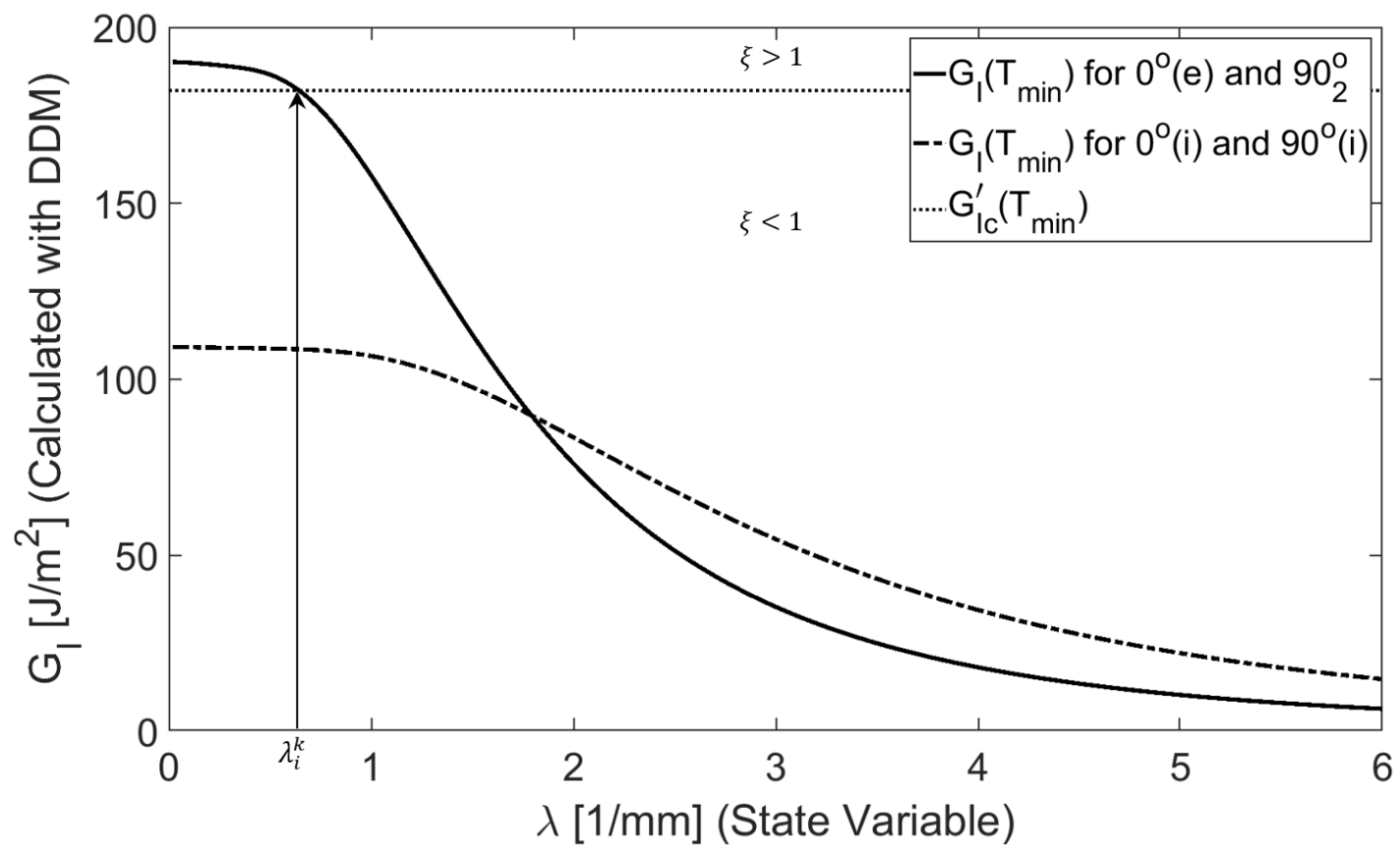

Figure 5.19: $G_{I}$ vs. crack density for $N=1$ and $T_{\min }=-156$ during cooling for $\left[(0 / 90)_{2}\right]_{s}$ P75/1962 with $R_{T}=-156 / 121$.

When uncracked laminas are subjected to thermal cycling loads, $f(N)$ cannot be calculated by a Master Paris's law. Note that only the crack growth rate $\lambda$ can be predicted by modified Paris's law. But, $\lambda$ remains equal to zero until damage initiation and thus $G_{I c}(N)$ using equation (5.5) is useless. However, the number of cycles $N_{d i}$, for which transverse damage initiation occurs can be calculated with a Master Paris's law. Therefore, while the exact shape of $f(N)$ is unknown until $N_{d i}, f(N)$ can be calculated using (5.14) for all $N \geq N_{d i}$.

Some authors $[23,296]$ reported a linear phenomenological equation for $f(N)$ in the range $0<\zeta<1$. Therefore, $f(N)$ will be assumed in this study to evolve linearly with $\log N$ until $N_{d i}$ is reached. In any case, transverse damage does not occur until $N_{d i}$ is reached and thus, $f(N)$ in the range $1<N<N_{d i}$ is irrelevant because no cracks 
propagate in that region. The number of cycles for damage initiation $N_{d i}$ can be calculated as follows:

1. The initial crack densities $\lambda_{1}^{k}$ of uncracked laminas are calculated with a quasi-static cooling analysis forcing $\zeta=1$.

2. The $\Delta G_{I}$ needed to reach $\lambda_{1}^{k}$ under quasi-static cooling $(N=1)$ is calculated with DDM using both equations (2.37) and (2.39), and the initial crack densities $\lambda_{1}^{k}$ from previous step.

3. Finally, the number of cycles $N_{d i}$ is calculated using (5.14). Inserting $\Delta G_{I}$ in (5.14) we get $d \lambda / d N$. Then, the number of cycles $N_{d i}$ is calculated using the algorithm illustrated in Figure 5.18.

Once a quasi-static cooling and $N_{d i}$ for each lamina for which $\zeta<1$ have been computed, the fatigue resistance $f(N)$ can be calculated using the Master Paris's Law (5.14) as shown in Figure 5.18. Note that $f(N)$ is controlled by $\Delta G_{I}$ which in turn depends on lamina thickness, LSS, lamina orientation, $R_{T}$, etc. Therefore, $\Delta G_{I}$ must be calculated for each lamina $k$. Furthermore, the material parameters $A$ and $\alpha$ of Master Paris's law (5.14) must be calculated as shown in Figure 5.17 for the material of interest prior to thermal fatigue analysis.

Thermal fatigue analysis as in Figure 5.18 can be done using numerical methods for a fixed $\Delta \lambda^{k}$. That is, $\Delta \lambda^{k}$ is imposed so that the number of cycles $N_{i+1}^{k}$ to develop an expected crack density $\lambda_{i+1}^{k}$ can be predicted using a Master Paris's law (5.14). A small $\Delta \lambda^{k}$ for laminas $k$ with $\zeta>1$ can be imposed in order to obtain as many data points as possible. Also, an initial $\lambda_{1}^{k}$ of uncracked laminas at $N=1$ should be imposed until $N_{d i}$ is reached. Then, a small $\Delta \lambda^{k}$ can be used after transverse damage initiation occurs $(\zeta>1)$ as shown in Figure 5.18.

The expected $N_{i+1}^{k}$ is computed by calculating $\Delta G_{I c}^{k}$ in each lamina $k$ as shown in Figure 5.18. Then, the number of cycles $N$ to be simulated with DDM correspond to the lowest $N_{i+1}^{k}$ at which the imposed $\Delta \lambda^{k}$ is generated. $G_{I c}$ in (5.5) is adjusted using (5.3) to later obtain $f(N)$ as shown in Figure 5.18. The thermal fatigue analysis proceeds to the next iteration by updating $\lambda_{i}^{k}$ and $N_{i}^{k}$ in each lamina. The analysis finishes when CDS for all laminas or infinite life $\left(N=10^{6}\right)$ is reached in the laminate.

In order to illustrate the versatility of Master Paris's law, fatigue resistance $f(N)$ for $\left[(0 / 90)_{2}\right]_{s}$ P75/1962 subjected to $R_{T}=-156 / 121$ is shown in Figure 5.20. The algorithm used is shown in Figure 5.18. Unlike $f(N)$ shown in Figures 5.3, 5.4, and 5.12, fatigue resistance $f(N)$ does not evolves linearly with $N$ in a semi-log scale. Instead, $f(N)$ fits 
with nonlinear fourth order rational function in terms of $\log _{10}(N)$ using SLR as follows

$$
\hat{y}=\frac{\beta_{0} \log _{10}^{2}(N)+\beta_{1} \log _{10}(N)+\beta_{2}}{\log _{10}^{4}(N)+\beta_{3} \log _{10}^{3}(N)+\beta_{4} \log _{10}^{2}(N)+\beta_{5} \log _{10}(N)+\beta_{6}}
$$

where residuals are assumed to follow a normal distribution $\sim\left(0, \sigma^{2}\right)$ of mean equal to zero and variance $\sigma^{2}$. Note that the fatigue resistance $f(N)$ generally fits well with a nonlinear quadratic rational function. However, $f(N)$ for very low $R_{T}$ needs a higher order polynomial. In any case, a fourth order rational function can be always reduced to quadratic order if possible. The coefficients in (5.16) are shown in Table 5.3.

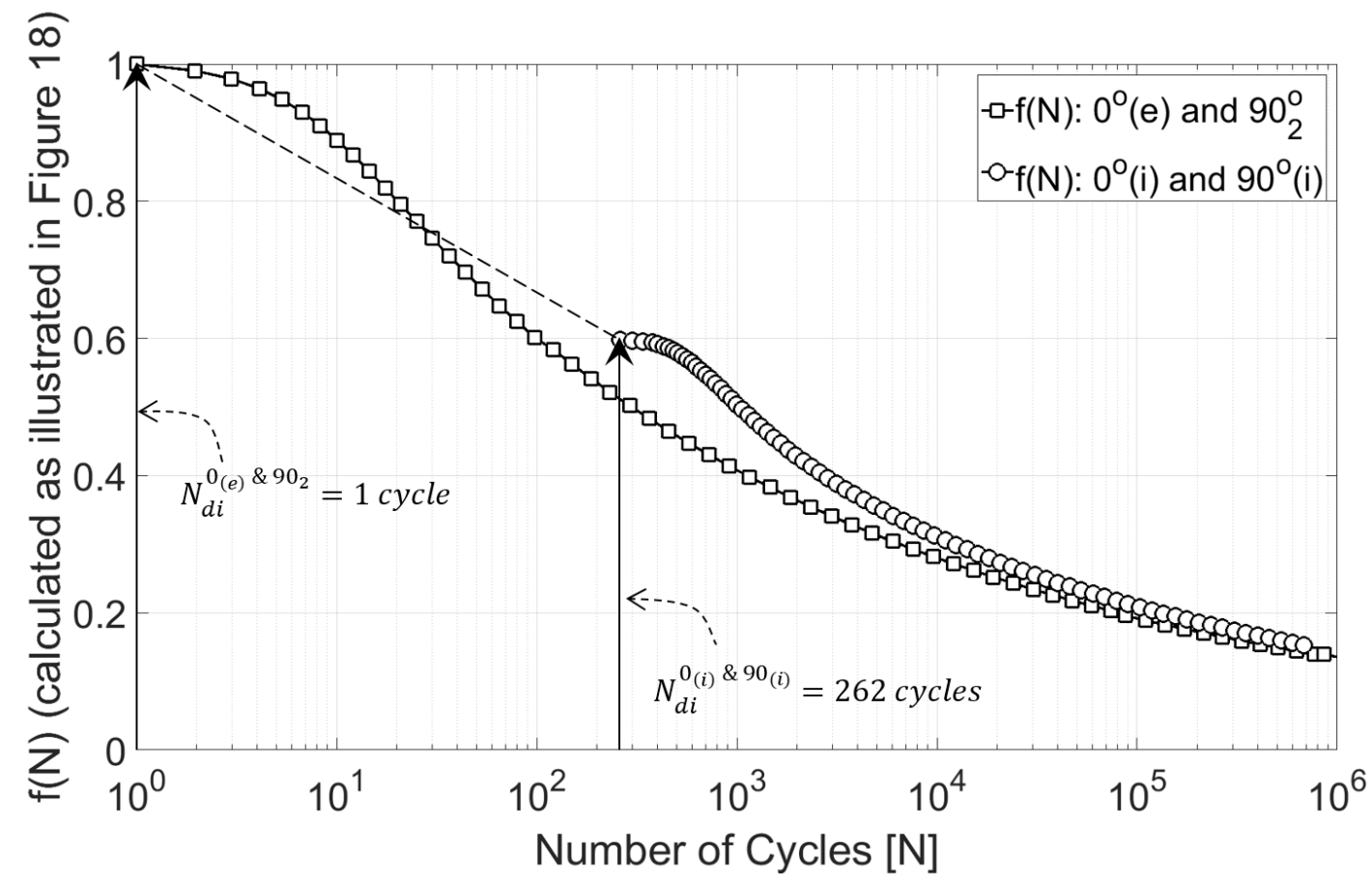

Figure 5.20: Fatigue resistance $f(N)$ for $\left[(0 / 90)_{2}\right]_{s}$ P75/1962 with $R_{T}=-156 / 121$ obtained through Paris's law and DDM model as illustrated in Figure 5.18.

Looking at Figure 5.20, as the crack density increases with $N$, both $f(N)$ for exterior and interior laminas trend to get closer at infinite life $\left(N=10^{6}\right)$. This is because $\lambda_{i}^{k}$ for each lamina is very high so that the calculated $\Delta G_{I}$ for all laminas are very similar. For this LSS, both exterior $0^{\circ}$ and middle $90_{2}^{\circ}$ laminas have $\zeta>1$ at first cycle so that $f(N)$ is calculated by a Master Paris's law. A small $\Delta \lambda$ is used to construct Figure 5.20 as showing by open-square symbols. The coefficients in (5.16) are shown in Table 5.3.

For interior $0^{\circ}$ and $90^{\circ}$ laminas with initial $\zeta<1$, transverse damage initiation occurs at $N_{d i}=262$. The unknown $f(N)$ in the range $1<N<262$ cycles is assumed to decrease linearly with $N$. Hereinafter, $f(N)$ is calculated by a Master Paris's law and a small $\Delta \lambda$ until infinite life is reached. Therefore, $f(N)$ must be computed separately for each lamina $k$ because the driving force $\Delta G_{I}$ varies for each lamina. 


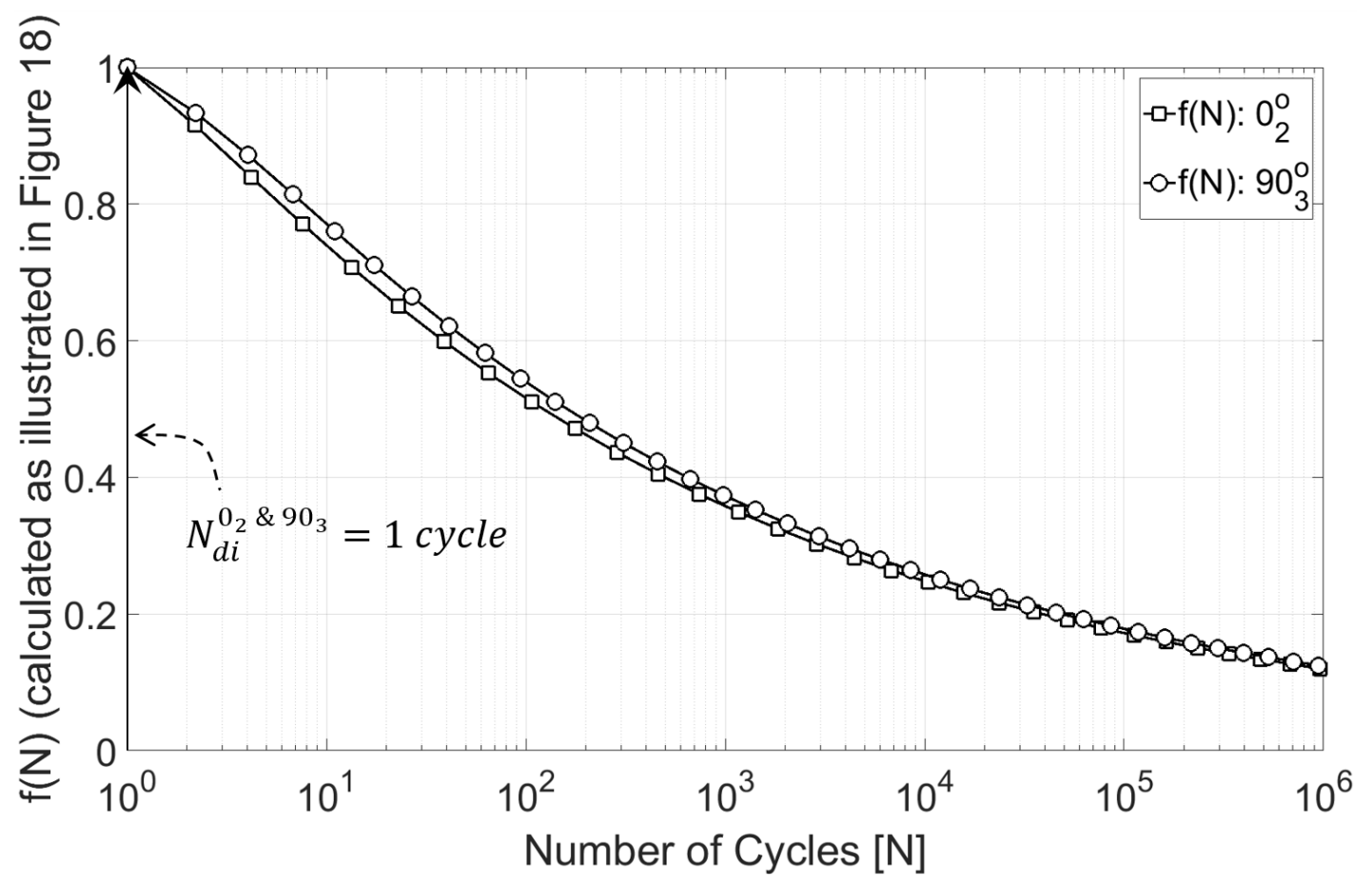

Figure 5.21: Fatigue resistance $f(N)$ for $\left[0_{2} / 90_{3}\right]_{s}$ P75/1962 with $R_{T}=-156 / 121$ obtained through Paris's law and DDM model as illustrated in Figure 5.18.

Fatigue degradation function $f(N)$ for $\left[0_{2} / 90_{3}\right]_{s}$ P75/1962 subjected to $R_{T}=-156 / 121$ is shown in Figure 5.21. The algorithm used is shown in Figure 5.18. The coefficients in (5.16) are shown in Table 5.3. Both $f(N)$ for $0_{2}^{o}$ and $90_{3}^{\circ}$ laminas appears to be almost identical during the whole thermal fatigue analysis. Since both laminas satisfy the condition $\zeta>1$ during quasi-static cooling, damage initiation occurs at $N_{d i}=1$. Therefore, $f(N)$ is calculated using a Master Paris's law and a small $\Delta \lambda$ until infinite life is reached.

Fatigue degradation function $f(N)$ for $\left[0_{2} / \pm 45 / 90_{3}\right]_{s}$ P75/1962 subjected to $R_{T}=$ $-60 / 50$ is shown in Figure 5.22. The algorithm used is shown in Figure 5.18. The coefficients in (5.16) are shown in Table 5.3. Both $0_{2}^{\circ}$ and $90_{3}^{\circ}$ laminas crack at $N_{d i}=1$ cycle with $\zeta \geq 1$. Therefore, $f(N)$ is calculated using a Master Paris's law and a small $\Delta \lambda$ until infinite life is reached.

For \pm 45 laminas that initially have $\zeta<1$, damage initiation occurs at $N_{d i}^{45}=2906$ and $N_{d i}^{-45}=2044$, respectively. The unknown $f(N)$ is assumed to decrease linearly until first crack appears. Since damage evolution $\lambda$ evolves differently for both $0_{2}^{\circ}$ and $90_{3}^{\circ}$ laminas, a different fatigue resistance function $f(N)$ is required for each lamina.

For \pm 45 laminas, $f(N)$ is slightly different in each angle-ply. This difference leads to a small ERR $G_{I I}$ in mode II. However, $G_{I I}$ is negligible compared with $G_{I}$ because crack density in \pm 45 laminas are almost identical once both \pm 45 laminas crack. In fact, $f(N)$ for \pm 45 laminas appears to be almost identical during thermal fatigue except in the range $N_{d i}^{-45^{\circ}}<N<N_{d i}^{45^{\circ}}$ where only one lamina has cracked. 


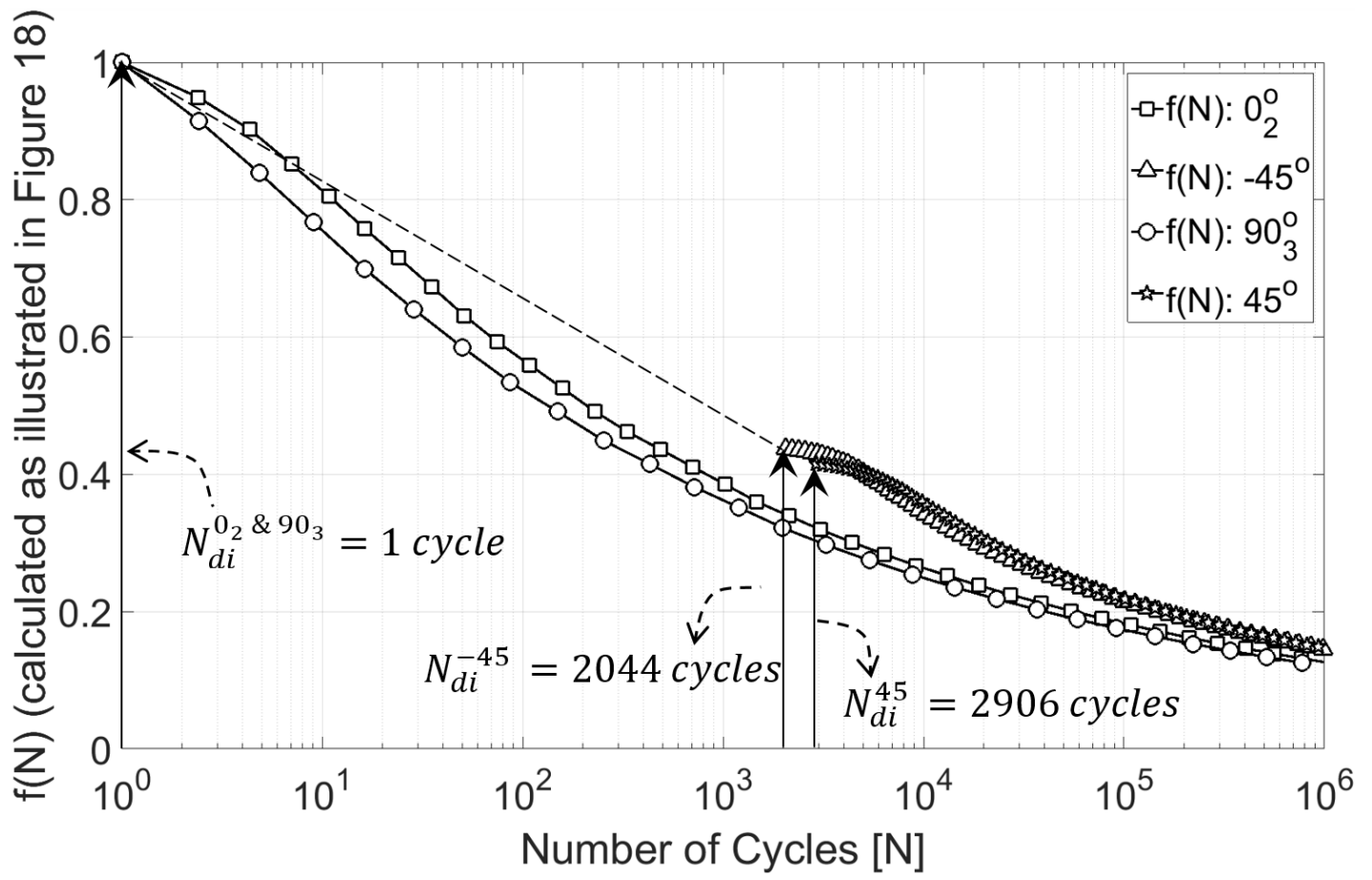

Figure 5.22: Fatigue resistance $f(N)$ for $\left[0_{2} / \pm 45 / 90_{3}\right]_{s}$ P75/1962 with $R_{T}=-60 / 50$ obtained through Paris's law and DDM model as illustrated in Figure 5.18.

Tables 5.3: $f(N)$ parameters of P75/1962 ( $V_{f}=0.52$ [10]). Subscript (e) and (i) represents exterior and interior laminas, respectively. Layup: A) $\left.\left.\left[(0 / 90)_{2}\right]_{s} ; B\right)\left[0_{2} / 90_{3}\right]_{s} ; C\right)\left[0_{2} / \pm\right.$ $\left.45 / 90_{3}\right]_{s}$.

\begin{tabular}{|c|c|c|c|c|c|c|c|c|c|}
\hline \multicolumn{10}{|c|}{ SLR parameters in equation $(5.16)$} \\
\hline Layup & Laminas & $\beta_{0}$ & $\beta_{1}$ & $\beta_{2}$ & $\beta_{3}$ & $\beta_{4}$ & $\beta_{5}$ & $\beta_{6}$ & $R_{T}$ \\
\hline \multirow{2}{*}{$A$} & $0_{(e)}^{o} \& 90_{2}^{o}$ & 0 & 0.082 & 5.301 & 0 & 1 & -0.169 & 5.287 & \multirow{2}{*}{$-156 / 121$} \\
\hline & $0_{(i)}^{o} \& 90_{(i)}^{o}$ & 0 & 0.579 & -0.872 & 0 & 1 & -3.999 & 4.705 & \\
\hline \multirow{2}{*}{$B$} & $0_{2}^{o}$ & 0 & -0.752 & 11.691 & 0 & 1 & 1.575 & 11.635 & \multirow{2}{*}{$-156 / 121$} \\
\hline & $90_{3}^{\circ}$ & 0 & -1.083 & 14.552 & 0 & 1 & 2.718 & 14.561 & \\
\hline \multirow{4}{*}{$C$} & $0_{2}^{o}$ & 0 & -0.342 & 8.254 & 0 & 1 & 0.535 & 8.218 & \multirow{4}{*}{$-60 / 50$} \\
\hline & $45^{\circ}$ & 0 & 0.447 & -1.128 & 0 & 1 & -5.715 & 8.774 & \\
\hline & $-45^{\circ}$ & 0 & 0.447 & -1.138 & 0 & 1 & -6.032 & 9.724 & \\
\hline & $90_{3}^{o}$ & 0 & -0.699 & 11.372 & 0 & 1 & 1.818 & 11.336 & \\
\hline
\end{tabular}


Crack density evolution $\lambda$ for $\left[(0 / 90)_{2}\right]_{s},\left[0_{2} / 90_{3}\right]_{s}$, and $\left[0_{2} / \pm 45 / 90_{3}\right]_{s}$ P75/1962 subjected to $R_{T}=-156 / 121, R_{T}=-156 / 121$, and $R_{T}=-60 / 50$, respectively, are shown in Figure 5.23, 5.24, and 5.25. The coefficients of fatigue resistance $f(N)$ according to (5.16) are listed in Table 5.3.

Experimental crack density $\lambda^{\exp }$ compares well with transverse cracking for $90_{2}^{o}$ in Figure 5.23. Note that no data is reported in the literature for the remaining laminas. Damage initiation for interior laminas ( $0 \& 90)$ is delayed until $N_{d i}=262$ is reached. However, once started, the crack growth rate is greater than those for surface $0^{\circ}$ and exterior $90_{2}^{\circ}$ laminas.

The $0_{2}^{\circ}$ and $90_{3}^{\circ}$ laminas start cracking at the first cycle in Figure 5.24. Due to larger thickness, both laminas reach high crack density $\lambda$ during the first cycles but the CDS reached is lower than for $\left[0_{2} / 90_{3}\right]_{s}$ laminate.

Finally, crack density evolution $\lambda$ for $\left[0_{2} / \pm 45 / 90_{3}\right]_{s}$ is slower than the previous cases (Figure 5.23 and 5.24) because the temperature range is lower $\left(R_{T}=-60 / 50\right)$. Furthermore, the CDS reached is lower due to low crack growth rate. For \pm 45 laminas, damage initiation is delayed but $\lambda$ increases faster once the first cracks propagate.

Similar to Figures 5.3, 5.4, and 5.12, fatigue resistance $f(N)$ decreases with number of cycles as shown in Figures 5.20, 5.21, and 5.22 so that higher crack densities can be predicted as illustrated in Figures 5.23, 5.24, and 5.25. Based on the crack density predictions, saturation crack density (CDS) for high-cycle data predictions would increase about $25-40 \%$ until infinite life $(N=1 E 6)$ is reached depending on the thermal ratio (Figures 5.23, 5.24, and 5.25). Therefore, CDS under thermal cyclic loads is smaller than those under mechanical fatigue or static tests, and crack density for thermal low-cycle data approaches saturation quickly. 


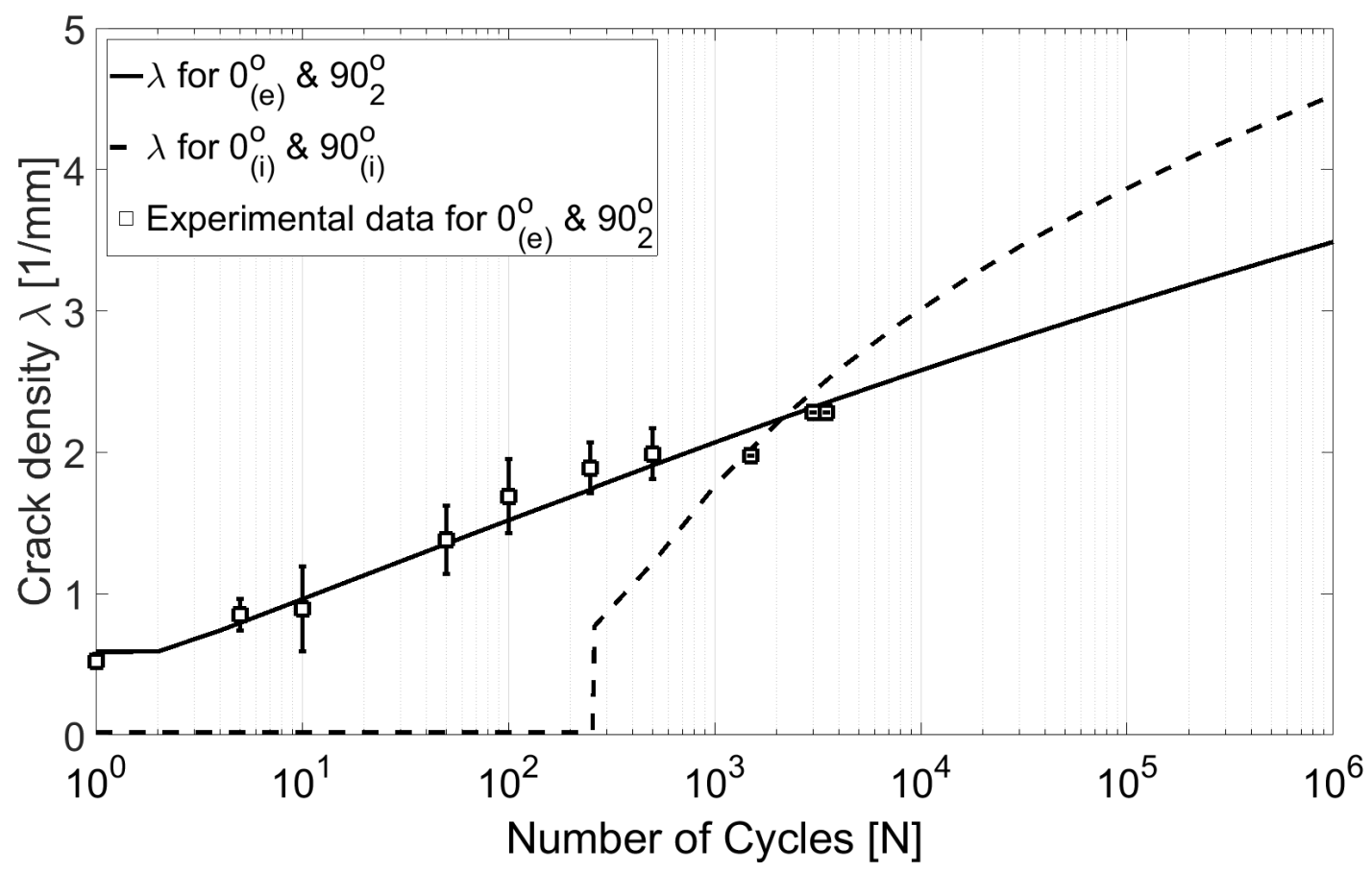

Figure 5.23: Crack density evolution $\lambda$ vs. number of cycles $N$ for $\left[(0 / 90)_{2}\right]_{s}$ Pr5/1962 with $R_{T}=-156 / 121$ calculated with $D D M$ and $f(N)$ reported in (5.16) and Table 5.3. Experimental data only available for middle $90_{2}^{\circ}$ lamina and low-cycle fatigue.

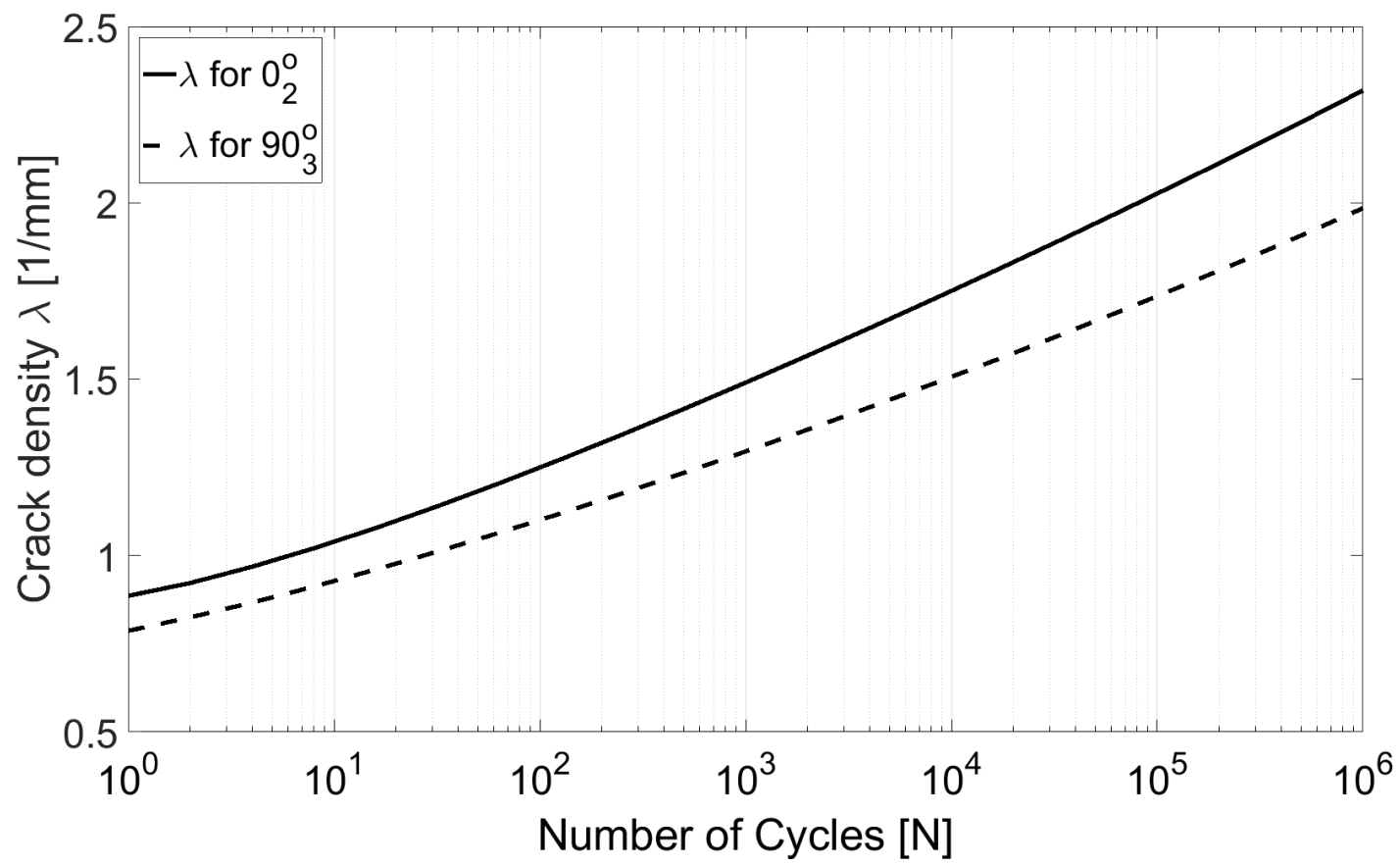

Figure 5.24: Crack density evolution $\lambda$ vs. number of cycles $N$ for $\left[0_{2} / 90_{3}\right]_{s}$ P75/1962 with $R_{T}=-156 / 121$ calculated with $D D M$ and $f(N)$ reported in (5.16) and Table 5.3. No experimental data is available to compare. 


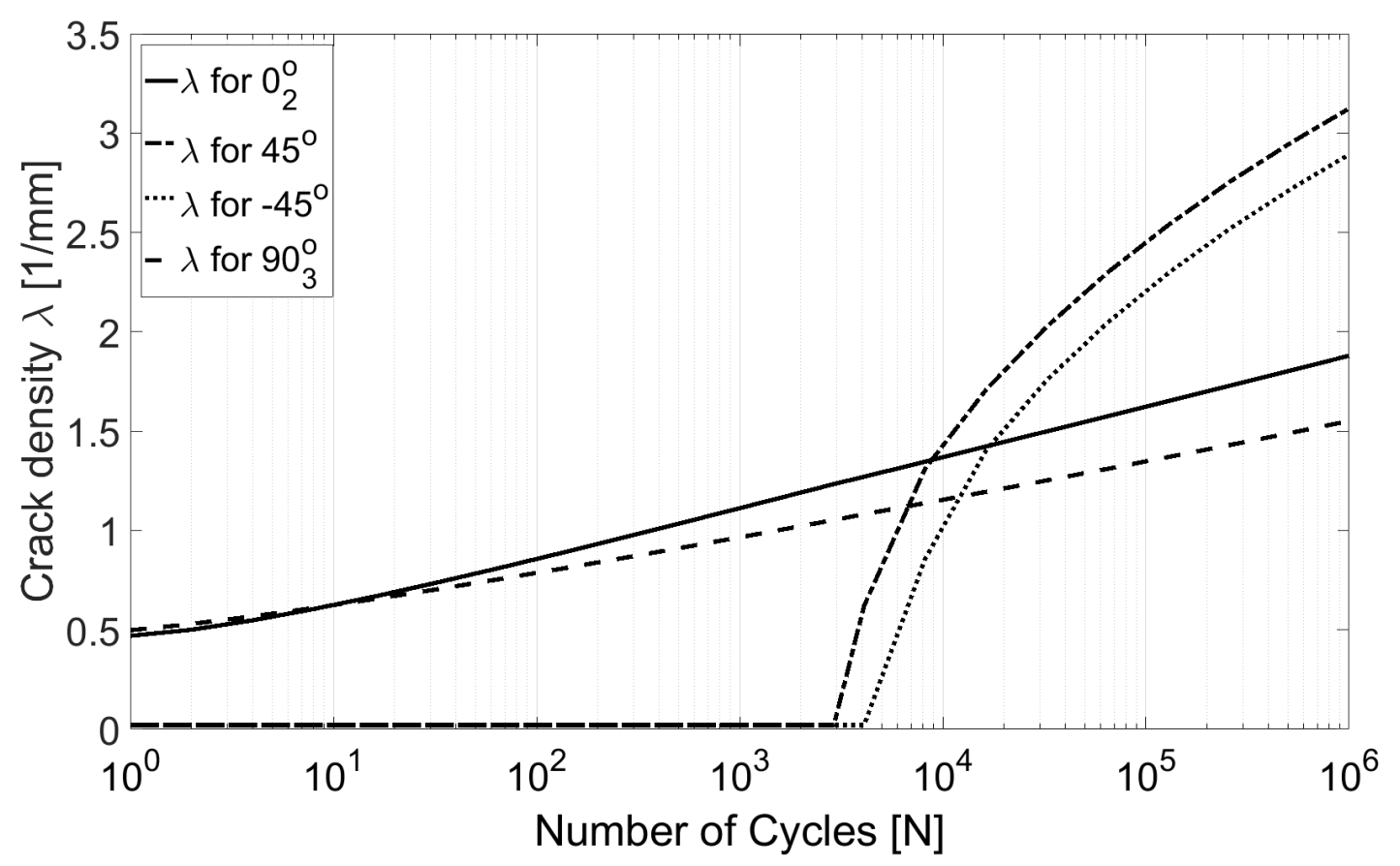

Figure 5.25: Crack density evolution $\lambda$ vs. number of cycles $N$ for $\left[0_{2} / \pm 45 / 90_{3}\right]_{s}$ P75/1962 with $R_{T}=-60 / 50$ calculated with $D D M$ and $f(N)$ reported in (5.16) and Table 5.3. No experimental data is available to compare.

\subsection{Conclusions}

Since crystalline polymers develop crazes which become an ideal path to propagate new cracks, the onset and growth of new cracks is caused by thermal cyclic loads until the craze size reaches a critical value $a_{c}$. Once the critical value $a_{c}$ has been reached, the critical ERR $G_{I c}$, which is a material property, can be used to predict transverse cracking. Since the craze size is impossible to measure, an analytical parametrization $f(N)$ is proposed as a measure of fatigue resistance $f(N)$. A decreasing $f(N)$ allows $G_{I c}$ in equation (5.5) to decrease with number of cycles and thus, higher crack densities can be predicted for larger number of cycles $N$.

For low cycle data $f(N)$ can be calculated by separation of variables using (5.5). On one hand, higher crack densities are generated at lowest temperature and thus $G_{I c}^{\prime}\left(T_{\text {min }}\right)$ can be adjusted at first cycle where no fatigue phenomenon exists. On the other hand, $G_{I c}$ as function of number of cycles in (5.5) can obtained using (5.3). Therefore, $f(N)$ can be adjusted using SLR (5.6) in a semi-logarithmic scale.

However, transverse cracking evolution beyond $N$ for the last experimental data point cannot be predicted because no experimental evidence exists beyond that number of cycles. Furthermore, the $f(N)$ calculated using low-cycle data $[1,10,186]$ is restricted to specific LSS, $R_{T}$, low number of cycles $(\approx 4000)$, and it does not account for lamina orientation. A Master Paris law is proposed that solves these problems. 
A Master Paris's law using (5.14) is proposed to predict thermal fatigue damage regardless of layup and $R_{T}$ as shown in Figure 5.15 and 5.17. $\Delta G_{I}$ is the only driving force to predict transverse damage. The fatigue resistance $f(N)$ can be easily predicted for each lamina at any $R_{T}$ and for number of cycles $N$. In addition, this methodology can be extended to other type of laminated composites including thermoplastic polymers or glass fibers only if a precise knowledge of the temperature-dependent properties has been previously calculated.

Unlike $f(N)$ adjusted from low-cycle data, fatigue resistance can be predicted regardless of LSS, $R_{T}$, and number of cycles. Furthermore, available data shows the following. First, saturation crack density (CDS) under thermal cyclic loads is smaller than those under mechanical fatigue or static tests. Second, crack density for thermal low-cycle data approaches saturation quickly.

Although experimental tests must be performed to obtain the Master Paris's law for each material system, the understanding of the Paris's plot allows us to predict fatigue resistance $f(N)$ using a small number of both specimens and thermal cycles. This is because $\Delta G_{I}$ decreases sharply as $\lambda$ increases. Therefore, it is proposed to map all the characteristic regions of Paris's curve (damage initiation, linear relation, and CDS as illustrated in Figure 5.15) combining a greater number of specimens with different $R_{T}$ and reducing the number of cycles to $20-30$ instead of $1500-4000$ reported in $[1,10,186]$. For instance, two LSS can be tested, one cross-ply (CP) and one quasi-isotropic (QI) laminates. Then, both layups subjected to very high $R_{T}$ with $5-10$ cycles (damage initiation), high $R_{T}$ with $10-20$ cycles and intermediate $R_{T}$ with $20-50$ cycles (linear relation), and very low $R_{T}$ until first crack propagate (CDS). This would drastically reduce the time and costs of experimentation because fatigue damage for any layup and $R_{T}$ of interest can be predicted without need to perform high-cycle fatigue tests. 


\section{Chapter 6}

\section{Thermo-Mechanical Equivalence}

Fatigue damage evolution is controlled by the evolving of critical $\operatorname{ERR}\left(G_{I c}\right)$, which can be decomposed into the quasi-static $\operatorname{ERR}\left(G_{I c}^{\prime}\left(T_{\min }\right)\right)$ and the fatigue resistance function $f(N)$ as follows

$$
G_{I c}\left(N, R_{T}, T_{\min }\right)=G_{I c}^{\prime}\left(T_{\min }\right) f\left(N, R_{T}\right)
$$

where $G_{I c}^{\prime}\left(T_{\min }\right)$ is the critical ERR at the lowest temperature $T_{\min }, f\left(N, R_{T}\right)$ accounts for void and craze nucleation that allows damage to increase with $N, T$ is the temperature, and $R_{T}=T_{\min } / T_{\max }$ is the thermal ratio. Then, transverse cracking initiation and evolution of polymer-matrix composites under thermal cycling loads can be successfully predicted using both a Master Paris's law and DDM as presented in Ch. 5. Through the use of a Master Paris's law for a specific material system, $f(N)$ can be calculated for a given LSS and thermal ratio $R_{T}$, as shown in Figures 5.20, 5.21, and 5.22.

Although the understanding of characteristic regions from a Master Paris law may drastically reduce the number of specimens to be tested, still some experimental data is required, but less than that deemed necessary in previous literature [8-10,14, 18,2224,27-30]. As a result, transverse cracking evolution for high-cycle fatigue tests can be predicted as shown in Figures 5.23, 5.24, and 5.25.

Despite the proposed high-cycle fatigue predictions using a Master Paris law, the lack of experimental data for a large number of thermal cycles $(N>4000)$ may call into question the analytical predictions. This is because transverse cracking often precedes catastrophic modes of damage such as delamination and fiber breakage, which may result in final failure prior to reach infinite life.

The thermal ratio $R_{T}$, which is used as independent variable, varies depending on the application. Furthermore, crack density data has to be measured during fatigue testing at several values of number of cycles $\mathrm{N}$. Unlike mechanical fatigue, thermal fatigue tests are time consuming. For instance, a 30-year satellite life at LEO (Low Earth Orbit with $\left.R_{T}=-101 / 65\right)$ with an average 90 -minute period requires 175,000 cycles. A thermal 
fatigue cycle takes about 14 minutes using ovens [10,21, 23, 27, 186,303] and about 18 minutes using liquid nitrogen $[9,21,22,30]$. Therefore, 175,000 cycles would take 6 years of testing. For this reason, all the experimental data presented in the literature $[1,8-$ $10,14,21-25,27,29,30,186,303$ ] only covers at most 4000 cycles ${ }^{1}$, and often much less. Instead, a mechanical fatigue test at room temperature (RT) can be performed much faster.

An effort to relate mechanical and thermal fatigue is proposed in order to use mechanical fatigue tests as surrogate for thermal fatigue tests. That is, the goal is to calculate equivalent mechanical strains to simulate an equivalent thermal fatigue test. Therefore, transverse damage evolution for both thermal and mechanical tests has to be identical i.e., same crack density as function of number of cycles $\mathrm{N}$.

\subsection{Material System}

The material system used in this study is Amoco P75/1962, and the material properties of the fiber and matrix are collected from [12]. Lamina mechanical properties are calculated using periodic microstructure model (PMM) [290, A.2] while lamina CTE's are obtained using Levin's Model (LM) [269, Sect.4.4,16] with a volume fraction $V_{f}=0.52[10,188,189]$.

\subsection{Methods}

The objective of this section is to develop a methodology to simulate a thermal fatigue test by using equivalent mechanical strains. The differences between thermal and mechanical fatigue tests are highlighted and the tools to carry out such tests are developed.

Since mechanical fatigue tests can be easily performed using mechanical testing machines at RT by controlling the applied cycling strain, equivalent mechanical strains $\epsilon_{T}^{m e}$ would drastically reduce costs and time of fatigue tests. For a given thermal ratio $R_{T}$, the subscript $T$ in $\epsilon_{T}^{m e}$ represents the equivalent mechanical strain for thermal fatigue at temperature in the range $\left[T_{\min }, T_{\max }\right]$. Furthermore, experimental data for high-cycles tests could be collected to ascertain if other modes of damage occur at a large number of cycles.

The proposed methodology is illustrated in Figure 6.1 by a flowchart. That is, given a thermal fatigue test with a fixed $R_{T}$, the objective is to calculate the equivalent mechanical strains that produce the same transverse damage for a given number of cycles $\mathrm{N}$ at $T_{\min }$. Since cracks propagate at lowest temperature (highest residual stresses), $T_{\min }$ becomes

\footnotetext{
${ }^{1}$ Assuming thermal cycling uninterupted and only one crack density measurement is made at 4000 cycles, the test would run for almost two months
} 
the critical temperature. Therefore, crack density $\lambda_{T_{m i n}}^{t h}$ in each lamina during thermal fatigue has to be equal as $\lambda_{R T}^{m e}$ generated by equivalent mechanical strains for all cycles $N=1, \ldots, 10^{6}$. The superscript th means thermal whereas me means mechanical.

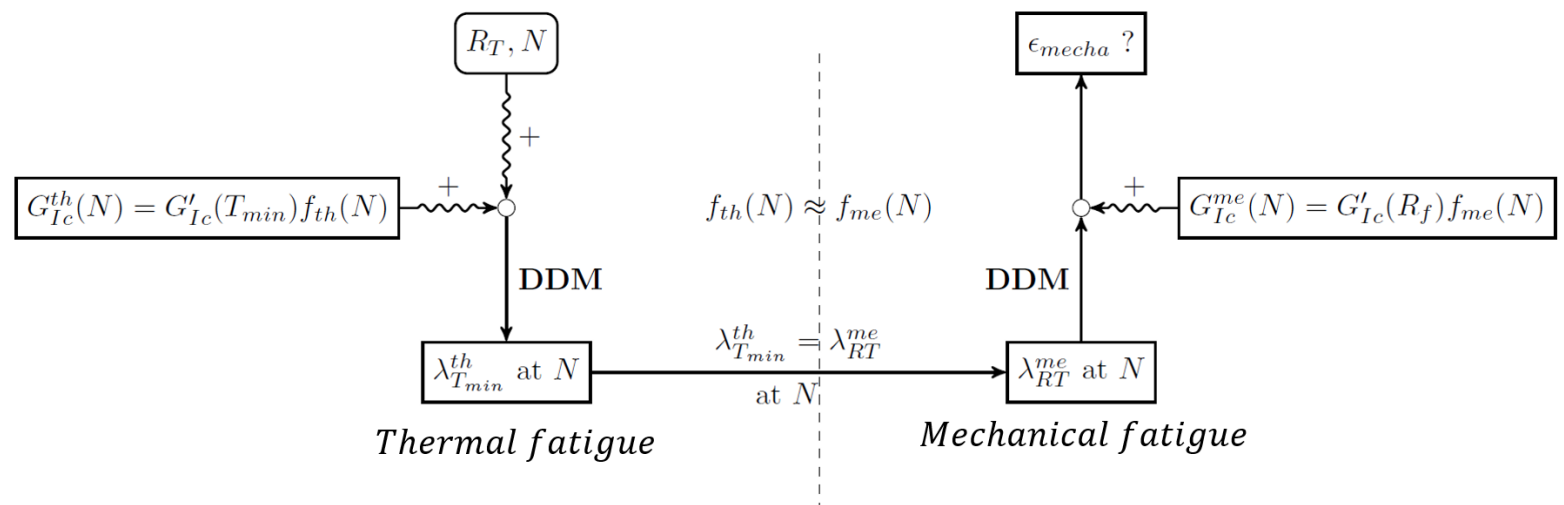

Figure 6.1: Proposed methodology to evaluate thermal fatigue through equivalent mechanical strains. Left side: Thermal fatigue. Right side: Mechanical fatigue.

On the left side of Figure 6.1 (thermal fatigue), given a fixed $R_{T}$ and number of cycles $N$, crack density $\lambda_{T_{m i n}}^{\text {th }}$ can be calculated using a Master Paris law and DDM model as explained in Ch. 5. Then, in order to obtain the equivalent mechanical strains $\epsilon_{T}^{m e}$ (right side in Figure 6.1), it is assumed that $\lambda_{T_{m i n}}^{t h}=\lambda_{R T}^{m e}$ for a given $N$. Since the thermo-mechanical properties of the material system are temperature-dependent, thermal strains $\epsilon^{t h}(T)$ under thermal cycling loads are different from mechanical strains $\epsilon_{T}^{m e}$ under mechanical cycling loads at room temperature $R T$. Note that the room temperature RT is often selected as reference temperature $T_{r}$ for mechanical testing because of its easy applicability, but other constant temperature $T_{r} \neq R T$ could be chosen as well as it will be explained later.

The fatigue resistance $f_{t h}(N)$ is temperature independent and thus, it is expected that $f_{t h}(N)$, which is only a function of $N$, can also be used to predict damage evolution in mechanical fatigue at RT. In order to simplify the proposed methodology, the analysis is split into stages: quasi-static cooling $(N=1)$ and thermal fatigue $(N \geq 2)$.

\subsubsection{Quasi-static cooling: $N=1$}

For a fixed thermal ratio $R_{T}=T_{\min } / T_{\max }$, crack density $\lambda_{T_{\min }}^{t h}$ in each lamina at the first cycle can be computed by cooling down to $T_{\min }$. This is because for $N=1$, the fatigue phenomenon is negligible so that $f\left(N=1, R_{T}\right)=1$. Thus, crack density evolution can be calculated with DDM when $G_{I} \geq G_{I c}$.

In order to simplify the problem, it is assumed that $G_{I c}^{\prime}\left(T_{\min }\right) \approx G_{I c}\left(T_{r}\right)$ which was shown to be a satisfactory approximation in Ch. 4 [12]. To satisfy identical crack density 
for both thermal and mechanical fatigue, equivalent mechanical strains at $T_{r}$ can be obtained satisfying the following conditions

$$
\begin{gathered}
\lambda_{T_{\text {min }}}^{\text {th }}=\lambda_{T_{r}}^{m e} \\
G_{I}^{t h}(T)=G_{I}^{m e}\left(\epsilon_{T}^{m e}, T_{r}\right)
\end{gathered}
$$

where $\epsilon_{T}^{m e}$ represent the equivalent mechanical strains for each temperature $T$ in the range $\left[T_{\min }, T_{\max }\right]$, and $T_{r}$ means reference temperature, which may be different from room temperature $R T$ as explained later. We use (6.3) instead of (6.2) to simplify the computations.

Since $G_{I c}^{\prime}$ is assumed to be temperature independent, ERR $G_{I}^{t h}$ and $G_{I}^{m e}$ of undamaged material have to be identical to satisfy equation (6.2). However, the material properties are temperature-dependent and consequently, the ERR $G_{I}(\lambda, T)$, with identical crack density $\lambda$, evolves differently with temperature $T$ than at temperature $T_{r}$ during mechanical fatigue. This fact can be seen in Figure 5.14 where $G_{I}$ at different $T$ differs even if crack density $\lambda$ is the same. Hence, for all $T \neq T_{r}$, the evolution of ERR for both thermal and mechanical differs and $\lambda_{T_{m i n}}^{t h}=\lambda_{T_{r}}^{t h}$ is not satisfied. This is because stiffness matrix $\bar{Q}_{i j}(\lambda, T)$, which is a direct function of $G_{I}$, changes with temperature and thus, the evolution of $G_{I}^{t h}(T)$ differ from $G_{I}^{m e}\left(\epsilon_{T}^{m e}, T_{r}\right)$.

In order to reduce discrepancies due to temperature-dependent properties and obtain the same fatigue resistance $f(N)$ in both thermal and mechanical fatigue, an additional condition is proposed

$$
\sigma_{2}^{t h}(T)=\sigma_{2}^{m e}\left(\epsilon_{T}^{m e}, T_{r}\right)
$$

where $\sigma_{2}^{\text {th }}$ and $\sigma_{2}^{m e}$ are the transverse stresses in each lamina. Cracks propagate in mode I so that only $\sigma_{2}$ is considered. Since stiffness matrix $\bar{Q}_{i j}(\lambda, T)$ changes with temperature and controls the evolution of $G_{I}(\lambda, T)$, the condition (6.4) forces equivalent mechanical strains $\epsilon_{T}^{m e}$ at $T_{r}$ to satisfy equation (6.3) as well. In other words, condition (6.4) must be satisfied during mechanical testing to ensure thermo-mechanical equivalence during thermal fatigue $(N>1)$ as explained later.

In order to obtain $\epsilon_{T}^{m e}$ at $T_{r}$ that best satisfy equations (6.3) and (6.4), a weighted residual method is proposed. Since a multitude of parameters must be considered $(\lambda, T$, $N, \bar{Q}_{i j}(\lambda, T), T_{r}$, etc.), a normalized objective residual function is minimized to obtain $\epsilon_{T}^{m e}$ by solving an optimization problem using a Nelder-Mead algorithm [304]. The objective residual function $\mathrm{L}$ involves the residual combination of both ERR $G_{I}(6.3)$ and stress $\sigma_{2}$ 
(6.4) for each lamina as follows

$$
L(T)=\left[\sum_{j=1}^{2 M} w_{j}^{f}\left(\frac{f_{j}(T)-Z_{j}^{*}(T)}{Z_{j}^{*}(T)}\right)^{2}\right]^{(1 / 2)}
$$

where

$$
Z_{j}^{*}=\left\{G_{I}^{t h}(T), \sigma_{2}^{t h}(T)\right\}
$$

are the pair of thermal ERR and stress values at each $T$ calculated with DDM cooling down to $T_{\min }$ with $j=1, \ldots, M$, and

$$
f_{j}=\left\{G_{I}^{m e}\left(\epsilon_{T}^{m e}, T_{r}\right), \sigma_{2}^{m e}\left(\epsilon_{T}^{m e}, T_{r}\right)\right\}
$$

are the pair of mechanical ERR and stress values calculated at each $T$ using $\epsilon_{T}^{m e}$ at $T_{r}$ with $j=1, \ldots, M$. The $w_{j}^{f}$ parameter are the user-supplied weights for each objective function $f_{j}$. Therefore, for $M$ number of symmetric laminas, both equation (6.3) and (6.4) must be satisfied leading to $2 M$ residual functions computed as unique objective function L (6.5) using the weights $w_{j}^{f}$.

The weights $w_{j}^{f}$ are computed for each pair functions $f_{j}(6.7)$ in each symmetric lamina so that the sum of all $w_{j}^{f}$ are equal to unit value. Furthermore, all $M$ of $w_{j}^{f}$ that correspond to one of the two objective functions $f_{j}$, namely the ERR $G_{I}(6.3)$ and stress $\sigma_{2}(6.4)$, are assumed to be equal. As a results, the constraint equations for $w_{j}^{f}$ are

$$
\sum_{j=1}^{2 M} w_{j}^{f}=1
$$

and

$$
w_{j=1}^{G_{I}}=\ldots=w_{j=M}^{G_{I}} \quad ; \quad w_{j=1}^{\sigma_{2}}=\ldots=w_{j=M}^{\sigma_{2}}
$$

Operating equations (6.8) and (6.9), all constraints can be reduced to one single equation as follows

$$
w_{j}^{\sigma_{2}}=\frac{1}{M}-w_{j}^{G_{I}}
$$

with $j=1, \ldots, M$ and thus, only $w_{j}^{G_{I}}$ needs to be calculated while minimizing (6.5) using the Nelder-Mead algorithm.

\subsubsection{Thermal cycling loads: $N>1$}

Under thermal cycling loads, void and craze nucleation must occur to propagate new cracks as the number of cycles increases. Such cracks come from initial flaws whose 
length size $a \leq a_{c}$. Therefore, as soon as larger number of cycles are performed, initial flaws length size $a$ increases until they reach a critical size $a_{c}$.

However, as soon as new cracks propagate and $\lambda$ increases, stiffness degradation leads to lower $G_{I}$ and consequently, lower stress $\sigma_{2}$ also. Therefore, according to equation (5.9) given by Linear Elastic Fracture Mechanics (LEFM), existing flaws will propagate under lower stress only if they reach a larger critical size $a_{c}$. That is, critical size $a_{c}(N)$ must increase with $\mathrm{N}$.

As explained in Ch. 5 , the fatigue resistance $f(N) \propto 1 / a_{c}$ and thus, as soon as $a_{c}(N)$ increases, $f(N)$ decreases with number of cycles in the range $1>f(N)>0$ as shown in Figures 5.20, 5.21, and 5.22. In other words, the fatigue resistance $f(N)$ describes the effect of void nucleation. Consequently, in order to satisfy equation (6.2) for each $N$, it is required that the damage growth rate $d \lambda / d N$ with number of cycles for both thermal and mechanical fatigue be identical.

Since $f(N)$ is temperature independent for a fixed $R_{T}$ (see equation 5.5), then the crack-growth rate $d \lambda / d N$ on laminates subjected to either thermal or mechanical loads evolves identically under same transverse loading conditions $\sigma_{2}$. Hence, crack density $\lambda$ under thermal or mechanical cycling loads as function of number of cycles $N$ is expected to be identical. Note that the stress is independent of how such stress is developed (thermal or mechanical loads) and thus it is a common parameter for both fields: mechanical and thermal.

In consequence, $f_{t h}(N)=f_{m e}(N)$ is automatically satisfied when $\lambda_{T_{r}}^{m e}=\lambda_{T_{\min }}^{\text {th }}$ is achieved under same transverse loading conditions $\sigma_{2}$, i.e. equation (6.4). However, the challenge is to find equivalent mechanical strains $\epsilon_{T}^{m e}(N)$ at each $T$ for which both equation (6.2) and (6.4) are satisfied, which is the same as satisfying (6.3) and (6.4).

Therefore, given a fixed $R_{T}$ and number of cycles $N \geq 2$, crack density $\lambda$ for both mechanical and thermal fatigue evolves identically at $N$ if conditions (6.3) and (6.4) are satisfied in the range $1<N<10^{6}$. In that case, similar to quasi-static cooling, equivalent mechanical strains $\epsilon_{T}^{m e}(N)$ at $T_{r}$ can be calculated by minimizing a normalized objective residual function L using a Nelder-Mead algorithm [304] as follows

$$
L(T, N)=\left[\sum_{j=1}^{2 M} w_{j}^{f}\left(\frac{f\left(T, \lambda_{T_{\min }}^{N-1}\right)-Z_{j}^{*}\left(T, \lambda_{T_{m i n}}^{N-1}\right)}{Z_{j}^{*}\left(T, \lambda_{T_{\min }}^{N-1}\right)}\right)^{2}\right]^{(1 / 2)}
$$

where

$$
Z_{j}^{*}=\left\{G_{I}^{t h}\left(T, \lambda_{T_{\min }}^{N-1}\right), \sigma_{2}^{t h}\left(T, \lambda_{T_{m i n}}^{N-1}\right)\right\}
$$

are the pair of thermal ERR and stress values at each $T$ calculated with DDM cooling down to $T_{\min }$ and updating the crack density $\lambda_{T_{m i n}}^{N-1}$ from previous cycle with $j=1, \ldots, M$, 
and

$$
f_{j}=\left\{G_{I}^{m e}\left(\epsilon_{T}^{m e}, T_{r}, \lambda_{T_{m i n}}^{N-1}\right), \sigma_{2}^{m e}\left(\epsilon_{T}^{m e}, T_{r}, \lambda_{T_{m i n}}^{N-1}\right)\right\}
$$

are the pair of mechanical ERR and stress values calculated at each $T$ using $\epsilon_{T}^{m e}(N)$ at $T_{r}$ updating the crack density $\lambda_{T_{m i n}}^{N-1}$ from previous cycle with $j=1, \ldots, M$. The updated $\lambda_{T_{\min }}^{N-1}$ from previous cycle $(N-1)$ can be calculated using the Master Paris Law for the material system of interest as explained in Ch.5 (for instance, see Figure 5.17, 5.20, and $5.23)$.

The $w_{j}^{f}$ parameter are the user-supplied weights for each objective function $f_{j}$. For $M$ number of symmetric laminas, both conditions (6.3) and (6.4) must be satisfied leading to $2 M$ residual functions computed as unique objective function $\mathrm{L}(6.11)$ using the weights $w_{j}^{f}$. Similar to quasi-static cooling, only $w_{j}^{G_{I}}$ needs to be calculated by solving the optimization problem. The rest of $w_{j}^{f}$ are calculated using equation (6.10).

\subsection{Biaxial Thermo-Mechanical Equivalence}

According to Figure 6.1, equivalent mechanical fatigue test (right side) can be calculated from thermal fatigue predictions (left side) so that equation (6.2) is satisfied for all life values $N$.

In order to achieve this, both conditions (6.3) and (6.4) must be satisfied by minimizing an objective residual function given by equations (6.5) and (6.11) for quasi-static cooling and thermal fatigue, respectively. Thermo-mechanical equivalence can be calculated if condition (6.4) is first satisfied with the best possible estimate during quasi-static cooling obtaining the set $\epsilon_{T}^{m e}$.

In this section, an equivalent biaxial mechanical fatigue test is developed for the entire laminate. This is because under thermal cycling loads there is no reference coordinate system and thus, all laminas are exposed to crack propagation. In addition, the thermomechanical equivalence is studied with the highest thermal ratio $R_{T}=-156 / 121$ and mechanical reference temperature $T_{r}=R T$, but a different reference temperature could be used as explained later. A uniaxial mechanical fatigue test is developed in Sect. 6.4.

\subsubsection{Laminate $\left[(0 / 90)_{2}\right]_{s}$}

In Figure 6.2, crack density evolution $\lambda^{\text {th }}$ for laminate $\left[(0 / 90)_{2}\right]_{s}$ P75/1962 with $R_{T}=$ $-156 / 121$ is compared with $\lambda^{m e}$ for the same laminate subjected to equivalent mechanical strains $\epsilon_{T}^{t h}$ at RT for all laminas. In the same way, $G_{I}^{t h}(T)$ is compared with $G_{I}^{m e}\left(\epsilon_{T}^{m e}, R T\right)$ in Figure 6.3, $\sigma_{1}^{\text {th }}(T)$ is compared with $\sigma_{1}^{m e}\left(\epsilon_{T}^{m e}, R T\right)$ in Figure 6.4, and $\sigma_{2}^{\text {th }}(T)$ is compared with $\sigma_{2}^{m e}\left(\epsilon_{T}^{m e}, R T\right)$ in Figure 6.5 for all laminas. 


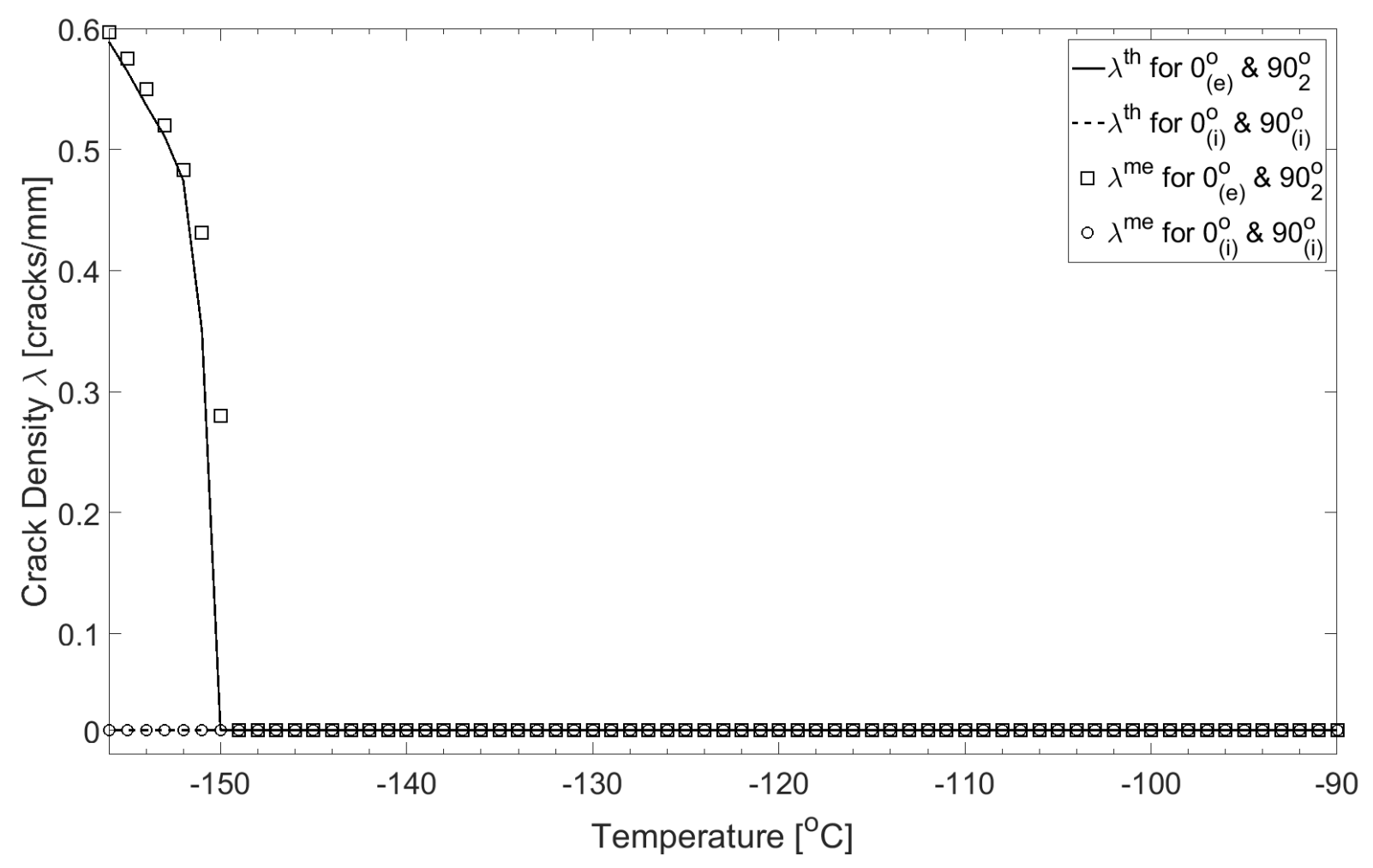

Figure 6.2: Comparison between crack density evolution $\lambda^{\text {th }}$ for $R_{T}=-156 / 121$ vs. crack density evolution $\lambda^{\text {me }}$ subjected to equivalent mechanical strains $\epsilon_{T}^{m e}$ at $R T$ for laminate $\left[(0 / 90)_{2}\right]_{s}$ P75/1962 in the range $\left[T_{\max }, T_{\min }\right]$.

As it can be seen in Figure $6.2, \lambda^{\text {th }}$ compares very well with $\lambda^{\text {me }}$ for all temperatures values $T$ and $\lambda_{R T}^{m e}=\lambda_{T_{m i n}}^{t h}$ is satisfied. Therefore, it is concluded that weighted residual method can be used to successfully to minimize the objective residual function (6.5). The weights $w_{j}^{f}$ that best satisfy the conditions (6.2), (6.3), and (6.4) are found to be 0.14375 for $G_{I}$ and 0.10625 for $\sigma_{2}$ in each symmetric lamina. Crack density occurs only on surface $0^{\circ}$ and middle $90_{2}^{\circ}$ laminas because $G_{I}>G_{I c}$ in those laminas.

In the same way, $G_{I}^{t h}(T)$ compares very well with $G_{I}^{m e}\left(\epsilon_{T}^{m e}, R T\right)$ during cooling as shown in Figure 6.3 satisfying the condition (6.3). However, some discrepancies occur for the stress state as it can be seen in Figures 6.4 and 6.5.

Looking into Figure 6.4, longitudinal stresses $\sigma_{1}$ in all laminas when laminate is subjected to thermal loads totally disagree with respect to those $\sigma_{1}$ obtained through equivalent mechanical strains $\epsilon_{T}^{m e}$. This is due to the following reasons.

According to Figure 5.1, each lamina in a polymer-matrix composite with carbon fibers expands along fiber direction (negative CTE) whereas it contracts perpendicular to fibers (positive CTE) during cooling. Therefore, the average $\bar{\epsilon}_{i}$ at $T_{\min }$ emerges from internal equilibrium between laminas. As a result, $\sigma_{1}$ becomes negative in all laminas while $\sigma_{2}$ are positive leading to transverse cracking regardless of lamina orientation. In addition, $\sigma_{1}$ are small in the range $0>\sigma_{1}>-90 \mathrm{MPa}$. 


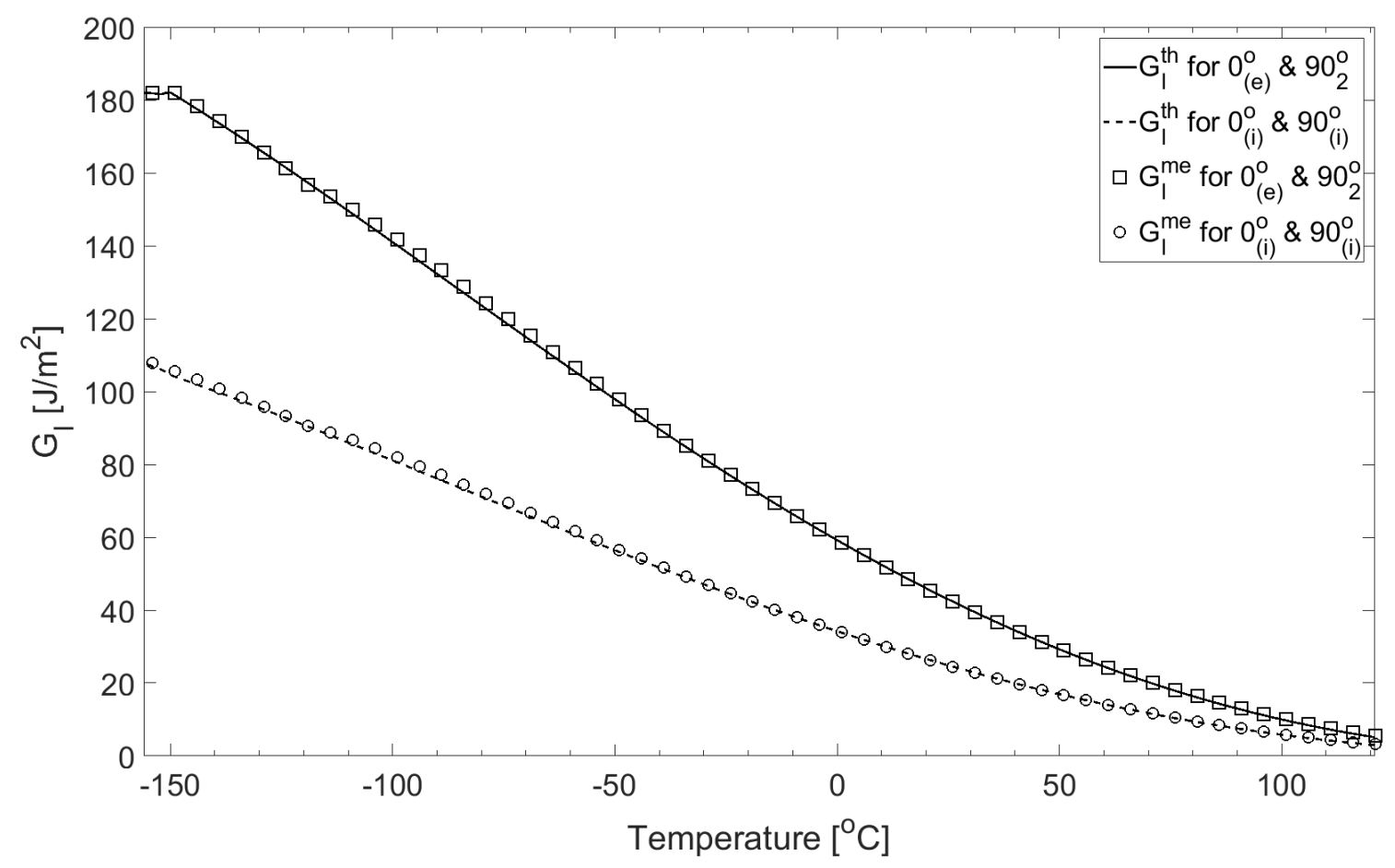

Figure 6.3: Comparison between ERR $G_{I}^{\text {th }}$ for $R_{T}=-156 / 121$ vs. $G_{I}^{m e}$ at RT subjected to equivalent mechanical strains $\epsilon_{T}^{m e}$ for laminate $\left[(0 / 90)_{2}\right]_{s}$ P 75/1962 in the range $\left[T_{\min }, T_{\max }\right]$.

Unlike thermal loading, the average $\bar{\epsilon}_{i}$ at RT caused by equivalent mechanical strains $\epsilon_{T}^{m e}$ are imposed in both directions, $\mathrm{x}$ and $\mathrm{y}$. Therefore, both stresses $\sigma_{1}$ and $\sigma_{2}$ are either positive or negative. In other words, it is physically impossible to replicate $\sigma_{1}$ from a free thermal expansion through equivalent mechanical strains. However, $\sigma_{1}$ are supported by the fibers and damage is controlled by $\sigma_{2}$, so only $\sigma_{2}$ need to be matched.

The longitudinal stress $\sigma_{1}$ obtained through equivalent mechanical strains $\epsilon_{T}^{m e}$ reach values close to ultimate tension and compression strength at $T_{\min }$ and $T_{\max }$, respectively as shown in Figure 6.4. Therefore, the testing conditions must be carefully selected in order to reduce the probability of fiber breakage. For instance, lower temperature range may need to be used for mechanical testing.

Transverse stresses $\sigma_{2}^{m e}$ using equivalent mechanical strains slightly differ at temperatures close to $T_{\min }$ and $T_{\max }$ as shown in Figure 6.5. Thus, no combination of $\epsilon_{T}^{m e}$ exist that exactly satisfies simultaneously the conditions given by equations (6.3) and (6.4) for all $T$. However, using equation (6.5), it is possible to find specific combination of $\epsilon_{T}^{m e}$ that satisfy at least $\lambda_{R T}^{m e}=\lambda_{T_{m i n}}^{t h}$. In this case, crack density evolution compares very well during cooling (Figure 6.2).

As temperature decreases, the error induced by the difference between $\epsilon_{T}^{m e}$ and $\bar{\alpha}_{i}(T)(T-$ $R T$ ) increases leading to higher discrepancy as $T$ move away from RT. At $T_{\text {max }}$, the error is $11 \%$ while at $T_{\min }$ is $7 \%$. However, for the material system used, $\Delta \sigma_{2}$ at $T_{\min }$ is less than $8 \mathrm{MPa}$ while $\Delta \sigma_{2}$ at $T_{\max }$ is less than $2 \mathrm{MPa}$. Therefore, smaller errors can be 


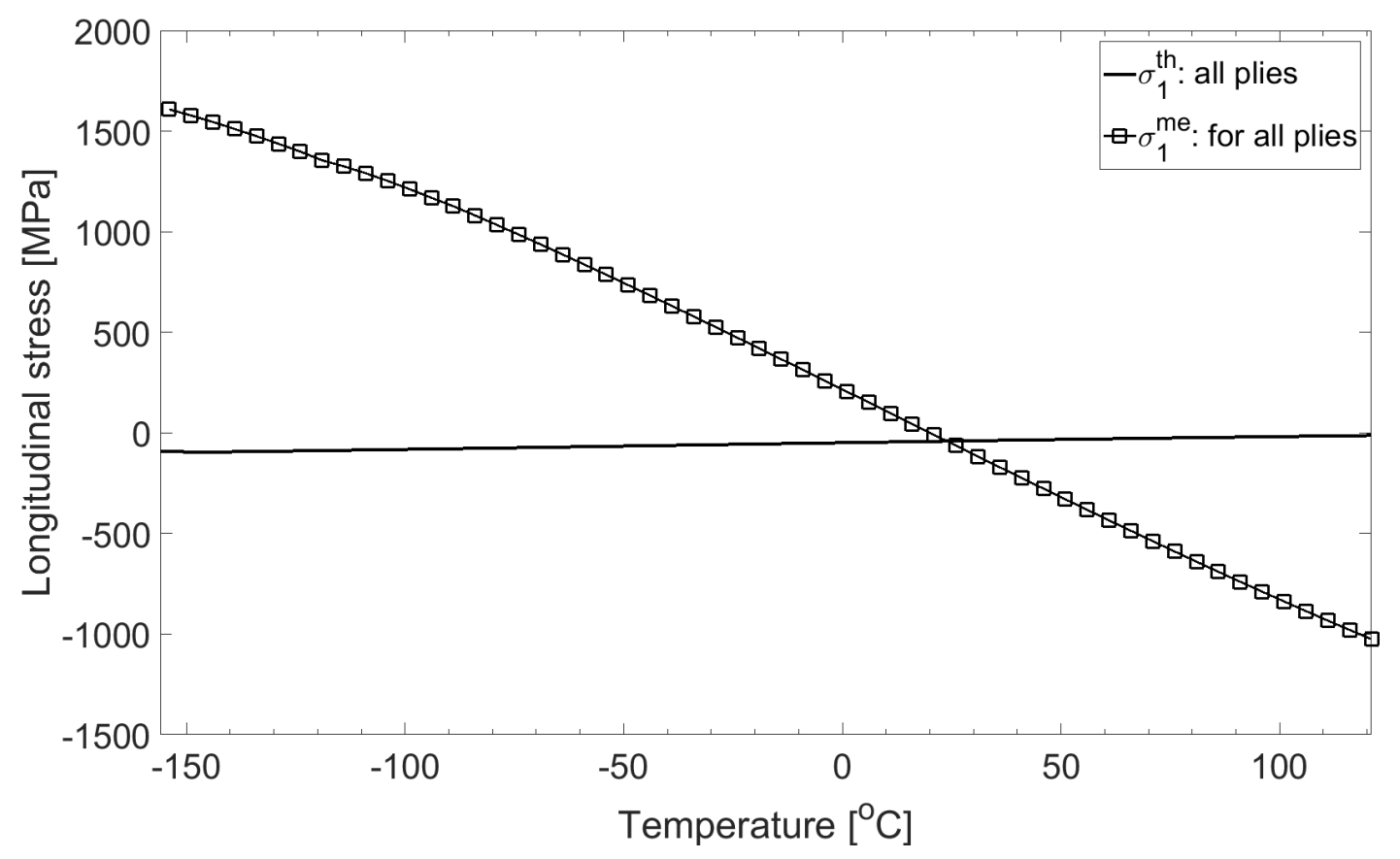

Figure 6.4: Comparison between longitudinal stress $\sigma_{1}^{\text {th }}$ for $R_{T}=-156 / 121$ vs. $\sigma_{1}^{m e}$ at $R T$ subjected to equivalent mechanical strains $\epsilon_{T}^{m e}$ for laminate $\left[(0 / 90)_{2}\right]_{s}$ Pr5/1962 in the range $\left[T_{\min }, T_{\max }\right]$.

achieved if the mechanical testing temperature is close to $T_{m i n}$, that is, if $T_{r}$ is chosen to be lower than RT. This is because higher residual stresses are generated at lowest temperature.

The equivalent mechanical strains $\epsilon_{T}^{m e}$ calculated using (6.5) are shown in Figure 6.6 with RT as reference temperature. Since laminate is cross-ply and no shear stress is generated, the calculated $\epsilon_{x}^{m e}(T)$ and $\epsilon_{y}^{m e}(T)$ are found to be identical.

Looking into Figure 6.5, the larger error occurs at $T_{\min }$ where larger residual stresses are generated $(\approx 8 \mathrm{MPa})$. Thus, a reference temperature set closer to $T_{\min }$ would lead a drastic reduction of $\Delta \sigma_{2}$. However, the equivalent mechanical strains $\epsilon_{T}^{m e}$ needed to simulate the thermal fatigue would become negative as illustrated in Figure 6.6 using RT as reference temperature where $\epsilon_{T}^{m e}$ are negative in the range $R T<T \leq T_{\max }$. Furthermore, the equivalent mechanical fatigue test would be performed applying compression which, on the other hand, it would make its execution more complex from a practical point of view. For this reason, a reference temperature equal to RT is desirable. If for a given $R_{T}, T_{\max }$ is close to RT, the error induced close to $T_{\max }$ is reduced and $\epsilon_{T}^{m e}$ become positive. However, larger errors would be induced at temperatures close to $T_{\min }$.

\subsubsection{Laminate $[0 / \pm 45 / 90]_{s}$}

Crack density evolution $\lambda^{\text {th }}$ for laminate $[0 / \pm 45 / 90]_{s} \mathrm{P} 75 / 1962$ with $R_{T}=-156 / 121$ is compared with $\lambda^{m e}$ for the same laminate subjected to equivalent mechanical strains $\epsilon_{T}^{t h}$ 


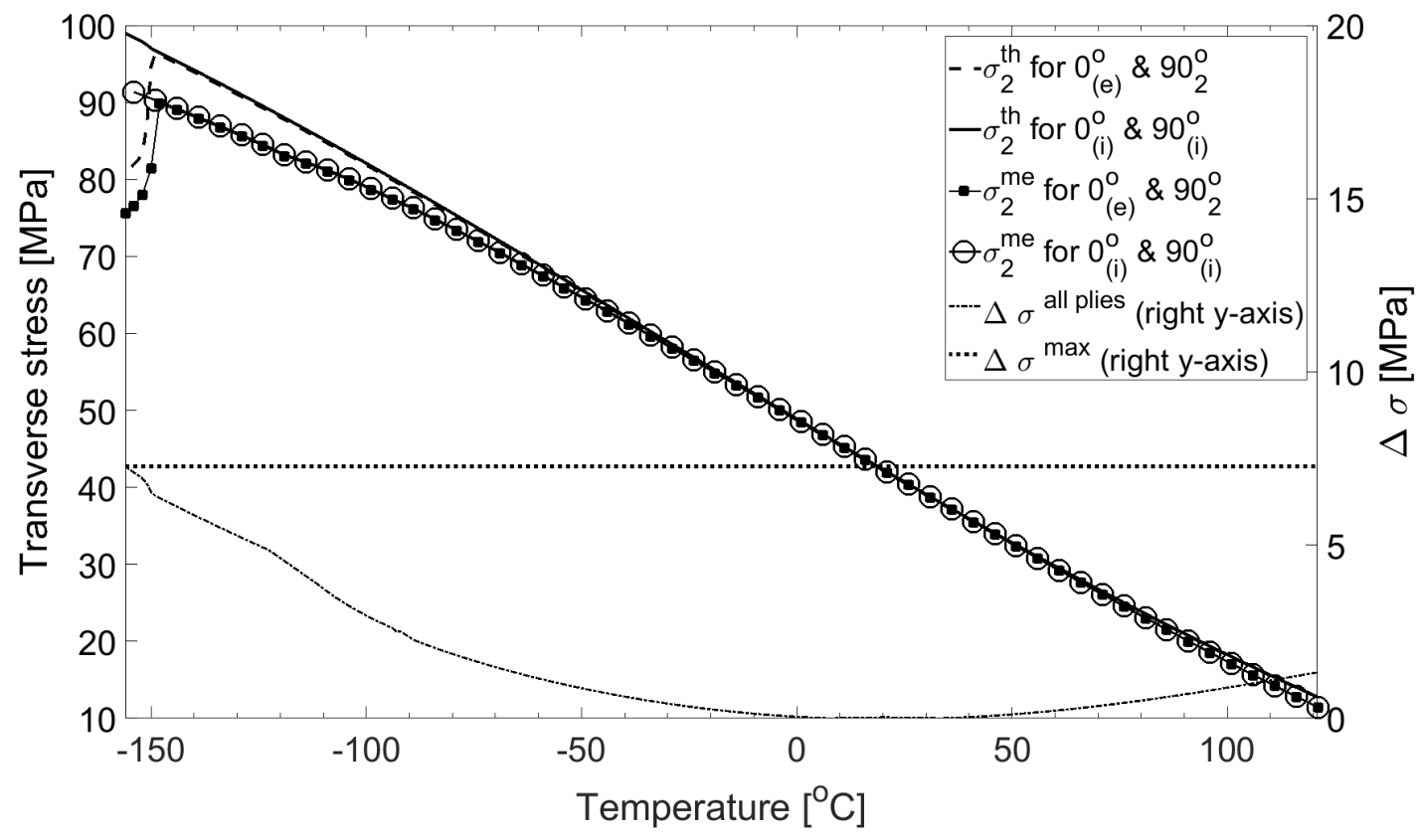

Figure 6.5: Comparison between transverse stress $\sigma_{2}^{\text {th }}$ for $R_{T}=-156 / 121$ vs. $\sigma_{2}^{\text {me }}$ at $R T$ subjected to equivalent mechanical strains $\epsilon_{T}^{m e}$ for laminate $\left[(0 / 90)_{2}\right]_{S} P 75 / 1962$ in the range $\left[T_{\min }, T_{\max }\right]$.

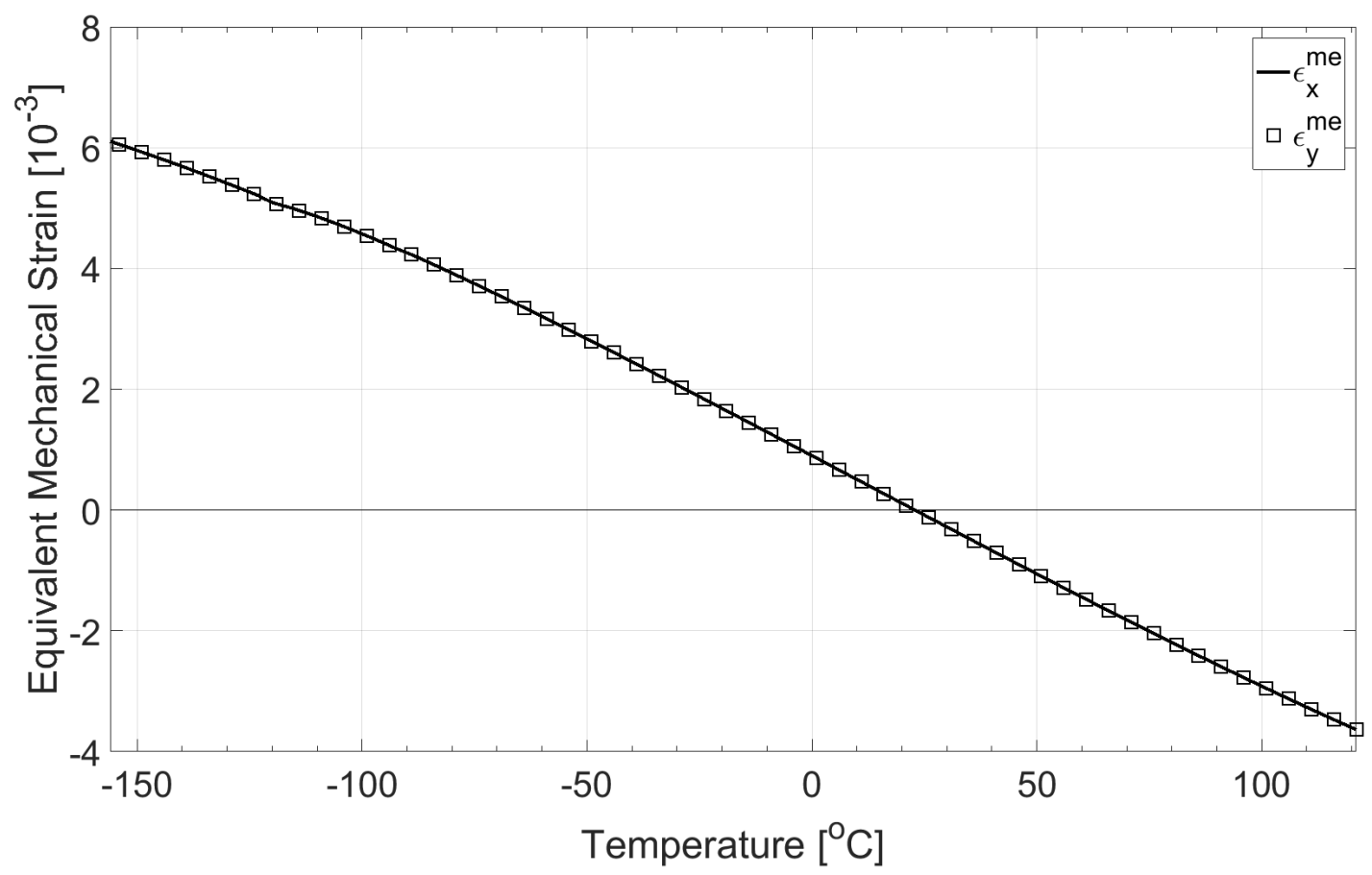

Figure 6.6: Evolution of equivalent mechanical strains $\epsilon_{T}^{m e}$ with $T$ for laminate $\left[(0 / 90)_{2}\right]_{s}$ $P^{r 5} / 1962$ in the range $\left[-156,121^{\circ} \mathrm{C}\right]$. Reference temperature is set to $R T=23^{\circ} \mathrm{C}$. 


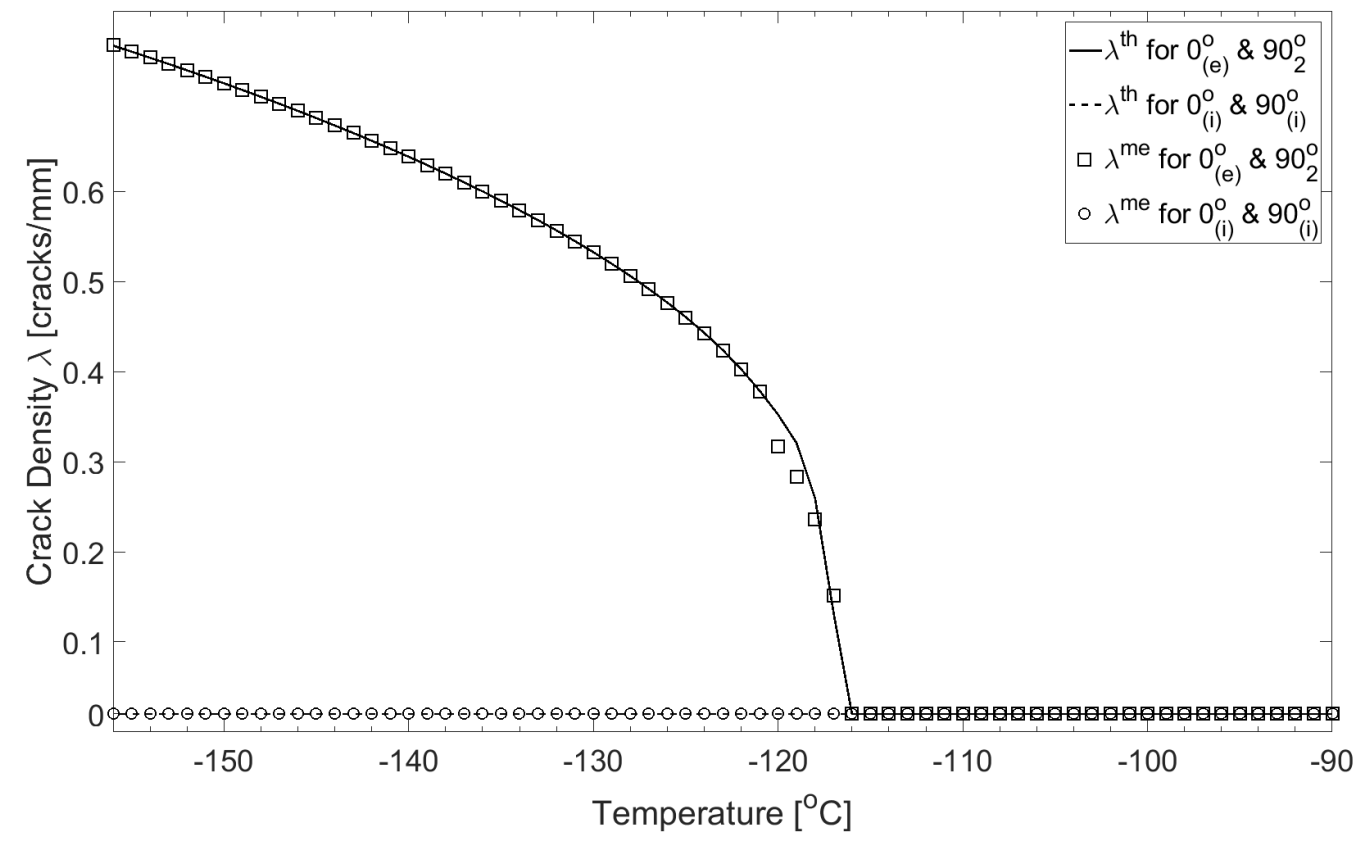

Figure 6.7: A comparison between crack density evolution $\lambda^{\text {th }}$ with $R_{T}=-156 / 121$ vs. crack density evolution $\lambda^{\text {me }}$ subjected to equivalent mechanical strains $\epsilon_{T}^{m e}$ at RT for laminate $[0 / \pm 45 / 90]_{s} P^{75} / 1962$ in the range $\left[T_{\min }, T_{\max }\right]$.

at RT as shown in Figure 6.7 for all laminas. In the same way, $\sigma_{2}^{t h}(T)$ is compared with $\sigma_{2}^{m e}\left(\epsilon_{T}^{m e}, R T\right)$ in Figure 6.8 for all laminas.

As it can be seen in Figure 6.7, $\lambda^{\text {th }}$ compares very well with $\lambda^{m e}$ at each temperature $T$ achieving the same crack density even though the laminate is quasi-isotropic (QI). The weights $w_{j}^{f}$ are set again to 0.14375 for $G_{I}$ and 0.10625 for $\sigma_{2}$ in each lamina because they are the combination that best satisfy the conditions given by equations (6.3), and (6.4). Damage occurs only on surface $0^{\circ}$ and middle $90_{2}^{\circ}$ laminas because $G_{I}>G_{I c}$ only for those laminas. Similar to Figure $6.3, G_{I}^{t h}(T)$ matches $G_{I}^{m e}\left(\epsilon_{T}^{m e}, R T\right)$ satisfying the condition given by equation (6.3).

The longitudinal stress $\sigma_{1}$ obtained through equivalent mechanical strains $\epsilon_{T}^{m e}$ reach similar values as those in Figure 6.4 for laminate $\left[(0 / 90)_{2}\right]_{s}$. Therefore, fiber breakage may occur unless the testing temperature range is reduced.

Similar to transverse stresses in Figure 6.5 for laminate $\left[(0 / 90)_{2}\right]_{s}$, small discrepancies can be found for $\sigma_{2}^{m e}$ close to $T_{\min }$ and $T_{\max }$ as shown in Figure 6.8. Thus, it does exist any combination of $\epsilon_{T}^{m e}$ that exactly satisfy simultaneously the conditions given by equations (6.3) and (6.4). However, using equation (6.5), it is possible to find a specific combination of $\epsilon_{T}^{m e}$ that satisfies at least $\lambda_{R T}^{m e}=\lambda_{T_{\min }}^{t h}$. In this case, crack density evolution compares very well during cooling (Figure 6.7). 


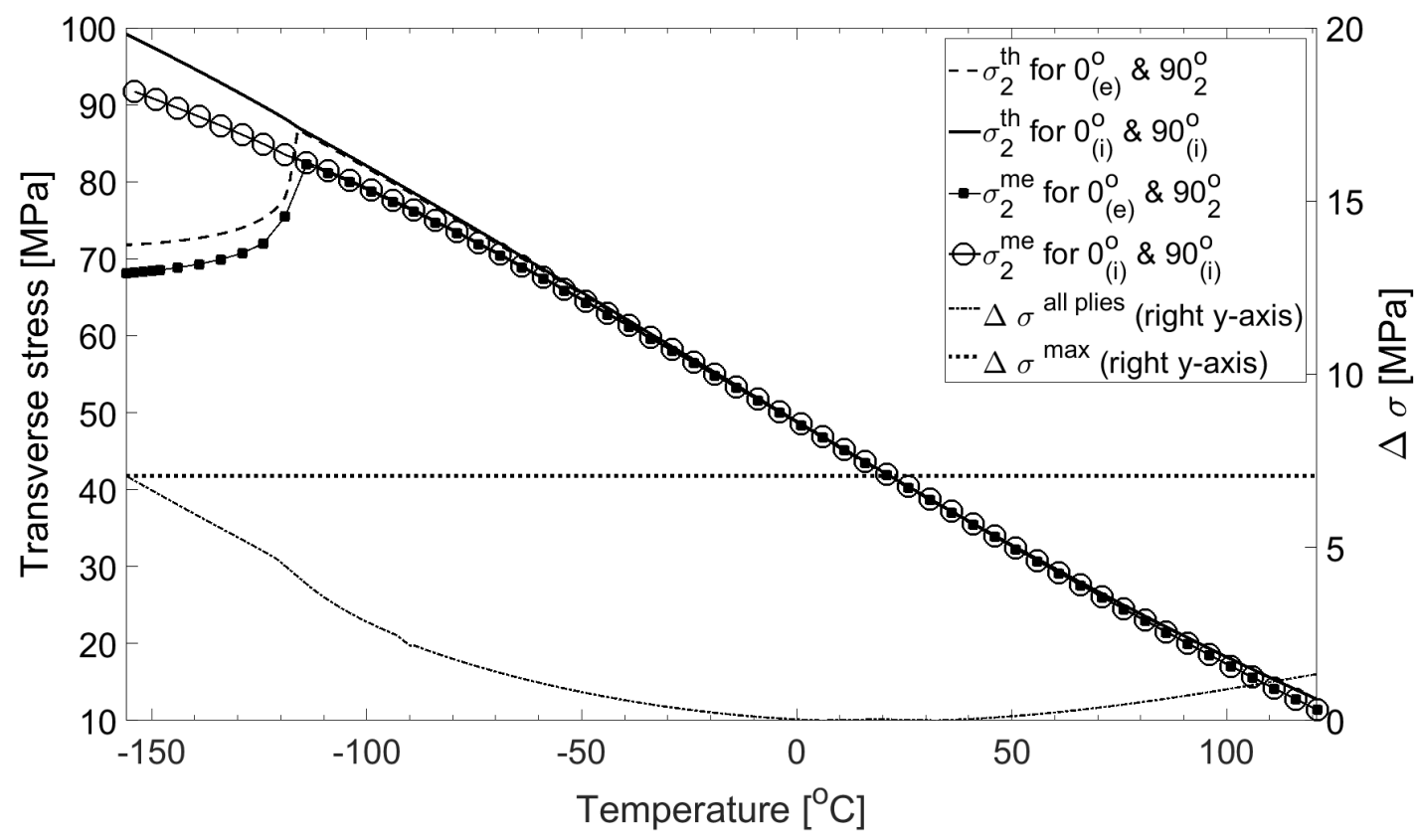

Figure 6.8: A comparison between transverse stress $\sigma_{2}^{\text {th }}$ with $R_{T}=-156 / 121$ vs. $\sigma_{2}^{\text {me }}$ at $R T$ subjected to equivalent mechanical strains $\epsilon_{i}^{m e}$ for laminate $[0 / \pm 45 / 90]_{s}$ P75/1962 in the range $\left[T_{\min }, T_{\max }\right]$.

\subsection{Unialxial Thermo-Mechanical Equivalence}

Based on Figures 6.4, 6.5, 6.7, and 6.8, a combination of $\epsilon_{T}^{m e}$ does not exist for which both $\lambda_{T_{r}}^{m e}=\lambda_{T_{m i n}}^{t h}$ and identical the stress field, namely $\sigma_{1}$ and $\sigma_{2}$ are obtained. This is due to two reasons.

First, the material system is temperature-dependent so that the stress field is only identical at the reference temperature $T_{r}$. Second, the imposed equivalent mechanical strains $\epsilon_{T}^{m e}$ are applied in a different way (Figure ??) compared to those $\epsilon^{\text {th }}$ obtained under thermal fatigue.

Although same crack density $\lambda$ can be achieved as shown in Figures 6.2 and 6.7 during the first cycle, for $N \geq 2 \lambda_{T_{r}}^{m e}=\lambda_{T_{m i n}}^{t h}$ will only be satisfied if all laminas are subjected to same transverse loading $\sigma_{2}$, so that same void and craze nucleation with the number of cycles $\mathrm{N}$ can be achieved in both, thermal and mechanical fatigue.

In order to reduce discrepancies for $\sigma_{2}$, the problem studied is simplified to accomplish $\lambda_{T_{r}}^{m e}=\lambda_{T_{m i n}}^{t h}$ focusing just in one lamina. The middle lamina is selected because it is the first lamina to crack due to its higher thickness. Reference temperature $T_{r}$ is set to room temperature $R T$ for equivalent mechanical test, but a different reference temperature could be used as explained later. 


\subsubsection{Thermo-Mechanical Equivalence: Middle lamina}

The thermo-mechanical equivalence that satisfies $\lambda_{T_{r}}^{m e}=\lambda_{T_{m i n}}^{t h}$ in the middle lamina allows us to simplify the problem from a biaxial mechanical test to uniaxial test making easier its practical execution. Thus, only $\epsilon_{T}^{m e}$ along x-direction needs to be considered.

Similar to previous cases, crack density $\lambda_{90_{2}}^{t h}$ for laminate $\left[(0 / 90)_{2}\right]_{s}$ P75/1962 with $R_{T}=-156 / 121$ is compared with $\lambda_{90_{2}}^{m e}$ for the same laminate subjected to equivalent mechanical strains $\epsilon_{x}^{m e}$ at RT as shown in Figure 6.9 for middle $90_{2}^{\circ}$ lamina. In the same way, $G_{I}^{\text {th }}(T)$ is compared with $G_{I}^{m e}\left(\epsilon_{x}^{m e}, R T\right)$ in Figure $6.10, \sigma_{1}^{\text {th }}(T)$ is compared with $\sigma_{1}^{m e}\left(\epsilon_{x}^{m e}, R T\right)$ in Figure 6.11, and $\sigma_{2}^{\text {th }}(T)$ is compared with $\sigma_{2}^{m e}\left(\epsilon_{x}^{m e}, R T\right)$ in Figure 6.12 for middle $90_{2}^{\circ}$ lamina.

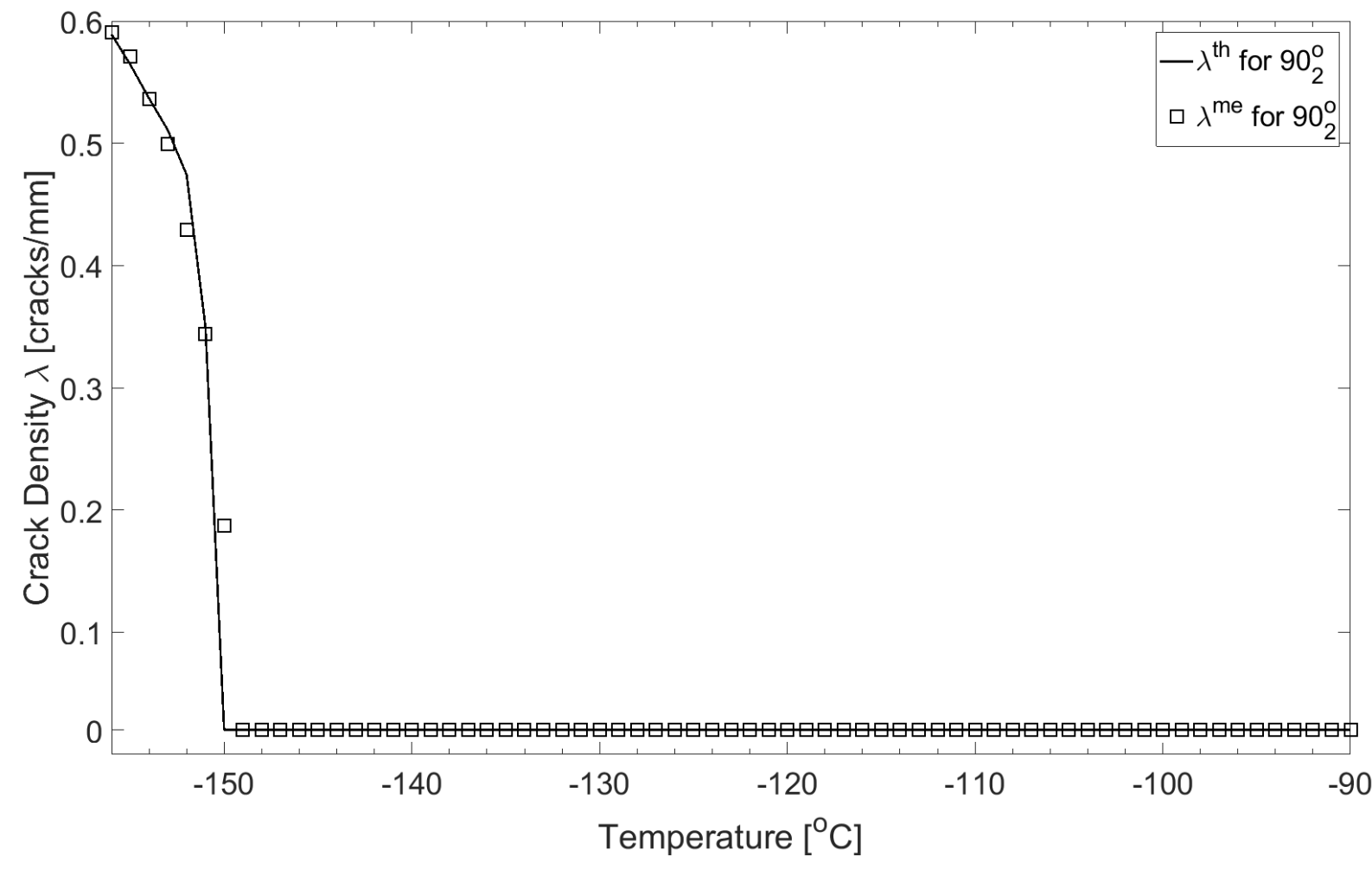

Figure 6.9: Comparison between crack density evolution $\lambda_{90_{2}}^{\text {th }}$ for $R_{T}=-156 / 121$ vs. crack density evolution $\lambda_{90_{2}}^{\text {me }}$ subjected to uniaxial mechanical strains $\epsilon_{x}^{m e}$ at $R T$ for laminate $\left[(0 / 90)_{2}\right]_{s}$ P $75 / 1962$ in the range $\left[T_{\min }, T_{\max }\right]$.

As it can be seen in Figure 6.9, $\lambda_{90_{2}}^{t h}$ compares very well with $\lambda_{90_{2}}^{m e}$ for all temperature values $T$ and thus $\lambda_{R T}^{m e}=\lambda_{T_{m i n}}^{t h}$. The weighted residual method is used to successfully minimize the objective residual function (6.5). The weights $w_{90_{2}}^{f}$ that best satisfy the conditions given by equations (6.2), (6.3), and (6.4) are 0.575 for $G_{I}$ and 0.425 for $\sigma_{2}$ in middle $90_{2}^{\circ}$ lamina. Since only the middle lamina is taken into account $(M=1)$, two $w_{j}^{f}$ are computed using (6.10). Crack density occurs because $G_{I}>G_{I c}$ for middle $90_{2}^{o}$ lamina.

In the same way, $G_{I}^{t h}(T)$ compares very well with $G_{I}^{m e}\left(\epsilon_{x}^{m e}, R T\right)$ during cooling for 


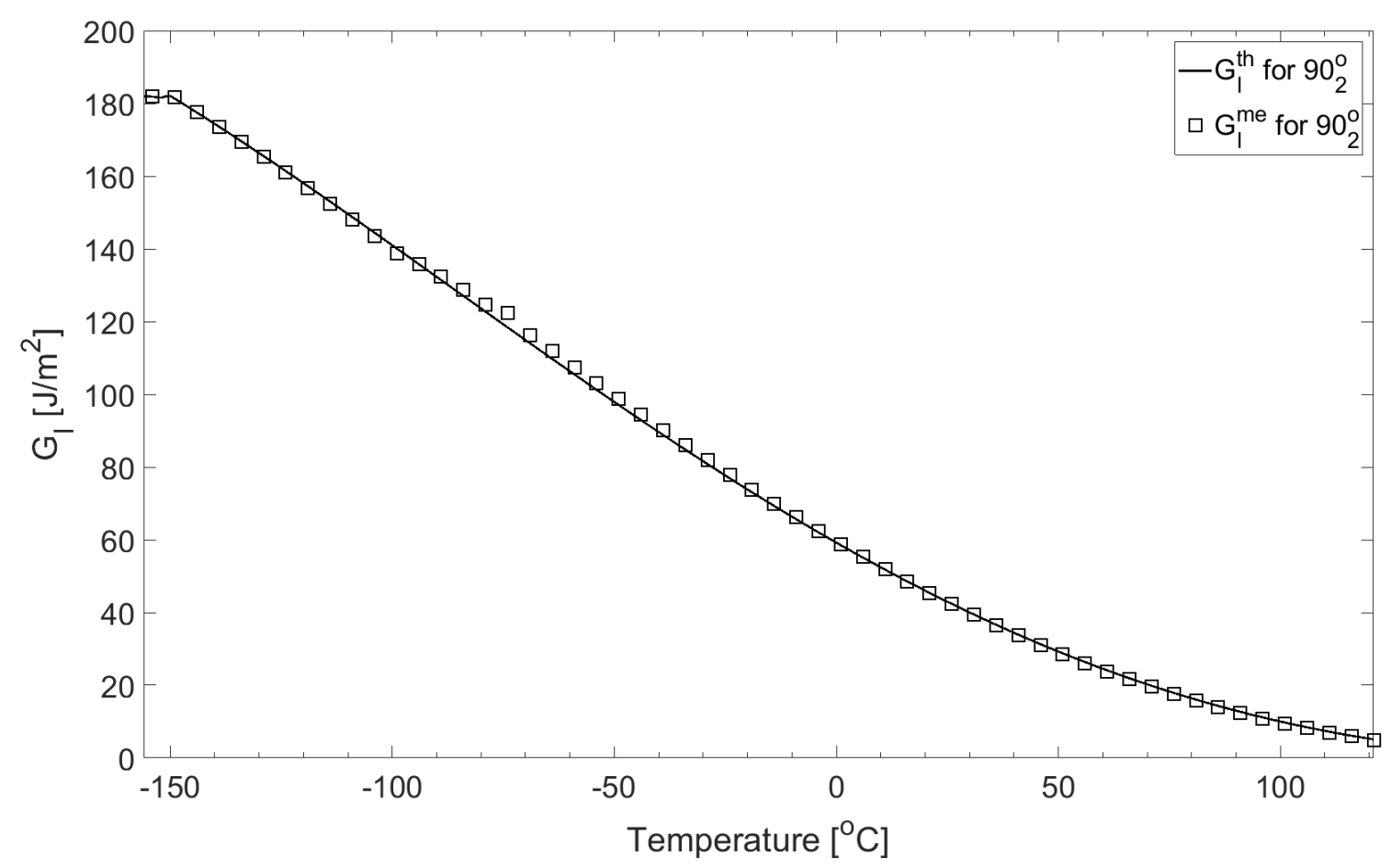

Figure 6.10: Comparison between ERR $G_{I}^{\text {th }}$ with $R_{T}=-156 / 121$ vs. $G_{I}^{\text {me }}$ at $R T$ for middle $90_{2}^{\circ}$ lamina subjected to equivalent mechanical strains $\epsilon_{x}^{m e}$ for laminate $\left[(0 / 90)_{2}\right]_{s}$ P $75 / 1962$ in the range $\left[T_{\min }, T_{\max }\right]$.

$90_{2}^{\circ}$ lamina as shown in Figure 6.10. However, some discrepancies still occur on the stress field as it can be seen in Figures 6.11 and 6.12.

Looking into Figure 6.11, longitudinal stresses $\sigma_{1}$ for $\left[(0 / 90)_{2}\right]_{s}$ P75/1962 using uniaxial equivalent mechanical strains $\epsilon_{x}^{m e}$ still disagree with respect to those $\sigma_{1}$ obtained under thermal cycling loads. Although the thermo-mechanical equivalence is focused on $90_{2}^{\circ}$ lamina, both surface and interior $0^{\circ}$ laminas still reach values close to ultimate tension and compression strength at $T_{\min }$ and $T_{\max }$, respectively as shown in Figure 6.11. Therefore, there still exists risk of fiber breakage unless the testing temperature range is reduced.

Similar to Figure 6.5, the transverse stress $\sigma_{2}^{m e}$ on $90_{2}^{o}$ lamina using uniaxial $\epsilon_{x}^{m e}$ are still slightly different at temperatures close to $T_{\min }$ and $T_{\max }$ as shown in Figure 6.12. Thus, even looking at one single lamina, no $\epsilon_{x}^{m e}$ exists that ensuring $\lambda_{90_{2}}^{m e}=\lambda_{90_{2}}^{t h}$ at $T_{\text {min }}$ exactly satisfy the conditions given by equation (6.4) for all $T_{i}$. Furthermore, the largest error is obtained at $T_{\min }$ being around $13 \mathrm{MPa}$, which is even worse than previous cases illustrated in Figure 6.5 and 6.8.

Therefore, a thermo-mechanical equivalence looking at the middle lamina would make easier its execution but, it still presents similar problems as those studied previously using biaxial mechanical strains $\epsilon_{T}^{m e}$ in both directions. 


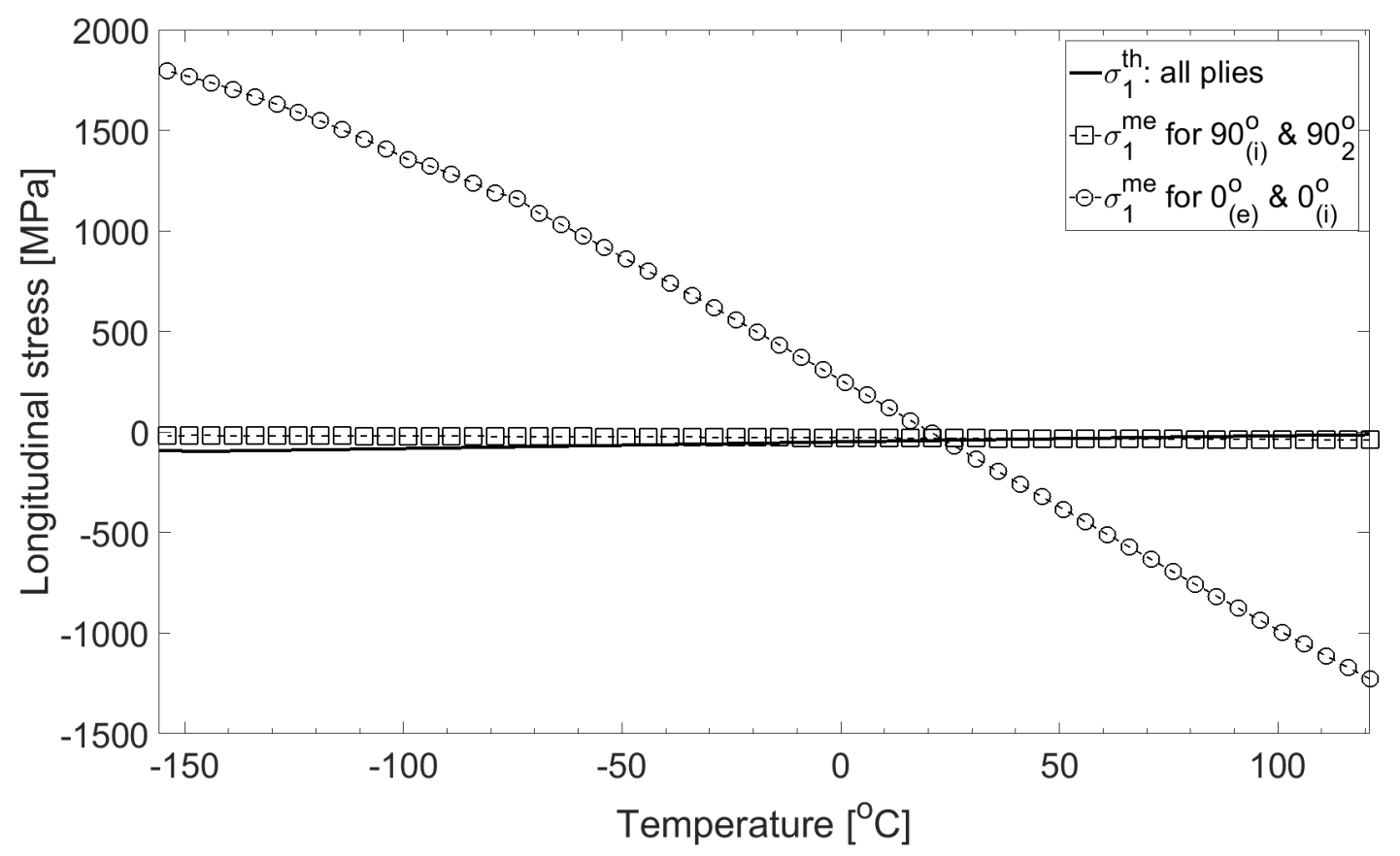

Figure 6.11: Comparison between longitudinal stress $\sigma_{1}^{\text {th }}$ with $R_{T}=-156 / 121$ vs. $\sigma_{1}^{\text {me }}$ at $R T$ for middle $90_{2}^{\circ}$ lamina subjected to equivalent uniaxial mechanical strains $\epsilon_{x}^{m e}$ for laminate $\left[(0 / 90)_{2}\right]_{s}$ P $75 / 1962$ in the range $\left[T_{\min }, T_{\max }\right]$.

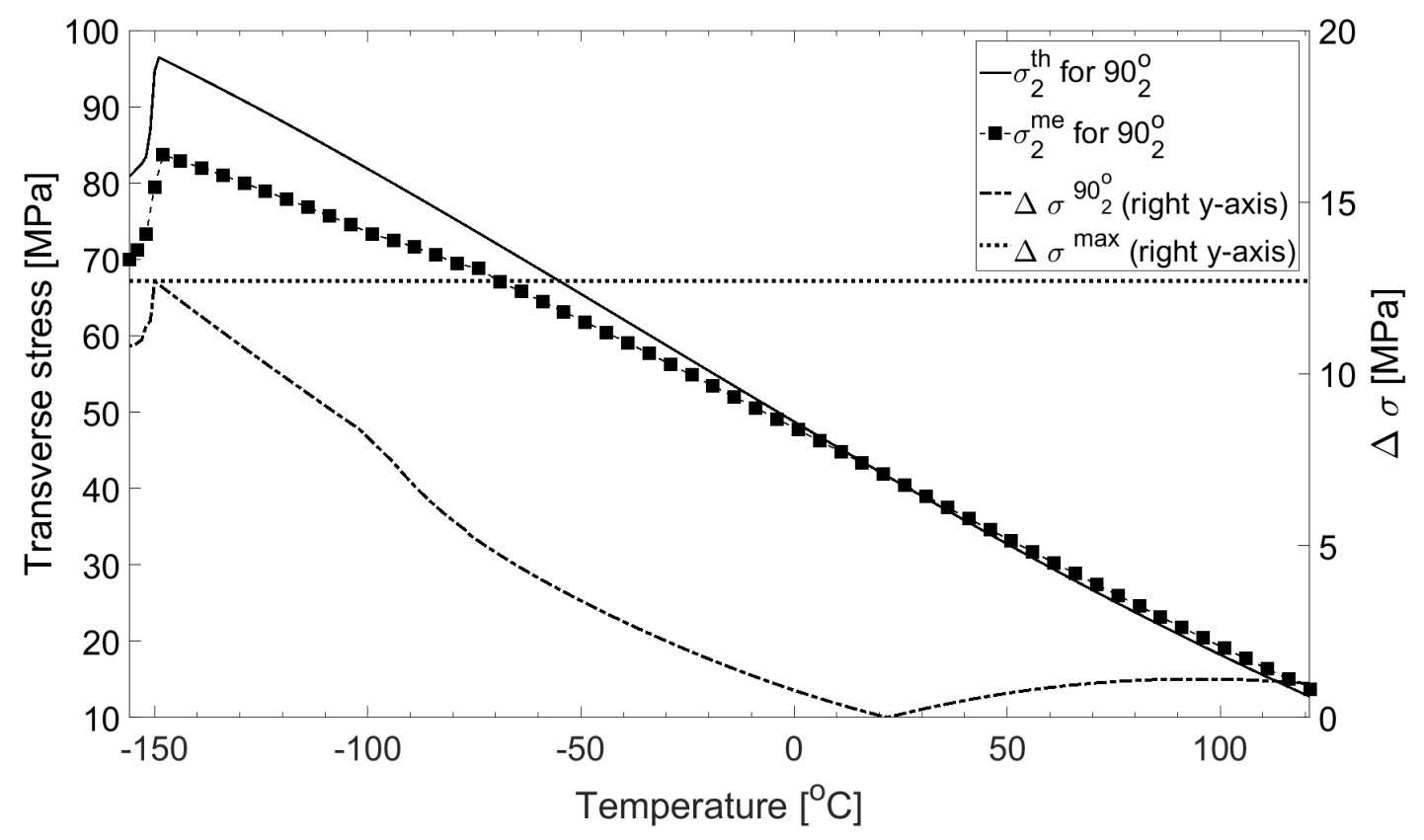

Figure 6.12: Comparison between transverse stress $\sigma_{2}^{\text {th }}$ with $R_{T}=-156 / 121$ vs. $\sigma_{2}^{m e}$ at RT for middle $90_{2}^{\circ}$ lamina subjected to equivalent uniaxial mechanical strains $\epsilon_{x}^{\text {me }}$ for laminate $\left[(0 / 90)_{2}\right]_{s}$ Pr5/1962 in the range $\left[T_{\min }, T_{\max }\right]$. 


\subsubsection{Equivalent mechanical thickness}

The main problem of the thermo-mechanical equivalence lies on the temperature-dependent properties. Such temperature-dependence lead to discrepancies on the transverse stresses $\sigma_{2}$ as shown in Figures 6.5, 6.8 and 6.12 even though $\lambda_{T_{r}}^{m e}=\lambda_{T_{m i n}}^{t h}$ is achieved by minimizing the objective residual function (6.5). According to Section $6.2 .2, \lambda_{T_{r}}^{m e}=\lambda_{T_{m i n}}^{t h}$ for $N \geq 2$ is expected to be accomplished during thermal fatigue if transverse stresses $\sigma_{2}$ are identical for both, thermal and mechanical fatigue. Therefore, it is mandatory to generate the same stress field $\sigma_{2}$ as closely as possible.

Ideally, the conditions given by equations (6.3) and (6.4) could be satisfied varying the lamina thickness at each $T$ but this is impossible because the thickness cannot change during cooling. Therefore, an equivalent mechanical thickness $t_{m e}$ is calculated at the most critical or relevant temperature.

According to Paris's law (5.14), $\Delta G_{I}$ is the only driving force for crack density growth and the fatigue resistance $f(N)$ depends only on the stress field. Since the most critical temperature at which both ERR and stress field are maximum is $T_{\min }$ leading to highest $\Delta G_{I}$, equivalent mechanical thickness $t_{m e}$ is calculated at coolest temperature, where the largest errors occurred previously (Figures 6.5, 6.8 and 6.12).

In order to produce a stress field as close as possible between thermal and mechanical fatigue, equivalent mechanical thickness $t_{m e}$ at $T_{\min }$ is calculated by minimizing the objective residual function $(6.5)$ at $T_{\min }$ and $N=1$, with variable $t_{m e}$. Thus, the $f_{i}$ objective functions in (6.7) become

$$
f_{i}=\left\{G_{I}^{m e}\left(\epsilon_{x}^{m e}, t_{m e}, R T\right), \sigma_{2}^{m e}\left(\epsilon_{x}^{m e}, t_{m e}, R T\right)\right\}
$$

where $t_{m e}$ is found to be equal to $0.70 t_{k}$ i.e., about $70 \%$ of the total middle lamina thickness for laminate $\left[(0 / 90)_{2}\right]_{s}$ P75/1962.

In Figure 6.13, crack density evolution $\lambda_{90_{2}}^{t h}$ for laminate $\left[(0 / 90)_{2}\right]_{s}$ P75/1962 with $R_{T}=-156 / 121$ is compared with $\lambda_{90_{70 \%}}^{m e}$ for the same laminate subjected to equivalent mechanical strains $\epsilon_{x}^{m e}$ at RT using $t_{m e}$ for middle lamina. In the same way, $G_{I}^{t h}(T)$ is compared with $G_{I}^{m e}\left(\epsilon_{x}^{m e}, t_{m e}, R T\right)$ in Figure $6.14, \sigma_{1}^{\text {th }}(T)$ is compared with $\sigma_{1}^{m e}\left(\epsilon_{x}^{m e}, t_{m e}, R T\right)$ in Figure 6.15, and $\sigma_{2}^{t h}(T)$ is compared with $\sigma_{2}^{m e}\left(\epsilon_{x}^{m e}, t_{m e}, R T\right)$ in Figure 6.16 using $t_{m e}$ for middle lamina.

As it can be seen in Figure 6.13, $\lambda_{90_{2}}^{\text {th }}$ compares reasonable well with $\lambda_{90_{70 \%}}^{m e}$ at each temperature $T$, but worse than Figure 6.2, 6.7, and 6.9. Despite of this less accuracy for crack density evolution $\lambda$ between thermal and mechanical fatigue, it still satisfy $\lambda_{T_{r}}^{m e}=\lambda_{T_{\min }}^{\text {th }}$ given by equation (6.2). That is, at $T=T_{\min }$, both $\lambda$ are equal and $T_{\min }$ is the critical temperature at which cracks propagate. When $t_{m e}$ is used, the crack 


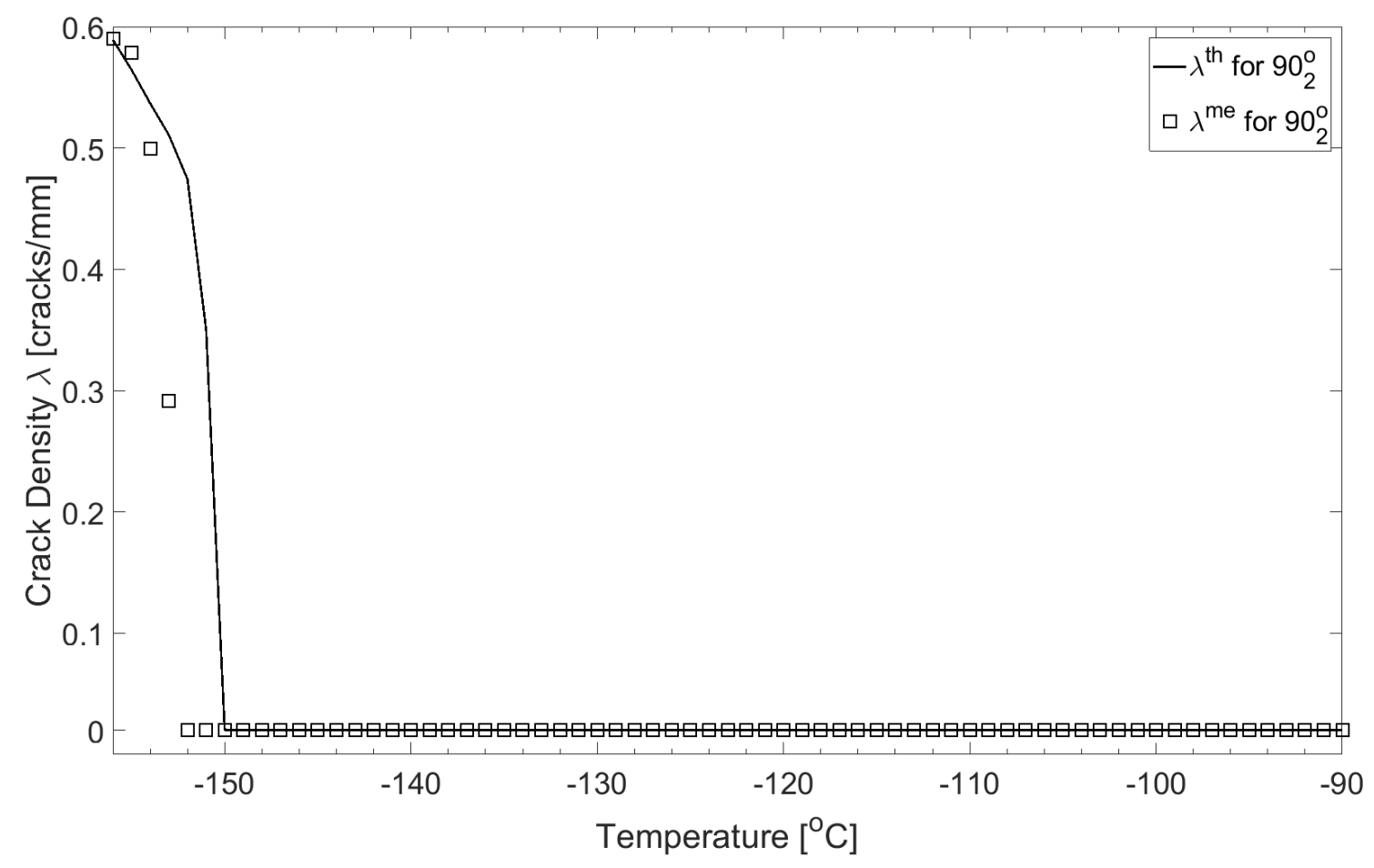

Figure 6.13: Comparison between crack density evolution $\lambda_{90_{2}}^{\text {th }}$ for $R_{T}=-156 / 121$ vs. crack density evolution $\lambda_{90_{70 \%}}^{m e}$ subjected to uniaxial equivalent mechanical strains $\epsilon_{x}^{m e}$ at RT for laminate $\left[(0 / 90)_{2}\right]_{s} P^{r 5} / 1962$ in the range $\left[T_{\min }, T_{\max }\right]$.

initiation is delayed a few celsius degrees but stress $\sigma_{2}$ is improved as seen later. The weighted residual method is successfully used to minimize the objective residual function (6.5). The weights $w_{90_{70 \%}}^{f}$ that best satisfy the conditions given by equations (6.2), (6.3), and (6.4) are 0.575 for $G_{I}$ and 0.425 for $\sigma_{2}$ using $t_{m e}$ for middle lamina. Crack density occurs in the middle lamina because $G_{I}>G_{I c}$.

In the same way, $G_{I}^{t h}(T)$ compares well with $G_{I}^{m e}\left(\epsilon_{x}^{m e}, t_{m e}, R T\right)$ during cooling for $90_{70 \%}^{\circ}$ lamina as shown in Figure 6.14. However, some discrepancies still occur on the stress field as it can be seen in Figures 6.15 and 6.16, but less than in Figure 6.12.

Looking into Figure 6.15, longitudinal stresses $\sigma_{1}$ for $\left[0 / 90 / 0 / 90_{70 \%}\right]_{s}$ P75/1962 using equivalent mechanical strains $\epsilon_{x}^{m e}$ again disagree with respect to those $\sigma_{1}$ obtained under thermal cycling loads. Both surface and interior $0^{\circ}$ laminas still reach close values to ultimate tension and compression strength at $T_{\min }$ and $T_{\max }$, respectively as shown in Figure 6.15. Therefore, there still exists risk of fiber breakage unless the testing temperature range is reduced.

According to Figure 6.16, transverse stress $\sigma_{2}^{m e}$ using $\epsilon_{x}^{m e}$ for middle $90_{70 \%}^{o}$ lamina approaches to the real values obtained during thermal fatigue. Since $t_{m e}$ is adjusted at $T_{m i n}$, there is no error at $T_{\min }$ as shown in Figure 6.16. Larger errors at high temperatures during cooling are obtained but they are smaller (less than $6 \mathrm{MPa}$ ) compared with those shown in Figure 6.5, 6.8, 6.12. 


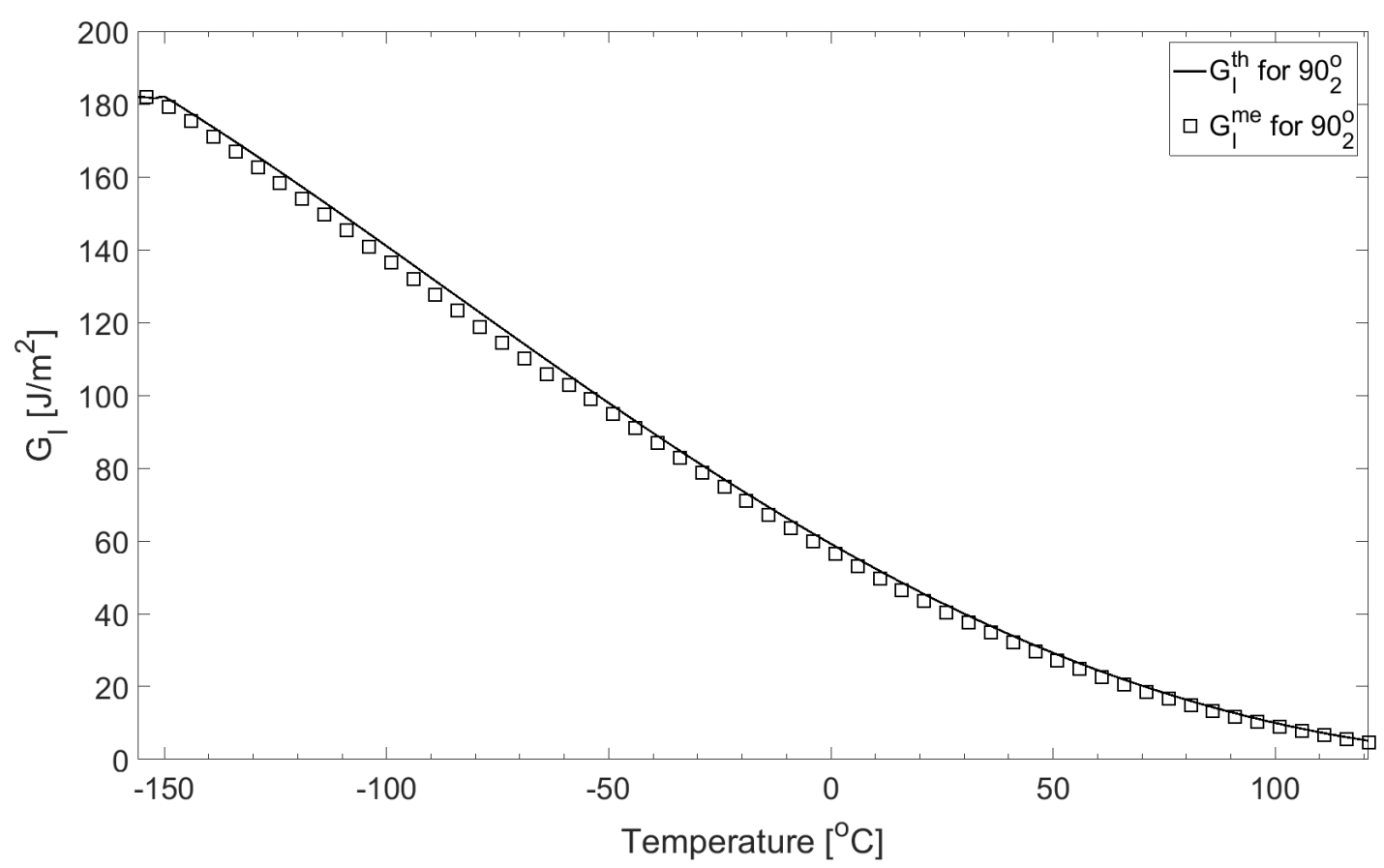

Figure 6.14: Comparison between ERR $G_{I}^{\text {th }}$ for $R_{T}=-156 / 121$ vs. $G_{I}^{\text {me }}$ at RT subjected to equivalent mechanical thickness $t_{m e}=0.70 t_{k}$ and strains $\epsilon_{x}^{m e}$ at $R T$ for laminate $\left[(0 / 90)_{2}\right]_{s}$ P75/1962 in the range $\left[T_{\min }, T_{\max }\right]$.

Therefore, the use of an equivalent mechanical thickness $t_{m e}$ allows to obtain $\lambda_{T_{r}}^{m e}=$ $\lambda_{T_{m i n}}^{t h}$ with accurate stress field $\sigma_{2}$ at critical temperature $T_{m i n}$ where highest crack density occur. Furthermore, the use of $t_{m e}$ replicate better the stress field $\sigma_{2}$. That means that $f(N)$ is the same for thermal and mechanical fatigue.

\subsection{Thermo-mechanical equivalence}

Based on the results shown in Figure 6.16, equivalent mechanical thickness $t_{m e}$ is the best approach to satisfy $\sigma_{2}^{m e}=\sigma_{2}^{t h}$. Furthermore, the conditions given by equations (6.2) and (6.3) compares well as shown in Figure 6.13 and 6.14, particularly at $T_{\text {min }}$, which is the most important temperature because it is the temperature at which cracks grow. Since thermo-mechanical equivalence only focuses on the middle lamina, the fatigue testing is reduced to uniaxial mechanical test.

However, there still remains two problematic matters that appear in all previous cases. First, the longitudinal stress $\sigma_{1}$ obtained using $\epsilon_{x}^{m e}$ reaches values close to ultimate tension and compression at $T_{\min }$ and $T_{\max }$, respectively. Therefore, a lower temperature range must be used for testing. For instance, a lower $R_{T}=-40 / 30$ is selected.

Second, compression mechanical strains $\epsilon_{x}^{m e}$ are needed to simulate a thermal fatigue in the range $R T<T<T_{\max }$. To avoid negative strains, $T_{\max }$ is selected to be the reference temperature $T_{r}$ during mechanical test. Since mechanical tests at specific temperature 


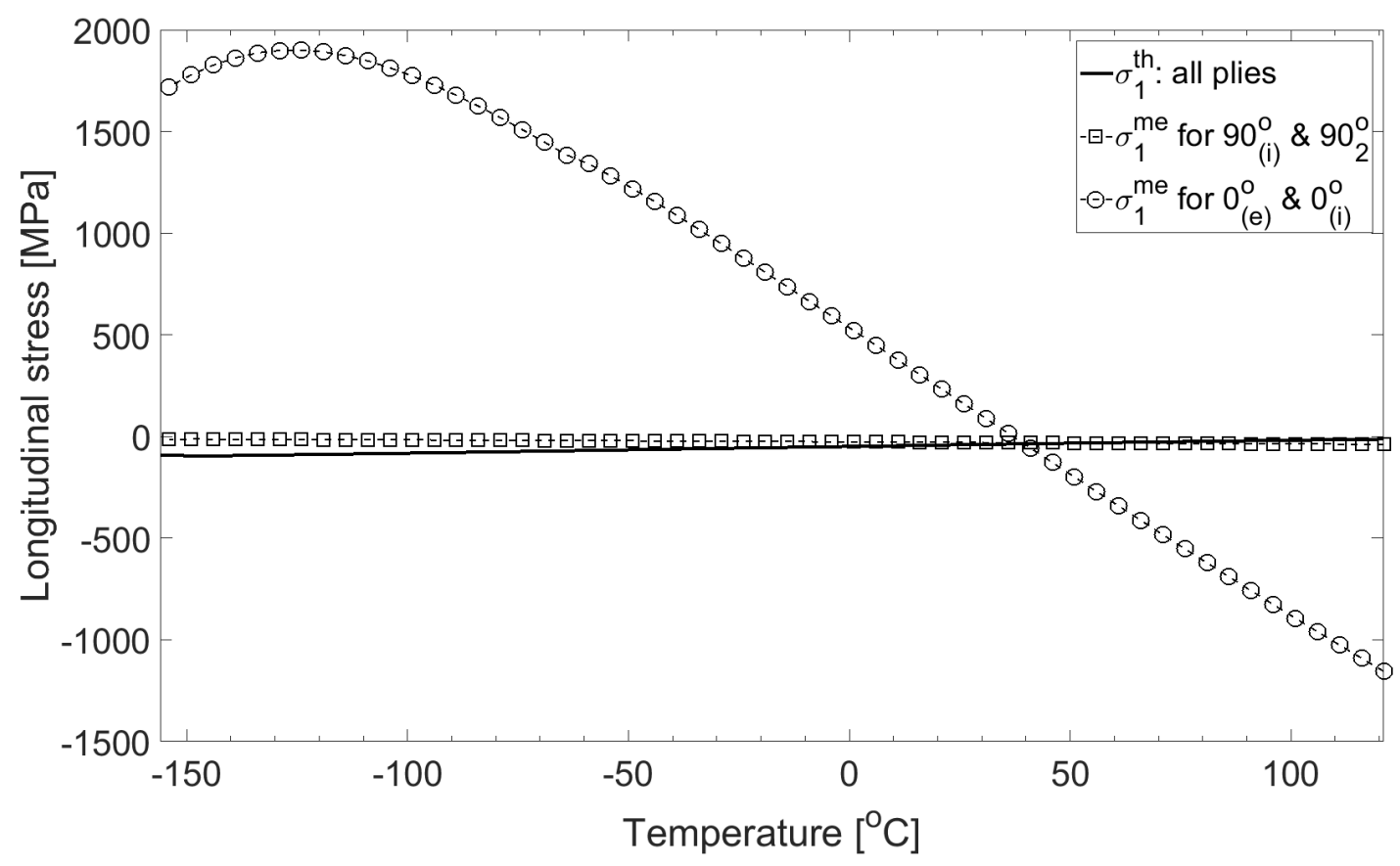

Figure 6.15: Comparison between longitudinal stress $\sigma_{1}^{\text {th }}$ for $R_{T}=-156 / 121$ vs. $\sigma_{1}^{m e}$ at $R T$ subjected to uniaxial equivalent mechanical strains $\epsilon_{x}^{m e}$ at $R T$ and $t_{m e}=0.70 t_{k}$ for laminate $\left[(0 / 90)_{2}\right]_{s}$ P $75 / 1962$ in the range $\left[T_{\min }, T_{\max }\right]$.

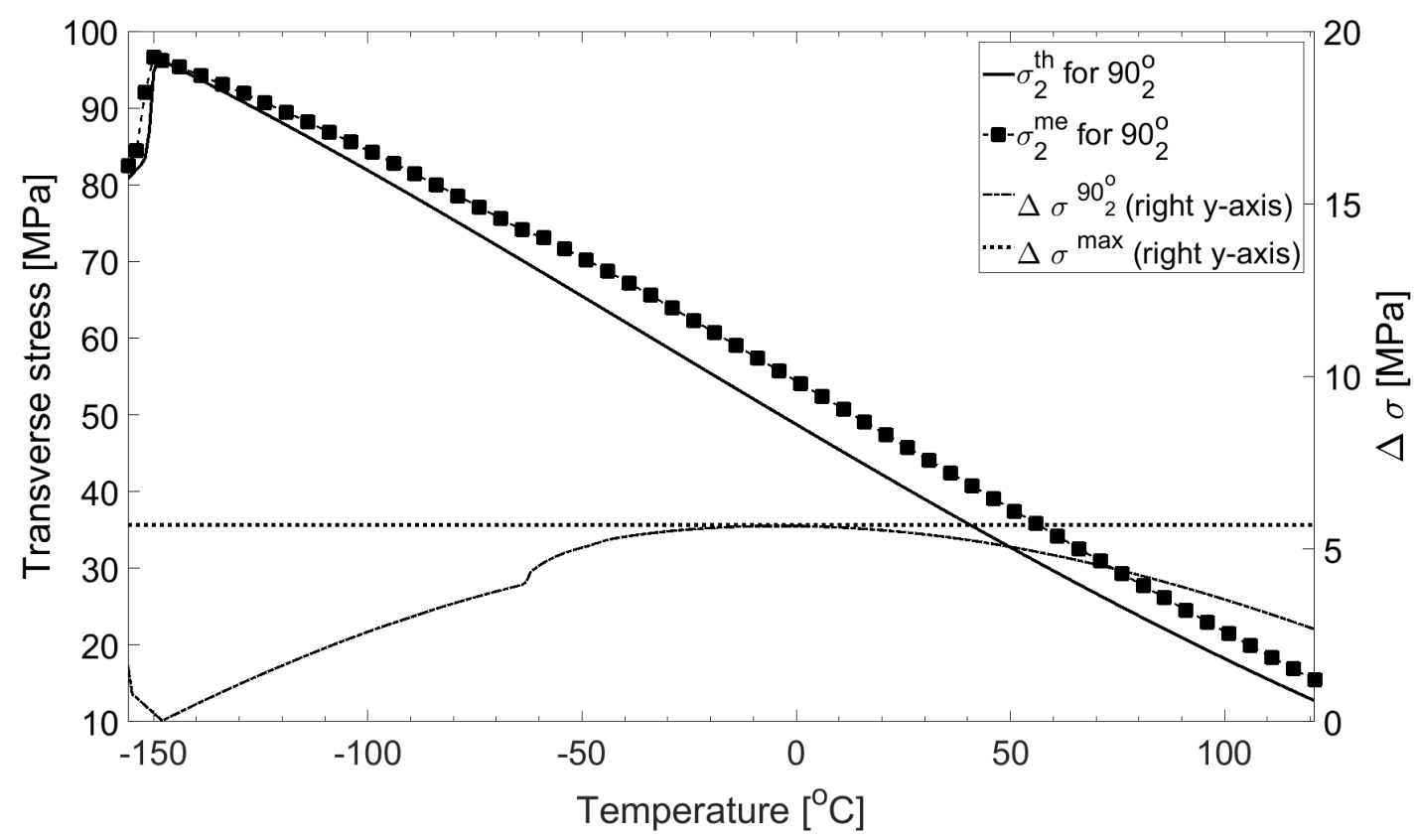

Figure 6.16: Comparison between transverse stress $\sigma_{2}^{\text {th }}$ for $R_{T}=-156 / 121$ vs. $\sigma_{2}^{\text {me }}$ subjected to uniaxial equivalent mechanical strains $\epsilon_{x}^{m e}$ at $R T$ with $t_{m e}=0.70 t_{k}$ for laminate $\left[(0 / 90)_{2}\right]_{s}$ P75/1962 in the range $\left[T_{\min }, T_{\max }\right]$. 


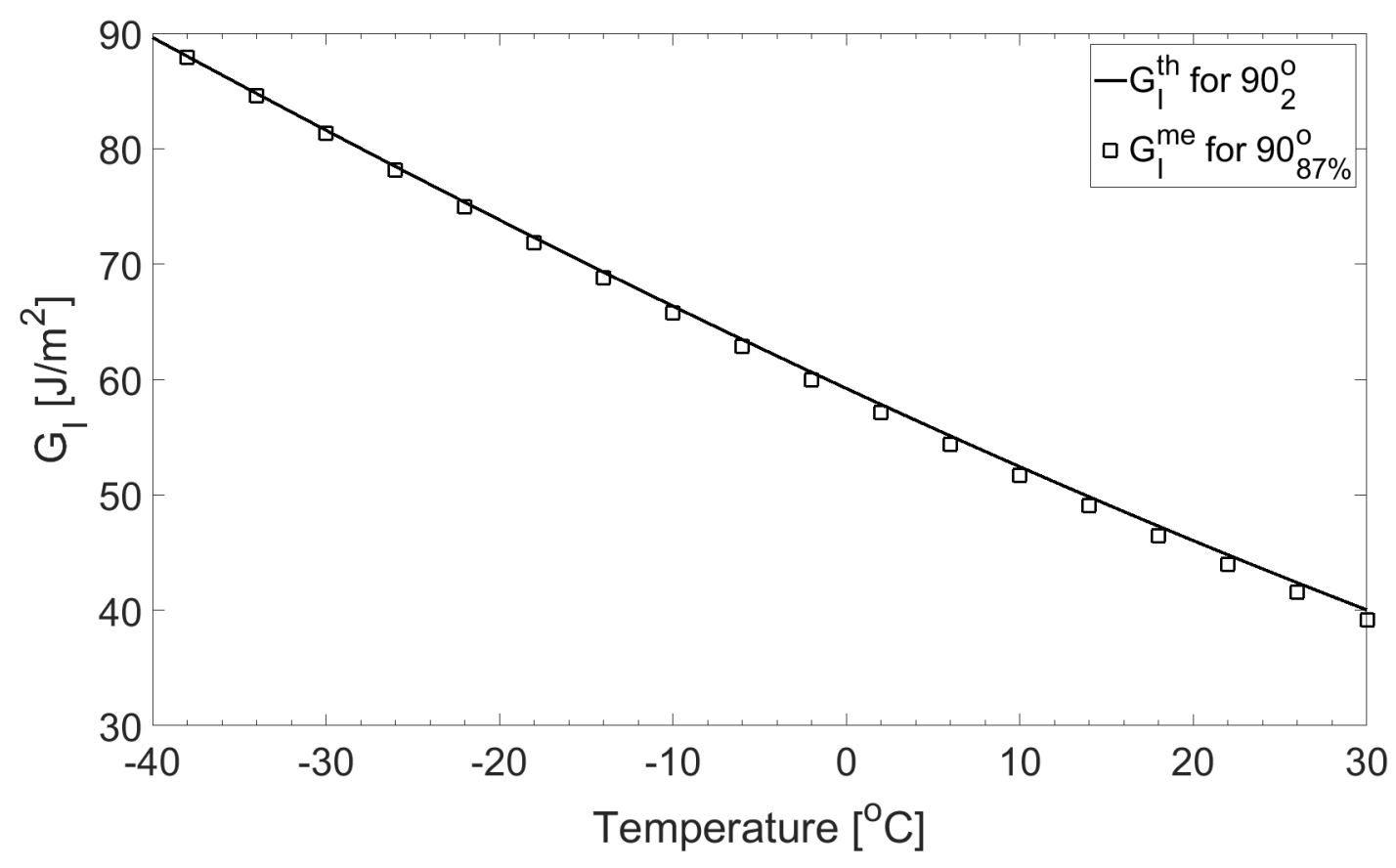

Figure 6.17: Comparison between ERR $G_{I}^{\text {th }}$ for $R_{T}=-40 / 30$ vs. $G_{I}^{m e}$ at $T_{r}=30^{\circ} \mathrm{C}$ subjected to equivalent mechanical strains $\epsilon_{x}^{m e}$ with $t_{m e}=0.87 t_{k}$ for laminate $\left[(0 / 90)_{2}\right]_{s}$ $P$ 75/1962 in the range $\left[T_{\min }, T_{\max }\right]$.

$T_{r}>R T$ are still easy to perform, it becomes a good alternative to avoid compression strains, which are difficult to apply.

\subsubsection{Quasi-static Cooling Using $T_{r}=T_{\max }$}

Equivalent mechanical thickness $t_{m e}$ is calculated by minimizing the objective residual function (6.5) at $T_{\min }=-40^{\circ} \mathrm{C}$ and $N=1$. Furthermore, in order to avoid compression loads during mechanical testing, the reference temperature $T_{r}$ is selected to be $T_{\max }=$ $30^{\circ} \mathrm{C}$. Thus, the objective functions $f_{i}$ in (6.7) become

$$
f_{i}=\left\{G_{I}^{m e}\left(\epsilon_{x}^{m e}, t_{m e}, 30^{\circ} C\right), \sigma_{2}^{m e}\left(\epsilon_{x}^{m e}, t_{m e}, 30^{\circ} C\right)\right\}
$$

where $t_{m e}$ is found to be equal to $0.87 t_{k}$ i.e., about $87 \%$ of the total middle lamina thickness.

For quasi-static cooling, crack density evolution $\lambda$ for both thermal and mechanical test does not occur because $G_{I}<G_{I c}$ for middle lamina. Therefore, larger number of cycles $\mathrm{N}$ are necessary to produce void nucleation. The ERR $G_{I}^{\text {th }}(T)$ compares very well with $G_{I}^{m e}\left(\epsilon_{x}^{m e}, t_{m e}, 30^{\circ} \mathrm{C}\right)$ during cooling for $90_{87 \%}^{\circ}$ lamina as shown in Figure 6.17.

Looking into Figure 6.18, longitudinal stresses $\sigma_{1}$ for $\left[0 / 90 / 0 / 90_{87 \%}\right]_{s}$ P75/1962 using equivalent mechanical thickness $t_{m e}$ and strains $\epsilon_{x}^{m e}$ reach values that are below the ultimate tensile strength of P75/1962 lamina [305]. Furthermore, the ultimate tensile 


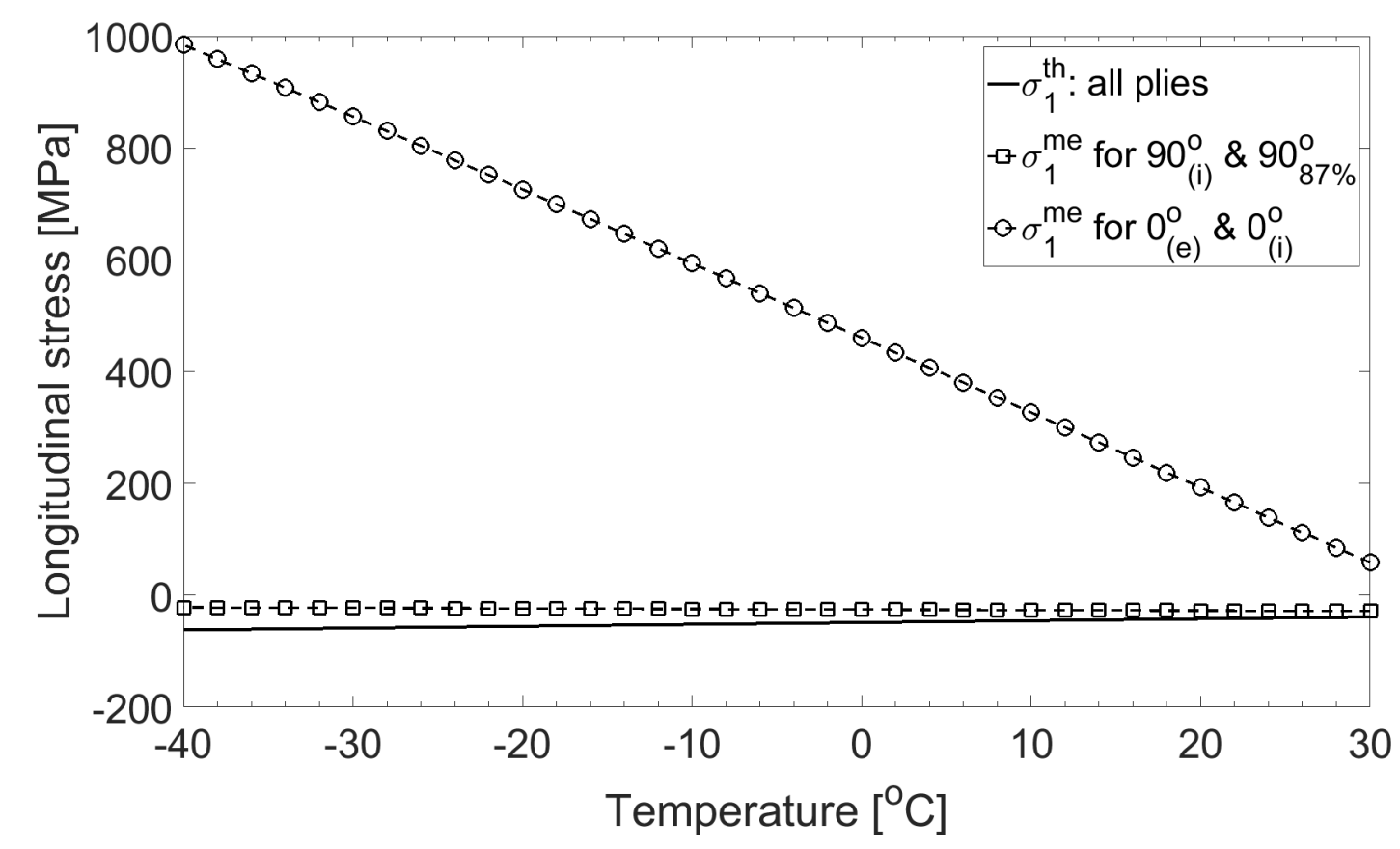

Figure 6.18: Comparison between longitudinal stress $\sigma_{1}^{\text {th }}$ for $R_{T}=-40 / 30$ vs. $\sigma_{1}^{\text {me at }}$ $T_{r}=30^{\circ} \mathrm{C}$ subjected to equivalent mechanical strains $\epsilon_{x}^{m e}$ with $t_{m e}=0.87 t_{k}$ for laminate $\left[(0 / 90)_{2}\right]_{s}$ P75/1962 in the range $\left[T_{\min }, T_{\max }\right]$.

strain $\epsilon_{x}^{u l t}$ of P75 fiber must be considered to avoid fiber breakage when $\epsilon_{x}^{m e}$ are applied for uniaxial mechanical test.

Assuming the P75 fiber elastic and transversely isotropic [12, Ch. 5], $\epsilon_{x}^{u l t}$ is found to be equal to $3.998 \cdot 10^{-3}$ using the modulus of elasticity $E=517 G P a$ and ultimate tensile strength $\sigma_{u l t}^{x}=2068 \mathrm{MPa}$ from [10]. The largest $\epsilon_{x}^{m e}$ is produced at $T_{\min }$ being equal to $3.7296 \cdot 10^{-3}$ and thus, $\epsilon_{x}^{u l t}$ is not reached. Furthermore, all $\epsilon_{x}^{m e}$ are positive as shown in Figure 6.18 because $T_{r}=T_{\text {max }}$.

The transverse stress $\sigma_{2}^{m e}$ using $\epsilon_{x}^{m e}$ at $T_{r}=30^{\circ} \mathrm{C}$ for middle $90_{87 \%}^{\circ}$ lamina compare well at cryogenic temperatures as shown in Figure 6.19. This is because $t_{m e}$ is adjusted at $T_{\min }$ so that the induced error at $T_{\min }$ is null. Small discrepancies (less than $2 \mathrm{MPa}$ ) are obtained at high temperatures during cooling compared with $\sigma_{2}^{\text {th }}$ due to temperaturedependent properties. However, according to Master Paris law (5.14), the most critical temperature is $T_{\min }$ where $\Delta G_{I}$ is maximum. Therefore, the error induced at $T_{r}$ can be assumed to be negligible compare with $\sigma_{2}^{\text {th }}$ at lowest temperature.

\subsubsection{Thermal Fatigue Using $T_{r}=T_{\max }$}

For thermal fatigue equivalence $t_{m e}$ and $\epsilon_{x}^{m e}$ at $N=1$ must be calculated first for monotonic cooling with $T_{r}=T_{\max }=30^{\circ} \mathrm{C}$ and $T_{\min }=-40^{\circ} \mathrm{C}$. Then, equivalent mechanical strains $\epsilon_{x}^{m e}(N)$ for $N \geq 2$ can be calculated.

According to Figure 6.1, equivalent mechanical test (right side) that satisfies $\lambda_{T_{r}}^{m e}=$ 


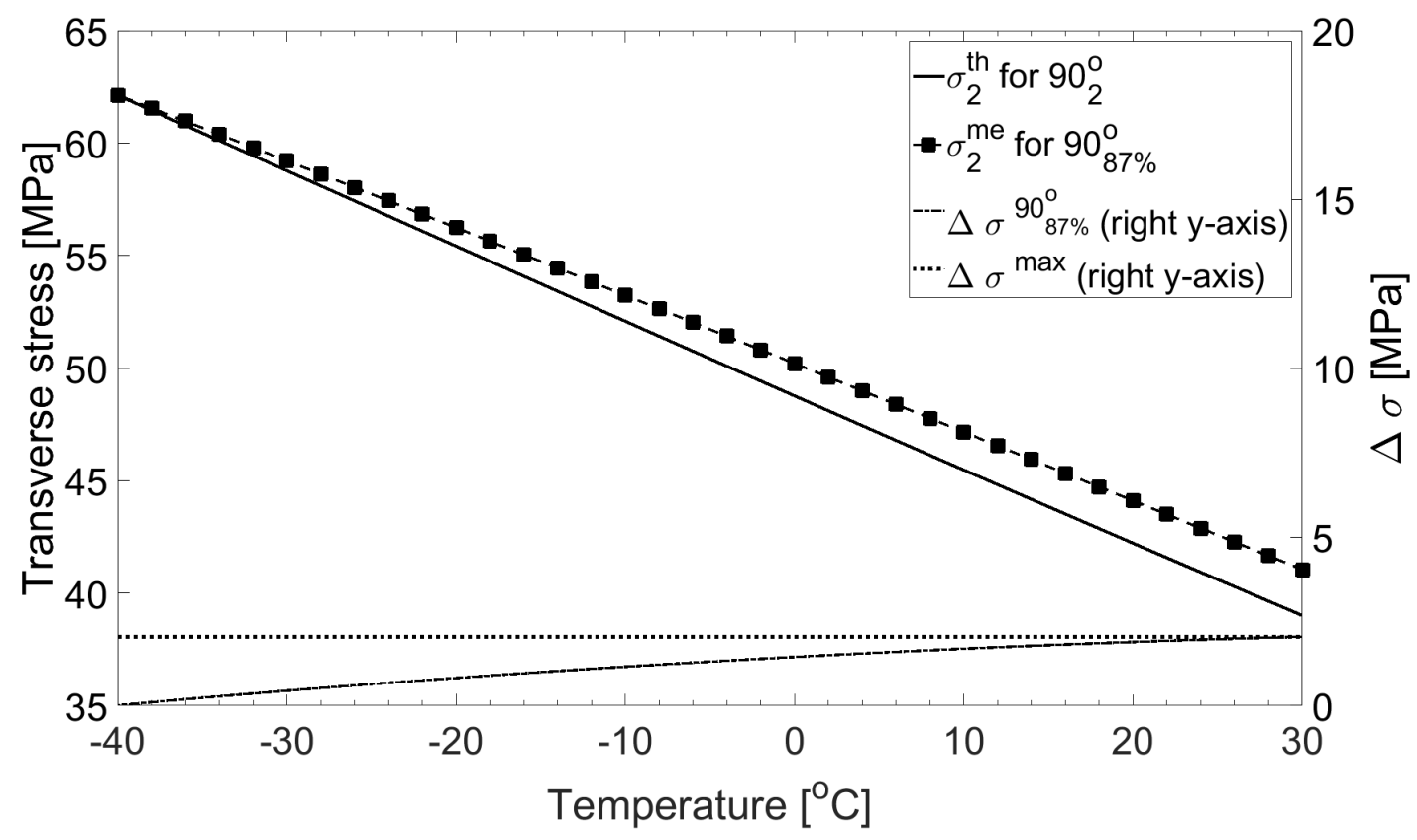

Figure 6.19: Comparison between transverse stress $\sigma_{2}^{\text {th }}$ for $R_{T}=-40 / 30$ vs. $\sigma_{2}^{m e}$ at $T_{r}=$ $30^{\circ} \mathrm{C}$ subjected to equivalent mechanical strains $\epsilon_{x}^{m e}$ at $R T=30^{\circ} \mathrm{C}$ and $t_{m e}=0.87 t_{k}$ for laminate $\left[(0 / 90)_{2}\right]_{s}$ P75/1962 in the range $\left[T_{\min }, T_{\max }\right]$.

Tables 6.1: $f(N)$ parameters of P75/1962 $\left(V_{f}=0.52\right)$ under thermal fatigue. Subscript (e) and (i) represents exterior and interior laminas, respectively. Layup $(A):\left[(0 / 90)_{2}\right]_{s}$.

\begin{tabular}{cccccccccc}
\hline \multicolumn{10}{c}{ SLR parameters in equation $(5.16)$} \\
\hline \multirow{2}{*}{ Layup } & Laminas & $\beta_{0}$ & $\beta_{1}$ & $\beta_{2}$ & $\beta_{3}$ & $\beta_{4}$ & $\beta_{5}$ & $\beta_{6}$ & $R_{T}$ \\
\hline \multirow{2}{*}{$A$} & $0_{(e)}^{o} \& 90_{2}^{o}$ & 3.28 & -24.69 & 47.5 & -12.6 & 65.93 & -173.5 & 192.2 & \multirow{2}{*}{$-40 / 30$} \\
\cline { 2 - 11 } & $0_{(i)}^{o} \& 90_{(i)}^{o}$ & 0 & 0.311 & -1.49 & 0 & 1 & -9.48 & 22.71 & \\
\hline
\end{tabular}

$\lambda_{T_{m i n}}^{t h}$ can be calculated from thermal fatigue test (left side). Since $\lambda_{T_{r}}^{m e}$ have to be equal to $\lambda_{T_{m i n}}^{t h}$ for all $N$, then $f_{t h}(N)=f_{m e}(N)$ is automatically satisfied if same transverse loading conditions $\sigma_{2}$ is accomplished as explained in Sec 6.2.2. Therefore, $\lambda^{\text {th }}(N)$ as function of number of cycles $\mathrm{N}$ must be calculated first prior to calculate equivalent mechanical strains $\epsilon_{x}^{m e}(N)$.

In order to predict $\lambda^{t h}(N)$ for laminate $\left[(0 / 90)_{2}\right]_{s}$ P75/1962 with $R_{T}=-40 / 30$, the fatigue resistance $f_{t h}(N)$ is calculated by the Master Paris's law (5.14) of Figure 5.17 using DDM model as illustrated the Figure 5.18. Fatigue resistance $f_{t h}(N)$ and crack density evolution $\lambda^{\text {th }}(N)$ for $\left[(0 / 90)_{2}\right]_{s}$ P75/1962 with $R_{T}=-40 / 30$ is shown in Figure 6.20 and 6.21, respectively. The coefficients of nonlinear function $f_{t h}(N)$ (5.16) are shown in Table 6.1.

Based on the results shown in Figure 6.20 and $6.21, G_{I}<G_{I c}$ for all laminas so that damage initiation occurs at $N_{d i}=1215$ for exterior $0^{\circ}$ and middle $90_{2}^{\circ}$ laminas whereas $N_{d i}=198746$ for interior $0^{\circ}$ and $90^{\circ}$ laminas. This is expected because for lower $R_{T}=$ $-40 / 30$ (Figure 5.14), the $\Delta G_{I}$ is small leading to low crack-growth rate (Figure 5.17) 


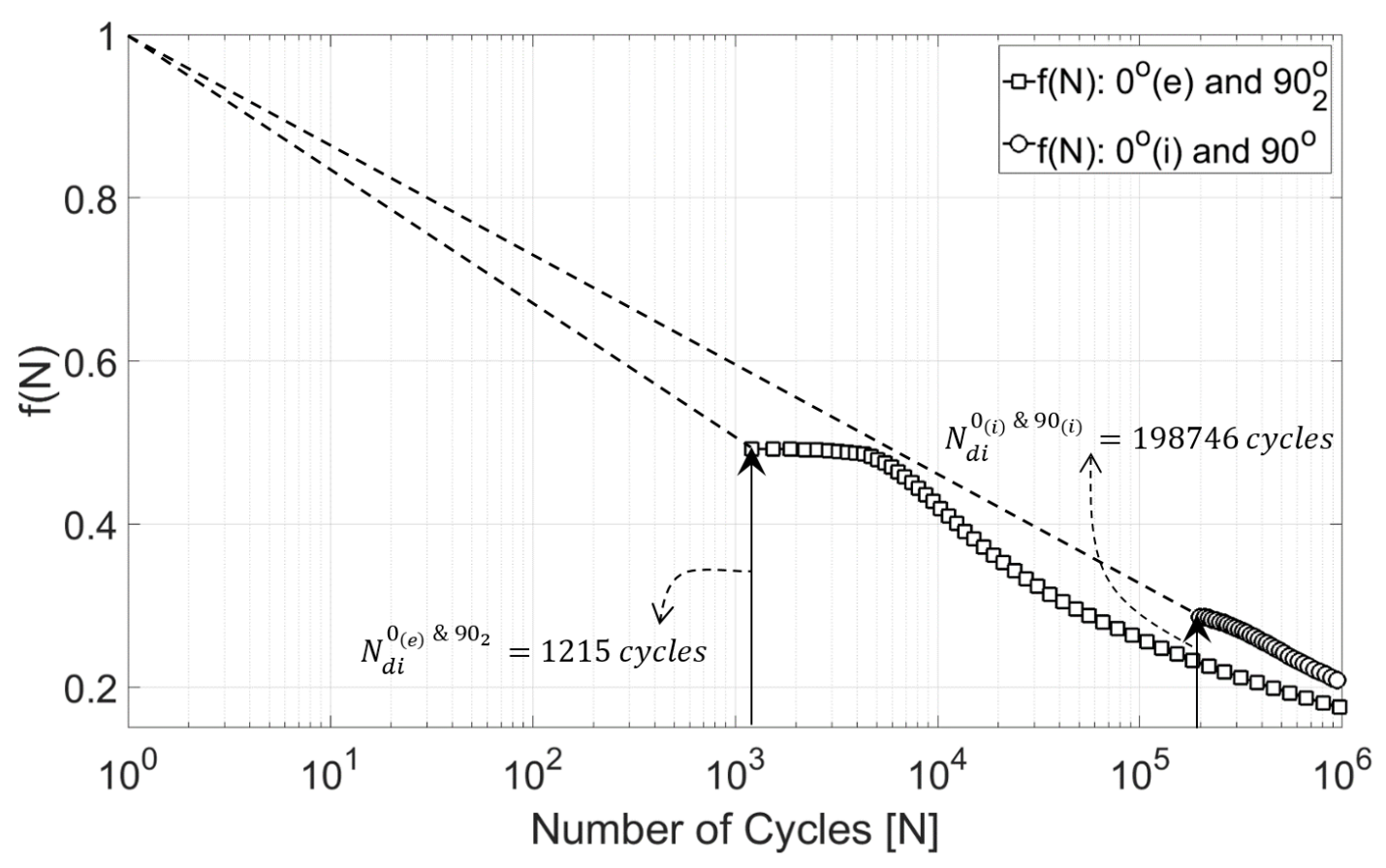

Figure 6.20: Fatigue degradation $f_{\text {th }}(N)$ for $\left[(0 / 90)_{2}\right]_{s}$ Pr5/1962 with $R_{T}=-40 / 30$ calculated by Master Paris's law and DDM.

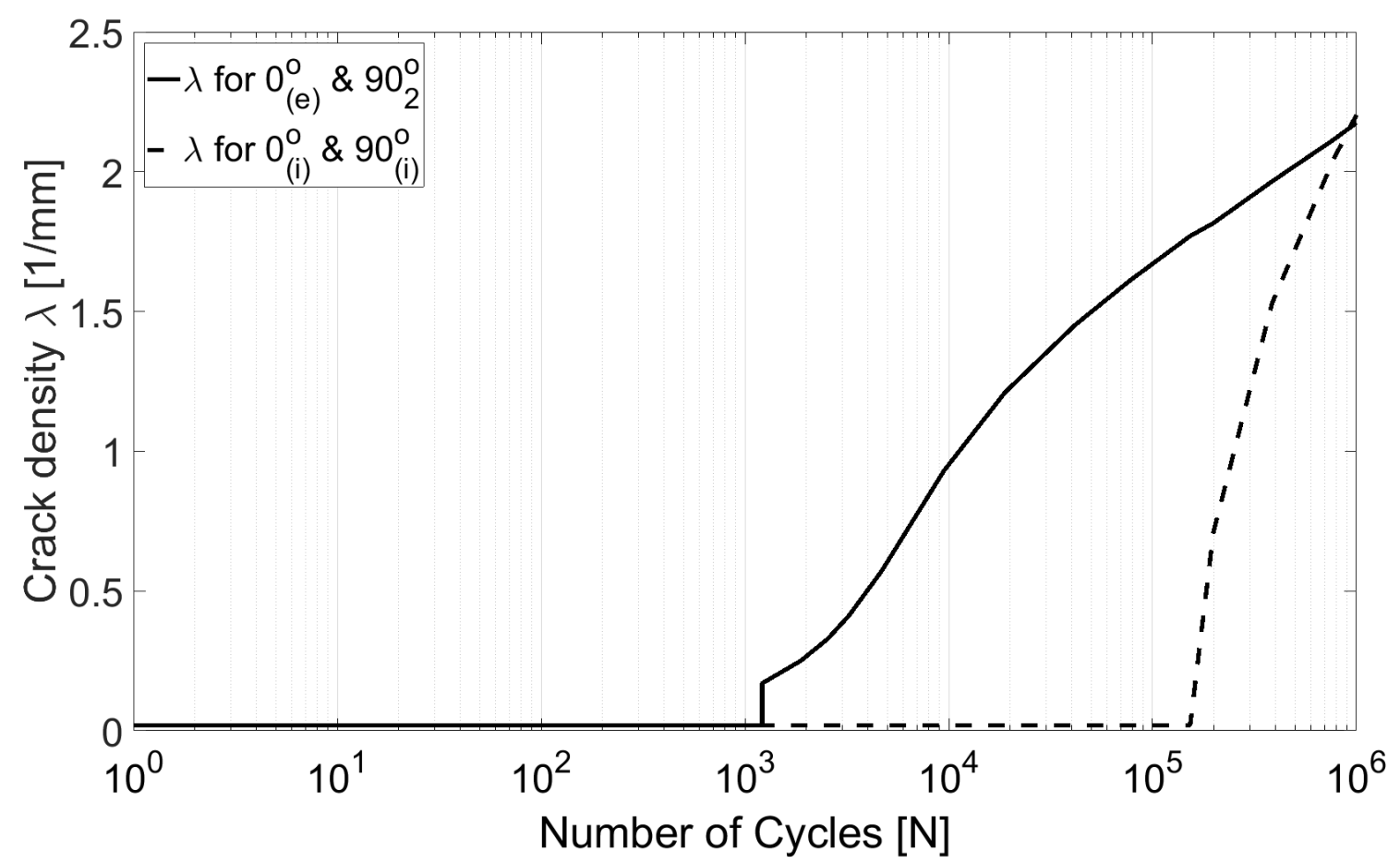

Figure 6.21: Crack density evolution $\lambda_{i}^{\text {th }}(N)$ vs. number of cycles $N$ for $\left[(0 / 90)_{2}\right]_{s}$ Pr5/1962 with $R_{T}=-40 / 30$ calculated with DDM model. 


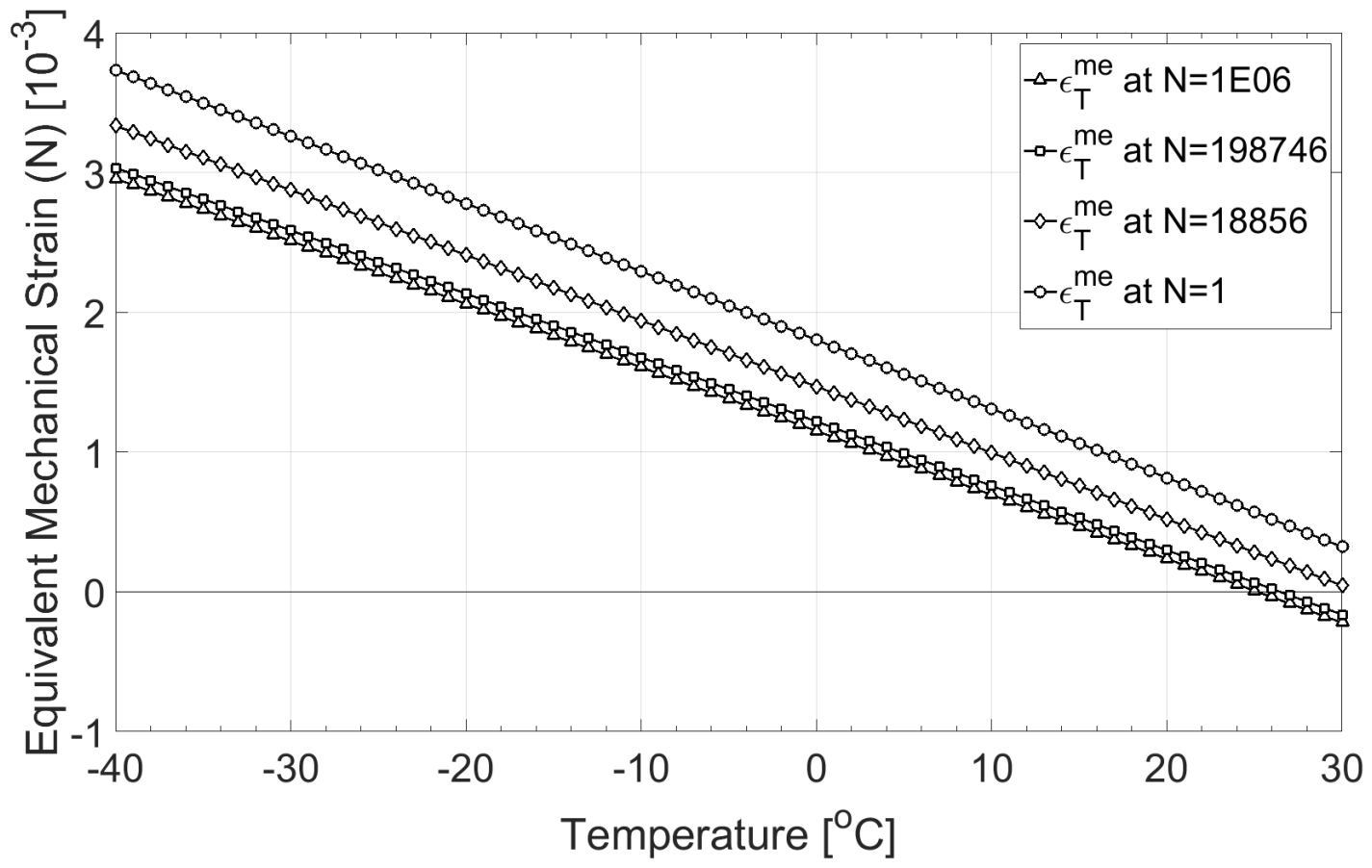

Figure 6.22: Uniaxial equivalent mechanical strains $\epsilon_{x}^{\text {me }}$ at discrete number of cycles $N$ vs. $T$ in the range $\left[-40,30^{\circ} \mathrm{C}\right]$ for laminate $\left[0 / 90 / 0 / 90_{87 \%}\right]_{s}$ P75/1962. Reference temperature is set to $T_{r}=30^{\circ} \mathrm{C}$. Results at $N=1285$ almost identical to $N=1$.

and thus, large number of cycles $\mathrm{N}$ must be performed to propagate first crack as shown in Figures 6.20 and 6.21. Although, $f(N)$ is unknown until $N_{d i}$ is reached, no cracks are propagated meanwhile and thus, it is irrelevant. However, $f(N)$ in the range $1<N<N_{d i}$ is plotted assuming it to be linear as explained in Ch. 5 .

With $\lambda^{\text {th }}(N)$ calculated, equivalent mechanical strains $\epsilon_{x}^{m e}(N)$ at $T_{r}=30^{\circ} C$ for $N \geq 2$ can be calculated by minimizing the objective residual function (6.11). Since highest crack density is reached at $T_{m i n}$, updated crack density $\lambda_{T_{m i n}}^{N-1}$ from previous cycle is used to calculate the equivalent mechanical strains $\epsilon_{x}^{m e}(N)$ for current $N$ cycles. Therefore, $\lambda_{T_{m i n}}^{N-1}$ can be easily collected from Figure 6.21.

Due to stiffness degradation, as $\lambda^{t h}(N)$ increases, $\epsilon_{x}^{m e}(N)$ varies for each $N$ for which higher crack density is predicted. Therefore, $\epsilon_{x}^{m e}(N)$ for thermal fatigue must be calculated for all $N$. Then, the equivalent mechanical strains history can be applied to the mechanical testing machine as input. Since fatigue life can reach up to one million cycles, $\epsilon_{x}^{m e}(N)$ at discrete number of cycles $N$ is calculated to illustrate the methodology as shown in Figure 6.22.

As it can be seen in Figure 6.22, equivalent mechanical strains $\epsilon_{x}^{m e}(N)$ decreases with number of cycles $N$ because $\lambda_{T_{r}}^{m e}(N)$ increases and laminate undergoes stiffness degradation. For very large $N$, a small compression $\epsilon_{x}^{m e}$ should be applied, but it is unlikely that failing to apply such small compression has any effect. Crack propagation takes place at temperature near $T_{\min }$ where strains are tensile. 


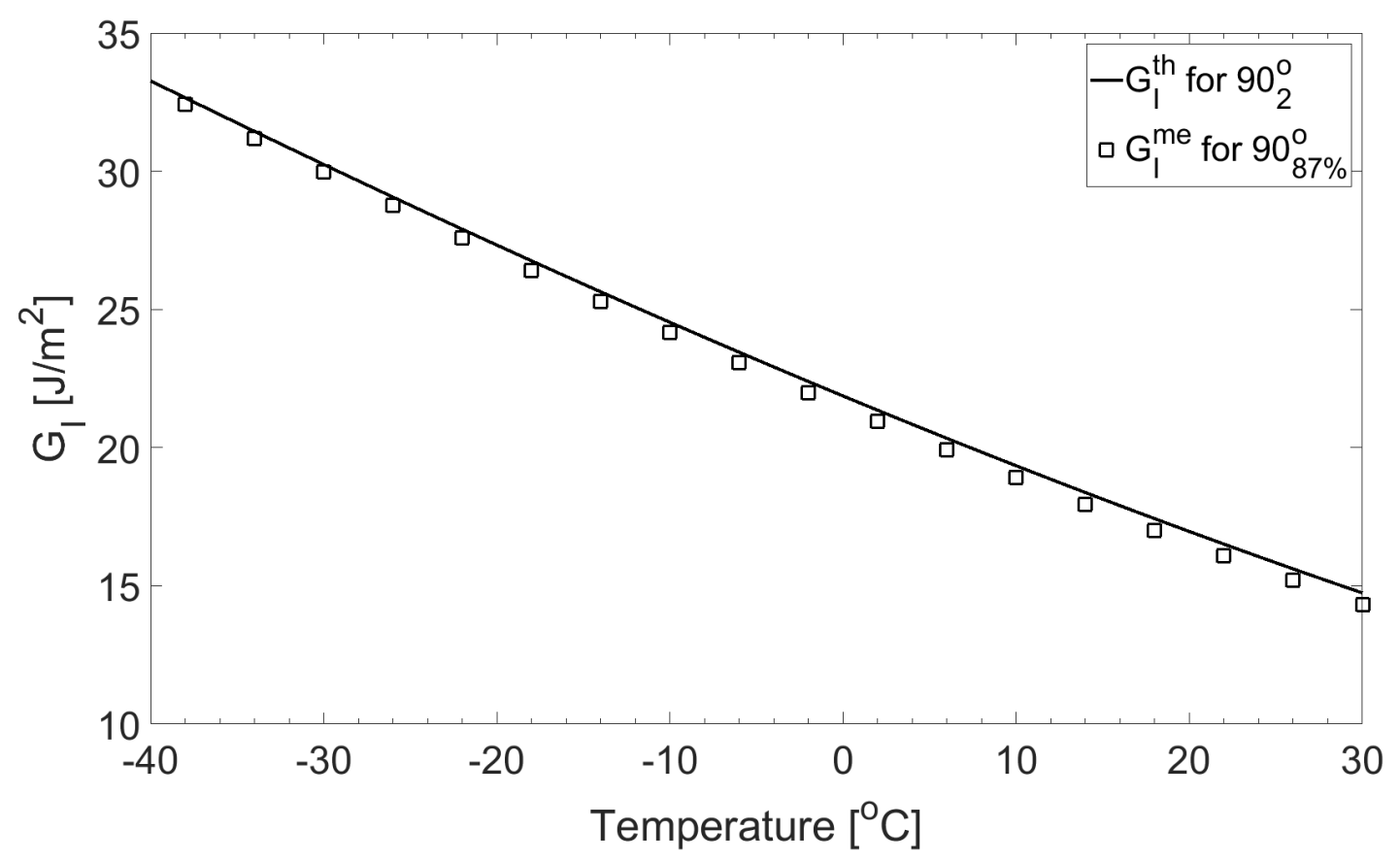

Figure 6.23: Comparison between ERR $G_{I}^{\text {th }}$ for $R_{T}=-156 / 121$ vs. $G_{I}^{m e}$ at $T_{r}=30^{\circ} C$ with equivalent mechanical thickness $t_{m e}=0.87 t_{k}$ and strains $\epsilon_{x}^{m e}$ for laminate $\left[(0 / 90)_{2}\right]_{s}$ P75/1962 with $N=198746$ cycles.

In order to check the conditions given by equations (6.3) and (6.4), the $\operatorname{ERR} G_{I}^{t h}(T)$ is compared with $G_{I}^{m e}\left(\epsilon_{x}^{m e}, t_{m e}, 30^{\circ} C\right)$ in Figure $6.23, \sigma_{1}^{t h}(T)$ is compared with $\sigma_{1}^{m e}\left(\epsilon_{x}^{m e}, 30^{\circ} C\right)$ in Figure 6.24, and $\sigma_{2}^{\text {th }}(T)$ is compared with $\sigma_{2}^{m e}\left(\epsilon_{x}^{m e}, 30^{\circ} C\right)$ in Figure 6.25 for middle $90_{87 \%}^{o}$ lamina at $N=1,000,000$ cycles.

The ERR $G_{I}^{t h}(T)$ compares very well with $G_{I}^{m e}\left(\epsilon_{x}^{m e}, t_{m e}, 30^{\circ} C\right)$ during cooling for $90_{87 \%}^{o}$ lamina as shown in Figure 6.23. Furthermore, the highest $\sigma_{1}$ in the laminate decreases about $200 \%$ and thus, there is not risk of fiber breakage (see Figure 6.25).

The transverse stress $\sigma_{2}^{m e}$ using $\epsilon_{x}^{m e}$ for middle $90_{87 \%}^{\circ}$ lamina approaches thermal fatigue values. As $\lambda_{T_{m i n}}^{t h}(N)$ increases, $\sigma_{2}$ decreases and thus the error induced in the thermo-mechanical equivalence decreases, being less than $1 \mathrm{MPa}$. Therefore, the approximation is very good. 


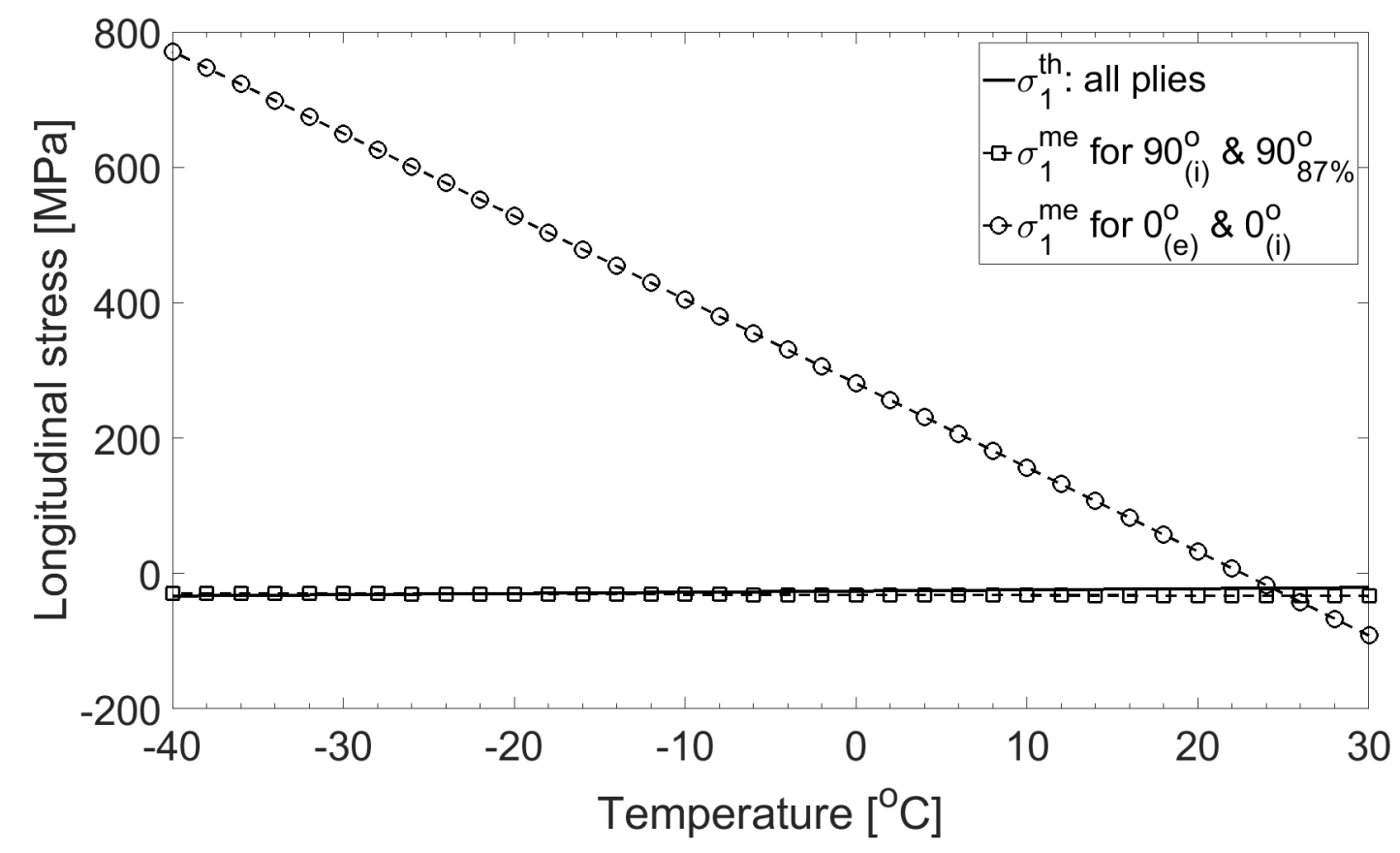

Figure 6.24: Comparison between longitudinal stress $\sigma_{1}^{\text {th }}$ for $R_{T}=-40 / 30$ vs. $\sigma_{1}^{\text {me }}$ at $T_{r}=30^{\circ} \mathrm{C}$ subjected to uniaxial equivalent mechanical strains $\epsilon_{x}^{\text {me }}$ and $t_{m e}=0.87 t_{k}$ for laminate $\left[(0 / 90)_{2}\right]_{s}$ P75/1962 with $N=198746$ cycles.

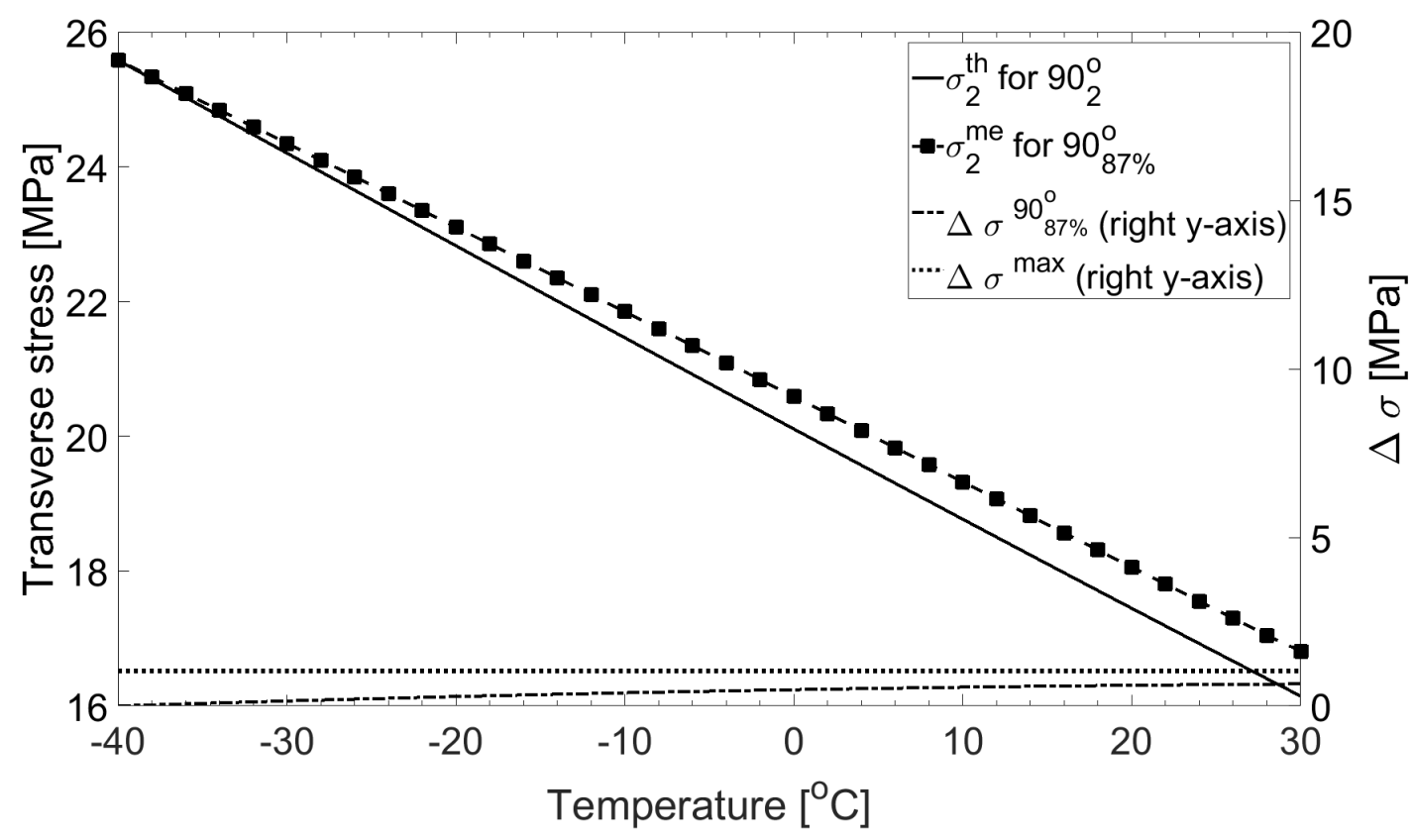

Figure 6.25: Comparison between transverse stress $\sigma_{2}^{\text {th }}$ for $R_{T}=-40 / 30$ vs. $\sigma_{2}^{m e}$ at $T_{r}=$ $30^{\circ} \mathrm{C}$ subjected to equivalent mechanical strains $\epsilon_{x}^{\text {me }}$ and $t_{m e}=0.867$ for laminate $\left[(0 / 90)_{2}\right]_{s}$ P75/1962 with $N=198746$ cycles. 


\subsection{Conclusions}

Equivalent mechanical fatigue test to simulate a thermal fatigue can be accomplished if the same transverse loading conditions $\sigma_{2}$ are satisfied for all life values $N$. Unfortunately, an exact combination of equivalent mechanical strains $\epsilon_{T}^{m e}$ does not exist to exactly satisfy the conditions (6.3) and (6.4). This is due to two reasons.

First, the temperature-dependent properties. During thermal fatigue, properties vary at each $T$ while thermo-mechanical properties are constant during mechanical fatigue test at reference temperature $T_{r}$. Second, physical differences between imposed mechanical strains $\epsilon_{T}^{m e}$ compared with thermal strains $\epsilon^{\text {th }}$ that emerges from equilibrium.

However, equivalent mechanical strains $\epsilon_{T}^{m e}$ that satisfy $\lambda_{T_{r}}^{m e}=\lambda_{T_{m i n}}^{t h}$ can be accomplished under certain conditions. Among these conditions, the most critical is the thermal ratio $R_{T}$. Since the error on $\sigma_{2}$ become larger as $T_{\text {min }}$ moves away from $T_{r}$, a not so high $R_{T}$ must be selected. The selection range of thermal ratio $R_{T}$ depends on the material properties. Furthermore, $R_{T}$ must be selected so that the strain to failure $\epsilon_{x}^{u l t}$ is not reached during mechanical testing.

A biaxial equivalent mechanical test can be performed to simulate a thermal fatigue test. However, its execution is very complex from a practical point of view. In order to simplify the fatigue testing and achieve the best approximation for stress field $\sigma_{2}$, an equivalent mechanical thickness $t_{m e}$ can be used to accomplish $\lambda_{T_{r}}^{m e}=\lambda_{T_{\min }}^{\text {th }}$ in the middle lamina with number of cycles $N$. In that way, the fatigue testing is reduced to uniaxial test with tensile $\epsilon_{x}^{m e}$. Since $\epsilon_{x}^{m e}$ vary with $N$, transverse damage needs to be updated from thermal fatigue predictions using a Master Paris law (5.14) and DDM. 


\section{Chapter 7}

\section{Conclusions and Future work}

Transverse damage initiation and evolution in laminated composites subjected to monotonic cooling and thermal cyclic loads require a precise knowledge of the temperaturedependent properties and a careful characterization of material system for any thermal ratio $R_{T}$. Such thermo-mechanical properties need to be back calculated from adequate experimental tests. Then, transverse damage predictions can be successfully predicted using discrete damage mechanics (DDM) model.

Since elastic and CTE properties of polymers are temperature-dependent, they induce temperature-dependency on all the effective properties of laminas and laminates. However, the temperature dependency of fiber-dominated properties is small because the fiber-properties are virtually independent of temperature or their variation with temperature is very small. The temperature dependence of matrix dominated properties can be accurately represented by a quadratic function and in some cases, the variation is so small that a linear function suffices.

Although the experimental data is scarce or non-existent in some cases and displays great scatter in other cases, a systematic procedure is developed and applied to extract in-situ properties for both fibers and polymers encompassing four composite material systems while taking into account their temperature dependence.

Finite element analysis confirms the accuracy of the analytical micromechanics model selected for this study. Once the fiber and polymer properties are found, micromechanics allows computation of all lamina effective properties for the temperature range of interest. However, care should be taken not to extrapolate outside the temperature range of the experimental data used for material characterization, particularly when nonlinear equations are used to model the data. Predictions outside this range are thus made assuming constant values for all properties outside the temperature range of the experimental data.

When laminates are mechanically loaded, damage initiation and accumulation up to crack saturation are characterized by two values of critical ERR in modes I (opening) and 
II (shear). However, cooling of quasi-isotropic laminates produces only mode I cracking because the thermal contraction is the same in every direction, and cross-ply laminates crack in mode I only because there is no shear induced. Therefore, only $G_{I c}$ was used in for this study.

The critical ERR $G_{I c}$ is easily obtained by minimizing the error between crack density prediction and available data. A constant value of critical ERR produces satisfactory predictions of crack density vs. temperature. To eliminate the small discrepancy on saturation crack density at cryogenic temperature requires adjusting the critical ERR with a quadratic equation. From a practical point of view, being able to produce satisfactory estimates of damage with a constant value of critical ERR is advantageous because it reduces the amount of experimentation needed to adjust the critical ERR.

Some of the experimental crack-density data is inconclusive about crack saturation for some material systems, namely AS4/3501-6 and T300/5208. In other words, for those material systems the temperature at which data is available is not low enough to show crack density leveling off. However, model predictions clearly show that crack saturation is likely in all cases. This is because the critical ERR does not change much with cooling, but transverse CTE drops significantly with cooling (Figures 3.14-3.15), thus depriving the system from the main driver for thermo-mechanical transverse cracking.

For thermal fatigue of laminated composites at low temperatures, polymers become more rigid and tend to a brittle crystalline molecular structure. Since crystalline polymers develop crazes which become an ideal path to propagate new cracks, the onset and growth of new cracks is caused by thermal cyclic loads until the craze size reaches a critical value $a_{c}$. Once the critical value $a_{c}$ has been reached, the critical ERR $G_{I c}$, which is a material property, can be used to predict transverse cracking. Since the craze size is impossible to measure, an analytical parametrization $f(N)$ is proposed as a measure of fatigue resistance $f(N)$. A decreasing $f(N)$ allows $G_{I c}$ in (5.5) to decrease with number of cycles and thus, higher crack densities can be predicted for larger number of cycles $N$.

For low cycle data $f(N)$ can be calculated by separation of variables using (5.5). On one hand, higher crack densities are generated at lowest temperature and thus $G_{I c}^{\prime}\left(T_{\min }\right)$ can be adjusted at first cycle where no fatigue phenomenon exists. On the other hand, $G_{I c}$ as function of number of cycles in (5.5) can obtained using (5.3). Therefore, $f(N)$ can be adjusted using SLR (5.6) in a semi-logarithmic scale.

However, transverse cracking evolution beyond $N$ for the last experimental data point cannot be predicted because no experimental evidence exists beyond that number of cycles. Furthermore, the $f(N)$ calculated using low-cycle data $[1,10,186]$ is restricted to specific LSS, $R_{T}$, low number of cycles $(\approx 4000)$ and it does not account for lamina orientation. A Master Paris law is proposed that solves these problems. 
A master Paris's law using (5.14) predict thermal fatigue damage regardless of layup and $R_{T}$ as shown in Figure 5.15 and 5.17. $\Delta G_{I}$ is the only driving force to predict transverse damage. The fatigue resistance $f(N)$ can be easily predicted for each lamina at any $R_{T}$ and number of cycles $N$.

Unlike $f(N)$ adjusted from low-cycle data, fatigue resistance can be predicted regardless of LSS, $R_{T}$, and number of cycles. Furthermore, available data shows the following. First, saturation crack density (CDS) under thermal cyclic loads is smaller than those under mechanical fatigue or static tests. Second, saturation crack density for thermal low-cycle data approaches CDS quickly.

Although experimental tests must be performed to obtain the master Paris's law for each material system, the understanding of the Paris's plot allows us to predict fatigue resistance $f(N)$ using a small number of both specimens and thermal cycles. This is because $\Delta G_{I}$ decreases sharply as $\lambda$ increases. Therefore, it is proposed to map all the characteristic regions of Paris's curve (damage initiation, linear relation, and CDS as illustrated in Figure 5.15) combining a greater number of specimens with different $R_{T}$ and reducing the number of cycles to $20-30$ instead of $1500-4000$ reported in $[1,10,186]$. For instance, two LSS can be tested, one cross-ply (CP) and one quasi-isotropic (QI) laminates. Then, both layups subjected to very high $R_{T}$ with $5-10$ cycles (damage initiation), high $R_{T}$ with $10-20$ cycles and intermediate $R_{T}$ with $20-50$ cycles (linear relation), and very low $R_{T}$ until first crack propagate (CDS). This would drastically reduce the time and costs of experimentation because fatigue damage for any layup and $R_{T}$ of interest can be predicted without need to perform high-cycle fatigue tests.

Despite the proposed high-cycle fatigue predictions using a master Paris Law, the lack of experimental data in thermal fatigue calls into question analytical predictions. This is because transverse cracking often precedes other catastrophic modes of damages. This fact combined with the time consuming to complete a real fatigue life testing, it makes that equivalent mechanical fatigue tests become a good alternative as a surrogate for thermal fatigue tests.

Equivalent mechanical fatigue test to simulate a thermal fatigue can be accomplished if the same transverse loading conditions $\sigma_{2}$ are satisfied for all life values $N$. Unfortunately, it does not exist an exact combination of equivalent mechanical strains $\epsilon_{T}^{m e}$ that operating together satisfy exactly such condition. This is due to two reasons.

First, the temperature-dependent properties. During thermal fatigue, properties vary at each $T$ while thermo-mechanical properties are constant during mechanical fatigue test at reference temperature $T_{r}$. Second, physical differences between imposed mechanical strains $\epsilon_{T}^{m e}$ compared with thermal strains $\epsilon^{\text {th }}$ that emerges from equilibrium.

However, equivalent mechanical strains $\epsilon_{T}^{m e}$ that satisfy $\lambda_{T_{r}}^{m e}=\lambda_{T_{m i n}}^{t h}$ can be accom- 
plished under certain conditions. Among these conditions, the most critical is the thermal ratio $R_{T}$. Since the error on $\sigma_{2}$ become larger as $T_{\text {min }}$ moves away from $T_{r}$, a not so high $R_{T}$ must be selected. The selection range of $R_{T}$ depends on the material properties. Furthermore, $R_{T}$ must be selected so that the strain to failure $\epsilon_{x}^{u l t}$ is not reached during mechanical testing.

A biaxial equivalent mechanical test can be performed to simulate a thermal fatigue test. However, its execution is very complex from a practical point of view. In order to simplify the fatigue testing and achieve the best approximation for stress field $\sigma_{2}$, an equivalent mechanical thickness $t_{m e}$ can be used to accomplish $\lambda_{T_{r}}^{m e}=\lambda_{T_{\min }}^{\text {th }}$ in the middle lamina for all life values $N$. In that way, the fatigue testing is reduced to uniaxial test with tensile $\epsilon_{x}^{m e}$. Since $\epsilon_{x}^{m e}$ vary with $N$, transverse damage needs to be updated from thermal fatigue predictions using a Master Paris law (5.14) and DDM.

Therefore, equivalent mechanical fatigue test to simulate thermal fatigue for low $R_{T}$ using equivalent mechanical thickness $t_{m e}$ can be easily accomplished, and it will be faster than conventional thermal fatigue tests. Even though not so high $R_{T}$ can be selected, transverse damage evolution can be tested for low $R_{T}$ using $\epsilon_{x}^{m e}$ and $t_{m e}$ to construct part of Master Paris Law. This is because according to Master Paris Law (5.14), laminates subjected to low $R_{T}$ (Figure 5.14) predict very low crack-growth rate (Figure 5.17) and thus, it is a very useful methodology to obtain crack density data that require large number of applied cycles. Furthermore, equivalent mechanical tests is useful to find out if other damage mechanisms appear or, conversely, the CDS is reached similar to endurance limit in metals.

For high or any thermal ratio, the Master Paris Law can still be used to successfully correlate transverse damage predictions despite equivalent mechanical tests can not be totally accomplished with accuracy. Since for high $R_{T}$ the $\Delta G_{I}$ is high (Figure 5.14), very fast crack-growth rate (Figure 5.17) is generated and a few number of cycles are only necessary to apply in order to obtain crack density data. Thus, from a practical point of view, experimental thermal tests are practicable in this case. Similar to previous case, no more than $20-50$ cycles are needed in order to obtian crack density data when $\Delta G_{I}$ yields a linear relation.

\subsubsection{Future work}

Since Master Paris law is based on $\Delta G$ as unique driving force that controls the fatigue resistance under thermal cyclic loads, $\Delta G_{I}$ is proposed in this dissertation because cracks propagate in mode I for cross-ply (CP) and quasi-isotropic (QI) laminates. However, it is expected that the fatigue resistance for angle-ply (AP) laminates can be calculated using a Master Paris law computing the total $\Delta G=\Delta G_{I}+\Delta G_{I I}$. This is because a mixed 
crack opening in mode I and II occurs in angle-ply laminates as reported in [300] under mechanical loads.

Since temperature-dependent properties are computed and $\Delta G$ makes not distinction between thermal or mechanical loads, a correlation between thermal only and mechanical only fatigue tests using master Paris law is expected to be correlated. For that, both the temperature dependence of $G_{I c}^{\prime}\left(T_{\min }\right)$ and thermo-mechanical properties as wells as the residual stresses $\Delta T=T_{\min }-S F T$ must be computed.

Furthermore, it is expected that outstanding failure theories can be implemented into DDM model to predict other failure modes such as fiber-matrix debonding and delamination. 


\section{Appendix A}

\section{Supplemental material}

A brief explanation about Python scripts and its use is included in this section. First, some libraries such as Numpy, Math and Xlsxwriter are needed to perform elemental and advanced math operations required to run the attached Python Scripts. Installation of these or other libraries to extend Abaqus functionality is described in detail on [35]. Note that the Numpy or Math library are already installed in Abaqus by default, so these library versions cannot be changed. However, Xlsxwriter library is required to handle or create new tables using an Excel file extension, and it must be installed as follows:

- Determine the Phyton version ( $\gg$ import sys) using the windows command from the installed Abaqus version.

- Install the correct Python version determined previously. Onwards, any necessary library to be used by Abaqus except Numpy or Math library must be first installed in the Python folder.

- Install the Xlsxwriter module in the Python folder. Check Xlsxwriter compatibility in [306] for Python version installed. Once the Xlsxwriter library is already installed in Python folder, it must be copied/moved to the Abaqus library folder, similar to the following path: C: \SIMULIA \Abaqus $\backslash 6.14-2 \backslash$ tools $\backslash$ SMApy $\backslash$ python $2.7 \backslash$ Lib $\backslash$ site-packages. This procedure can be followed for any other type of library if needed.

Once all the libraries needed are properly installed, the FEA model to obtain lamina CTE can be run keeping in the same folder the following scripts:

1. LaminaName.py: creates a RVE with identical mesh through the thickness in order to apply the PBC as well as material properties, steps, thermal loads and job. 
2. PBC.py: a function script which detects a basic geometry (it can be another one such as cube, polygonal shape,...) and creates the PBC constraints. This script is good because you can apply PBC independently of the RVE shape (may be not to much complicate).

3. ParameterIntegrator.py: special function with 4 sub-functions which can calculate the value of a function, the integral of a second order polynomial, and the accumulated thermal strain given the tangent lamina CTE, typically as one can find in the literature.

4. ExcelProperties.py: script to obtain an Excel table with the temperature-dependent properties

5. Epsilonrecover.py: script to obtain the accumulated thermal strain once the FEA model has been submitted.

The FEA model can be run as follows:

- Run this script with the constituent properties and settings

1. Set your work directory and run LaminaName.py

2. Select 'Part-1' in Part section to see the RVE

3. Go to 'Interaction' section to check that all the PBC have been created. The PBC must be shown as small yellow circles.

4. Go to 'Job' section and submit the Job

- Once 'Job-1' has been completed successfully, run the 'Epsilonrecover.py' script

- Wait until appear the message 'All calculations finished', on Message Area

- In current folder, the excel file with the accumulated thermal strain should appear as 'Alpha.xlsx'

- The excel file with matrix properties is optional. You can run 'Excelproperties.py' once LaminaName.py script has been run.

Free-edge stress analysis can be run as follows:

- Keep in the same folder the following scripts:

1. 3DFreeEdge.py 
2. 3DFreeEdge-2.py

3. PBC-FreeEdge.py

- Select the work directory in 3DFreeEdge.py file (line 48)

- Run 3DFreeEdge.py script

1. Select 'Part-1' in Part section to see the composite laminate

- Due to high complexity, you must refine the mesh close to edge using Abaqus GUI

- Once '3DFreeEdge' was run, 3DFreeEdge-2 should be run

- Once 'Job-1' has been completed successfully, path lines are created to verify the edge stresses

- XY data can be easily obtained in Postprocessor using the previous path created

\section{A.1 LaminaName}

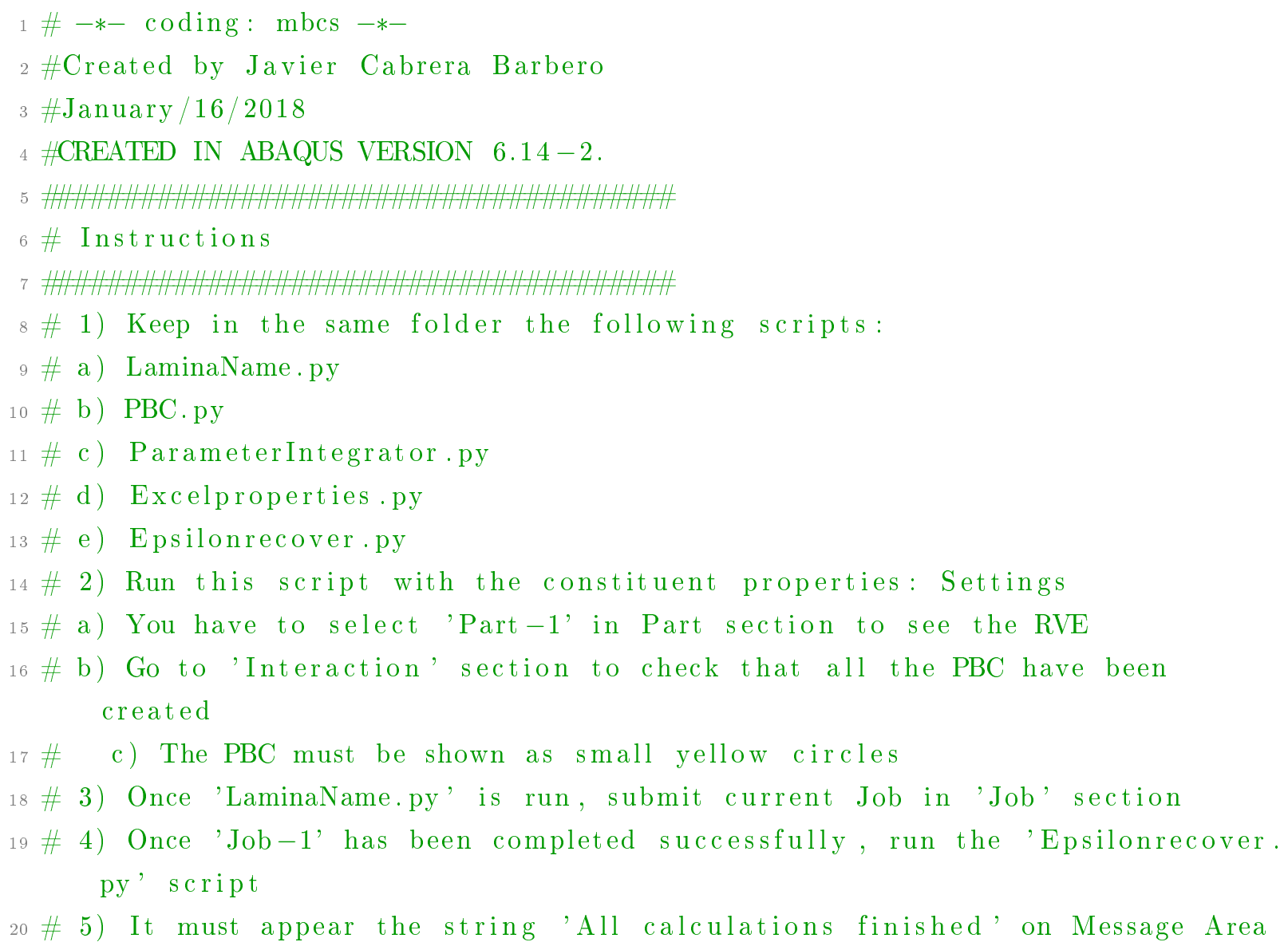


21 \# 6) In current folder, the excel file with the accumulated thermal strain should appear

22 as 'Alpha.xlsx'

23 \# 7) The excel file with matrix properties is optional. You can run,

Excelproperties.py'

24 \# once this script has been run.

25

26

27

28 \#FUNCTION TO CREATE RVE, MATERIAL PROPERTIES, PBC, STEPS, MESH, LOAD AND CREATE JOB :

29 \# All the libraries needed to obtain the lamina CTE are imported

30 from abaqus import

31 from abaqusConstants import *

2 from section import *

from part import *

4 from material import *

from assembly import *

6 from step import *

7 from interaction import *

from load import *

from mesh import *

from job import *

from sketch import *

from visualization import *

from optimization import *

4 from connectorBehavior import *

5 from abaqusConstants import*

import xlsxwriter

import math

import os

import numpy

50 \# All scripts needed are imported

51 import $\mathrm{PBC}$

52 import ParameterIntegrator

53 reload (ParameterIntegrator)

54 from ParameterIntegrator import *

$55 \#$

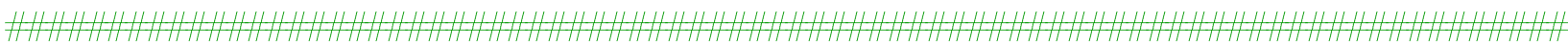

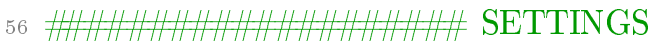

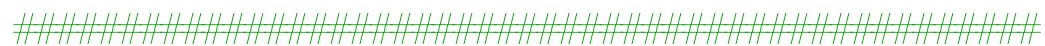

$57 \#$ 


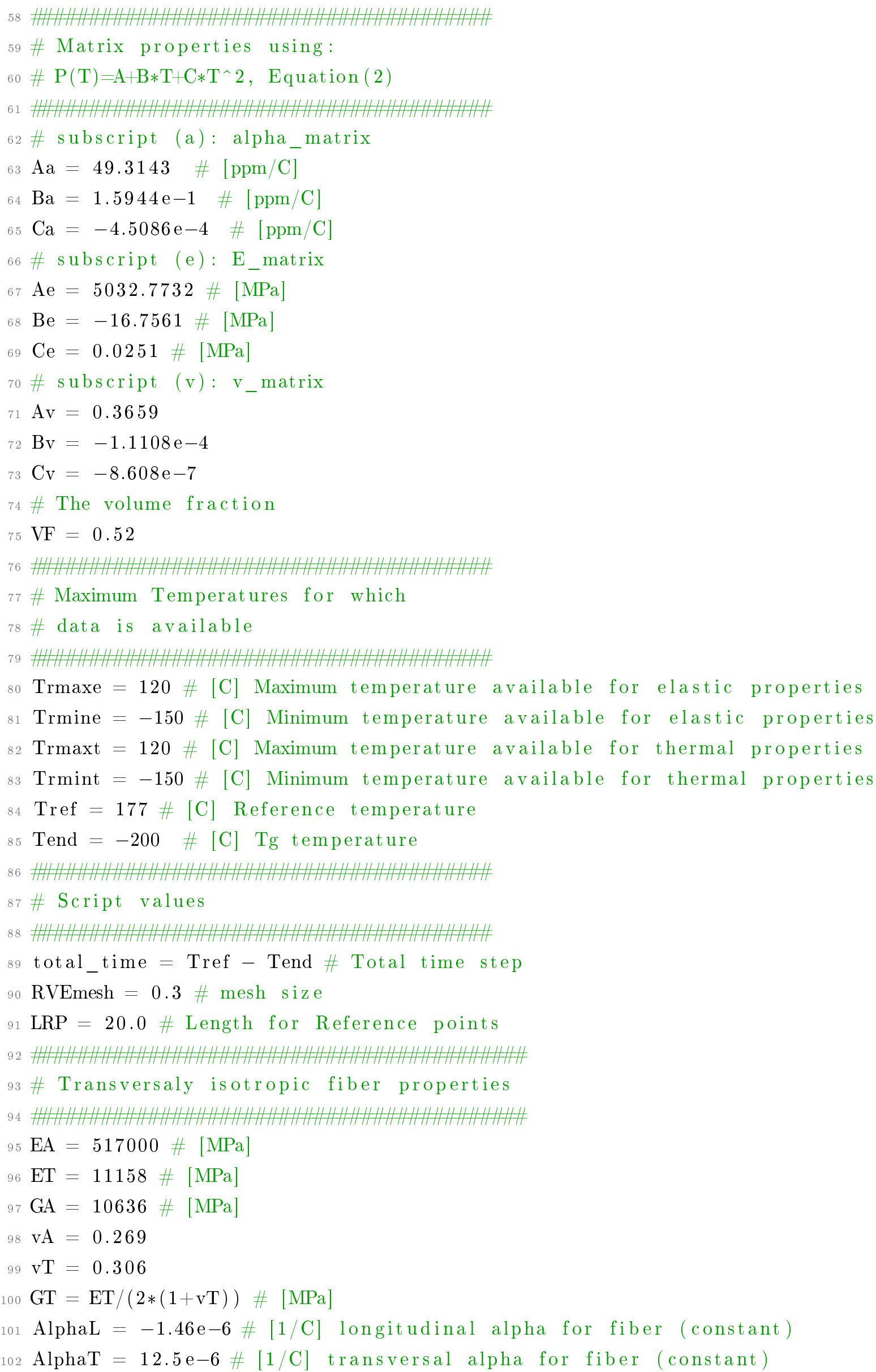




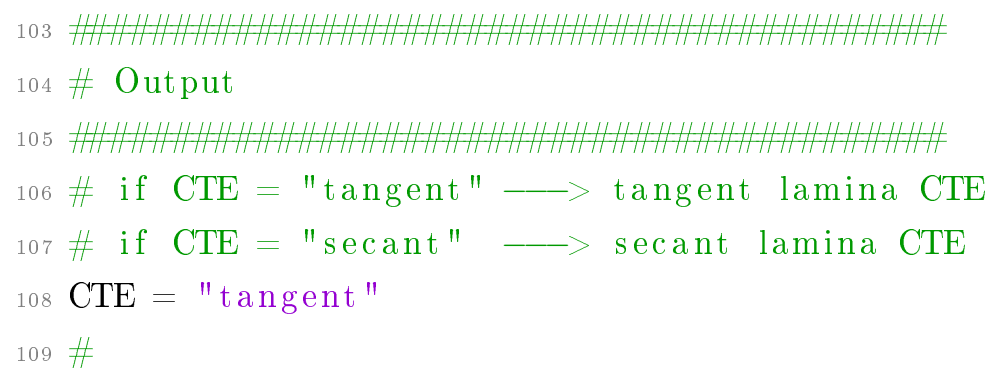


140 mdb. models ['Model-1'] p parts ['Part-1'] · projectReferencesOntoSketch (filter=

COPLANAR_EDGES, sketch=mdb.models ['Model-1'].sketches ['__profile_- '])

142 mdb. models ['Model-1']. sketches ['__profile_-'] . CircleByCenterPerimeter ( center $=($

$143 \quad 0.0,0.0)$, point $1=(0.0$, rf $))$

144 mdb. models ['Model-1']. sketches ['__profile_-']. CircleBy CenterPerimeter ( center $=($

$145 \mathrm{a} 2, \mathrm{a} 3)$, point $1=(\mathrm{a} 2+\mathrm{rf}, \mathrm{a} 3))$

146 mdb. models ['Model-1'] parts ['Part-1']. PartitionCellBySketch ( cells=

147 mdb.models['Model-1'] p parts ['Part-1']. cells .getSequenceFromMask (('[\#1 ] ',

148 ), ), sketch $=$ mdb. models ['Model-1']. sketches ['__profile_-'], sketchPlane=

149 mdb.models ['Model-1'].parts ['Part-1'].faces [4], sketchUpEdge $=$

150 mdb.models ['Model-1'].parts ['Part-1']. edges [7])

151 del mdb. models ['Model-1']. sketches ['__profile_-_']

152 mdb. models ['Model-1'] · parts ['Part-1']. PartitionCellByExtrudeEdge ( cells=

153 mdb.models ['Model-1']. parts ['Part-1']. cells . getSequenceFromMask (('[\#1 ] ',

$154), \quad)$, edges $=(\operatorname{mdb} \cdot \operatorname{models}[$ 'Model -1 '] $\cdot \operatorname{parts}[$ 'Part -1 ']. edges [3],

155 mdb.models ['Model-1']. parts ['Part-1'].edges [4],

156 mdb.models ['Model-1'].parts ['Part -1 '] $\cdot$ edges [5]), line $=$

mdb. models ['Model-1'] parts ['Part-1']. Partition CellByExtrudeEdge ( cells= mdb.models ['Model-1'].parts ['Part-1']. cells .getSequenceFromMask (('[\#2 ]',

$160), \quad)$, edges $=(\operatorname{mdb} \cdot \operatorname{models}[$ 'Model -1 '] . parts ['Part -1 '] . edges [11],

161 mdb.models['Model-1'].parts ['Part-1'].edges [12],

162 mdb.models ['Model-1']. parts ['Part-1']. edges [13]), line $=$

163 mdb. models['Model-1']. parts ['Part-1']. edges [16], sense=REVERSE)

67 \#CREATE MATERIAL

$168 \#$

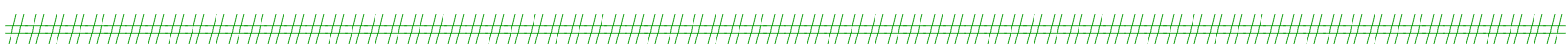

169

170 \# CREATE MATRIX TEMPERATURE DEPENDENT PROPERTIES FOR 'MATERIAL-1'

71 \# We assign the names to obtain $\mathrm{E}, \mathrm{v}$ or alpha values given the parameters

172 alphaS $=$ ParameterIntegrator $(\mathrm{Aa} * 1 \mathrm{e}-6$, Ba $* 1 \mathrm{e}-6, \mathrm{Ca} * 1 \mathrm{e}-6$, Trmaxt,

Trmint, Tref $)$ in $[1 / \mathrm{C}]$ units

73 calcE $=$ ParameterIntegrator $(A e, B e$, Ce, Trmaxe, Trmine, Tref $)$

$74 \mathrm{calcV}=$ ParameterIntegrator (Av, Bv, Cv, Trmaxe, Trmine, Tref $)$

175

176 \# To get Excel with properties go to Excelproperties.py

177 \# Tables with Elastic and Thermal properties are created 


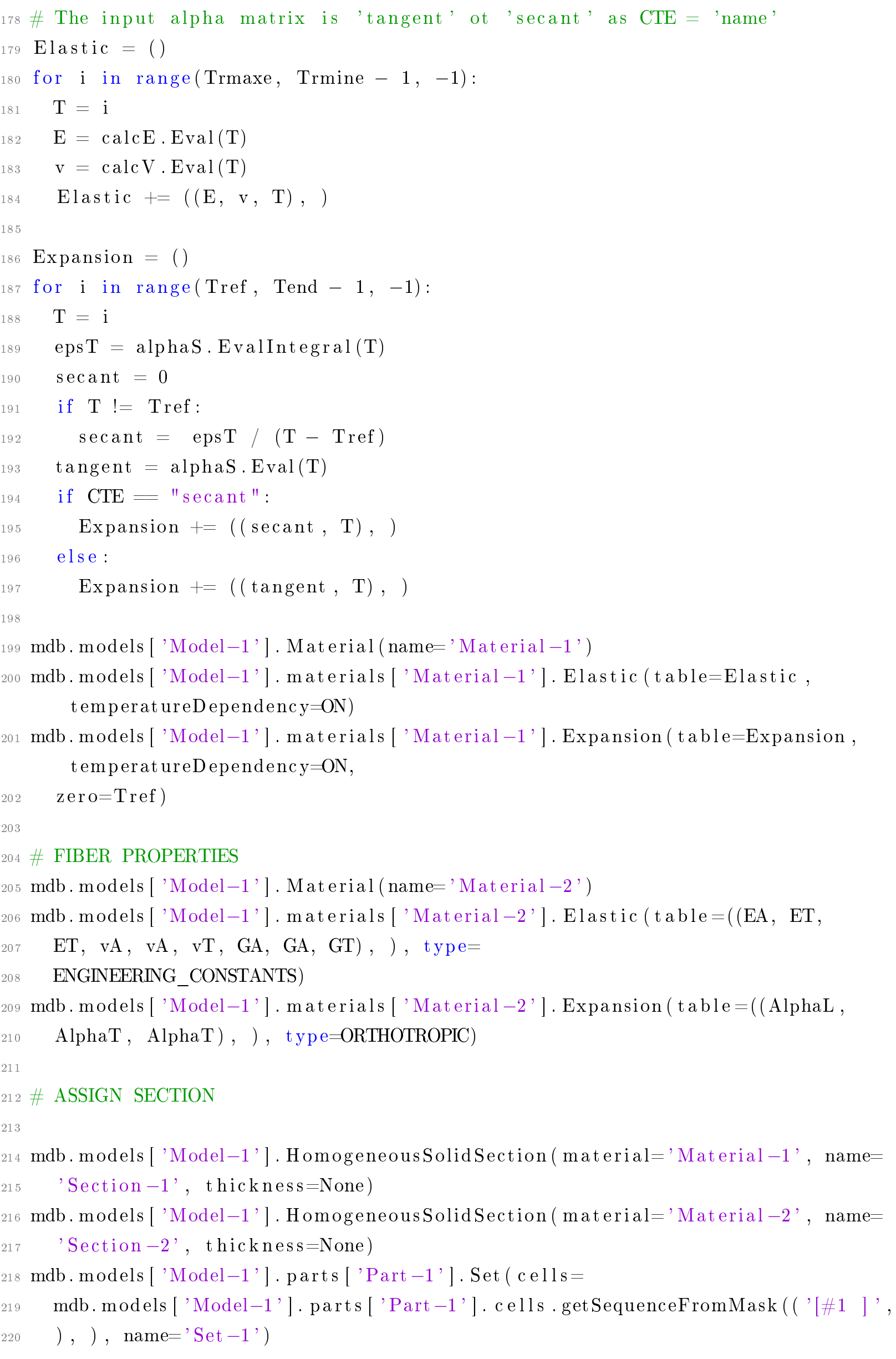


21 mdb. models ['Model-1'] . parts ['Part-1']. Section Assignment ( offset $=0.0$, offsetField=', , offsetType=MIDDLE_SURFACE, region= mdb. models ['Model -1 '] $\cdot$ parts ['Part -1 '] . sets ['Set -1 '], sectionName= 'Section -1', thicknessAssignment=FROM_SECTION)

mdb. models ['Model-1']. parts ['Part-1']. Set ( cells= mdb. models ['Model-1'] parts ['Part-1']. cells .getSequenceFromMask (( '[\#6 ] ', ), ), name='Set $-2^{\prime}$ )

mdb. models ['Model-1'] . parts ['Part-1']. Section Assignment ( offset $=0.0$, offsetField=', , offsetType=MIDDLE_SURFACE, region= mdb. models ['Model-1']. parts ['Part-1']. sets ['Set-2'], sectionName= 'Section-2', thicknessAssignment=FROM_SECTION)

233 \# CREATE A CSYS

234 mdb. models ['Model-1'] . parts ['Part -1 '] . Datum CsysByThreePoints (coordSysType= CARTESIAN, name $={ }^{\prime}$ Datum $\operatorname{csys}-1$ ', origin $=(0.0,0.0$, a1 $)$, point $1=(0.0,0.0$, $\mathrm{a} 1+1)$, point $2=(1.0,0.0,0.0))$

\section{8 \# MATERIAL ORIENTATION}

239 mdb. models ['Model-1']. parts ['Part-1']. MaterialOrientation( additionalRotationField=' ', additionalRotationType=ROTATION_NONE, angle $=0.0$

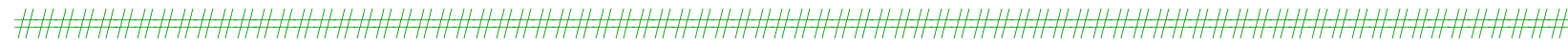

248 \#CREATE ASSEMBLY

249 \# 
259

260 mdb. models ['Model-1']. StaticStep ( initialInc =1.0, $\operatorname{maxInc}=1.0$, maxNumInc $=1000$,

261

262

284 \#CREATE PERIODIC BOUNDARY CONDITIONS

285 \#

286

287 mdb. models ['Model-1']. root Assembly . Set $($ nodes $=($

288 mdb.models ['Model-1'].rootAssembly.instances ['Part-1-1'].nodes, ), name=' PerBound') 'S ', 'PE', 'PEEQ' , 'PEMAG', 'LE', 'U' , 'RF' , 'CF' , 'CSTRESS', 'CDISP', 'IVOL', 'THE') )

HCREATE MESH

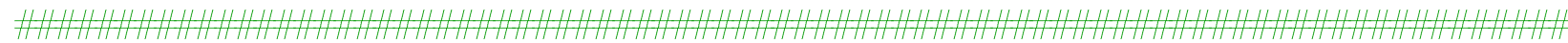

mdb. models ['Model-1'].parts ['Part-1']. seedPart (deviationFactor $=0.1$, minSizeF actor $=0.1$, size=RVEmesh)

mdb. models ['Model-1'].parts ['Part-1'] . setElementType ( elem Types=(ElemType( elem Code=C3D8R, elemLibrary $=$ STANDARD, secondOrderAccuracy=OFF, kinemat icSplit=AVERAGE_STRAIN, hourglas sControl=DEFAULT, distortionControl=DEFAULT)，ElemType( elemCode=C3D6, elemLibrary=STANDARD)

Elem Type ( elem Code=C3D4, elemLibrary=STANDARD) ) , regions $=($ mdb. models ['Model-1'].parts ['Part-1'] . cells .getSequenceFromMask (( '[\#1 ]' , )$, \quad)$ )

mdb.models ['Model-1'] parts ['Part-1'].generateMesh ()

mdb. models ['Model-1'].rootAssembly.regenerate ()

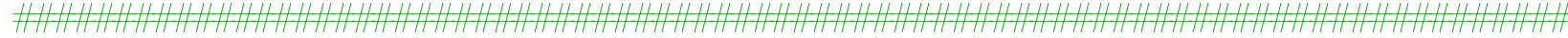

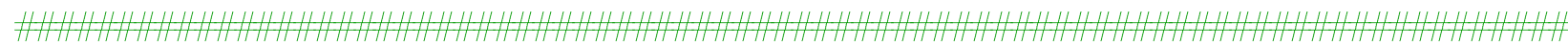

289 \# PBC.py is called to build the PBC constraints 
293 \# SYMMETRIC BOUNDARY CONDITIONS

294 \#

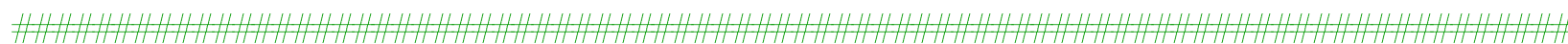

29

296 mdb. models ['Model-1'] $\operatorname{root}$ Assembly. Set ( faces=

297

298

300

313 \# LOAD TEMPERATURE getSequenceFrom Mask (

('[\#420 ]', ) , ), name $=$ 'Set -2340 ') name $=$

mdb. models ['Model-1'].rootAssembly. Set ( faces=

getSequenceFrom Mask (

('[\#2080 ]', ) , ), name='Set -2341 ') name $=$

mdb. models ['Model-1'].rootAssembly. Set (faces= getSequenceFrom Mask (

$('[\# 8044] ', \quad), \quad)$, name $=$ 'Set -2342 ') name $=$

mdb. models ['Model-1'] r rootAssembly.instances ['Part-1-1']. faces .

mdb. models ['Model-1'].XsymmBC(createStepName='Initial ', localCsys=None,

'XSYM', region=mdb. models ['Model-1']. rootAssembly.sets ['Set -2340'])

mdb. models ['Model-1']. root Assembly.instances ['Part-1-1']. faces .

mdb. models ['Model-1'].YsymmBC( createStepName='Initial ', localCsys=None,

'YSYM', region=mdb. models ['Model-1']. rootAssembly.sets ['Set -2341'])

mdb. models ['Model-1']. root Assembly.instances ['Part-1-1']. faces .

mdb. models ['Model-1'].ZsymmBC( createStepName=' In itial ', localCsys=None,

'ZSYM', region=mdb. models ['Model-1']. rootAssembly.sets ['Set -2342'])

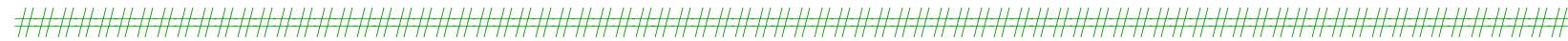

$314 \#$

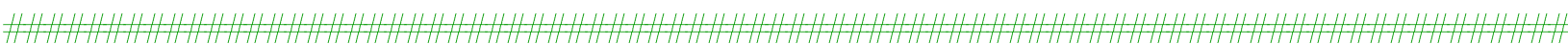

315

316 mdb. models ['Model-1'] $\cdot \operatorname{root}$ Assembly.Set ( c ells $=$

mdb. models ['Model-1']. root Assembly. instances ['Part-1-1']. cells .

getSequenceFrom Mask (

$('[\# 7] ', \quad), \quad)$, edges $=$ 
mdb. models [ 'Model-1']. root Assembly.instances ['Part-1-1']. edges . getSequenceFrom Mask (

('[\#ffffff ]', ), ), faces $=$ mdb. models ['Model-1']. root Assembly . instances ['Part-1-1']. faces . getSequenceFrom Mask ( ('[\#ffff ]', ), ), name='Set -2343 ', vertices $=$ mdb. models ['Model-1']. root Assembly. instances ['Part-1-1']. vertices . getSequenceFrom Mask (

('[\#ffff ]', ), ))

mdb. models ['Model-1']. Temperature (createStepName=' Initial ', cross SectionDistribution=CONSTANT_THROUGH_THICKNESS, distribution Type= UNIFORM, magnitudes $=($ Tref , ) , name='Predefined Field -1 ', region= mdb. models ['Model-1']. root Assembly . sets ['Set -2343'])

mdb. models ['Model-1']. rootAssembly. Set ( cells= mdb. models ['Model-1']. root Assembly.instances ['Part-1-1']. cells . getSequenceFrom Mask ( $('[\# 7] ', \quad), \quad)$, edges $=$ mdb. models ['Model-1']. root Assembly. instances ['Part-1-1']. edges . getSequenceFrom Mask ( ('[\#ffffffe]', ), ), faces $=$ mdb. models ['Model-1']. root Assembly. instances ['Part-1-1']. faces . getSequenceFrom Mask ( $($ '[\#ffff ]', ), ), name='Set -2344 ', vertices $=$ mdb. models ['Model-1']. root Assembly. instances ['Part-1-1']. vertices . getSequenceFrom Mask (

('[\#ffff ]', ), ))

mdb. models ['Model-1']. Temperature (createStepName='Step -1 ', cross SectionDistribution=CONSTANT_THROUGH_THICKNESS, distribution Type= UNIFORM, magnitudes $=$ (Tend, ), name $=$ 'Predefined Field -2 ', region $=$ mdb.models ['Model-1']. root Assembly.sets ['Set -2344'])

mdb.Job(atTime=None, contactPrint=OFF, description=', , echoPrint=OFF, explicitPrecision=SINGLE, getMemoryFromAnalysis=True, historyPrint=OFF, memory $=90$, memoryUnits=PERCENTAGE, model='Model-1', modelPrint=OFF, multiprocessing Mode $=$ DEFAULT, name $=$ 'Job -1 ', nodalOutputPrecision=SINGLE, numCpus $=1$, numGPUs $=0$, queue $=$ None, resultsFormat $=$ ODB, $\operatorname{scratch}=$ ', , type $=$ 
ANALYSIS, userSubroutine=', , waitHours $=0$, waitMinutes $=0$ )

\section{A.2 PBC}

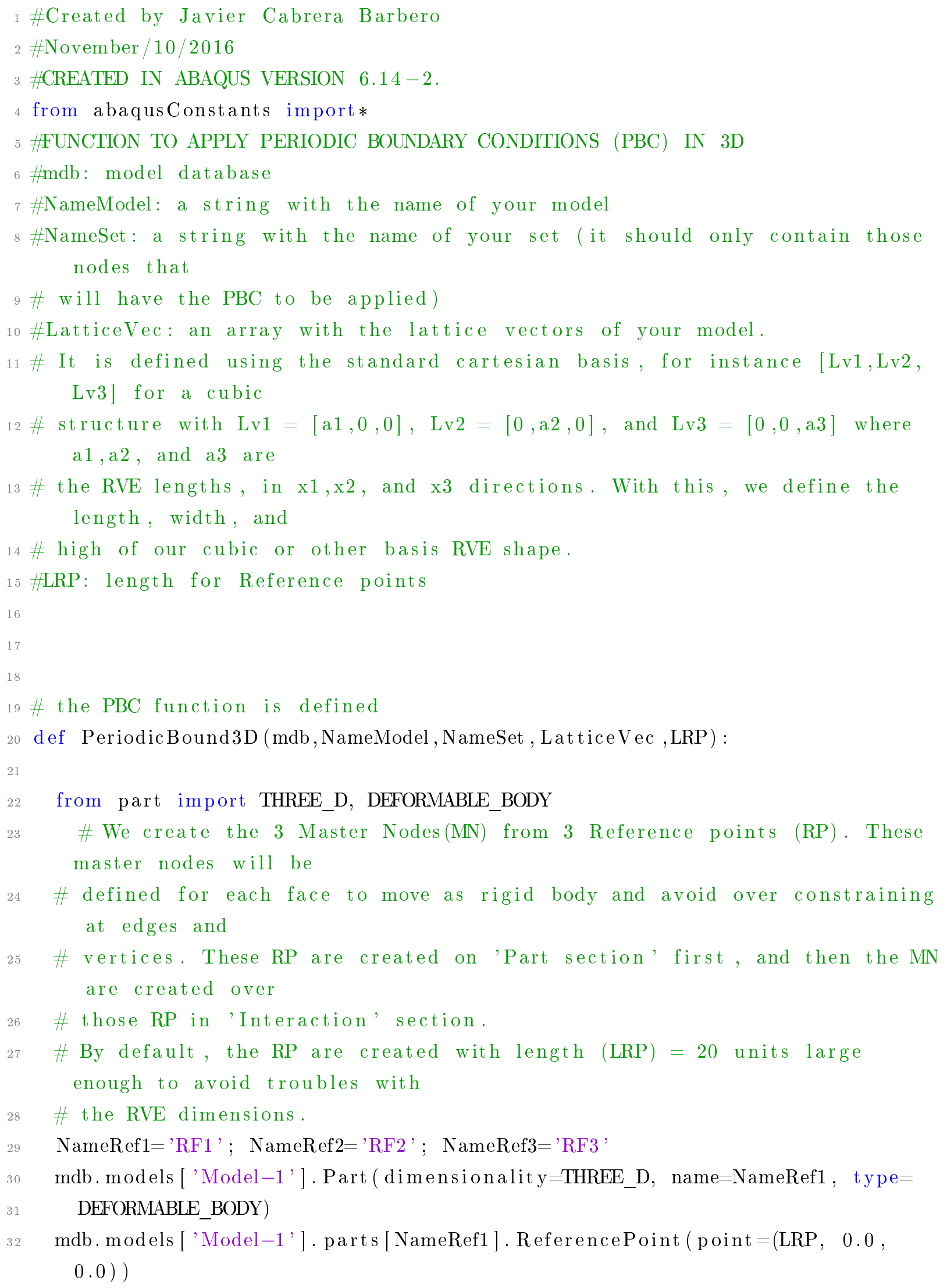


mdb. models ['Model-1']. Part (dimensionality=THREE_D, name=NameRef2, type=

\section{DEFORMABLE_BODY)}

mdb. models ['Model-1']. parts [NameRef2]. ReferencePoint ( point $=(0.0$, LRP,

$0.0))$

mdb. models ['Model-1']. Part (dimensionality=THREE_D, name=NameRef3, type= DEFORMABLE_BODY)

mdb. models ['Model-1']. parts [NameRef3]. ReferencePoint (point $=(0.0,0.0$, LRP )) mdb. models ['Model-1'] $\operatorname{root}$ Assembly. Instance (dependent=ON, name=NameRef1, part $=$ mdb. models ['Model -1 '] . parts [NameRef1]) mdb. models ['Model-1'] $\operatorname{root}$ Assembly. Instance (dependent=ON, name=NameRef2, part $=$ mdb. models ['Model -1 ']. parts [NameRef2]) mdb. models ['Model-1'] $\operatorname{root}$ Assembly. Instance (dependent=ON, name=NameRef3, part $=$ mdb. models ['Model -1 '] . parts [NameRef3]) mdb. models ['Model-1'] $\operatorname{root}$ Assembly. Set (name=NameRef1, referencePoints $=($ mdb. models ['Model-1']. root Assembly. instances [NameRef1]. referencePoints $[1])$,

mdb. models ['Model-1'] $\operatorname{root}$ Assembly. Set (name=NameRef2, referencePoints $=($ mdb. models ['Model-1']. root Assembly. instances [NameRef2]. referencePoints $[1])$,

mdb. models ['Model-1'] $\operatorname{root}$ Assembly. Set (name=NameRef3, referencePoints $=($ mdb. models ['Model-1'] root Assembly. instances [NameRef3]. referencePoints $[1])$,

mdb. models [ 'Model-1'] $\operatorname{root}$ Assembly . Set ( nodes $=($

mdb. models ['Model-1']. root Assembly.instances ['Part-1-1']. nodes , ) , name= 'PerBound')

\# We create a vector 'nodesALL' where is keept all the nodes with its coordinates

nodes All=mdb. models [ 'Model-1'] . rootAssembly. sets ['PerBound'] . nodes nodesAllCoor $=[]$

for nod in mdb.models['Model-1']. rootAssembly. sets ['PerBound']. nodes: nodes AllCoor . append (nod. coordinates)

\# We start markers

repConst $=0$

repConst $1=0$

\# Find periodically located nodes and apply equation constraints. Notes that we just establish

\# the three surfaces opposites to symmetric $\mathrm{BC}$ so we will have to set the $\mathrm{BC}$ in our python script later.

ranNodes=range $(0$, len $($ nodesAll $))$ \#Index array of nodes not used in equations constraint

\# Planesi $i=1, \ldots, 3$ set the coordinate from each plane or surface

plane $1=$ LatticeVec $[0]$

plane $2=$ LatticeVec [1]

plane 3 = LatticeVec[2] 


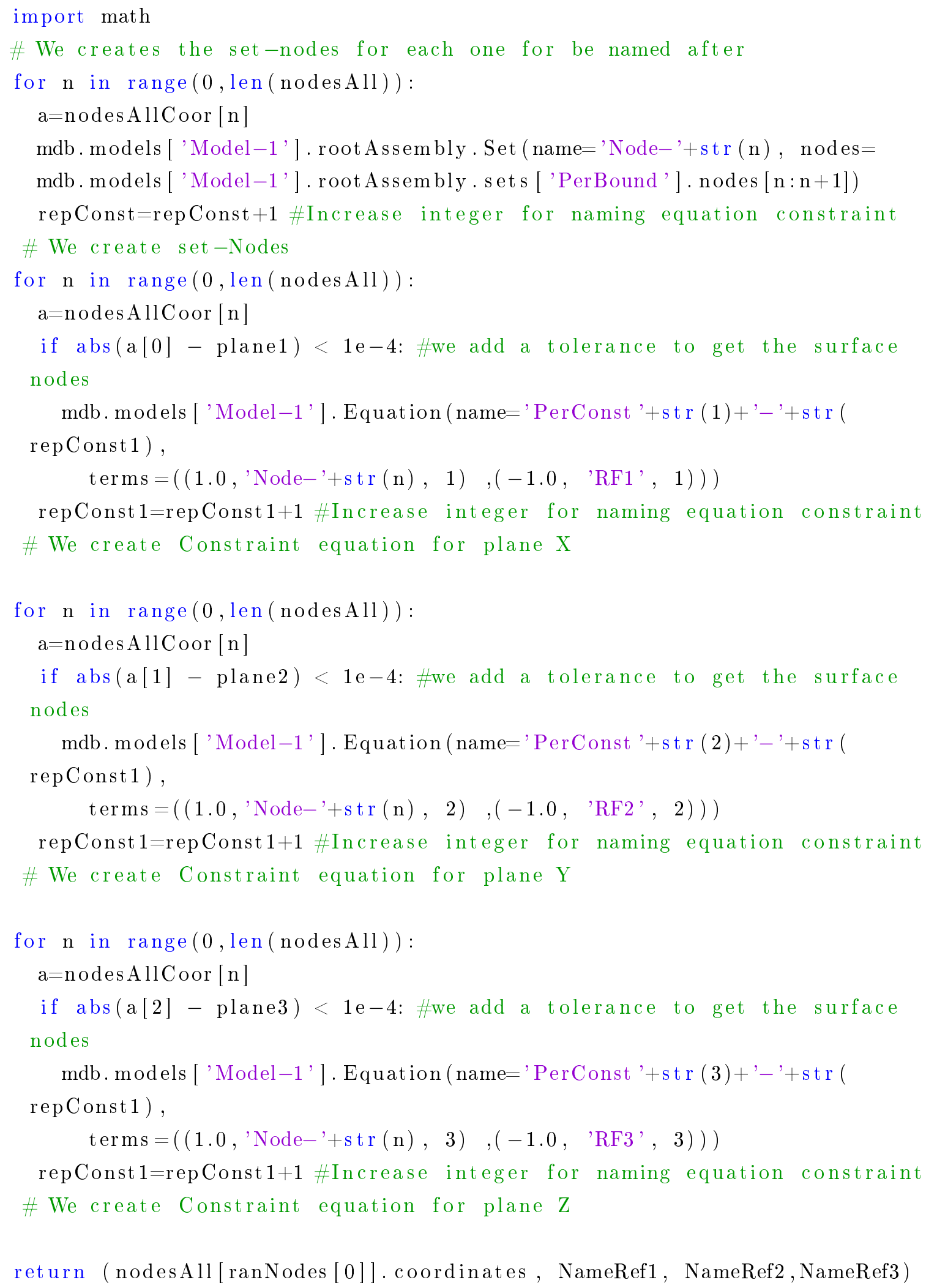

\section{A.3 ParameterIntegrator}

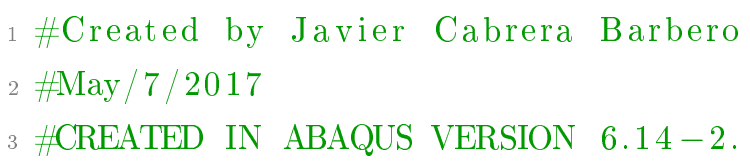




\section{4 \#FUNCTION TO OBTAIN 4 SUB-FUNCTIONS:}

5 \# a) the function value given the a second order polynomial

\# b) the integral given the a second order polynomial

\# c) the piece-wise function value given the a second order polynomial

\# and assuming constant outside the temperature range for which

\# data is available

10 \# c) the piece-wise integral value given the a second order polynomial

11 \# and assuming constant outside the temperature range for which

12 \# data is available

13 \#mdb: model database

14

class ParameterIntegrator:

def__init__(self, A, B, C, Trmax, Trmin, Tref):

self $. A=A$

self. $\mathrm{B}=\mathrm{B}$

self.C $=\mathrm{C}$

self. Trmax $=$ Trmax

self. Trmin $=$ Trmin

self. Tref $=$ Tref

self.C0 $=$ self.CalcPoly $($ Trmax $)$

self.C1 $=$ self.CalcPoly (Trmin)

def CalcPoly (self, T):

return (self.A + self. $\mathrm{B} * \mathrm{~T}+$ self. $\mathrm{C} * \mathrm{~T} * \mathrm{~T})$;

def CalcPolyI( self, T) :

return (self. $\mathrm{A} * \mathrm{~T}+$ self. $\mathrm{B} * \mathrm{~T} * \mathrm{~T} / 2+$ self. $* \mathrm{~T} * \mathrm{~T} * \mathrm{~T} / 3$ );

def Eval(self, T):

if $\mathrm{T}>$ self.Trmax:

return self.C0

elif $\mathrm{T}>$ self.Trmin:

return self.CalcPoly $(\mathrm{T})$

else :

return self.C1

def EvalIntegral (self, T):

if $\mathrm{T}>$ self. Trmax:

return self.C0 * $(\mathrm{T}-$ self. Tref $)$

elif $\mathrm{T}>$ self.Trmin:

return self.C0 * (self.Trmax - self.Tref $)+$ self.CalcPolyI $(\mathrm{T})-$ self.

CalcPolyI ( self . Trmax)

else:

return self.C0 * (self.Trmax - self.Tref $)+$ self.CalcPolyI(self.Trmin 
) - self.CalcPolyI(self.Trmax $)+$ self.C1 $*(\mathrm{~T}-$ self.Trmin $)$

\section{A.4 ExcelProperties}

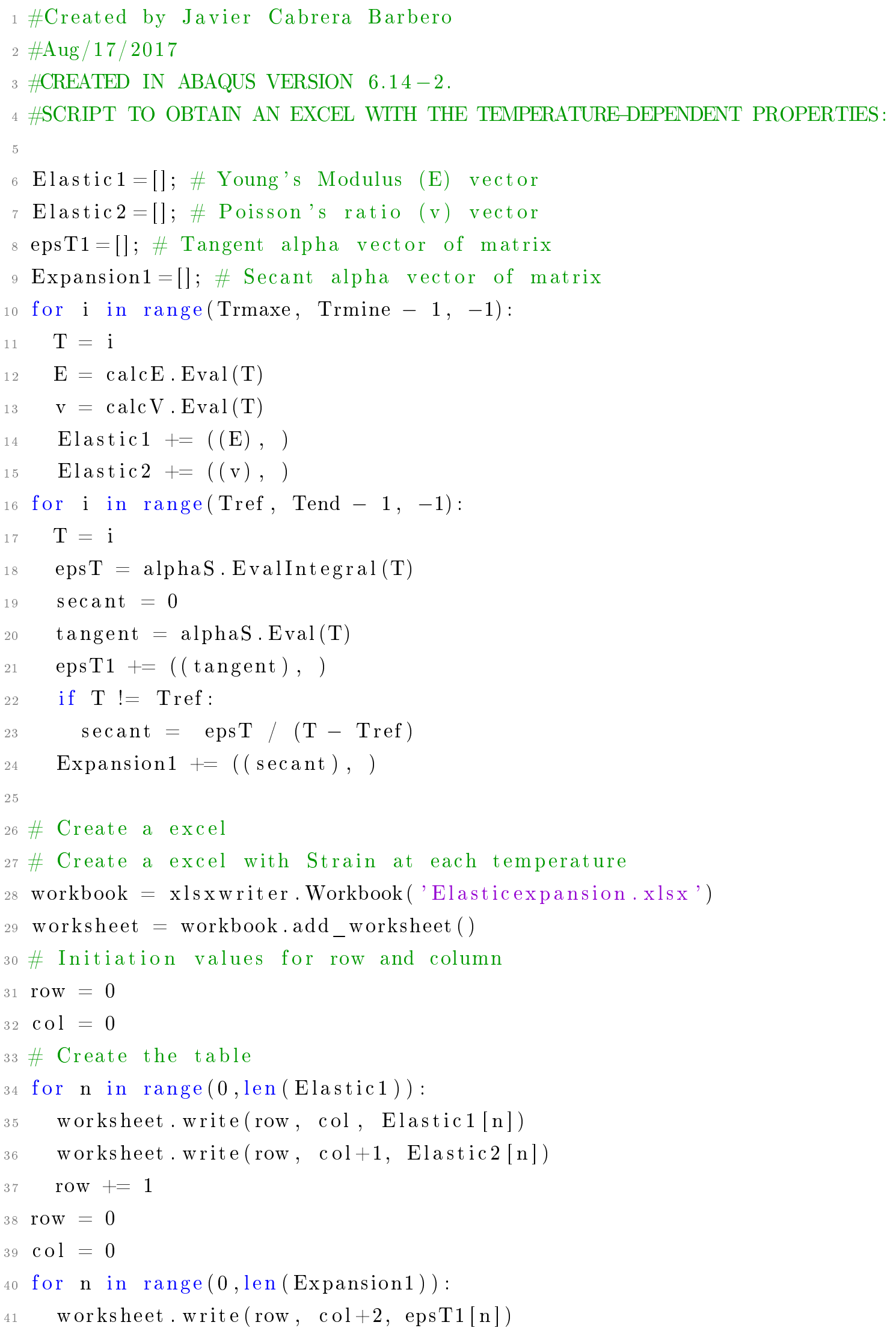




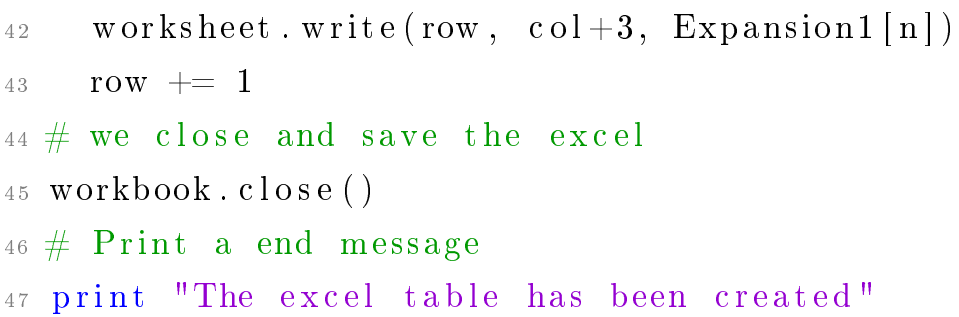

\section{A.5 Epsilonrecover}

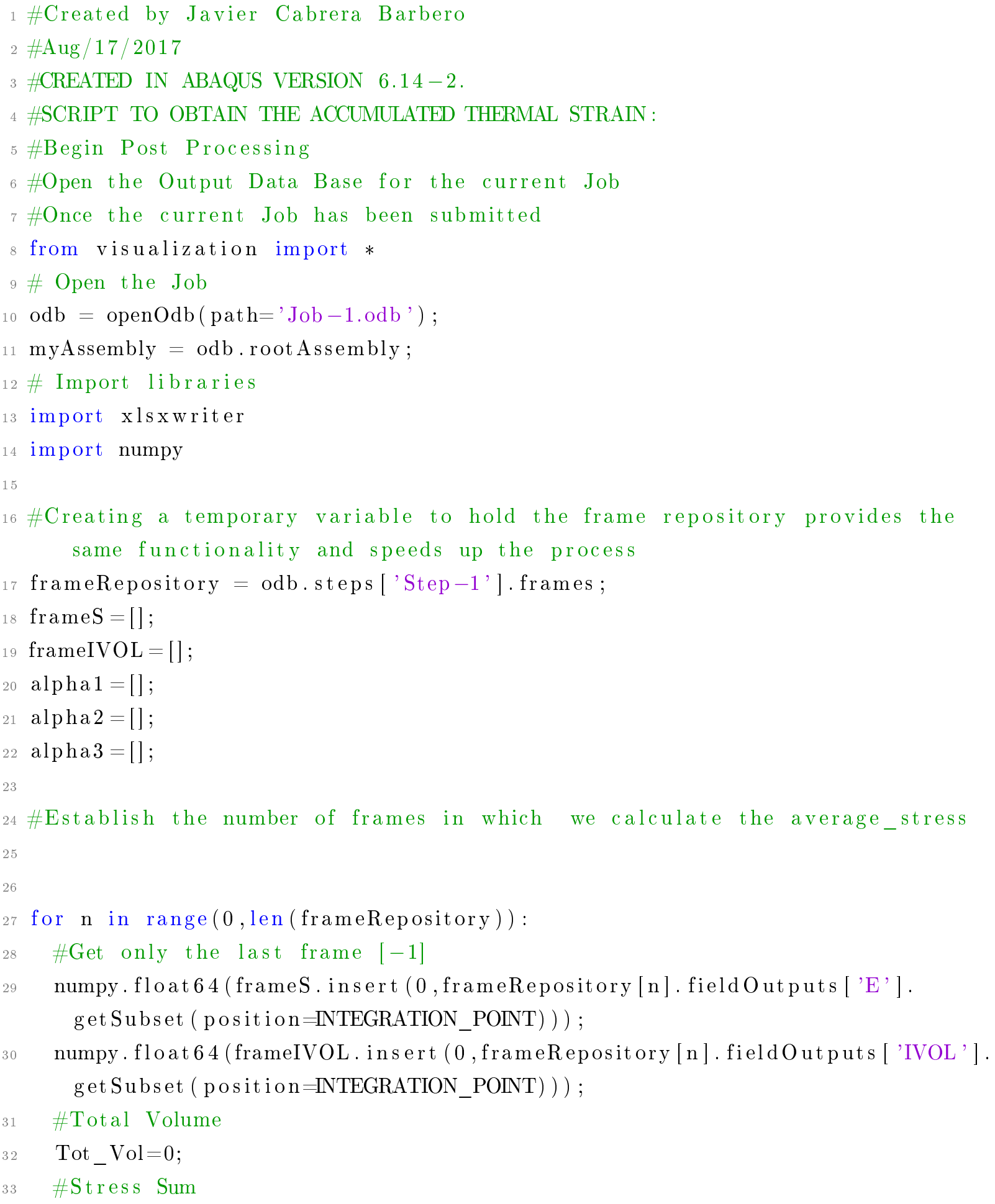




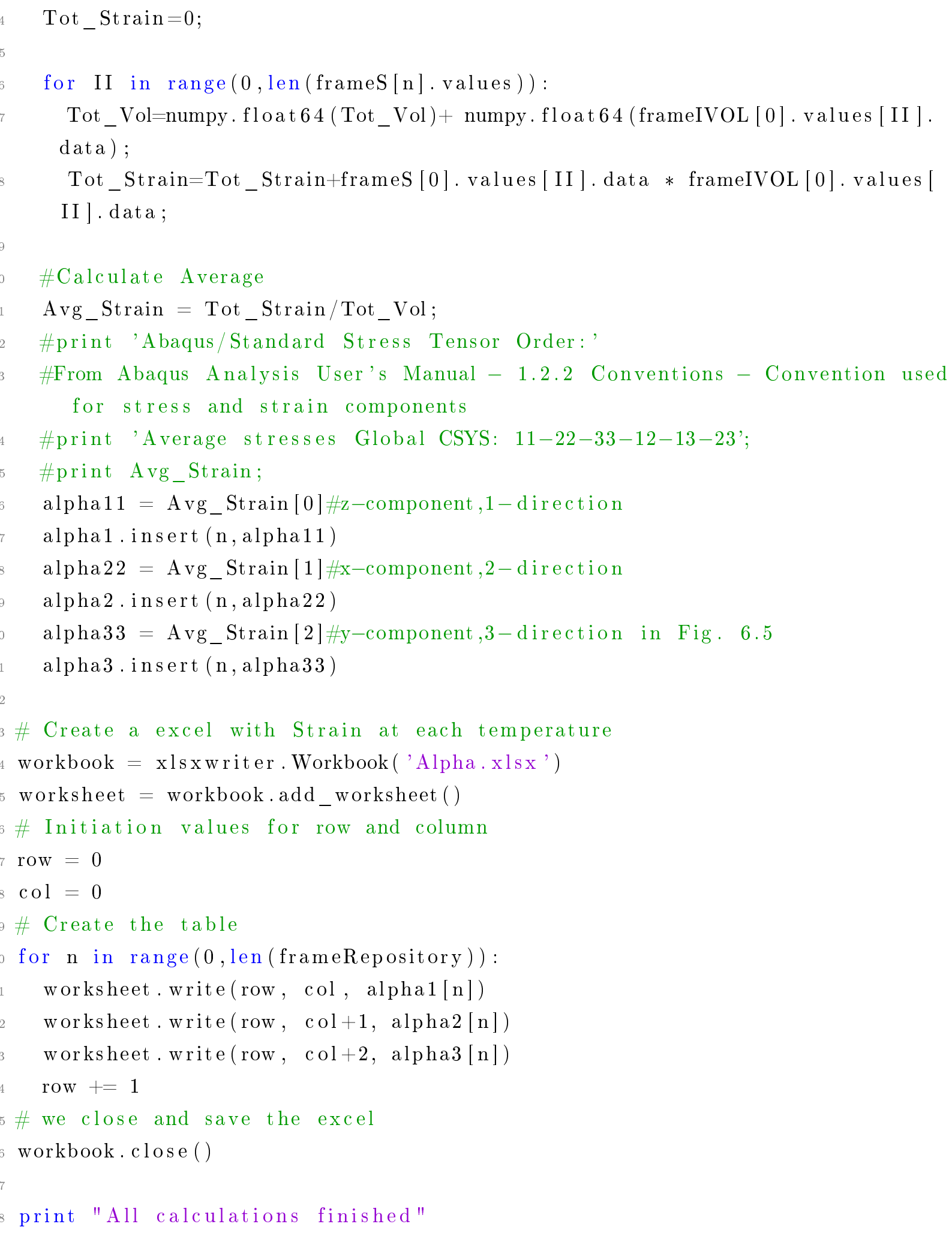

\section{A.6 3DFreeEdge}

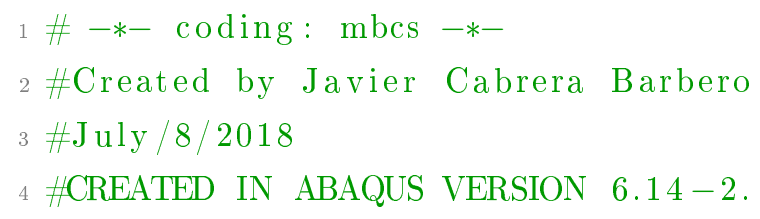




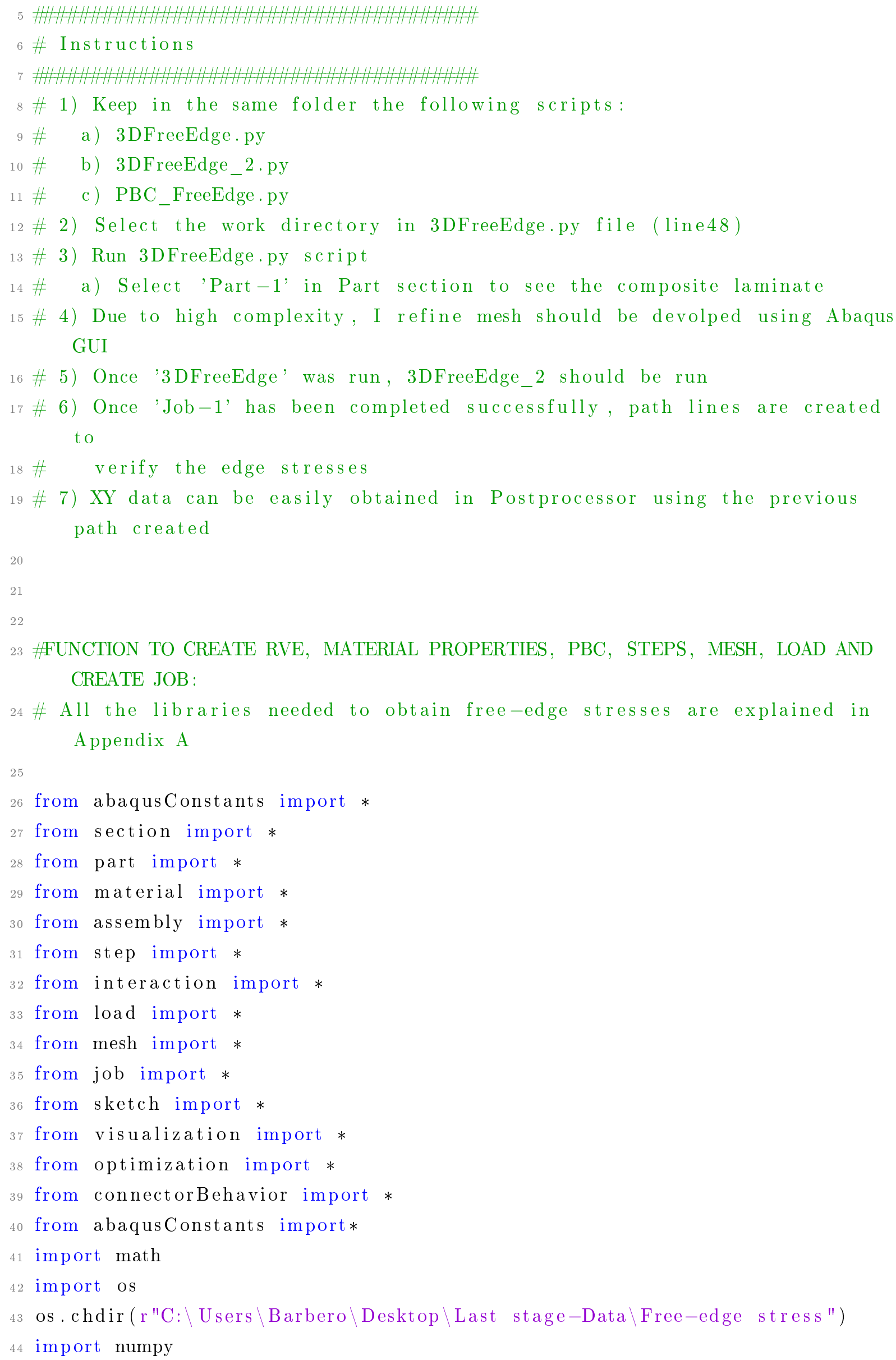




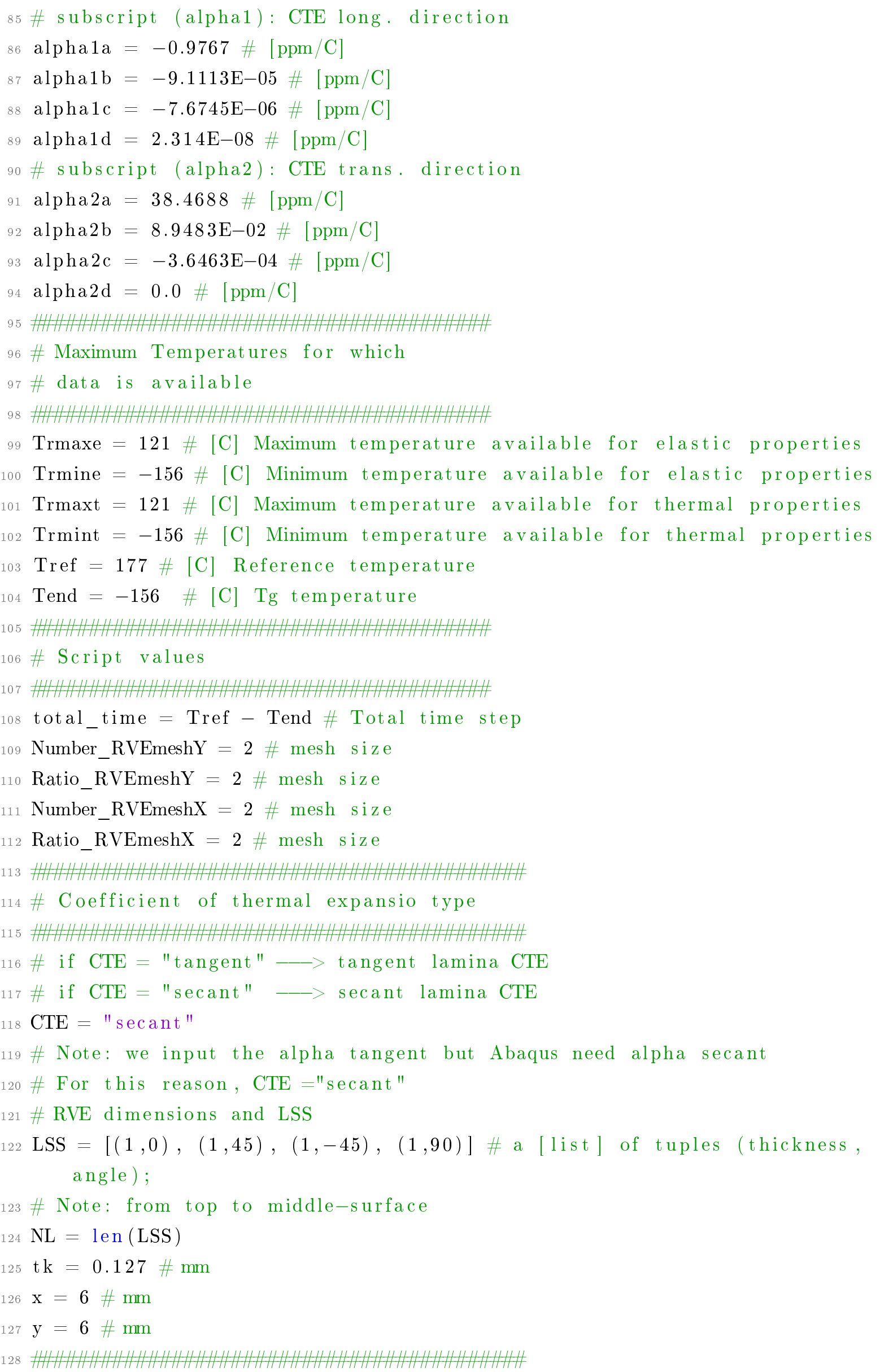




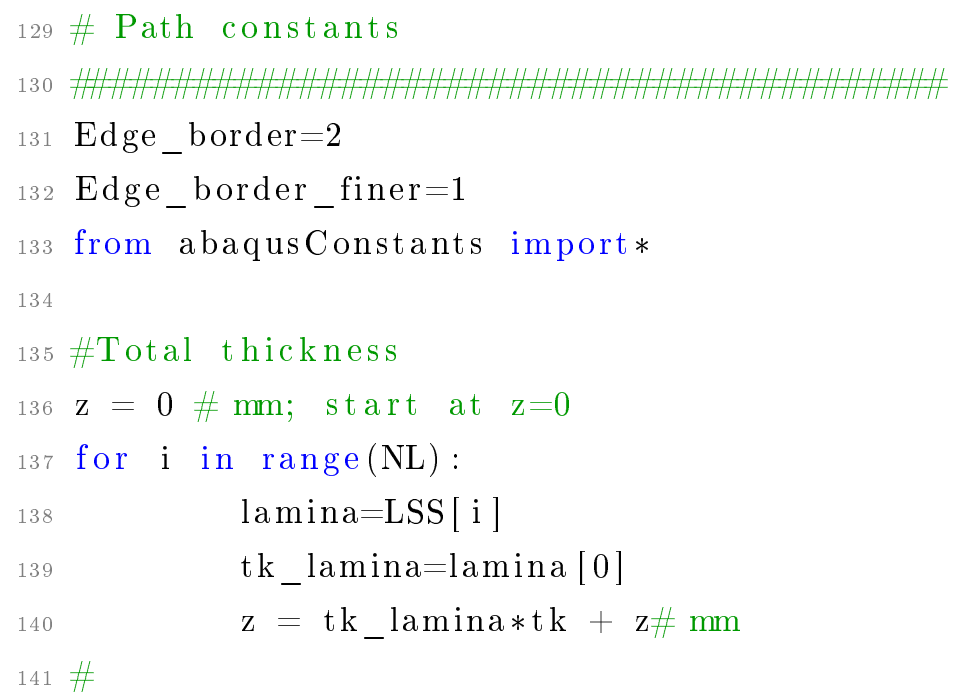

142

$143 \mathrm{Mdb}()$

144 \#It copy and write the AbaqusScriptFunc2_4 that we need in order to create the constraint equations

$145 \#$

146 \#CREATE PART

$147 \#$

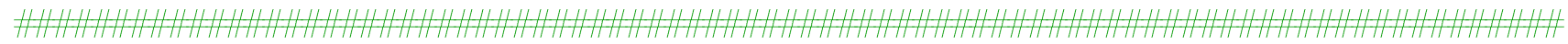

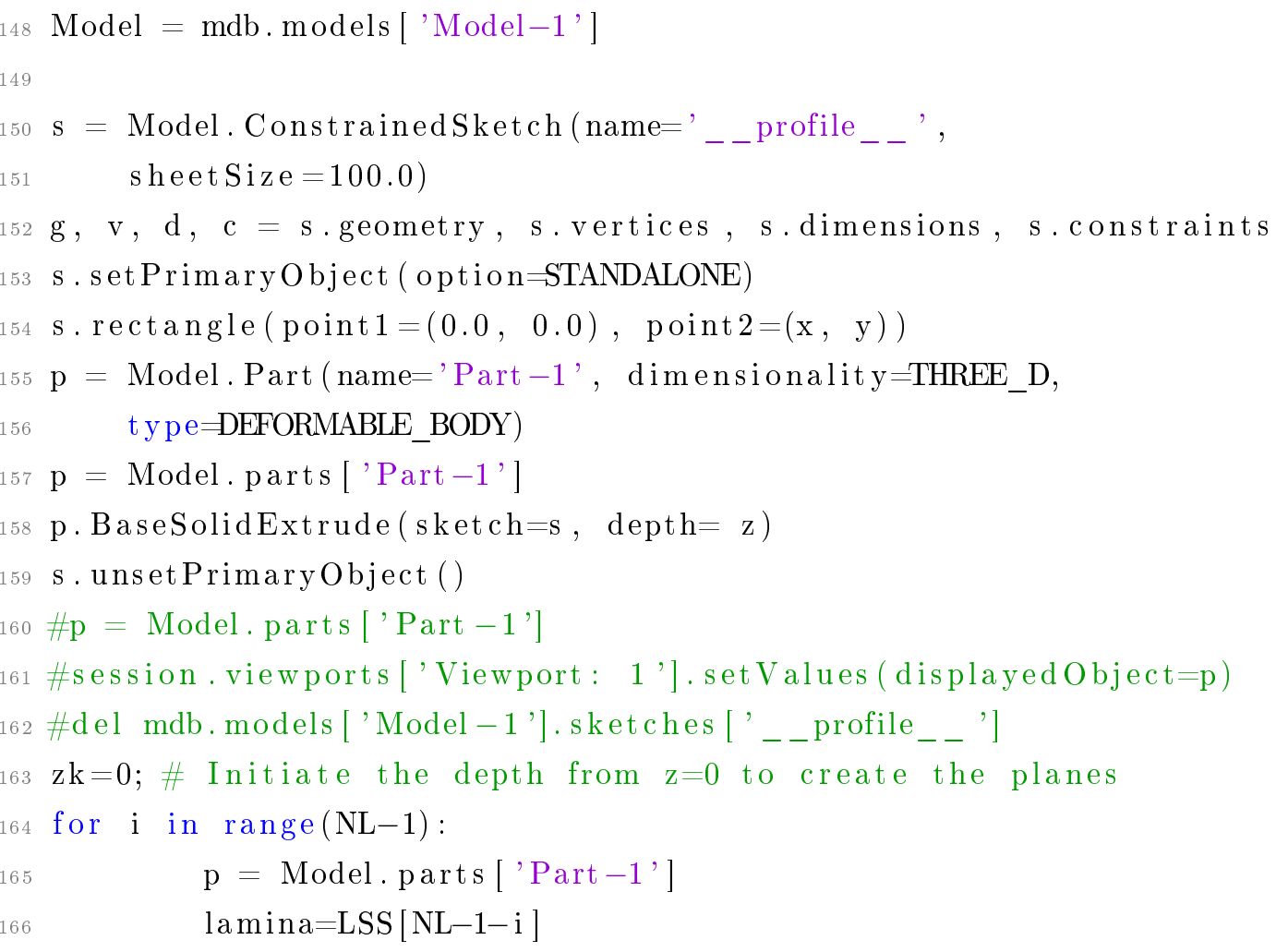


182

183 \# CREATE MATRIX TEMPERATURE DEPENDENT PROPERTIES FOR 'MATERIAL-1'

184 \# We assign the names to obtain E1, E2, G12, Nu12, Nu23 or alpha values given the parameters

185 alpha $1=$ ParameterIntegrator (alpha1a * 1e -6, alpha1b * 1e -6, alpha1c * 1e -6 , alpha1d * 1e-6, Trmaxt, Trmint, Tref) \# in [1/C] units

186 alpha $2=$ ParameterIntegrator (alpha $2 \mathrm{a} * 1 \mathrm{e}-6$, alpha2b * 1e -6, alpha $2 \mathrm{c} * 1 \mathrm{e}$ -6, alpha2d*1e-6, Trmaxt, Trmint, Tref) \# in [1/C] units

187 calcE1 = ParameterIntegrator (E1a, E1b, E1c, E1d, Trmaxe, Trmine, Tref) \# in [MPa] units

188 calcE $2=$ ParameterIntegrator (E2a, E2b, E2c, E2d, Trmaxe, Trmine, Tref) \# in $[\mathrm{MPa}]$ units

189 calcG12 = ParameterIntegrator(G12a, G12b, G12c, G12d, Trmaxe, Trmine, Tref) $\#$ in $[\mathrm{MPa}]$ units

190 calcNu12 = ParameterIntegrator (Nu12a, Nu12b, Nu12c, Nu12d, Trmaxe, Trmine, Tref) \# in $[\mathrm{MPa}]$ units

191 calcNu23 = ParameterIntegrator (Nu23a, Nu23b, Nu23c, Nu23d, Trmaxe, Trmine, Tref) \# in $[\mathrm{MPa}]$ units

192

193 \# To get Excel with properties go to Excelproperties.py

194 \# Tables with Elastic and Thermal properties are created

195 \# The input alpha matrix is 'tangent' ot 'secant' as CTE = 'name'

196 Elastic $=()$

197 for $\mathrm{i}$ in range(Trmaxe, Trmine $-1,-1)$ :

$198 \quad \mathrm{~T}=\mathrm{i}$

$199 \quad \mathrm{E} 1=\operatorname{calcE} 1 . \mathrm{Eval}(\mathrm{T})$ 


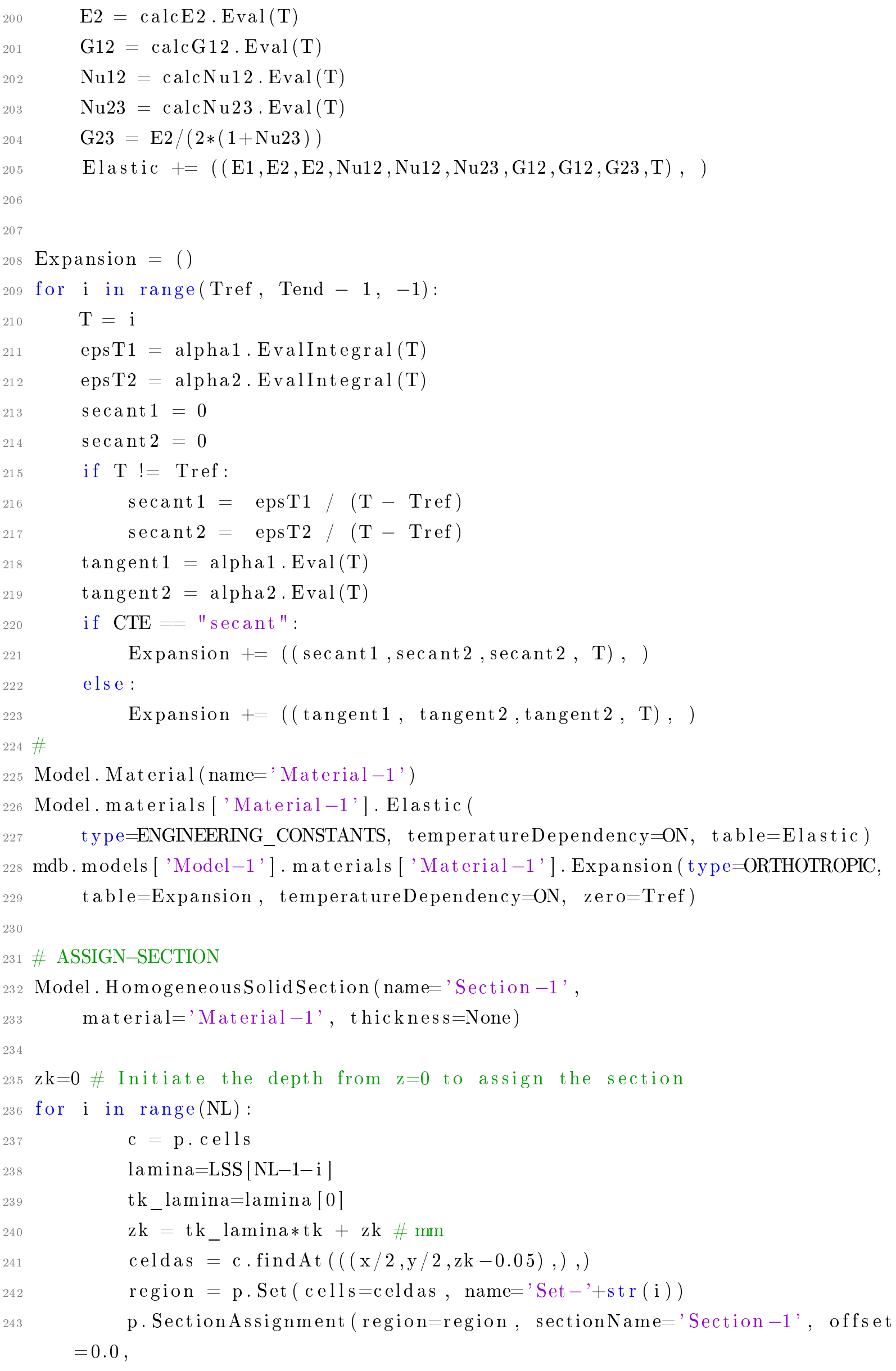






mdb. models ['Model-1'].parts ['Part -1 ']. MaterialOrientation( region= region ,

$268 \mathrm{a}=\operatorname{mdb}$. models ['Model-1']. root Assembly

269 a. Datum CsysByDefault (CARTESIAN)

270 MyInstance $=\mathrm{a}$. Instance $($ name $=$ 'Part $-1-1$ ', part $=\mathrm{p}, \quad$ dependent $=\mathrm{OFF})$

271

$272 \#$

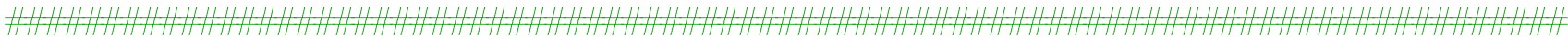

273 \#CREATE STEP AND FIELD OUTPUT

$274 \#$

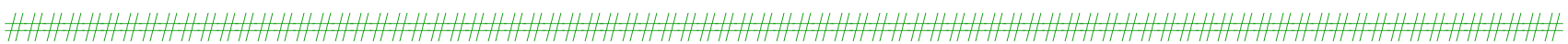

275

276 mdb. models ['Model-1']. StaticStep (name='Step-1', previous='Initial ', 
timePeriod $=333.0, \operatorname{maxNumInc}=1000, \quad$ in itial In $\mathrm{c}=1.0, \min \operatorname{Inc}=0.00333$, $\max \operatorname{Inc}=1.0)$

mdb.models ['Model-1']. fieldOutputRequests ['F-Output-1']. setValues (variables $=$ 'S ', 'E', 'PE', 'PEEQ', 'PEMAG', 'LE', 'U', 'RF', 'CF'))

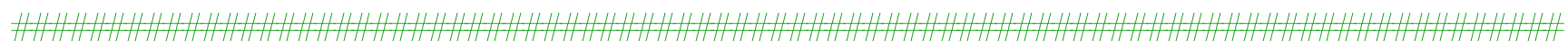

\section{A.7 3DFreeEdge-2}

$1 \#$

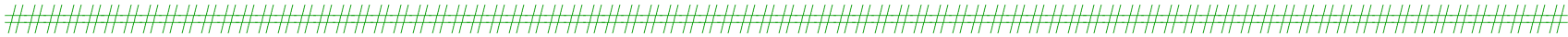

2 \#CREATE PERIODIC BOUNDARY CONDITIONS

$3 \#$

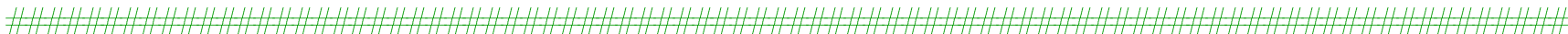

a. Set $($ nodes $=($ mdb.models ['Model-1'].rootAssembly.instances ['Part-1-1']. nodes, ), name= 'PerBound')

8 $($ CoorFixNode $)=$ PBC. PeriodicBound3D (mdb, 'Model-1' , 'PerBound ' , [round $(\mathrm{x}, 10)$, round $(\mathrm{y}, 10)$, round $(\mathrm{z}, 10)]$, NL, LSS , tk $)$

$10 \#$

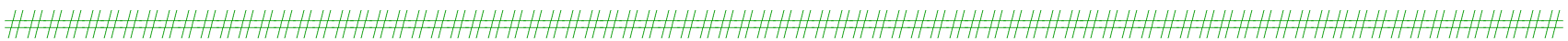

11 \# SYMMETRIC BOUNDARY CONDITIONS

$12 \#$

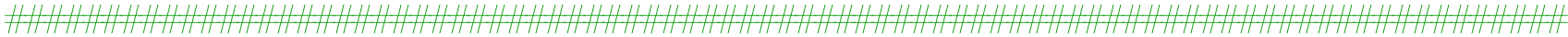

$13 \# \#$ Zsymmetry

faces $Z=[]$

$\mathrm{f}=$ MyInstance.faces

facesz $=\mathrm{f}$.findAt $(((\mathrm{x} / 2, \mathrm{y} / 2,0))$,

faces $Z$. append ( facesz )

facesz $=\mathrm{f}$.findAt $(((\mathrm{x}-1, \mathrm{y} / 2,0))$,

faces $Z$. append ( facesz)

facesz $=\mathrm{f}$.find At $(((\mathrm{x} / 2, \mathrm{y}-1,0))$,

faces Z . append ( facesz)

facesz $=\mathrm{f}$. find $\mathrm{At}(((\mathrm{x}-1, \mathrm{y}-1,0))$, 


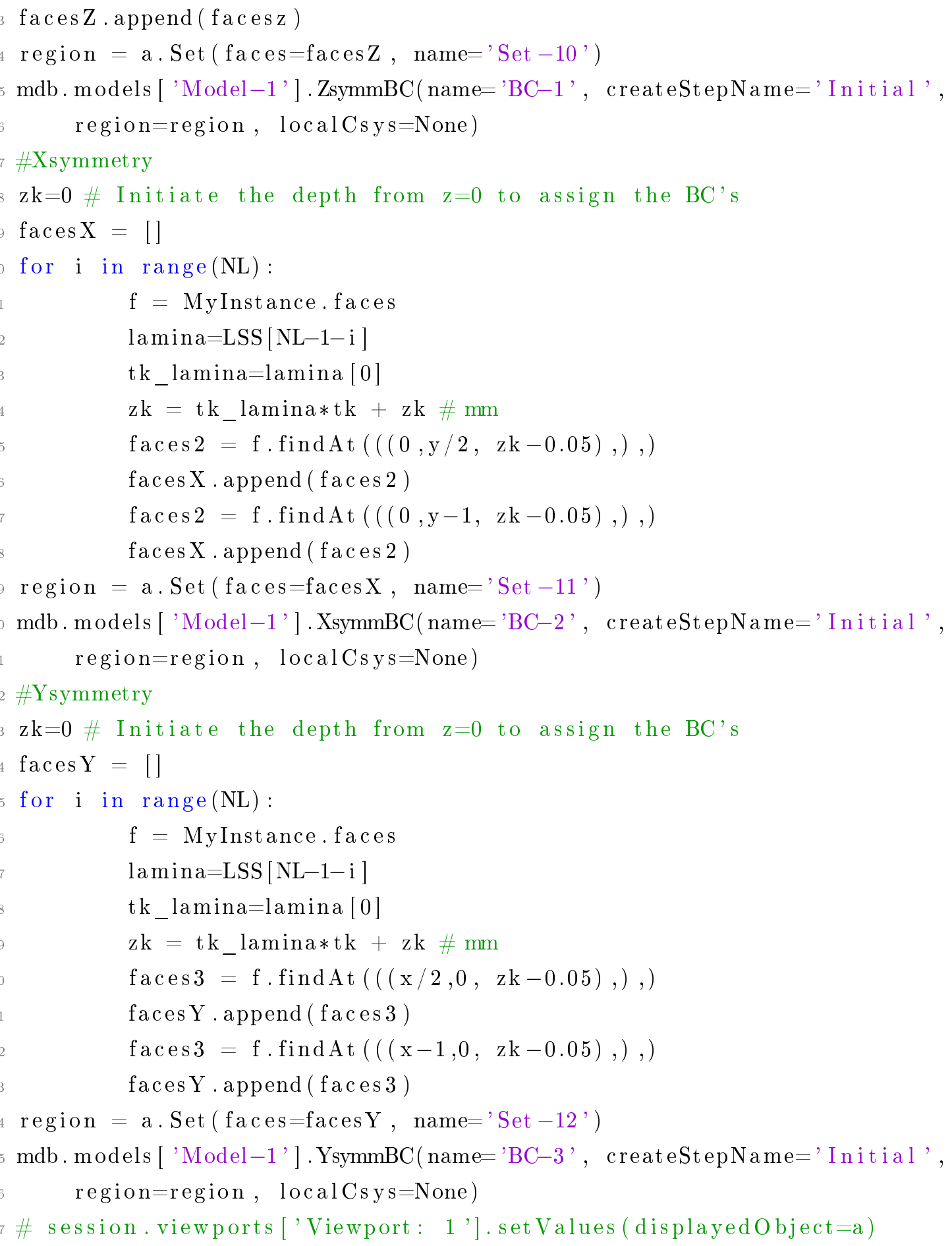


${ }_{64} \mathrm{f} 1=$ MyInstance.faces

6 e1 $=$ MyInstance.edges

$6 \mathrm{v} 1=$ MyInstance. vertices

region $=$ a.Set $($ vertices $=$ v1, edges $=$ e1, faces $=f 1$, cells $=\mathrm{c} 1$, name $=$ 'Set -4 ')

mdb. models ['Model-1']. Temperature (name='Predefined Field -1 ', createStepName='Initial', region=region, distributionType=UNIFORM, crossSectionDistribution=CONSTANT_THROUGH_THICKNESS, magnitudes=(Tref , ))

session . viewports ['Viewport: 1']. setValues (displayedObject=a)

\#Tend - PREDEFINED FIELD

region $=$ a.Set $($ vertices $=$ v1, edges $=$ e1, faces $=f 1$, cells $=$ c1, name $=$ 'Set -5 ')

mdb. models ['Model-1']. Temperature (name='Predefined Field-2', createStepName='Step -1 ', region=region, distribution Type=UNIFORM, crossSectionDistribution=CONSTANT_THROUGH_THICKNESS, magnitudes=(Tend , )$)$

80

$81 \#$

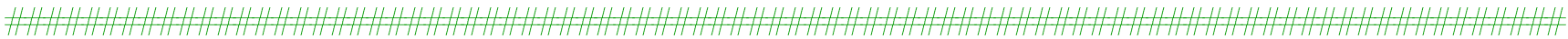

Job $=\operatorname{mdb.Job}($ name='Job -1 ', model='Model-1', description=' , , type=ANALYSIS, atTime $=$ None, waitMinutes $=0$, waitHours $=0$, queue $=$ None, memory $=95$, memoryUnits=PERCENTAGE, getMemoryFromAnalysis=True, explicitPrecision=SINGLE, nodalOutputPrecision=SINGLE, echoPrint=OFF, modelPrint=OFF, contactPrint=OFF, historyPrint=OFF, userSubroutine=' , scratch=' , , resultsFormat $=$ ODB, multiprocessingMode=DEFAULT, numCpus $=4$, numDomains $=4$, numGPUs $=0$ )

Job.submit ()

Job.waitForCompletion()

92

$93 \#$

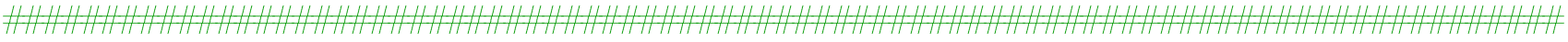

94 \# RESULTS

$95 \#$

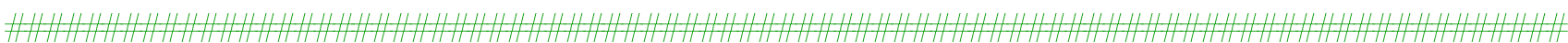

96

97 \# Postprocessor: Path lines to obtained free-edge data

98

99 from visualization import *

$100 \mathrm{odb}=\operatorname{openOdb}($ path $=$ 'Job -1. odb ' $)$; 


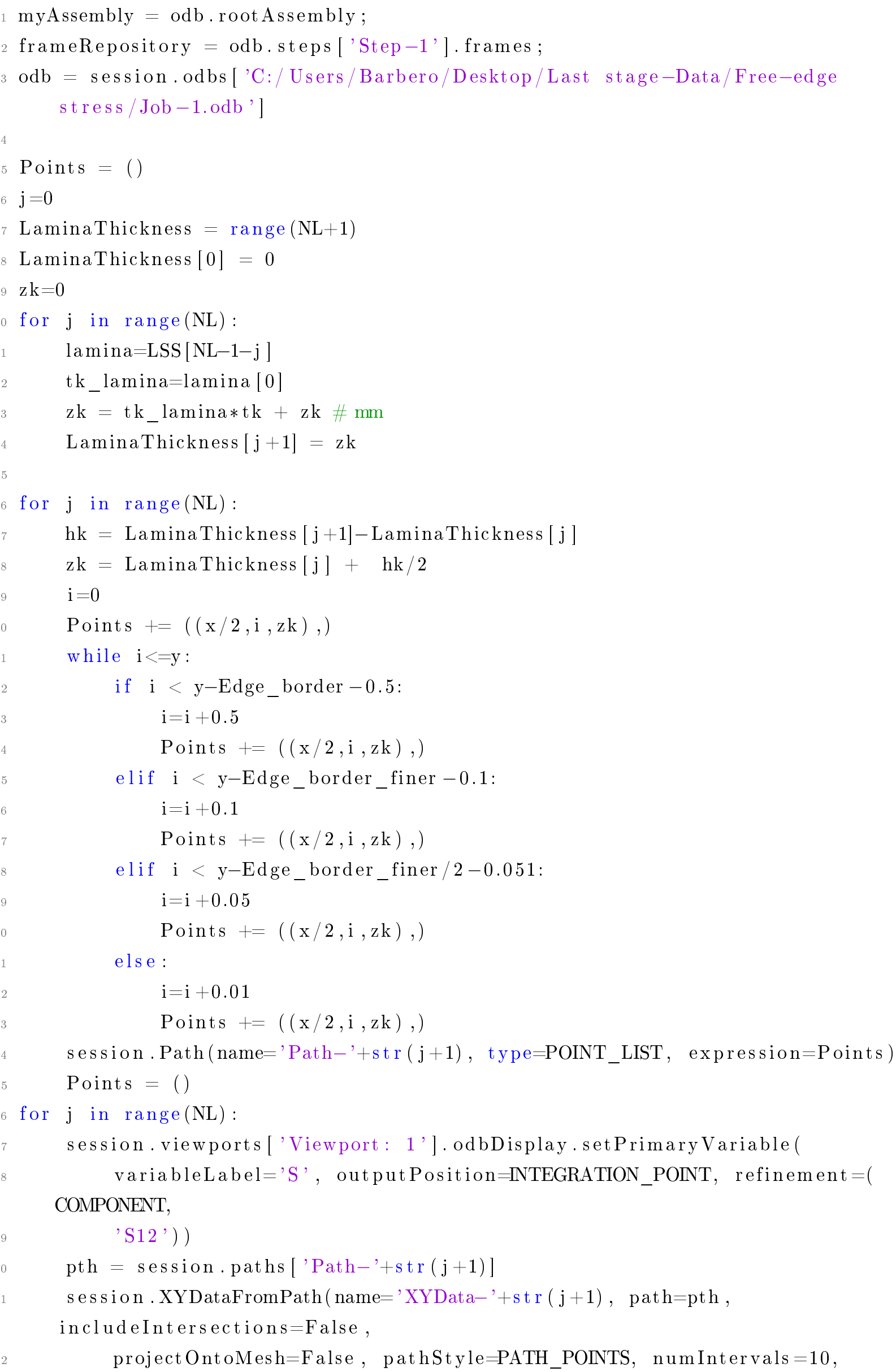


44 \#Other direction

${ }_{5}$ Points $=()$

$\mathrm{j}=0$

LaminaThickness $=$ range $(\mathrm{NL}+1)$

LaminaThickness $[0]=0$

$\mathrm{zk}=0$

for $j$ in range(NL):

lamina $=$ LSS [NL $-1-\mathrm{j}$ ]

tk_lamina=lamina $[0]$

$\mathrm{zk}=\mathrm{tk} \_$lamina $* \mathrm{tk}+\mathrm{zk} \# \mathrm{~mm}$

LaminaThickness $[\mathrm{j}+1]=\mathrm{zk}$

for $j$ in range(NL):

$\mathrm{hk}=$ LaminaThickness $[\mathrm{j}+1]-$ LaminaThickness $[\mathrm{j}]$

$\mathrm{zk}=$ LaminaThickness $[\mathrm{j}]+\mathrm{hk} / 2$

$\mathrm{i}=0$

Points $+=((\mathrm{i}, \mathrm{y} / 2, \mathrm{zk})$,

while $\mathrm{i}<=\mathrm{y}$ :

if $\mathrm{i}<\mathrm{y}$-Edge_border -0.5 :

$\mathrm{i}=\mathrm{i}+0.5$

Points $+=((\mathrm{i}, \mathrm{y} / 2, \mathrm{zk})$,

elif $\mathrm{i}<\mathrm{y}$-Edge_border_finer-0.1:

$\mathrm{i}=\mathrm{i}+0.1$

Points $+=((\mathrm{i}, \mathrm{y} / 2, \mathrm{zk})$,

elif $\mathrm{i}<\mathrm{y}$-Edge_border_finer $/ 2-0.051$ :

$\mathrm{i}=\mathrm{i}+0.05$

Points $+=((\mathrm{i}, \mathrm{y} / 2, \mathrm{zk})$,

else :

$\mathrm{i}=\mathrm{i}+0.01$

Points $+=((\mathrm{i}, \mathrm{y} / 2, \mathrm{zk})$,

session.Path $\left(\right.$ name $=$ 'Path $-{ }^{\prime}+\operatorname{str}(j+1)$, type=POINT_LIST, expression=Points $)$

Points $=()$

for $j$ in range (NL) :

session . viewports ['Viewport: 1'] . odbDisplay . setPrimaryVariable (

variableLabel='S', outputPosition=INTEGRATION_POINT, refinement=(

COMPONENT,

'S12') )

pth $=$ session $\cdot$ paths ['Path-'+str $(j+1)]$

session. XYDataFromPath (name $=$ 'XYData-' + str $(j+1)$, path $=$ pth,

includeIntersections $=$ False ,

projectOntoMesh=False, pathStyle=PATH_POINTS, numIntervals $=10$,

projection Tolerance $=0$, shape=UNDEFORMED, la belType=TRUE_DISTANCE) 


\section{A.8 PBC-FreeEdge}

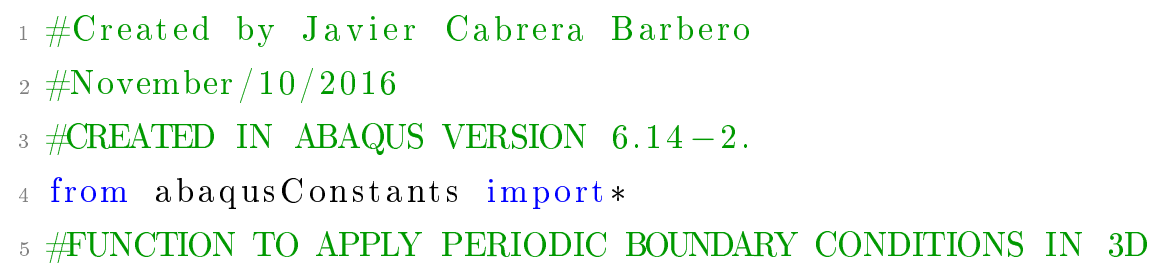




\section{$\mathrm{a}=$ nodes AllCoor $[\mathrm{n}]$}

mdb. models ['Model-1'] $\operatorname{root}$ Assembly . Set (name $=$ 'Node-' $+\operatorname{str}(\mathrm{n})$, nodes= mdb. models ['Model-1'].rootAssembly.sets ['PerBound'] . nodes [n:n+1]) repConst=repConst +1 \#Increase integer for naming equation constraint

\# We create set-Nodes $\#$

$$
\text { ZkThickness }=\text { range }(\mathrm{NL}+1)
$$$$
\text { ZkThickness }[0]=0
$$

$\mathrm{zk}=0$

for $j$ in range (NL) :

lamina $=$ LSS $[\mathrm{NL}-1-\mathrm{j}]$

tk_lamina=lamina $[0]$

$\mathrm{zk}=\mathrm{tk} \_$lamina $* \mathrm{tk}+\mathrm{zk} \# \mathrm{~mm}$

ZkThickness $[\mathrm{j}+1]=\mathrm{zk}$

for $\mathrm{j}$ in range $(\mathrm{NL}+1)$ :

amodel $=$ mdb. models [ 'Model-1'] . rootAssembly

n1=amodel . instances ['Part $-1-1$ '] . nodes

delta $=1.0 \mathrm{e}-4$

xmin, ymin, $z$ min $=$ LatticeVec[0]-delta, LatticeVec[1]-delta,

ZkThickness $[\mathrm{j}]-$ delta

xmax, $y \max , \quad z \max =$ LatticeVec $[0]+$ delta, LatticeVec $[1]+$ delta,

ZkThickness $[j]+$ delta

node $=n 1$. getByBoundingBox (xmin, ymin, zmin, $x \max , y \max , z \max )$

amodel. Set ( nodes=node, name='ReferenceNode $\left.-{ }^{\prime}+\operatorname{str}(j)\right)$

for $n$ in range $(0$, len $($ nodes All $))$ :

$\mathrm{a}=$ nodesAllCoor $[\mathrm{n}]$

if $\operatorname{abs}(\mathrm{a}[2]-$ ZkThickness[j] $)<$ delta and abs(a[1] - LatticeVec

$[1])<$ delta and abs(a[0] - LatticeVec[0]) < delta:

NoEquation $=\mathrm{n}$

for $n$ in range $(0$, len $($ nodes All $))$ :

$\mathrm{a}=$ nodes AllCoor $[\mathrm{n}]$

if $(\operatorname{abs}(\mathrm{a}[2]-$ ZkThickness $[\mathrm{j}])<$ delta and $\mathrm{n} !=$ NoEquation and $\mathrm{j}$ $!=0)$ :

mdb. models [ 'Model-1'] . Equation (name='Lamina-' + str $(j)+$ '

ConstZ-' + str $(3)+{ }^{\prime}-'+\operatorname{str}($ rep Const 1$)$, terms $=\left(\left(1.0\right.\right.$, ' Node $\left.-^{-}+\operatorname{str}(\mathrm{n}), 3\right),(-1.0$, ' ReferenceNode - '

$+\operatorname{str}(\mathrm{j}), 3)))$

repConst $1=$ repConst $1+1 \quad$ \#Increase integer for naming

equation constraint

if (abs(a[2] - ZkThickness[j])< delta and abs(a[1] -

LatticeVec[1]) < delta and $n !=$ NoEquation $)$ :

mdb. models [ 'Model-1']. Equation (name='Lamina-' $+\operatorname{str}(j)+$ '

ConstY-'+str (2)+'- '+str (repConst 1$)$, 
terms $=\left(\left(1.0\right.\right.$, 'Node $\left.-^{-}+\operatorname{str}(\mathrm{n}), 2\right),(-1.0$, 'ReferenceNode - ' $+\operatorname{str}(j), 2)))$

repConst $1=$ repConst $1+1 \quad$ \#Increase integer for naming

equation constraint if $(\operatorname{abs}(\mathrm{a}[2]-$ ZkThickness $[\mathrm{j}])<$ delta and abs $(\mathrm{a}[0]-$ LatticeVec $[0])<$ delta and $n !=$ NoEquation $):$ mdb. models ['Model-1']. Equation (name='Lamina-' + str $(j)+$ ' ConstX-' +str $(1)+{ }^{\prime}-'+\operatorname{str}(\operatorname{rep} \operatorname{Const} 1)$, terms $=\left(\left(1.0\right.\right.$, ' Node $\left.-^{-}+\operatorname{str}(\mathrm{n}), 1\right),(-1.0$, 'ReferenceNode - ' $+\operatorname{str}(j), 1)))$

repConst $1=$ repConst $1+1$ \#Increase integer for naming

equation constraint

for $\mathrm{i}$ in range(NL):

mdb. models [ 'Model-1']. Equation (name='ReferenceNodesConst-X'+'-zk'+ $\operatorname{str}(j)+{ }^{\prime}-'+\operatorname{str}($ repConst 1$)$, terms $=\left(\left(1.0,{ }^{\prime}\right.\right.$ ReferenceNode $\left.-{ }^{\prime}+\operatorname{str}(\mathrm{i}), 1\right),(-1.0$, 'ReferenceNode$'+\operatorname{str}(\mathrm{NL}), 1)))$

repConst $1=$ repConst $1+1 \quad$ \#Increase integer for naming equation constraint

mdb. models [ 'Model-1']. Equation (name='ReferenceNodesConst-Y'+'-zk'+ $\operatorname{str}(j)+{ }^{\prime}-'+\operatorname{str}($ repConst 1$)$, terms $=\left(\left(1.0,{ }^{\prime}\right.\right.$ ReferenceNode $\left.-{ }^{\prime}+\operatorname{str}(\mathrm{i}), 2\right),(-1.0$, ' ReferenceNode $'+\operatorname{str}(\mathrm{NL}), 2)))$ repConst1=repConst $1+1 \quad$ \#Increase integer for naming equation constraint

return (nodesAll[ranNodes [0]]. coordinates) 


\section{Bibliography}

[1] S. Tompkins. Effects of thermal cycling on composite materials for space structures. 2(19890014181):447-470, May 011989.

[2] E. J. Barbero. Finite element analysis of composite materials using Abaqus. CRC Press, 2013.

[3] G.C. Grimes. In composite materials: testing and design. Number 617, pages 106-119, Philadelphia, 1977. A.S.T.M, American society for testing and materials.

[4] J.T. Ryder and E.K. Walker. Infatigue of filamentary materials. Number 636. A.S.T.M. S.T.P., American Society for testing and materials, 1977.

[5] V. Carvelli and V. Lomov. Fatigue of textile composites. Elsevier, 2015.

[6] A.P. Vassilopoulos. Fatigue life prediction of composites and composite structures. Elsevier, 1rd edition, 2010.

[7] R. Schaff and D. Davidson. Life prediction methodology for composite structures. Part I-constant amplitude and two-stress level fatigue. Journal of composite materials, 31(2):128-157, 1997.

[8] S. Adams, E. Bowles, and T. Herakovich. Thermally induced transverse cracking in graphite-epoxy cross-ply laminates. Journal of reinforced Plastics and composites, $5(3): 152-169,1986$.

[9] S. Kobayashi, K. Terada, and N. Takeda. Evaluation of long-term durability in high temperature resistant cfrp laminates under thermal fatigue loading. Composites Part B: Engineering, 34(8):753-759, 2003.

[10] T. L. Brown. The effect of long-term thermal cycling on the microcracking behavior and dimensional stability of composite materials. PhD thesis, Virginia Polytechnic Institute and State University, 1997. 
[11] M.C. Lafarie-Frenot and N.Q. Ho. Influence of free edge intralaminar stresses on damage process in cfrp laminates under thermal cycling conditions. Composites science and technology, 66(10):1354-1365, 2006.

[12] E.J. Barbero and J. Cabrera Barbero. Damage initiation and evolution during monotonic cooling of laminated composites. Journal of Composite Materials, 2018.

[13] S.S. Tompkins. Thermal expansion of selected graphite-reinforced polyimide-, epoxy-, and glass-matrix composites. Journal of Thermophysical Properties and Thermophysics and its Applications., 8:119-132, 1987.

[14] B.J. Knouff, S.S. Tompkins, and N. Jayaraman. The effect of graphite fiber properties on microcracking due to thermal cycling of epoxy-cyanate matrix laminates. Composite Materials: Fatigue and Fracture, ASTM STP 1230, 5:268-282, 1994.

[15] S. Tompkins and G. Funk. Effects of changes in composite lamina properties on laminate coefficient of thermal expansion. International SAMPE Electronics Conference, 24:867 - 878, 1992.

[16] C.H. Park and H.L. McManus. Thermally induced damage in composite laminates: predictive methodology and experimental investigation. Composites Science and Technology, 56(10):1209-1219, 1996.

[17] S.P Rawal, M.S. Misra, and R.G. Wendt. Composite materials for space applications. Technical Report N96-187472, NASA, 1990.

[18] R. Maddocks. Microcracking in composite laminates under thermal and mechanical loading. Technical Report N96-16101, NASA, 1995.

[19] MIL17. Composite Materials Handbook. Department of Defense, Vol. 2 of 5, 2002.

[20] D.S. Adams, D.E. Bowles, and C.T. Herakovich. Characteristics of thermallyinduced transverse cracks in graphite epoxy composite laminates. Technical Report NASA-TM-85429, NASA, 1983.

[21] S. Tompkins and S.L. Williams. Effects of thermal cycling on mechanical properties of graphite polyimide. Journal of Spacecraft and Rockets, 21(3):274-280, 1984.

[22] S. Kobayashi and N. Takeda. Experimental and analytical characterization of transverse cracking behavior in carbon/bismaleimide cross-ply laminates under mechanical fatigue loading. Composites part B: Engineering, 33(6):471-478, 2002. 
[23] M.C. Lafarie-Frenot, C. Henaff-Gardin, and D. Gamby. Matrix cracking induced by cyclic ply stresses in composite laminates. Composites science and technology, 61(15):2327-2336, 2001.

[24] M.C. Lafarie-Frenot. Damage mechanisms induced by cyclic ply-stresses in carbon-epoxy laminates: Environmental effects. International Journal of Fatigue, 28(10):1202-1216, 2006.

[25] P. Ladeveze and G. Lubineau. An enhanced mesomodel for laminates based on micromechanics. Composites Science and Technology, 62(4):533-541, 2002.

[26] J.M. Augl. Prediction and verification of moisture effects on carbon fiber-epoxy composites. Technical report, DTIC Document: ADA034787, 1979.

[27] M. Hajikazemi, M.H. Sadr, and J. Varna. Analysis of cracked general cross-ply laminates under general bending loads: A variational approach. Journal of Composite Materials, 51(22):3089-3109, 2017.

[28] P. Ladevèze, O. Allix, J.F. Deü, and D. Lévêque. A mesomodel for localisation and damage computation in laminates. Computer Methods in Applied Mechanics and Engineering, 183(1-2):105-122, 2000.

[29] C. Henaff-Gardin, M.C. Lafarie-Frenot, and D. Gamby. Doubly periodic matrix cracking in composite laminates part 2: Thermal biaxial loading. Composite Structures, 36(1-2):131-140, 1996.

[30] R. W. Dalgarno, M. R. Garnich, and E. W. Andrews. Thermal fatigue cracking of an im7/5250-4 cross ply laminate: Experimental and analytical observations. Journal of composite materials, 43(23):2699-2715, 2009.

[31] E. J. Barbero and D. H. Cortes. A mechanistic model for transverse damage initiation, evolution, and stiffness reduction in laminated composites. Composites Part $B, 41: 124-132,2010$.

[32] E. J. Barbero, F. A. Cosso, and X. Martinez. Identification of fracture toughness for discrete damage mechanics analysis of glass-epoxy laminates. Applied Composite Materials, November:1-18, 2013.

[33] E. J. Barbero and F. A. Cosso. Benchmark solution for degradation of elastic properties due to transverse matrix cracking in laminated composites. Composite Structures, 98:242-252, 2013. 
[34] E. J. Barbero and F. A. Cosso. Determination of material parameters for discrete damage mechanics analysis of carbon-epoxy laminates. Composites Part B, 56:638$646,2014$.

[35] Ever J. Barbero. Introduction to Composite Materials Design. CRC Press, 3rd edition, 2018.

[36] R. Talreja. Fatigue of composite materials. Technomic, 1rd edition, 1987.

[37] R. Talreja. Damage mechanics and fatigue life assessment of composite materials. International Journal of Damage Mechanics, 8, 339-354.

[38] R. Talreja and C. V Singh. Damage and failure of composite materials. Cambridge University Press, 2012.

[39] C.K. Dharan. Fatigue of composite materials. American society for testing and materials, 568:171-188, 1977.

[40] Z. Hashin and A.J. Rotem. Journal of Composite Materials, 7:448-464, 1973.

[41] H.T. Hahn and R.Y. Kim. Journal composite materials, 10:156-180, 1976.

[42] L.E. Asp, L.A. Berglund, and R. Talreja. A criterion for crack initiation in glassy polymers subjected to a composite-like stress state. Composites Science and Technology, 56:1291-1301, 1996.

[43] L.E. Asp, L.A. Berglund, and R. Talreja. Prediction of matrix-initiated transverse failure in polymer composites. Composites Science and Technology, 56:1089-1097, 1996.

[44] L.E. Asp, L.A. Berglund, and R. Talreja. Effects of fiber and interphase on matrix initiated transverse failure in polymer composites. Composites Science and Technology, 56:657-665, 1996.

[45] E.K. Gamstedt and B.A. Sjögren. Micromechanisms in tension-compression fatigue of composite laminates containing transverse plies. Composites Science and Technology, 59(2):167-178, 1999.

[46] J. Awerbuch and H.T. Hahn. In fatigue of filamentary composite materials. 636:248$266,1977$.

[47] K. L. Reifsnider. Fatigue of composite materials, volume 4. Elsevier, 1991. 
[48] J. Varna, R. Joffe, and R. Talreja. A synergistic damage mechanics analysis of transverse cracking in $\left[ \pm \theta / 90_{4}\right]_{s}$ laminates. Composites Science and Technology, 61(5):657-665, 2001.

[49] A. M. Abad Blazquez, M. Herraez Matesanz, C. Navarro Ugena, , and E. J. Barbero. Acoustic emission characterization of intralaminar damage in composite laminates. In MATCOMP XIII, pages 33-38, 2013.

[50] T. K. O'Brien. Characterization of delamination onset and growth in a composite laminate. In Damage in Composite Materials: Basic Mechanisms, Accumulation, Tolerance, and Characterization. ASTM International, 1982.

[51] A.L. Highsmith and K. L. Reifsnider. Internal load distribution effects during fatigue loading of composite laminates. In Composite Materials: Fatigue and Fracture. ASTM International, 1986.

[52] C.V. Singh and R. Talreja. Evolution of ply cracks in multidirectional composite laminates. International Journal of Solids and Structures, 47(10):1338-1349, 2010.

[53] J.N. Yang. Fatigue and residual strength degradation for graphite/epoxy composites under tension-compression cyclic loadings. Journal of Composite Materials, 12(1):19-39, 1978.

[54] G.P. Sendeckyj. Life prediction for resin-matrix composite materials. Journal of Composite materials, 4:431-483, 1991.

[55] D.G. Harlow and S.L. Phoenix. Bounds on the probability of failure of composite materials. International Journal of Fracture, 15(4):321-336, 1979.

[56] R.G. Budynas, J.K. Nisbett, et al. Shigley's mechanical engineering design. McGraw-Hill New York, 8rd edition, 2008.

[57] J.F. Mandell, F.J. McGarry, A.J. Hsieh, and C.G. Li. Tensile fatigue of glass fibers and composites with conventional and surface compressed fibers. Polymer Composites, 6(3):168-174, 1985.

[58] ASTM Committee et al. Standard practices for statistical analysis of linear or linearized stress-life $(\mathrm{sn})$ and strain-life $(\varepsilon-\mathrm{n})$ fatigue data. designation: E739-91 (reapproved 2004). ASTM International, West Conshohocken, PA, USA, 2004.

[59] I.M. Daniel, J.M. Whitney, and R.B. Pipes. Experimental mechanics of fiber reinforced composite materials. Experimental Techniques, 7(3):25-25, 1983. 
[60] G.P. Sendeckyj. Fitting models to composite materials fatigue data. In Test methods and design allowables for fibrous composites. ASTM International, 1981.

[61] S.V. Ramani and D.P. Williams. Notched and unnotched fatigue behavior of angleply graphite/epoxy composites. In Fatigue of filamentary composite materials. ASTM International, 1977.

[62] B. Harris. Fatigue behaviour of polymer-based composites and life prediction methods. Durability Analysis of Structural Composite Systems, AH Cardon (Ed), Balkema, Rotterdam, pages 49-84, 1996.

[63] M. Kawai. A phenomenological model for off-axis fatigue behavior of unidirectional polymer matrix composites under different stress ratios. Composites Part A: applied science and manufacturing, 35(7-8):955-963, 2004.

[64] M.P. Ansell, I.P. Bond, and P.W. Bonfield. Constant life diagrams for wood composites and polymer matrix composites. ICCM/9. Composites Behaviour., 5:692-699, 1993.

[65] A.P. Vassilopoulos, B.D. Manshadi, and T. Keller. Piecewise non-linear constant life diagram formulation for frp composite materials. International journal of fatigue, 32(10):1731-1738, 2010.

[66] V.A. Passipoularidis and T.P. Philippidis. A study of factors affecting life prediction of composites under spectrum loading. International Journal of Fatigue, 31(3):408417, 2009.

[67] M. Kawai and M. Koizumi. Nonlinear constant fatigue life diagrams for carbon/epoxy laminates at room temperature. Composites Part A: Applied Science and Manufacturing, 38(11):2342-2353, 2007.

[68] J.B. Sturgeon. Fatigue and creep testing of unidirectional CFRP. in proceedings of the 28th annual technical conference of the society of the plastics industry. pages 12-13, 1973.

[69] M.J. Owen. Fracture and fatigue. Composite Materials, 5:313-340, 1974.

[70] A. Rotem and Z. Hashin. Fatigue Failure of angle ply laminates. AIAA. 14:868-872, 1976.

[71] M.J. Owen. Static and fatigue strength of glass chopped strand mat/polyester resin laminates. (772):64-84, 1982. 
[72] L. Broutman and S. Sahu. Proceedings of the 24th conference of the society of the plastic industry. pages $1-12,1969$.

[73] Ever J. Barbero. Prediction of compression strength of unidirectional polymer matrix composites. Journal of composite Materials, 32(5):483-502, 1998.

[74] B. Budiansky. Micromechanics. Computers and Structures, 16(1):3-12, 1983.

[75] P.M. Jelf and N.A. Fleck. Compression failure mechanisms in unidirectional composites. Journal of Composite Materials, 26:2706-2726, 1992.

[76] P.M. Moran, X.H. Liu, and C.F. Shih. Kink band formation and band broadening in fiber composites under compression loading. Acta Metallurgica et Materialia, 43:2943-2958, 1995.

[77] T.J. Vogler, S.Y. Hsu, and S Kyriakides. On the initiation and growth of kink bands in fiber composites . part ii: analysis. International Journal of Solids and Structures, 38:2653-2682., 2001.

[78] Y.X. Zhou and P.K. Mallick. Fatigue strength characterization of e-glass fibers using fiber bundle test. Journal of Composite Materials, 38(22):2025-2035, 2004.

[79] S.L. Phoenix. Stochastic strength and fatigue of fiber bundle. International Journal of Fracture, 14(3):327-344, 1978.

[80] O. Konur and F.L. Matthews. Effect of the properties of the constituents on the fatigue performance of composites: a review. Composites, 20(4):317-328, 1989.

[81] A.R. Bunsell, J.W. Hearle, and R.D. Hunter. Apparatus for fatigue-testing of fibres. Journal of Physics E-Scientific Instruments, 4(11):868-872, 1971.

[82] J.W. Hearle. Fatigue in fibres and plastics (a review). Journal of Materials Science, 2(5):474-488, 1967.

[83] G.M. Cai, X.G. Wang, X.J. Shi, and W.D. Yu. Evaluation of fatigue properties of high-performance fibers based on fixed-point bending fatigue test. Journal of Composite Materials, 46(7):833-840, 2012.

[84] C. Qian, R.P. Nijssen, D.D. Samborsky, C. Kassapoglou, Z. GâĆňurdal, and G.Q Zhang. Tensile fatigue behavior of single fibres and fibre bundles. In In European conference of composite materials, Budapest, Hungary, 2010. 
[85] I. Severin, R. El Abdi, M. Poulain, and G. Amza. Fatigue testing procedures of silica optical fibres. Journal of Optoelectronics and Advanced Materials, 7(3):1581-1587, 2005.

[86] J.M. Herrera Ramirez, P. Colomban, and A. Bunsell. Micro-raman study of the fatigue fracture and tensile behaviour of polyamide (pa 66) fibres. Journal of Raman Spectroscopy, 35(12):1063-1072, 2004.

[87] Y. Arao, N. Taniguchi, T. Nishiwaki, N. Hirayama, and H. Kawada. Strain-rate dependence of the tensile strength of glass fibers. Journal of Materials Science, 47(12):4895-4903, 2012.

[88] S. Feih, K. Manatpon, Z. Mathys, A.G. Gibson, and A.P. Mouritz. Strength degradation of glass fibers at high temperatures. Journal of Materials Science, 44(2):392400, 2009.

[89] M.J. Matthewson and C.R. Kurkjian. Environmental effects on the static fatigue of silica optical fiber. Journal of the American Ceramic Society, 71(3):177-183, 1988.

[90] A.R. Bunsell and A. Somer. The tensile and fatigue behavior of carbon-fibers. Plastics Rubber and Composites Processing and Applications, 18(4):263-267, 1992.

[91] Y.X. Zhou, M.A. Baseer, H. Mahfuz, and S. Jeelani. Statistical analysis on the fatigue strength distribution of t700 carbon fiber. Composites Science and Technology, 66(13):2100-2106, 2006.

[92] Y. Zhou and L. Nicolais. Fatigue strength distribution of carbon fiber. John Wiley and Sons, I., 2011.

[93] M. Quaresimin and L. Susmel. Multiaxial fatigue behaviour of composite laminates. In Key Engineering Materials, volume 221, pages 71-80. Trans Tech Publ, 2002.

[94] M. Quaresimin, L. Susmel, and R. Talreja. Fatigue behaviour and life assessment of composite laminates under multiaxial loadings. International Journal of Fatigue, 32(1):2-16, 2010.

[95] M.J. Owen and J.R. Griffiths. Evaluation of biaxial stress failure surfaces for a glass fabric reinforced polyester resin under static and fatigue loading. Journal of Materials Science, 13(7):1521-1537, 1978.

[96] N. Himmel. Fatigue life prediction of laminated polymer matrix composites. International journal of fatigue, 24(2-4):349-360, 2002. 
[97] D.F. Sims and V.H. Brogdon. Fatigue behavior of composites under different loading modes. In Fatigue of filamentary composite materials. ASTM International, 1977.

[98] M. Kawai, S. Yajima, A. Hachinohe, and Y. Takano. Off-axis fatigue behavior of unidirectional carbon fiber-reinforced composites at room and high temperatures. Journal of Composite Materials, 35(7):545-576, 2001.

[99] T.P Philippidis and A.P. Vassilopoulos. Complex stress state effect on fatigue life of grp laminates.: part i, experimental. International journal of fatigue, 24(8):813$823,2002$.

[100] E.J. Barbero and M. Shahbazi. Determination of critical energy release rates for discrete damage mechanics analysis in ANSYS. Theoretical and Applied Fracture Mechanics, 92:99 - 112, 2017.

[101] M. Shahbazi and E.J. Barbero. Determination of material properties for discrete damage mechanics analysis in ANSYS. In CAMX, The Composites and Advanced Materials Expo, Anaheim, CA, 2016.

[102] S. Li, S. R. Reid, and P. D. Soden. A continuum damage model for transverse matrix cracking in laminate fiber-reinforced composites. Philosophical Transaction of the Royal Society of London. A, 356:2379-2412, 1998.

[103] K.L. Reifsnider and Z. Gao. A micromechanics model for composites under fatigue loading. International Journal of Fatigue, 13(2):149-156, 1991.

[104] M-HR. Jen and C-H. Lee. Strength and life in thermoplastic composite laminates under static and fatigue loads. Part I- experimental. International journal of fatigue, 20(9):605-615, 1998.

[105] T.P. Philippidis and A.P. Vassilopoulos. Fatigue strength prediction under multiaxial stress. Journal of Composite Materials, 33(17):1578-1599, 1999.

[106] C.M. Lawrence Wu. Thermal and mechanical fatigue analysis of cfrp laminates. Composite Structures, 25(1-4):339-344, 1993.

[107] I.P. Bond. Fatigue life prediction for grp subjected to variable amplitude loading. Composites Part A: Applied Science and Manufacturing, 30(8):961-970, 1999.

[108] Z. Fawaz and F. Ellyin. Fatigue failure model for fibre-reinforced materials under general loading conditions. Journal of Composite Materials, 28(15):1432-1451, 1994. 
[109] F. Ellyin and H. El-Kadi. A fatigue failure criterion for fiber reinforced composite laminae. Composite Structures, 15(1):61-74, 1990.

[110] E.W. Smith and K.J. Pascoe. Biaxial fatigue of a glass-fibre reinforced composite. part iiâĂŤfailure criteria for fatigue and fracture. In ICBMFF2, 1989.

[111] R.S. Sandhu, R.L Gallo, and G.P. Sendeckyj. Initiation and accumulation of damage in composite laminates. In Composite Materials: Testing and Design (6th Conference). ASTM International, 1982.

[112] M.M. Shokrieh and L.B. Lessard. Multiaxial fatigue behaviour of unidirectional plies based on uniaxial fatigue experiments -I. modelling. International Journal of Fatigue, 19(3):201-207, 1997.

[113] M.M. Shokrieh and F. Taheri-Behrooz. A unified fatigue life model based on energy method. Composite Structures, 75(1-4):444-450, 2006.

[114] N. Gathercole, H. Reiter, T. Adam, and B. Harris. Life prediction for fatigue of t800/5245 carbon-fibre composites: I. constant-amplitude loading. International Journal of Fatigue, 16(8):523-532, 1994.

[115] R. D. Jamison, K. Schulte, K. L. Reifsnider, , and W. W Stinchcomb. Characterization and analysis of damage mechanisms in tension-tension fatigue of graphite/epoxy laminates. In Effects of defects in composite materials. American Society for Testing and Materials, 836:21-55, 1984.

[116] R.D. Jamison and K.L. Reifsnider. Advanced fatigue damage development in graphite epoxy laminates. Technical Report AFWAL-TR-82-3103, Virginia Polytechnich Institute and State University Blacksburg, 1982.

[117] W. Hwang and Kyung S. Han. Fatigue of compositesâĂ Ťfatigue modulus concept and life prediction. Journal of Composite Materials, 20(2):154-165, 1986.

[118] W. Van Paepegem, J. Degrieck, and P. De Baets. Finite element approach for modelling fatigue damage in fibre-reinforced composite materials. Composites Part B: Engineering, 32(7):575-588, 2001.

[119] F. Sidoroff and B. Subagio. Fatigue damage modelling of composite materials from bending tests. In Sixth International Conference on Composite Materials (ICCMVI) \& Second European Conference on Composite Materials (ECCM-II), volume 4, pages 4-32. Elsevier London, UK, 1987. 
[120] H. Mao and S. Mahadevan. Fatigue damage modelling of composite materials. Composite Structures, 58(4):405-410, 2002.

[121] H.A. Whitworth. A stiffness degradation model for composite laminates under fatigue loading. Composite structures, 40(2):95-101, 1997.

[122] A. Poursartip, M.F. Ashby, and P.W.R. Beaumont. The fatigue damage mechanics of a carbon fibre composite laminate: I -development of the model. Composites Science and Technology, 25(3):193-218, 1986.

[123] G.F. Abdelal, A. Caceres, and E.J. Barbero. A micro-mechanics damage approach for fatigue of composite materials. Composite Structures, 56(4):413-422, 2002.

[124] W. Van Paepegem and J. Degrieck. A new coupled approach of residual stiffness and strength for fatigue of fibre-reinforced composites. International Journal of Fatigue, 24(7):747-762, 2002.

[125] C. Henaff-Gardin and M.C. Lafarie-Frenot. The use of a characteristic damage variable in the study of transverse cracking development under fatigue loading in cross-ply laminates. International Journal of Fatigue, 24(2-4):389-395, 2002.

[126] P.C. Chou and R. Croman. Residual strength in fatigue based on the strength-life equal rank assumption. Journal of Composite materials, 12(2):177-194, 1978.

[127] P.C. Chou and Robert Croman. Degradation and sudden-death models of fatigue of graphite/epoxy composites. In Composite Materials: Testing and Design (Fifth Conference). ASTM International, 1979.

[128] I.M. Daniel and A. Charewicz. Fatigue damage mechanisms and residual properties of graphite/epoxy laminates. Engineering Fracture Mechanics, 25(5-6):793-808, 1986.

[129] W.X. Yao and N. Himmel. A new cumulative fatigue damage model for fibrereinforced plastics. Composites science and technology, 60(1):59-64, 2000.

[130] Xiaoxue Diao, Lin Ye, and Yiu-Wing Mai. Simulation of fatigue performance of cross-ply composite laminates. Applied Composite Materials, 3(6):391-406, 1996.

[131] T.K. O’Brien and K.L Reifsnider. Fatigue damage evaluation through stiffness measurements in boron-epoxy laminates. Journal of composite materials, 15(1):55$70,1981$. 
[132] D. H. Cortes and E. J. Barbero. Stiffness reduction and fracture evolution of oblique matrix cracks in composite laminates. Annals of Solid and Structural Mechanics, 1(1):29-40, 2010.

[133] A. Rotem. Fatigue failure mechanism of composite laminates. In Mechanics of Composite Materials: Recent Advances, pages 421-435. Elsevier, 1983.

[134] M.M. Shokrieh and L.B. Lessard. Progressive fatigue damage modeling of composite materials, part I: Modeling. Journal of composite materials, 34(13):1056-1080, 2000.

[135] H.W. Bergmann and R. Prinz. Fatigue life estimation of graphite/epoxy laminates under consideration of delamination growth. International Journal for Numerical Methods in Engineering, 27(2):323-341, 1989.

[136] M. Kenane and M.L. Benzeggagh. Mixed-mode delamination fracture toughness of unidirectional glass/epoxy composites under fatigue loading. Composites Science and Technology, 57(5):597-605, 1997.

[137] T.W. Coats and C.E. Harris. Experimental verification of a progressive damage model for im7/5260 laminates subjected to tension-tension fatigue. Journal of Composite Materials, 29(3):280-305, 1995.

[138] S.M. Spearing, P.W.R. Beaumont, and M.F. Ashby. Fatigue damage mechanics of composite materials. ii: A damage growth model. Composites Science and Technology, 44(2):169-177, 1992.

[139] P. Ladeveze and E. LeDantec. Damage modelling of the elementary ply for laminated composites. Composites science and technology, 43(3):257-267, 1992.

[140] K.I. Tserpes, P. Papanikos, G. Labeas, and S.P. Pantelakis. Fatigue damage accumulation and residual strength assessment of cfrp laminates. Composite structures, $63(2): 219-230,2004$.

[141] H.T. Hahn. Fatigue behavior and life prediction of composite laminates. In Composite materials: testing and design (Fifth Conference). ASTM International, 1979.

[142] T. Adam, R.F. Dickson, C.J. Jones, H. Reiter, and B. Harris. A power law fatigue damage model for fibre-reinforced plastic laminates. Proceedings of the Institution of Mechanical Engineers, Part C: Journal of Mechanical Engineering Science, 200(3):155-166, 1986. 
[143] M.M. Shokrieh and L.B. Lessard. Progressive fatigue damage modeling of composite materials, part ii: Material characterization and model verification. Journal of Composite materials, 34(13):1081-1116, 2000.

[144] K.L. Reifsnider and W.W. Stinchcomb. A critical-element model of the residual strength and life of fatigue-loaded composite coupons. In Composite Materials: Fatigue and Fracture. ASTM International, 1986.

[145] S.B. Batdorf. Tensile strength of unidirectionally reinforced compositesâĂ ̌̌i. Journal of reinforced plastics and composites, 1(2):153-164, 1982.

[146] D.G. Harlow and S. L.H Phoenix. Probability distributions for the strength of fibrous materials under local load sharing i: two-level failure and edge effects. $A d-$ vances in Applied Probability, 14(1):68-94, 1982.

[147] G. J. Dvorak, N. Laws, and M. Hejazi. Analysis of progressive matrix cracking in composite laminates i. thermoelastic properties of a ply with cracks. Journal of Composite Materials, 19(3):216-234, 1985.

[148] J. Zhang, J.and Fan and C. Soutis. Analysis of multiple matrix cracking in $\left[ \pm \theta / 90_{n}\right]_{s}$ composite laminates, Part I, inplane stiffness properties. Composites, 23(5):291304, 1992.

[149] Z. Hashin. Analysis of cracked laminates: a variational approach. Mechanics of Materials, 4(121):121-136, 1985.

[150] L. N. McCartney. Energy-based prediction of progressive ply cracking and strength of general symmetric laminates using an homogenisation method. Composites Part A: Applied Science and Manufacturing, 36(2):119-128, 2005.

[151] L. N. McCartney. Energy-based prediction of failure in general symmetric laminates. Engineering Fracture Mechanics, 72:909-930, 2005.

[152] E. J. Barbero and L. DeVivo. Constitutive model for elastic damage in fiberreinforced pmc laminae. International Journal of Damage Mechanics, 10(1):73-93, 2001.

[153] K.L. Reifsnider. The critical element model: a modeling philosophy. Engineering Fracture Mechanics, 25(5-6):739-749, 1986.

[154] P. Camanho and C. Davila. Mixed-mode decohesion finite elements for the simulation of delamination in composite materials. NASA/TM, pages 1-37, 2002. 
[155] Simulia. Abaqus Theory Manual, http://abaqus.software.polimi.it/v6.14/index.html.

[156] E.J. Barbero, J. Cabrera Barbero, and C. Navarro Ugena. Analytical solution for plane stress/strain deformation of laminates with matrix cracks. Composite Structures, 132:621-632, 2015.

[157] J. Varna, R. Joffe, N. Akshantala, and R. Talreja. Damage in composite laminates with off-axis plies. Composites Science and Technology, 59:2139-2147, 1999.

[158] J. A. Nairn. The strain energy release rate of composite microcracking: A variational approach. Journal of Composite Materials, 23:1106-1129, 1989.

[159] P. Lonetti, R. Zinno, F. Greco, and E. J. Barbero. Interlaminar damage model for polymer matrix composites. Journal of Composite Materials, 37(16):1485-1504, 2003.

[160] E. J. Barbero, F. Greco, and P. Lonetti. Continuum damage-healing mechanics with application to self-healing composites. International Journal of Damage Mechanics, $14(1): 51-81,2005$.

[161] R. J. Nuismer and S. C. Tan. Constitutive relations of a cracked composite lamina. Journal of Composite Materials, 22:306-321, 1988.

[162] S. C. Tan and R. J. Nuismer. A theory for progressive matrix cracking in composite laminates. Journal of Composite Materials, 23:1029-1047, 1989.

[163] S. Liu and J. A. Nairn. Formation and propagation of matrix microcracks in crossply laminates. J Reinf Plas Compos, pages 158-178, 1992.

[164] E. Adolfsson and P. Gudmundson. Matrix crack initiation and progression in compositelaminatessubjected to bending and extension. International journal of Solids and Structures, 36(21):3131-3169, 1999.

[165] S.R. Kim and J.A. Nairn. Fracture mechanics analysis of coating/substrate systems: Part II: Experiments in bending. Engineering fracture mechanics, 65(5):595-607, 2000.

[166] J.A. Nairn. Finite fracture mechanics of matrix microcracking in composites. $A p$ plication of fracture mechanics to polymers, Adhesives and composites, Elsevier, pages 207-212, 2004.

[167] J-W. Lee, D.H. Allen, and C.E. Harris. Internal state variable approach for predicting stiffness reductions in fibrous laminated composites with matrix cracks. Journal of Composite Materials, 23(12):1273-1291, 1989. 
[168] Z. Gao. A cumulative damage model for fatigue life of composite laminates. Journal of reinforced plastics and composites, 13(2):128-141, 1994.

[169] A.W. Wharmby, F. Ellyin, and J.D. Wolodko. Observations on damage development in fibre reinforced polymer laminates under cyclic loading. International journal of fatigue, 25(5):437-446, 2003.

[170] N. Takeda and S. Ogihara. Initiation and growth of delamination from the tips of transverse cracks in cfrp cross-ply laminates. Composites science and technology, 52(3):309-318, 1994.

[171] C. Henaff-Gardin, M.C. Lafarie-Frenot, and D. Gamby. Doubly periodic matrix cracking in composite laminates part 1: general in-plane loading. Composite structures, 36(1-2):113-130, 1996.

[172] X. Feng, M.D. Gilchrist, A.J. Kinloch, and F.L. Matthews. Development of a method for predicting the fatigue life of cfrp components. In Int Conf on Fatigue of Composites. Proc, pages 3-5, 1997.

[173] C. Henaff-Gardin, M.C. Lafarie-Frenot, and I. Goupillaud. Prediction of cracking evolution under uniaxial fatigue loading in cross-ply composite laminates. In Int Conf on Fatigue of Composites: Proc, pages 3-5, 1997.

[174] S.L. Ogin, P.A. Smith, and P.W.R. Beaumont. Matrix cracking and stiffness reduction during the fatigue of a (0/90)s GFRP laminate. Composites Science and Technology, 22(1):23-31, 1985.

[175] J. Bartley-Cho, Seung Lim, H. Thomas Hahn, and Peter Shyprykevich. Damage accumulation in quasi-isotropic graphite/epoxy laminates under constant-amplitude fatigue and block loading. Composites Science and Technology, 58(9):1535-1547, 1998.

[176] N. Blanco, E.K. Gamstedt, L.E. Asp, and J. Costa. Mixed-mode delamination growth in carbon-fibre composite laminates under cyclic loading. International journal of solids and structures, 41(15):4219-4235, 2004.

[177] Leif E. Asp, Anders Sjögren, and Emile S. Greenhalgh. Delamination growth and thresholds in a carbon/epoxy composite under fatigue loading. Journal of Composites, Technology and Research, 23(2):55-68, 2001.

[178] G.S. Dahlen, C.and Springer. Delamination growth in composites under cyclic loads. Journal of Composite Materials, 28(8):732-781, 1994. 
[179] S.and Fujita Y.and Kawada H. Hosoi, A.and Sakuma. Prediction of initiation of transverse cracks in cross-ply cfrp laminates under fatigue loading by fatigue properties of unidirectional cfrp in 90Âř direction. Composites Part A: Applied Science and Manufacturing, 68:398-405, 2015.

[180] B. Mohammadi, B. Fazlali, and D. Salimi-Majd. Development of a continuum damage model for fatigue life prediction of laminated composites. Composites Part A: Applied Science and Manufacturing, 93:163-176, 2017.

[181] R.M. Christensen. A physically based cumulative damage formalism. In Major Accomplishments in Composite Materials and Sandwich Structures, pages 51-65. Springer, 2009.

[182] M.J. Owen and R.J. Howe. The accumulation of damage in a glass-reinforced plastic under tensile and fatigue loading. Journal of Physics D: Applied Physics, 5(9):1637, 1972.

[183] R.Y. Kim, A.S. Crasto, and G.A. Schoeppner. Dimensional stability of composite in a space thermal environment. Science and Technology, 60(12):2601-2608, 2000.

[184] V.T. Bechel, J.D. Camping, and R.Y. Kim. Cryogenic/elevated temperature cycling induced leakage paths in pmcs. Composites Part B: Engineering, 36(2):171-182, 2005.

[185] H.L. McManus and C. Park. Thermally induced damage in composite laminates: predictive methodology and experimental inverstigation. Composites Science and Technology, 56:1209-1219, 1996.

[186] H.L. McManus. Prediction of thermal cycling induced matrix cracking. Journal of Reinforced Plastics and Composites, 15:124-140, 1996.

[187] J. Varna. Modelling mechanical performance of damaged laminates. Journal of composite materials, 47(20-21):2443-2474, 2013.

[188] H.L. McManus. Prediction of thermal cycling induced cracking in polymer matrix composites. Technical Report NAG-1-1493, NASA Langley Research Center, Hampton, VA, United States.

[189] S.S Tompkins, J.Y. Shen, and A.J. Lavoie. Thermal cycling of thin and thick ply composites. 1(15:111552):326-335, Jan 011994. 
[190] G. Lubineau, P. Ladevèze, and D. Violeau. Durability of cfrp laminates under thermomechanical loading: A micro-meso damage model. Composites Science and Technology, 66(7-8):983-992, 2006.

[191] H. Zrida, P. Fernberg, Z. Ayadi, and J. Varna. Microcracking in thermally cycled and aged carbon fibre/polyimide laminates. International Journal of Fatigue, 94:121-130, 2017.

[192] Ever J. Barbero and Javier Cabrera Barbero. Analytical solution for bending of laminated composites with matrix cracks. Composite Structures, 135:140-155, 2016.

[193] Ever J. Barbero. Finite Element Analysis of Composite Materials Using Abaqus. CRC Press, first edition.

[194] Ever J. Barbero. Finite Element Analysis of Composite Materials Using ANSYS. CRC Press, second edition.

[195] J.A. Nairn. On the use of shear-lag methods for analysis of stress transfer in unidirectional composites. Journal of Mechanics of Materials, 26:63-80, 1977.

[196] E. Kreyszig. Advanced Engineering Mathematics. Wiley, 10th edition, 2011.

[197] J. Rebiere and D. Gamby. A decomposition of the strain energy release rate associated with the initiation of transverse cracking, longitudinal cracking and delamination in cross-ply laminates. Composite Structures, 84:186-197, 2008.

[198] H. Tada, P.C. Paris, and G. Irwin. The stress analysis of cracks handbook. ASME Press, 2000.

[199] H.T. Hahn. A mixed-mode fracture criterion for composite materials. Composites Technology, 5:26-29, 1983.

[200] Ever J. Barbero. Introduction to Composite Materials Design. CRC Press, 3rd edition, 2018.

[201] M. M. Moure, S. Sanchez-Saez, E. Barbero, and E. J. Barbero. Analysis of damage localization in composite laminates using a discrete damage model. Composites Part B, 66:224-232, 2014.

[202] E. J. Barbero, F. A. Cosso, R. Roman, and T. L. Weadon. Determination of material parameters for Abaqus progressive damage analysis of E-Glass Epoxy laminates. Composites Part B:Engineering, 46:211-220, 2013. 
[203] E.J. Barbero, G. Sgambitterra, A. Adumitroaie, and X. Martinez. A discrete constitutive model for transverse and shear damage of symmetric laminates with arbitrary stacking sequence. Composite Structures, 93(2):1021 - 1030, 2011.

[204] G. Sgambitterra, A. Adumitroaie, E.J. Barbero, and A. Tessler. A robust threenode shell element for laminated composites with matrix damage. Composites Part B: Engineering, 42(1):41 - 50, 2011.

[205] M. Ghayour, H. Hosseini-Toudeshky, M. Jalalvand, and E.J. Barbero. Micro/macro approach for prediction of matrix cracking evolution in laminated composites. Journal of Composite Materials, 50(19):2647-2659, 2016.

[206] M.M. Moure, F. Otero, S.K. Garcia-Castillo, S. Sanchez-Saez, E. Barbero, and E.J. Barbero. Damage evolution in open-hole laminated composite plates subjected to in-plane loads. Composite Structures, 133:1048 - 1057, 2015.

[207] M.M. Moure, S.K. Garcia-Castillo, S. Sanchez-Saez, E. Barbero, and E.J. Barbero. Influence of ply cluster thickness and location on matrix cracking evolution in openhole composite laminates. Composites Part B: Engineering, 95:40 - 47, 2016.

[208] M.M. Moure, S.K. Garcia-Castillo, S. Sanchez-Saez, E. Barbero, and E.J. Barbero. Matrix cracking evolution in open-hole laminates subjected to thermo-mechanical loads. Composite Structures, 183(-):510-520, 2018.

[209] E.J. Barbero and M. Shahbazi. Determination of material properties for ANSYS progressive damage analysis of laminated composites. Composite Structures, 176:768-779, 2017.

[210] E. M. Silverman. Space environmental effects on spacecraft: Leo materials selection guide. Technical Report NSA1-19291, NASA Langley Research Center, Hampton, VA, United States, August 1995.

[211] E.G. Wolff. Stiffness-thermal expansions relationships in high modulus carbon fibers. Journal of Composite Materials, 21:81-97, 1987.

[212] D.E. Bowles. Micromechanics analysis of space simulated thermal deformations and stresses in continuous fiber reinforced composites. Technical Report 90N21140, NASA, 1990.

[213] D.F. Adams. Finite element micromechanical analysis of a unidirectional composite including longitudinal shear loading. Journal of Computers and Structures, 18(6):1153-1165, 1984. 
[214] T.R. King, D.M. Blackketter, D.E. Walrath, and D.F. Adams. Micromechanics prediction of the shear strength of carbon fiber/epoxy matrix composites: The influence of the matrix and interface strengths. Journal of Composite Materials, 26(4):558-573, 1992.

[215] D.F. Adams. A micromechanics analysis of the influence of the interface on the performance of polymer-matrix composites. Journal of Reinforced Plastics and Composites, 6(1):66-88, 1987.

[216] Choi Sukjoo and B.V. Sankar. Micromechanical analysis of composite laminates at cryogenic temperature. Journal of Composite Materials, 0:1-15, 2005.

[217] Y.Y. Ran Zhiguo, Q.Z. Li Jianfeng, and L. Yang. Determination of thermal expansion coefficients for unidirectional fiber-reinforced composites. Chinese Journal of Aeronautics, 27(5):1180-1187, 2014.

[218] X. Song, Y. Chen, S. Chen, E. J. Barbero, E. L. Thomas, and P. Barnes. Significant enhancement of electrical transport properties of thermoelectric ca3co4o $+\mathrm{d}$ through yb doping,. Solid State Comm., 152(16):1509-1512, 2012.

[219] X. Song, D. McIntyre, X. Chen, E.J. Barbero, and Y. Chen. Phase evolution and thermoelectric performance of calcium cobaltite upon high temperature aging. Ceramics International, 41(9, Part A):11069-11074, 2015.

[220] S.M. Milkovich M.W. Hyer, C.T. Herakovich and J.S. Short. Temperature dependence of mechanical and thermal expansion properties of T300/5208 graphite/epoxy. Composites, 14(3):276-280, 1983.

[221] S.M. Milkovich and C.T. Herakovich. Temperature dependence of elastic and strength properties of T300/5208 graphite-epoxy. Technical Report NASA-CR$173616,1984$.

[222] D.E. Bowles. Effect of microcracks on the thermal expansion of composite laminates. Journal of composite materials, 18(2):173-187, 1984.

[223] D.F. Adams, R.S. Zimmerman, and E.M. Odom. Polymer matrix and graphite fiber interface study. Technical Report NASA-CR-165632, NASA, 1985.

[224] R.R. Johnson, M.H. Kural, and G.B. Mackey. Thermal expansion properties of composite materials. Technical Report NASA-CR-177357, NASA, 1981. 
[225] D.S. Cairns and D.F. Adams. Moisture and thermal expansion properties of unidirectional composite materials and the epoxy matrix. Journal of Reinforced Plastics and Composites, 2(4):239-255, 1983.

[226] M.J. Michno. High modulus composite properties. In AIAA Space Systems Technology Conference, AIAA-1187, pages 126-131, 1986.

[227] S.M. Milkovich, C.T. Herakovich, and G.F. Sykes. Space radiation effects on graphite-epoxy composite materials. Technical Report NASA Report, TM 85478, NASA Langley Research Center, Virginia Polythechnic Institute and State University, 1984.

[228] G. Yaniv, I.M. Daniel, S. Cokeing, and G.M. Martinez. Temperature effects on high strain rate properties of graphite/epoxy composites. Technical Report NASACR-189082, NASA, 1991.

[229] E.J. Barbero and R. Luciano. Micromechanical formulas for the relaxation tensor of linear viscoelastic composites with transversely isotropic fibers. International Journal of Solids and Structures, 32(13):1859 - 72, 1995/07/.

[230] R. Luciano and E.J. Barbero. Formulas for the stiffness of composites with periodic microstructure. International Journal of Solids and Structures, 31(21):2933 - 44, 1994.

[231] R. Luciano and E.J. Barbero. Analytical expressions for the relaxation moduli of linear viscoelastic composites with periodic microstructure. Transactions of the ASME. Journal of Applied Mechanics, 62(3):786 - 93, 1995.

[232] Computer Aided Design Environment for Composites (CADEC). http://en.cadeconline.com.

[233] J.W. Weeton, D.M. Peters, and K.L. Thomas. Engineers' Guide to Composite Materials. American Society for Metals, 1987.

[234] J.R. Strife and K.M. Prewo. Thermal expansion behavior of unidirectional and bidirectional kevlar/epoxy composites. Journal of Composite Materials, 13:264277, October, 1979.

[235] V.M. Levin. Determining the elasticity and thermoelasticity constants of composite materials. Izvestiya Akademii Nauk SSSR, Mekhanika Tverdogo Tela, (6):137 - 45, 1976. 
[236] B.W. Rosen and Z. Hashin. Effective thermal expansion coefficients and specific heats of composite materials. International Journal of Engineering Science, 8:157$173,1984$.

[237] D.E. Bowles and S.S. Tompkins. Prediction of coefficients of thermal expansion for unidirectional composites. Journal of Composite Materials, 23(4):370 - 88, 1989.

[238] S.K. Shao-Yun Fu. Polymers at Cryogenic Temperatures. Springer, first edition edition, 2013.

[239] I. Perepechko. Low-Temperature Properties of Polymers. Elsevier Science and Technology, 1980.

[240] G. Hartwing. Polymer Properties at Room and Cryogenic Temperatures. Springer US, 1994.

[241] P.K. Dutta. Behavior of materials at cold regions temperatures. Cold regions research and engineering laboratory, US Army Corps of Engineers, 1988.

[242] J. B. Titus. Effect of low temperature $(0$ to $-65 \mathrm{~F})$ on the properties of plastic. Technical report, Plastics Technical Evolution Center, 1967.

[243] M.M. Pavlick, W.S. Johnson, Brian Jensen, and Erik Weiser. Evaluation of mechanical properties of advanced polymers for composite cryotank applications. Composites: Part A, 2009.

[244] G. Hartwing. Fibre-epoxy composites at low temperature. Cryogenics, 24(11):639647, 1984.

[245] U. Saburo, H. Ejima, T. Suzuki, and K. Asano. Cryogenic small-flaw strength and creep deformation of epoxy resins. Cryogenics, 39:729-738, 1999.

[246] B. Kneifel. Nonmpolymer materials and composites at low temperatures, volume Fracture properties of Epoxi resins at low temperatures of 123-129. 1979.

[247] V.B. Gupta, L.T. Drzal, and C. Lee. The temperature dependence of some mechanical properties of a cured epoxy resin suystem. Polymer engineering and science, 25(13):812-823, 1985.

[248] Z.Bo, L. Jing-wei, L. Shu-guang, L. Yi-hui, and H. Ju-tao. Relationship between fracture toughness and temperature in epoxy coatings. Polimery, 60(4):258-263, 2015. 
[249] M.B. Kase. Mechanical and thermal properties of filamentary-reinforced structural composites at cryogenic temperatures. Cryogenics, 15:327-249, 1975.

[250] 1987 Thornel Carbon Fiber Data Sheets, Amoco. Amoco, 1987.

[251] 2012 Thornel Carbon Fiber Data Sheets, Cytec. Cytec, 2012.

[252] Hexcel Hercules Carbon Fiber Data Sheets. Hextow , inc., 1984.

[253] S.S. Tompkins and J.G. Funk. Sensitivity of the coefficient of thermal expansion of selected graphite reinforced composite laminates to lamina thermoelastic properties. S.A.M.P.E. quarterly, 23(3):55 - 61, 1992.

[254] MathWorks. Matlab tolerances and stopping criteria. http://docs. scipy. org/ doc/scipy-0.17. O/ reference/ generated/scipy. optimize. fmin. html .

[255] A.S. Crasto and R.Y. Kim. On the determination of residual stresses in fiberreinforced thermoset composites. Journal of Reinforced Plastics and Composites, 12(5):545-558, 1993.

[256] C.T. Sun and K.J. Yoon. Mechanical properties of graphite/epoxy composites at various temperatures. Technical Report HTMIAC 9, US DoD, 1988.

[257] J.M. Augl. Prediction and verification of moisture effects on carbon fiber-epoxy composites. Technical report, DTIC ADA079557, 1979.

[258] J.M. Augl. Moisture effects on the mechanical properties of hercules 3501-6 epoxy resin. Technical report, DTIC ADA078616, 1979.

[259] J.F. Derek, C.T. Herakovich, and G.F. Sykes. Space enviromental effects on graphite-epoxy composite properties and epoxy tensile properties. Technical Report NASA Report, TM 89297, NASA Langley Research Center, Virginia Polythechnic Institute and State University, 1987.

[260] MatWeb. Material property data. http://www. matweb. com/ search/ DataSheet. aspx, 2013.

[261] S.W. Tsai. Composite Design. Think Composites, 3rd edition, 1987.

[262] S. Kawabata. Measurement of the transverse mechanical properties of highperformance fibres. Journal of the Textile Institute, 81(4):432-447, 1990.

[263] D. Belitskus. Fiber and Whisker Reinforced Ceramics for Structural Applications. CRC Press, 1993. 
[264] Y Huang and RJ Young. Effect of fibre microstructure upon the modulus of pan-and pitch-based carbon fibres. Carbon, 33(2):97-107, 1995.

[265] L.A. Pilato and M.J. Michno. Advanced Composite Materials. Springer Science and Business Media, 1994.

[266] N.P. Bansal and J. Lamon. Ceramic Matrix Composites: Materials, Modeling and Technology. John Wiley and Sons, 2014.

[267] P. Arsenovic. Nonlinear elastic characterization of carbon fibers. Fiber, Matrix, and Interface Properties, ASTM., pages 2-8, 1996.

[268] B. Christopher and J. Zakrzewski. Moisture absorption and mechanical properties for high modulus pitch 75 graphite fiber/modified cyanate ester resin laminates. Journal of Design and Optical Instruments, 1690, 1992.

[269] V.M. Levin. Thermal expansion coefficients of heterogeneous materials. Mechanics of Solids, 2:58-61, 1967.

[270] J. F. Helmer and R. J. Diefendorf. Transverse thermal expansion of carbon fiber/epoxy matrix composites. In Proceedings of 5th International Symposium on Composite Metallic Materials, pages 15-20, 1983.

[271] K. Y. Donaldson and D. P. H. Hasselman H. D. Bhatt. Role of interfacial gaseous heat transfer and percolation in the effective thermal conductivity of two uniaxial carbon-fiber-reinforced glass matrix composites. In Proceedings of 17th Annual Conference on Composites and Advanced Ceramic Materials, pages 335-340, 1993.

[272] D.F. Adams. Laminate analyses, micromechanical creep response, and fatigue behavior of polymer matrix composite materials. Technical report, 1982.

[273] E. Orowan. Energy criteria of fracture. Technical Report N5ori-07870, MIT, Cambridge, MA, 1954.

[274] G.R. Irwin. Analysis of stresses and strains near the end of a crack traversing a plate. Journal of applied mechanics, 24(3):361-364, 1957.

[275] C.G. Davila, C.A. Rose, and E.V. Iarve. Mathematical Methods and Models in Composites, volume 5, chapter Modeling fracture and complex crack networks in laminated composites, pages 297 - 347. World Scientific, 2014.

[276] A. Adumitroaie and E.J. Barbero. Intralaminar damage model for laminates subjected to membrane and flexural deformations. Mechanics of Advanced Materials and Structures, 22(9):705 - 716, 2015. 
[277] E. Tschegg, K. Humer, and H. W. Weber. Mechanical properties and fracture behaviour of polyimide (sintimid) at cryogenic temperatures. Cryogenics, 31(10):878883, 1991.

[278] K. Humer, E. K. Tschegg, and H.W. Weber. Tensile, fracture and thermal properties of polyarylates at room and cryogenic temperatures. Cryogenics, 33(7):686-691, 1993.

[279] A. J. Kinlonch. Fracture Behaviour of polymers. Applied Sciences Publishers, 1983.

[280] U. Saburo, H. Ejima, T. Suzuki, and K. Asano. Cryogenic small-flaw strength and creep deformation of epoxy resins. Cryogenics, 39:729-738, 1999.

[281] F. Sawa. Evaluation of fracture toughness of epoxy resin for cryogenic use. Journal of Cryogenics and Superconductivity Society of Japan, 30:129-134, 1999.

[282] Hartwing. Fracture properties of polymers and composites at cryogenic temperatures. Advances in cryogenic engineering, 1986.

[283] G. Hartwing. Low-temperature properties of resins and their correlations. Advances in cryogenic engineering, 22:283-290, 1977.

[284] L.E. Evseeva and S.A. Tanaeva. Thermophysical properties of epoxy composite materials at low temperatures. Cryogenics, 35:277-279, 1995.

[285] H.L. McManus. The effect of long-term thermal cycling on the microcracking behavior and dimensional stability of composite materials. Journal of Reinforced Plastics and Composites, 15:124-140, 1996.

[286] N.V. Bhat and P.A. Lagace. An analytical method for the evaluation of interlaminar stresses due to material discontinuities. Journal of Composite Materials, 28(3):190$210,1994$.

[287] SR. Soni. Elastic properties of T300/5208 bidirectional symmetric laminates. Technical Report ADA093227, Air Force, Defense Technical Information Center, 1980.

[288] A. Adumitroiaie and E.J. Barbero. Intralaminar damage model for laminates subjected to membrane and flexural deformations. Mechanics of Advanced Materials and Structures, 2013.

[289] C. Henaff-Gardin, J. Desmeuzes, and D. Gaillot. Damage development due to cyclic thermal loading in cross-ply carbon/epoxy laminates. Fatigue under thermal and mechanical loading, 1:285-293, 1996. 
[290] EJ Barbero. Introduction to Composite Materials Design. CRC Press, second edition edition, 2011.

[291] D.C. Montgomery, E.A. Peck, and G.G. Vining. Introduction to linear regression analysis, volume 821. John Wiley \& Sons, first edition edition, 2012.

[292] MathWorks. Matlab diagnostic of simple linear regression. https: //www. mathworks. com/help/stats/ summary-of-output-and-diagnostic-statistics. html.

[293] R.W. Hertzberg. Deformation and fracture mechanics of engineering materials. Wiley, fouth edition edition, 1989.

[294] S.S. Sternstein. Yielding in glassy polymers. Polymeric materials, pages 369-410, 1975.

[295] H.H. Kausch. Polymer fracture, polymers, properties and applications, 1978.

[296] C. Henaff-Gardin and M.C. Lafarie-Frenot. The use of a characteristic damage variable in the study of transverse cracking development under fatigue loading in cross-ply laminates. International Journal of Fatigue, 24(2-4):389-395, 2002.

[297] R. Talreja. Damage characterization by internal variables. In R Talreja and J A E Manson, editors, Damage Mechanics of Composite Materials, pages 53-78. Elsevier Science, Amsterdam, 1994.

[298] N. Pugno, M. Ciavarella, P. Cornetti, and A. Carpinteri. A generalized parisâĂŹ law for fatigue crack growth. Journal of the Mechanics and Physics of Solids, 54(7):1333-1349, 2006.

[299] S. Kobayashi, K. Terada, S. Ogihara, and N. Takeda. Damage-mechanics analysis of matrix cracking in cross-ply CFRP laminates under thermal fatigue. Composites Science and Technology, 61(12):1735-1742, 2001.

[300] M. Quaresimin, P.A. Carraro, L.P. Mikkelsen, N. Lucato, L. Vivian, P. Brøndsted, B.F. Sørensen, J. Varna, and R. Talreja. Damage evolution under cyclic multiaxial stress state: Aa comparative analysis between glass/epoxy laminates and tubes. Composites Part B: Engineering, 61:282-290, 2014.

[301] J. Nairn. Matrix microcracking in composites. In R Talreja and J A E Manson, editors, Polymer Matrix Composites, volume 2 of Comprehensive Composite Materials, pages 403-432. Elsevier, Amsterdam, 2000. 
[302] E.J. Barbero, J. Cabrera Barbero, and U. Carlos Navarro. Analytical solution for plane stress/strain deformation of laminates with matrix cracks. Composite Structures, 132:621-632, 2015.

[303] C.T. Herakovich and M.W. Hyer. Damage-induced property changes in composites subjected to cyclic thermal loading. Engineering Fracture Mechanics, 25(5-6):779791, 1986.

[304] Nelder-Mead method. http://docs.scipy.org/doc/scipy0.17.0/reference/generated/scipy.optimize.fmin.html.

[305] M.M. Moure, S.K. García-Castillo, S. Sanchez-Saez, E. Barbero, and E.J. Barbero. Matrix cracking evolution in open-hole laminates subjected to thermo-mechanical loads. Composite Structures, 183:510-520, 2018.

[306] Python. Xlsxwriter module documentation. https://pypi.python. org/pypi/ XlsxWriter. 